\title{
Atlas of the Spectrum of a Platinum/Neon Hollow-Cathode Reference Lamp in the Region 1130-4330 A
}

Jean E. Sansonetti, Joseph Reader, Craig J. Sansonetti, and Nicolo Acquista

National Institute of Standards and Technology, Gaithersburg, MD 20899, USA
The spectrum of a platinum hollowcathode lamp containing neon carrier gas was recorded photographically and photoelectrically with a $10.7 \mathrm{~m}$ normalincidence vacuum spectrograph. Wavelengths and intensities were determined for about 5600 lines in the region $1130-4330 \AA$. An atlas of the spectrum is given, with the spectral lines marked and their intensities, wavelengths, and classifications listed. Lines of impurity species are also identified. The uncertainty of the photographically measured wavelengths is estimated to be $\pm 0.0020 \AA$. The uncertainty of lines measured in the photoelectric scans is
$0.01 \AA$ for wavelengths shorter than $2030 \AA$ and $0.02 \AA$ for longer wavelengths. Ritz-type wavelengths are given for many of the classified lines of $P t$ II with uncertainties varying from \pm 0.0004 to $\pm 0.0025 \AA$. The uncertainty of the relative intensities is estimated to be about $20 \%$.

Key words: hollow-cathode lamp; neon; platinum; spectral atlas; spectrum; wavelength.

Accepted: November 21, 1991

\section{Introduction}

The deployment of the Hubble Space Telescope (HST) on April 24, 1990, launched a new era in astronomy. With the HST, stars and other astronomical objects are being observed with unprecedented clarity. The improvement over ground-based telescopes is most significant in the ultraviolet region of the spectrum, where the earth's atmosphere absorbs most of the radiation. Although the muchpublicized spherical aberration in the HST's primary mirror [1] greatly reduces the quality of star images, many experiments of a spectroscopic nature are not severely affected because they do not require high spatial resolution. For example, for the Goddard High Resolution Spectrograph (GHRS), the highest resolution spectrograph on HST, the spherical aberration in the primary mirror does not degrade the spectral resolution noticeably when the small science aperture is used [2]. However, because of enlargement of the point spread function, the exposure time must be increased by a factor of about 5 to produce the signal-to-noise ratio of prelaunch expectations [2]. Nevertheless, spectra of very high quality have been obtained [2].

The region of observation of GHRS is 1100 $3200 \AA$. In its echelle mode it has a resolving power of 90,000 and a wavelength accuracy of a few parts in $10^{6}$. Line-of-sight velocities of stellar objects can thus be determined to an accuracy of about $1 \mathrm{~km} / \mathrm{s}$. In order to achieve this accuracy, of course, an accurate wavelength scale must be established. This is accomplished by illuminating the spectrograph with an onboard platinum/neon hollow-cathode lamp during periods in which stellar observations are not being made [3]. The use of a $\mathrm{Pt} / \mathrm{Ne}$ lamp for this purpose and its space-qualified design are due to Mount, Yamasaki, Fowler, and Fastie [4], who originally suggested it for wavelength calibration of the International Ultraviolet Explorer (IUE) satellite. 
To achieve the accuracy for which GHRS was designed, the calibration wavelengths must be accurate to about $0.002 \AA$. However, tests carried out in our laboratory in 1983 indicated that the best available wavelengths for $\mathrm{Pt}[5]$ had errors ranging to about $0.015 \AA$. We thus began a program to measure the spectra emitted by a $\mathrm{Pt} / \mathrm{Ne}$ hollowcathode lamp similar to the one to be used with GHRS. This work was carried out with our high resolution $10.7 \mathrm{~m}$ normal-incidence vacuum spectrograph at NIST. At about the same time Engleman [6] recorded the spectrum of a $\mathrm{Pt}$ hollow-cathode lamp with a Fourier-transform spectrometer. $\mathrm{He}$ obtained accurate wavelengths for 320 lines of $\mathrm{Pt} \mathrm{I}$ in the region 2200-7220 $\AA$, optimized the energy level values, and calculated accurate Ritz-type wavelengths for 81 lines in the region 1724-2250 $\AA$. Many of these lines were used in calibrating our grating measurements.

Some of the results of our work have appeared in two previous papers. In the first [7] we determined accurate values for 100 energy levels of Pt II by combining our new grating measurements for over $500 \mathrm{Pt}$ II lines in the ultraviolet with measurements of lines at longer wavelengths made by Engleman by Fourier transform spectroscopy. In the second [8] we reported wavelengths with accuracies of $0.002 \AA$ or better for some 3000 lines emitted by a $\mathrm{Pt} / \mathrm{Ne}$ lamp in the region 1032 $4100 \AA$. In this second report we also provided relative intensities of the spectral lines of the $\mathrm{Pt} / \mathrm{Ne}$ lamp that were determined by recording the spectra photoelectrically with the same spectrograph used for the wavelength measurements.

Our wavelengths for the $\mathrm{Pt} / \mathrm{Ne}$ lamp are currently being used for calibration of GHRS as well as for wavelength calibration of the Faint Object Spectrograph on HST, which uses a $\mathrm{Pt}-\mathrm{Cr} / \mathrm{Ne}$ hollow-cathode lamp for both wavelength and radiometric calibration [9]. Our data are also being used for revised calibrations of spectra from the IUE satellite [10], and for calibration of spectra obtained with sounding rockets, which also use onboard $\mathrm{Pt} / \mathrm{Ne}$ hollow cathode lamps [11]. In a different type of application, the data are being used to interpret the spectra of stars that contain $\mathrm{Pt}$ in anomalously high abundances [12].

In the present paper we present a comprehensive report of our observations of the $\mathrm{Pt} / \mathrm{Ne}$ hollowcathode lamp. For completeness we give a full account of the experimental work and data analysis. Some of this information has been given in our previous papers.
Our results are presented in the form of an atlas of the spectrum emitted by a $\mathrm{Pt} / \mathrm{Ne}$ hollow-cathode lamp in the region $1130-4330 \AA$. The atlas consists of plots of the spectrum accompanied by tables that include the wavelengths, wave numbers, intensities, and identifications or classifications where known for more than 5600 lines. We have attempted to provide the best available wavelength data, substituting values from the literature or calculated Ritz-type wavelengths where these are more accurate than our measurements.

The line list developed in this work was communicated to J. Blaise and J.-F. Wyart of the Laboratoire Aimé Cotton, Orsay, France, who have used it to substantially extend the energy level analysis of Pt II. Based on our measurements they have located nearly 150 new Pt II levels. Their report on the analysis appears as a companion paper in the same issue of this journal [13]. Blaise and Wyart have also located about 100 new levels of $\mathrm{Pt}$. The new line identifications for $\mathrm{Pt} I$ and II have been provided to us and are incorporated in the atlas.

The data included in this atlas should be of use not only for astronomical spectroscopy but also for the calibration of general laboratory spectra obtained with medium to high resolution diffraction grating spectrographs. No other source provides such a dense and complete coverage of this spectral region with lines suitable for use as reference wavelengths. The $\mathrm{Pt} / \mathrm{Ne}$ hollow cathode is easy to operate and is commercially available at moderate cost.

\section{Photographic Observations}

Our observations were made with the $10.7 \mathrm{~m}$ normal-incidence vacuum spectrograph at the National Institute of Standards and Technology. Two different gratings were used, the first blazed at $1200 \AA$ in first order and the second blazed at $3000 \AA$ in first order. Both gratings were ruled with 1200 lines $/ \mathrm{mm}$. All measurements were made in the first order, the plate factor being $0.78 \AA / \mathrm{mm}$. The slit width was $0.023 \mathrm{~mm}$. With this slit width the resolving limit throughout the region of observation was about $0.020 \AA$. Photographic exposures were made on Kodak SWR plates. ${ }^{1}$

\footnotetext{
${ }^{1}$ Certain commercial equipment, instruments, or materials are identified in this paper to specify adequately the experimental procedure. Such identification does not imply recommendation or endorsement by the National Institute of Standards and Technology, nor does it imply that the materials or equipment identified are necessarily the best available for the purpose.
} 
Two different light sources were used. The first was a windowless, demountable hollow-cathode lamp having a solid copper cathode containing a helical platinum wire and some chips of silicon and germanium. The general design of the lamp was similar to that of Reader and Davis [14]. In the version used in the present work the O-ring assembly at the front of the lamp was replaced by a large ball joint by which the lamp could be connected directly to the spectrograph. The lamp was operated in series with a $300 \Omega$ ballast resistor at a dc voltage of $250 \mathrm{~V}$ and a current of $90 \mathrm{~mA}$. The cathode was cooled with flowing water. The carrier gas consisted of flowing helium with a trace of neon at a total pressure of approximately $266 \mathrm{~Pa} \mathrm{(2}$ Torr). With this gas mixture the spectra of both $\mathrm{Cu}$ and $\mathrm{Pt}$ could be excited simultaneously. This could not be accomplished when only a single gas was used. Exposure times for this lamp were about 15 $\min$.

The second source was a sealed hollow-cathode lamp similar to the one used by GHRS. It has a platinum hollow cathode with neon carrier gas and is sealed with a magnesium fluoride window. The lamp was manufactured by the Westinghouse Corporation (Model WL34045). It was connected to the spectrograph by a quick-disconnect flange. The cathode was located $215 \mathrm{~mm}$ from the slit. The lamp was operated with a $5000 \Omega$ ballast resistor at a dc voltage of $310 \mathrm{~V}$ and a current of $20 \mathrm{~mA}$. Exposure times ranged from 2 to $150 \mathrm{~min}$.

In the first phase of the wavelength reductions of the photographic data, the spectra of Pt observed with the demountable $\mathrm{Pt}-\mathrm{Cu}$ lamp were measured with respect to lines of $\mathrm{Cu}$ II, Si I, Si II, Ge I, Ge II, $\mathrm{Ne}$, and $\mathrm{Ne}$ II to determine accurate wavelengths for a select group of $\mathrm{Pt}$ lines. Wavelengths for $\mathrm{Cu}$ II were Ritz values derived from the level values of Ross [15]. Wavelengths for most Ne I and II lines above $2780 \AA$ were taken from the Fourier-transform measurements of Palmer and Engleman [16]. Wavelengths for other Ne II lines above $2780 \AA$ and all Ne II lines below this wavelength were Ritz values given by Persson [17]. $\mathrm{Ne} \mathrm{I}, \mathrm{Si}$, and $\mathrm{Ge}$ wavelengths were taken from the compilation of reference wavelengths by Kaufman and Edlén [18]. The measurements made with the demountable Pt$\mathrm{Cu}$ lamp provided accurate values for about 1500 lines of $\mathrm{Pt} I$ and II extending from 1032 to $2885 \AA$.

In the second phase of the reductions the spectra of all lines observed with the sealed $\mathrm{Pt} / \mathrm{Ne}$ lamp were measured with respect to the above group of $\mathrm{Pt}$ lines, lines of $\mathrm{Ne} I$ and II, and lines of $\mathrm{Pt} I$ reported by Engleman [6]. In the region above
$2885 \AA$, our reference spectra consisted solely of lines of $\mathrm{Ne}$ I, Ne II, and Pt I with wavelengths taken from the sources cited above.

Next, our values for lines of $\mathrm{Pt}$ II with known classifications were combined with values for classified lines of Pt II measured by Engleman by means of Fourier-transform spectroscopy to determine accurate values for 28 even and 72 odd energy levels of Pt II [7]. Using these level values we calculated Ritz-type wavelengths for almost all of the classified lines of Pt II. For some of these levels the energy or $J$ value has been revised as a result of the work of Blaise and Wyart [13]. For those levels that have not been changed, the Ritz values have been substituted for the measured values in the final list of wavelengths.

\section{Photoelectric Observations}

To determine the relative intensities of lines emitted by the $\mathrm{Pt} / \mathrm{Ne}$ lamp and to observe lines weaker than those recorded on the photographic plates, we recorded the spectrum by translating an exit slit and photomultiplier tube along the focal curve of the $10.7 \mathrm{~m}$ vacuum spectrograph. The entrance and exit slit widths were $0.050 \mathrm{~mm}$. The line intensities were measured by photon counting. Signals from the photomultiplier were amplified and processed by a discriminator and logarithmic ratemeter. The analog output signal from the ratemeter was sampled at $1 \mathrm{~Hz}$ by a computer, which digitized and stored the data. This acquisition rate corresponded to a wavelength interval of $0.0086 \AA$ per sample. Prior to each scan the analog response of the ratemeter was calibrated by using a pulse generator to simulate the amplified pulse signal from the photomultiplier tube. The response of the ratemeter was digitized and recorded for pulse frequencies ranging from $10 / \mathrm{s}$ to $10^{6} / \mathrm{s}$ by decades.

The resolution limit for the scans was about $0.07 \AA$. The spectrum was scanned in overlapping $650 \AA$ segments, each segment corresponding to a different rotational setting of the grating. Each scan lasted $20 \mathrm{~h}$. Two scans were made for each region above $1685 \AA$, the first a normal scan and the second a scan at reduced sensitivity to record very intense lines that were saturated at normal recording conditions. The sensitivity was reduced by introducing a one decade offset in the logarithmic ratemeter. In addition, for the region above $2000 \AA$, the source intensity was attenuated by reflecting the lamp from an uncoated glass plate.

Four different $\mathrm{Pt} / \mathrm{Ne}$ lamps were used in the course of the experiments. Two lamps were used 
for the photographic exposures. One of these and two additional ones were used for the photoelectric scans. The longest use of any lamp was during the photoelectric scans, where one of the lamps was run for about $250 \mathrm{~h}$. After this time the cavity of the cathode had become noticeably enlarged.

The position and intensity of each spectral line in the photoelectric scans was determined by using a computer line-finding algorithm. First, the recorded signal at each point in the spectrum was converted to absolute counts/s by using the calibration information mentioned above. Then these data were scanned by the computer to locate peaks in the spectrum. The position of each peak was determined by calculating the quadratically smoothed first derivative of the data in the vicinity of the maximum intensity point and linearly interpolating the zero crossing of the derivative. The wavelength was then calculated by making a linear fit of wavelength versus position for the local spectral region, using as standards four lines accurately measured from the photographic observations on either side of the line to be determined.

The intensities derived from the raw data for each scan were adjusted to produce a consistent set of values over the whole spectral region. First, using the measured intensities for lines of moderate strength in the overlapping regions of the various scans, a set of multiplicative factors was determined to bring the separate scans onto the same relative scale. Then the spectral response of the spectrograph/detector combination as a function of wavelength was calibrated by using accurate radiance values for about 80 lines of platinum measured by Klose [19] in a similar $\mathrm{Pt} / \mathrm{Ne}$ hollowcathode lamp. All of the spectral data were corrected for this instrumental response. Thus the intensities plotted in the atlas are on a true relative scale.

The number of lines observed by photon counting was much greater than observed photographically. Whereas the weakest photographic lines produced count rates of about 500 photons/s, lines having signals as low as about 10 photons/s could be observed photoelectrically. The most intense lines produced counts of about 2,000,000 photons/ s. In all scans we observed a residual background count in excess of the photomultiplier dark count. This background was only a few counts/s at low wavelengths but increased to about 60 counts/s at the highest wavelengths. This increasing background is apparent in the atlas plots. The background count has been subtracted from the measured line intensities printed in the table so that the value reported accurately reflects the count rate due to the spectral line.

\section{Description of the Atlas}

The atlas is a series of tables and plots that provides a comprehensive description of the spectrum of the Pt/Ne hollow-cathode lamp in the region $1128-4333 \AA$. Each page of plots depicts a $32 \AA$ section of the spectrum. Every spectral line for which a wavelength and intensity have been determined is indicated with a tic mark at the bottom of the plot. The wavelengths, wave numbers, and relative intensities for these lines are listed in the table on the page facing the plot.

The wavelengths and intensities of Rowland ghosts (spurious lines caused by imperfections in the ruling of the grating) were predicted from the known properties of the gratings. Ghost lines are marked on the plots with a carat instead of a tic mark to distinguish them from true spectral lines. They are not listed in the table.

Wavelengths of lines measured on our photographic plates, taken from the literature, or calculated from optimized Pt II energy levels are given to four decimal places. Lines measured in the photoelectric data only are given to two decimal places. Wavelengths below $2000 \AA$ are given in vacuum; wavelengths above $2000 \AA$ are given in air. For lines originally observed in vacuum, conversion of the wavelengths from vacuum to standard air was carried out by using the three-term formula of Peck and Reeder [20] for the index of refraction of air.

Also listed in the table under the column heading CODE are the sources for wavelengths of various lines emitted by the Pt/Ne lamp that we have taken from the literature, mainly $\mathrm{Pt} I, \mathrm{Ne} I$, and Ne 11. Most of these lines were used as wavelength standards. Literature values were also substituted for lines of impurity species such as $\mathrm{HI}, \mathrm{C}_{\mathrm{I}}, \mathrm{O}$, $\mathrm{Si}$ I, $\mathrm{Al}$ I, and $\mathrm{Al}$ II. The presence of additional impurity lines of $\mathrm{Mg} \mathrm{I}, \mathrm{Mg}$ II, Fe I, Cr I, Pd I, Rh I, $\mathrm{Au}$ I, Ag I, Ni I, Ca I, and Ca II were subsequently pointed out by J. Blaise. These lines are identified in the table. Literature values for their wavelengths have been substituted only for $\mathrm{Ca}$ II and $\mathrm{Fe}$ I.

The intensity of impurity lines varies greatly from lamp to lamp. For example, we did not observe the intense Al I lines at 3944 and $3961 \AA$ on our photographic plates. However, in a lower wavelength exposure using a different lamp the normally less intense lines at 3082 and $3092 \AA$ did appear. For this reason we have given no intensities for the impurity lines. 
The energy level designations for classified lines of $\mathrm{Pt} \mathrm{I}$ and II correspond to the integer parts of the level energies and are given with the even parity level first. Classifications and wavelengths for $\mathrm{Pt} I$ lines with CODES D and E were taken from Engleman [6]. Pt I lines with CODE $\mathrm{N}$ and $\mathrm{Pt}$ II lines with CODE $\mathrm{K}$ are newly classified by Blaise and Wyart [13]; the wavelengths are from the present work. Classifications for other $\mathrm{Pt}$ II lines were taken from Shenstone [5], with level values given by Reader, Acquista, Sansonetti, and Engleman [7]; a number given in the CODE column is the wavelength uncertainty of the Ritz wavelength in units of $0.0001 \AA$ (see Sec. 5).

The intensities in the atlas are a uniform set of relative values covering the entire region of observation. For lines that were blended on the photoelectric scans but resolved or nearly resolved on the photographic exposures, the intensities were estimated visually from the photographic plates by comparison with nearby well-resolved lines. In a few places a real spectral line is blended with a grating ghost. This is noted with an $M$ in the CODE column in the table. The intensities measured for such lines are probably affected by the presence of the ghost. As mentioned, the spectral sensitivity of the spectrometer and detector combination was taken into account by using the accurate radiance values of Klose [19] for about 80 of the lines to normalize the observations. From the reproducibility of our measurements and comparisons with the data of Klose we estimate the relative intensities for a given species (element and stage of ionization) to be accurate to about $20 \%$. A prime factor in possible variation of the relative intensities is the length of time that a particular lamp has been used. Over many hours of use the intensities of the $\mathrm{Ne}$ lines are observed to change relative to the Pt lines. However, for a given atom and ionization stage the relative intensities should be reliable within our estimated uncertainty. For most lines the present intensities are identical to those given by Reader, Acquista, Sansonetti, and Sansonetti [8]. The intensities of a few lines have been slightly revised in the present work.

Our relative intensities for lines emitted by the $\mathrm{Pt} / \mathrm{Ne}$ lamp are potentially useful for calibration of the spectral response of spectrographic systems in other laboratories. In general, the values are sufficiently reliable to provide a good semi-quantitative calibration. Of course the accuracy that can be obtained is limited by the degree to which other $\mathrm{Pt} / \mathrm{Ne}$ lamps might vary from those we used. We found only small variations in the relative intensi- ties of lines in our lamps, all of which were purchased separately over a 5 year period. Nevertheless, it is not certain that other lamps would exhibit identical properties. In particular, comparison of lines in the $1130-1300 \AA$ region with lines in higher wavelength regions could be affected by variation in the low wavelength transmission of the magnesium fluoride windows of different lamps. Since we used only a small number of lamps and did not scan each lamp over the entire spectral region, we can make no definitive statement regarding lamp to lamp variation. Further investigation would be needed to evaluate the importance of such systematic variations.

\section{Accuracy of Wavelengths}

Our estimate of the uncertainty of the photographically measured wavelengths is based on several considerations:

a. The standard deviation of our polynomial fits for the $\mathrm{Cu}$ II reference lines in the $\mathrm{Pt} / \mathrm{Cu}$ lamp was typically $0.0010 \AA$.

b. The standard deviation of our polynomial fits for the Pt lines used as internal standards for measurements in the $\mathrm{Pt} / \mathrm{Ne}$ lamp was typically $0.0015 \AA$.

c. A comparison of a group of about 100 lines measured by different operators on different plates and taken with different grating rotations in the region $1470-1520 \AA$ showed an average deviation of $0.0001 \AA$ and an rms difference of $0.0014 \AA$. In general, our separate measurements of the wavelengths of individual lines agreed to about this level of accuracy.

d. A comparison of the wavelengths of 37 lines of Pt II in the region 2247-3700 $\AA$ that were measured in this work and independently by Engleman [7] shows an average deviation of $0.0003 \AA$ and an rms difference of $0.0019 \AA$.

e. For the 508 lines of $\mathrm{Pt}$ II whose wavelengths can be calculated from the optimized level values, the rms difference between the calculated and observed wavelengths is about $0.0015 \AA$.

f. A comparison of our measured wavelengths for impurity lines appearing in the $\mathrm{Pt} / \mathrm{Ne}$ lamp with standard wavelengths for these lines shows an average deviation of $0.0003 \AA$ and an rms difference of $0.0015 \AA$.

Based on these comparisons we estimate an uncertainty of $\pm 0.0020 \AA$ for the wavelengths measured photographically. 
As mentioned above, the wavelengths of classified lines of $\mathrm{Pt} I \mathrm{I}$ in the atlas which have numbers in the CODE column are those derived from the optimized level values. The uncertainties of these wavelengths are taken to be the square root of the sum of the squares of the uncertainties of the combining levels as given by Reader, Acquista, Sansonetti, and Engleman [7]. They are listed in the far right column under the heading CODE in units of $0.0001 \AA$.

The uncertainties of the photoelectrically measured lines were estimated by comparing the measured wavelengths of $\mathrm{Pt}$ II lines observed only in the photoelectric scans with calculated Ritz wavelengths for the same lines. The standard deviation of the differences was about $0.006 \AA$ for lines below $2030 \AA$ and about $0.015 \AA$ for lines at longer wavelengths. Based on these comparisons we estimate the uncertainty to be $\pm 0.01 \AA$ for lines below $2030 \AA$ and $\pm 0.02 \AA$ for lines above $2030 \AA$.

The uncertainties of lines whose wavelengths have been taken from the literature are discussed in some detail in the notes to the atlas. Most of these uncertainties are less than $0.001 \AA$ and virtually all are less than $0.002 \AA$.

The cathodes of the lamps used in this work and with GHRS contain isotopes of Pt in their natural abundances. Some lines of Pt I and II show appreciable isotope and magnetic hyperfine structure (hfs). At the resolution of our spectrograph (and also GHRS) almost all Pt lines appear sharp and symmetric. A few lines show evidence of unresolved structure and appear wide, hazy, or asymmetric on the photographic plates. These lines are noted (W, H, L, or S) adjacent to their intensities in the atlas. Lines showing partially resolved structure are noted in the atlas as being complex (C). A few hyperfine patterns occurred in the photographic data as three fully resolved features and were measured as separate lines.

For GHRS and other instruments with resolving power of $10^{5}$ or less, the existence of hfs in some lines should present no problem in using the present list of Pt lines for wavelength calibration. To achieve the highest accuracy, lines with notations indicating detectable unresolved structure should not be used. For instruments with resolving limits significantly below $0.02 \AA$, structure may be observed in many additional Pt lines, and our present wavelength list may not be adequate for calibration purposes. Thus, for calibration of spectrographs having much higher resolution, it may be desirable to develop calibration wavelengths based on a lamp whose cathode contains a single even isotope of $\mathrm{Pt}$.

\section{Acknowledgments}

This investigation was undertaken at the suggestion of William C. Martin, who realized that the hollow-cathode spectrum of platinum would probably have to be newly measured in order for the Goddard High Resolution Spectrograph to meet its design goals. His encouragement and suggestions throughout the work are gratefully acknowledged. Our photoelectric scans of the Pt/Ne lamp on the $10.7 \mathrm{~m}$ spectrograph owe much of their success to suggestions of Richard Deslattes regarding photon counting techniques. We thank him for lending us his expertise as well as much of the equipment required to carry out the experiment. Many of the impurity lines in our list were identified by Jean Blaise. We thank him and Jean-François Wyart for making available their new classifications in Pt $\mathrm{I}$ and Pt II for inclusion in the atlas. This work was supported in part by the National Aeronautics and Space Administration.

\section{References}

[1] C. J. Burrows, J. A. Holtzman, S. M. Faber, P. Y. Bely, H. Hasan, C. R. Lynds, and D. Schroeder, Astrophys. J. 369, L21 (1991).

[2] G. M. Wahlgren, D. S. Leckrone, S. N. Shore, D. J. Lindler, R. L. Gilliland, and D. C. Ebbets, Astrophys. J. 377, L41 (1991).

[3] S. R. Heap, B. Altner, D. Ebbets, I. Hubeny, J. B. Hutchings, R. P. Kudritzki, S. A. Voels, S. Haser, A. Pauldrach, J. Puls, and K. Butler, Astrophys. J. 377, L29 (1991).

[4] G. H. Mount, G. Yamasaki, W. Fowler, and W. G. Fastie, Appl. Opt. 16, 591 (1977).

[5] A. G. Shenstone, Philos. Trans. R. Soc. London Ser. A 237, 453 (1938).

[6] R. Engleman, Jr., J. Opt. Soc. Am. B 2, 1934 (1985).

[7] J. Reader, N. Acquista, C. J. Sansonetti, and R. Engleman, Jr., J. Opt. Soc. Am. B 5, 2106 (1988).

[8] J. Reader, N. Acquista, C. J. Sansonetti, and J. E. Sansonetti, Astrophys. J. Suppl. 72, 831 (1990).

[9] G. A. Kriss, G. F. Hartig, L. Armus, W. P. Blair, S. Caganoff, and L. Dressel, Astrophys. J. 377, L13 (1991).

[10] T. R. Ayres, E. Jensen, and O. Engvold, Astrophys. J. Suppl. 66, 51 (1988).

[11] D. M. Hassler, G. J. Rottman, and F. Q. Orrall, Astrophys. J. 372, 710 (1991).

[12] D. S. Leckrone, G. M. Wahlgren, and S. G. Johansson, Astrophys. J. 377, L37 (1991).

[13] J. Blaise and J.-F. Wyart, J. Res. Natl. Inst. Stand. Technol. 97, 217 (1992) and private communication.

[14] J. Reader and S. P. Davis, J. Opt. Soc. Am. 53, 431 (1963).

[15] C. B. Ross, Los Alamos Scientific Laboratory Rep. 4498, National Technical Information Service, Springfield, VA (1970). 
[16] B. A. Palmer and R. Engleman, Jr., Los Alamos National Laboratory Rep. 9615, National Technical Information Service, Springfield, VA (1983).

[17] W. Persson, Phys. Scr. 3, 133 (1971).

[18] V. Kaufman and B. Edlén, J. Phys. Chem. Ref. Data 3, 825 (1974).

[19] J. Klose, Appl. Opt. 29, 2951 (1990).

[20] E. R. Peck and K. Reeder, J. Opt. Soc. Am. 62, 958 (1972).

About the authors: Jean E. Sansonetti is a Guest Researcher in the Atomic Physics Division of the NIST Physics Laboratory. Joseph Reader and Craig J. Sansonetti are physicists in the Atomic Physics Division. Nicola Acquista is recently retired from the Atomic Physics Division. The National Institute of Standards and Technology is an agency of the Technology Administration, U.S. Department of Commerce. 


\section{Spectral Atlas of a Platinum/Neon Hollow-Cathode Reference Lamp}

Wavelength $\AA$

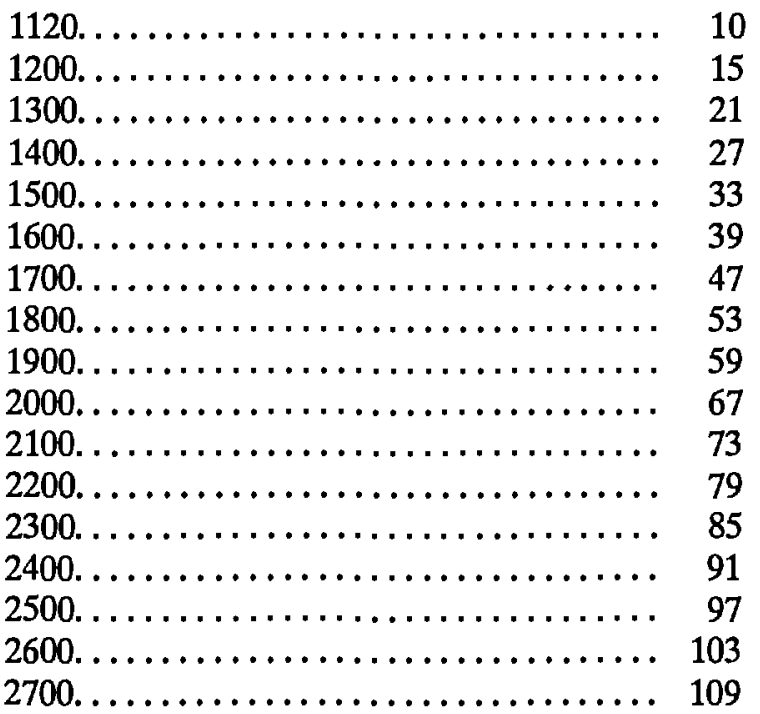

\section{Explanatory Notes}

Wavelengths are given in $\AA$. Wave numbers are given in $\mathrm{cm}^{-1}$. Energy level designations for the classified lines of Pt I and II correspond to the integer parts of the level energies and are given with the even parity level first. A letter appearing in the CODE column indicates the source of a literature value reported for the wavelength or a note pertaining to the line. A number appearing in the CODE column is the uncertainty of the Pt II wavelength determined from the optimized Pt II energy levels (Ritz wavelength) in units of $0.0001 \AA$.

The following protocols were used in substituting literature values for our measured wavelengths. For each spectrum the various literature sources are listed in order of preference. For all doublyclassified lines our experimental wavelength is given.

\section{Pt I}

1) Ritz wavelength from Table 4 of R. Engleman, Jr., J. Opt. Soc. Am. B 2, 1934 (1985).

2) Measured wavelength from Table 1 of R. Engleman, Jr., J. Opt. Soc. Am. B 2, 1934 (1985).

Pt II

1) Wavelength calculated from the optimized level values given by J. Reader, N. Acquista,
Wavelength $\AA$

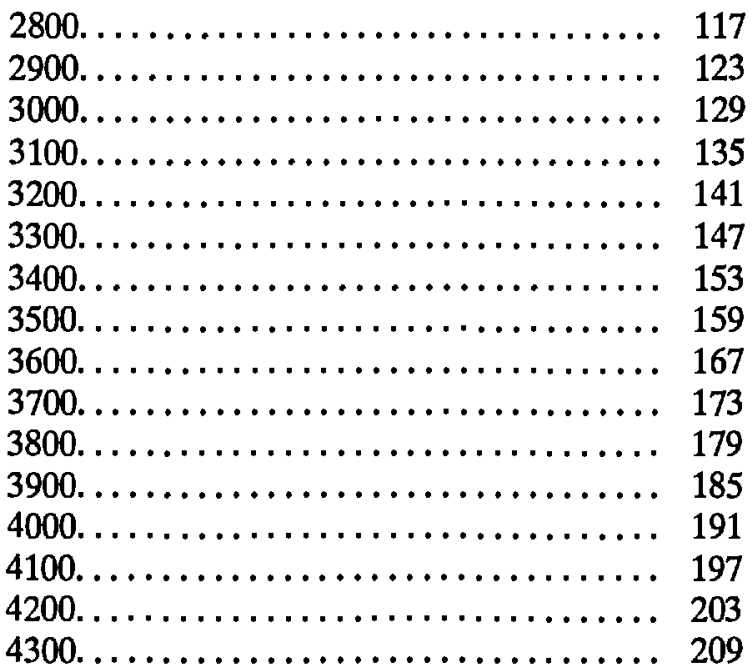

C. J. Sansonetti, and R. J. Engleman, Jr., J. Opt. Soc. Am. B 5, 2106 (1988) except where the energy or $J$ value of one of the combining levels was changed by J. Blaise and J.-F. Wyart, J. Res. Natl. Inst. Stand. Technol. 97, 217 (1992).

$\mathrm{Ne}$ I

1) B. A. Palmer and R. Engleman, Jr., Los Alamos National Laboratory Rep. 9615, National Technical Information Service, Springfield, VA (1983) except for a few lines that may be blended with lines of thorium.

2) V. Kaufman and B. Edlén, J. Phys. Chem. Ref. Data 3, 825 (1974).

3) K. Burns, K. Adams, and J. Longwell, J. Opt. Soc. Am. 40, 6 (1950).

Ne II

1) B. A. Palmer and R. Engleman, Jr., Los Alamos National Laboratory Rep. 9615, National Technical Information Service, Springfield, VA (1983) except for a few lines that may be blended with lines of thorium.

2) Ritz wavelength from W. Persson, Phys. Scr. 3, 133 (1971). 
$\mathrm{Fe} I$

1) R. C. M. Learner and A. P. Thorne, J. Opt. Soc. Am. B 5, 2045 (1988).

2) T. R. O'Brian, M. E. Wickliffe, J. E. Lawler, W. Whaling, and J. W. Brault, J. Opt. Soc. Am. B 8, 1185 (1991).

3) H. M. Crosswhite, J. Res. Natl. Bur. Stand. (U.S.) 79A, 17 (1975).

Line character descriptors (appear to right of intensity):

C - Complex

D - Double; central position of two close lines not resolved on the measuring comparator

H - Hazy

L - Asymmetric, tail toward longer wavelengths

$P$ - Perturbed by close line

S - Asymmetric, tail toward shorter wavelengths

$\mathrm{U}$ - Unresolved from close line; shoulder on stronger line

W - Wide

\section{CODES:}

A - Doubly classified line. The wavelength is the present experimental value.

B - V. Kaufman and B. Edlén, J. Phys. Chem. Ref. Data 3, 825 (1974). Uncertainty is less than $0.002 \AA$.

C - Value determined from optimized Ne II level values; W. Persson, Phys. Scr. 3, 133 (1971). For lines below $2000 \AA$ the uncertainty in wavelength corresponds to a wave number uncertainty of about $0.03 \mathrm{~cm}^{-1}$, which is $0.0004 \AA$ at $1200 \AA$ and $0.001 \AA$ at $2000 \AA$. The uncertainty for lines above $2000 \AA$ appears to be about $0.002 \AA$.

D - Value determined from optimized Pt I level values; R. Engleman, Jr., J. Opt. Soc. Am. B 2, 1934 (1985). The wavelength uncertainty is $0.0005 \AA$.

E - R. Engleman, Jr., J. Opt. Soc. Am. B 2, 1934 (1985). The wavelength uncertainty corresponds to a wave number uncertainty of 0.01 $\mathrm{cm}^{-1}$, which is $0.0005 \AA$ at $2250 \AA$ and $0.0017 \AA$ at $4095 \AA$.

F - Value determined from optimized $\mathrm{Al}$ I level values; K. B. S. Eriksson and H. B. S. Isberg, Ark. Fys. 23, 527 (1963). Uncertainty is less than $0.002 \AA$.
G - B. A. Palmer and R. Engleman, Jr., Los Alamos National Laboratory Rep. 9615, National Technical Information Service, Springfield, VA (1983). The wavelength uncertainty is $0.0001 \AA$.

$\mathrm{H}$ - Measured component of hyperfine pattern of a Pt I line.

I - K. Burns, K. Adams, and J. Longwell, J. Opt. Soc. Am. 40, 6 (1950). The wavelength uncertainty is $0.0004 \AA$.

$\mathrm{J}$ - Measured component of the incomplete hyperfine pattern of the Pt II line 3648461190.

K - Newly identified Pt II line. J. Blaise and J.-F. Wyart, J. Res. Natl. Inst. Stand. Technol. 97, 217 (1992). For photographically measured lines the wavelength uncertainty is $\pm 0.002 \AA$. For lines found only in the photoelectric scans (two decimal digits) the uncertainty is $\pm 0.01 \AA$ below $2030 \AA$ and $\pm 0.02 \AA$ above $2030 \AA$.

L - W. Persson, C.-G. Wahlström, L. Jönsson, and H. O. DiRocco, Phys. Rev. A 43, 4791 (1991). The wavelength is the experimental value from the present work.

M - Probably blended with a grating ghost; the intensity may be affected.

N - Newly identified Pt I line. J. Blaise, private communication (1990). For photographically measured lines the wavelength uncertainty is $\pm 0.002 \AA$. For lines found only in the photoelectric scans the uncertainty is $\pm 0.01 \AA$ below $2030 \AA$ and $\pm 0.02 \AA$ above $2030 \AA$.

$P$ - Pt II line for which a Ritz wavelength was given in J. Reader, N. Acquista, C. J. Sansonetti, and J. E. Sansonetti, Astrophys. J. Suppl. 72, 831 (1990). The experimental value is given here because the energy or $J$ value of a combining level was changed in the analysis of J. Blaise and J.-F. Wyart, J. Res. Natl. Inst. Stand. Technol. 97, 217 (1992).

Q - R. C. M. Learner and A. P. Thorne, J. Opt. Soc. Am. B 5, 2045 (1988).

R - T. R. O'Brian, M. E. Wickliffe, J. E. Lawler, W. Whaling, and J. W. Brault, J. Opt. Soc. Am. B 8, 1185 (1991). Some additional measured wavelengths not included in this reference were communicated privately by the authors.

S - H. M. Crosswhite, J. Res. Natl. Bur. Stand. (U.S.) 79A, 17 (1975).

T - N. E. Wagman, U. Pitt. Bull. 34, 1 (1937). 


\begin{tabular}{llrlrl} 
WAVELENGTH & WAVE NUMBER & INTENSITY & \multicolumn{2}{c}{ CLASSIFICATION } & CODE \\
\hline 1131.87 & 88349.4 & 41 & & & \\
1132.8322 & 88274.326 & 2300 & Pt II & $9356-97630$ & 05 \\
1134.66 & 88132.1 & 22 & Pt II & $18097-106229$ & $\mathrm{~K}$ \\
1135.4782 & 88068.623 & 2400 & Pt II & $13329-101397$ & 05 \\
1136.06 & 88023.5 & 35 & Pt II & $8419-96443$ & $\mathrm{~K}$ \\
1136.2004 & 88012.640 & 22 & Pt II & $13329-101341$ & 05 \\
1138.39 & 87843.4 & 120 & & & \\
1138.83 & 87809.4 & 330 & & & \\
1139.62 & 87748.5 & 99 & & & \\
1139.75 & 87738.5 & 140 & & & \\
1140.6146 & 87672.034 & 53 & Pt II & $15791-103463$ & 07 \\
1140.65 & 87669.3 & 53 & & & \\
1140.79 & 87658.6 & 39 & & &
\end{tabular}

\begin{tabular}{|c|c|c|c|c|c|}
\hline AVELENGTH & WAVE NUMBER & INTENSI TY & \multicolumn{2}{|c|}{ CLASSI FI CATION } & CODE \\
\hline $\begin{array}{l}1141.17 \\
1141.8885 \\
1143.2957 \\
1144.60\end{array}$ & $\begin{array}{l}87629.4 \\
87574.227 \\
87466.439 \\
87366.8\end{array}$ & $\begin{array}{r}91 \\
1400 \\
5800 \\
93\end{array}$ & $\begin{array}{l}\text { Pt } \text { II } \\
\text { Pt II } \\
\text { Pt } \\
\text { II }\end{array}$ & $\begin{array}{l}15791-103421 \\
13329-100903 \\
13329-100795\end{array}$ & $\begin{array}{l}K \\
06 \\
06\end{array}$ \\
\hline 1145.00 & 87336.2 & 49 & Pt II & $16820-104158$ & $\begin{array}{l}K \\
05\end{array}$ \\
\hline $\begin{array}{l}1145.7055 \\
1145.87\end{array}$ & $\begin{array}{l}87282.468 \\
87269.9\end{array}$ & $\begin{array}{l}800 \\
310\end{array}$ & $\begin{array}{l}\text { Pt I I } \\
\text { Pt II }\end{array}$ & $\begin{array}{l}13329-100611 \\
16820-104092\end{array}$ & $\begin{array}{l}05 \\
K\end{array}$ \\
\hline $\begin{array}{l}1147.62 \\
1150.1564\end{array}$ & $\begin{array}{l}87136.9 \\
86944.697\end{array}$ & $\begin{array}{r}120 \\
1500\end{array}$ & $\begin{array}{l}\text { Pt II } \\
\text { Pt II }\end{array}$ & $\begin{array}{r}8419-95557 \\
18097-105042\end{array}$ & $\begin{array}{l}K \\
k\end{array}$ \\
\hline 1150.6130 & 86910.194 & 7200 & Pt II & $13329-100239$ & 05 \\
\hline 1150.9198 & 86887.027 & 1700 & Pt II & $15791-102678$ & $k$ \\
\hline $\begin{array}{l}1150.9689 \\
1151.59\end{array}$ & $\begin{array}{l}86883.321 \\
86836.5\end{array}$ & $\begin{array}{r}1800 \\
200\end{array}$ & $\begin{array}{l}\text { Pt II } \\
\text { Pt II }\end{array}$ & $\begin{array}{r}4786-91669 \\
24879-111716\end{array}$ & $\begin{array}{l}K \\
K\end{array}$ \\
\hline
\end{tabular}



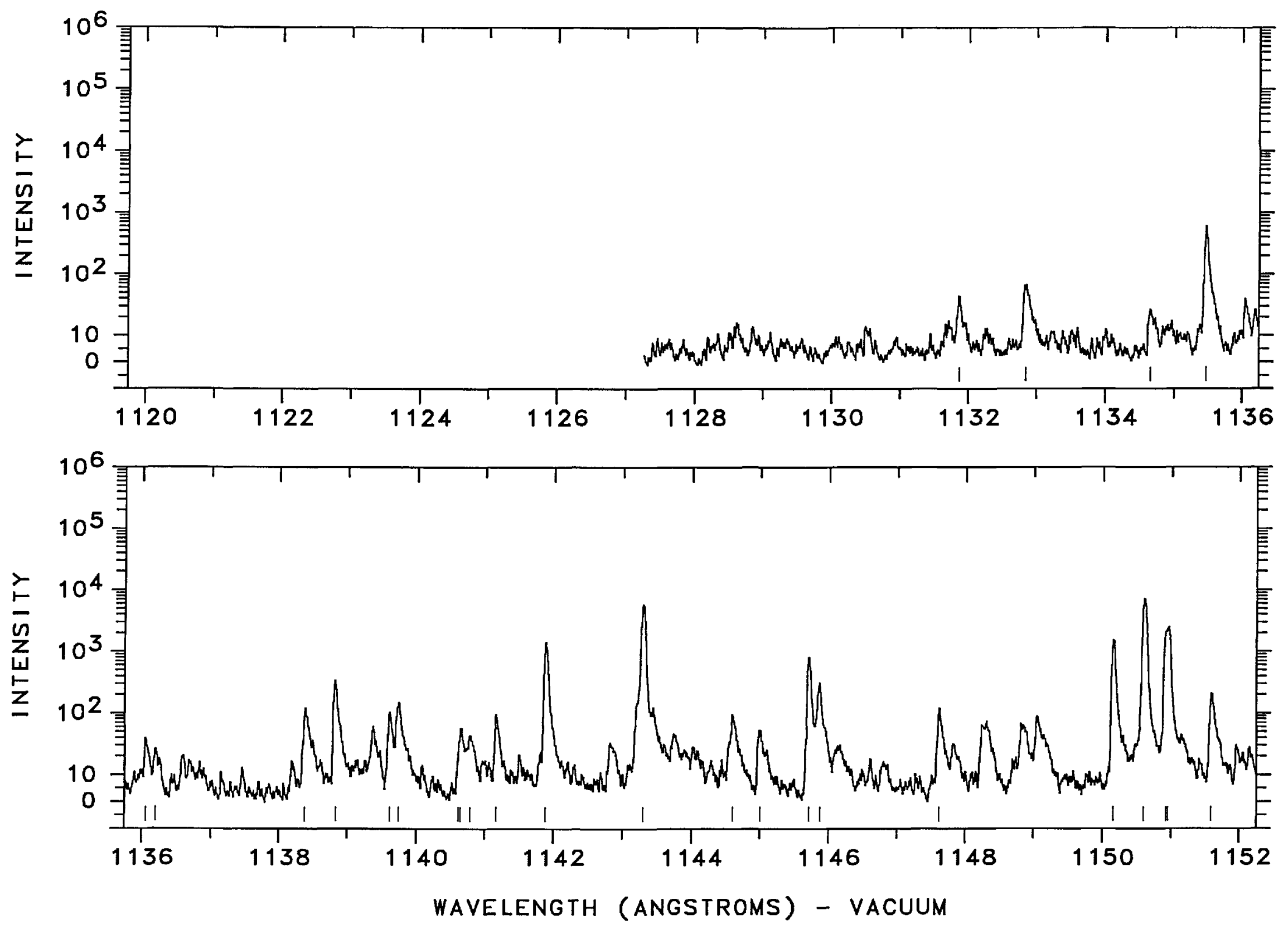


\section{WAVELENGTH WAVE NUMBER INTENSITY}

CLASSI FI CATION

CODE

\begin{tabular}{|c|c|c|c|c|c|}
\hline 1152.4079 & 86774.830 & 8100 & Pt I I & $9356-96131$ & K \\
\hline 1152.58 & 86761.9 & 500 & Pt II & $23875-110638$ & K \\
\hline 1152.86 & 86740.8 & 600 & Pt I I & $23461-110202$ & K \\
\hline $\begin{array}{l}1153.4526 \\
1154.03\end{array}$ & $\begin{array}{l}86696.238 \\
86652.9\end{array}$ & $\begin{array}{r}1500 \\
150\end{array}$ & Pt I I & $16820-103517$ & 08 \\
\hline 1154.1691 & 86642.416 & 50 & Pt II & $16820-103463$ & 07 \\
\hline 1154.4201 & 86623.581 & 230 & Pt II & $15791-102414$ & 06 \\
\hline 1155.69 & 86528.4 & 240 & & & \\
\hline 1156.4898 & 86468.551 & 990 & Pt II & $13329-99797$ & 05 \\
\hline 1156.89 & 86438.6 & 77 & Pt II & $21717-108155$ & $k$ \\
\hline 1157.13 & 86420.7 & 300 & & & \\
\hline 1157.26 & 86411.0 & 83 & & & \\
\hline 1157.43 & 86398.3 & 470 & Pt II & $9356-95754$ & K \\
\hline 1157.84 & 86367.7 & 61 & & & \\
\hline 1158.48 & 86320.0 & 110 & Pt I I & $23875-110196$ & AK \\
\hline 1158.48 & 86320.0 & 110 & Pt II & $21717-108038$ & AK \\
\hline 1159.03 & 86279.0 & 180 & & & \\
\hline 1159.1308 & 86271.541 & 860 & Pt II & $23461-109733$ & K \\
\hline 1159.2760 & 86260.735 & 1700 & Pt II & 9356- 95617 & K \\
\hline 1159.40 & 86251.5 & 180 & & & \\
\hline 1159.96 & 86209.9 & 570 & Pt II & $23875-110085$ & $k$ \\
\hline 1160.87 & 86142.3 & 39 & & & \\
\hline 1161.90 & 86065.9 & 450 & Pt II & $23461-109528$ & $k$ \\
\hline 1161.9681 & 86060.882 & 1700 & Pt II & $18097-104158$ & $k$ \\
\hline 1162.15 & 86047.4 & 360 & & & \\
\hline 1162.30 & 86036.3 & 66 & & & \\
\hline 1162.50 & 86021.5 & 28 & & & \\
\hline 1162.95 & 85988.2 & 110 & & & \\
\hline 1164.4184 & 85879.784 & 11000 & Pt II & 13329- 99209 & 06 \\
\hline 1164.5543 & 85869.762 & 3100 & Pt II & $9356-95226$ & $k$ \\
\hline 1164.7198 & 85857.560 & 1000 & Pt II & $16820-102678$ & K \\
\hline $\begin{array}{l}1164.8721 \\
1165.81\end{array}$ & $\begin{array}{l}85846.335 \\
85777.3\end{array}$ & $\begin{array}{r}1000 \\
390\end{array}$ & Pt I I & 23461-109307 & K \\
\hline 1166.32 & 85739.8 & 480 & Pt II & $13329-99068$ & $k$ \\
\hline 1166.47 & 85728.7 & 52 & & & \\
\hline 1166.8635 & 85699.827 & 12000 & Pt II & $16820-102520$ & $k$ \\
\hline 1167.0766 & 85684.179 & 1500 & Pt II & $21168-106852$ & $k$ \\
\hline 1167.52 & 85651.6 & 110 & Pt II & $23875-109528$ & $\ddot{k}$ \\
\hline
\end{tabular}

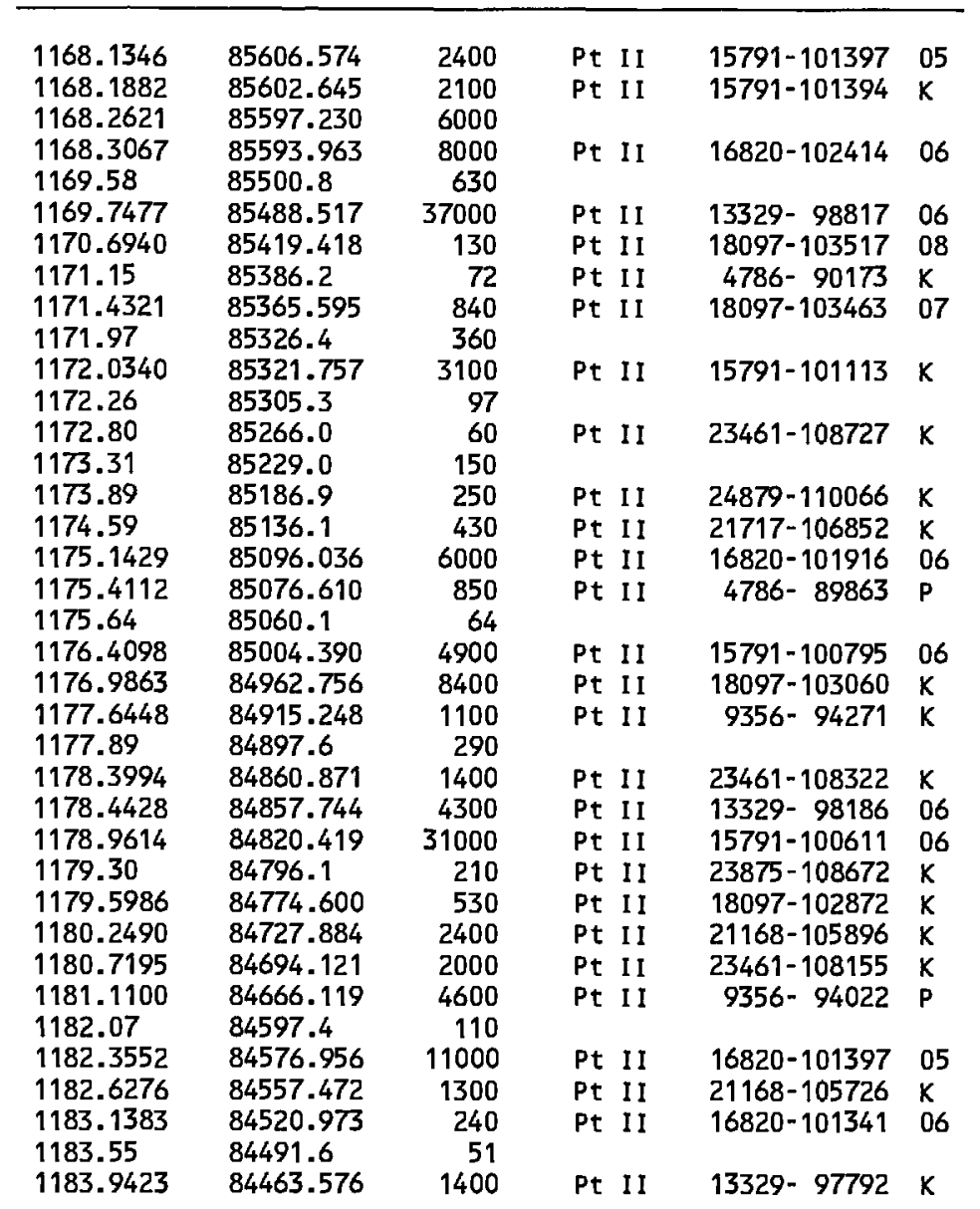




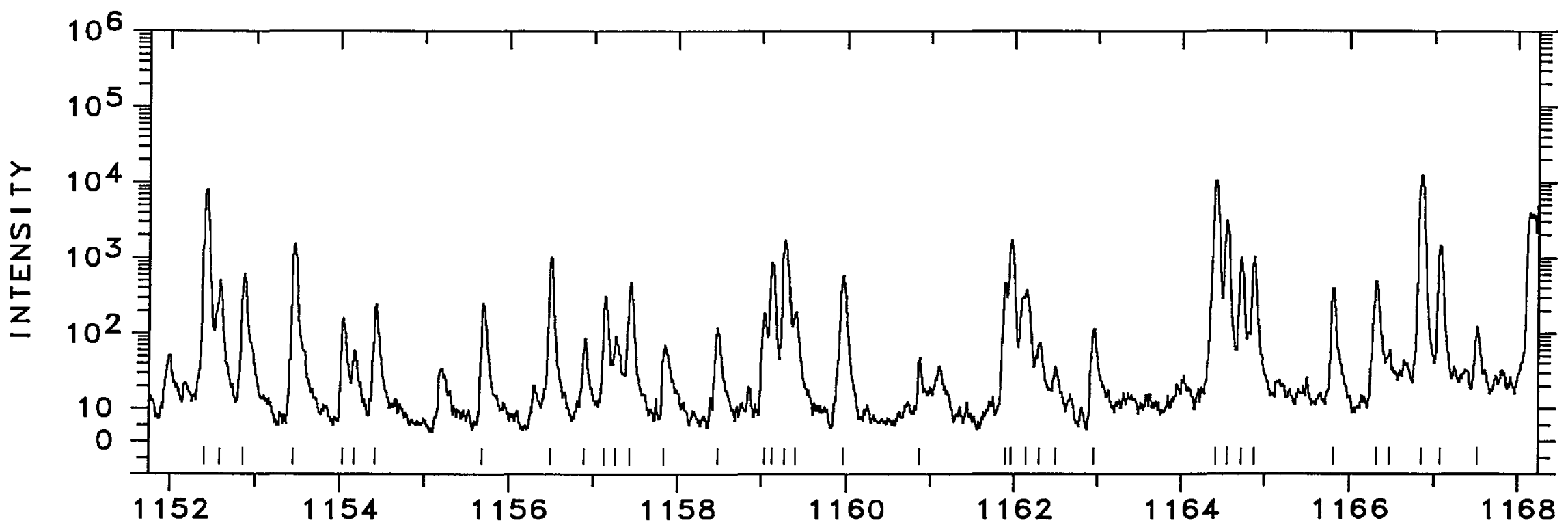

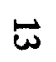

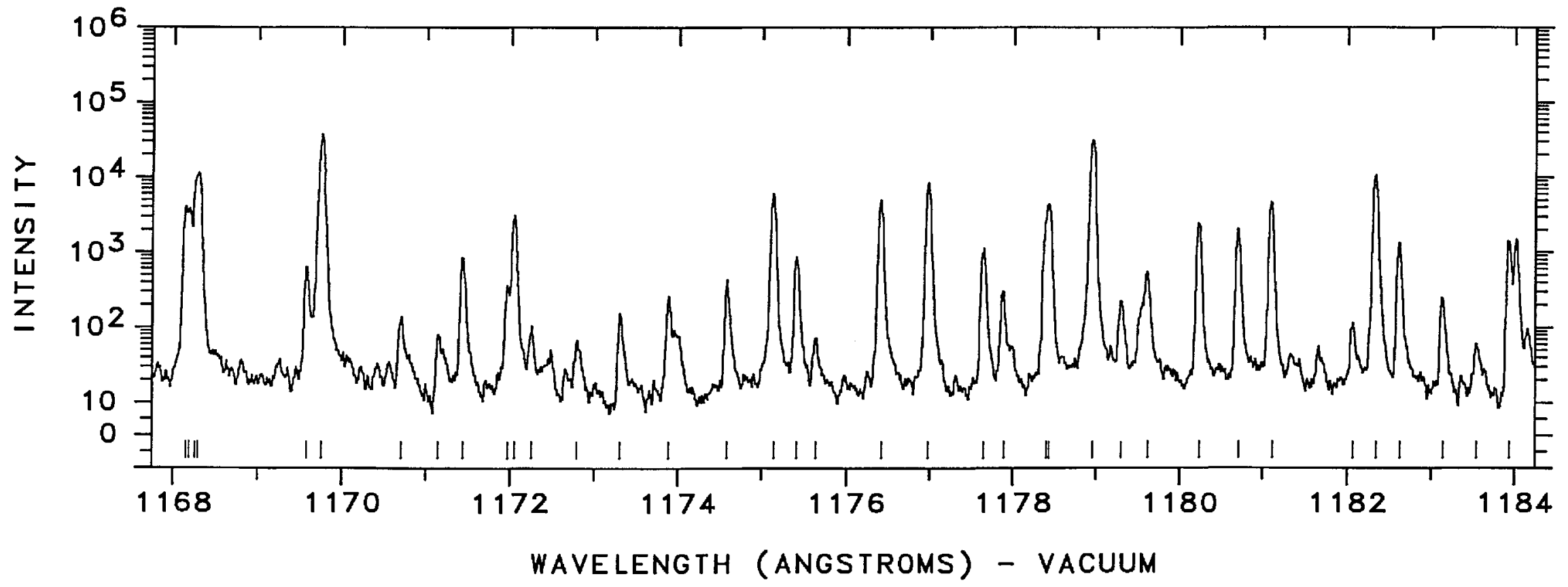




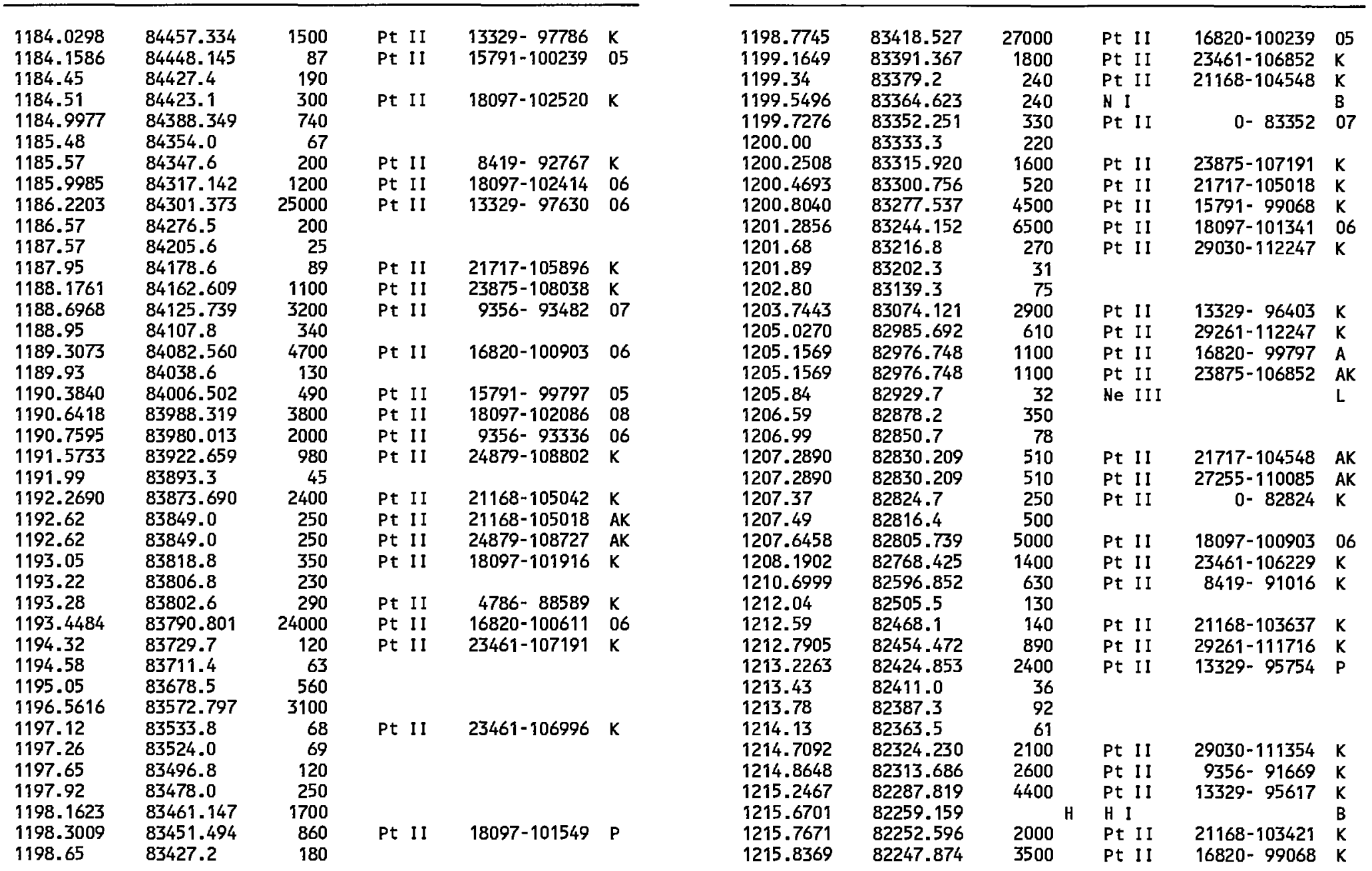




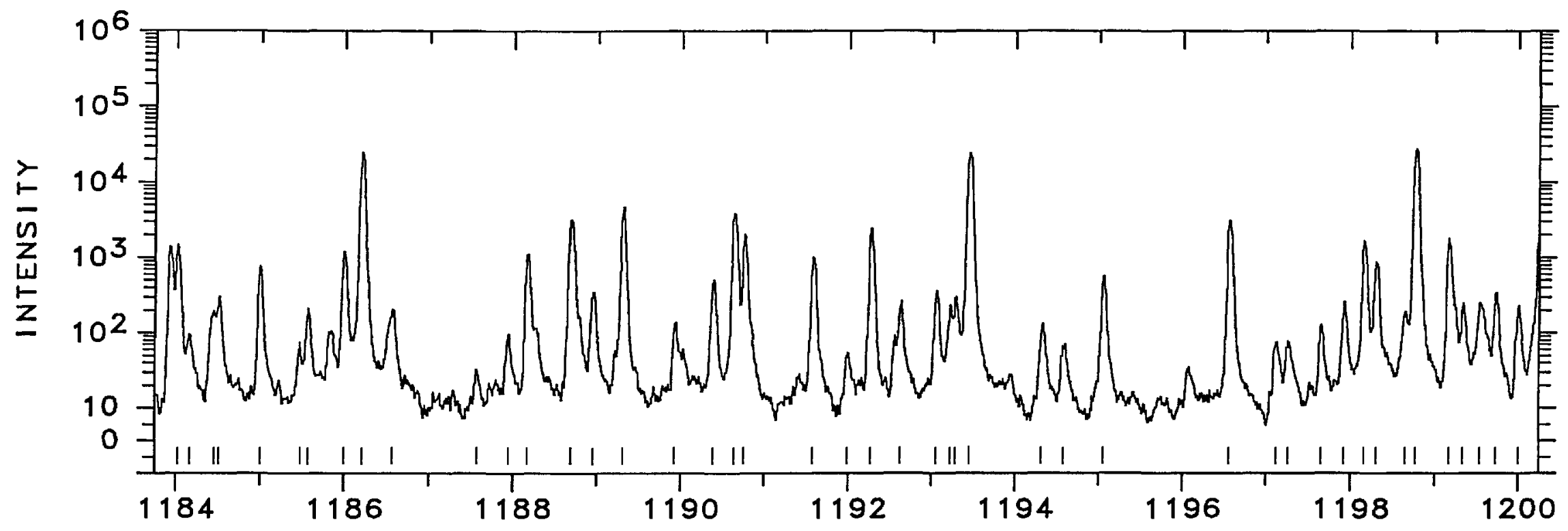

$\bar{n}$

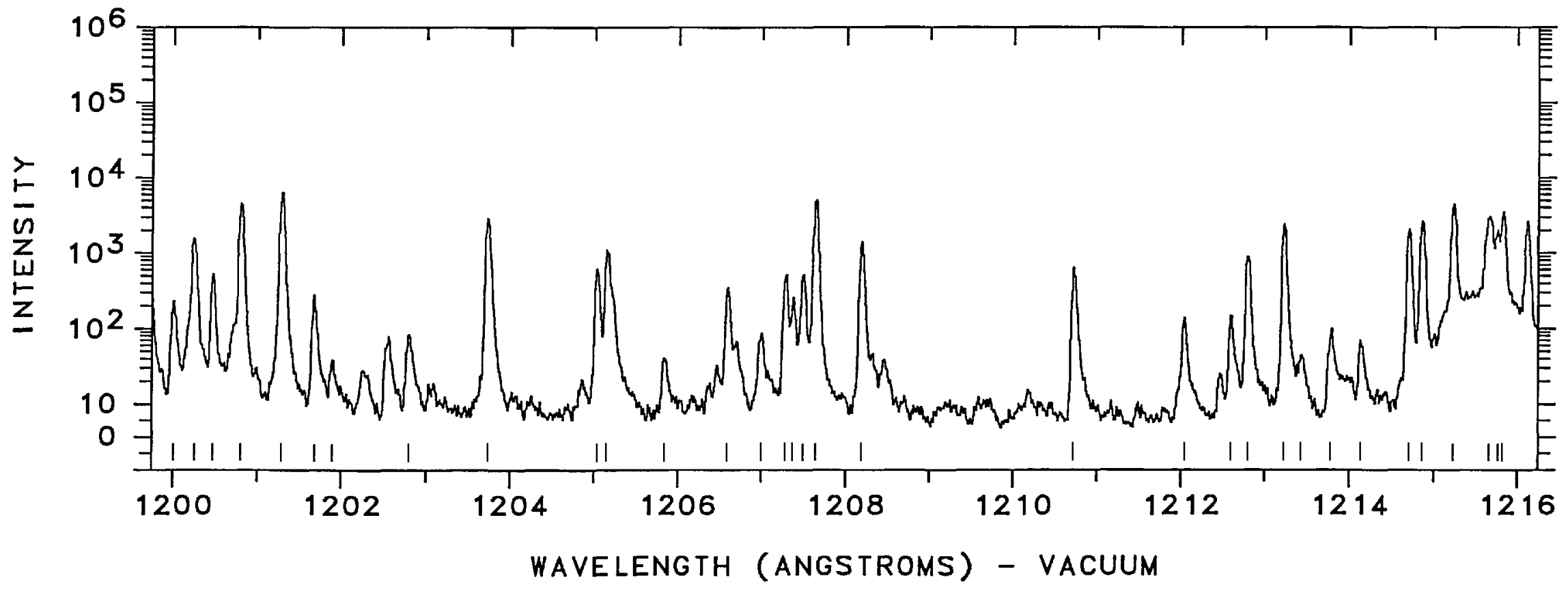




\begin{tabular}{|c|c|c|c|c|c|}
\hline $\begin{array}{l}1216.1236 \\
1216.63\end{array}$ & $\begin{array}{l}82228.484 \\
82194\end{array}$ & $\begin{array}{r}2600 \\
130\end{array}$ & Pt II & $13329-95557$ & $k$ \\
\hline 1217.4951 & 82135.854 & 2200 & Pt II & $23461-105597$ & K \\
\hline 1217.7927 & 82115.782 & 1500 & Pt II & $24879-106995$ & K \\
\hline $\begin{array}{l}1218.15 \\
1219.00\end{array}$ & $\begin{array}{l}82091.7 \\
82034.5\end{array}$ & $\begin{array}{r}230 \\
51\end{array}$ & & & \\
\hline 1219.4931 & 82001.284 & 43000 & Pt II & $15791-97792$ & K \\
\hline 1219.5786 & 81995.535 & 900 & Pt II & $15791-97786$ & K \\
\hline 1220.09 & 81961.2 & 130 & & & \\
\hline 1220.47 & 81935.6 & 240 & & & \\
\hline 1220.7795 & 81914.875 & 3900 & Pt II & $9356-91271$ & $k$ \\
\hline 1221.04 & 81897.4 & 150 & & & \\
\hline 1221.7369 & 81850.683 & 760 & Pt II & $23875-105726$ & $\mathrm{k}$ \\
\hline 1223.1214 & 81758.033 & 1200 & & & \\
\hline 1223.5053 & 81732.380 & 1600 & Pt II & $29030-110762$ & $\mathrm{~K}$ \\
\hline 1223.6648 & 81721.726 & 530 & Pt II & $23875-105597$ & $\mathrm{k}$ \\
\hline 1223.98 & 81700.7 & 240 & & & \\
\hline 1224.1006 & 81692.632 & 380 & & & \\
\hline 1224.43 & 81670.7 & 170 & & & \\
\hline 1225.39 & 81606.7 & 56 & Pt II & $29030-110638$ & $\mathrm{~K}$ \\
\hline 1225.82 & 81578.0 & 360 & Pt II & $29030-110609$ & K \\
\hline 1226.7936 & 81513.304 & 1000 & Pt II & $13329-94842$ & $\mathrm{~K}$ \\
\hline 1226.9816 & 81500.815 & 860 & Pt II & $29261-110762$ & $\mathrm{~K}$ \\
\hline 1228.5930 & 81393.920 & $400 \mathrm{~L}$ & & & \\
\hline 1228.6470 & 81390.342 & 1300 & Pt II & $9356-90746$ & $\mathrm{~K}$ \\
\hline 1229.0134 & 81366.077 & 17000 & Pt II & $16820-98186$ & 06 \\
\hline 1229.2515 & 81350.318 & 1500 & Pt II & $24879-106229$ & $\mathrm{~K}$ \\
\hline 1229.3001 & 81347.102 & 4500 & Pt II & $29261-110609$ & K \\
\hline 1229.6873 & 81321.49 & 510 & Ne II & & c \\
\hline 1229.8367 & 81311.61 & 2400 & $\mathrm{Ne}$ II & & c \\
\hline 1229.9505 & 81304.085 & 630 & Pt II & $13329-94633$ & $\mathrm{~K}$ \\
\hline 1230.8272 & 81246.173 & 130 & Pt II & $21168-102414$ & 07 \\
\hline 1231.64 & 81192.6 & 97 & Ne III & & $\mathrm{L}$ \\
\hline 1231.82 & 81180.7 & 67 & $\mathrm{Ne}$ III & & $\bar{L}$ \\
\hline
\end{tabular}

\begin{tabular}{|c|c|c|c|c|c|}
\hline 1231.89 & 81176.1 & 42 & $\mathrm{Ne}$ III & & L \\
\hline 1232.0302 & 81166.842 & 420 & Pt II & $23875-105042$ & $\mathrm{~K}$ \\
\hline 1232.3983 & 81142.598 & 910 & Pt II & $23875-105018$ & $\ddot{k}$ \\
\hline 1232.8739 & 81111.296 & 14000 & Pt II & $18097-99209$ & 06 \\
\hline 1233.25 & 81086.6 & 100 & Pt II & $23461-104548$ & $\mathrm{~K}$ \\
\hline 1234.0154 & 81036.266 & 720 & Pt II & $29030-110066$ & $\ddot{k}$ \\
\hline 1234.4019 & 81010.893 & 1200 & & & \\
\hline 1234.5580 & 81000.650 & 4000 & & & \\
\hline 1235.0916 & 80965.655 & 4900 & Pt II & $16820-97786$ & K \\
\hline $\begin{array}{l}1235.1607 \\
1235.47\end{array}$ & $\begin{array}{l}80961.125 \\
80940.9\end{array}$ & $\begin{array}{l}600 \\
250\end{array}$ & Pt II & $21717-102678$ & K \\
\hline 1235.8863 & 80913.592 & 2700 & Pt II & $4786-85700$ & $P$ \\
\hline 1237.4751 & 80809.706 & 4300 & Pt II & $16820-97630$ & 06 \\
\hline 1238.4170 & 80748.246 & 450 & Pt II & $21168-101916$ & 06 \\
\hline 1238.8499 & 80720.029 & 36000 & Pt II & $18097-98817$ & 07 \\
\hline 1239.0156 & 80709.23 & 1200 & Ne II & & $\mathrm{c}$ \\
\hline 1239.1184 & 80702.538 & 350 & Pt II & $29030-109733$ & K \\
\hline 1239.2011 & 80697.152 & 990 & Pt II & $23461-104158$ & $\mathrm{~K}$ \\
\hline $\begin{array}{l}1239.5438 \\
1240.24\end{array}$ & 80674.842 & 890 & Pt II & $24879-105554$ & K \\
\hline $\begin{array}{l}1240.5098 \\
1240.9502\end{array}$ & $\begin{array}{l}80612.019 \\
80583.411\end{array}$ & $\begin{array}{r}1000 \\
620\end{array}$ & Pt II & $15791-96403$ & K \\
\hline 1242.1331 & 80506.670 & 580 & Pt II & $9356-89863$ & $p$ \\
\hline 1242.6815 & 80471.142 & 1100 & Pt II & $29261-109733$ & K \\
\hline 1244.3623 & 80362.448 & 980 & Pt II & $16820-97183$ & K \\
\hline 1244.8278 & 80332.396 & 2000 & Pt II & $27255-107588$ & K \\
\hline 1245.21 & 80307.7 & 160 & & & \\
\hline 1245.6812 & 80277.362 & 7500 & Pt II & $29030-109307$ & K \\
\hline 1246.0801 & 80251.662 & 670 & Pt II & $9356-89607$ & 07 \\
\hline 1246.3668 & 80233.203 & 650 & Pt II & $34647-114880$ & $\mathrm{~K}$ \\
\hline 1246.4295 & 80229.166 & 2900 & Pt II & $21168-101397$ & 06 \\
\hline 1246.6262 & 80216.508 & 1100 & Pt II & $23875-104092$ & K \\
\hline 1247.6173 & 80152.786 & 1300 & Pt II & $13329-93482$ & 08 \\
\hline
\end{tabular}




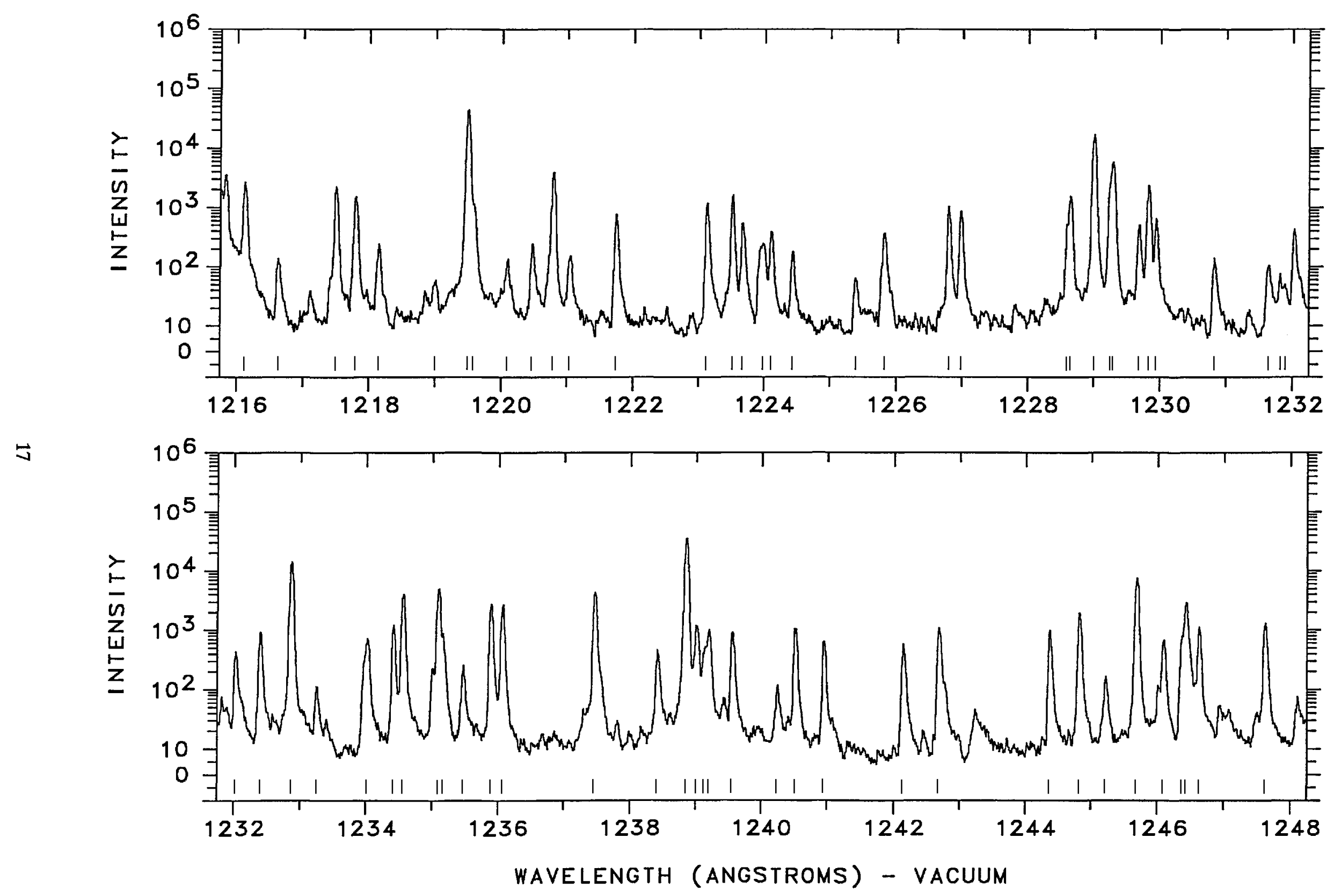


WAVELENGTH WAVE NUMBER INTENSITY

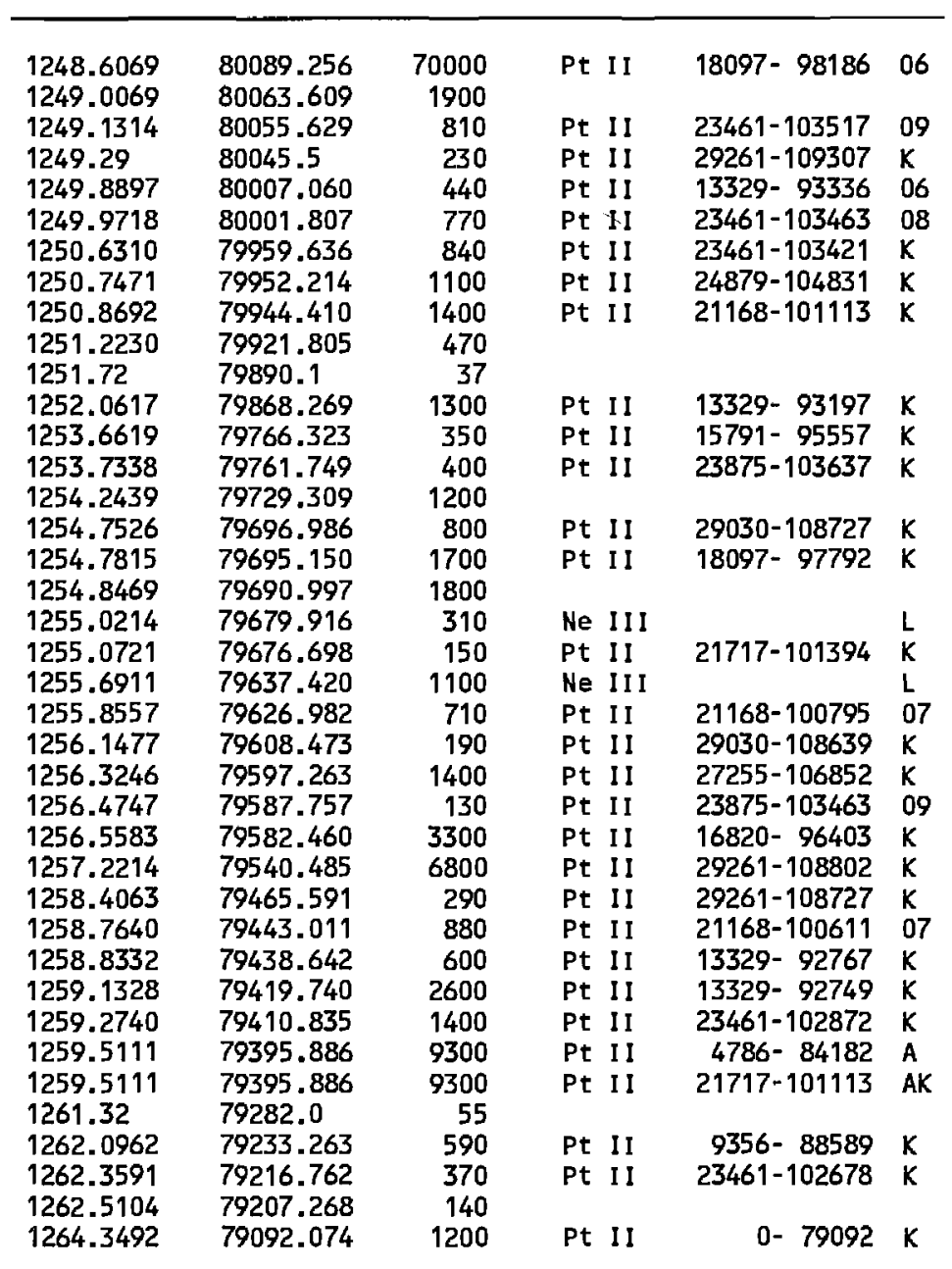

WAVELENGTH HAVE NUMBER INTENSITY CLASSIFICATION CODE

\begin{tabular}{|c|c|c|c|c|c|}
\hline $\begin{array}{l}1264.4898 \\
1264.5677 \\
1264.6904 \\
1264.8691 \\
1265.07\end{array}$ & $\begin{array}{l}79083.279 \\
79078.406 \\
79070.737 \\
79059.564\end{array}$ & $\begin{array}{c}130 \\
17000 \\
8800 \\
7200 \\
190\end{array}$ & $\begin{array}{ll}\text { Pt } & \text { II } \\
\text { Pt } & \text { II } \\
\text { Pt } & \text { I I } \\
\text { Pt } & \text { II }\end{array}$ & $\begin{array}{l}32237-111320 \\
21717-100795 \\
21168-100239 \\
23461-102520\end{array}$ & $\begin{array}{l}K \\
07 \\
06 \\
K\end{array}$ \\
\hline 1265.2074 & 79038.425 & 1400 & Pt II & $15791-94829$ & k \\
\hline 1265.7145 & 79006.759 & 3100 & Pt I I & $29030-108037$ & $\mathrm{~K}$ \\
\hline 1266.5706 & 78953.354 & 310 & Pt II & $23461-102414$ & 07 \\
\hline $\begin{array}{l}1266.8932 \\
1267.25\end{array}$ & $\begin{array}{l}78933.252 \\
78911.0\end{array}$ & 1100 & Pt II & $16820-95754$ & $\mathbf{P}$ \\
\hline 1267.5165 & 78894.435 & 310 & Pt II & $21717-100611$ & 07 \\
\hline 1268.3599 & 78841.975 & 450 & Pt II & $15791-94633$ & $\mathrm{~K}$ \\
\hline 1268.7589 & 78817.181 & 970 & Pt II & 9356- 88173 & K \\
\hline 1268.9912 & 78802.753 & 270 & Pt II & $23875-102678$ & K \\
\hline 1269.0742 & 78797.599 & $520 P$ & Pt II & $32918-111716$ & $k$ \\
\hline 1269.0973 & 78796.165 & 700 & Pt I I & $16820-95617$ & K \\
\hline 1269.4345 & 78775.234 & 530 & Pt II & $29261-108037$ & K \\
\hline 1269.8121 & 78751.809 & 540 & Pt II & 4786- 83538 & K \\
\hline 1271.34 & 78657.2 & 87 & & & \\
\hline 1271.7939 & 78629.094 & 15000 & Pt II & $21168-99797$ & 06 \\
\hline 1272.83 & 78565.1 & 44 & & & \\
\hline 1273.28 & 78537.3 & 94 & & & \\
\hline 1274.03 & 78491.1 & 66 & & & \\
\hline 1274.2091 & 78480.055 & 150 & Pt II & $15791-94271$ & $\mathrm{k}$ \\
\hline 1274.3665 & 78470.362 & 620 & Pt II & $27255-105726$ & $\mathrm{k}$ \\
\hline 1274.6091 & 78455.427 & 3800 & Pt II & $23461-101916$ & 07 \\
\hline 1274.6566 & 78452.502 & 900 & Pt II & $0-78452$ & $k$ \\
\hline 1274.7362 & 78447.604 & 300 & Pt II & $32237-110684$ & $\mathrm{k}$ \\
\hline 1274.9222 & 78436.159 & 250 & Pt II & $32918-111354$ & $\mathrm{k}$ \\
\hline 1275.4940 & 78400.996 & 890 & Pt II & $32237-110638$ & K \\
\hline 1276.2289 & 78355.850 & 730 & Pt II & $29030-107386$ & $\mathrm{k}$ \\
\hline 1276.4754 & 78340.719 & 320 & Pt II & $13329-91669$ & $\mathrm{k}$ \\
\hline 1277.0472 & 78305.641 & 700 & Pt II & $18097-96403$ & $k$ \\
\hline 1277.1026 & 78302.245 & 800 & Pt II & $21168-99471$ & $\mathrm{k}$ \\
\hline 1277.89 & 78254.0 & 120 & & & \\
\hline 1278.6998 & 78204.439 & 560 & & & \\
\hline $\begin{array}{l}1278.84 \\
1279.0832\end{array}$ & $\begin{array}{l}78195.9 \\
78180.997\end{array}$ & $\begin{array}{r}40 \\
510\end{array}$ & Pt II & $24879-103060$ & $\mathrm{k}$ \\
\hline 1279.4 & 78160.4 & 150 & & & \\
\hline
\end{tabular}




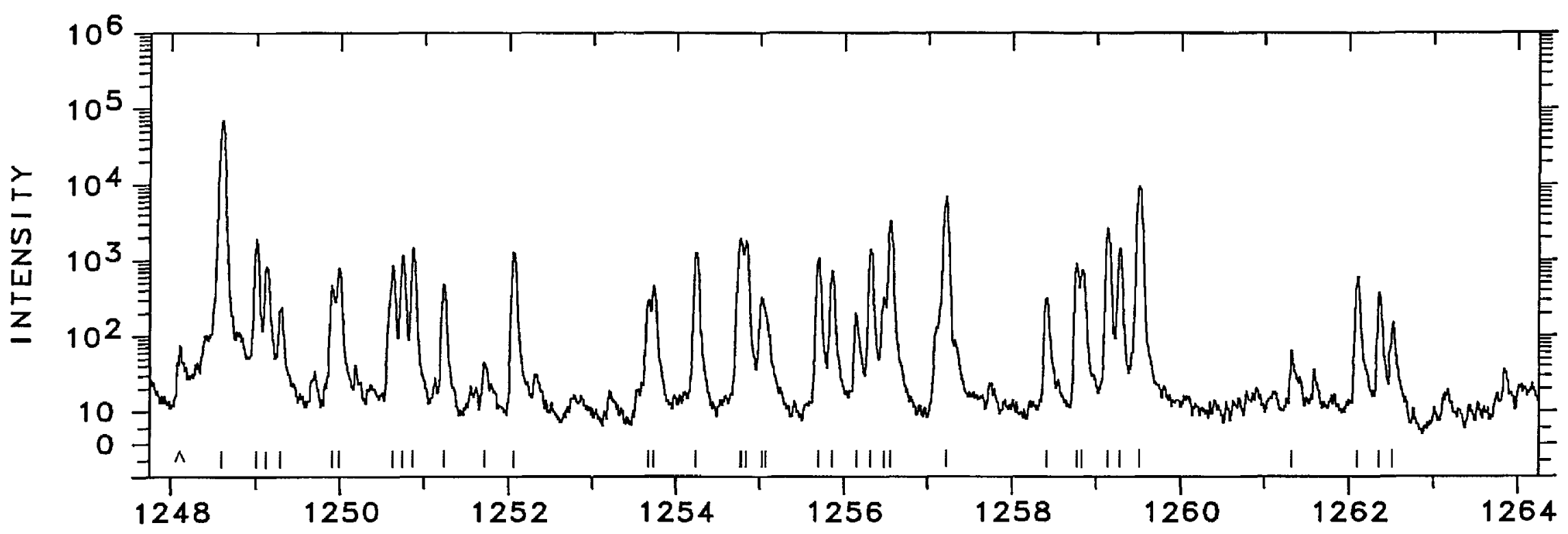

6

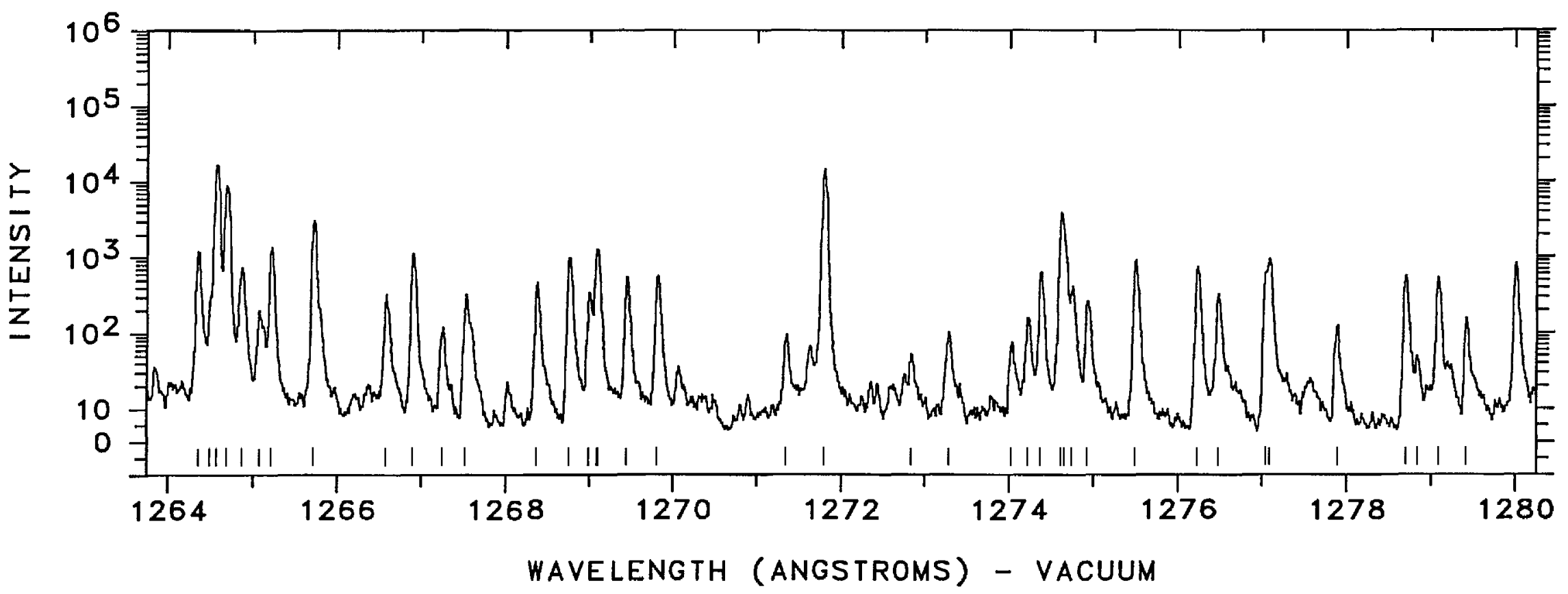




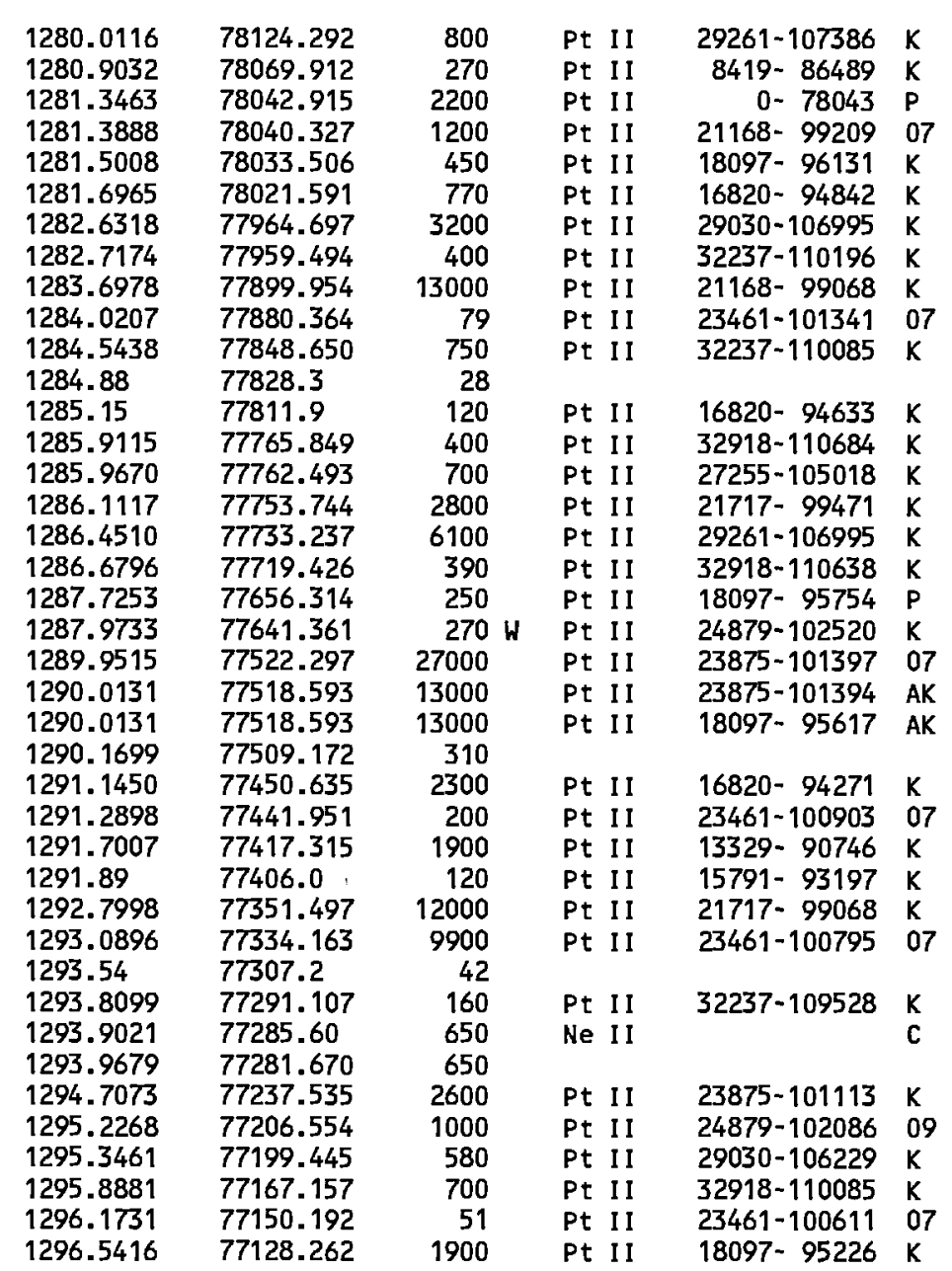

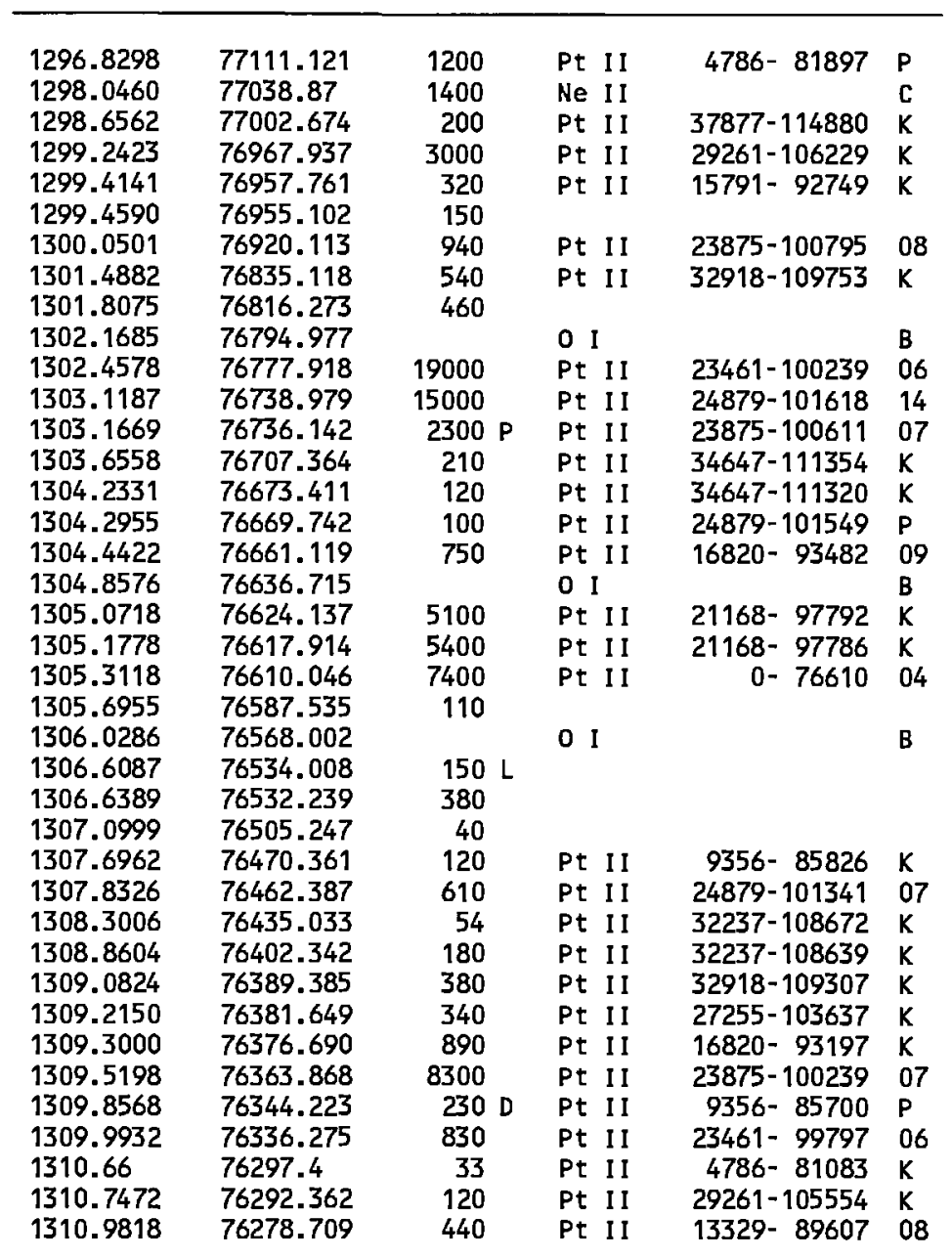




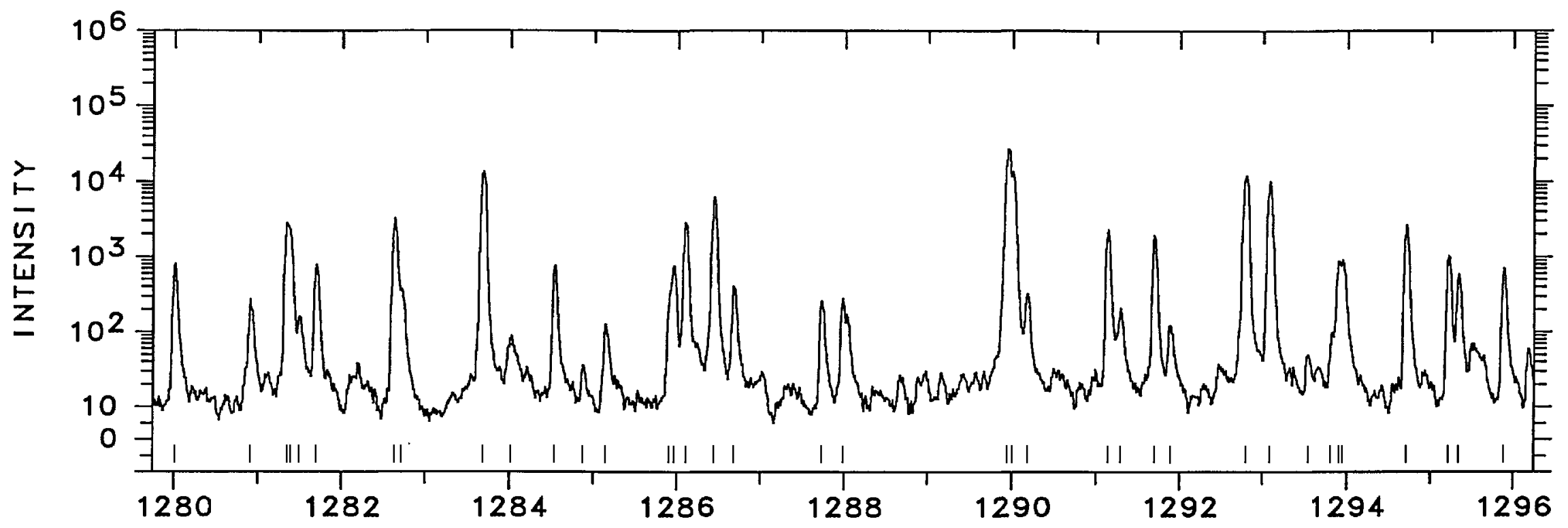

$N$

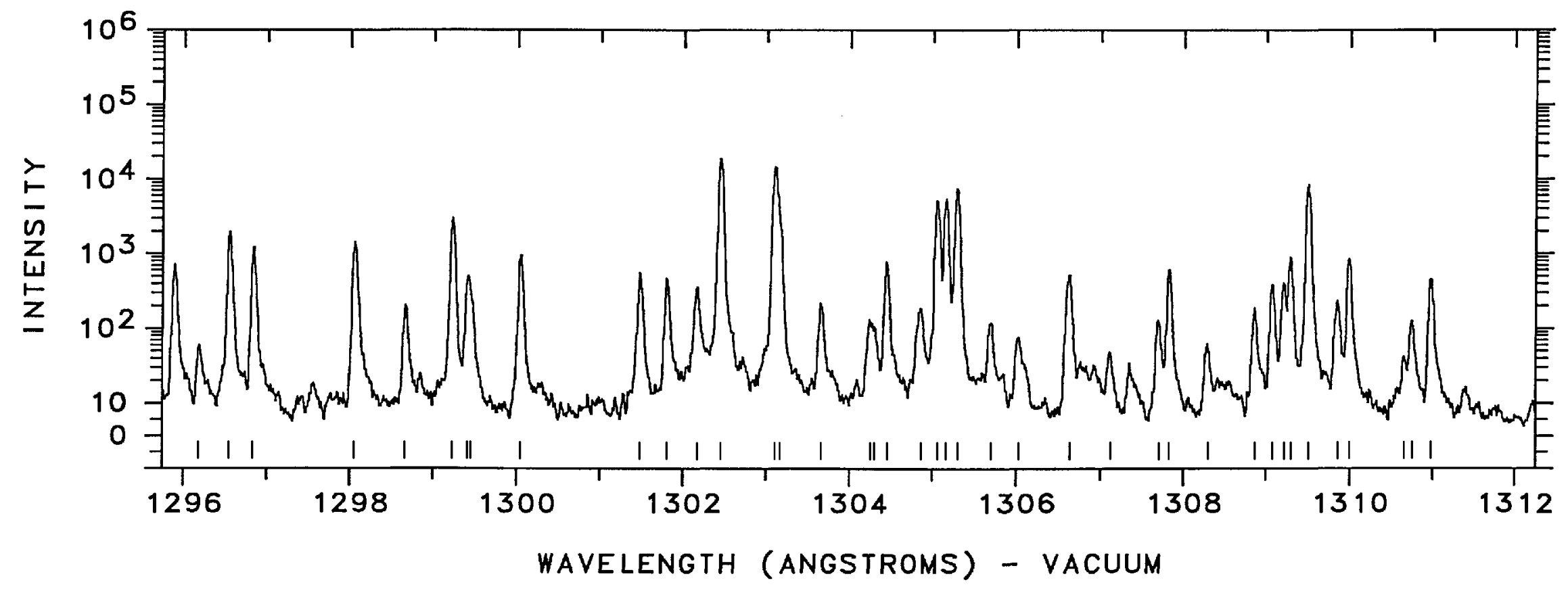




\begin{tabular}{|c|c|c|c|c|c|}
\hline $\begin{array}{l}1312.7858 \\
1312.9308 \\
1314.5907 \\
1315.40\end{array}$ & $\begin{array}{l}76173.889 \\
76165.477 \\
76069.304 \\
76022.5\end{array}$ & $\begin{array}{r}480 \\
1200 \\
1100 \\
42\end{array}$ & $\begin{array}{ll}\text { Pt II } \\
\text { Pt II } \\
\text { Pt II }\end{array}$ & $\begin{array}{l}18097-94271 \\
27255-103421 \\
21717-97786\end{array}$ & $\begin{array}{l}\mathrm{K} \\
\mathrm{K} \\
\mathrm{K}\end{array}$ \\
\hline 1315.5348 & 76014.713 & 2300 & Pt I I & $21168-97183$ & $\mathrm{~K}$ \\
\hline 1315.5810 & 76012.043 & $270 \mathrm{P}$ & Pt II & $29030-105042$ & $k$ \\
\hline & 75990.700 & 80 & Pt II & $34647-110638$ & $\mathrm{~K}$ \\
\hline $\begin{array}{l}1316.4501 \\
1316.7058\end{array}$ & $\begin{array}{l}75961.861 \\
75947.110\end{array}$ & $\begin{array}{l}410 \\
290\end{array}$ & $\begin{array}{l}\text { Pt II } \\
\text { Pt II }\end{array}$ & $\begin{array}{l}34647-110609 \\
16820-92767\end{array}$ & $\begin{array}{l}\mathrm{K} \\
\mathrm{K}\end{array}$ \\
\hline 1316.8913 & 75936.412 & 71 & & & \\
\hline 1317.0348 & 75928.138 & 290 & Pt I I & $16820-92749$ & $\mathrm{~K}$ \\
\hline 1317.2032 & 75918.431 & 740 & Pt II & $32237-108155$ & K \\
\hline 1317.3856 & 75907.919 & 100 & Pt II & $37877-113785$ & K \\
\hline $\begin{array}{l}1319.1080 \\
1319.1412\end{array}$ & $\begin{array}{l}75808.804 \\
75806.896\end{array}$ & $\begin{array}{r}84 U \\
270\end{array}$ & Pt II & $32918-108727$ & $\mathrm{k}$ \\
\hline 1319.2429 & 75801.052 & 140 & Pt I I & $29030-104831$ & AK \\
\hline 1319.2429 & 75801.052 & 140 & Pt II & $32237-108038$ & AK \\
\hline 1319.8553 & 75765.881 & 340 & Pt II & $13329-89095$ & $\mathrm{~K}$ \\
\hline 1320.1754 & 75747.508 & 3000 & Pt II & $23461-99209$ & 07 \\
\hline 1320.6427 & 75720.708 & 590 & Pt II & $32918-108639$ & $\mathrm{~K}$ \\
\hline 1322.8372 & 75595.092 & 7500 & Pt II & $23875-99471$ & AK \\
\hline 1322.8372 & 75595.092 & 7500 & Pt I I & $29030-104625$ & AK \\
\hline 1323.0765 & 75581.422 & 710 & Pt I I & $0-75581$ & 06 \\
\hline 1323.2831 & 75569.619 & 8900 & Pt II & $29261-104831$ & $\mathrm{~K}$ \\
\hline 1323.5388 & 75555.020 & 260 & Pt II & $34647-110202$ & $\mathrm{~K}$ \\
\hline 1323.6402 & 75549.232 & 280 & Pt II & $34647-110196$ & $\mathrm{~K}$ \\
\hline 1324.8562 & 75479.890 & 1900 & Pt II & $15791-91271$ & K \\
\hline $\begin{array}{l}1325.0971 \\
1325.6560\end{array}$ & 75466.168 & 2500 & Pt I I & $21717-97183$ & $\mathrm{~K}$ \\
\hline 1326.0723 & 75410.670 & 1600 & Pt I I & $4786-80197$ & K \\
\hline 1326.1916 & 75403.886 & 1700 & Pt II & $32918-108322$ & $\mathrm{k}$ \\
\hline 1326.5362 & 75384.299 & 30 & Pt I I & $18097-93482$ & 09 \\
\hline 1326.8620 & 75365.788 & 810 & Pt II & $0-75365$ & $\mathrm{~K}$ \\
\hline 1326.9041 & 75363.397 & 250 & Pt I I & $29261-104625$ & $\mathrm{~K}$ \\
\hline 1327.0301 & 75356.241 & 250 & Pt II & $23461-98817$ & 08 \\
\hline
\end{tabular}

\begin{tabular}{llrrrl}
\hline & & & & & \\
1327.0833 & 75353.220 & 800 & Pt I I & $24879-100232$ & $\mathrm{~K}$ \\
1327.1146 & 75351.443 & 44 U & Pt I I & $32237-107588$ & $\mathrm{~K}$ \\
1327.4314 & 75333.458 & 18000 & Pt I I & $23875-99209$ & 08 \\
1328.7227 & 75260.248 & 1600 & Pt I I & $13329-88589$ & $\mathrm{~K}$ \\
1329.1748 & 75234.649 & 830 & Pt I I & $21168-96403$ & $\mathrm{~K}$ \\
1329.3385 & 75225.385 & 320 & Pt I I & $15791-91016$ & $\mathrm{~K}$ \\
1329.9067 & 75193.245 & 2900 & Pt I I & $23875-99068$ & $\mathrm{~K}$ \\
1330.0547 & 75184.880 & 7700 & Pt I I & $0-75184$ & 04 \\
1330.79 & 75143.3 & 42 & & & \\
1331.2263 & 75118.708 & 440 & Pt I I & $32918-108037$ & $\mathrm{~K}$ \\
1331.5606 & 75099.849 & 270 & Pt I I & $18097-93197$ & $\mathrm{~K}$ \\
1334.1414 & 74954.574 & 1100 & Pt I I & $32237-107191$ & $\mathrm{~K}$ \\
1334.37 & 74941.7 & 49 & & & \\
1335.6420 & 74870.362 & 120 & & & \\
1335.7277 & 74865.558 & & C I I & & \\
1336.0355 & 74848.311 & 230 & & & \\
1336.2452 & 74836.564 & 670 & Pt I I & $36484-111320$ & $\mathrm{~K}$ \\
1337.2361 & 74781.110 & 530 & Pt I I & $13329-88110$ & $\mathrm{~K}$ \\
1337.8657 & 74745.916 & 4900 & Pt I I & $0-74745$ & 05 \\
1338.2103 & 74726.670 & 850 & Pt I I & $21717-96443$ & $\mathrm{~K}$ \\
1338.5127 & 74709.788 & 54 & & & \\
1338.5821 & 74705.915 & 64 & & & \\
1338.95 & 74685.4 & 46 & & & \\
1339.2328 & 74669.617 & 31 & Pt I I & $32918-107588$ & $\mathrm{~K}$ \\
1339.3918 & 74660.753 & 320 & Pt I I & $34647-109307$ & $\mathrm{~K}$ \\
1340.1393 & 74619.107 & 3000 & Pt I I & $0-74619$ & 05 \\
1340.7443 & 74585.437 & 610 & Pt I I & $21168-95754$ & $\mathrm{P}$ \\
1341.3300 & 74552.869 & 920 & Pt I I & $8419-82972$ & $\mathrm{P}$ \\
1341.9630 & 74517.703 & 40 & & & \\
1342.2101 & 74503.984 & 68 & & & \\
1342.5224 & 74486.653 & 1500 & Pt I I & $29030-103517$ & 11 \\
1343.18 & 74450.2 & 21 & Pt I I & $16820-91271$ & $\mathrm{~K}$ \\
1343.3742 & 74439.423 & 650 & Pt I I & $18097-92537$ & $\mathrm{~K}$ \\
1343.4932 & 74432.831 & 930 & Pt I I & $29030-103463$ & 09
\end{tabular}




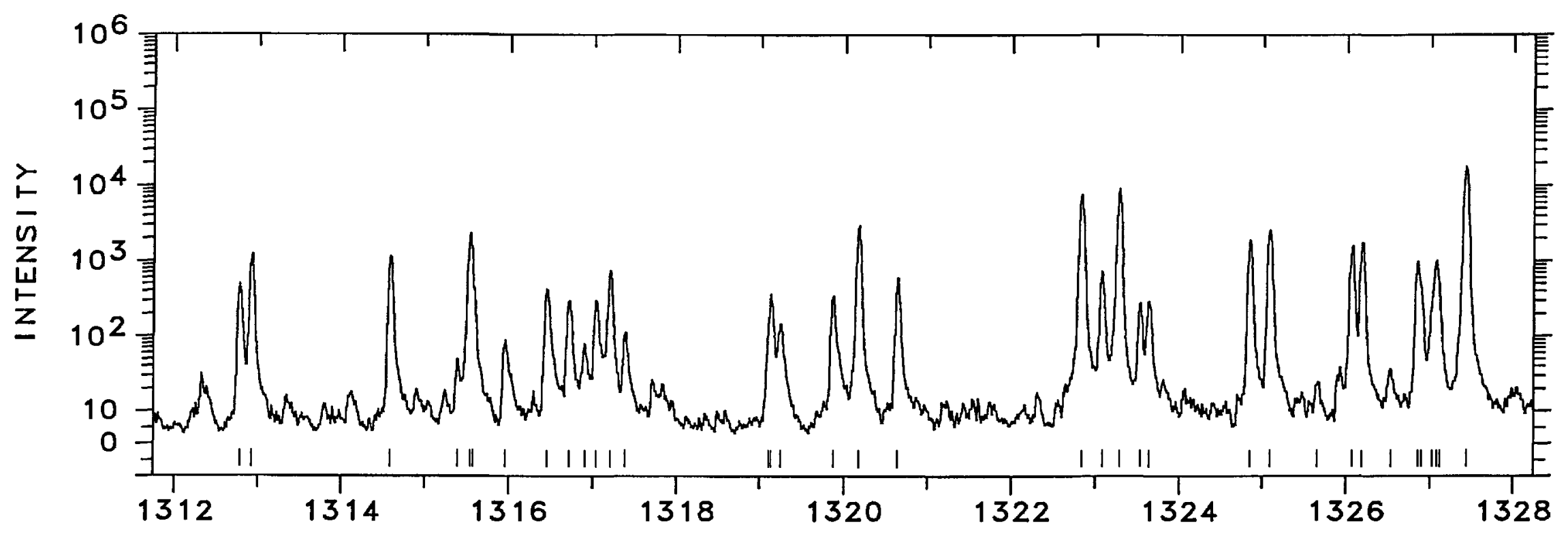

w

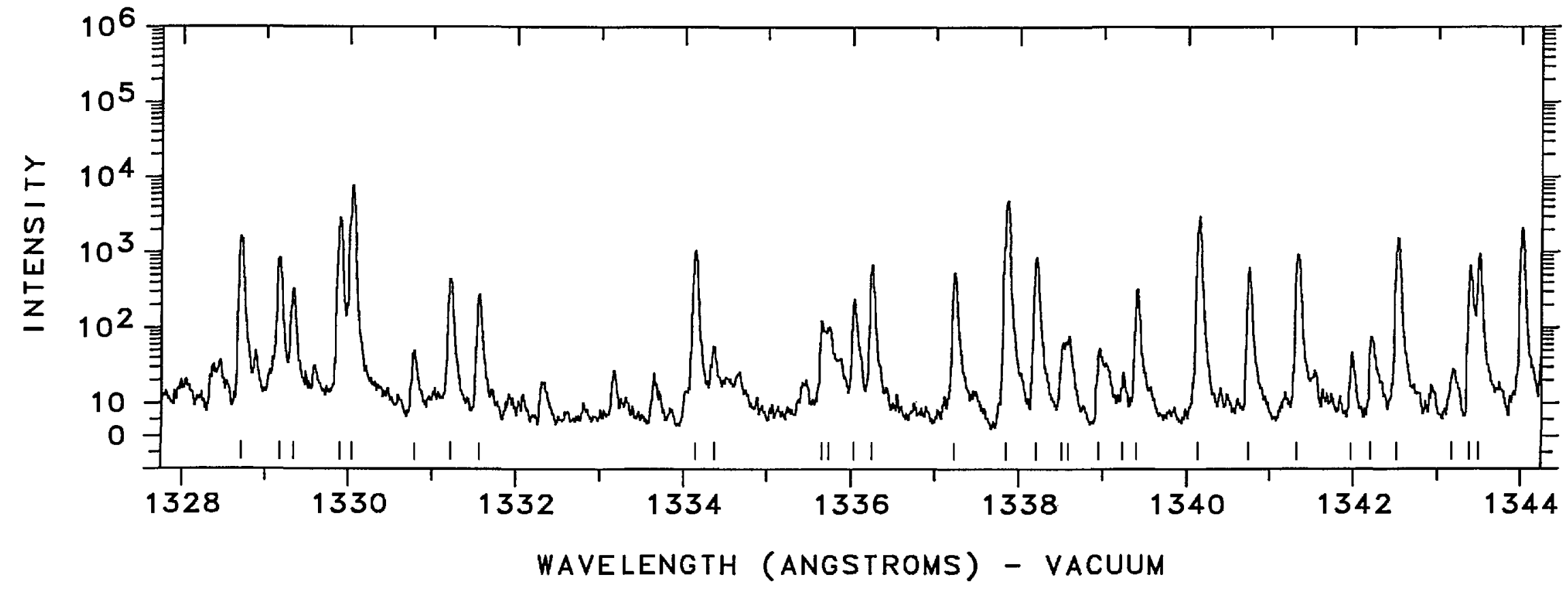




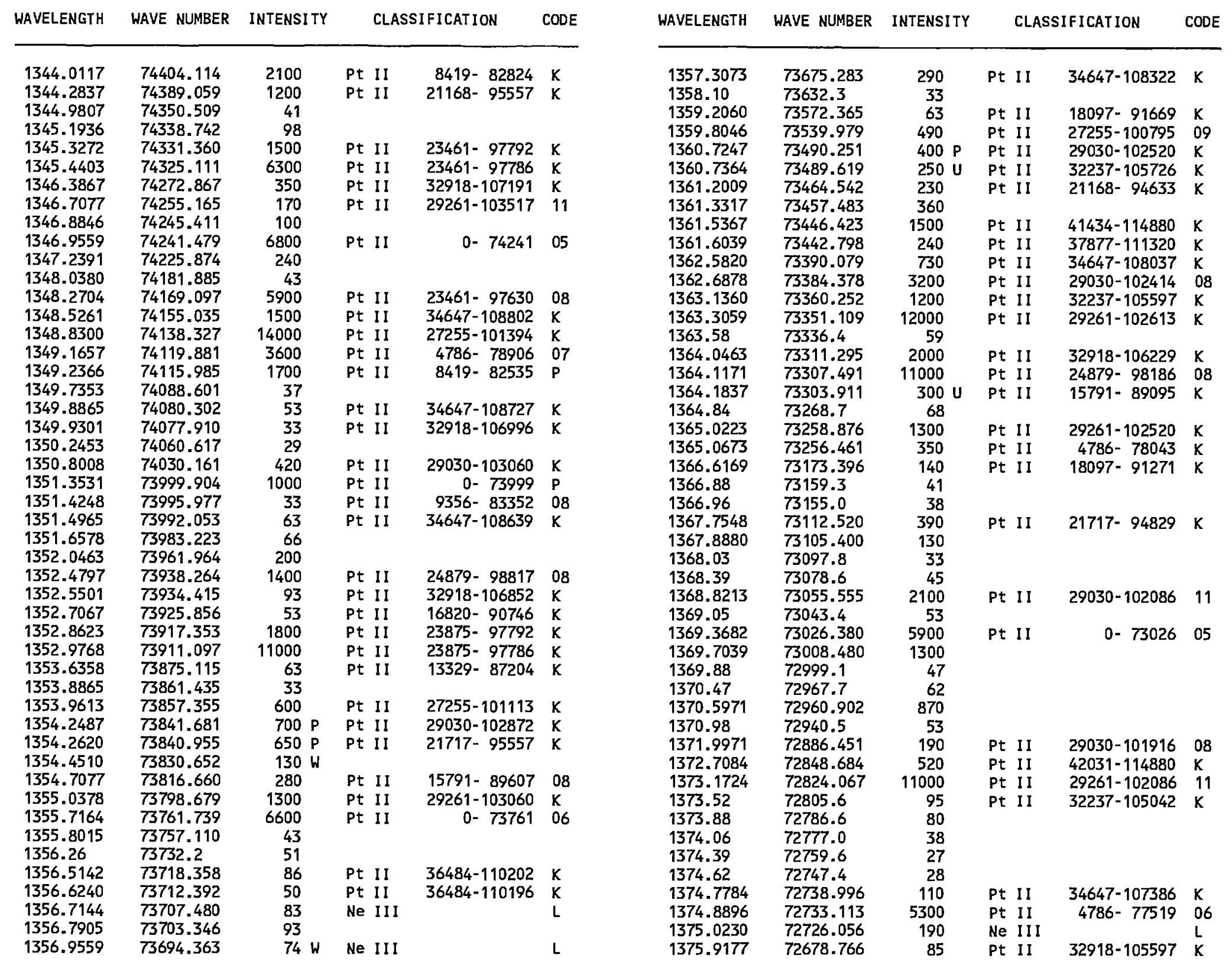




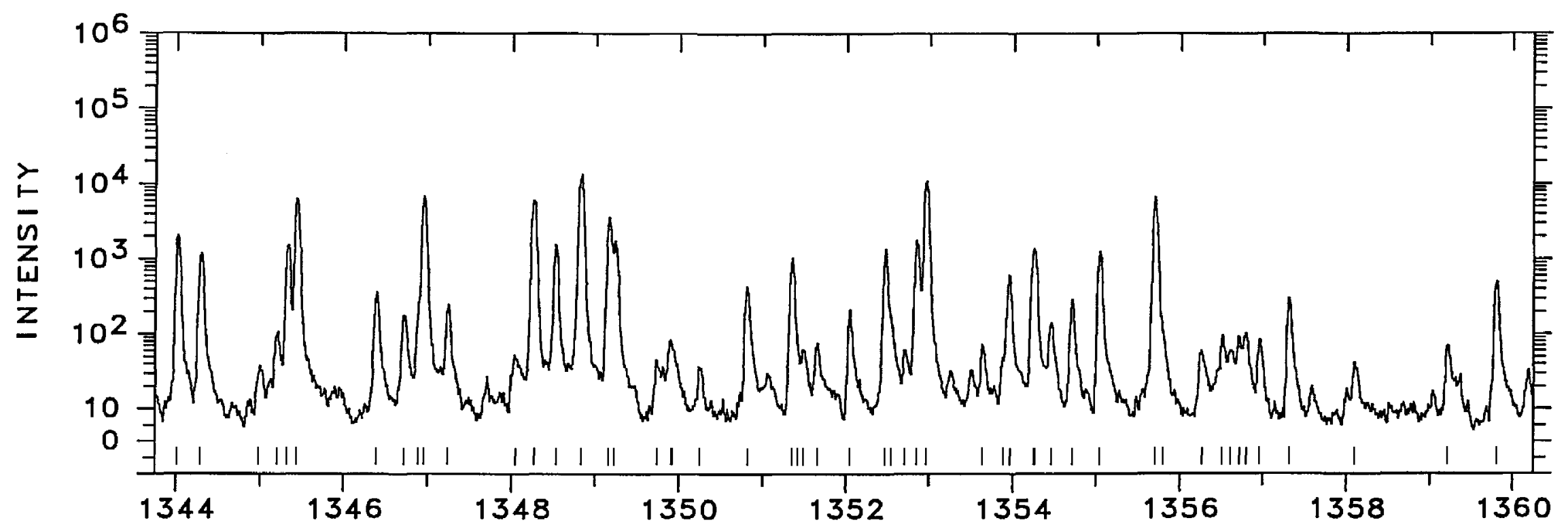

$\tilde{n}$

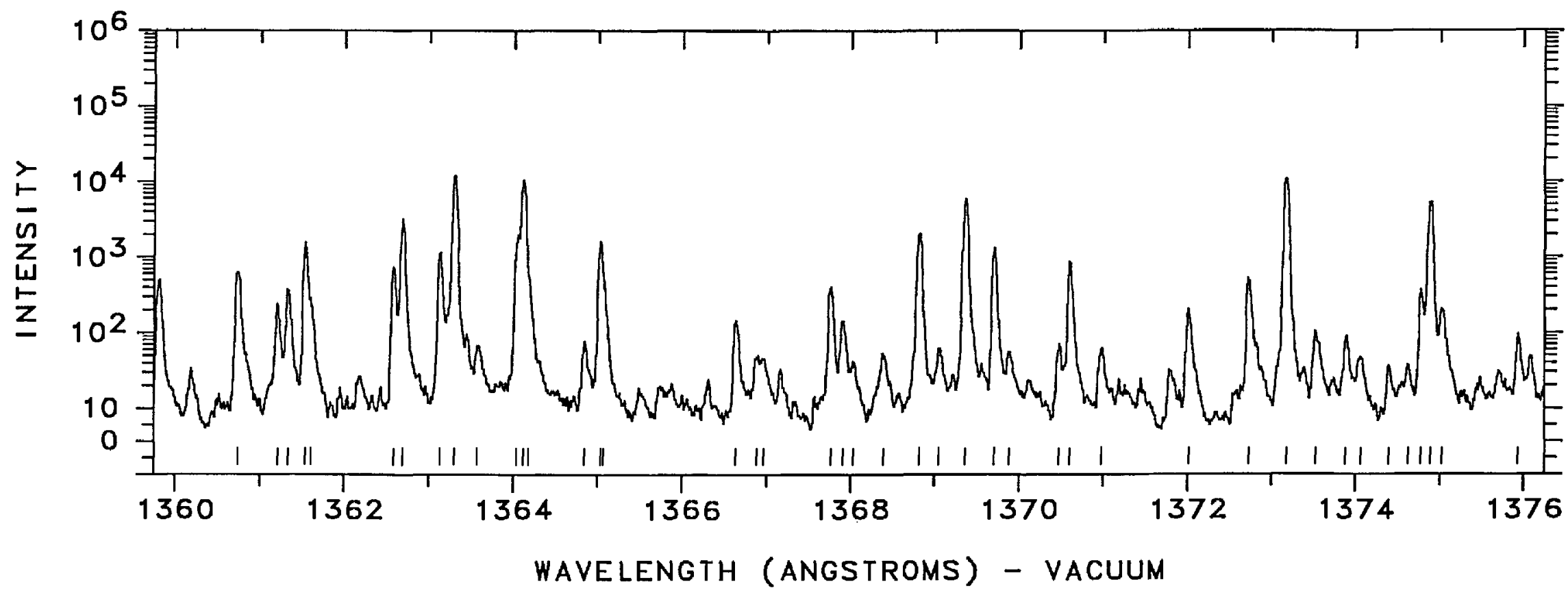


WAVELENGTH WAVE NUMBER INTENSITY

CLASSIF ICATION

CODE

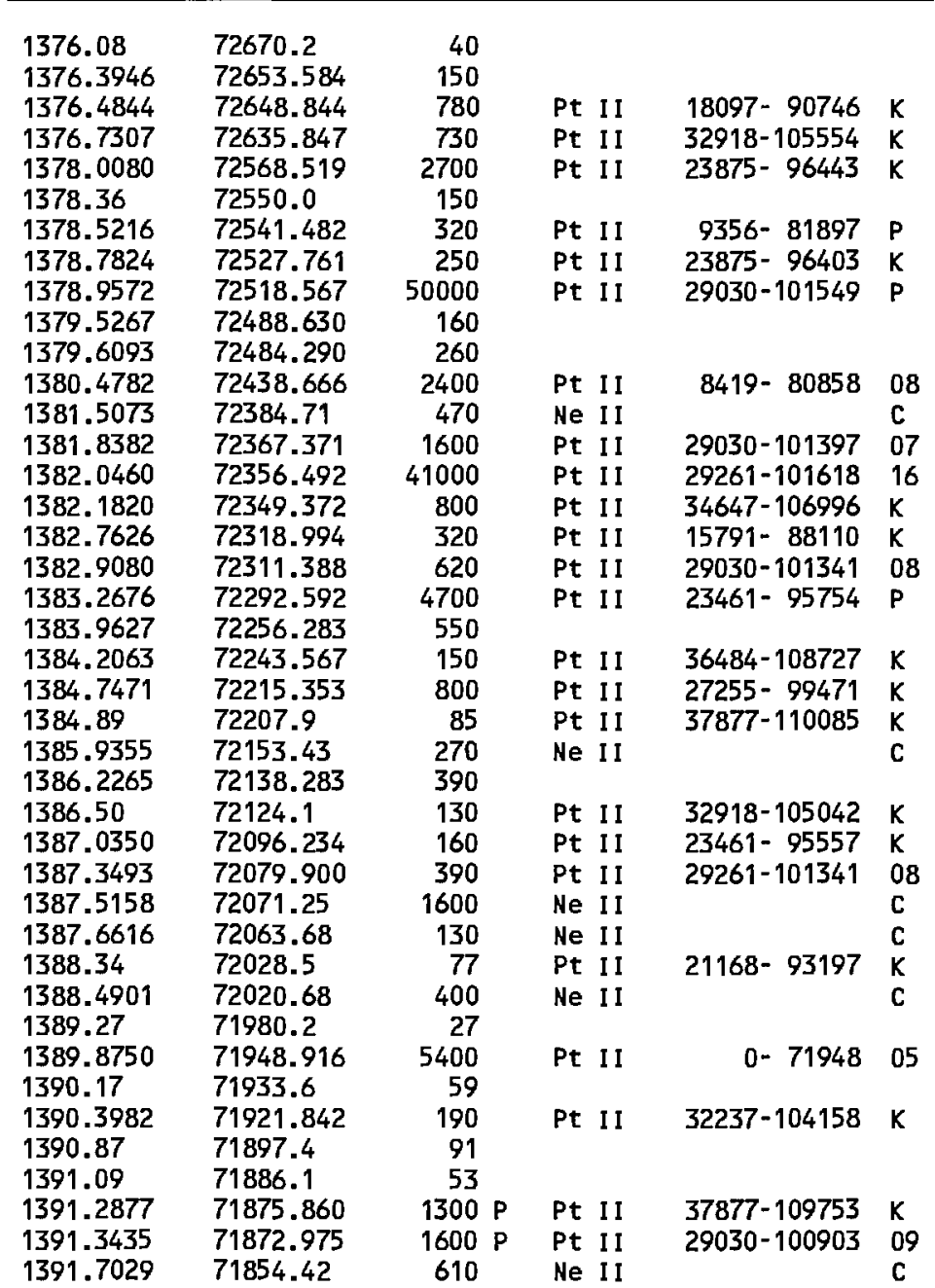

N
WAVELENGTH WAVE NUMBER INTENSITY CLASSIFICATION CODE

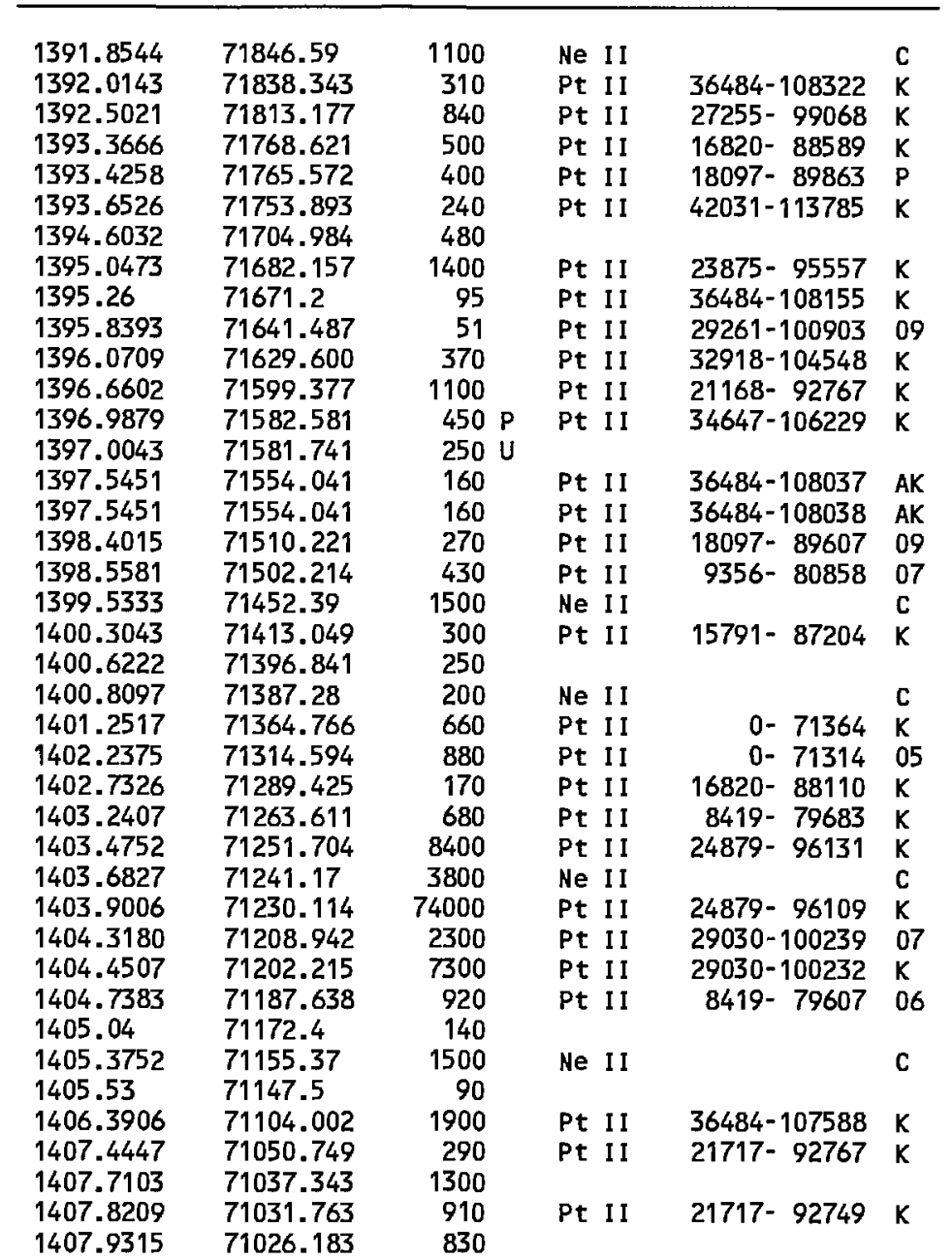




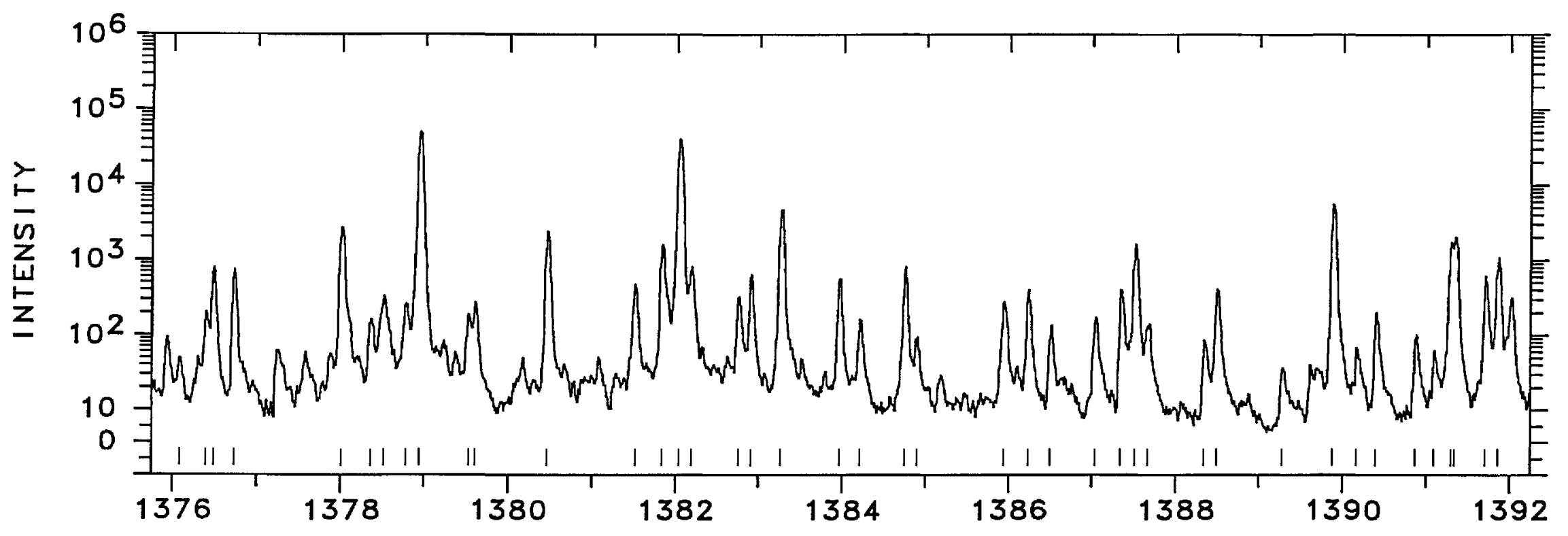

$N$

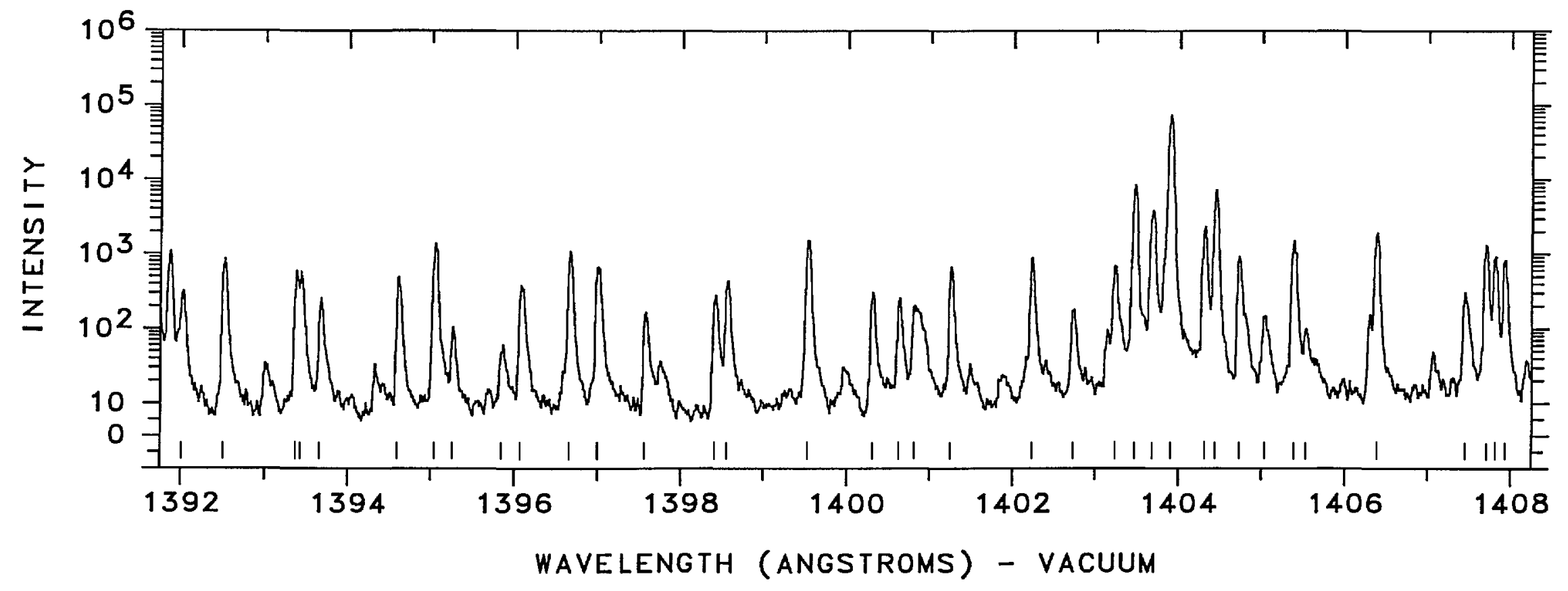




\begin{tabular}{|c|c|c|c|c|c|}
\hline $\begin{array}{l}1409.0315 \\
1409.3589 \\
1409.4407 \\
1409.7467 \\
1410.1346\end{array}$ & $\begin{array}{l}70970.734 \\
70954.247 \\
70950.129 \\
70934.73 \\
70915.216\end{array}$ & $\begin{array}{r}7400 \\
300 \\
840 \\
630 \\
18000\end{array}$ & $\begin{array}{ll}\mathrm{Pt} & \text { II } \\
\mathrm{Pt} & \text { II } \\
\mathrm{Pt} & \text { II } \\
\mathrm{Ne} & \text { I I }\end{array}$ & $\begin{array}{r}29261-100232 \\
23875-94829 \\
34647-105597\end{array}$ & $\begin{array}{l}K \\
K \\
K \\
C\end{array}$ \\
\hline $\begin{array}{l}1410.2951 \\
1410.50 \\
1411.09\end{array}$ & $\begin{array}{l}70907.146 \\
70896.8 \\
70867.2\end{array}$ & $\begin{array}{r}1700 \\
87 \\
48\end{array}$ & Pt II & $34647-105554$ & K \\
\hline 1411.3059 & 70856.36 & 160 & Ne II & & C \\
\hline $\begin{array}{l}1411.62 \\
1411.76 \\
1411.91\end{array}$ & $\begin{array}{l}70840.6 \\
70833.6 \\
70826.0\end{array}$ & $\begin{array}{r}120 \\
120 \\
62\end{array}$ & Pt II & $9356-80197$ & K \\
\hline $\begin{array}{l}1412.2278 \\
1412.5350 \\
1413.10\end{array}$ & $\begin{array}{l}70810.106 \\
70794.706 \\
70766.4\end{array}$ & $\begin{array}{r}180 \\
410 \\
97\end{array}$ & $\begin{array}{l}\text { Pt II } \\
\text { Pt II }\end{array}$ & $\begin{array}{l}23461-94271 \\
37877-108672\end{array}$ & $\begin{array}{l}K \\
K\end{array}$ \\
\hline $\begin{array}{l}1413.1988 \\
1413.2736 \\
1413.6768 \\
1413.9570 \\
1414.3241\end{array}$ & $\begin{array}{l}70761.453 \\
70757.708 \\
70737.526 \\
70723.51 \\
70705.152\end{array}$ & $\begin{array}{r}170 \\
1100 \\
3800 \\
1100 \\
390\end{array}$ & $\begin{array}{l}\text { Pt II } \\
\text { Pt II } \\
\text { Pt II } \\
\text { Ne II }\end{array}$ & $\begin{array}{l}37877-108639 \\
23875-94633 \\
24879-95617\end{array}$ & $\begin{array}{l}K \\
K \\
K \\
C\end{array}$ \\
\hline $\begin{array}{l}1414.4573 \\
1415.00\end{array}$ & $\begin{array}{l}70698.493 \\
70671.4\end{array}$ & $\begin{array}{r}230 \\
64\end{array}$ & Pt II & $15791-86489$ & K \\
\hline $\begin{array}{l}1415.7144 \\
1416.20 \\
1416.30\end{array}$ & $\begin{array}{l}70635.71 \\
70611.5 \\
70606.5\end{array}$ & $\begin{array}{r}1200 \\
82 \\
78\end{array}$ & Ne II & & C \\
\hline 1416.4593 & 70598.571 & 69 & Pt II & $32918-103517$ & 12 \\
\hline $\begin{array}{l}1417,5400 \\
1417,8186\end{array}$ & $\begin{array}{l}70544.749 \\
70530.885\end{array}$ & $\begin{array}{r}400 \\
4400\end{array}$ & $\begin{array}{l}\text { Pt II } \\
\text { Pt II }\end{array}$ & $\begin{array}{l}32918-103463 \\
27255-97786\end{array}$ & $\begin{array}{l}11 \\
K\end{array}$ \\
\hline 1418.1875 & 70512.538 & 200 & Pt II & $36484-106996$ & K \\
\hline $\begin{array}{l}1418.3779 \\
1418.7471\end{array}$ & $\begin{array}{l}70503.08 \\
70484.72\end{array}$ & $\begin{array}{l}3100 \\
2100\end{array}$ & $\begin{array}{l}\text { Ne II } \\
\text { Ne II }\end{array}$ & & $\begin{array}{l}\text { C } \\
\text { C }\end{array}$ \\
\hline $\begin{array}{l}1418.6908 \\
1419.6208 \\
1420.08\end{array}$ & $\begin{array}{l}70482.202 \\
70441.346 \\
70418.6\end{array}$ & $\begin{array}{l}550 \\
150\end{array}$ & Pt II & $32237-102678$ & $\mathrm{~K}$ \\
\hline $\begin{array}{l}1420.5511 \\
1420.89\end{array}$ & $\begin{array}{l}70395.215 \\
70378.4\end{array}$ & $\begin{array}{r}2000 \\
82\end{array}$ & Pt II & $34647-105042$ & K \\
\hline 1421.0852 & 70368.758 & 350 & Pt II & $36484-106852$ & K \\
\hline $\begin{array}{l}1421.5372 \\
1421.80\end{array}$ & 70346.383 & 2800 & & & \\
\hline 1422.84 & 70282.0 & 110 & & & \\
\hline
\end{tabular}

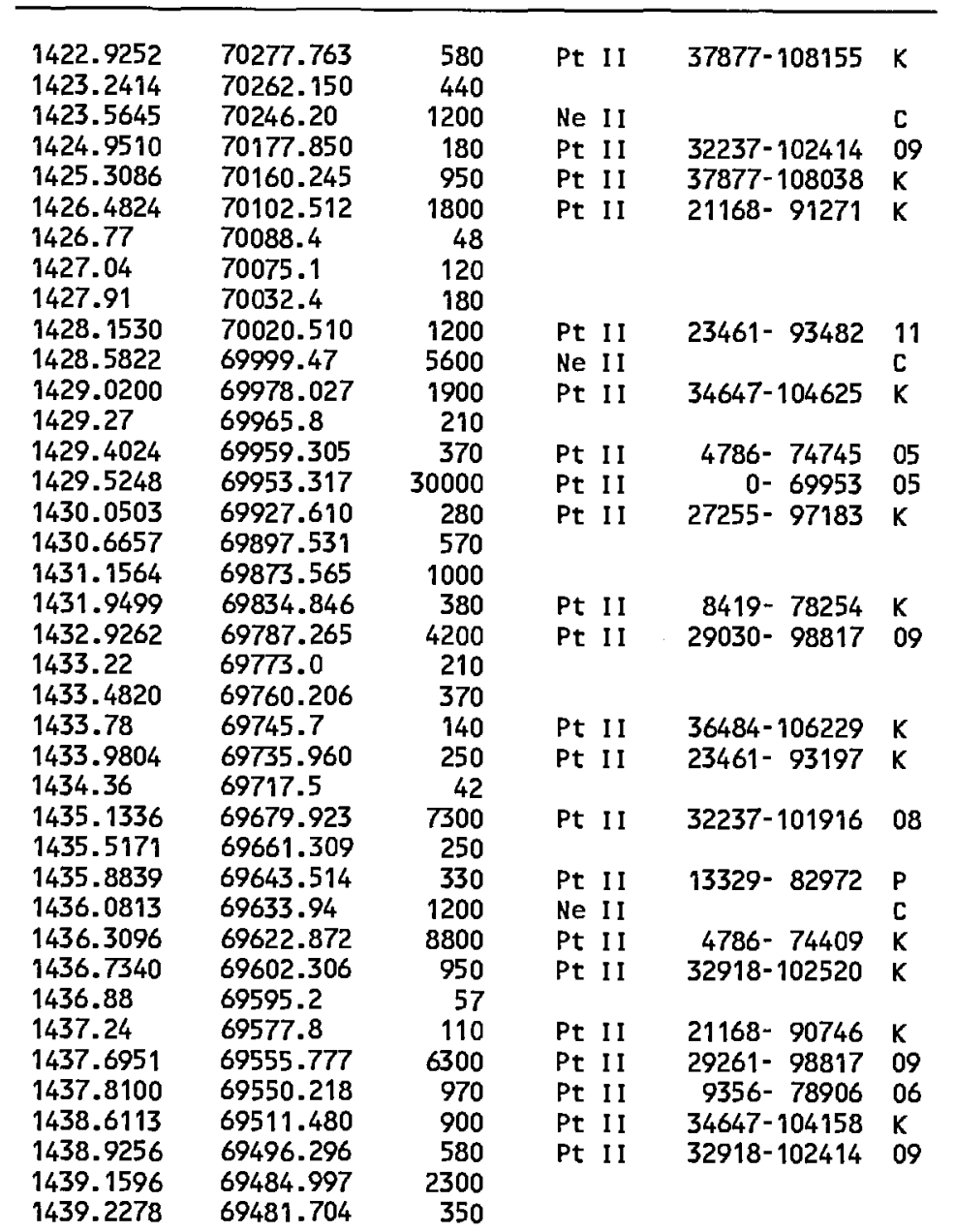




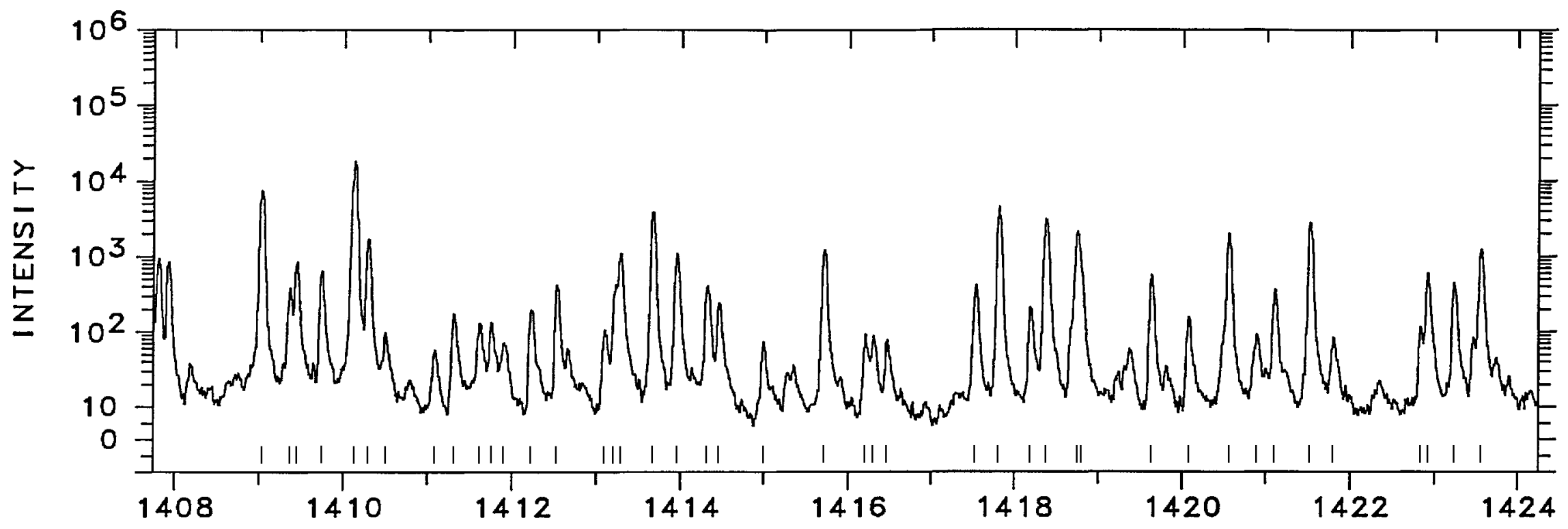

No

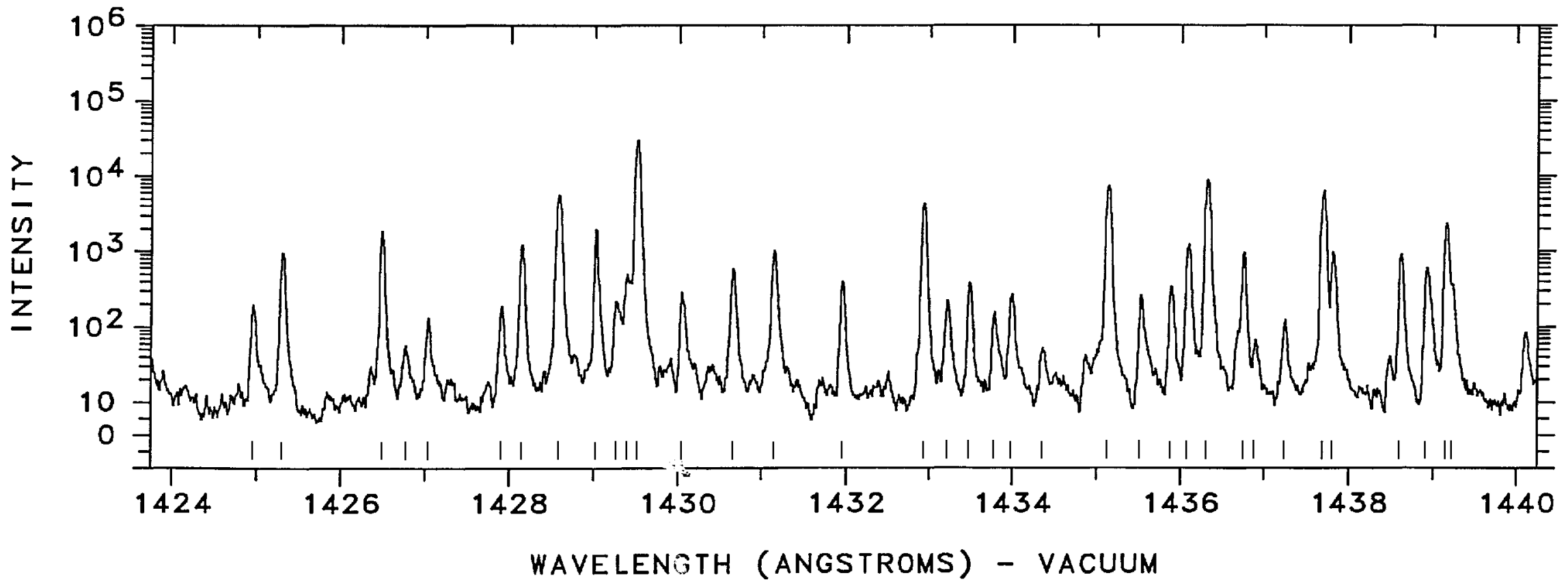


WAVELENGTH WAVE NUMBER INTENSITY

CLASSIFICATION

CODE

\begin{tabular}{llr}
\hline & & \\
1440.10 & 69439.6 & 72 \\
1440.51 & 69419.9 & 37 \\
1440.6635 & 69412.462 & 1000 \\
1441.1876 & 69387.22 & 200 \\
1441.28 & 69382.8 & 130 \\
1442.0911 & 69343.747 & 590 \\
1442.57 & 69320.7 & 71 \\
1442.7150 & 69313.759 & 230 \\
1442.8676 & 69306.428 & 360 \\
1443.0133 & 69299.431 & 360 \\
1443.25 & 69288.1 & 330 \\
1444.0351 & 69250.394 & 2100 \\
1444.38 & 69233.9 & 40 \\
1444.71 & 69218.0 & 100 \\
1445.01 & 69203.7 & 240 \\
1445.34 & 69187.9 & 53 \\
1445.9958 & 69156.492 & 2600 \\
1446.2820 & 69142.809 & 6900 \\
1446.7921 & 69118.431 & 420 \\
1446.9019 & 69113.186 & 2300 \\
1447.43 & 69088.0 & 37 \\
1447.8030 & 69070.171 & 16000 \\
1448.04 & 69058.9 & 190 \\
1449.16 & 69005.5 & 98 \\
1449.8015 & 68974.960 & 2200 \\
1449.8015 & 68974.960 & 2200 \\
1450.22 & 68955.1 & 120 \\
1450.55 & 68939.4 & 83 \\
1450.8523 & 68925.004 & 320 \\
1451.5382 & 68892.434 & 950 \\
1451.8840 & 68876.026 & 1800 \\
1452.0129 & 68869.911 & 1300 \\
1452.49 & 68847.3 & 300 \\
1453.1486 & 68816.089 & 350 \\
1453.5678 & 68796.241 & 870 \\
1453.81 & 68784.8 & 36 \\
1454.0865 & 68771.700 & 350 \\
1454.1586 & 68768.290 & 940 \\
1454.2866 & 68762.237 & 12000 \\
1454.2866 & 68762.237 & 12000 \\
1454.72 & 68741.8 & 32 \\
1454.97 & 68729.9 & 110 \\
1455.8872 & 68686.640 & 1600 \\
& &
\end{tabular}

$\begin{array}{lll}\text { Pt II } 36484-105896 & K \\ \text { Ne II } & & \text { C }\end{array}$

Pt II 8419- $77763 \mathrm{~K}$

Pt II $37877-107191 \quad K$

Pt II 23461-92767 K

Pt II 21717-91016 K

$\begin{array}{lll}\text { Pt II } & 23461-92749 & K \\ \text { Pt II } & 41434-110684 & K\end{array}$

Pt II 41434-110638 K

Pt II 27255-96443 K

Pt II 29030-98186 09

Pt II 24879- $94022 P$

Pt II 8419- $77538 \mathrm{~K}$

Pt II 36484-105597 K

Pt II 36484-105554 K

Pt II 16820-85826 K

Pt II 4786- 73761 A

Pt II 37877-106852 AK

Pt II 29261- $98186 \quad 09$

Pt II 23875- $92767 \mathrm{~K}$

Pt II 34647-103463 11

Pt II 41434-110202 K

Pt II 29030- 97792 AK

Pt II 41434-110196 AK

Pt II 9356- 78043 P $\begin{array}{lll}\text { Pt II } & 32237-101113 & K \\ \text { Pt II } & 34647-103517 & 12\end{array}$

WAVELENGTH WAVE NUMBER INTENSITY

CLASSIFICATION

CODE

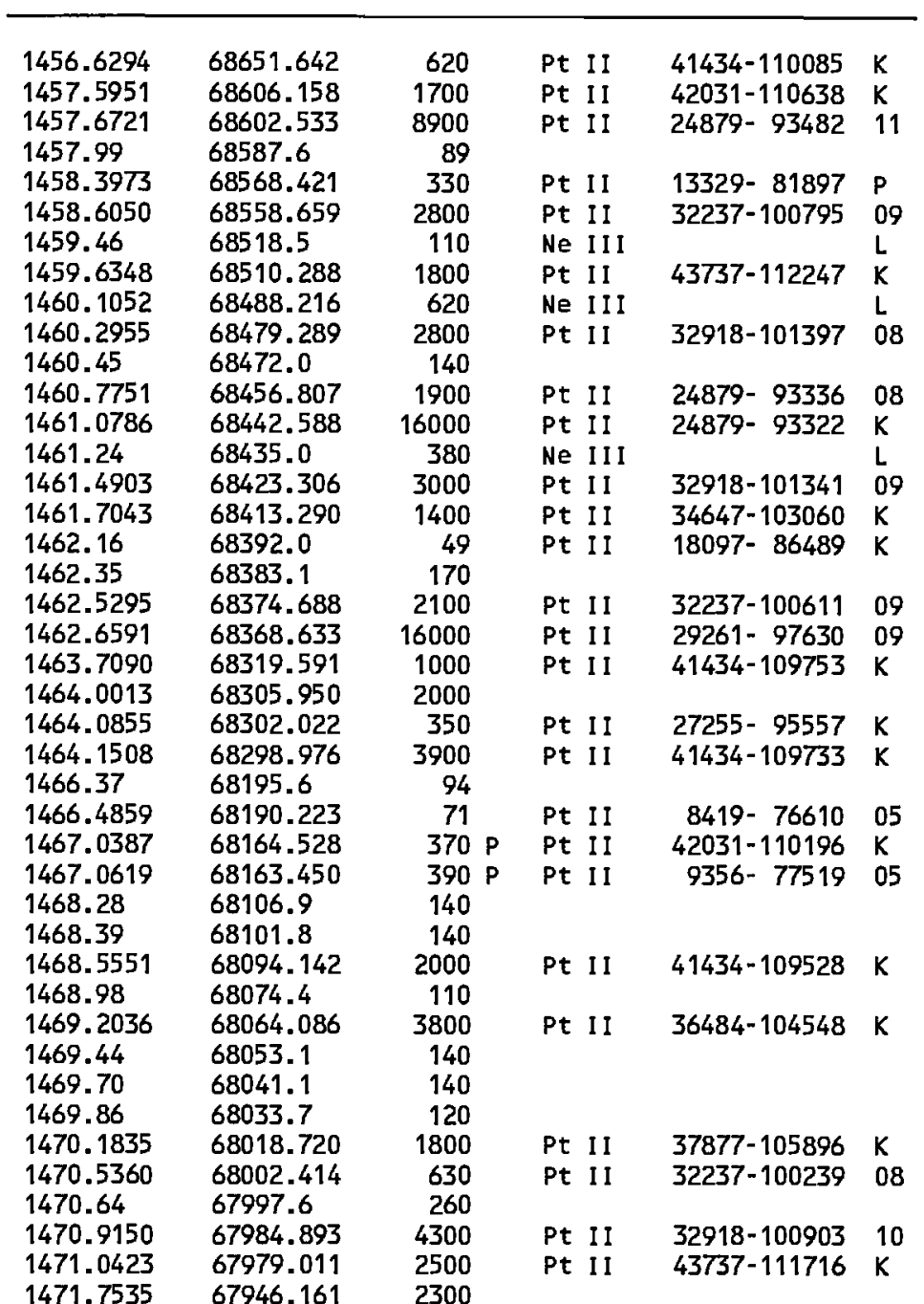



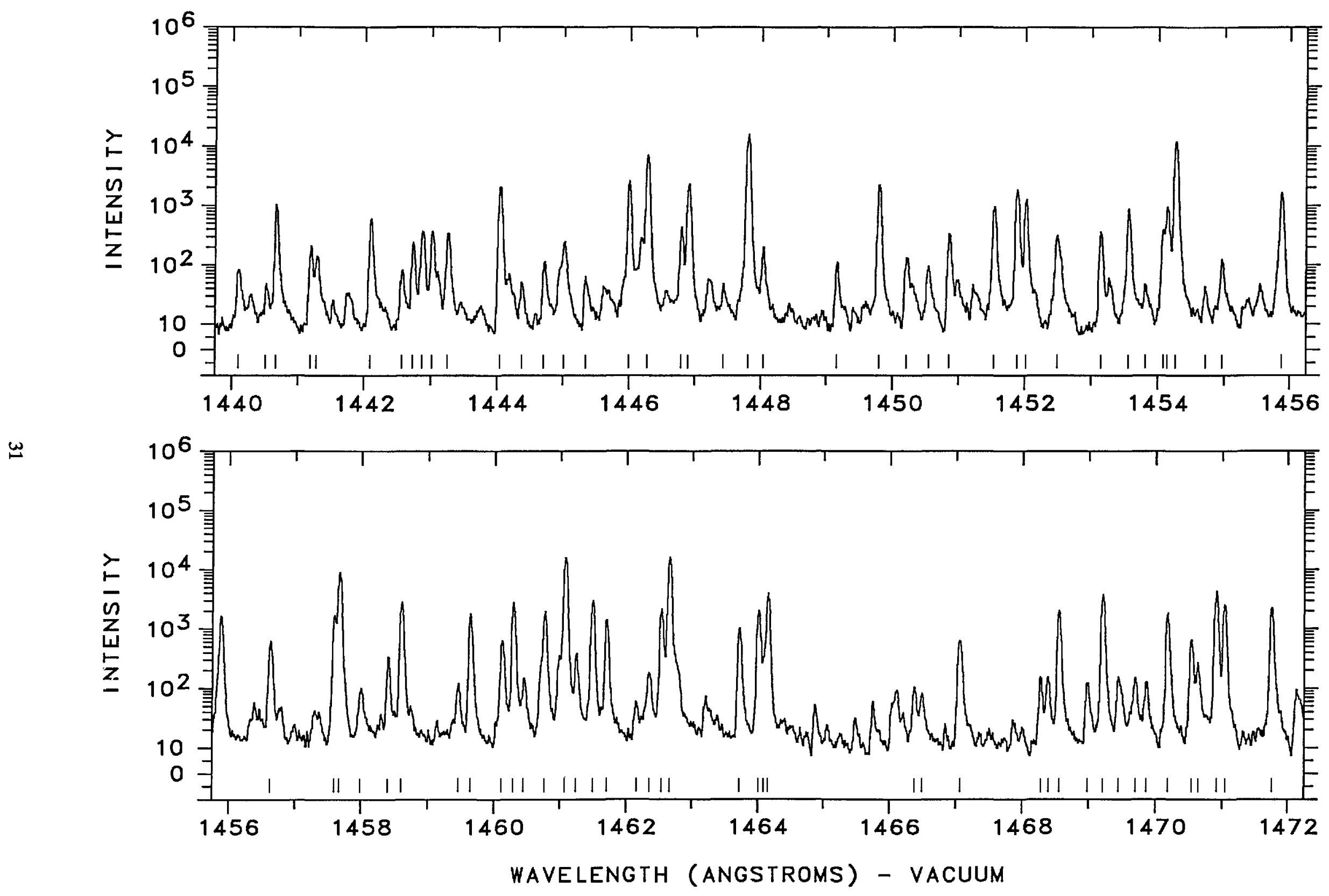


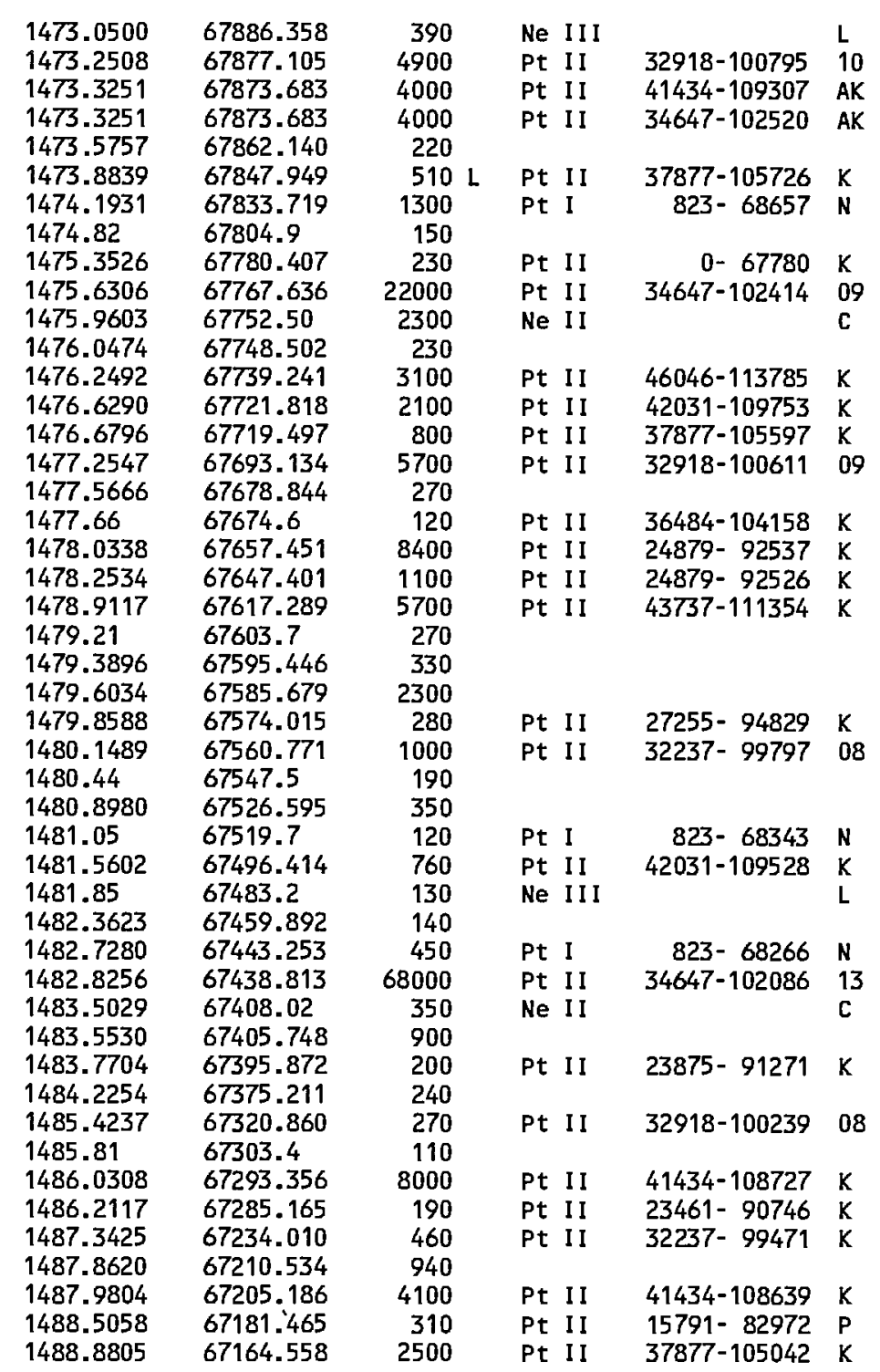

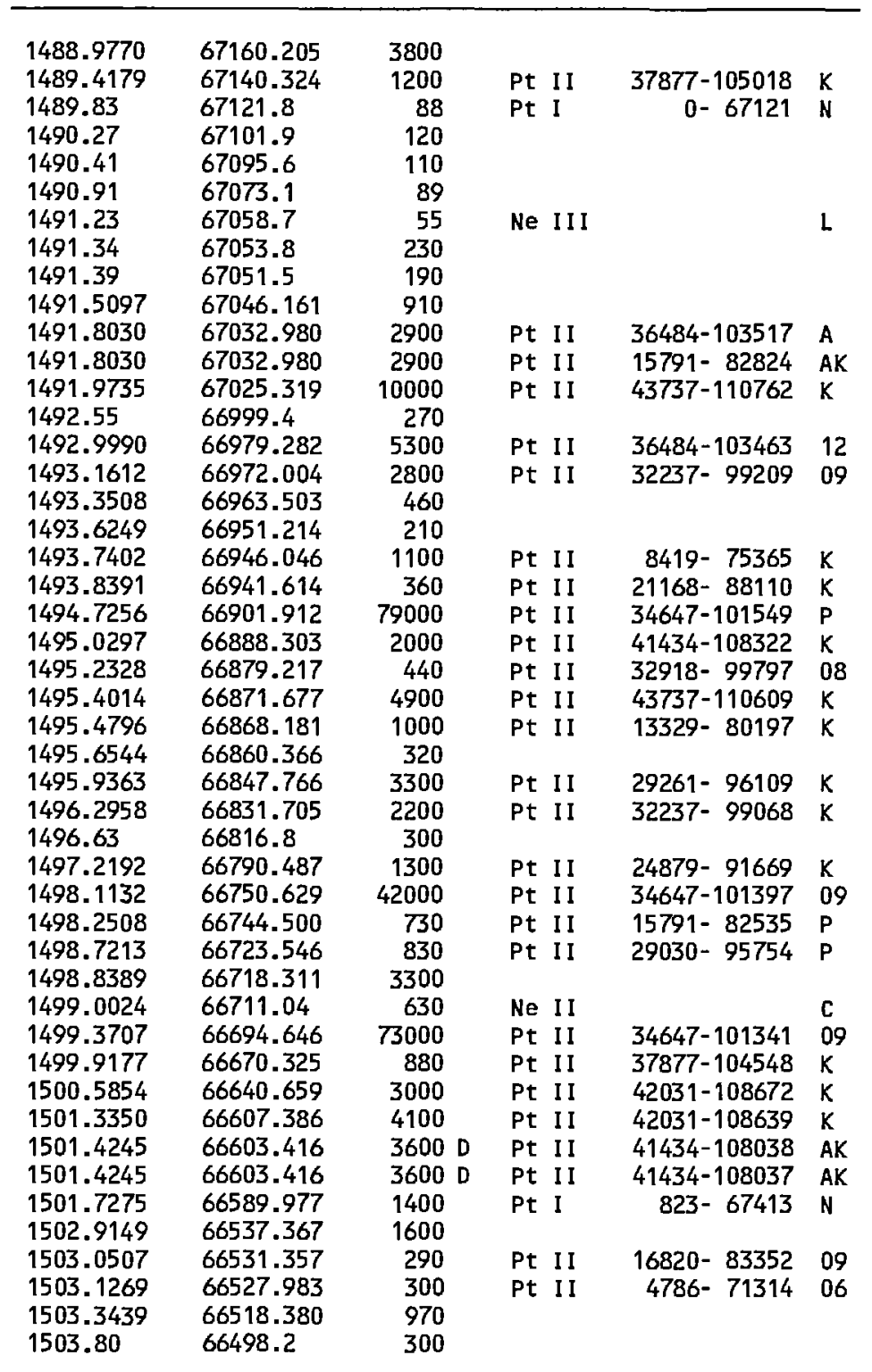




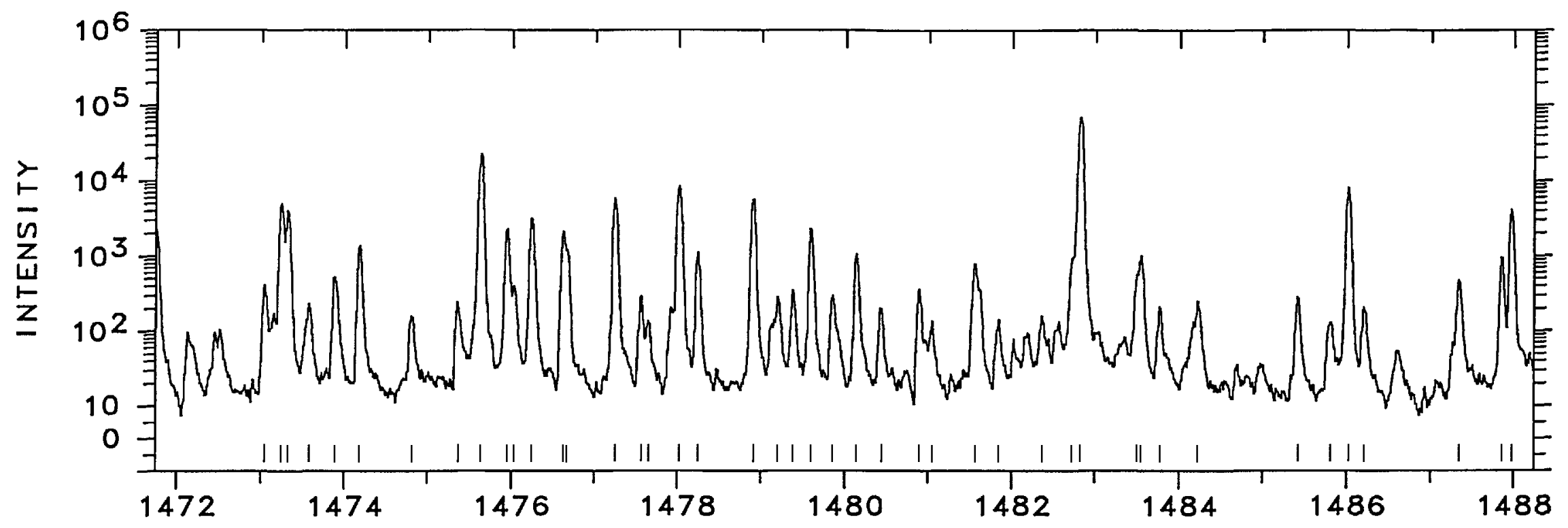

w

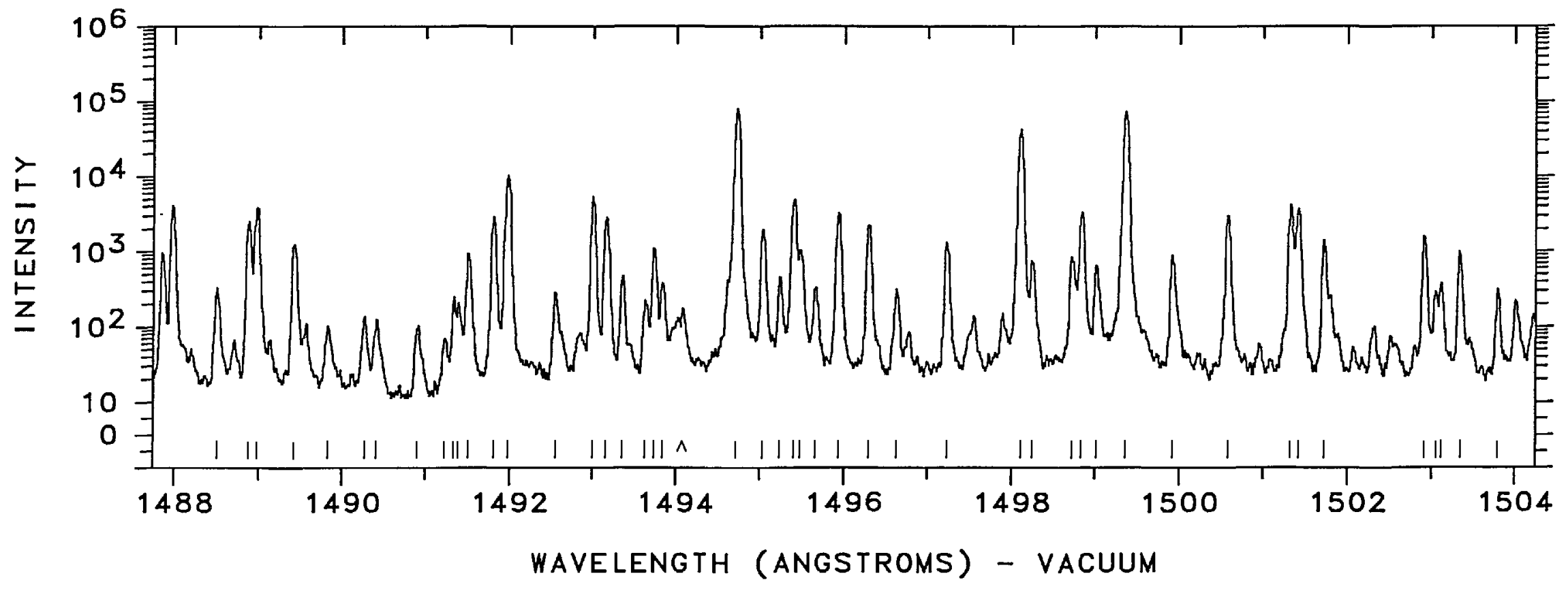




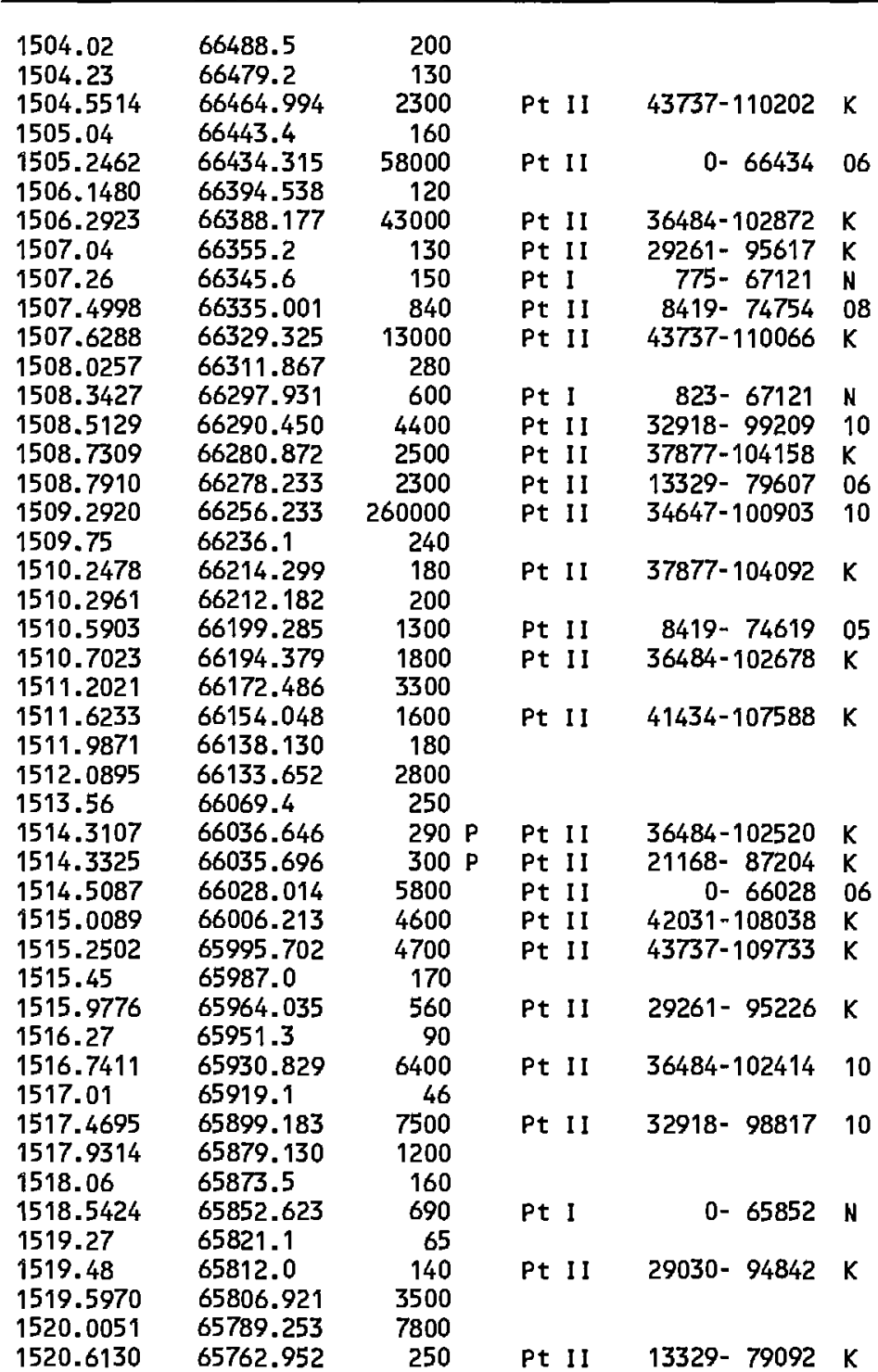

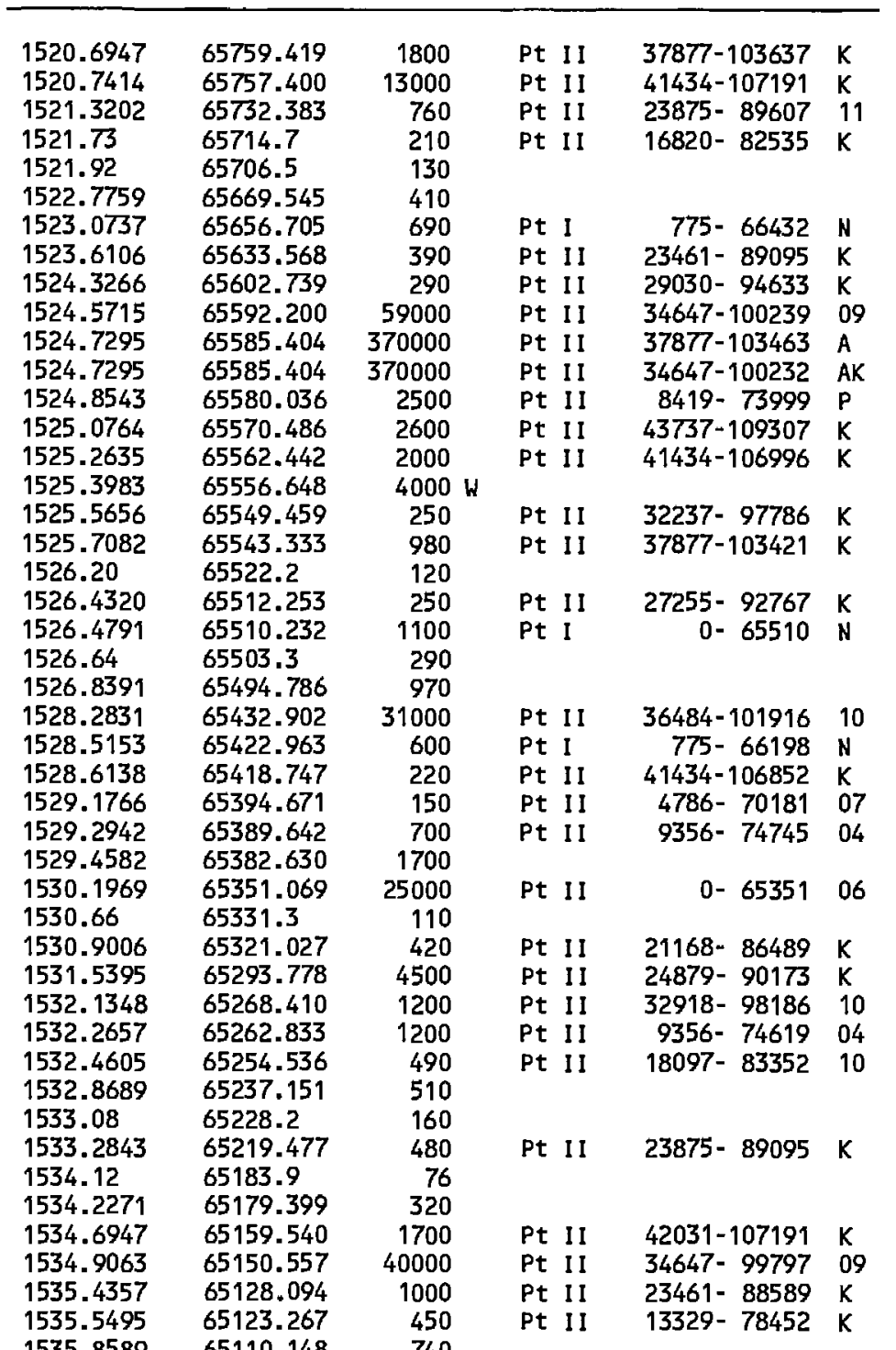




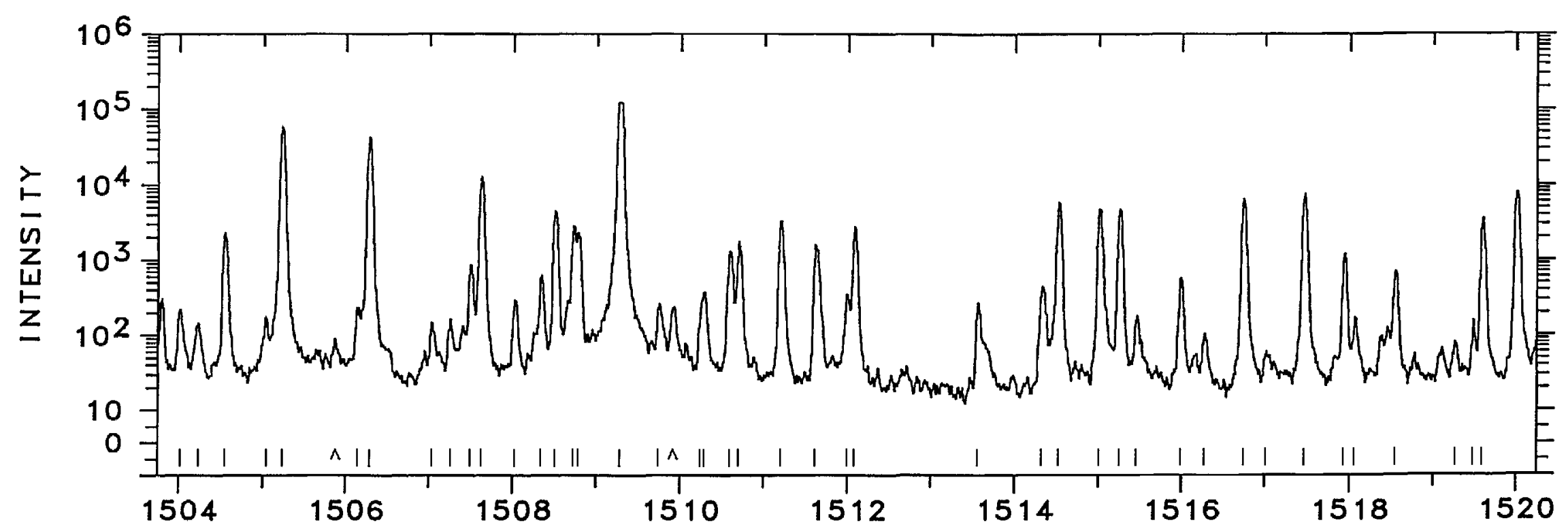

u

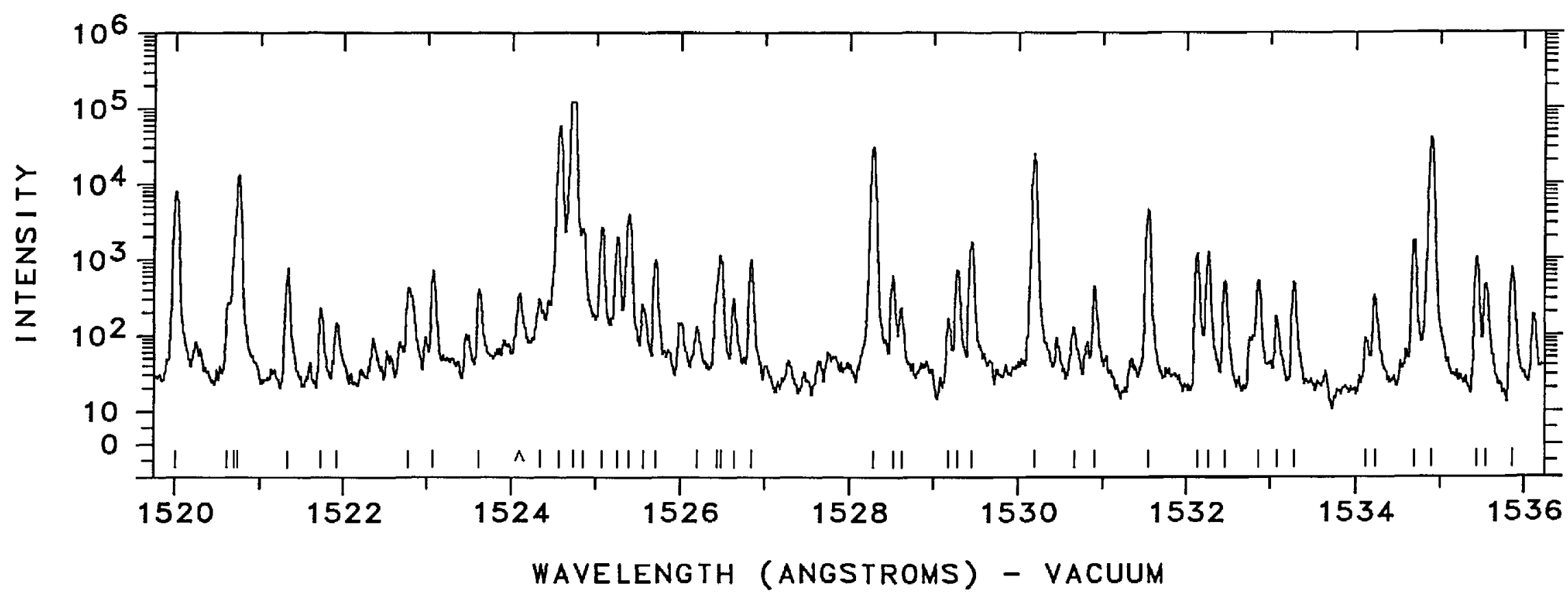


WAVELENGTH WAVE NUMBER INTENSITY

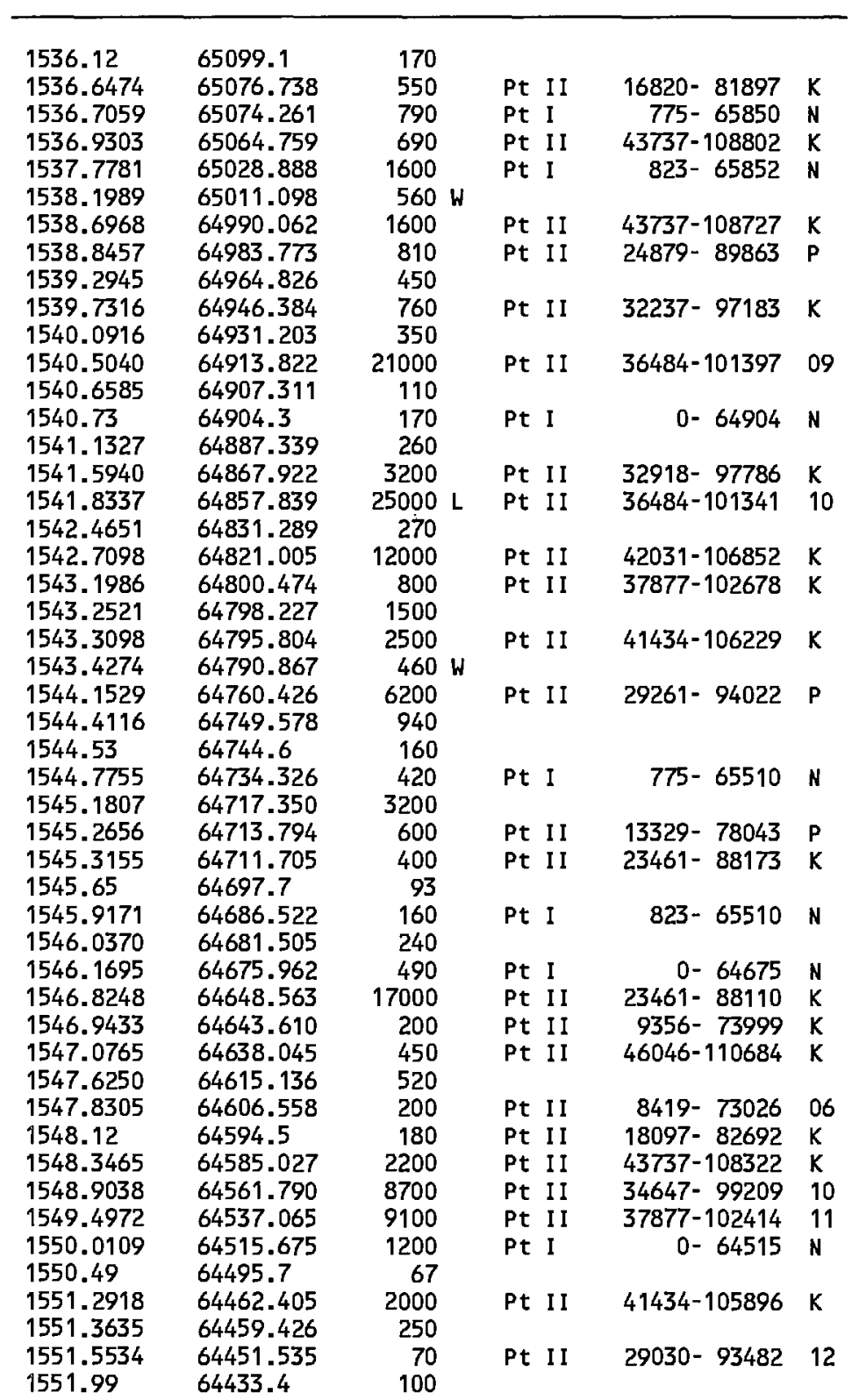

WAVELENGTH WAVE NUMBER INTENSITY CLASSIFICATION CODE

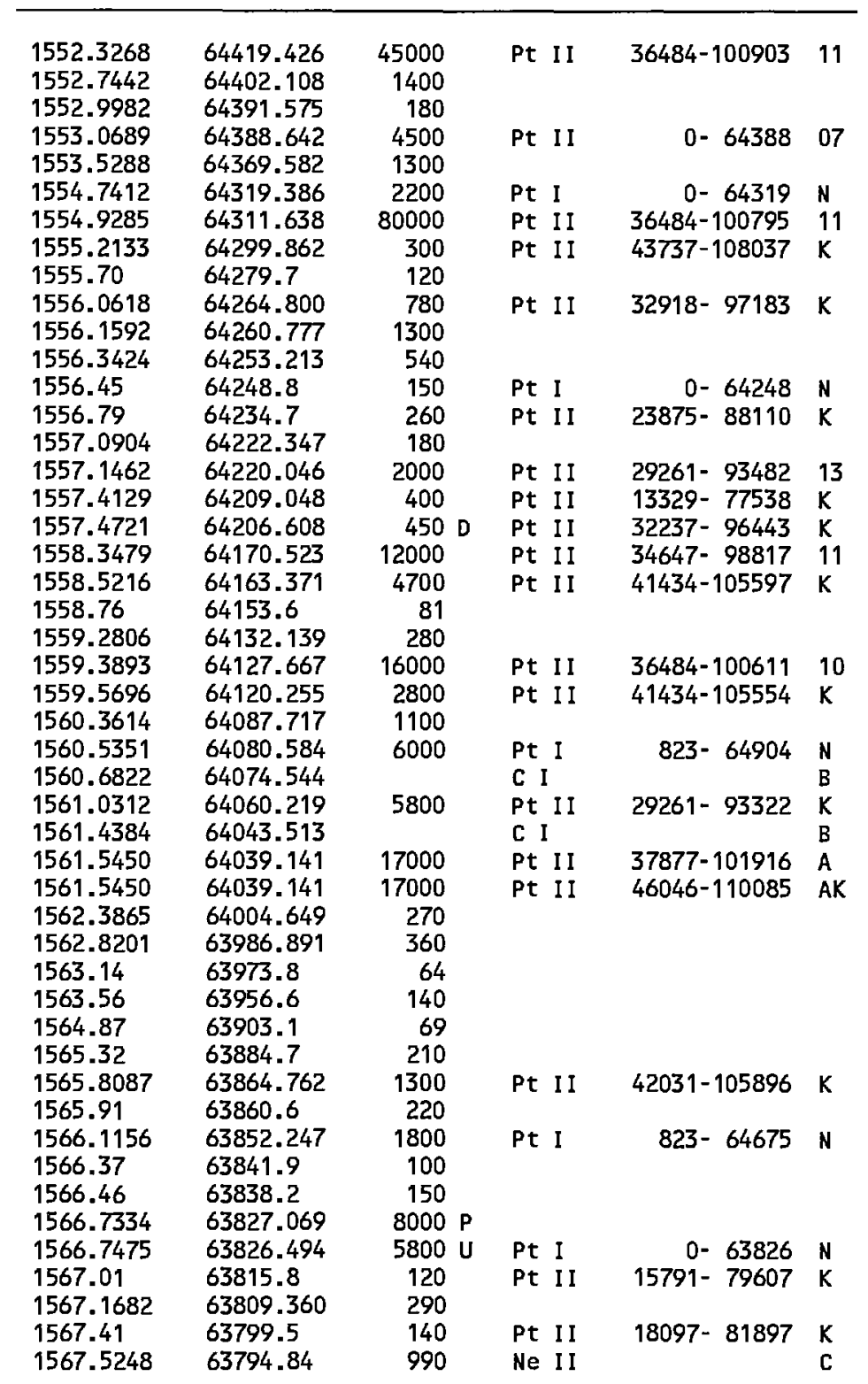




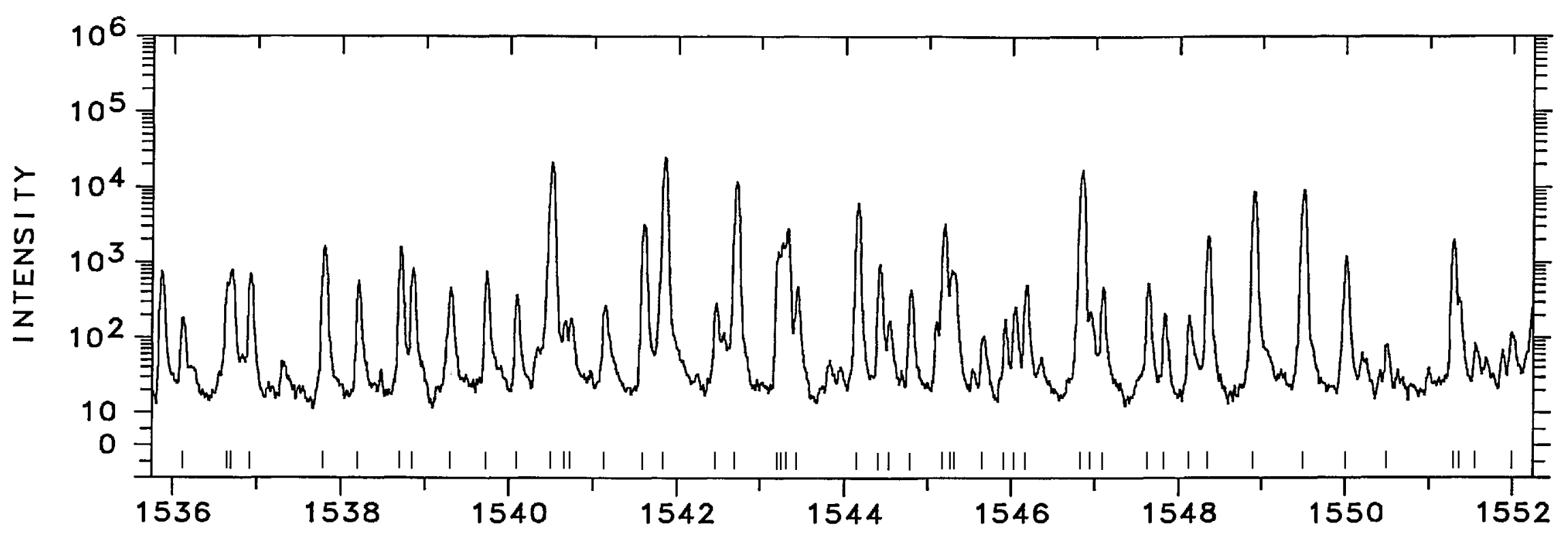

$w$

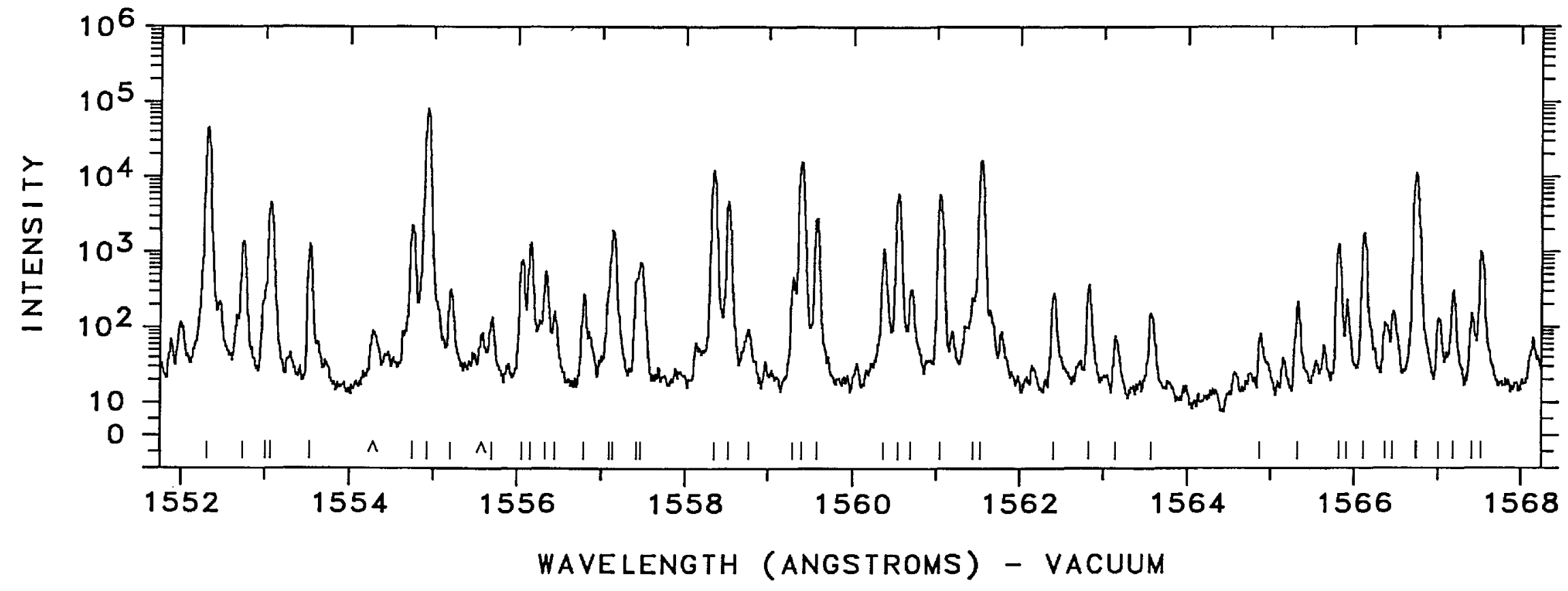


WAVELENGTH WAVE NUMBER INTENSITY

1568.3594

63760.896 63755.393

63738.1

63731.2

1568.92

1569.09
1569.6106

1569.7820

1570.30

1570.75

1570.9275

1571.8842

1572.1223

1572.1752

1572.56

1572.7201

1573.8180

1574.0819

1574.3059

1574.4002

1574.6393

1574.9089

1575.30

11000

11000
CLASSIFICATION CODE

Pt II 27255- $91016 \mathrm{~K}$

1575.4706

1575.62

1575.86

1576.48

1576.88
1577.07

1577.07
1577.2202

1577.3573

1577.8723

1578.17

1578.55

1578.75

1579.15

1579.4357

1579.9278
1579.9481

1580.4001

1580.6548

1580.7121

1580.8013

1580.8322

1581.3980

1583.095

1583.6406
63710.069

63703.113

63682.1

63648.878

63617.918

63608.283

63606.143

63590.6

63584.105

63539.750

63529.094

63520.058

63516.252

63506.608

63495.736
63480.0

63473.098

63467.1

63457.4

63432.5

63416.4

63408.7

63402.688

63397.177

63376.485

63364.5

63349.3

63341.3

63325.2

63313.750
63294.032

63294.032
63293.218

63275.116

63264.920

63262.627

63259.057

63257.821

63235.188

63167.391

63145.641
Pt II

$0-63738 \quad 06$

Pt II 24879-88589 K

Ne II I

$48591-112247 \quad \mathrm{~L}$

Pt II 43737-107386 K

$\begin{array}{lll}\text { Ne III } & & \text { L } \\ \text { Pt II } & 41434-105018 & K\end{array}$

Pt II 42031-105597 K

Pt II 34647-98186 10

Pt II 8419-71948 06

Pt II 37877-101397 10

Pt I I 37877-101394 K

Pt II 29030- $92537 \mathrm{~K}$

Pt I

823- $64319 \mathrm{~N}$

Pt I 775- 64248 N

Pt II 16820- 80197 K

1400

300
37

140

61

32000
550

$550 \mathrm{P}$ Pt II 36484- 9979709

$300 \mathrm{U}$

1200

1000

$3000 \mathrm{P}$

$1800 \mathrm{P}$

37000

1100

5200

Pt I

43737-106995 $\mathrm{k}$

$37877-101113 \quad K$

Pt II 34647- $97792 \mathrm{~K}$

WAVELENGTH WAVE NUMBER INTENSITY

CLASSIFICATION

CODE

Pt II 36484-100239 09

Pt II 117493- $53875 \mathrm{~K}$

Pt II 41434-105042 K

$1583.8462 \quad 63137.444 \quad 380$

$1583.9753 \quad 63132.298 \quad 160$

$1584.2474 \quad 63121.454 \quad 1900$

1584.625203113 .0

$1584.7233 \quad 63102.499$

$1585.13 \quad 63086.3$

1585.68

$1586.0312 \quad 63050.462$

1586.47

1586.59

1587.0368

1587.4559
1587.6482

1587.6482

1587.7205

1588.1904

1588.6920

1589.3735

1589.8128

1590.9851

1591.5069

1591.8192

1592.29

1592.6974

1594.0344

1594.2611

1594.47

1594.9347

1595.1388

1595.34

1595.8834

1596.3988

1596.4379

1596.7767

1597.30

1597.6295

1597.8343

1597.9705

1598.11

1598.11

1598.34
1599.14

1599.14
1599.5835

1599.7339
63064.4

63033.0

63028.3

63010.511

62993.876

62986.246

62986.246

62983.379

62944.863

62917.874

62900.487

62854.140

62833.532

62821.205

62802.6

62786.566

62746.779

62733.903

62724.983

62716.8

62698.492

62690.469

62682.6

62661.220

62658.039

62640.99

62639.455

62626.164

62605.6

62592.735

62592.735

62584.712

62579.38

62573.9

62564.9

62533.6

62516.274
62510.396
Pt II 13329- $76461 \quad 07$

$\begin{array}{lrl}\text { Pt II } & 13329-76461 & 07 \\ \text { Pt I } & 823-63945 & \text { N }\end{array}$

120
280
380

380

160

410

570

75

220
2200

2000

2500

2500

16000

270

2300
17000

400

3100

3100
500

130

250

250
420
15000

15000

14000

54

54
270
460
100

1100

2900

450

1100
2000

110

1100

1100

3500

3300

150

82
80
570

570
430

Pt I

42031-104548 K

$0-62510 \mathrm{~N}$

Ne III

Pt I

Pt II

Pt II

Pt II

Pt II

Pt II

Pt I

$\begin{array}{rl}37877-100795 & 12\end{array}$

Pt II

16820- $79607 \quad 07$

Pt

37877-100611 11

36484- 9920911

$\begin{array}{lrr}\text { Pt II } & 32918-95617 & \mathrm{~K} \\ \text { Pt I } & 775-63466 & \text { N }\end{array}$

Pt II 15791- $78452 \quad \mathrm{~K}$

Pt II

Ne II

t II

Pt II

Pt I

Pt II

Ne II

$\begin{array}{ll}- & -1 \\ & \end{array}$

36484- 99068 

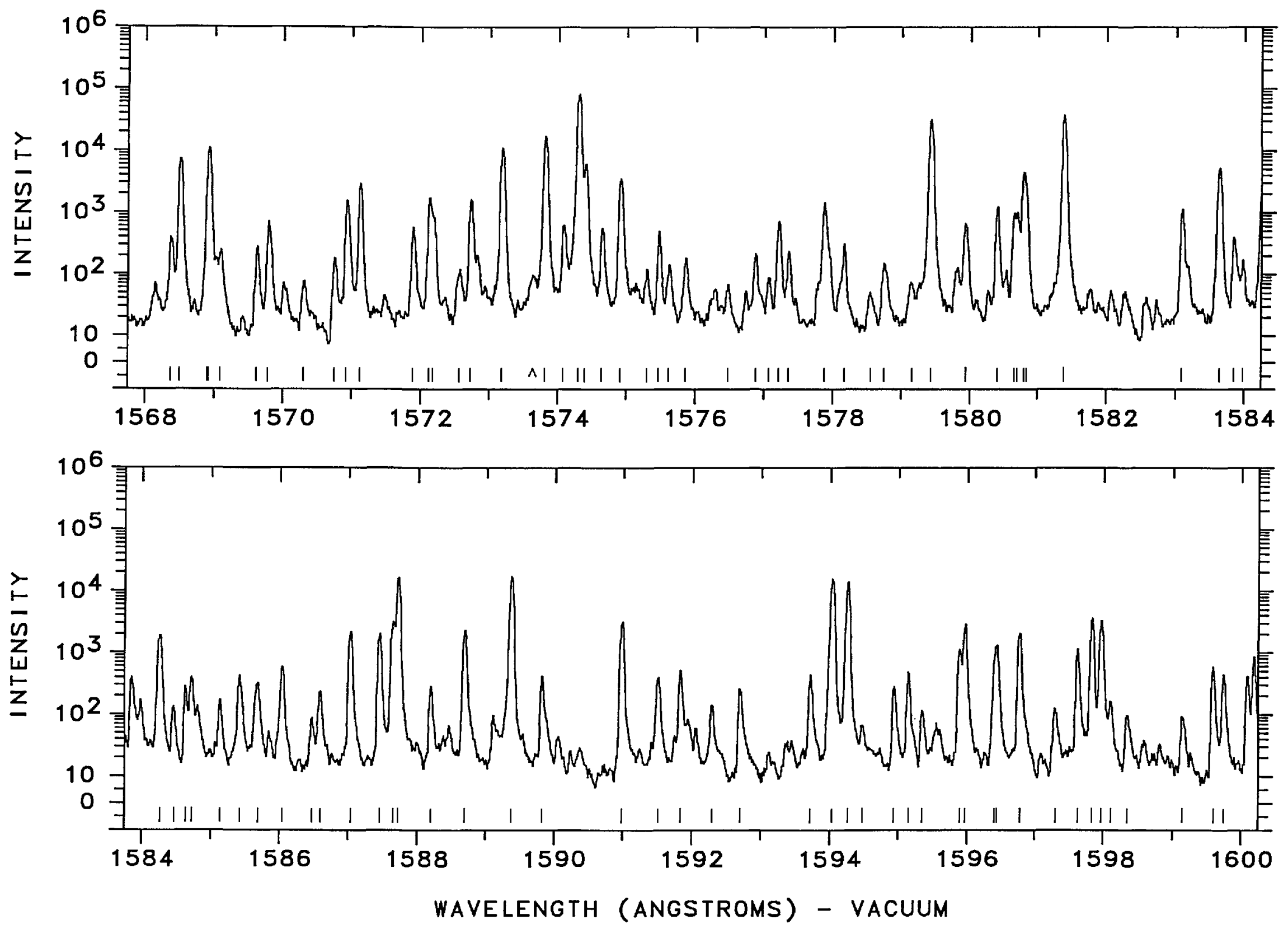


\begin{tabular}{|c|c|c|c|c|c|}
\hline $\begin{array}{l}1600.0814 \\
1600.1934 \\
1600.94 \\
1601.1825 \\
1601.2962 \\
1601.70 \\
1602.09\end{array}$ & $\begin{array}{l}62496.82 \\
62492.446 \\
62463.3 \\
62453.843 \\
62449.408 \\
62433.7 \\
62418.5\end{array}$ & $\begin{array}{r}400 \\
850 \\
73 \\
230 \\
750 \\
230 \\
40\end{array}$ & $\begin{array}{ll}\text { Ne II } \\
\text { Pt II } \\
\text { Pt II }\end{array}$ & $\begin{array}{l}43737-106229 \\
15791-78254\end{array}$ & $\begin{array}{l}c \\
K \\
K\end{array}$ \\
\hline $\begin{array}{l}1602.7837 \\
1603.4612\end{array}$ & $\begin{array}{l}62391.451 \\
62365.089\end{array}$ & $\begin{array}{r}9500 \\
200\end{array}$ & $\begin{array}{ll}\text { Pt } & \text { I } \\
\text { Pt } & \text { II }\end{array}$ & $\begin{array}{r}775-63167 \\
23461-85826\end{array}$ & $\begin{array}{l}\mathrm{N} \\
\mathrm{K}\end{array}$ \\
\hline $\begin{array}{l}1603.5502 \\
1604.0102\end{array}$ & $\begin{array}{l}62361.629 \\
62343.743\end{array}$ & $\begin{array}{r}6600 \\
14000\end{array}$ & $\begin{array}{l}\text { Pt II } \\
\text { Ne I I }\end{array}$ & $37877-100239$ & $\begin{array}{l}10 \\
A\end{array}$ \\
\hline $\begin{array}{l}1604.0102 \\
1604.0927\end{array}$ & $\begin{array}{l}62343.743 \\
62340.54\end{array}$ & $\begin{array}{r}14000 \\
300\end{array}$ & $\begin{array}{l}\text { Pt I } \\
\text { Ne I I }\end{array}$ & $823-63167$ & $\begin{array}{l}\text { AN } \\
\text { C }\end{array}$ \\
\hline 1604.2682 & 62333.716 & 1100 & Pt II & $36484-98817$ & 11 \\
\hline 1604.5702 & 62321.985 & 1100 & Pt I & 0- 62321 & $\mathbf{N}$ \\
\hline 1604.7337 & 62315.635 & 4100 & $\mathrm{Ne}$ III & & $\mathbf{L}$ \\
\hline $\begin{array}{l}1605.3536 \\
1605.58\end{array}$ & $\begin{array}{l}62291.572 \\
62282.8\end{array}$ & $\begin{array}{r}8800 \\
63\end{array}$ & Pt I & $775-63067$ & $\mathbf{N}$ \\
\hline 1606.1550 & 62260.492 & 1000 & & & \\
\hline $\begin{array}{l}1606.2741 \\
1606.54\end{array}$ & $\begin{array}{l}62255.875 \\
62245.6\end{array}$ & $\begin{array}{r}3700 \\
74\end{array}$ & & & \\
\hline $\begin{array}{l}1606.6658 \\
1608.30\end{array}$ & $\begin{array}{l}62240.698 \\
62177.5\end{array}$ & $\begin{array}{r}280 \\
72\end{array}$ & Pt II & $29030-91271$ & $\mathrm{k}$ \\
\hline 1608.5173 & 62169.05 & 220 & $\mathrm{Ne} I \mathrm{I}$ & & C \\
\hline 1609.6117 & 62126.785 & 200 & Pt II & $42031-104158$ & $\mathrm{~K}$ \\
\hline 1609.6647 & 62124.739 & 930 & & & \\
\hline $\begin{array}{l}1609.78 \\
1609.8562\end{array}$ & $\begin{array}{l}62120.3 \\
62117.349\end{array}$ & $\begin{array}{l}270 \\
190\end{array}$ & & & \\
\hline 1610.0697 & 62109.112 & 800 & Pt II & $46046-108155$ & K \\
\hline 1610.1405 & 62106.381 & 4800 & Pt I & $0-62106$ & $\mathbf{N}$ \\
\hline 1610.3173 & 62099.563 & 250 & Pt II & $18097-80197$ & $\mathrm{k}$ \\
\hline 1610.5649 & 62090.016 & 520 & Pt I & $6567-68657$ & $\mathbf{N}$ \\
\hline 1610.7448 & 62083.081 & 2600 & Pt II & $41434-103517$ & K \\
\hline 1610.7907 & 62081.312 & 1900 & & & \\
\hline 1611.2844 & 62062.290 & 600 & Pt I & $0-62062$ & $\mathbf{N}$ \\
\hline 1611.3397 & 62060.160 & 300 & Pt II & $42031-104092$ & K \\
\hline 1611.8840 & 62039.204 & 280 & Pt I & $6567-68606$ & N \\
\hline 1611.9533 & 62036.537 & 250 & Pt II & $13329-75365$ & $\mathrm{k}$ \\
\hline 1612.0071 & 62034.466 & 440 & Pt II & $32237-94271$ & $\mathrm{~K}$ \\
\hline 1612.44 & 62017.8 & 99 & Pt II & $48591-110609$ & K \\
\hline 1612.5934 & 62011.912 & 330 & Pt I & $823-62835$ & $\mathbf{N}$ \\
\hline $\begin{array}{l}1613.2389 \\
1613.41\end{array}$ & $\begin{array}{l}61987.099 \\
61980.5\end{array}$ & $\begin{array}{r}1200 \\
230\end{array}$ & Pt II & $41434-103421$ & $\mathrm{~K}$ \\
\hline 1613.9653 & 61959.201 & 800 & Pt II & $8419-70379$ & 08 \\
\hline 1613.9882 & 61958.320 & 4000 & Pt II & $9356-71314$ & 04 \\
\hline 1614.4078 & 61942.218 & 5200 & Pt I & 0- 61942 & $\mathbf{N}$ \\
\hline 1614.8843 & 61923.941 & 1300 & Pt I I & $32918-94842$ & K \\
\hline
\end{tabular}

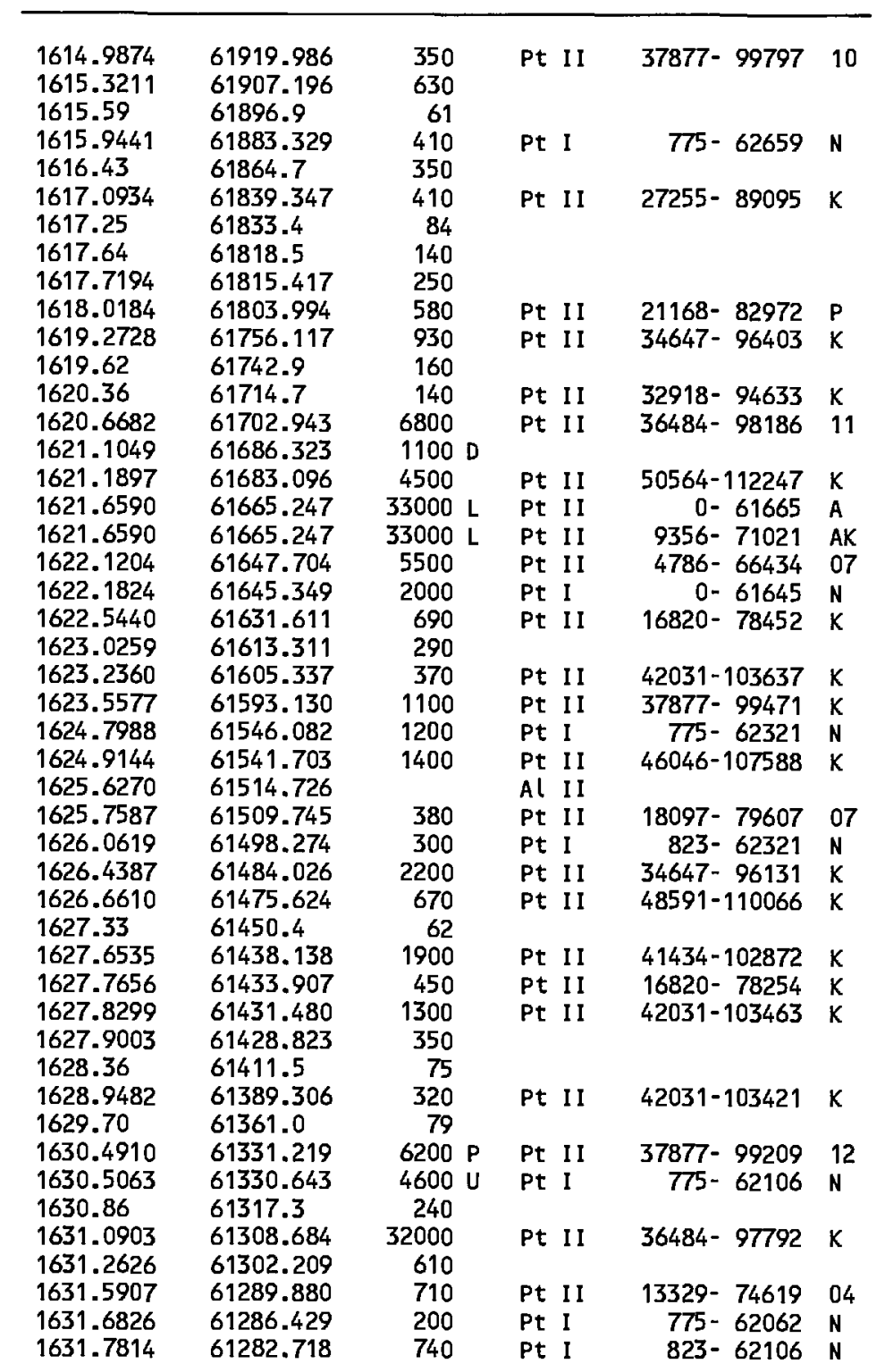



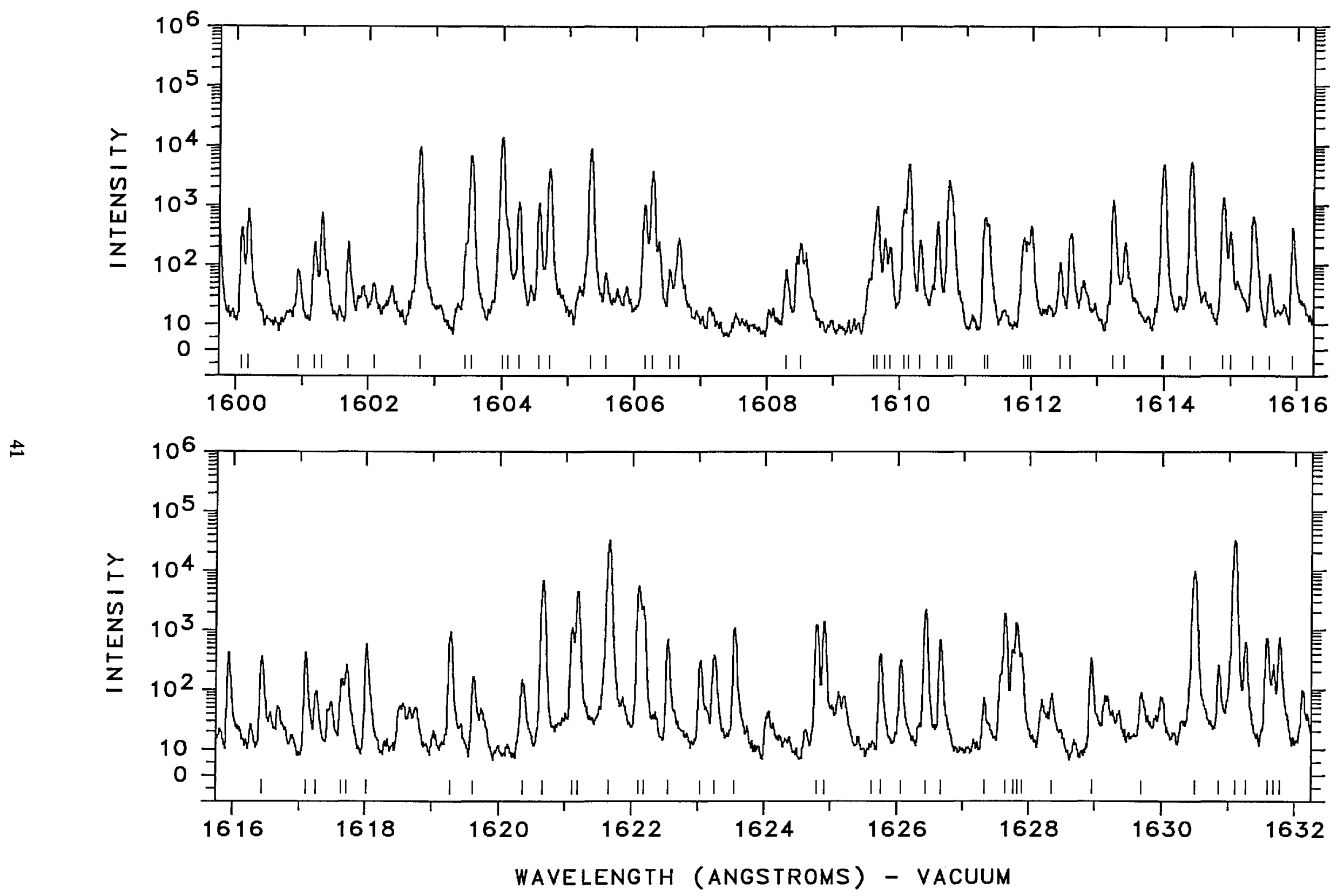
WAVELENGTH WAVE NUMBER INTENSITY

CLASSIFICATION

CODE

$\begin{array}{llr}1632.13 & 61269.6 & 87 \\ 1632.8049 & 61244.304 & 1100 \\ 1633.3302 & 61224.607 & 340 \\ 1634.2337 & 61190.759 & 46000 \\ 1635.2734 & 61151.854 & 500 \\ 1635.4147 & 61146.572 & 4600 \\ 1636.1647 & 61118.541 & 34000 \\ 1636.28 & 61114.2 & 310 \\ 1636.7302 & 61097.425 & 850 \\ 1636.8152 & 61094.252 & 3200 \\ 1637.0168 & 61086.728 & 6200 \\ 1638.04 & 61048.6 & 40 \\ 1638.18 & 61043.4 & 190 \\ 1638.7331 & 61022.749 & 310 \\ 1639.8606 & 60980.793 & 1300 \\ 1640.1553 & 60969.836 & 1300 \\ 1640.41 & 60960.4 & 130 \\ 1640.7691 & 60947.028 & 790 \\ 1641.7317 & 60911.293 & 1100 \\ 1642.15 & 60895.8 & 99 \\ 1642.8597 & 60869.471 & 450 \\ 1643.11 & 60860.2 & 110 \\ 1643.29 & 60853.5 & 70 \\ 1644.1761 & 60820.736 & 1300 \\ 1644.2292 & 60818.770 & 1100 \\ 1644.3084 & 60815.843 & 7900 \\ 1644.4634 & 60810.110 & 12000 \\ 1645.0044 & 60790.111 & 3400 \\ 1645.46 & 60773.3 & 170 \\ 1645.69 & 60764.8 & 100 \\ 1645.99 & 60753.7 & 200 \\ 1646.31 & 60741.9 & 56 \\ 1646.9762 & 60717.332 & 640 \\ 1647.49 & 60698.4 & 75 \\ 1647.83 & 60685.9 & 110\end{array}$

WAVELENGTH WAVE NUMBER INTENSI TY

CLASSIFICATION

CODE

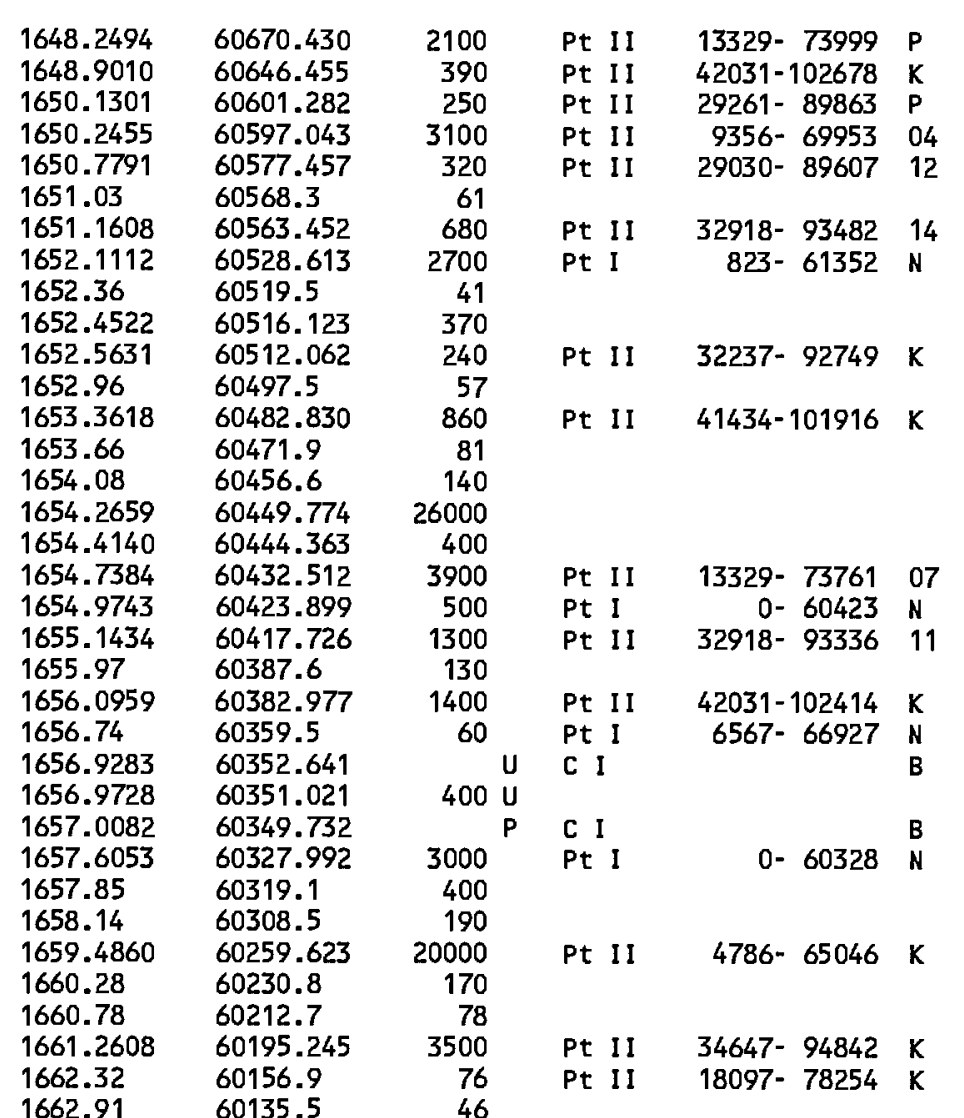



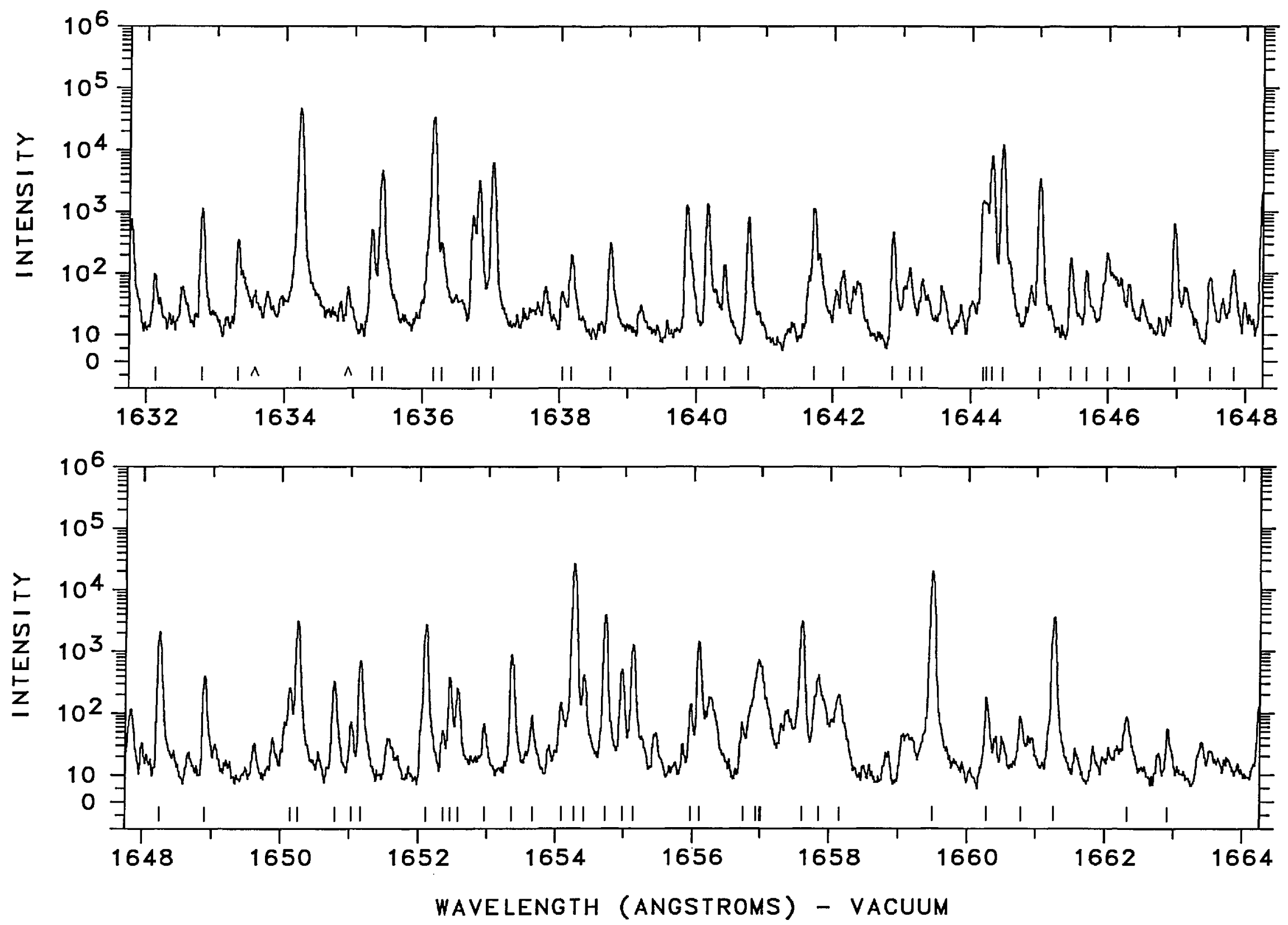
WAVELENGTH WAVE NUMBER INTENSITY

CLASSIFICATION

CODE

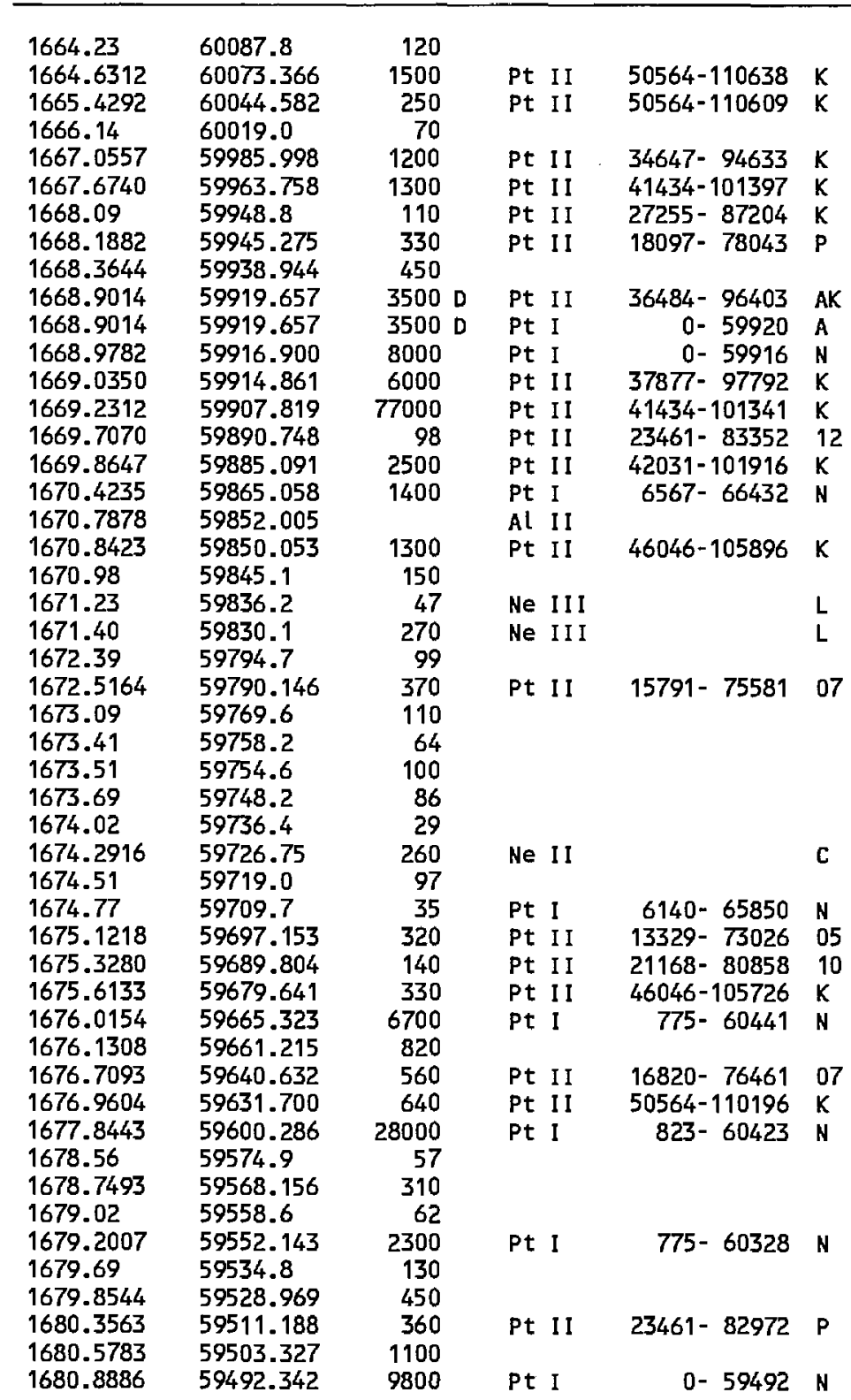

WAVELENGTH WAVE NUMBER INTENSITY CLASSIFICATION CODE

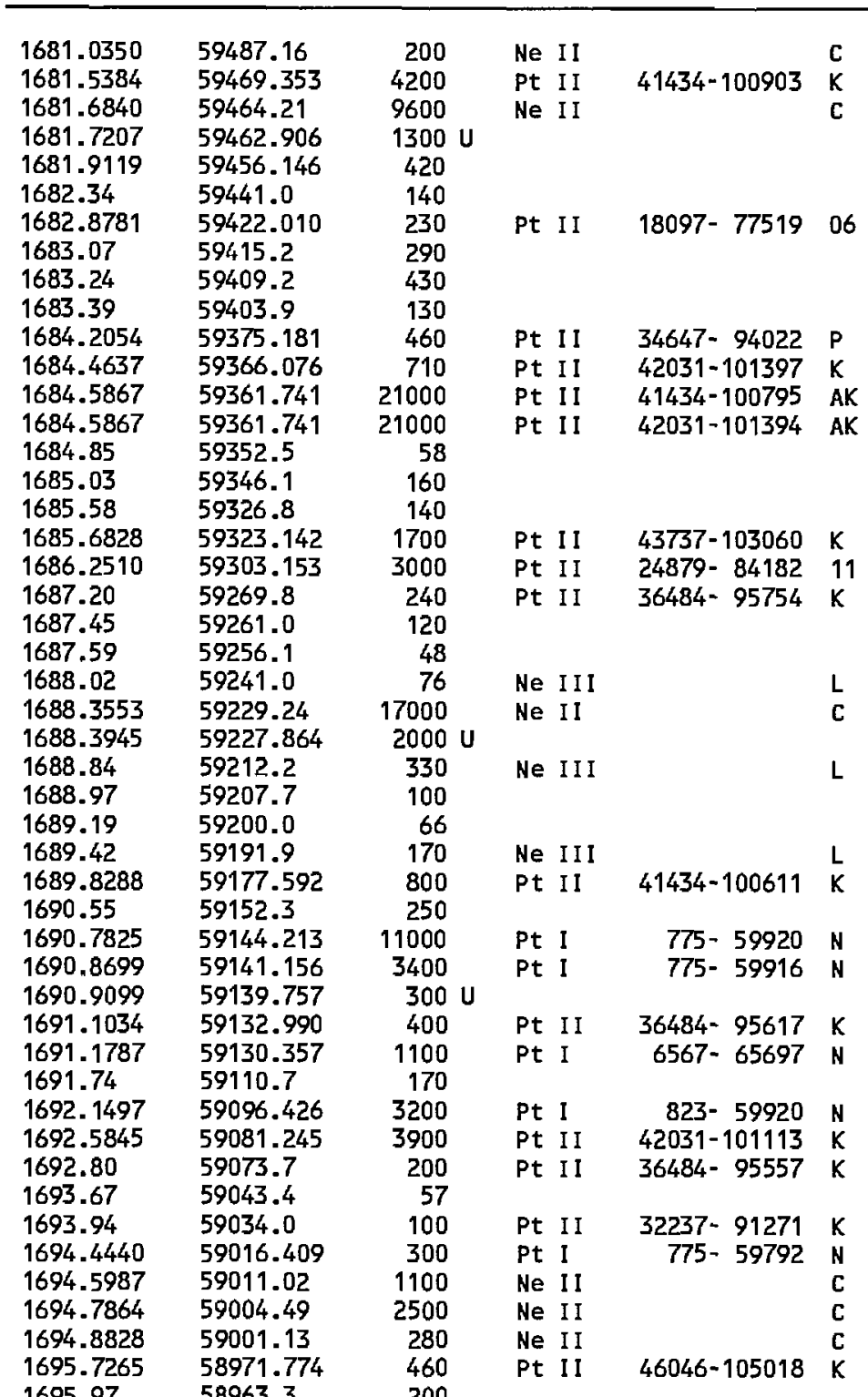




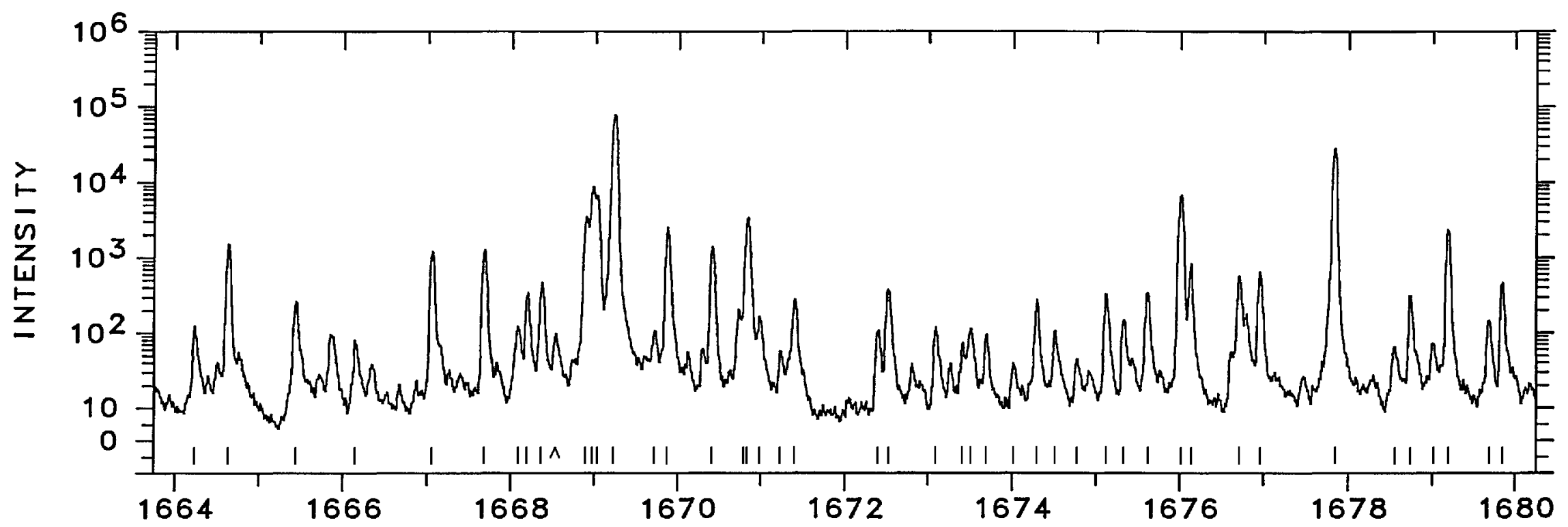

t

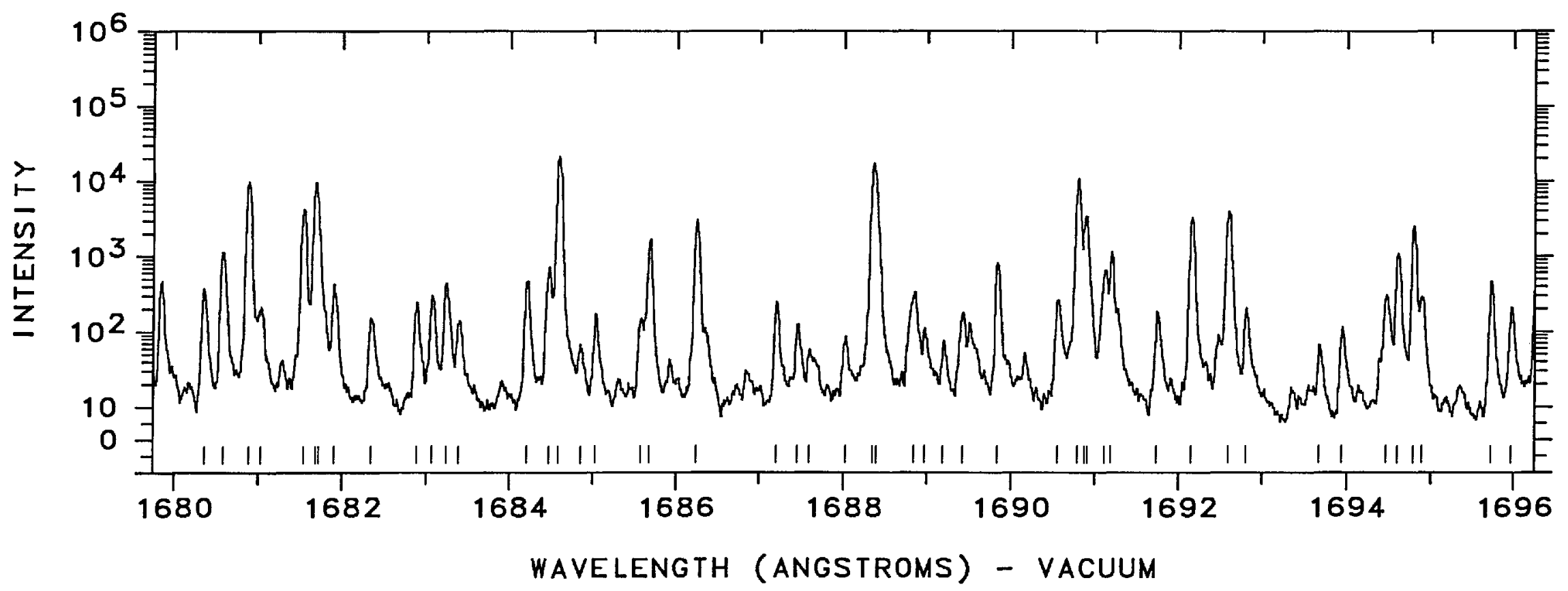


WAVELENGTH WAVE NUMBER INTENSITY

CLASSIFICATION

CODE

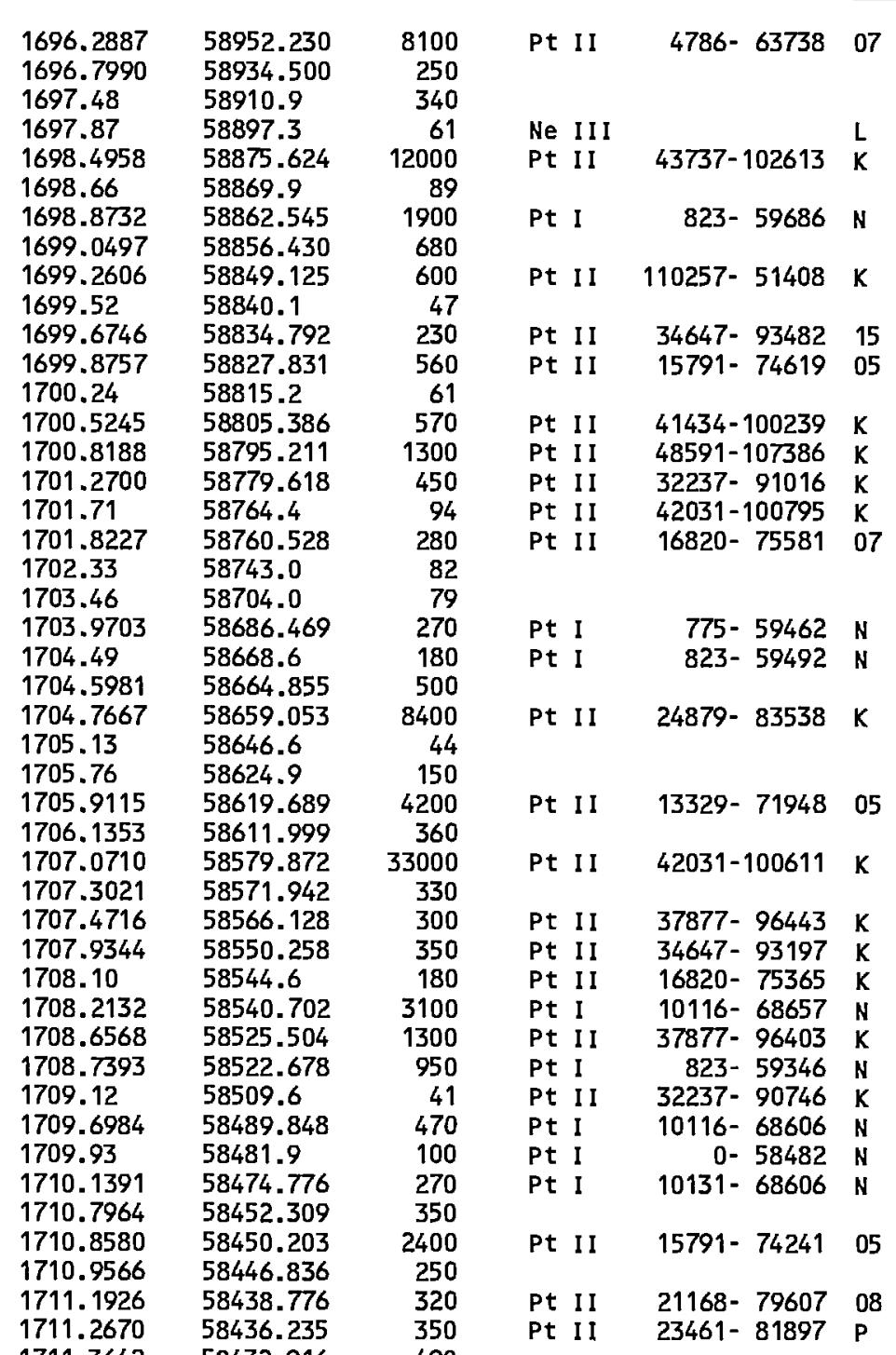

WAVELENGTH WAVE NUMBER INTENSITY CLASSIFICATION CODE

\begin{tabular}{|c|c|c|c|c|c|}
\hline $\begin{array}{l}1711.6209 \\
1712.0665\end{array}$ & $\begin{array}{l}58424.152 \\
58408.946\end{array}$ & $\begin{array}{l}1100 \\
1400\end{array}$ & Pt II & $9356-67780$ & $\mathrm{~K}$ \\
\hline 1712.2098 & 58404.058 & 1200 & Pt I I & $48591-106995$ & K \\
\hline 1712.6670 & 58388.467 & 7300 & Pt I & $0-58388$ & $\mathbf{N}$ \\
\hline 1713.3934 & 58363.713 & 3700 & Pt II & 41434- 99797 & $\mathrm{AK}$ \\
\hline 1713.3934 & 58363.713 & 3700 & Pt II & $18097-76461$ & A \\
\hline 1713.3934 & 58363.713 & 3700 & Pt II & $16820-75184$ & AK \\
\hline 1713.5477 & 58358.457 & 500 & Pt II & $36484-94842$ & $\mathrm{~K}$ \\
\hline 1713.7421 & 58351.837 & 400 & Pt I & $775-59127$ & N \\
\hline 1713.8364 & 58348.627 & 16000 & Pt I I & $43737-102086$ & $\mathrm{~K}$ \\
\hline 1714.1842 & 58336.788 & 2100 & Pt I & $6567-64904$ & $\mathrm{~N}$ \\
\hline 1714.4801 & 58326.720 & 10000 & Pt I & $0-58326$ & N \\
\hline 1715.49 & 58292.4 & 37 & & & \\
\hline 1715.7210 & 58284.535 & 300 & & & \\
\hline 1716.02 & 58274.4 & 140 & & & \\
\hline 1716.7118 & 58250.896 & 600 & & & \\
\hline 1717.1032 & 58237.618 & 1100 & Pt II & $50564-108802$ & $\mathrm{~K}$ \\
\hline 1717.9693 & 58208.258 & $1200 \mathrm{U}$ & Pt II & $15791-73999$ & $\mathbf{P}$ \\
\hline 1717.9888 & 58207.597 & $4400 \mathrm{P}$ & Pt II & $42031-100239$ & $\mathrm{~K}$ \\
\hline 1719.7159 & 58149.140 & 1500 & Pt II & $36484-94633$ & $\mathrm{~K}$ \\
\hline 1719.92 & 58142.2 & 120 & & & \\
\hline 1720.49 & 58123.0 & 26 & & & \\
\hline 1720.9199 & 58108.457 & 680 & Pt I & $6567-64675$ & N \\
\hline 1721.0604 & 58103.713 & $510 \mathrm{D}$ & & & \\
\hline 1721.2723 & 58096.560 & & Al II & & \\
\hline $\begin{array}{l}1721.9209 \\
1722.40\end{array}$ & $\begin{array}{l}58074.677 \\
58058.5\end{array}$ & $\begin{array}{l}310 \\
110\end{array}$ & Pt II & $50564-108639$ & K \\
\hline 1722.60 & 58051.8 & 220 & Pt I & $6567-64619$ & N \\
\hline 1722.87 & 58042.7 & 180 & Pt II & $115060-57018$ & K \\
\hline 1723.0983 & 58034.994 & 3500 & & & \\
\hline 1723.1314 & 58033.878 & 68000 & Pt II & $4786-62820$ & 07 \\
\hline 1723.3891 & 58025.20 & 190 & Ne II & & $\mathrm{C}$ \\
\hline 1723.9935 & 58004.859 & 3400 & Pt I & $775-58780$ & $\mathbf{N}$ \\
\hline 1724.5730 & 57985.367 & 4300 & Pt II & $13329-71314$ & 05 \\
\hline 1724.83 & 57976.7 & 63 & & & \\
\hline 1724.9840 & 57971.552 & & Al II & & \\
\hline 1725.15 & 57966.0 & 69 & Pt II & $21717-79683$ & $\mathrm{~K}$ \\
\hline 1725.26 & 57962.3 & 150 & & & \\
\hline 1725.58 & 57951.5 & 110 & & & \\
\hline 1725.69 & 57947.8 & 170 & Pt I & $6567-64515$ & $\mathbf{N}$ \\
\hline 1726.3697 & 57925.022 & 2500 & Pt II & $16820-74745$ & 05 \\
\hline 1726.5970 & 57917.395 & 420 & & & \\
\hline 1726.9376 & 57905.972 & 380 & & & \\
\hline 1727.4189 & 57889.838 & 1200 & Pt II & $34647-92537$ & K \\
\hline 1727.6799 & 57881.092 & 20000 & Pt II & $43737-101618$ & K \\
\hline 1727.8258 & 57876.205 & 1600 & Pt II & $37877-95754$ & $\mathbf{P}$ \\
\hline
\end{tabular}




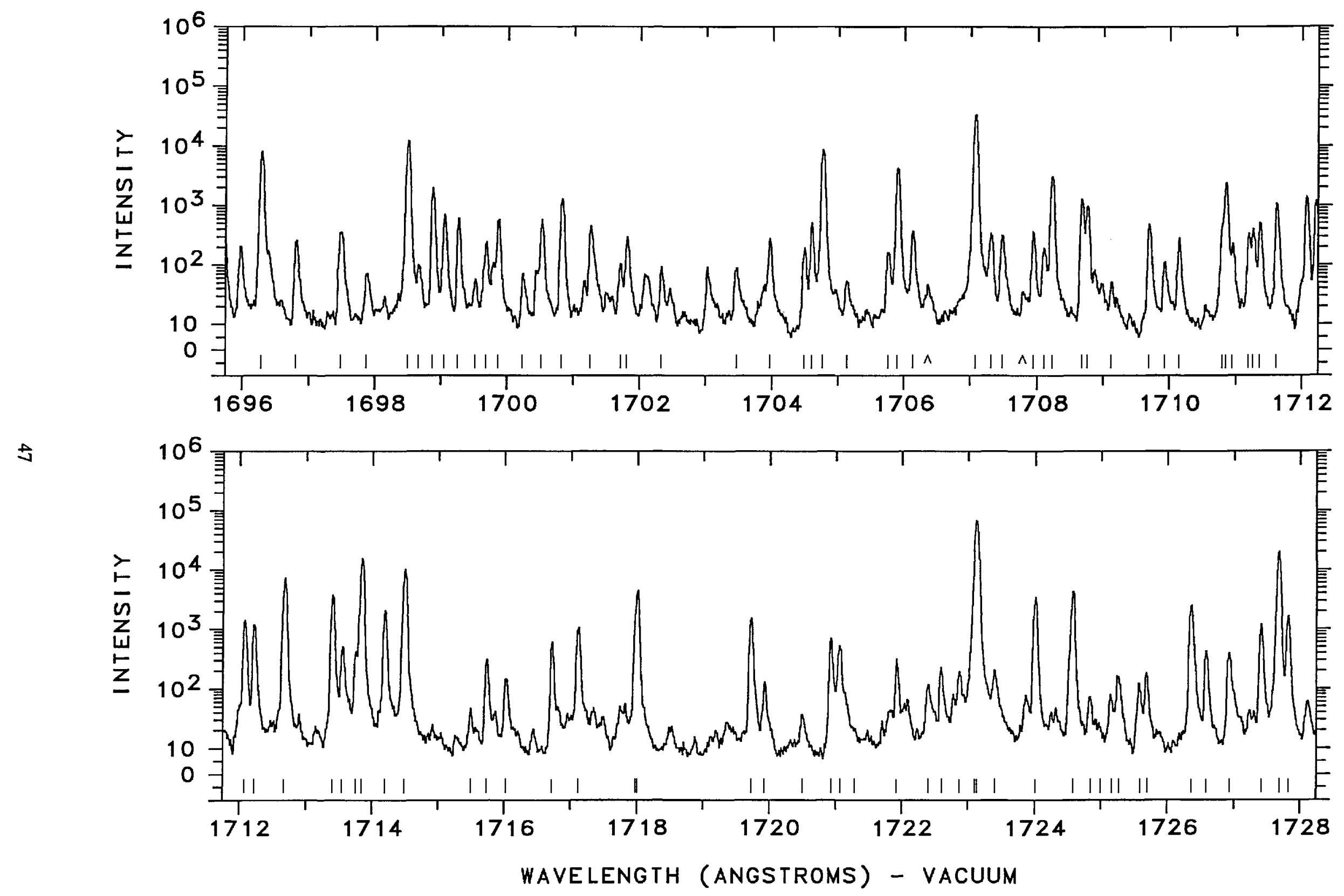




\begin{tabular}{|c|c|c|c|c|c|}
\hline $\begin{array}{l}1728.13 \\
1728.30 \\
1728.83\end{array}$ & $\begin{array}{l}57866.0 \\
57860.3 \\
57842.6\end{array}$ & $\begin{array}{l}52 \\
56 \\
44\end{array}$ & & & \\
\hline $\begin{array}{l}1729.26 \\
1729.7243\end{array}$ & $\begin{array}{l}57828.2 \\
57812.682\end{array}$ & $\begin{array}{r}63 \\
270\end{array}$ & $\begin{array}{l}\text { Pt II } \\
\text { Pt II }\end{array}$ & $\begin{array}{l}32918-90746 \\
24879-82692\end{array}$ & $\begin{array}{l}K \\
K\end{array}$ \\
\hline 1730.4798 & 57787.441 & 500 & Pt II & $36484-94271$ & $\hat{k}$ \\
\hline 1730.6473 & 57781.85 & 620 & Ne II & & C \\
\hline 1730.8544 & 57774.935 & 2000 & Pt II & 41434-99209 & $k$ \\
\hline 1731.1250 & 57765.904 & 1200 & Pt II & $42031-99797$ & $\mathrm{k}$ \\
\hline 1731.4175 & 57756.145 & 1100 & & & \\
\hline 1731.57 & 57751.1 & 85 & & & \\
\hline 1731.91 & 57739.7 & 42 & & & \\
\hline 1732.44 & 57722.1 & 58 & & & \\
\hline 1732.93 & 57705.7 & 83 & Pt I & $775-58482$ & $\mathbf{N}$ \\
\hline 1733.19 & 57697.1 & 150 & & & \\
\hline 1733.5407 & 57685.407 & 150 & & & \\
\hline 1733.7099 & 57679.777 & 350 & Pt II & $37877-95557$ & K \\
\hline 1733.93 & 57672.5 & 170 & & & \\
\hline 1735.24 & 57628.9 & 190 & & & \\
\hline 1735.8642 & 57608.192 & 36000 & Pt II & $8419-66028$ & 06 \\
\hline 1735.9774 & 57604.437 & 4100 & Pt II & $43737-101341$ & K \\
\hline 1736.39 & 57590.7 & 180 & Pt II & $46046-103637$ & $k$ \\
\hline 1737.1732 & 57564.784 & 21000 & Pt I & 823- 58388 & $N$ \\
\hline 1737.3402 & 57559.25 & 290 & Ne II & & C \\
\hline 1737.5956 & 57550.790 & 3600 & Pt I & $775-58326$ & $\mathbf{N}$ \\
\hline 1738.7356 & 57513.06 & 1600 & $\mathrm{Ne}$ II & & c \\
\hline 1738.9433 & 57506.187 & 1700 & Pt I & $0-57506$ & D \\
\hline 1739.76 & 57479.2 & 130 & & & \\
\hline 1739.9574 & 57472.672 & 320 & Pt II & $50564-108037$ & $\mathrm{k}$ \\
\hline 1740.3637 & 57459.254 & 720 & Pt II & $29030-86489$ & k \\
\hline 1740.9739 & 57439.115 & 480 & Pt II & 42031-99471 & $\mathrm{k}$ \\
\hline 1741.46 & 57423.1 & 180 & & & \\
\hline 1742.2518 & 57396.985 & 140 & Pt II & $23461-80858$ & 11 \\
\hline 1742.34 & 57394.1 & 89 & & & \\
\hline 1742.66 & 57383.5 & 110 & Pt II & $41434-98817$ & $\mathrm{k}$ \\
\hline 1742.8712 & 57376.59 & 910 & Ne II & & C \\
\hline 1743.40 & 57359.2 & 170 & & & \\
\hline 1744.17 & 57333.9 & 150 & & & \\
\hline 1744.2756 & 57330.39 & 290 & $\mathrm{Ne} I \mathrm{I}$ & & C \\
\hline
\end{tabular}

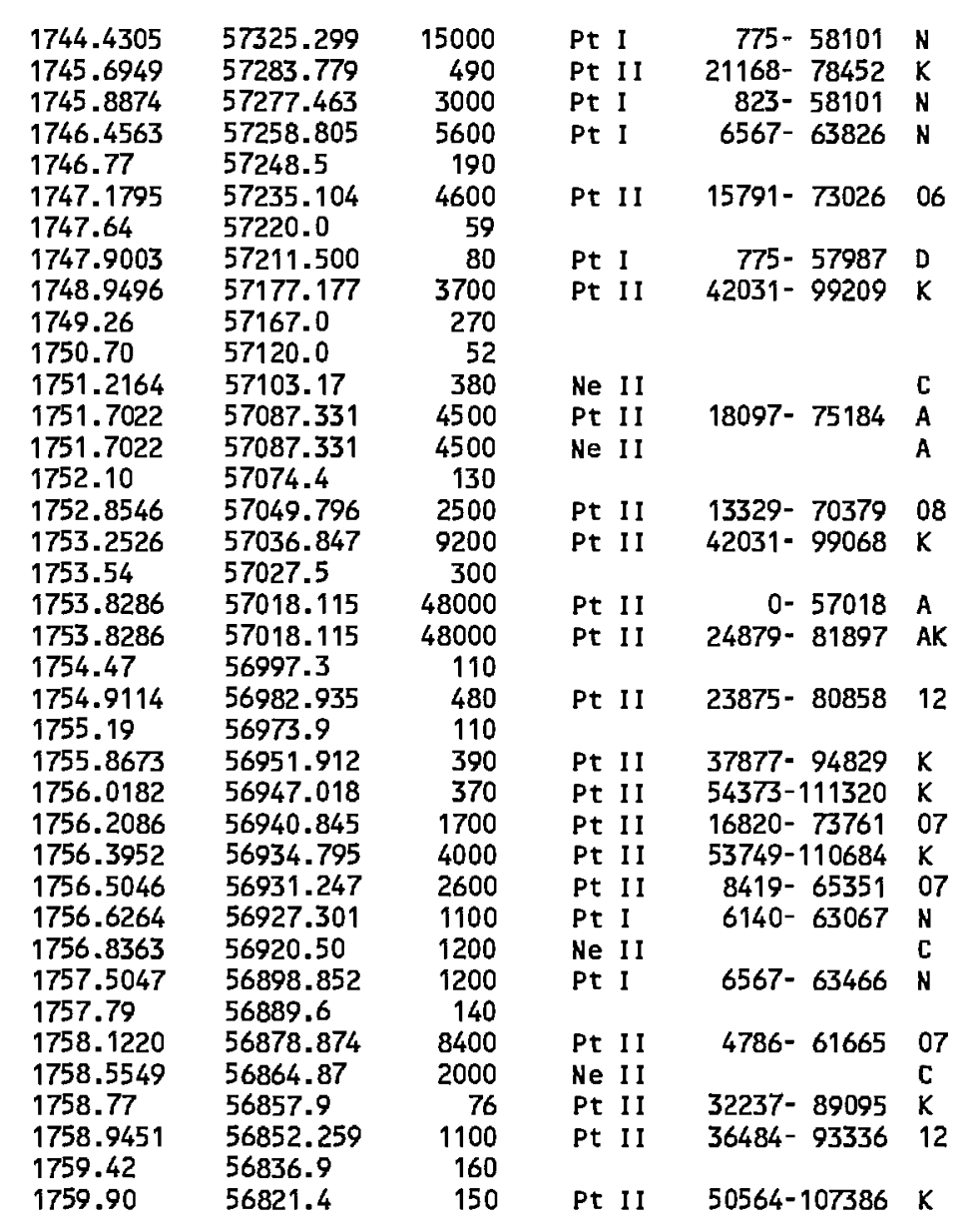



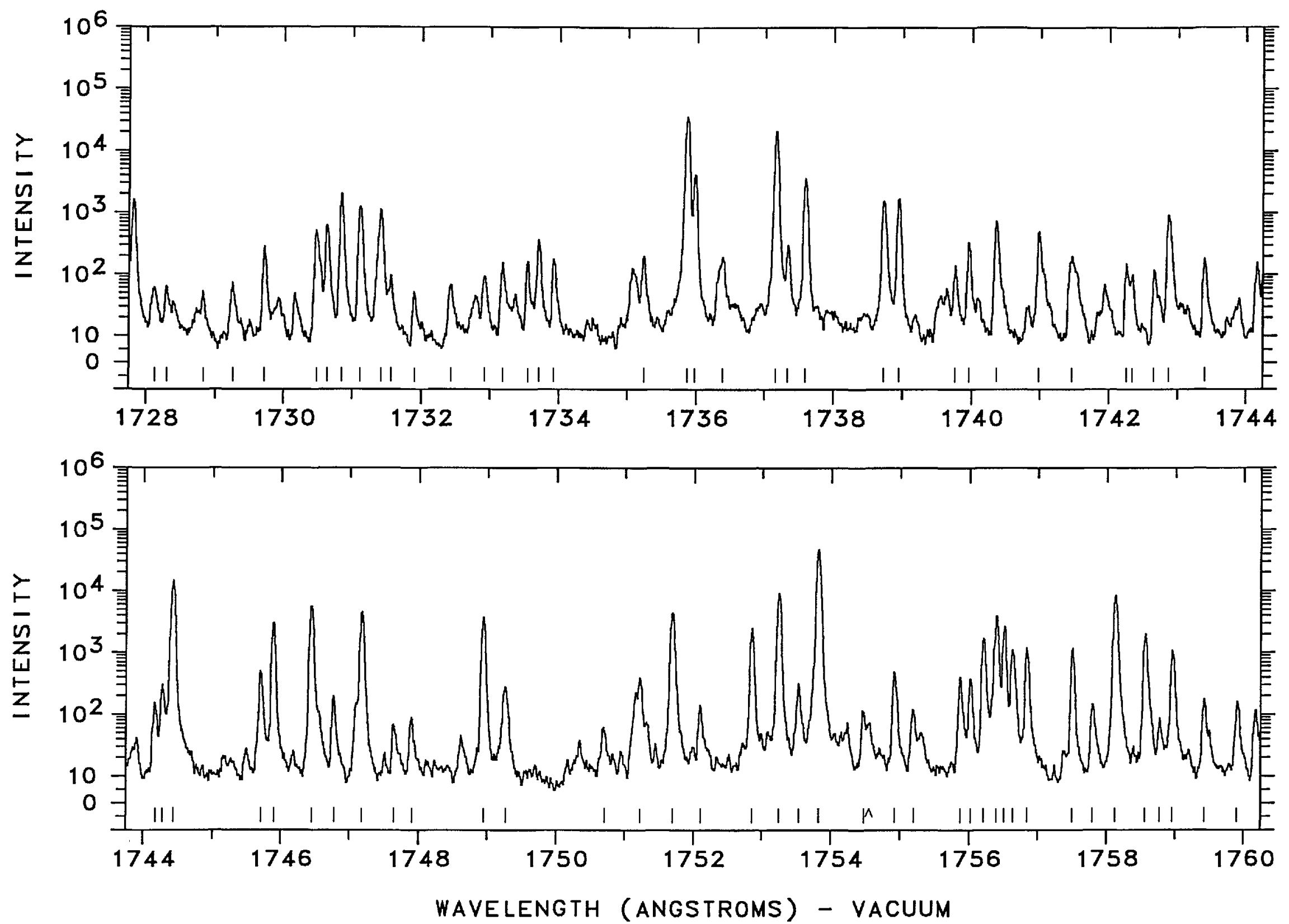
WAVELENGTH WAVE NUMBER INTENSITY

CLASSIFICATION

CODE

\begin{tabular}{|c|c|c|c|c|c|}
\hline $\begin{array}{l}1760.16 \\
1760.6896 \\
1760.6896 \\
1761.11 \\
1761.38 \\
1761.9446\end{array}$ & $\begin{array}{l}56813.0 \\
56795.928 \\
56795.928 \\
56782.4 \\
56773.7 \\
56755.474\end{array}$ & $\begin{array}{l}110 \\
960 \mathrm{D} \\
960 \mathrm{D} \\
120 \\
710 \\
1700\end{array}$ & $\begin{array}{l}\text { Pt I } \\
\text { Pt II }\end{array}$ & $\begin{array}{l}10131-66927 \\
29030-85826\end{array}$ & $\begin{array}{l}\text { AN } \\
\text { AK }\end{array}$ \\
\hline $\begin{array}{l}1762.4146 \\
1762.5591\end{array}$ & $\begin{array}{l}56740.338 \\
56735.686\end{array}$ & $\begin{array}{l}680 \\
3400 D\end{array}$ & $\mathrm{Pt} \mathrm{U}$ & $23461-80197$ & $A K$ \\
\hline 1762.5591 & 56735.686 & 3400 D & Pt II & $21717-78452$ & $A K$ \\
\hline $\begin{array}{l}1762.7266 \\
1763.27\end{array}$ & $\begin{array}{l}56730.295 \\
56712.8\end{array}$ & $\begin{array}{r}1000 \\
61\end{array}$ & Pt I & $775-57506$ & D \\
\hline $\begin{array}{l}1763.7269 \\
1764.01\end{array}$ & $\begin{array}{l}56698.12 \\
56689.0\end{array}$ & $\begin{array}{l}160 \\
210\end{array}$ & $\mathrm{Ne} I \mathrm{I}$ & & c \\
\hline 1764.2127 & 56682.509 & 2200 & Pt I & $823-57506$ & D \\
\hline $\begin{array}{l}1764.5948 \\
1764.84\end{array}$ & $\begin{array}{l}56670.234 \\
56662.4\end{array}$ & $\begin{array}{r}21000 \\
160\end{array}$ & Pt I & $0-56670$ & N \\
\hline $\begin{array}{l}1765.0132 \\
1765.35\end{array}$ & $\begin{array}{l}56656.80 \\
56646.0\end{array}$ & $\begin{array}{r}150 \\
72\end{array}$ & $\mathrm{Ne}$ II & & c \\
\hline 1765.8981 & 56628.41 & 810 & Ne II & & C \\
\hline 1766.0328 & 56624.090 & 10000 & Pt II & $13329-69953$ & 04 \\
\hline 1766.7883 & 56599.877 & 1700 & Pt I & $6567-63167$ & N \\
\hline $\begin{array}{l}1766.95 \\
1767.1612\end{array}$ & $\begin{array}{l}56594.7 \\
56587.934\end{array}$ & $\begin{array}{r}330 \\
47000\end{array}$ & $\begin{array}{l}\text { Pt II } \\
\text { Pt II }\end{array}$ & $\begin{array}{r}21168-77763 \\
0-56587\end{array}$ & $\begin{array}{l}K \\
07\end{array}$ \\
\hline 1767.89 & 56564.6 & 280 & Pt II & $29261-85826$ & $\mathrm{~K}$ \\
\hline $\begin{array}{l}1768.0852 \\
1768.31\end{array}$ & $\begin{array}{l}56558.36 \\
56551.2\end{array}$ & $\begin{array}{r}120 \\
55\end{array}$ & $\mathrm{Ne} I I$ & & C \\
\hline 1769.2416 & 56521.393 & 200 & Pt II & $18097-74619$ & 05 \\
\hline 1769.4841 & 56513.647 & 6600 & Pt II & $29261-85775$ & K \\
\hline 1769.9101 & 56500.045 & 730 & Pt I & $6567-63067$ & N \\
\hline 1770.0610 & 56495.228 & 5600 & Pt II & $43737-100232$ & $\mathrm{~K}$ \\
\hline & & 300 & & & \\
\hline 1770.90 & $\begin{array}{l}56468.5 \\
564643\end{array}$ & 110 & & & \\
\hline $\begin{array}{l}1771.03 \\
1771.26\end{array}$ & $\begin{array}{l}56464.3 \\
56457.0\end{array}$ & $\begin{array}{l}230 \\
110\end{array}$ & & & \\
\hline 1771.4140 & 56452.077 & 1600 & & & \\
\hline 1771.89 & 56436.9 & 130 & & & \\
\hline 1772.0902 & 56430.536 & 290 & Pt II & 50564-106995 & $K$ \\
\hline $\begin{array}{l}1772.80 \\
1774.0082\end{array}$ & $\begin{array}{l}56407.9 \\
56369.525\end{array}$ & $\begin{array}{r}65 \\
830\end{array}$ & Pt I & $21168-77538$ & $\mathbf{K}$ \\
\hline 1774.5470 & 56352.410 & 4800 & Pt II & $41434-97786$ & $\mathrm{~K}$ \\
\hline
\end{tabular}

WAVELENGTH WAVE NUMBER INTENSITY CLASSIfICATION CODE

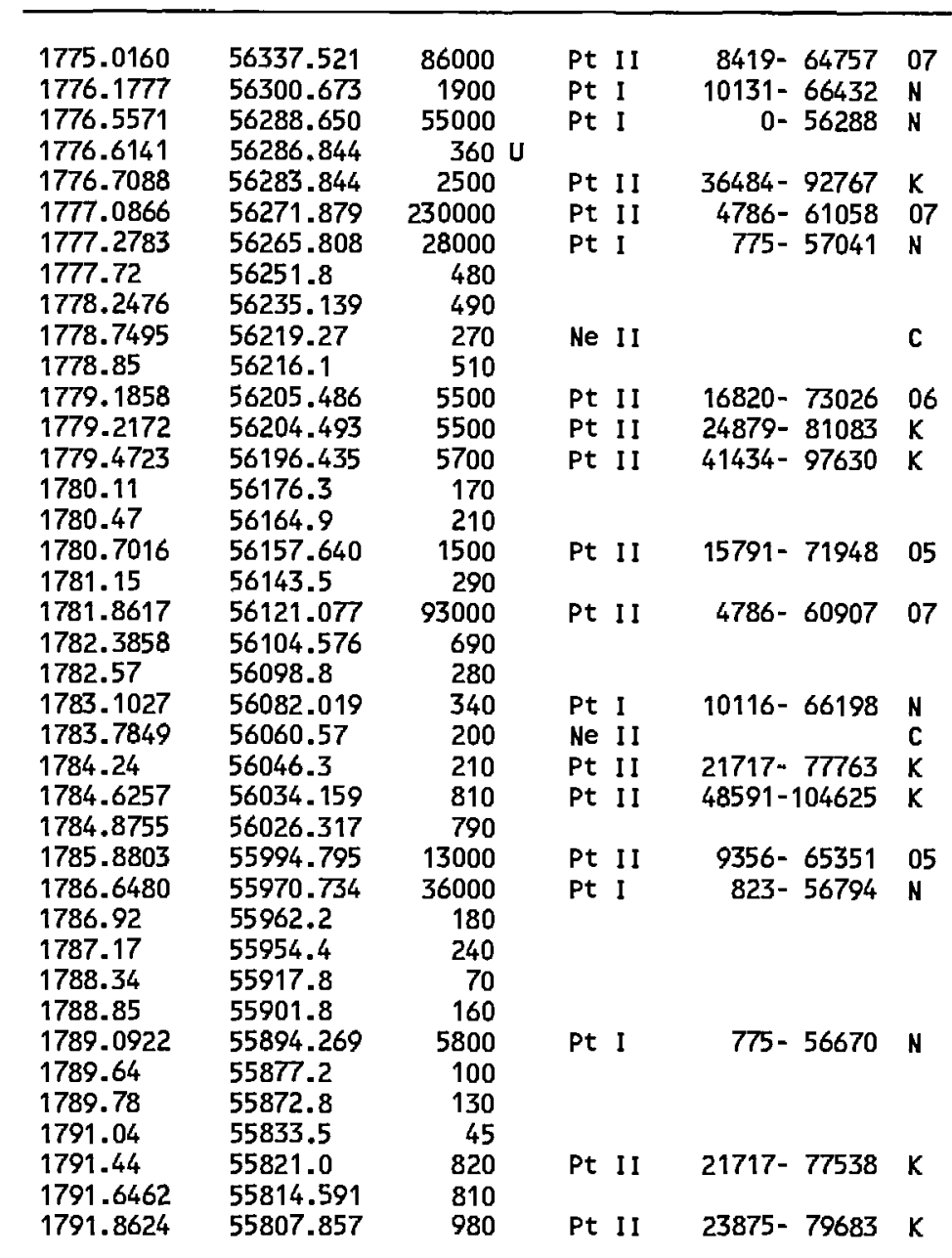




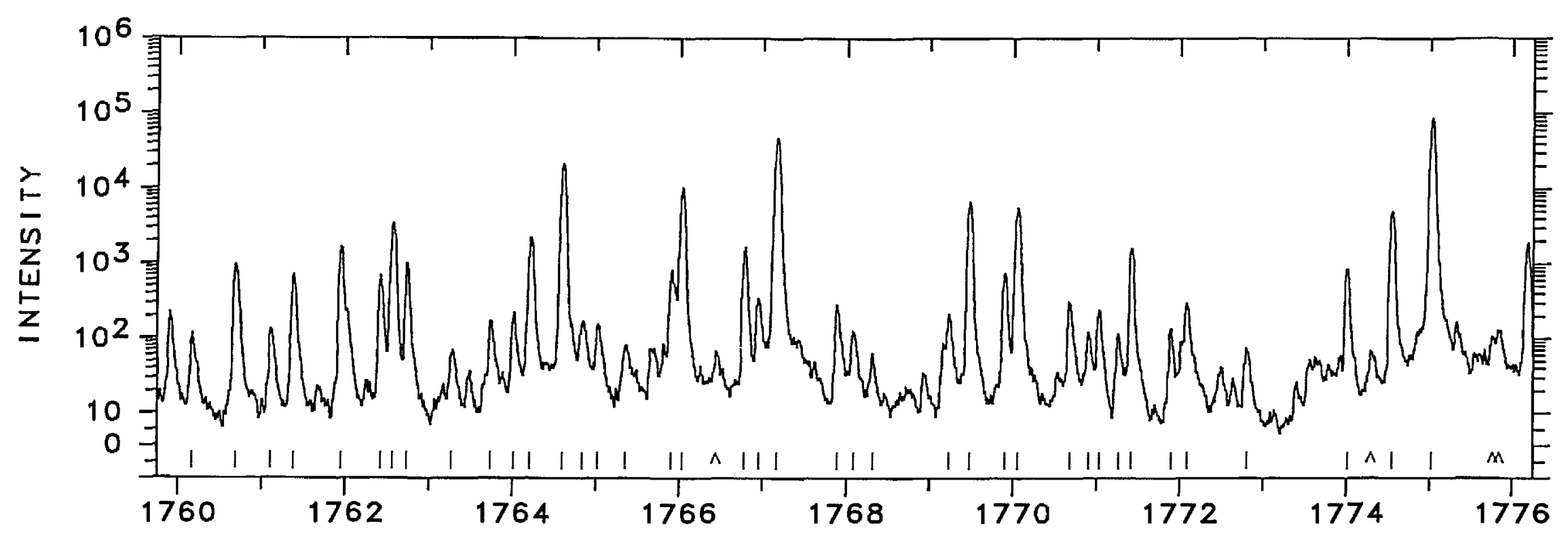

$\cong$

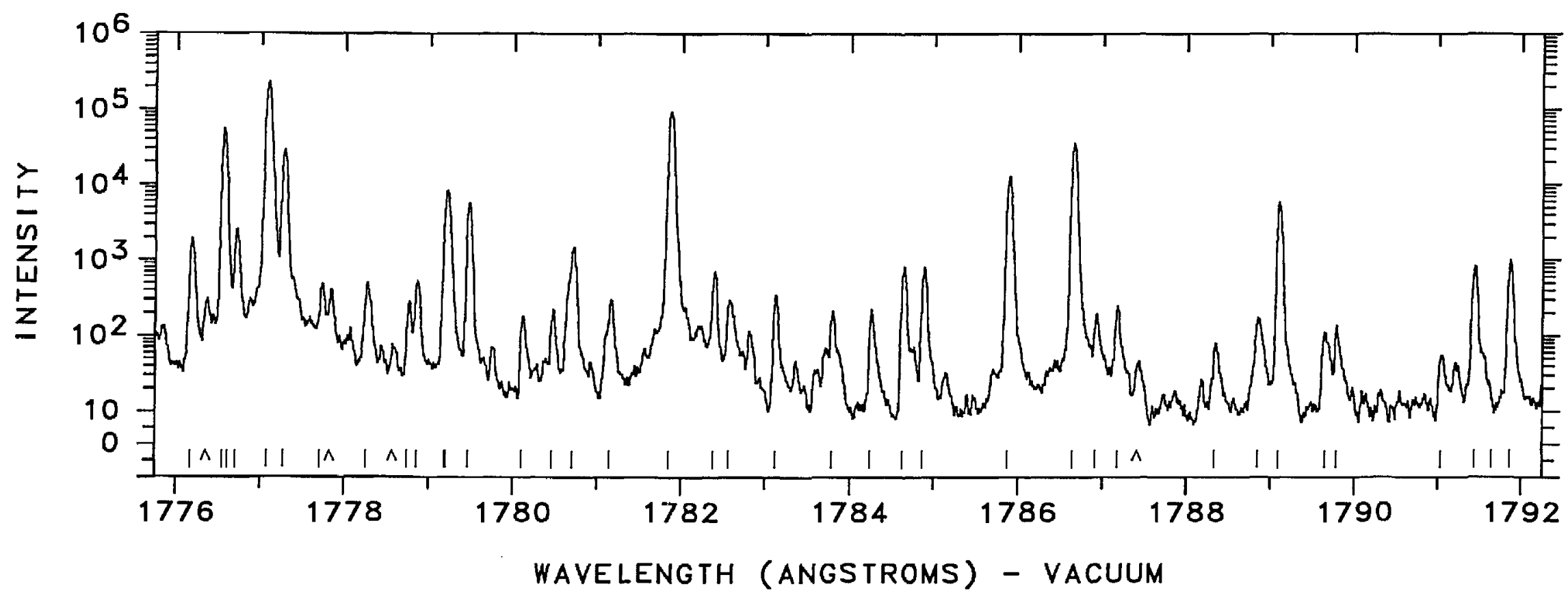


WAVELENGTH WAVE NUMBER INTENSITY

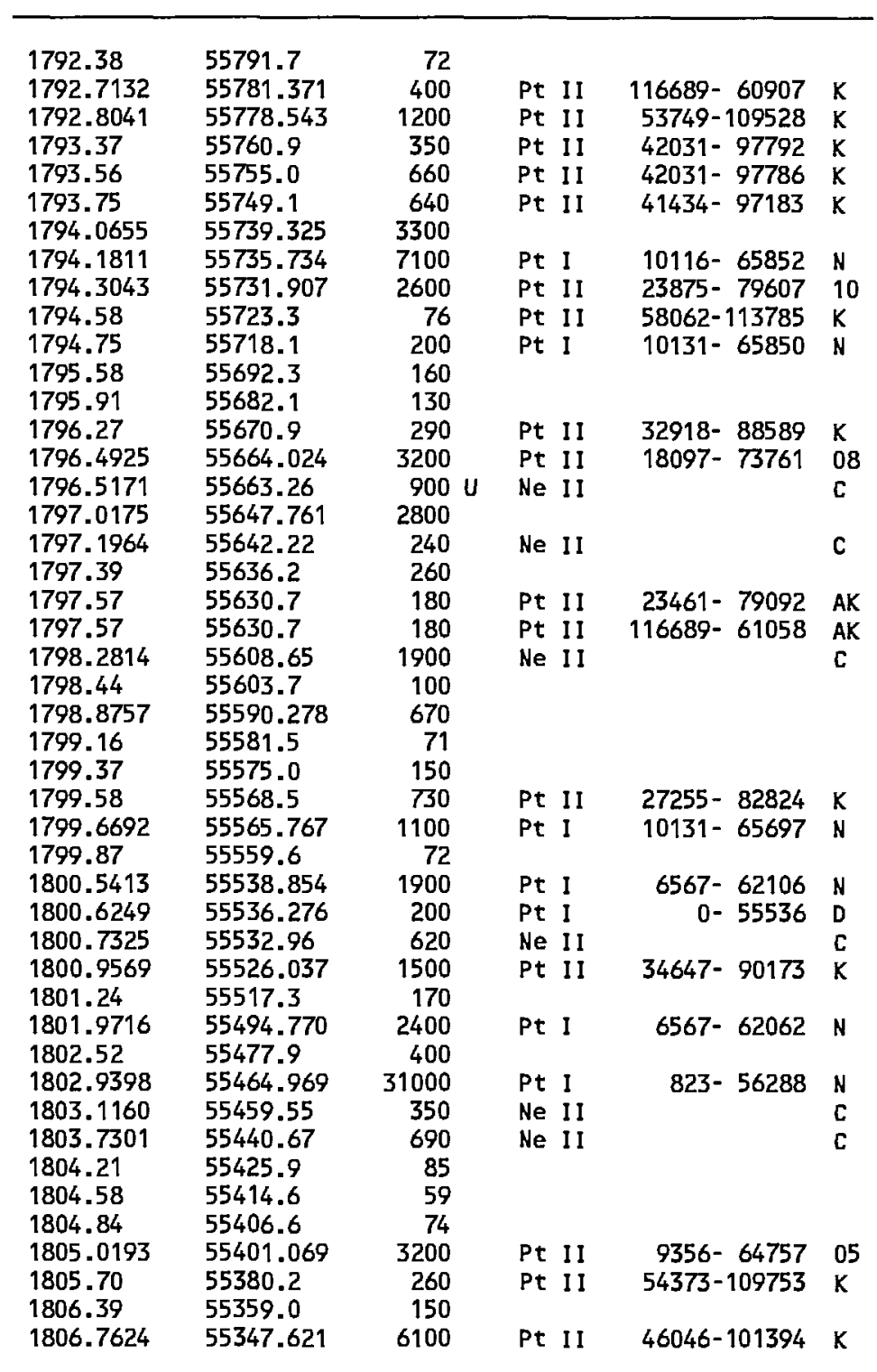

WAVELENGTH WAVE NUMBER INTENSITY CLASSIFICATION CODE

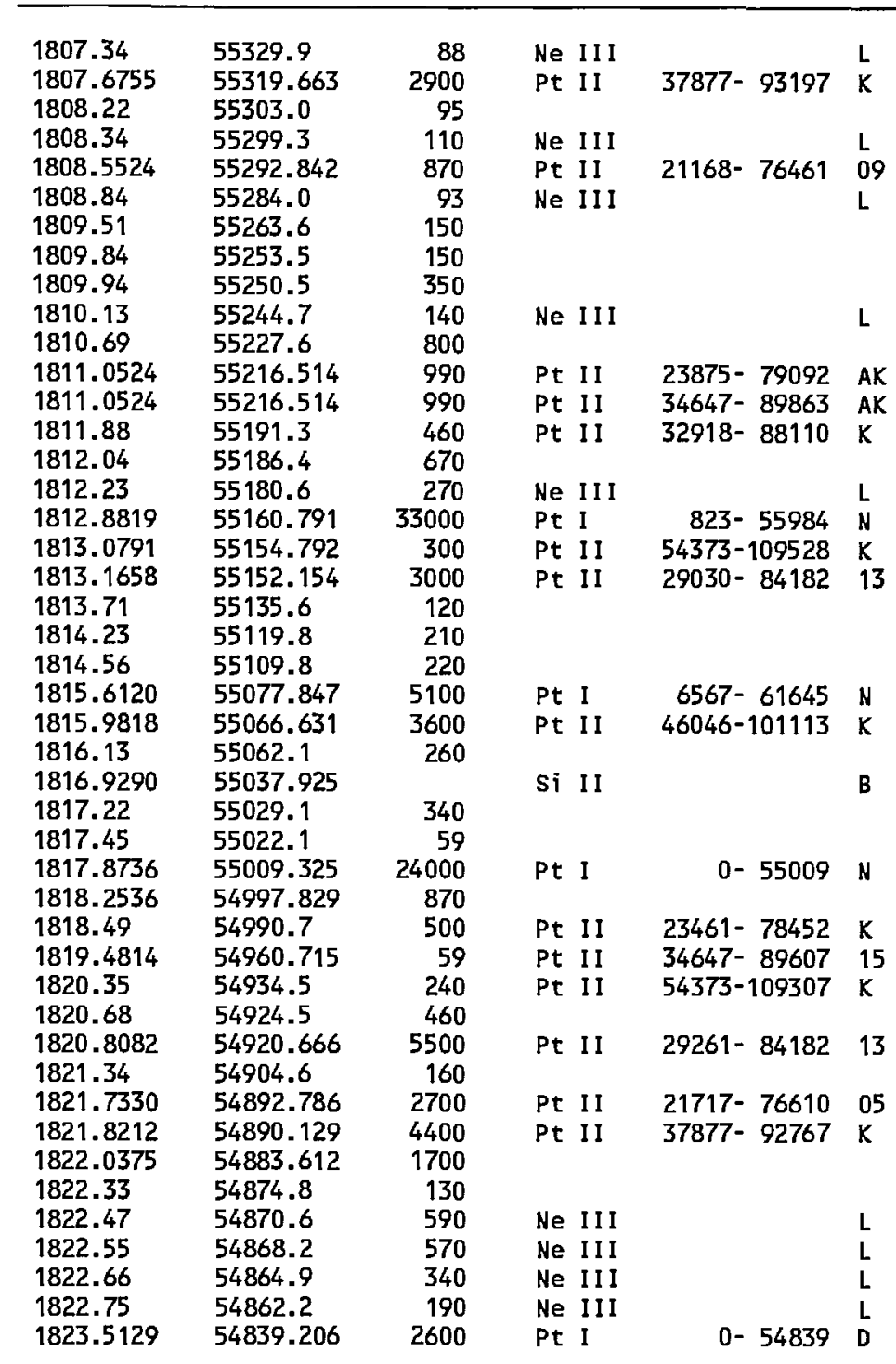




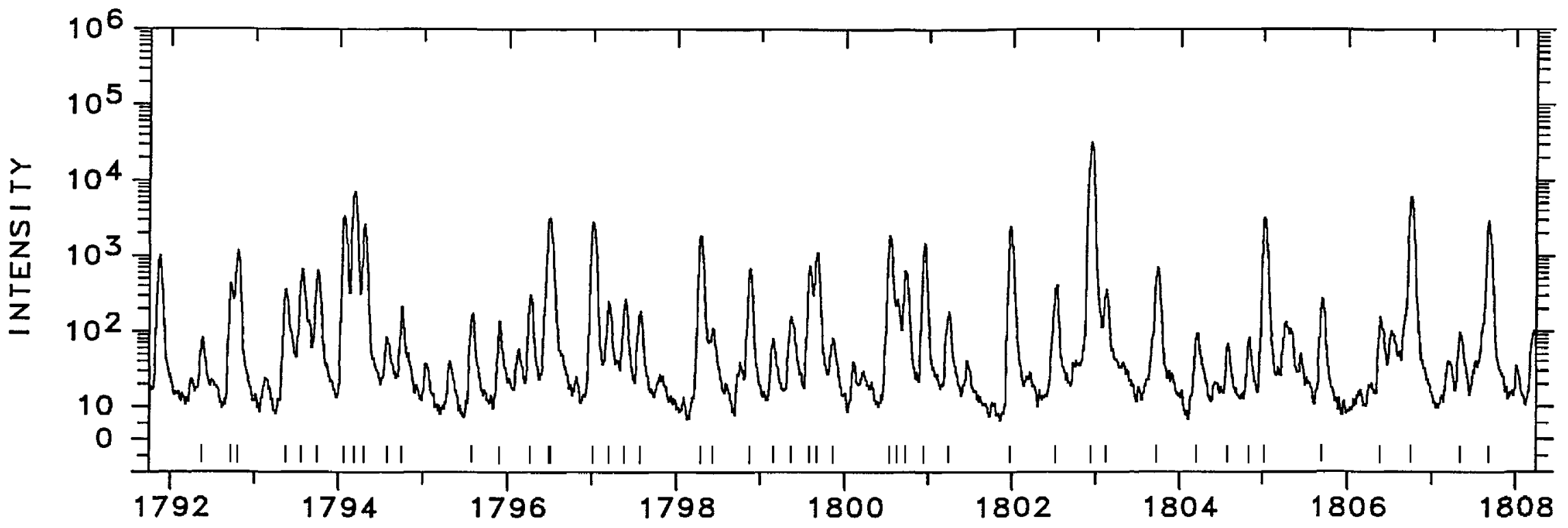

w

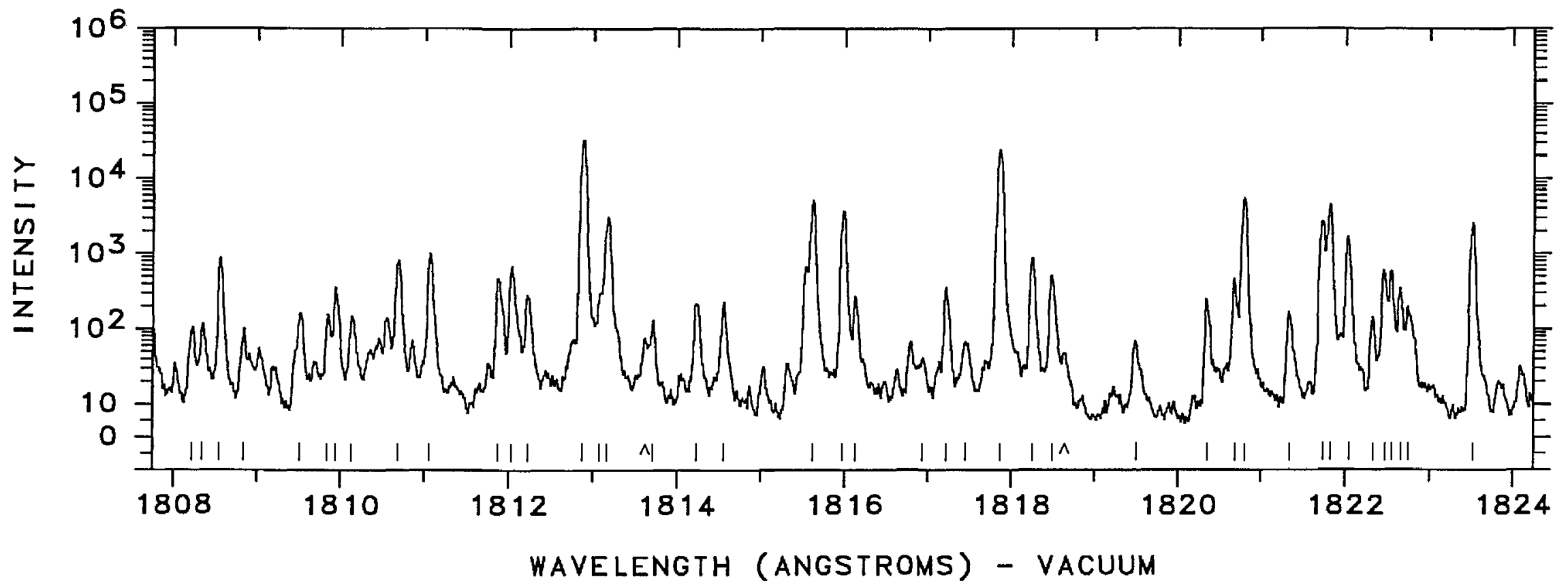




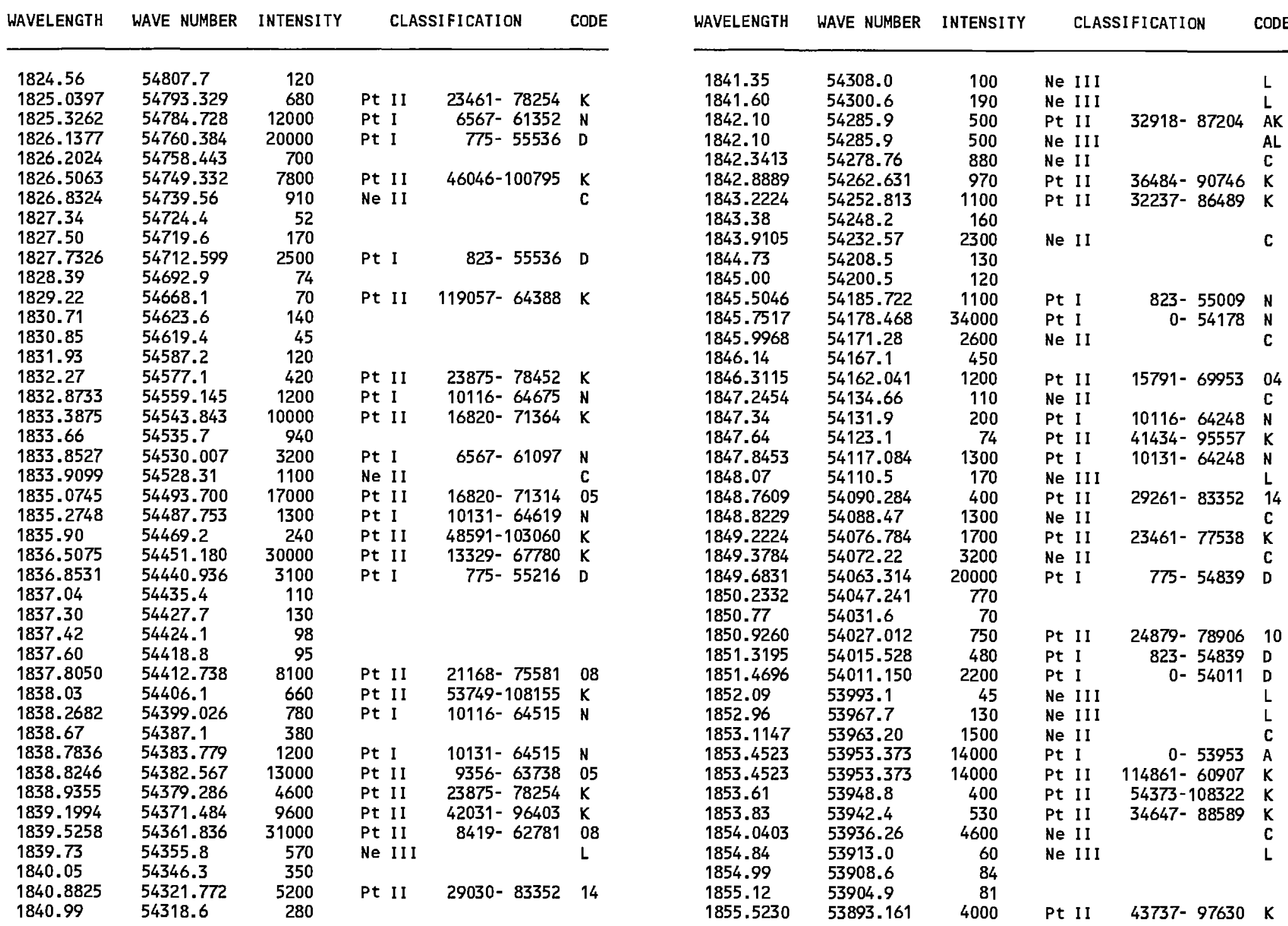




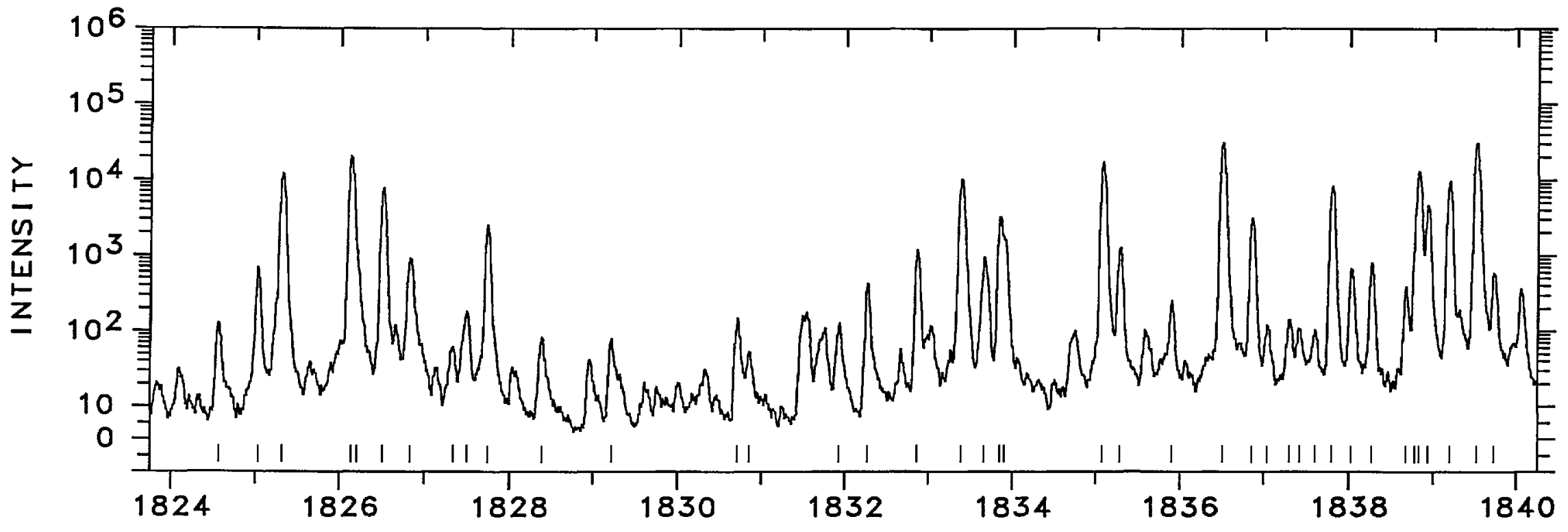

un

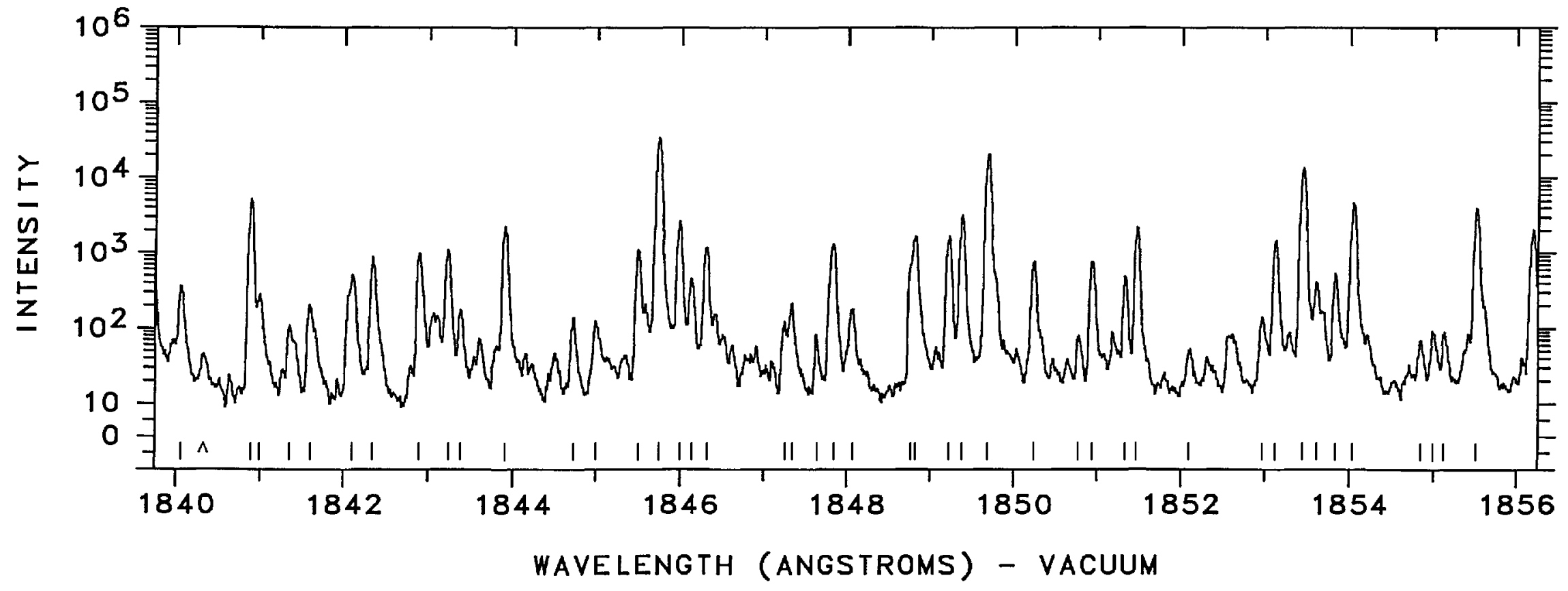




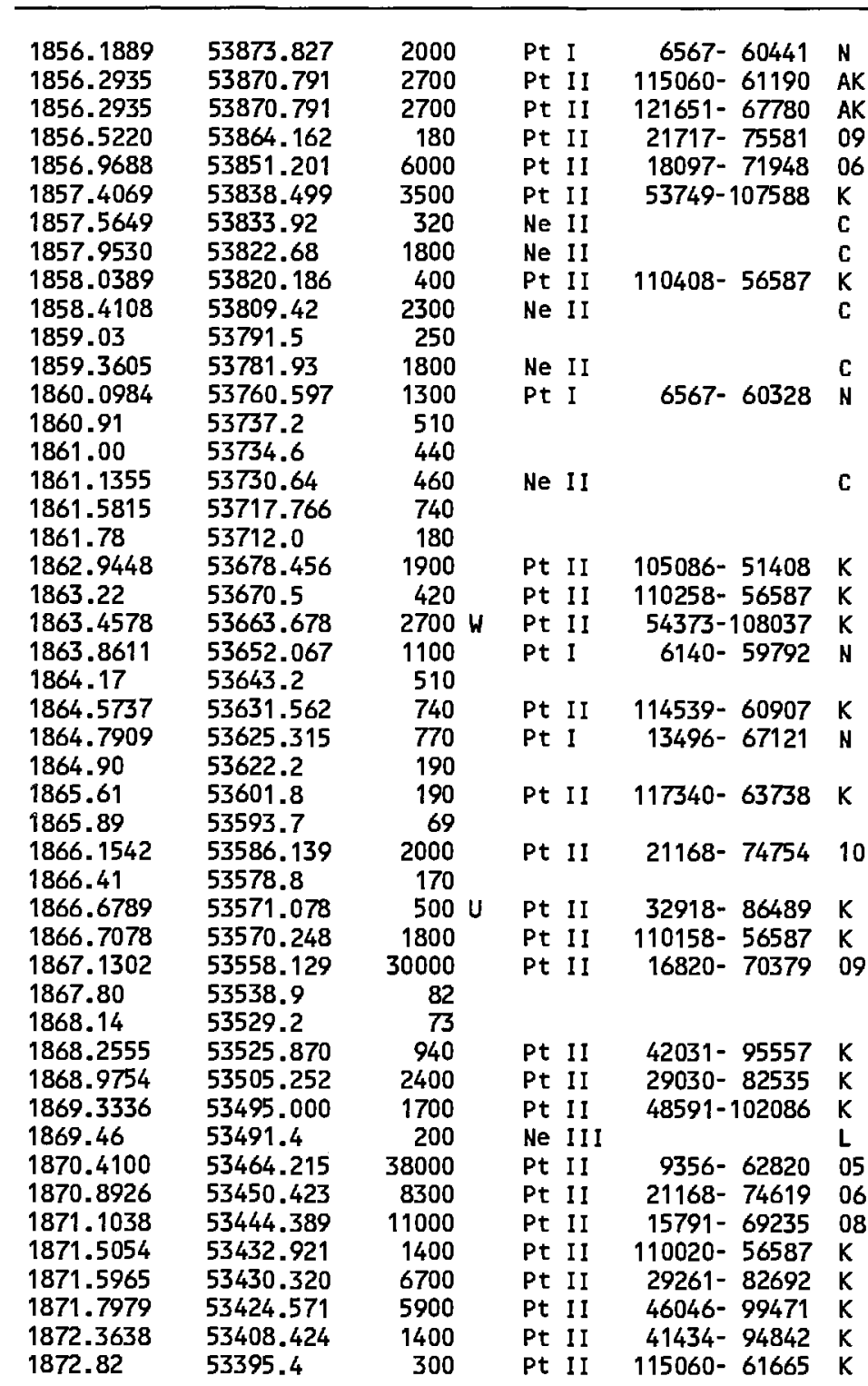

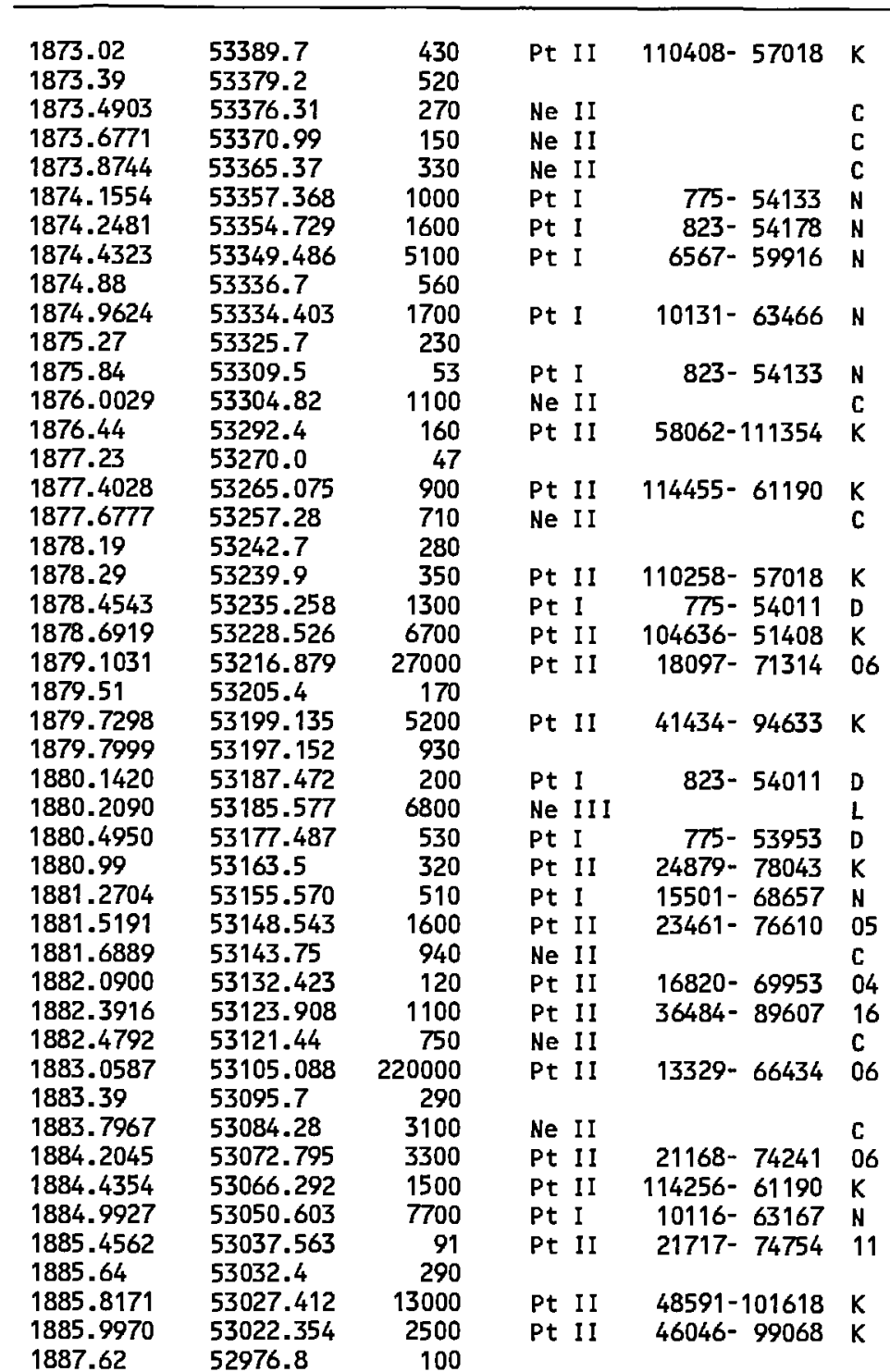



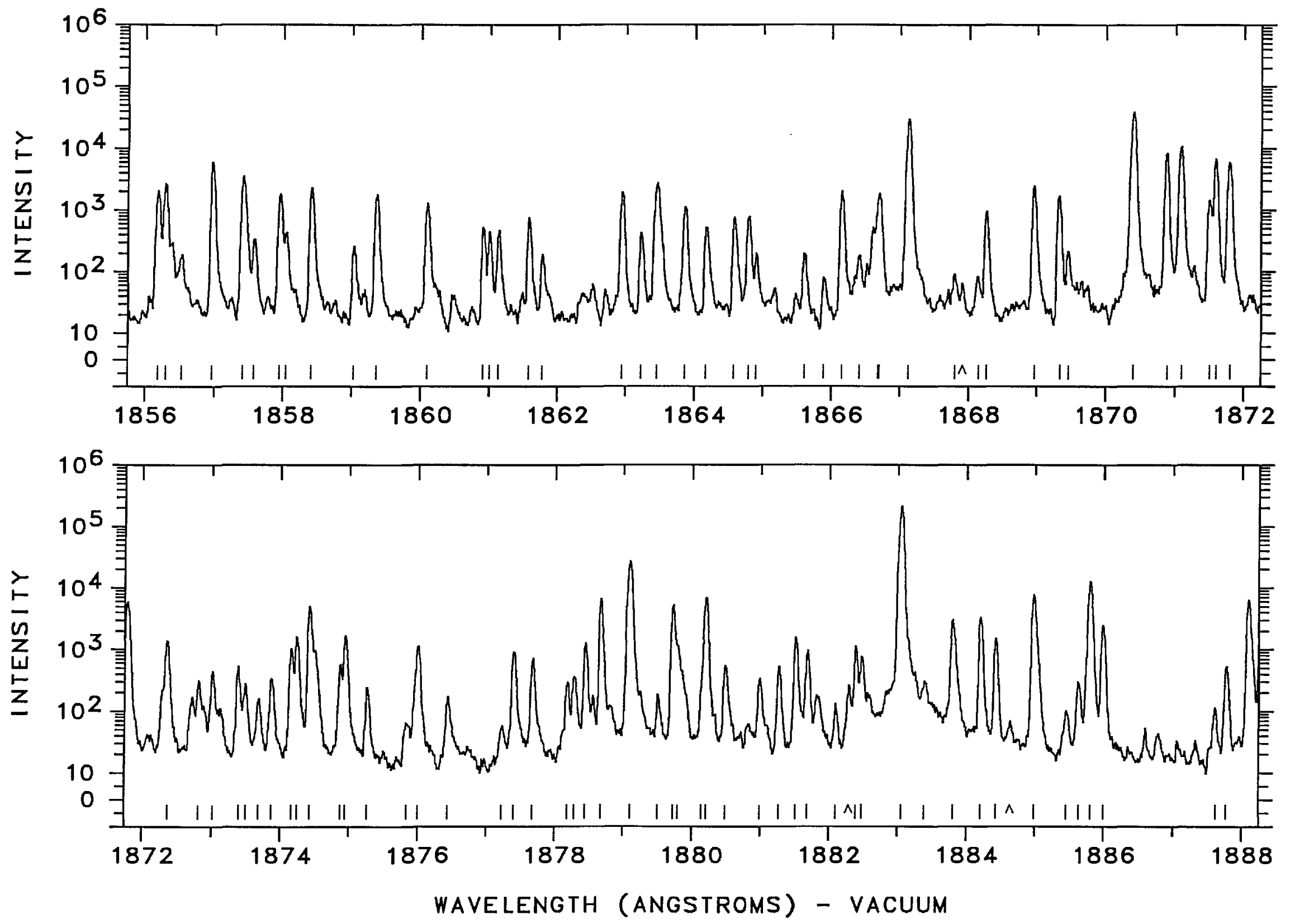
WAVELENGTH WAVE NUMBER INTENSITY

CLASSIFICATION CODE

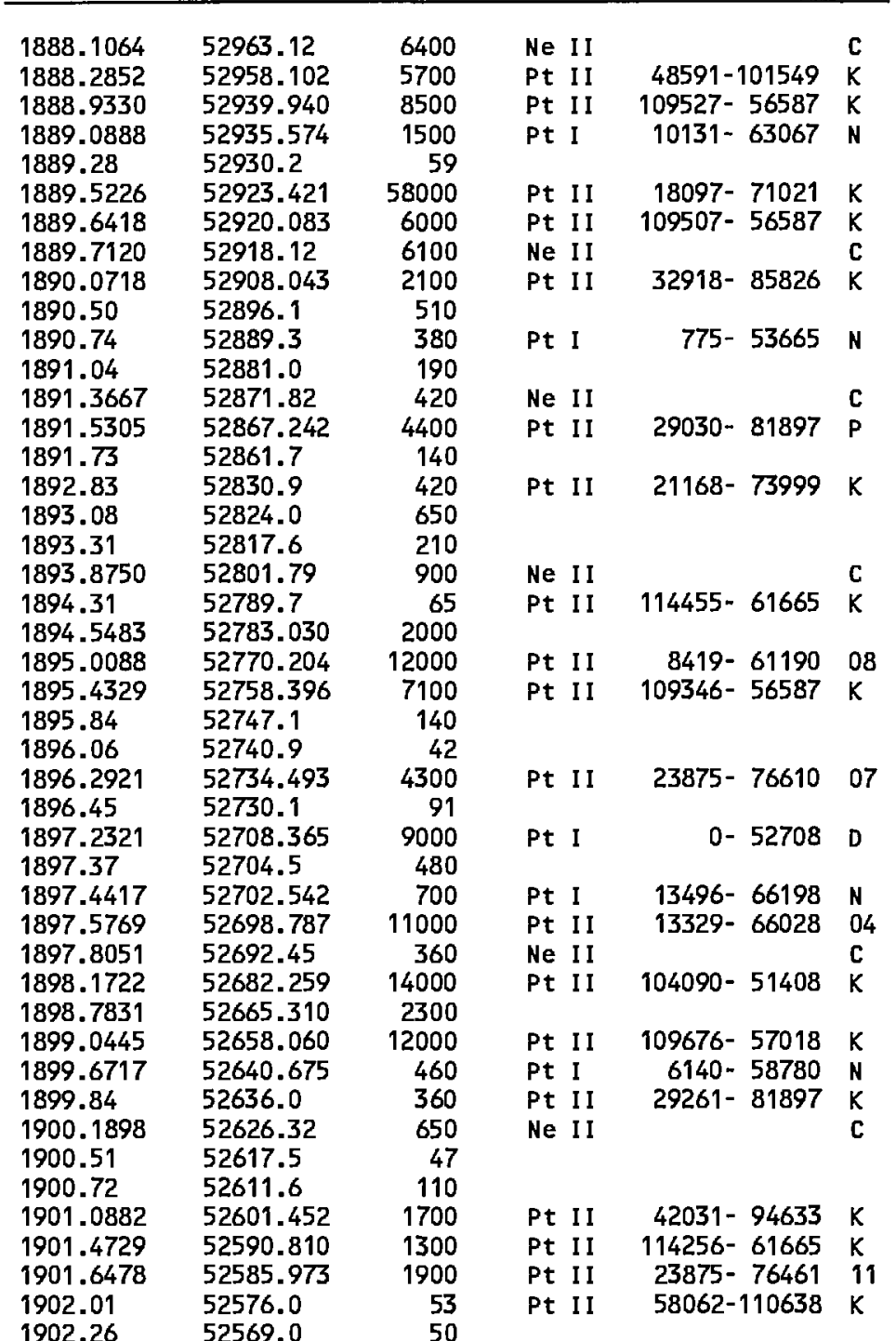

WAVELENGTH WAVE NUMBER INTENSITY CLASSIFICATION CODE

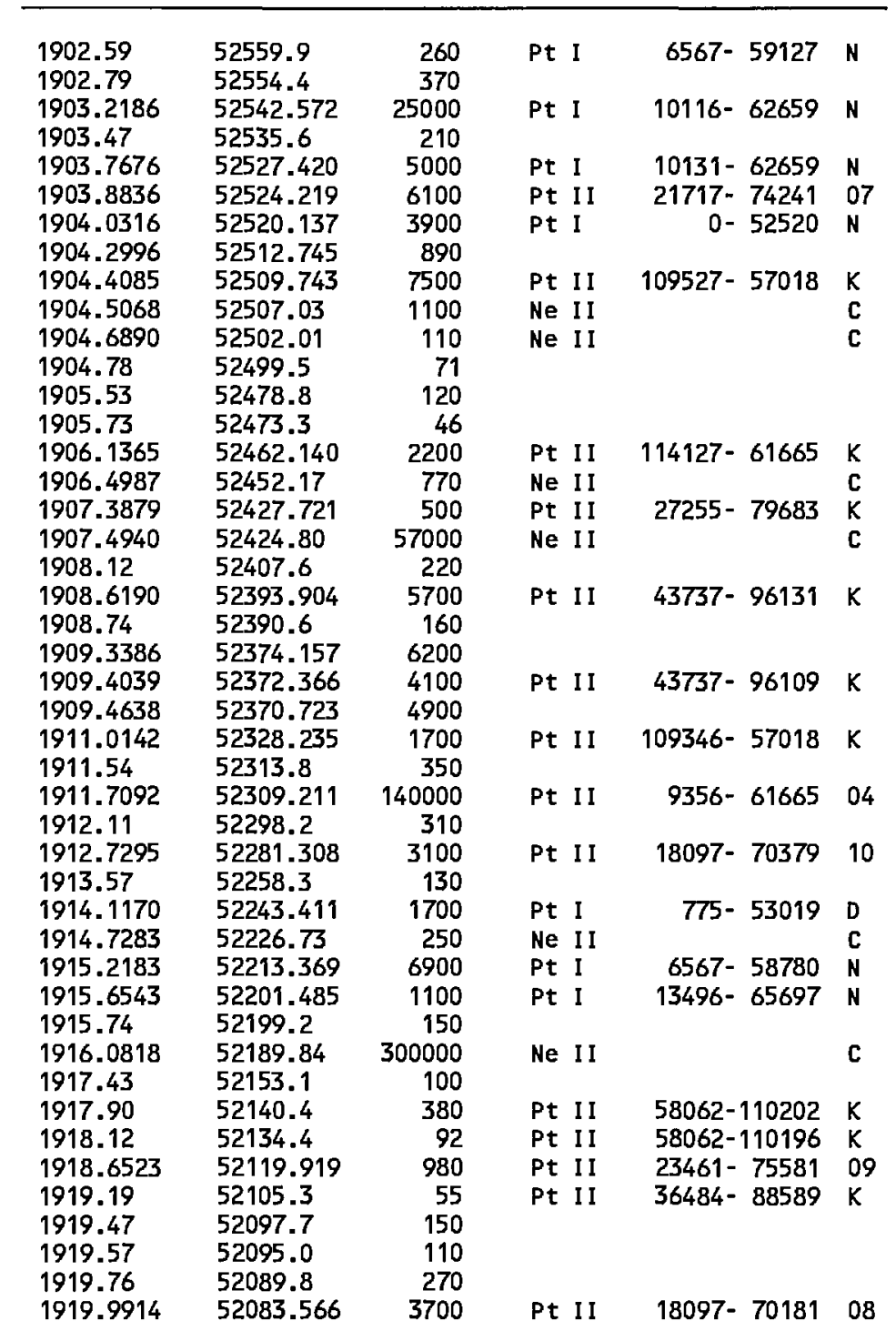



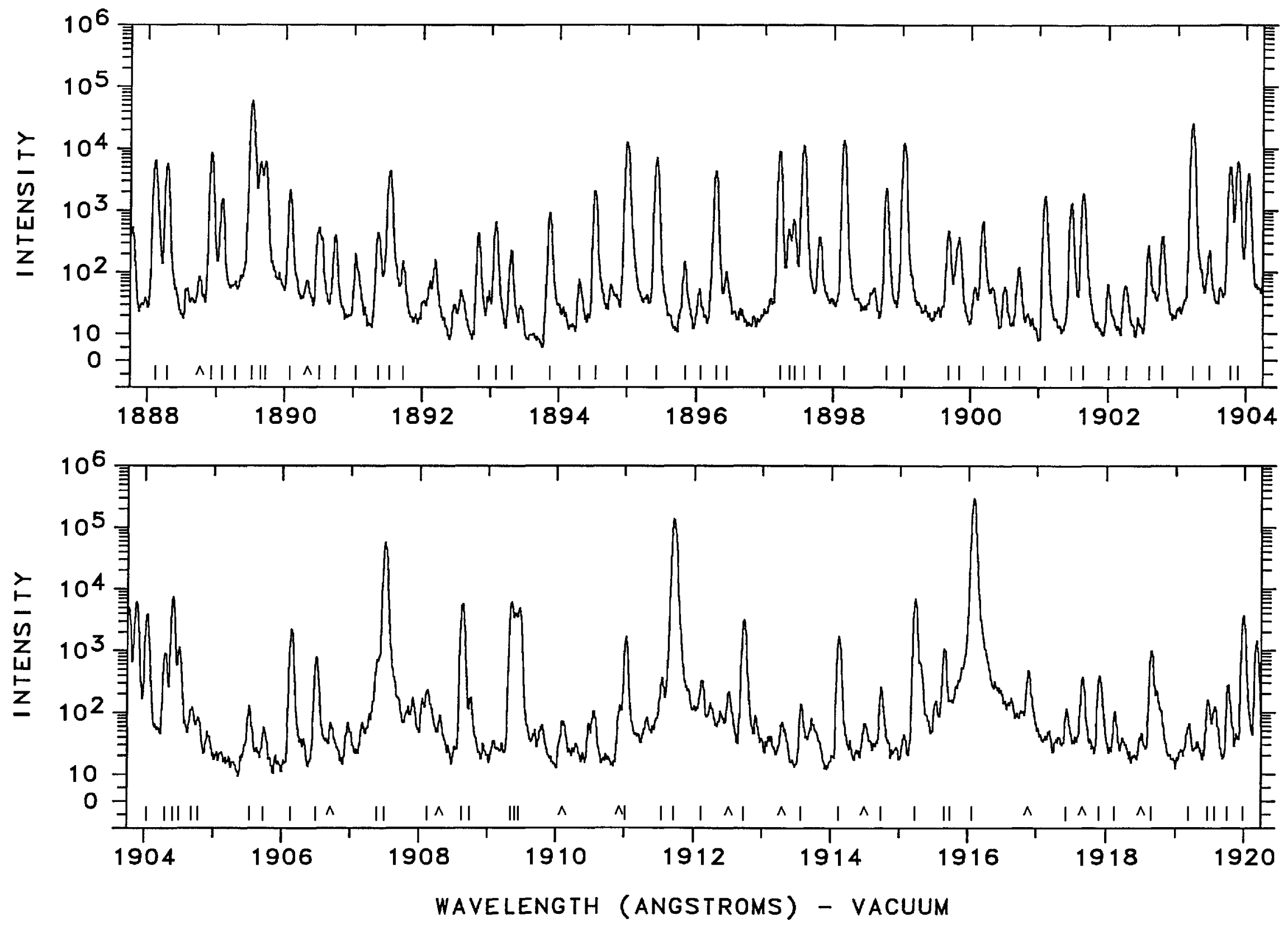
WAVELENGTH WAVE NUMBER INTENSITY CLASSIFICATION CODE

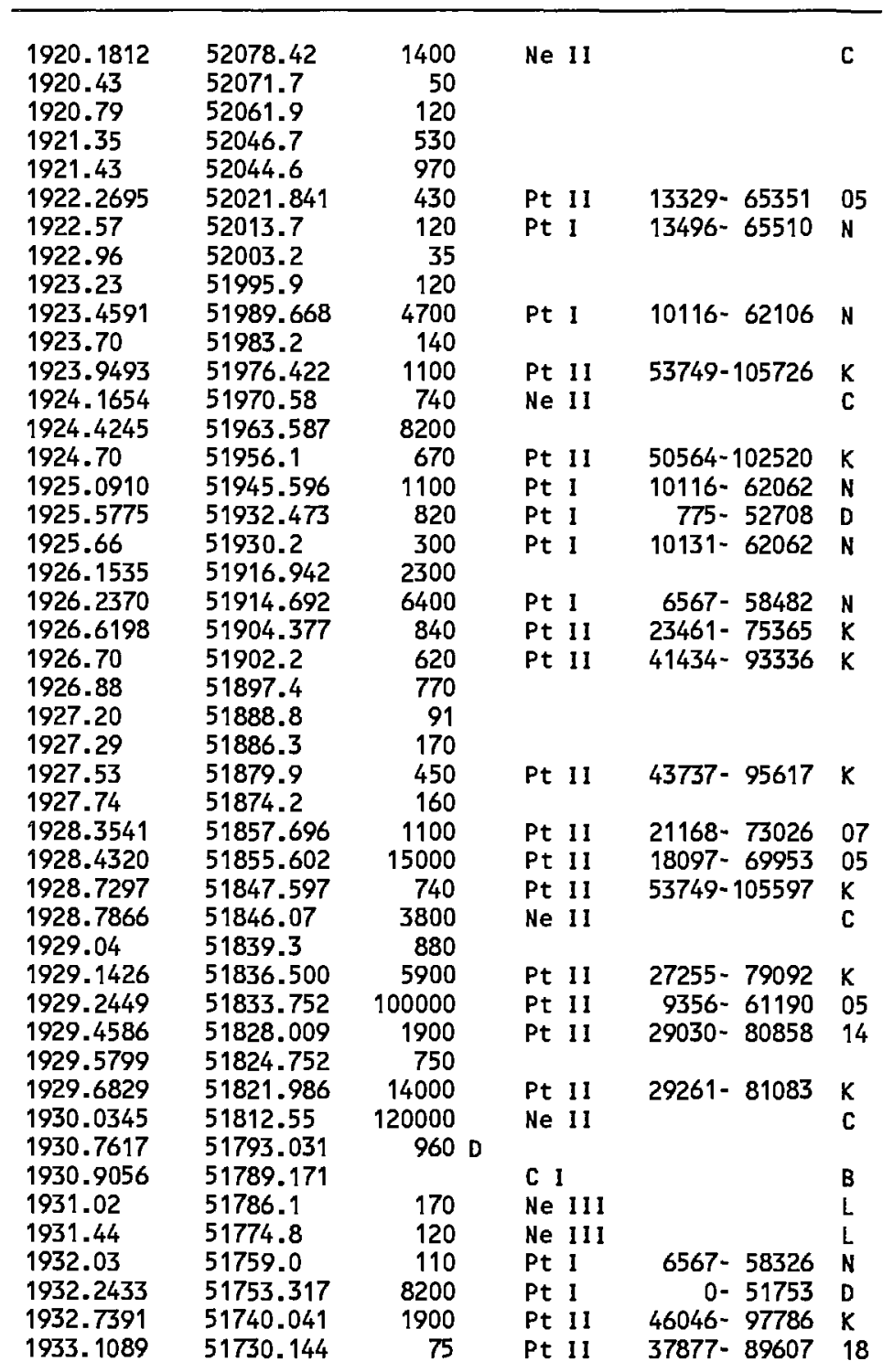

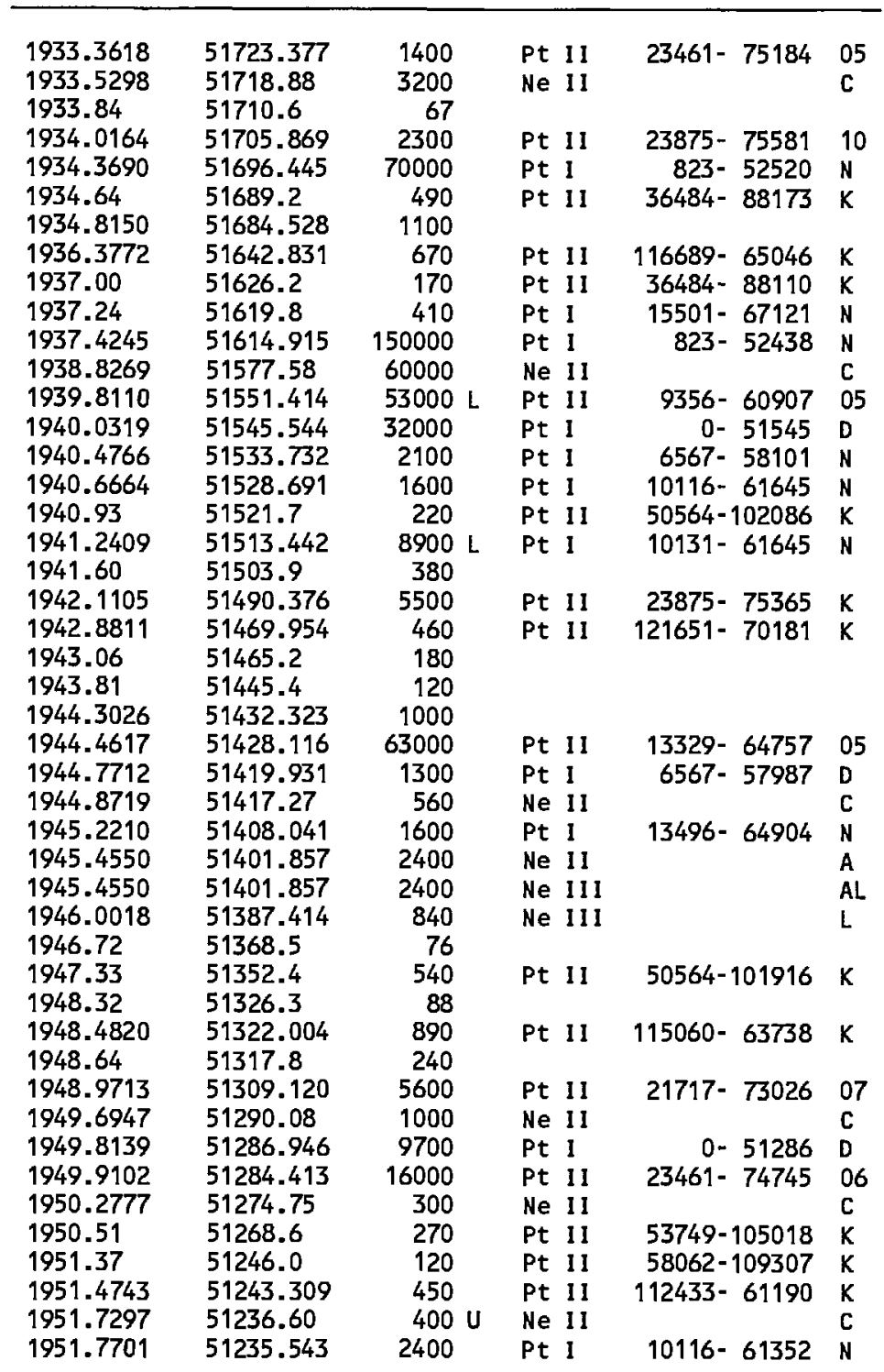



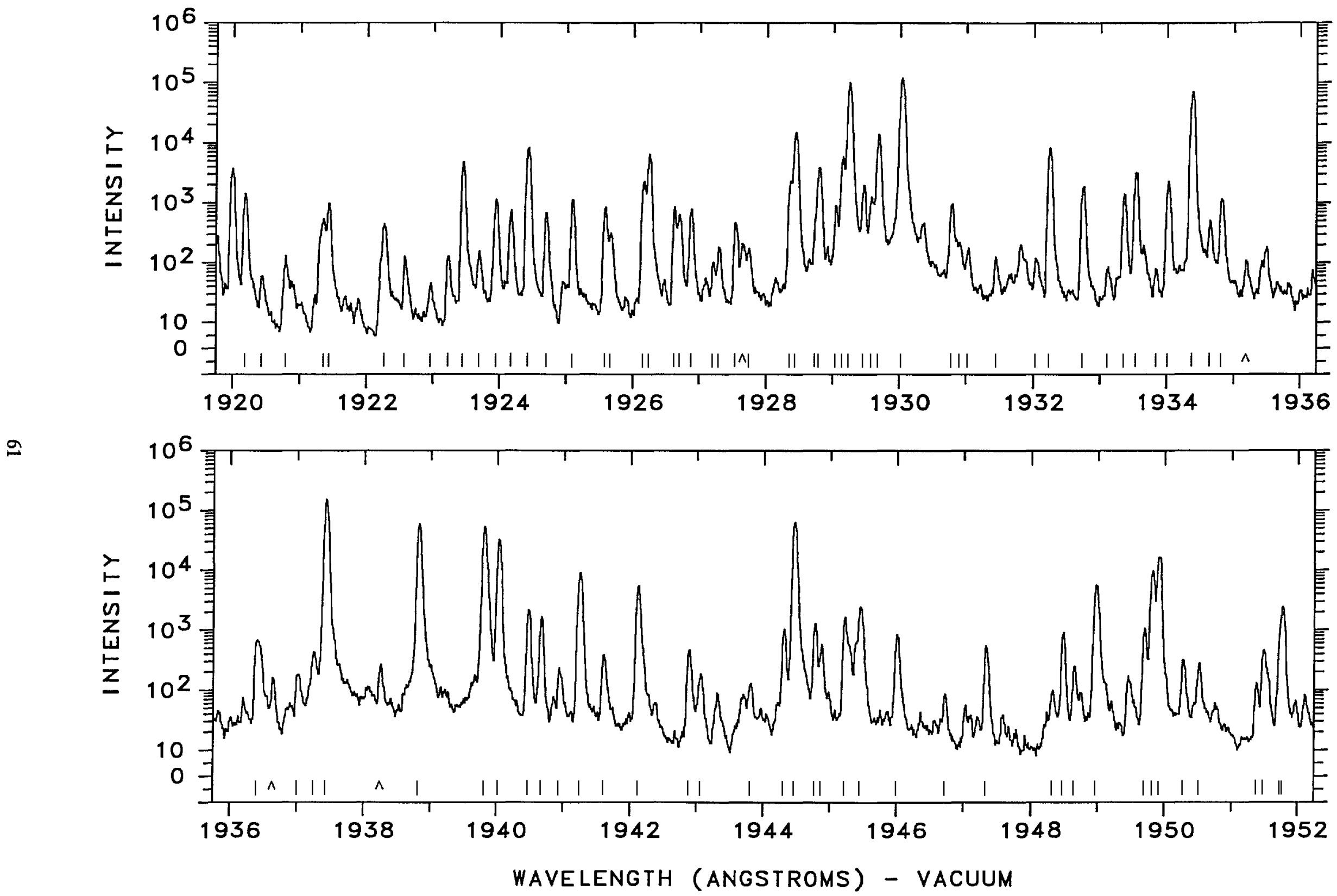


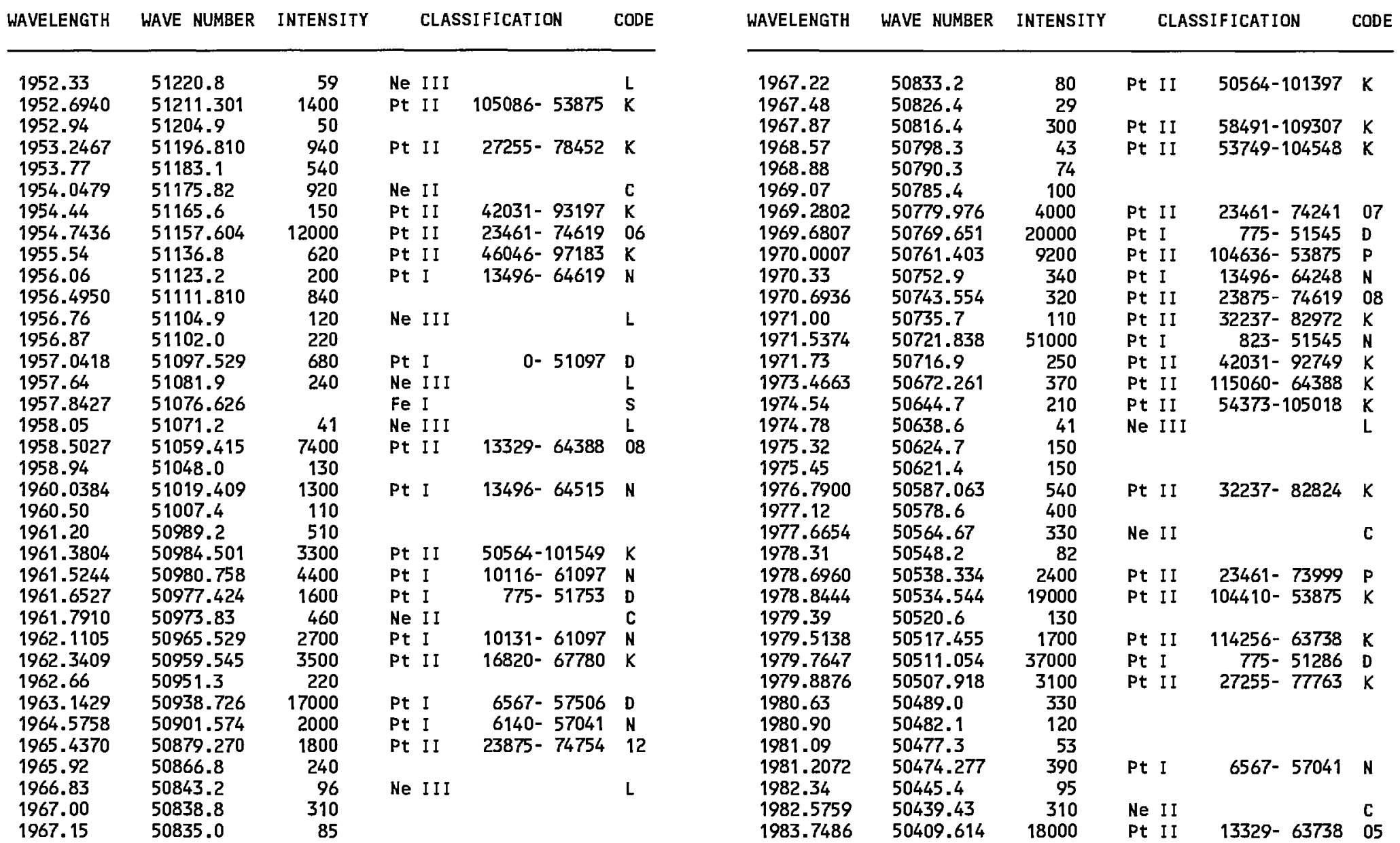




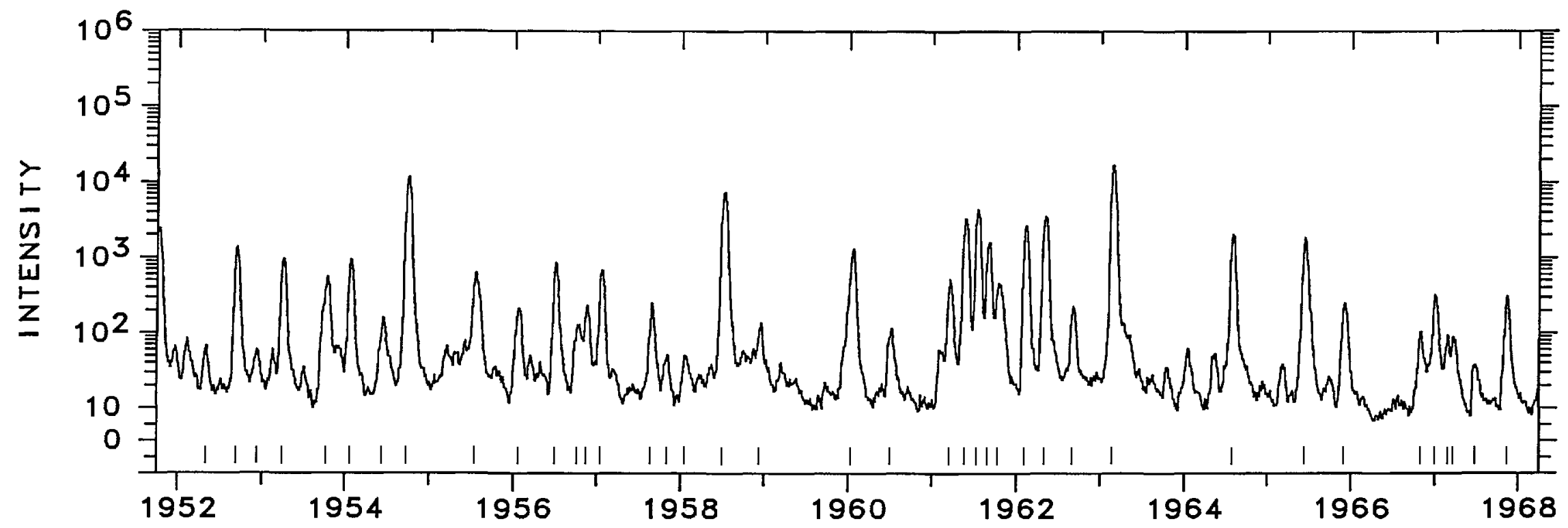

ธิ

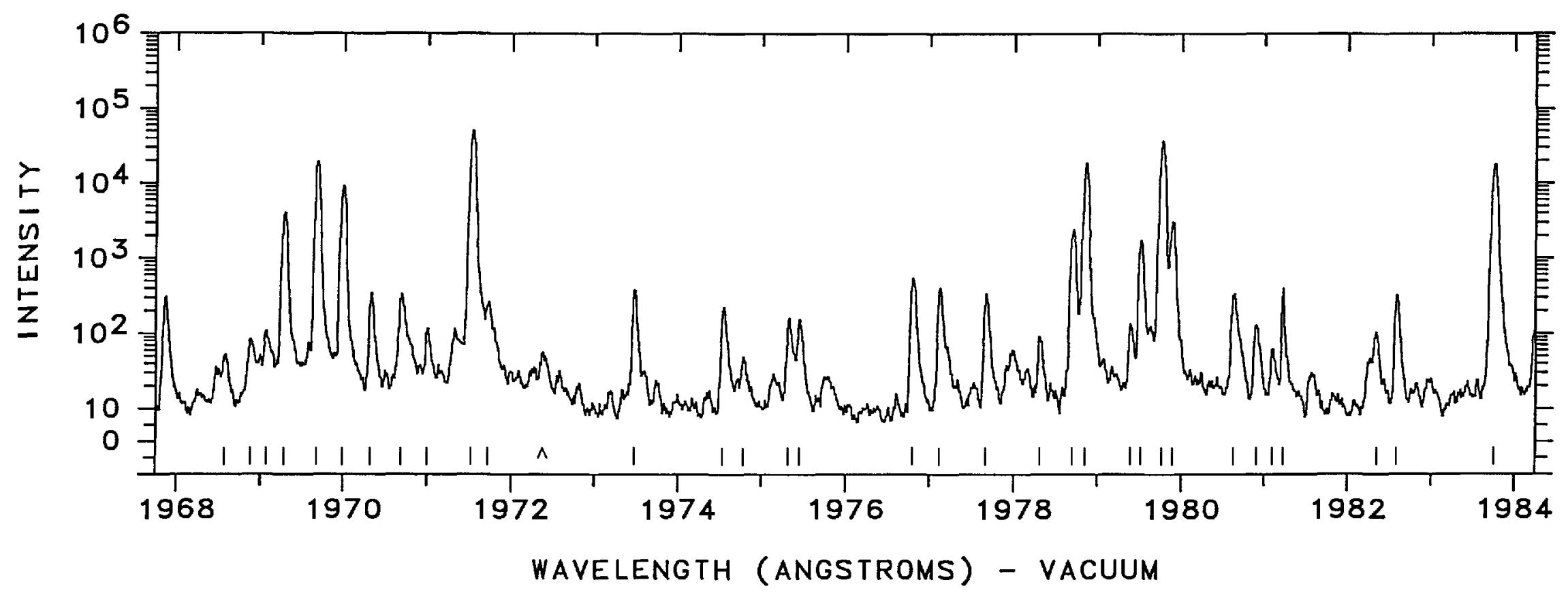




\begin{tabular}{|c|c|c|c|c|c|}
\hline \multirow{2}{*}{$\begin{array}{c}\text { WAVELENGTH } \\
1984.23\end{array}$} & WAVE NUMBER & INTENSITY & \multicolumn{2}{|c|}{ CLASSIFICATION } & CODE \\
\hline & $\begin{array}{l}50397.4 \\
50395.4\end{array}$ & $\begin{array}{l}96 \\
71\end{array}$ & Pt II & $46046-96443$ & $K$ \\
\hline & 50388.754 & 3700 & Pt II & $114127-63738$ & K \\
\hline .4693 & 50365.926 & 160 & Pt II & $23875-74241$ & 09 \\
\hline 198 & 50337 & 130 & Pt II & $113119-62781$ & $\mathrm{~K}$ \\
\hline & 50330 & 970 & Pt I & $13496-63826$ & $\mathrm{~N}$ \\
\hline & 503 & 1 & Pt I & $775-51097$ & D \\
\hline 987 & 50309.4 & 1500 & Pt I & $10131-60441$ & $\mathrm{~N}$ \\
\hline .7868 & 503 & 13000 & Pt I & $10116-60423$ & $\ddot{N}$ \\
\hline 19 & & 2200 & Pt 1 & $79-75184$ & 05 \\
\hline 19 & 503 & 5900 & Pt I & $23461-73761$ & 10 \\
\hline & 50283.9 & 230 & & & \\
\hline & & $1000 \mathrm{U}$ & $\mathrm{Ne} \mathrm{I}$ & & A \\
\hline & 50274.939 & U & Si I & & A \\
\hline 1989.1056 & 50273.852 & 23000 & Pt I & $823-51097$ & D \\
\hline & 50270.816 & 200 & & & \\
\hline 1989.65 & 50260.1 & & Pt I & $58062-108322$ & $k$ \\
\hline 1990.5751 & 50236.738 & 32000 & Pt I & $15791-66028$ & 05 \\
\hline & & 260 & Pt II & $37877-88110$ & K \\
\hline 1991.4283 & & & Pt II & $104090-53875$ & K \\
\hline & & & Pt I & $10116-60328$ & $\mathrm{~N}$ \\
\hline
\end{tabular}

WAVELENGTH WAVE NUMBER INTENSITY CLASSIFICATION

CODE

\begin{tabular}{llrlrl}
\hline 1991.8236 & 50205.249 & 2500 & & & \\
1992.1936 & 50195.924 & 1300 & Pt I & $15501-65697$ & AN \\
1992.1936 & 50195.924 & 1300 & Pt II & $21168-71364$ & AK \\
1993.52 & 50162.5 & 91 & & & \\
1994.0957 & 50148.05 & 2200 & Ne II & & C \\
1994.46 & 50138.9 & 190 & & & \\
1995.04 & 50124.3 & 220 & Pt II & $23875-73999$ & K \\
1995.2792 & 50118.30 & 240 & Ne II & & C \\
1995.8991 & 50102.733 & 17000 & Pt I & $6567-56670$ & N \\
1996.27 & 50093.4 & 270 & Pt II & $58062-108155$ & K \\
1996.80 & 50080.1 & 77 & & & \\
1997.10 & 50072.6 & 70 & & & \\
1997.36 & 50066.1 & 42 & & & \\
1997.8371 & 50054.131 & 590 & Pt II & $32918-82972$ & P \\
1998.16 & 50046.0 & 330 & & & \\
1998.41 & 50039.8 & 140 & Pt I & $18566-68606$ & $\mathrm{~N}$ \\
1998.6681 & 50033.32 & 190 & Ne II & & C \\
1998.86 & 50028.5 & 270 & & & \\
1999.28 & 50018.0 & 44 & & & \\
1999.5947 & 50010.135 & 280 & Pt I & $0-50010$ & N
\end{tabular}




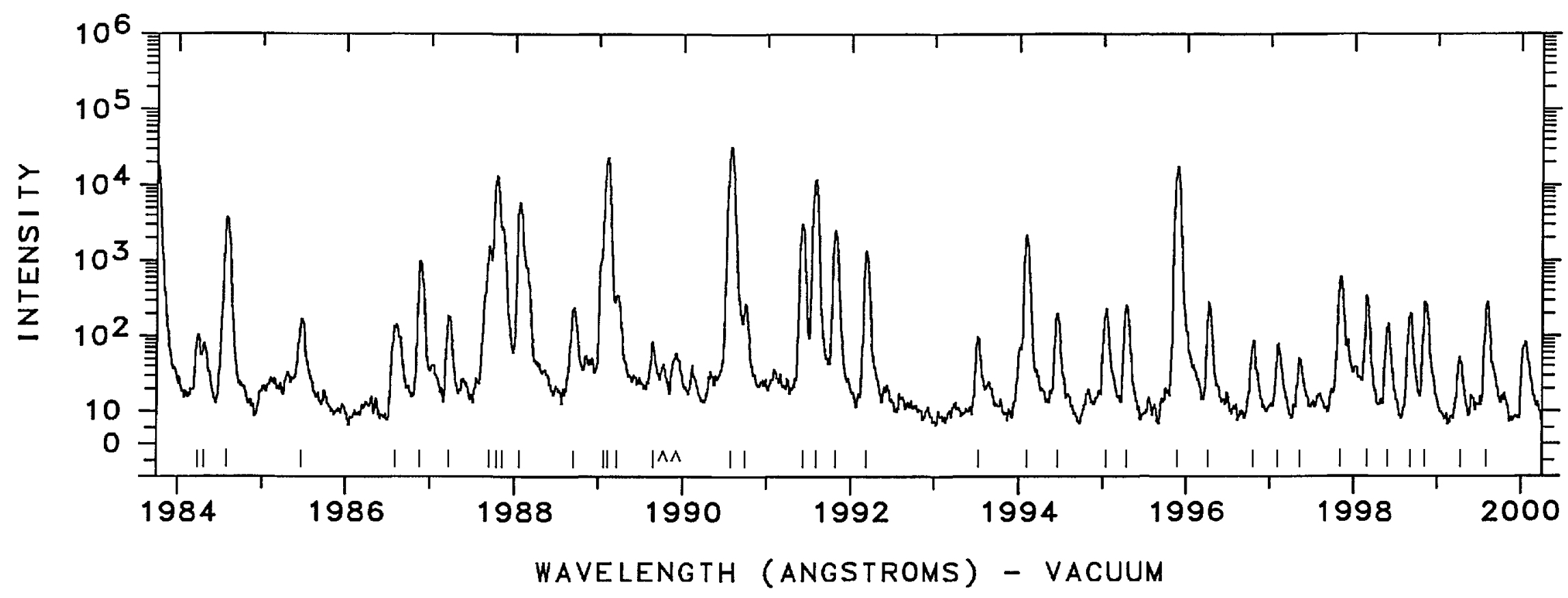




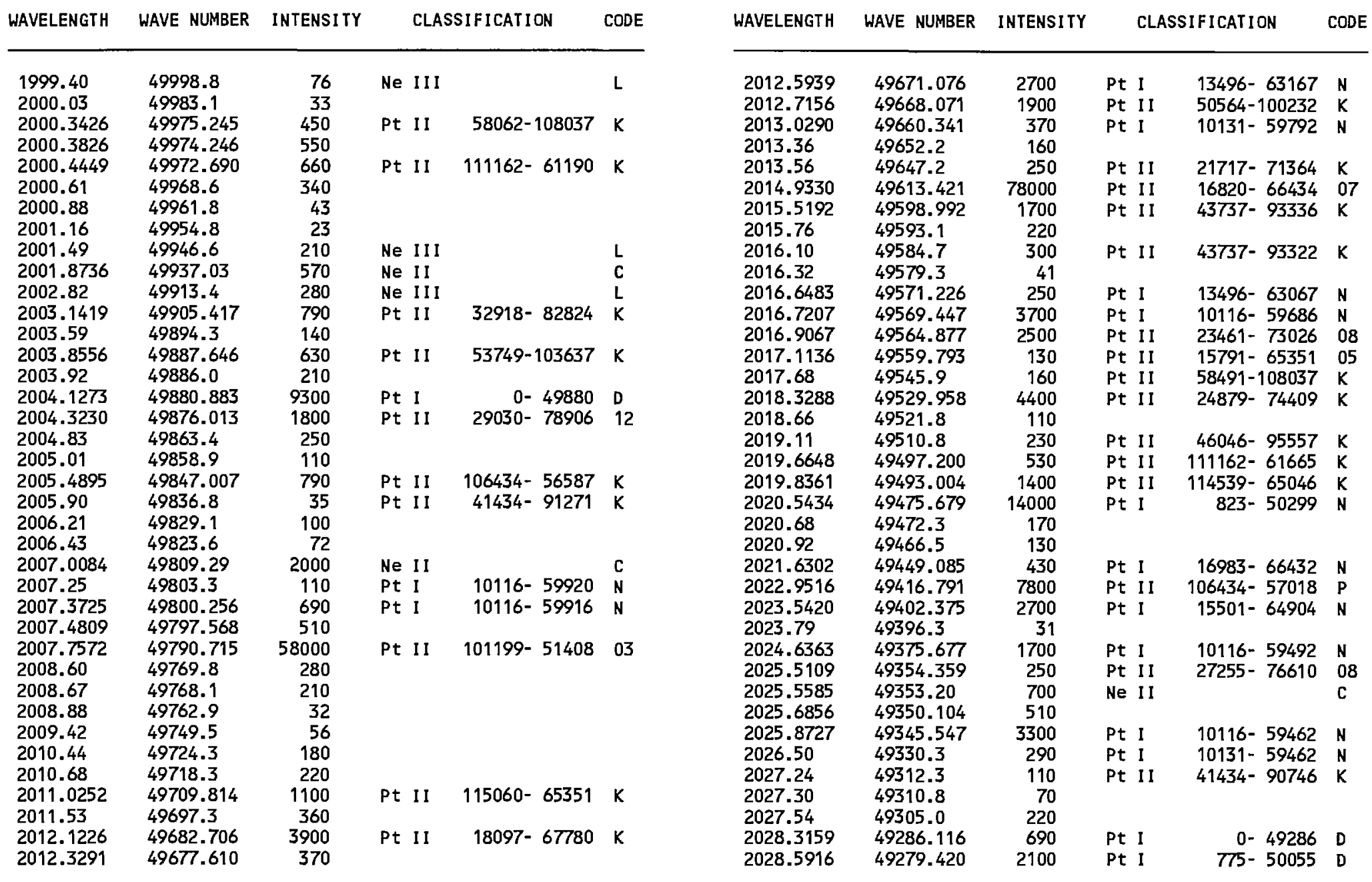




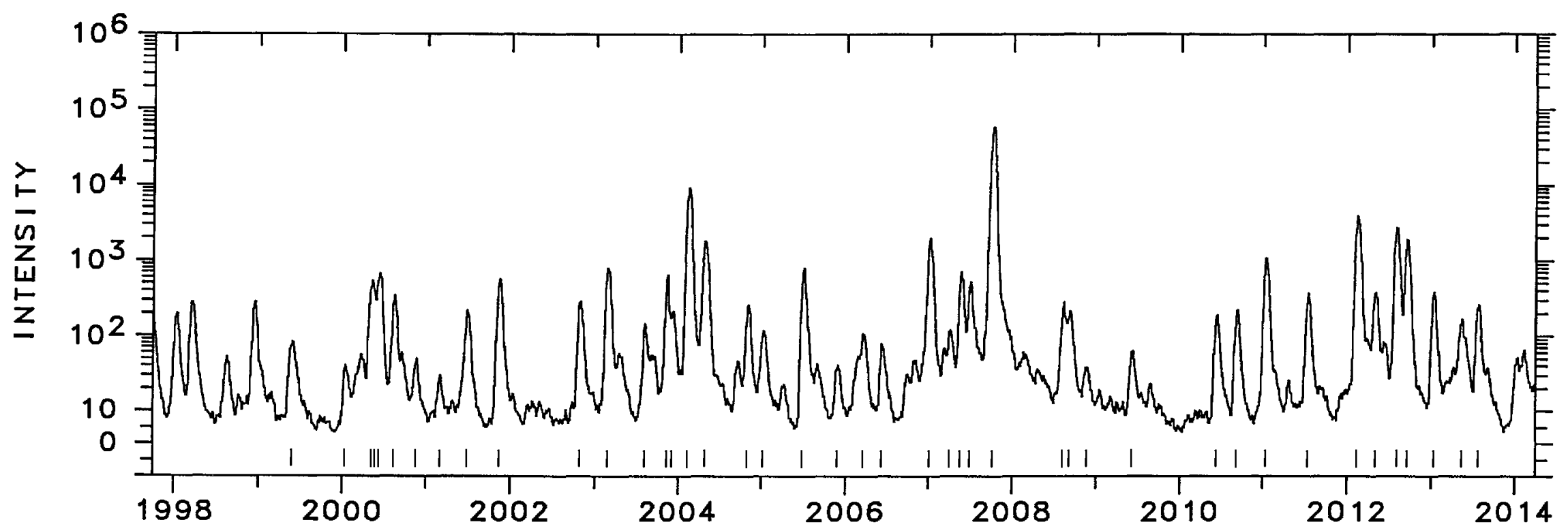

3

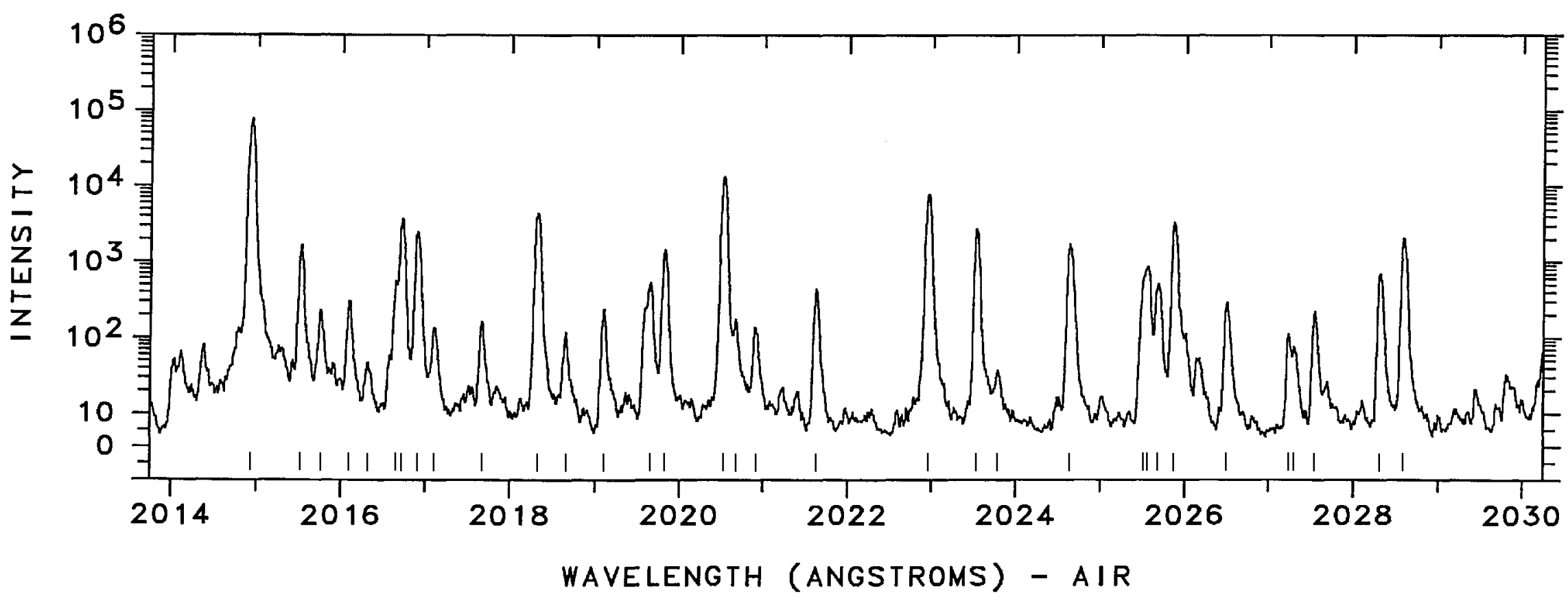




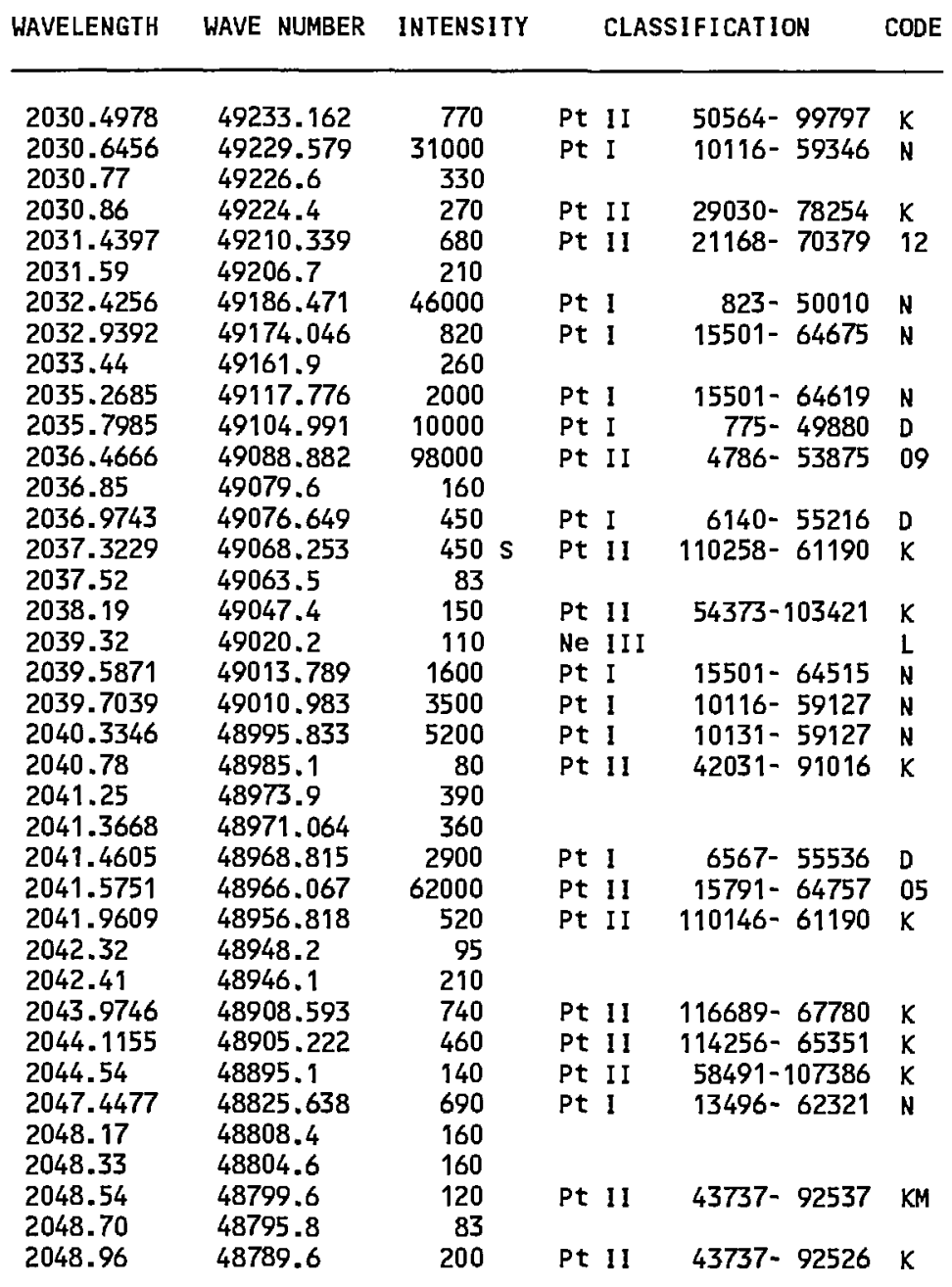

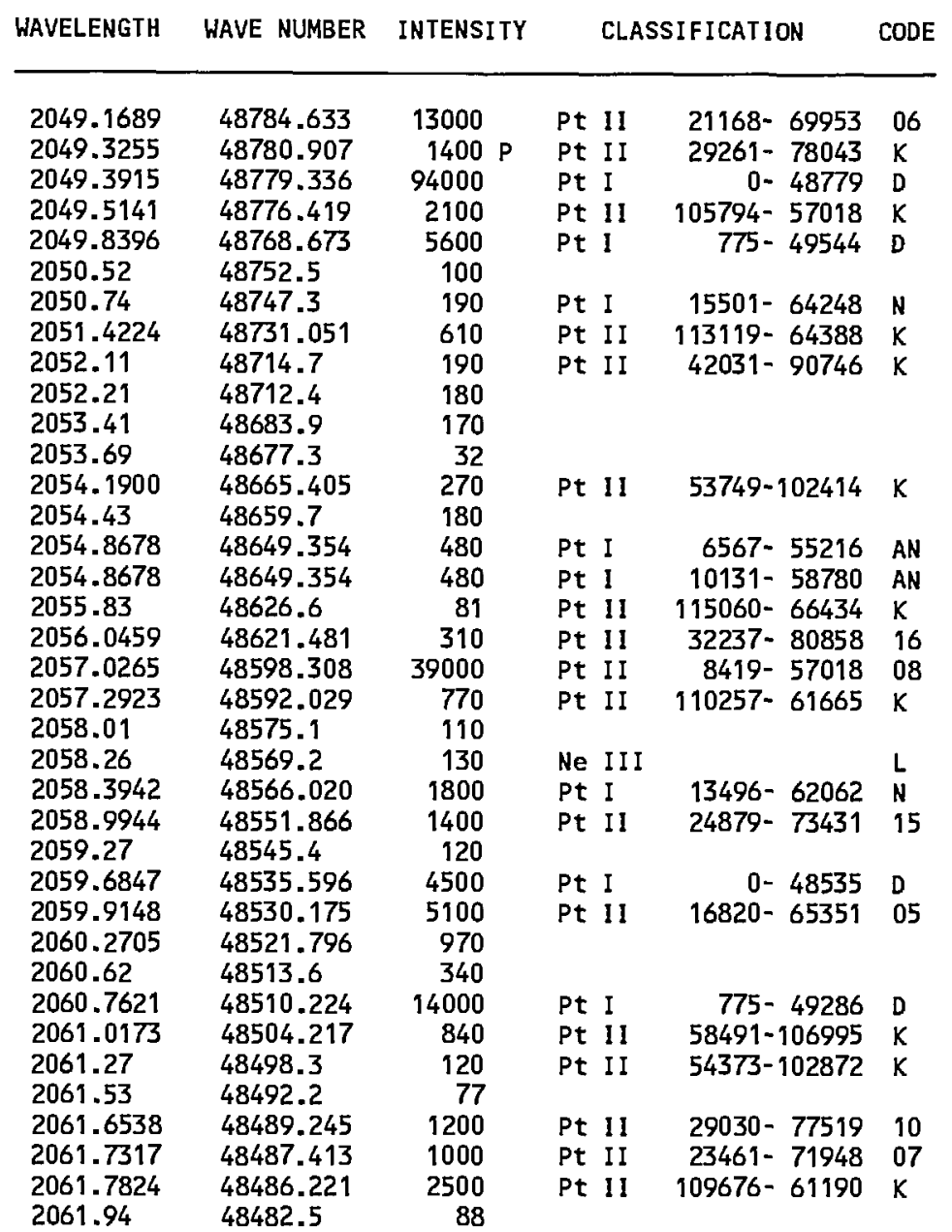




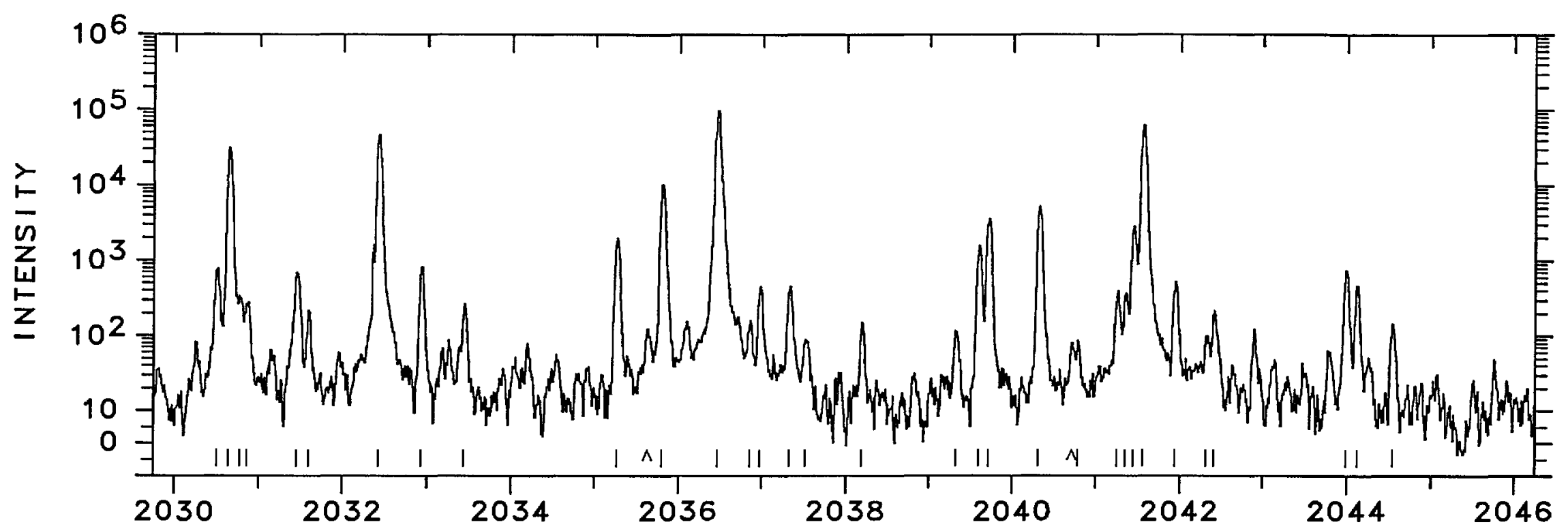

8

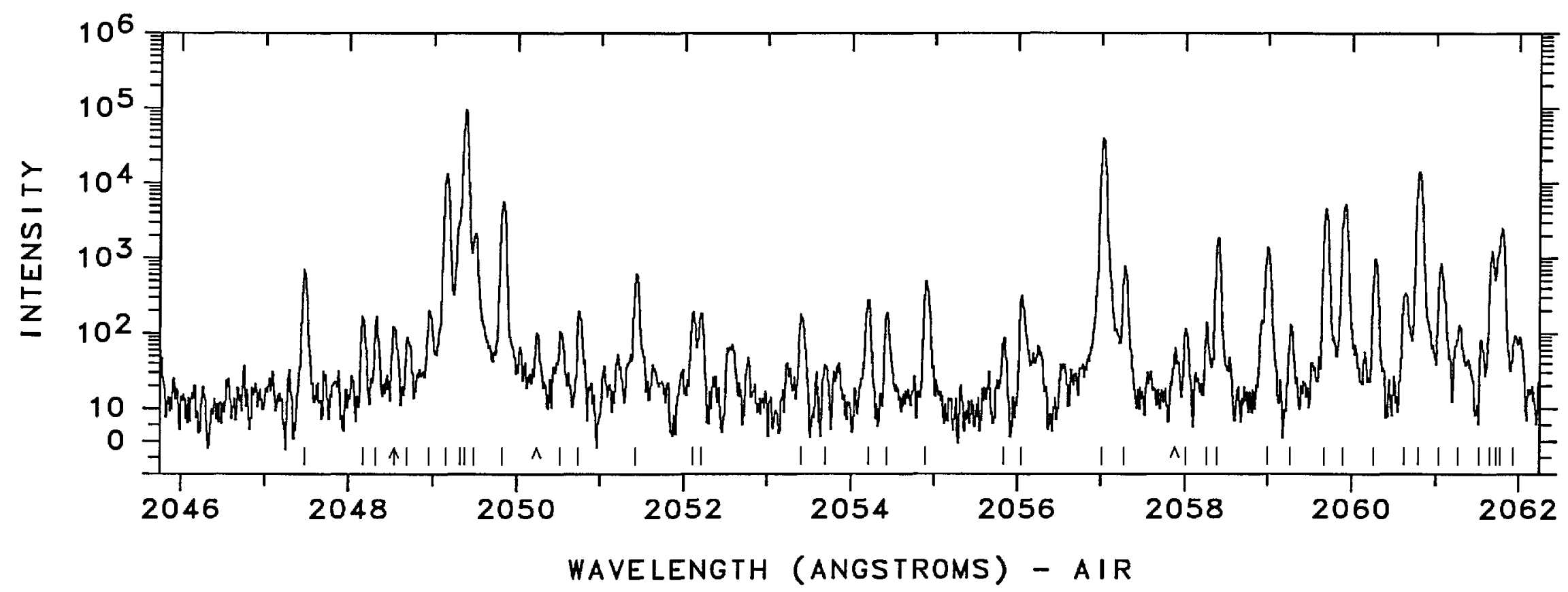




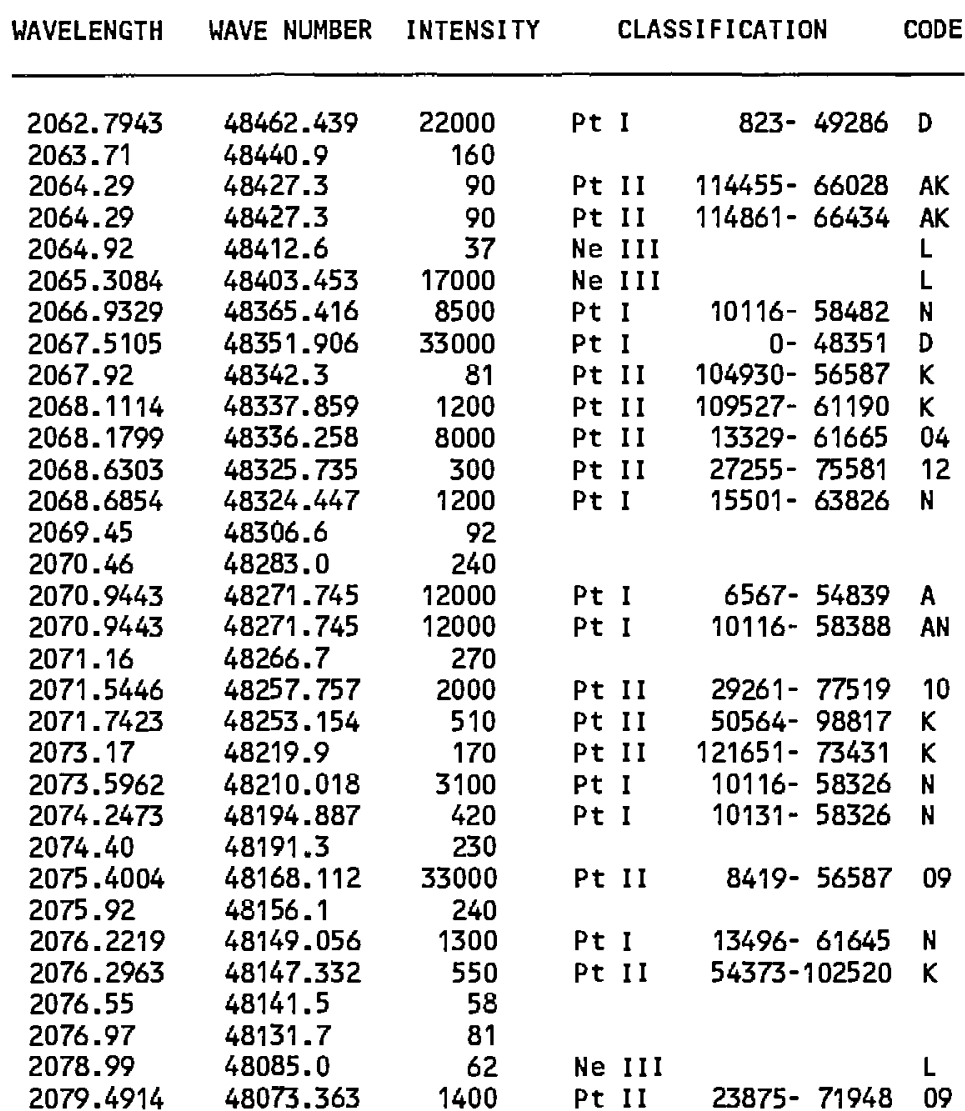

\begin{tabular}{llrlrl} 
WAVELENGTH & WAVE NUMBER & INTENSITY & \multicolumn{2}{c}{ CLASSIFICATION } & CODE \\
\hline & & & & & \\
2079.6934 & 48068.696 & 1100 & Pt II & $105086-57018$ & $\mathrm{~K}$ \\
2079.7676 & 48066.981 & 1700 & Pt II & $21168-69235$ & 11 \\
2080.16 & 48057.9 & 380 & & & \\
2080.7324 & 48044.696 & 950 & Pt II & $112433-64388$ & $\mathrm{~K}$ \\
2080.8762 & 48041.375 & 910 & Pt I I & $54373-102414$ & $\mathrm{~K}$ \\
2081.39 & 48029.5 & 190 & & & \\
2082.20 & 48010.8 & 180 & Pt II & $109676-61665$ & $\mathrm{~K}$ \\
2082.5207 & 48003.444 & 2900 & Pt I & $775-48779$ & $\mathrm{D}$ \\
2083.2782 & 47985.992 & 1000 & & & \\
2083.3453 & 47984.445 & 6900 & Pt I & $10116-58101$ & $\mathrm{~N}$ \\
2084.26 & 47963.4 & 130 & & & \\
2084.36 & 47961.1 & 320 & & & \\
2084.5960 & 47955.659 & 70000 & Pt I & $823-48779$ & $\mathrm{D}$ \\
2085.4315 & 47936.449 & 6900 & Pt II & $16820-64757$ & 05 \\
2085.4628 & 47935.73 & 37000 & Ne I I & & $\mathrm{C}$ \\
2086.4898 & 47912.138 & 1100 & Pt II & $104930-57018$ & $\mathrm{~K}$ \\
2086.8804 & 47903.173 & 1000 & Pt I I & $23461-71364$ & $\mathrm{~K}$ \\
2087.19 & 47896.1 & 160 & & & \\
2087.29 & 47893.8 & 430 & & & \\
2087.52 & 47888.5 & 43 & Pt II & $34647-82535$ & $\mathrm{~K}$ \\
2088.2978 & 47870.663 & 5600 & Pt I & $10116-57987$ & $\mathrm{D}$ \\
2088.48 & 47866.5 & 150 & Pt I & $18566-66432$ & $\mathrm{~N}$ \\
2088.7282 & 47860.799 & 9300 & Pt II & $13329-61190$ & 05 \\
2088.9388 & 47855.976 & 3400 & Pt I & $13496-61352$ & $\mathrm{~N}$ \\
2089.0647 & 47853.091 & 1800 & Pt II & $23461-71314$ & 07 \\
2090.44 & 47821.6 & 100 & & & \\
2091.1788 & 47804.719 & 650 & & & \\
2091.95 & 47787.1 & 86 & & & \\
2092.0837 & 47784.046 & 1800 & & & \\
2092.9456 & 47764.370 & 400 & & & \\
2093.82 & 47744.4 & 140 & & & \\
& & & & &
\end{tabular}



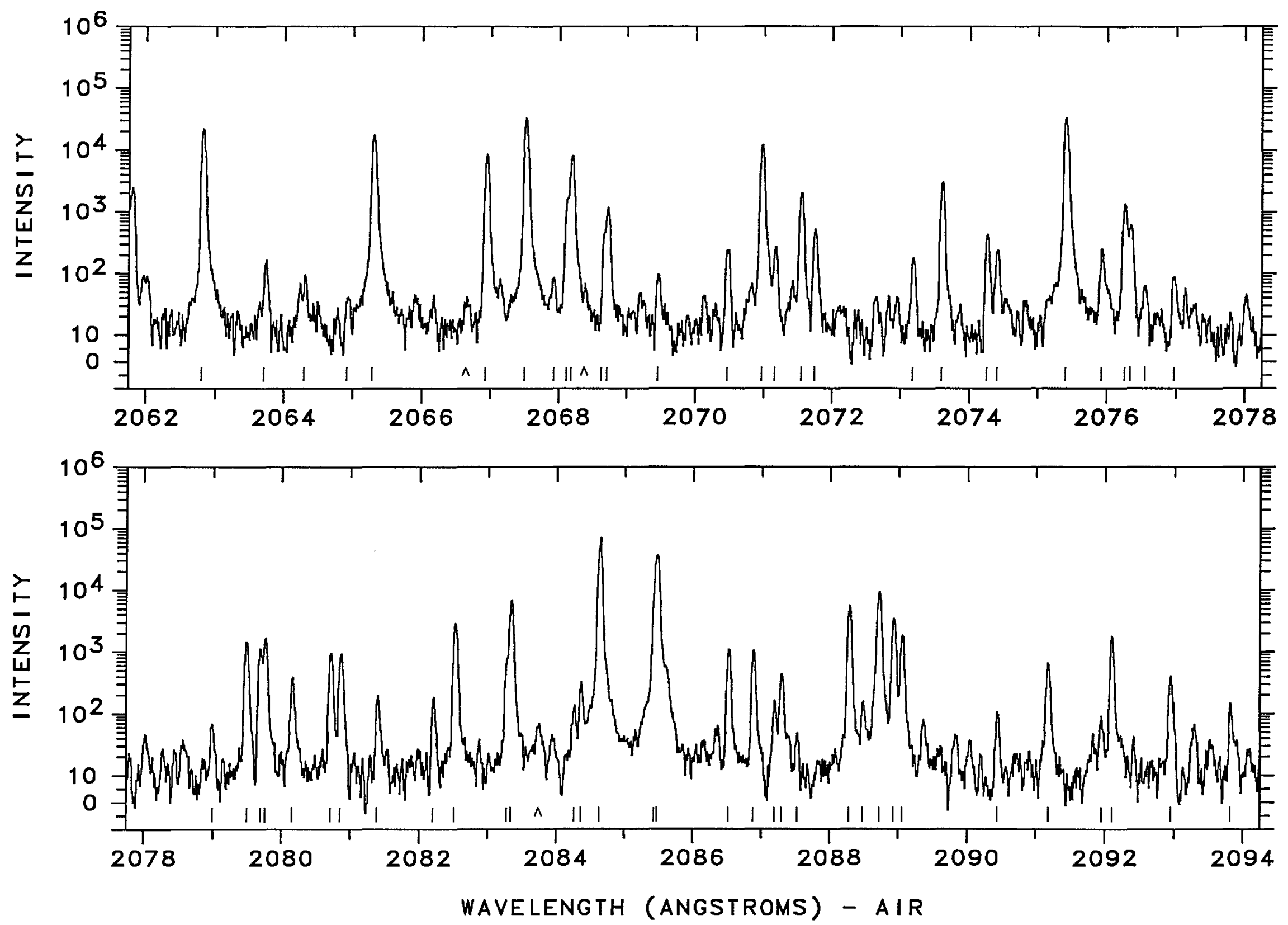
WAVELENGTH WAVE NUMBER INTENSITY

CLASSIFICATION

CODE

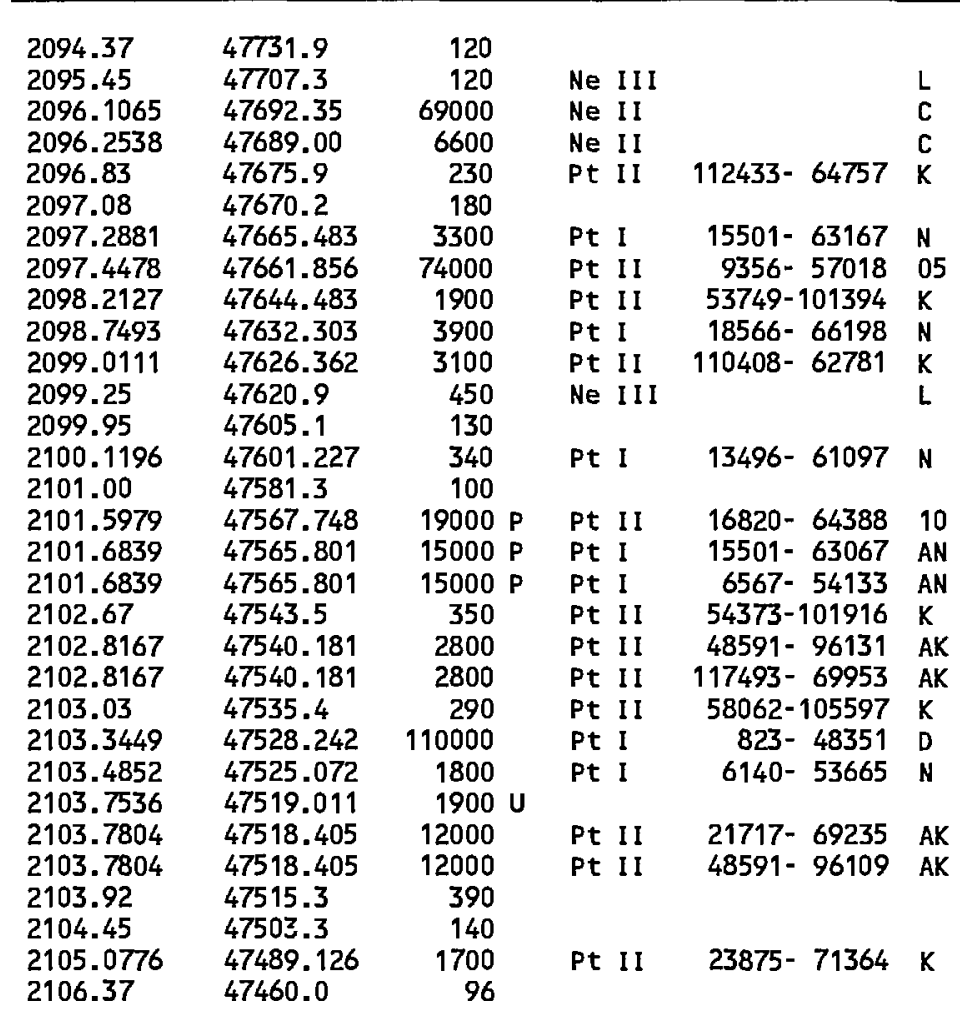

WAVELENGTH WAVE NUMBER INTENSITY

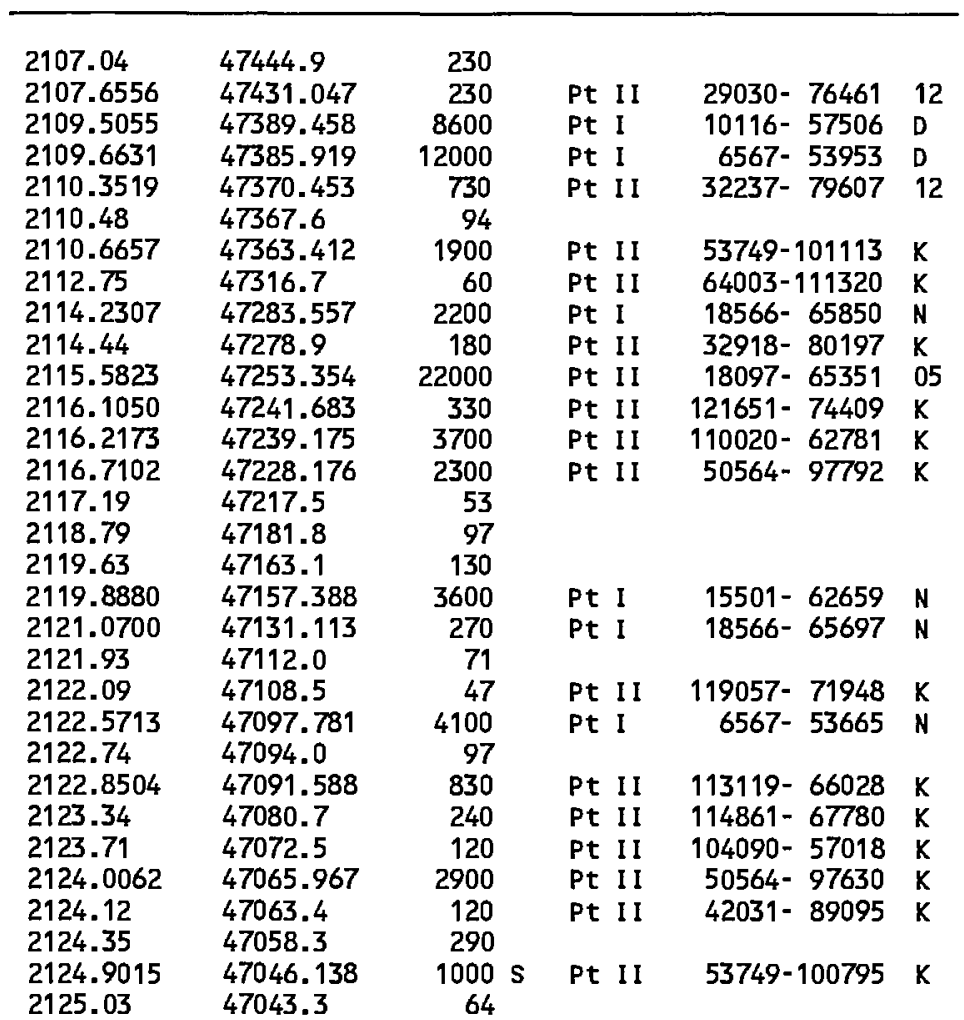



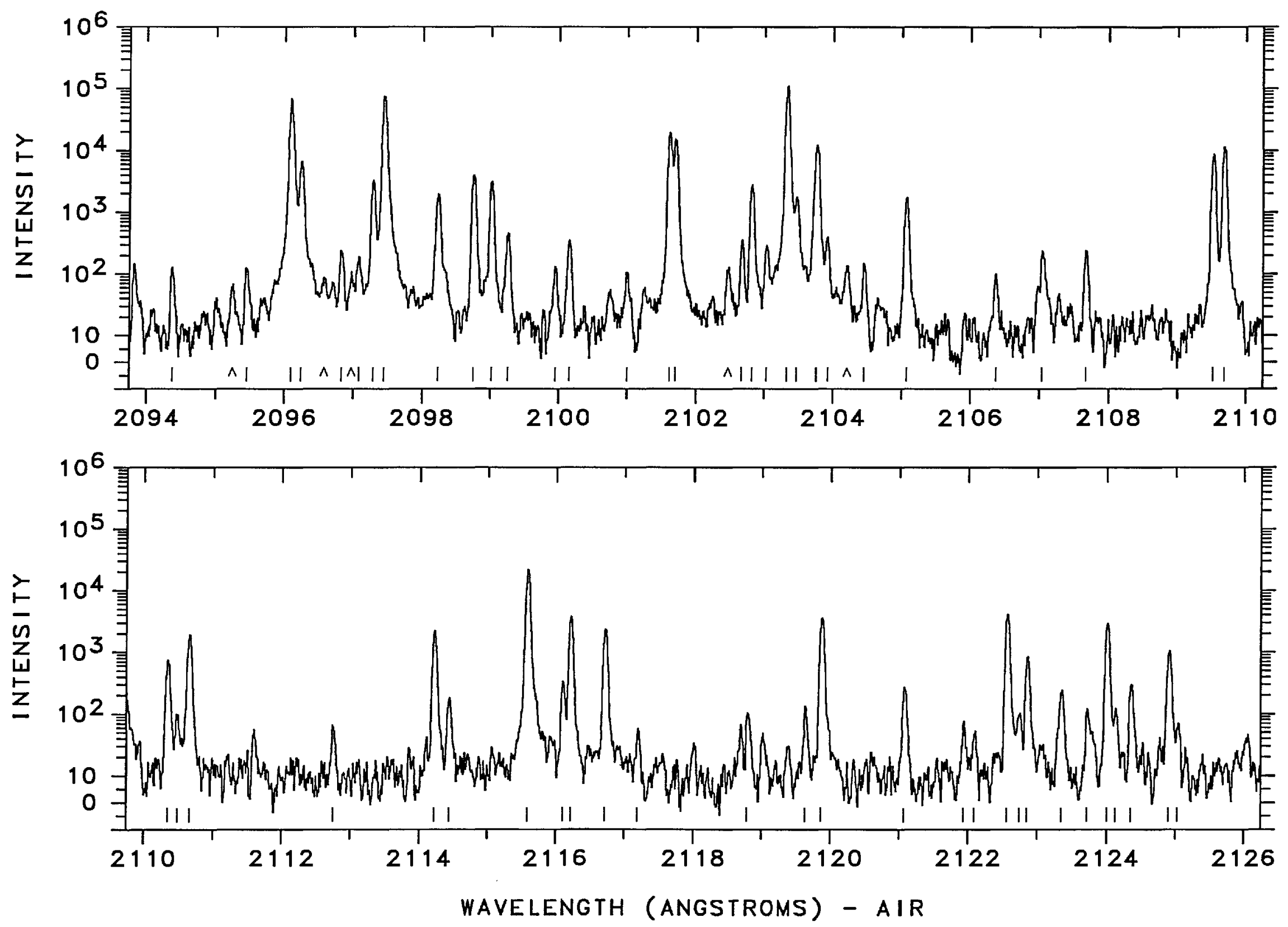


\begin{tabular}{|c|c|c|c|c|c|c|c|c|c|c|c|}
\hline JAVELENGTH & WAVE NUMBER & INTENSI TY & CLA & IFICATION & CODE & WAVELENGTH & WAVE NUMBER & INTENSITY & CLAS & SIFICATION & CODE \\
\hline $\begin{array}{l}2127.4231 \\
2128.07 \\
2128.4205 \\
2128.5878 \\
2128.6340 \\
2129.47 \\
2130.02 \\
2130.7079 \\
2131.0749 \\
2132.4727 \\
2132.7460 \\
2132.9687 \\
2133.2486 \\
2133.9737 \\
2134.6307 \\
2135.1631 \\
2135.3443 \\
2137.25 \\
2137.62 \\
2137.9562 \\
2138.59 \\
2139.4476 \\
2139.66 \\
2140.4367 \\
2141.02 \\
2141.1620 \\
2141.81 \\
2142.32 \\
2142.5054 \\
2143.08 \\
2143.62 \\
2144.2123 \\
2144.2458\end{array}$ & $\begin{array}{l}46990.382 \\
46976.1 \\
46968.364 \\
46964.673 \\
46963.654 \\
46945.2 \\
46933.1 \\
46917.947 \\
46909.869 \\
46879.123 \\
46873.116 \\
46868.223 \\
46862.073 \\
46846.153 \\
46831.737 \\
46820.061 \\
46816.087 \\
46774.3 \\
46766.3 \\
46758.900 \\
46745.0 \\
46726.308 \\
46721.7 \\
46704.718 \\
46692.0 \\
46688.899 \\
46674.8 \\
46663.7 \\
46659.628 \\
46647.1 \\
46635.4 \\
46622.489 \\
46621.759\end{array}$ & $\begin{array}{r}32000 \\
71 \\
3500 \\
40000 \mathrm{P} \\
130000 \\
400 \\
460 \\
26000 \\
470 \\
520 \\
400 \\
70 \\
1700 \\
1700 \\
2600 \\
10000 \\
390 \\
210 \\
220 \\
740 \\
61 \\
700 \\
59 \\
800 \\
65 \\
550 \\
77 \\
110 \\
3900 \\
230 \\
230 \\
200000 \mathrm{U} \\
350000 \mathrm{P}\end{array}$ & 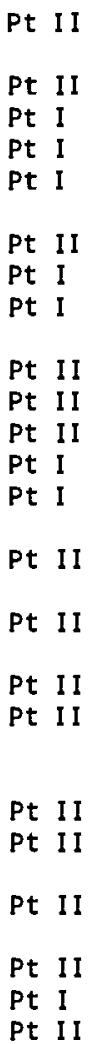 & $\begin{array}{r}15791-62781 \\
51 \\
54373-101341 \\
775-47740 \\
0-46963 \\
13496-60441 \\
\\
16820-63738 \\
10131-57041 \\
6140-53019 \\
\\
36484-83352 \\
53749-100611 \\
112433-65587 \\
13496-60328 \\
15501-62321 \\
\\
111162-64388 \\
64003-110762 \\
\\
109507-62781 \\
46046-92767\end{array}$ & $\begin{array}{l}06 \\
K \\
D \\
N \\
N \\
\\
05 \\
N \\
D \\
19 \\
K \\
K \\
N \\
N \\
K \\
K \\
K \\
K \\
\\
12 \\
K \\
06 \\
K \\
D \\
10\end{array}$ & $\begin{array}{l}2145.0395 \\
2145.1386 \\
2146.8767 \\
2147.0706 \\
2147.3909 \\
2147.50 \\
2148.09 \\
2148.4748 \\
2148.9998 \\
2149.5030 \\
2149.7007 \\
2149.8689 \\
2150.2397 \\
2150.6274 \\
2150.6567 \\
2151.2003 \\
2151.40 \\
2151.69 \\
2152.0902 \\
2152.32 \\
2152.8656 \\
2153.06 \\
2153.3933 \\
2153.5394 \\
2153.5684 \\
2154.14 \\
2154.2472 \\
2154.2472 \\
2154.76 \\
2157.2670 \\
2157.52 \\
2157.89\end{array}$ & $\begin{array}{l}46604.512 \\
46602.359 \\
46564.634 \\
46560.428 \\
46553.485 \\
46551.1 \\
46538.3 \\
46530.002 \\
46518.636 \\
46507.747 \\
46503.470 \\
46499.832 \\
46491.814 \\
46483.433 \\
46482.800 \\
46471.055 \\
46466.7 \\
46460.5 \\
46451.842 \\
46446.9 \\
46435.114 \\
46430.9 \\
46423.736 \\
46420.587 \\
46419.962 \\
46407.6 \\
46405.336 \\
46405.336 \\
46394.3 \\
46340.384 \\
46335.0 \\
46327.0\end{array}$ & $\begin{array}{r}5200 \\
300 \\
1200 \\
2400 \\
560 \\
260 \\
88 \\
1200 \\
1800 \\
780 \\
1100 \\
1000 \\
4000 \\
850 ~ U \\
4200 \\
790 \\
110 \\
190 \\
7500 \\
170 \\
47 \\
170 \\
1600 \\
7500 \\
6500 \\
130 \\
6200 \\
6200 \\
48 \\
580 \\
82 \\
46\end{array}$ & $\begin{array}{l}\text { Pt I } \\
\text { Pt I I } \\
\text { Pt I } \\
\text { Pt I } \\
\text { Pt I } \\
\text { Pt I I } \\
\text { Pt II } \\
\text { Pt I I } \\
\text { Pt I I } \\
\text { Ne I I } \\
\text { Pt I I } \\
\text { Ne I I } \\
\text { Pt I } \\
\text { Ne I I I } \\
\text { Pt I I } \\
\text { Ne I I } \\
\text { Pt I } \\
\text { Pt I I } \\
\text { Ne I I } \\
\text { Pt I } \\
\text { Pt I } \\
\text { Pt I } \\
\text { Pt I I } \\
\text { Pt I I } \\
\text { Pt I I } \\
\text { Pt I I }\end{array}$ & $\begin{array}{r}15501-62106 \\
109346-62781 \\
15501-62062 \\
10116-56670 \\
10131-56670 \\
54373-100903 \\
110257-63738 \\
116689-70181 \\
23875-70379 \\
\\
23461-69953 \\
\\
16983-63466 \\
121651-75184 \\
\\
6567-53019 \\
\\
24879-71314 \\
13496-59920 \\
13496-59916 \\
0-46419 \\
110146-63738 \\
112433-66028 \\
111162-64757 \\
58491-104831\end{array}$ & $\begin{array}{l}K \\
N \\
N \\
\\
N \\
K \\
K \\
K \\
14 \\
L \\
06 \\
L \\
N \\
L \\
K \\
L \\
D \\
\\
07 \\
L \\
N \\
N \\
D \\
K \\
\text { AK } \\
\text { AK }\end{array}$ \\
\hline
\end{tabular}




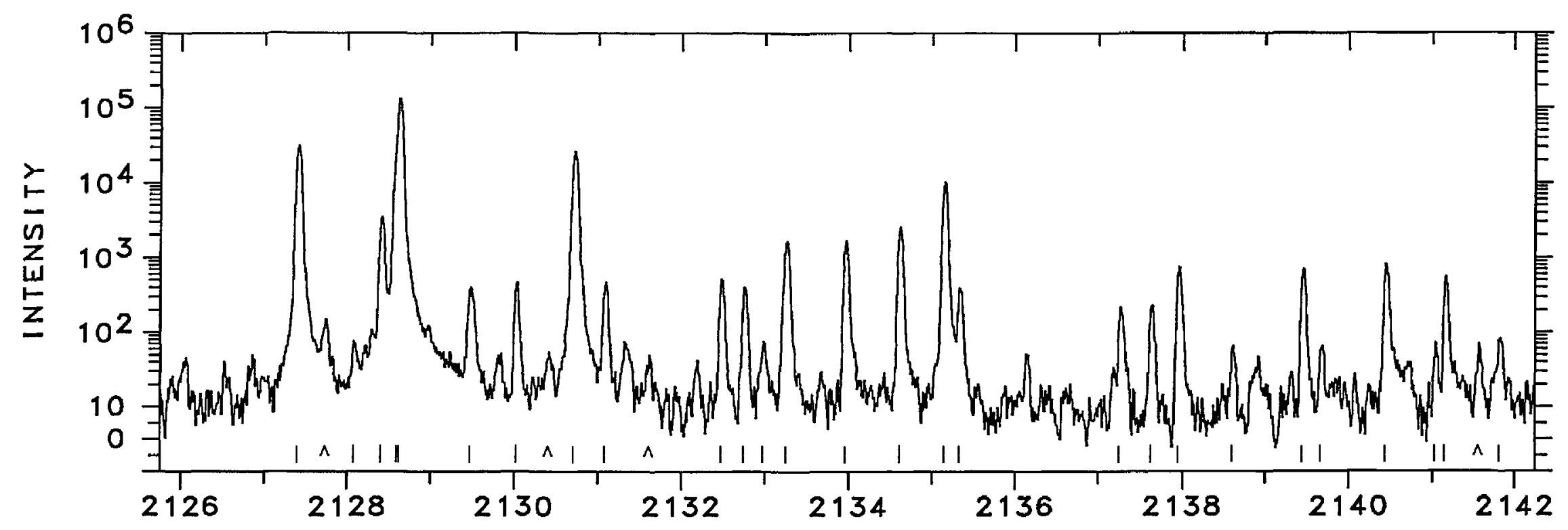

$\overrightarrow{\text { ch }}$

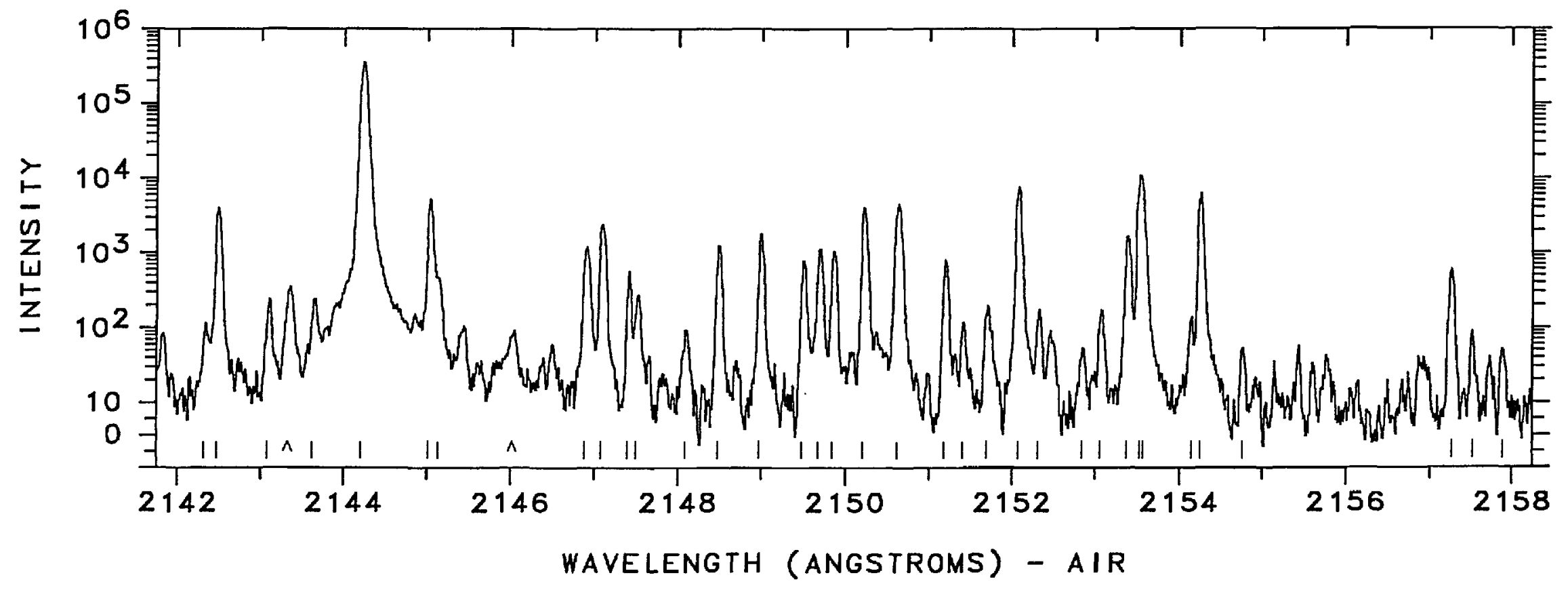




\section{WAVELENGTH WAVE NUMBER INTENSITY}

CLASSIFICATION

CODE

\begin{tabular}{|c|c|c|c|c|c|}
\hline $\begin{array}{l}2159.1595 \\
2159.29 \\
2159.3719 \\
2159.5153 \\
2159.8289 \\
2160.02 \\
2160.57\end{array}$ & $\begin{array}{l}46299.771 \\
46297.0 \\
46295.216 \\
46292.143 \\
46285.424 \\
46281.3 \\
46269.5\end{array}$ & $\begin{array}{r}500 \\
240 \\
530 \\
420 \\
590 \\
74 \\
120\end{array}$ & $\begin{array}{ll}\text { Pt } & \text { I } \\
\text { Ne III } \\
\text { Ne III } \\
\text { Ne III }\end{array}$ & $21967-68266$ & $\begin{array}{l}\mathbf{N} \\
\mathrm{L} \\
\mathrm{L} \\
\mathrm{L}\end{array}$ \\
\hline $\begin{array}{l}2160.82 \\
2160.9675\end{array}$ & $\begin{array}{l}46264.2 \\
46261.037\end{array}$ & $\begin{array}{l}170 \\
690\end{array}$ & $\begin{array}{l}\mathrm{Ne} \text { II I } \\
\mathrm{Ne} \text { III }\end{array}$ & & $\begin{array}{l}L \\
L\end{array}$ \\
\hline $\begin{array}{l}2161.1343 \\
2162.05\end{array}$ & $\begin{array}{l}46257.468 \\
46237.9\end{array}$ & $\begin{array}{r}1200 \\
340\end{array}$ & Ne II I & & $\mathrm{L}$ \\
\hline $\begin{array}{l}2163.6119 \\
2163.7345\end{array}$ & $\begin{array}{l}46204.503 \\
46201.886\end{array}$ & $\begin{array}{r}550 \\
2000\end{array}$ & $\begin{array}{l}\mathrm{Ne} \text { III } \\
\mathrm{Ne} \text { III }\end{array}$ & & $\begin{array}{l}\mathrm{L} \\
\mathrm{L}\end{array}$ \\
\hline $\begin{array}{l}2163.8965 \\
2164.18\end{array}$ & $\begin{array}{l}46198.426 \\
46192.4\end{array}$ & $\begin{array}{l}360 \\
210\end{array}$ & $\begin{array}{ll}\text { Pt } & \text { II } \\
\text { Pt } & \text { II }\end{array}$ & $\begin{array}{l}64003-110202 \\
64003-110196\end{array}$ & $\begin{array}{l}K \\
K\end{array}$ \\
\hline $\begin{array}{l}2164.2949 \\
2164.3955\end{array}$ & $\begin{array}{l}46189.923 \\
46187.776\end{array}$ & $\begin{array}{r}1300 \\
910\end{array}$ & Pt I & $13496-59686$ & N \\
\hline $\begin{array}{l}2165.1407 \\
2165.2108 \\
2165.8714\end{array}$ & $\begin{array}{l}46171.882 \\
46170.386 \\
46156.306\end{array}$ & $\begin{array}{r}8000 P \\
30000 P \\
400\end{array}$ & $\begin{array}{ll}\mathrm{Pt} & \mathrm{I} \\
\mathrm{Pt} & \mathrm{I}\end{array}$ & $\begin{array}{r}10116-56288 \\
0-46170\end{array}$ & $\begin{array}{l}\text { N } \\
\text { D }\end{array}$ \\
\hline 2165.9608 & 46154.401 & 1600 & Pt II & $29030-75184$ & 07 \\
\hline 2166.2045 & 46149.21 & 220 & $\mathrm{Ne} I I$ & & C \\
\hline $\begin{array}{l}2166.5655 \\
2166.6376\end{array}$ & $\begin{array}{l}46141.521 \\
46139.986\end{array}$ & $\begin{array}{r}300 \mathrm{U} \\
6500\end{array}$ & $\begin{array}{l}\text { Pt II } \\
\text { Pt I }\end{array}$ & $\begin{array}{r}24879-71021 \\
823-46963\end{array}$ & $\begin{array}{l}K \\
N\end{array}$ \\
\hline $\begin{array}{l}2167.30 \\
2168.56 \\
2168.70\end{array}$ & $\begin{array}{l}46125.9 \\
46099.1 \\
46096.1\end{array}$ & $\begin{array}{l}68 \\
63 \\
79\end{array}$ & Pt II & $43737-89863$ & K \\
\hline 2169.2711 & $\begin{array}{l}46090.1 \\
46083.977\end{array}$ & 3300 & Pt I & $16983-63067$ & N \\
\hline 2169.5637 & 46077.764 & 710 & Pt II & $23875-69953$ & 09 \\
\hline $\begin{array}{l}2170.5112 \\
2170.6696\end{array}$ & $\begin{array}{l}46057.65 \\
46054.29\end{array}$ & $\begin{array}{l}160 \\
300 u\end{array}$ & $\begin{array}{l}\mathrm{Ne} \text { II } \\
\mathrm{Ne} \text { II }\end{array}$ & & $\begin{array}{l}c \\
c\end{array}$ \\
\hline 2170.7267 & 46053.079 & 1100 & Pt I & $18566-64619$ & N \\
\hline 2171.79 & & 110 & Pt II & $119057-73026$ & K \\
\hline 2172.00 & 46026.1 & 110 & Pt II & $117340-71314$ & $k$ \\
\hline 2172.3162 & & 420 & Pt II & $110408-64388$ & $\mathrm{~K}$ \\
\hline 2172.3937 & 46017.744 & 1600 & Pt II & $32237-78254$ & K \\
\hline
\end{tabular}

WAVELENGTH WAVE NUMBER INTENSITY

CLASSIFICATION

CODE

\begin{tabular}{llrlrl}
\hline 2172.89 & 46007.2 & 170 & & & \\
2173.19 & 46000.9 & 57 & & & \\
2173.43 & 45995.8 & 110 & & & \\
2174.6853 & 45969.257 & 290000 & Pt I & $823-46792$ & $\mathrm{~N}$ \\
2174.8392 & 45966.005 & 1000 & Pt I & $13496-59462$ & $\mathrm{~N}$ \\
2175.32 & 45955.8 & 150 & & & \\
2175.52 & 45951.6 & 330 & & & \\
2176.19 & 45937.5 & 150 & Pt I I & $109676-63738$ & $\mathrm{~K}$ \\
2176.4730 & 45931.504 & 820 & Pt I & $6140-52071$ & $\mathrm{D}$ \\
2176.6289 & 45928.214 & 2600 & Ne II I & & $\mathrm{L}$ \\
2176.8802 & 45922.913 & 4200 & Pt II & $29261-75184$ & 07 \\
2177.6988 & 45905.652 & 5700 & Ne III & & $\mathrm{L}$ \\
2178.1182 & 45896.809 & & Fe I & & $\mathrm{S}$ \\
2178.6549 & 45885.508 & 2000 & Ne III & & $\mathrm{L}$ \\
2179.4123 & 45869.563 & 1000 & Pt I I & $110258-64388$ & $\mathrm{~K}$ \\
2179.5832 & 45865.967 & 1300 & Pt II & $54373-100239$ & $\mathrm{~K}$ \\
2179.83 & 45860.8 & 430 & & & \\
2180.3229 & 45850.408 & 4800 & Pt I & $15501-61352$ & $\mathrm{~N}$ \\
2180.5042 & 45846.596 & 17000 & Pt I & $775-46622$ & $\mathrm{D}$ \\
2180.8613 & 45839.090 & 11000 & Ne III & & $\mathrm{L}$ \\
2181.7748 & 45819.90 & 60 & Ne I I & & $\mathrm{C}$ \\
2182.1734 & 45811.532 & 250 & Pt II & $111162-65351$ & $\mathrm{~K}$ \\
2182.2632 & 45809.645 & 2000 & Ne III & & $\mathrm{L}$ \\
2182.59 & 45802.8 & 110 & & & \\
2182.7795 & 45798.811 & 7200 & Pt I & $823-46622$ & $\mathrm{D}$ \\
2183.23 & 45789.4 & 180 & Pt II & $109527-63738$ & $\mathrm{~K}$ \\
2183.47 & 45784.3 & 270 & Pt II & $111371-65587$ & $\mathrm{~K}$ \\
2184.1755 & 45769.542 & 7500 & Pt II & $110158-64388$ & $\mathrm{~K}$ \\
2185.17 & 45748.7 & 140 & & & \\
2186.4314 & 45722.325 & $600 \mathrm{P}$ & & & \\
2186.4768 & 45721.375 & $8900 \mathrm{~L}$ & Pt II & $53749-99471$ & $\mathrm{~K}$ \\
2188.3437 & 45682.374 & 840 & Pt I & $18566-64248$ & $\mathrm{~N}$ \\
2188.82 & 45672.4 & 120 & & & \\
2189.50 & 45658.3 & 250 & & & \\
2189.8625 & 45650.694 & 1400 & Pt II & $110408-64757$ & $\mathrm{~K}$
\end{tabular}




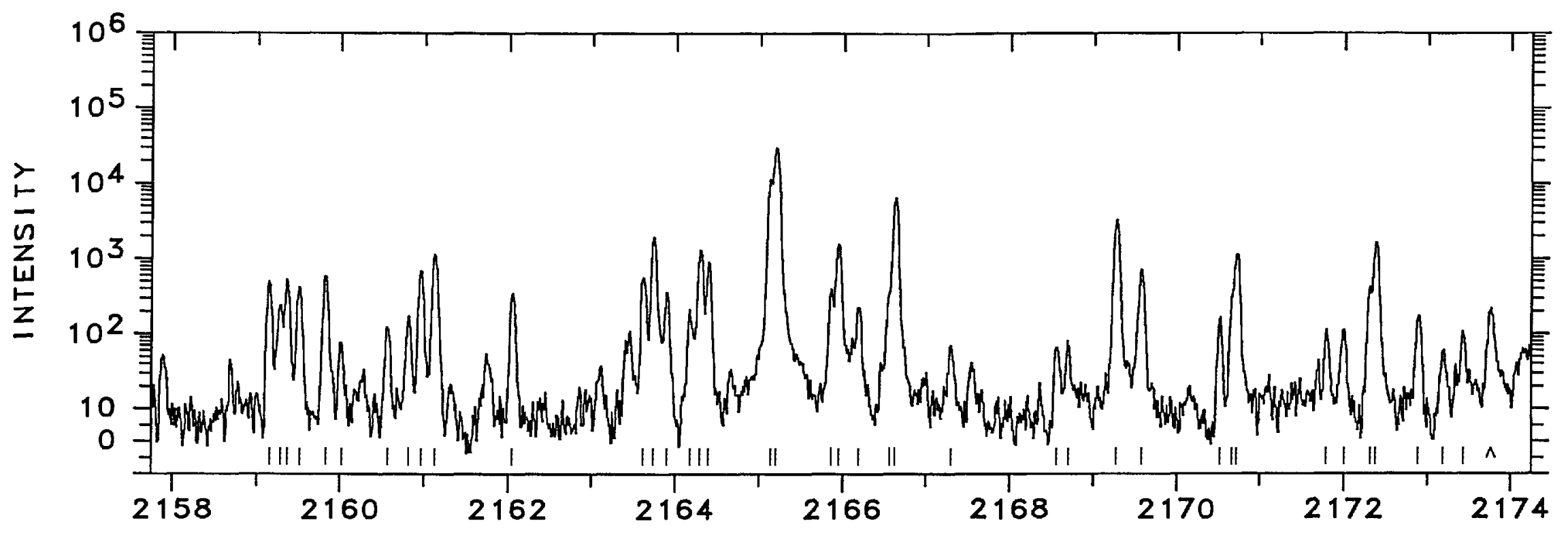

$\checkmark$

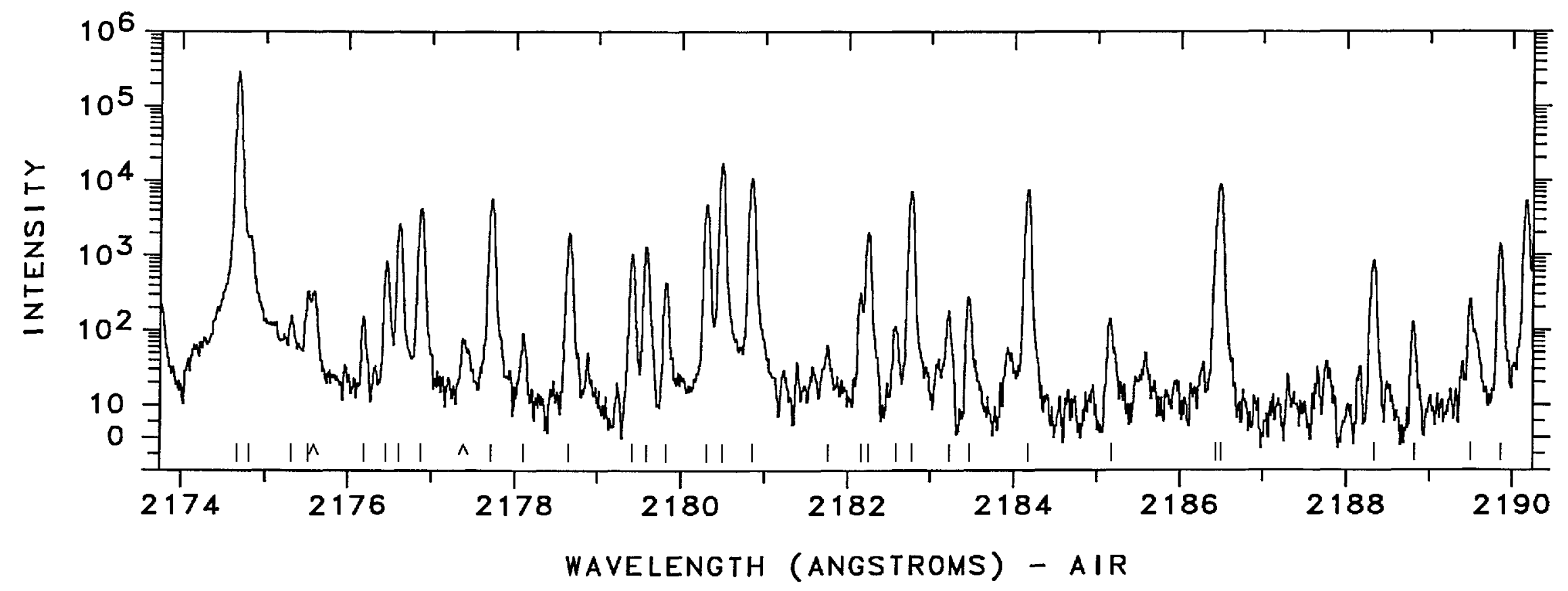




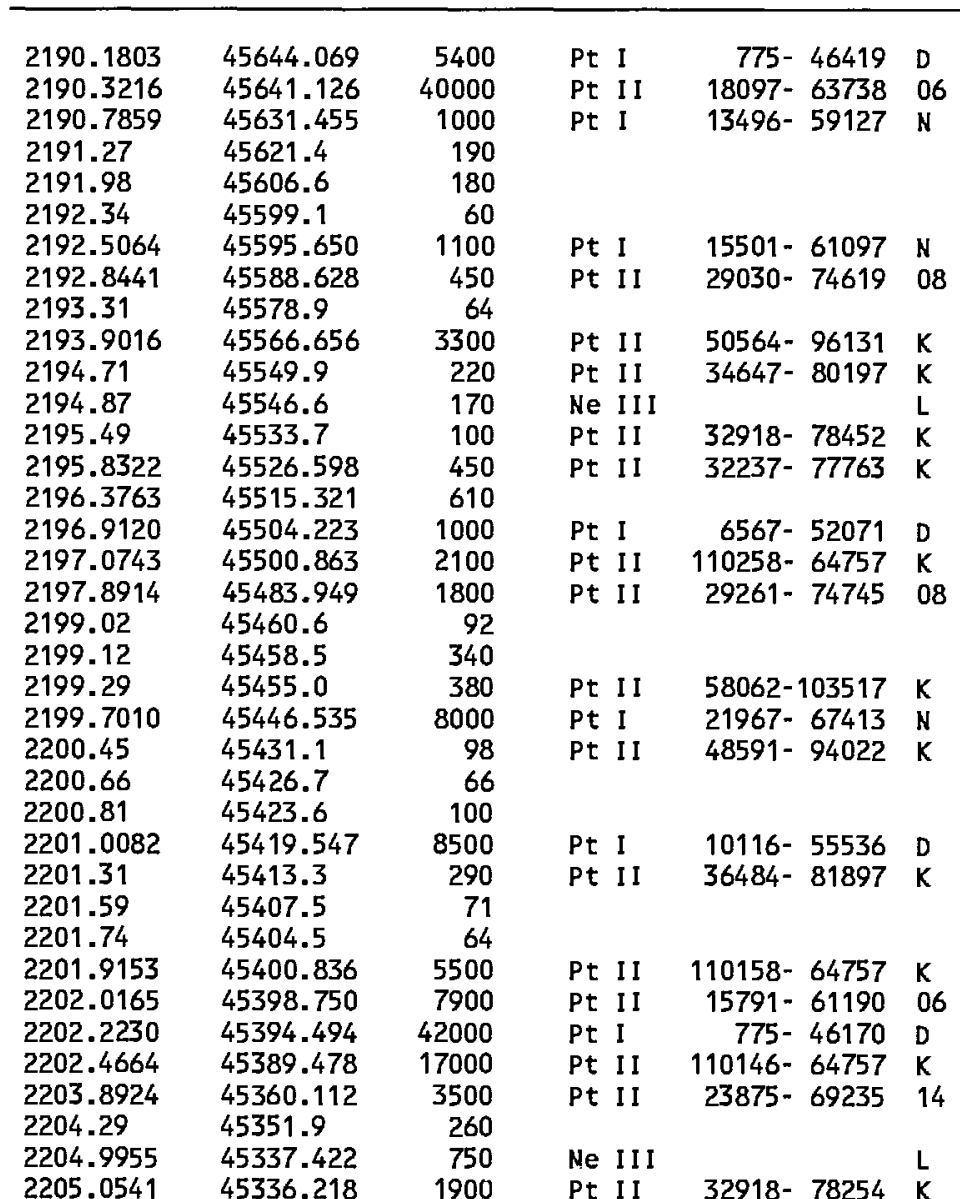

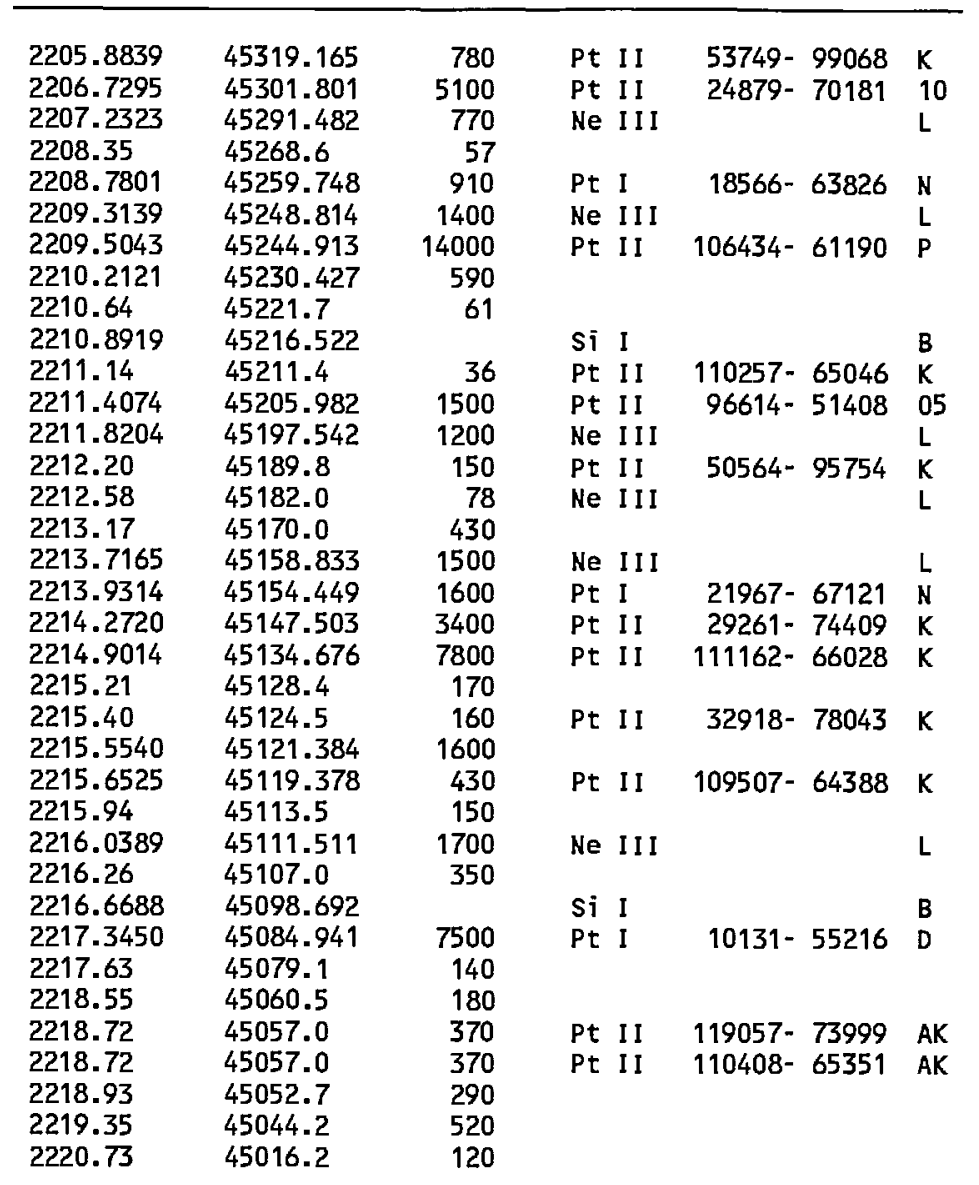




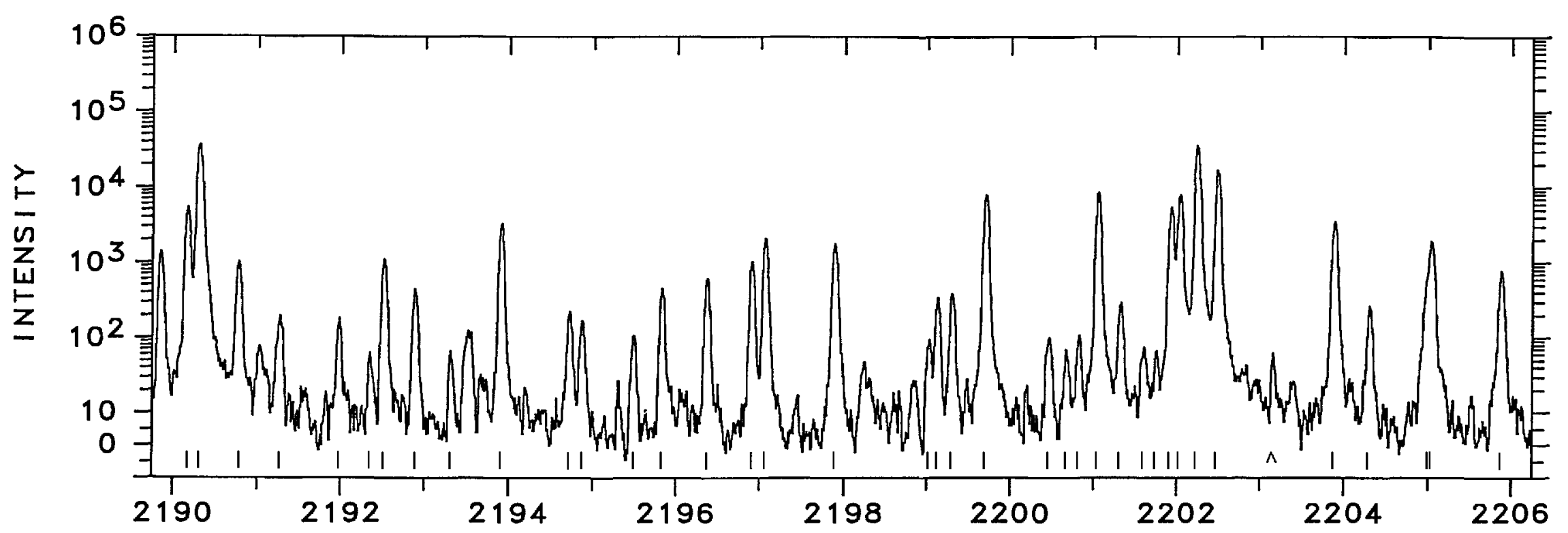

ซิ

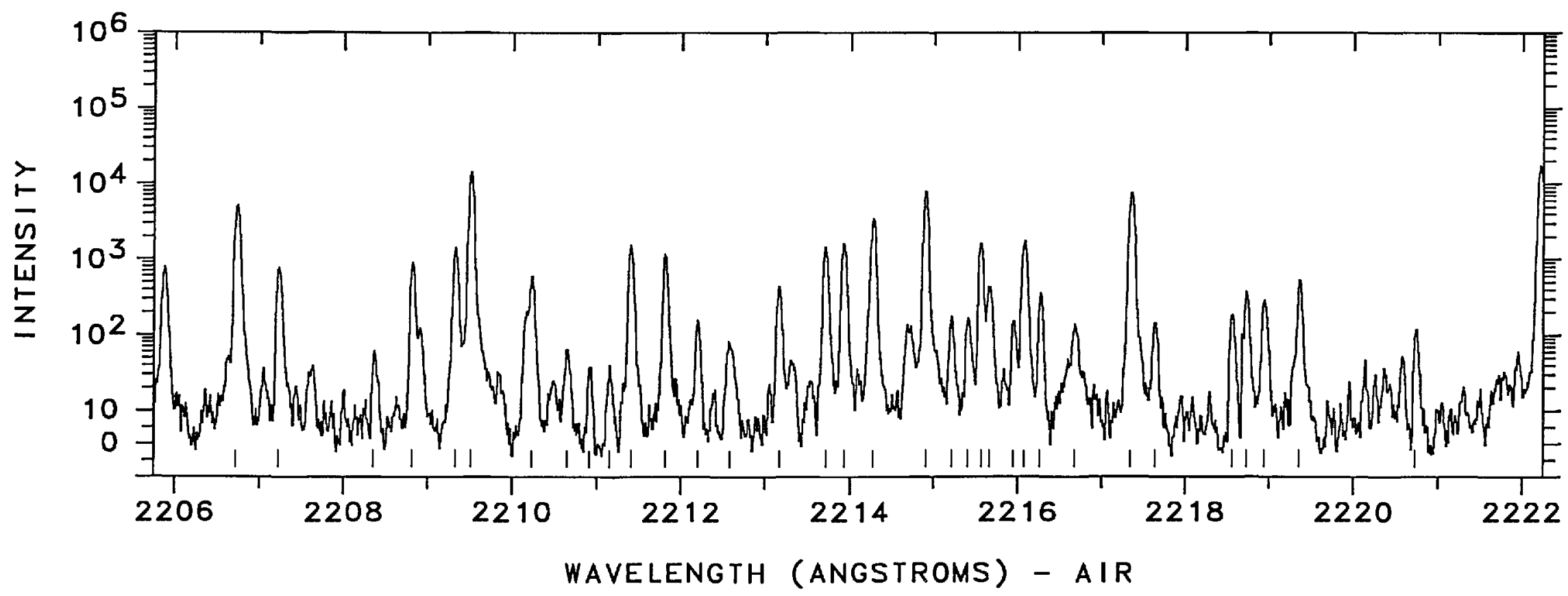




\section{WAVELENGTH WAVE NUMBER INTENSITY}

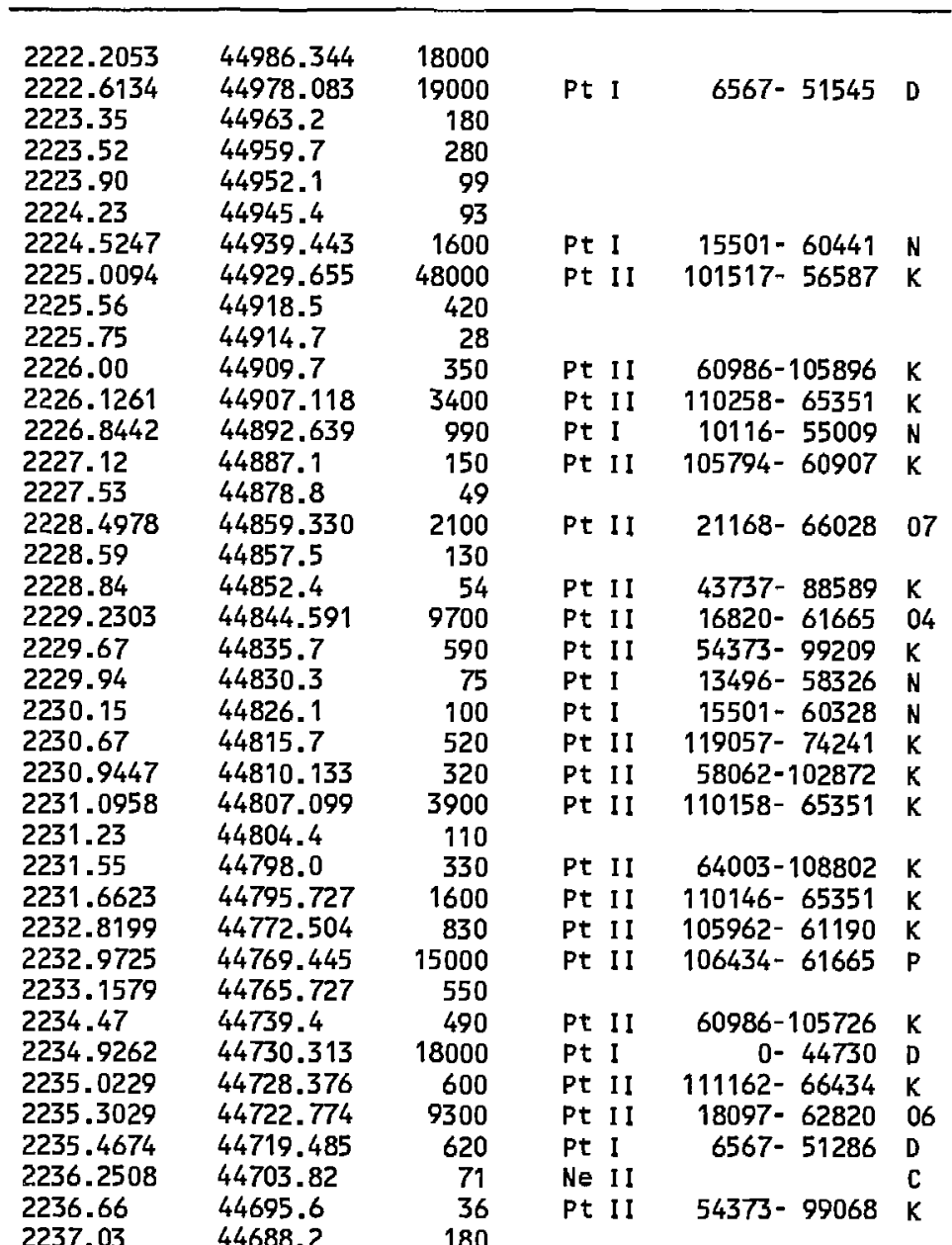

WAVELENGTH WAVE NUMBER INTENSITY CLASSIFICATION CODE

\begin{tabular}{llrlrll}
\hline 2237.3520 & 44681.818 & 1600 & Pt II & $115060-70379$ & $\mathrm{~K}$ \\
2237.8916 & 44671.046 & 2400 & Pt II & $110258-65587$ & $\mathrm{~K}$ \\
2238.04 & 44668.1 & 190 & & & & \\
2238.38 & 44661.3 & 310 & Pt II & $50564-95226$ & $\mathrm{~K}$ \\
2239.80 & 44633.0 & 370 & & & & \\
2240.3222 & 44622.586 & 1300 & Pt I & $775-45398$ & $\mathrm{D}$ \\
2240.6434 & 44616.189 & 560 & Pt II & $58062-102678$ & $\mathrm{~K}$ \\
2240.8965 & 44611.151 & 240000 & Pt II & $101199-56587$ & 04 \\
2241.2288 & 44604.537 & 15000 & Pt II & $105794-61190$ & $\mathrm{~K}$ \\
2242.00 & 44589.2 & 100 & Pt II & $109346-64757$ & $\mathrm{~K}$ \\
2242.63 & 44576.7 & 110 & & & & \\
2244.9773 & 44530.068 & 21000 & Pt I & $6567-51097$ & $\mathrm{D}$ \\
2245.5244 & 44519.219 & 170000 & Pt II & $9356-53875$ & 06 \\
2245.9752 & 44510.284 & 650 & & & \\
2246.0273 & 44509.252 & 650 & & & \\
2246.4630 & 44500.620 & 600 & Pt I & $18566-63067$ & $\mathrm{~N}$ \\
2246.5216 & 44499.460 & 110000 & Pt II & $101517-57018$ & $\mathrm{~K}$ \\
2246.7172 & 44495.585 & 5900 & & & & \\
2246.9427 & 44491.121 & 600 & Pt I & $13496-57987$ & $\mathrm{D}$ \\
2247.4822 & 44480.442 & 15000 & Pt II & $105388-60907$ & 11 \\
2248.15 & 44467.2 & 180 & & & \\
2248.32 & 44463.9 & 340 & & & \\
2248.42 & 44461.9 & 150 & & & \\
2248.59 & 44458.5 & 400 & Pt II & $58062-102520$ & $\mathrm{~K}$ \\
2249.3075 & 44444.350 & 24000 & Pt I & $0-44444$ & $\mathrm{~A}$ \\
2249.3075 & 44444.350 & 24000 & Pt II & $54373-98817$ & AK \\
2249.6320 & 44437.939 & 800 & Pt II & $119057-74619$ & $\mathrm{~K}$ \\
2249.8994 & 44432.659 & 16000 & Pt I & $0-44432$ & $\mathrm{E}$ \\
2250.38 & 44423.2 & 99 & & & \\
2250.6201 & 44418.431 & 9000 & Pt II & $21168-65587$ & 12 \\
2250.7883 & 44415.113 & 410 & Pt I & $15501-59916$ & $\mathrm{~N}$ \\
2251.5105 & 44400.867 & 19000 & Pt II & $29030-73431$ & 18 \\
2251.8084 & 44394.993 & 13000 & Pt II & $95803-51408$ & 05 \\
2252.09 & 44389.4 & 72 & & & \\
2252.32 & 44384.9 & 38 & & & \\
2252.5690 & 44380.005 & 6400 & Pt II & $110408-66028$ & $\mathrm{~K}$ \\
2252.8022 & 44375.41 & 200 & Ne II & & $\mathrm{C}$ \\
2253.1210 & 44369.132 & 3500 & Pt II & $16820-61190$ & 05 \\
2253.96 & 44352.6 & 110 & Pt II & $58062-102414$ & $\mathrm{~K}$
\end{tabular}




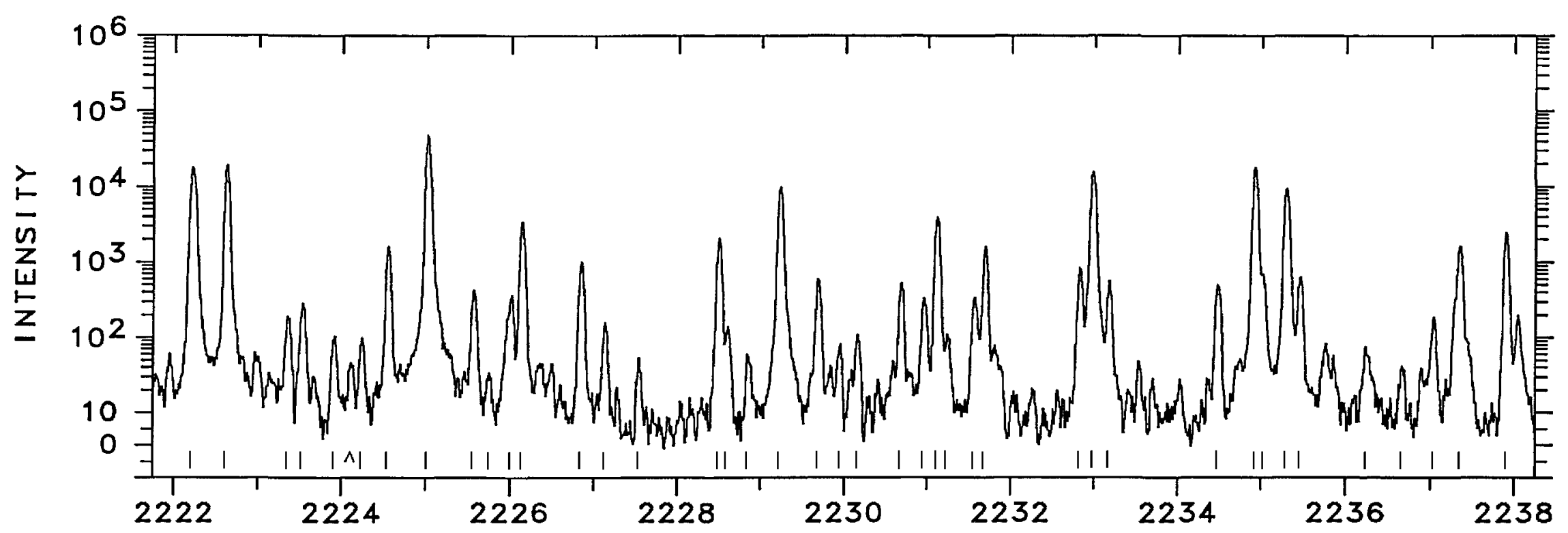

$\stackrel{\infty}{\circledR}$

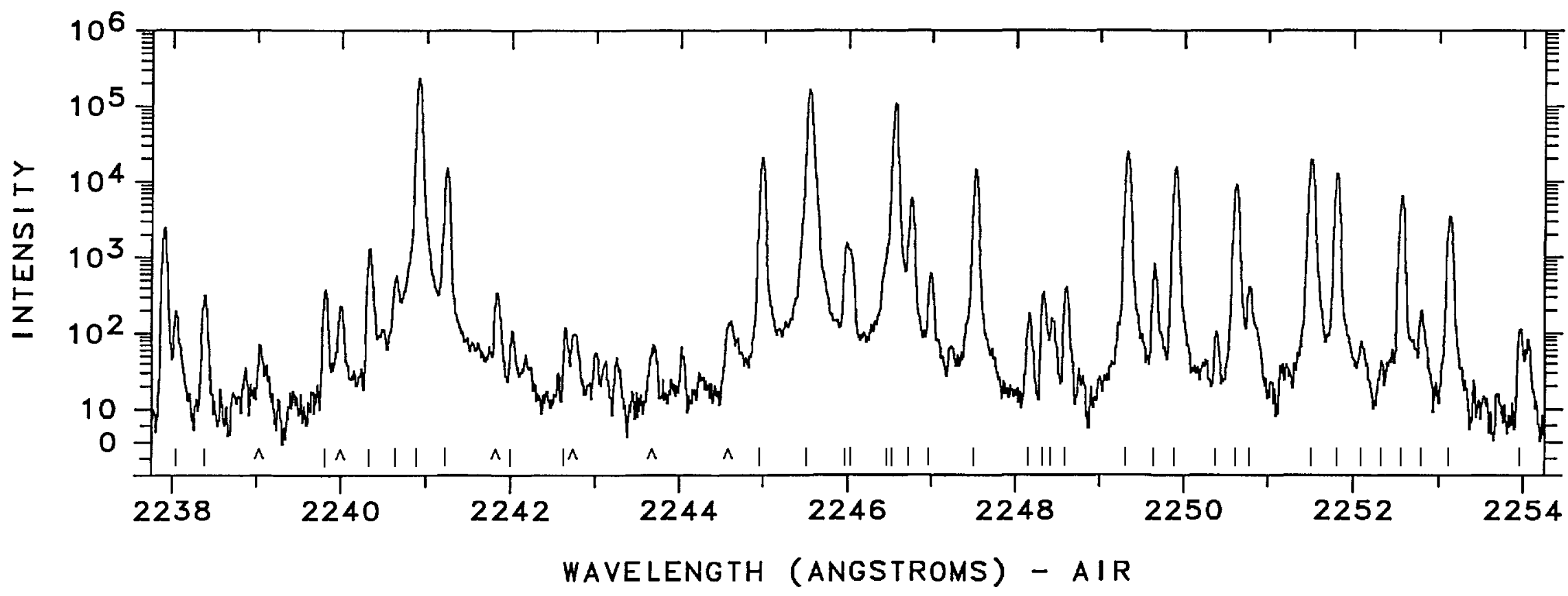




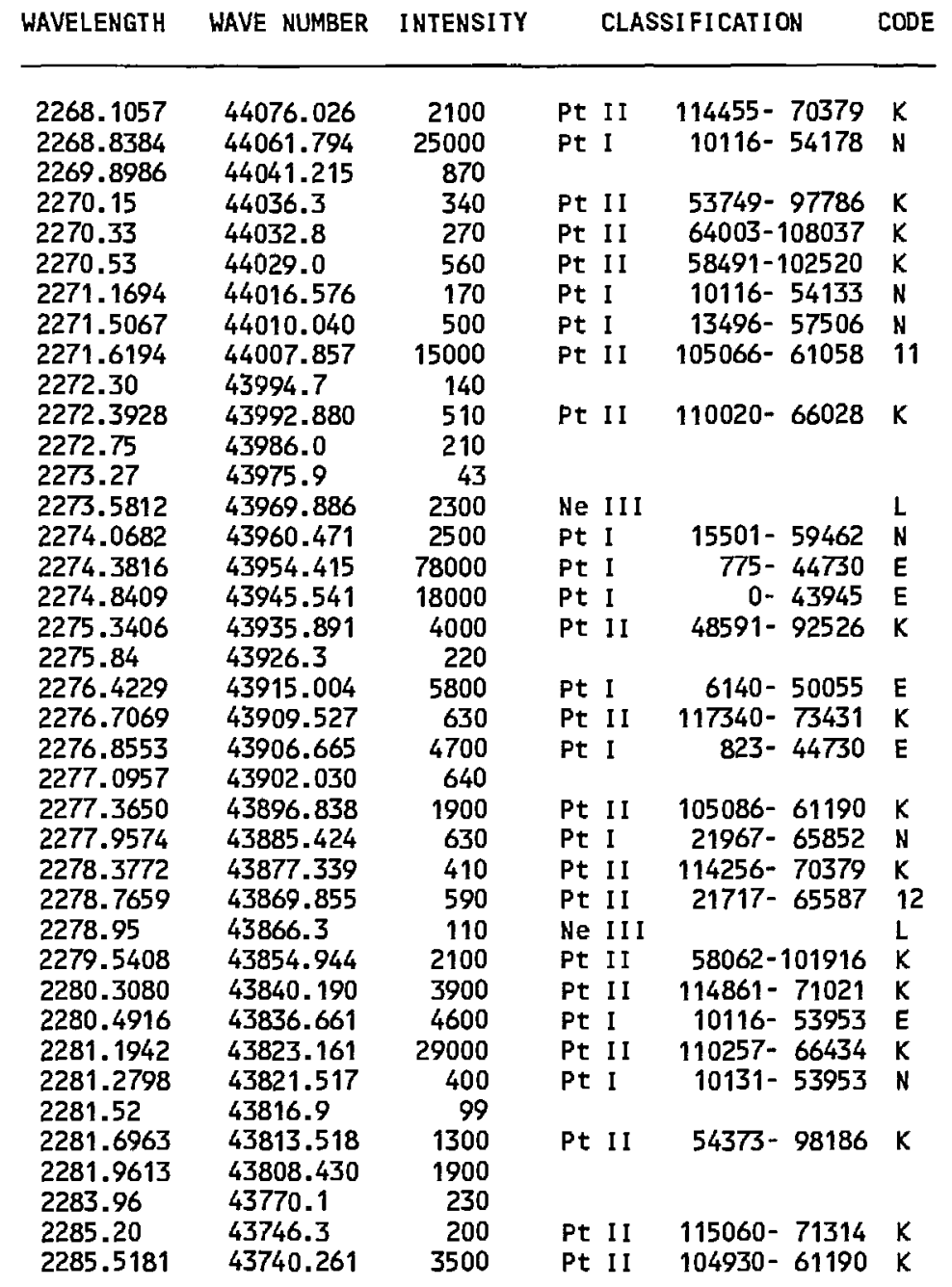




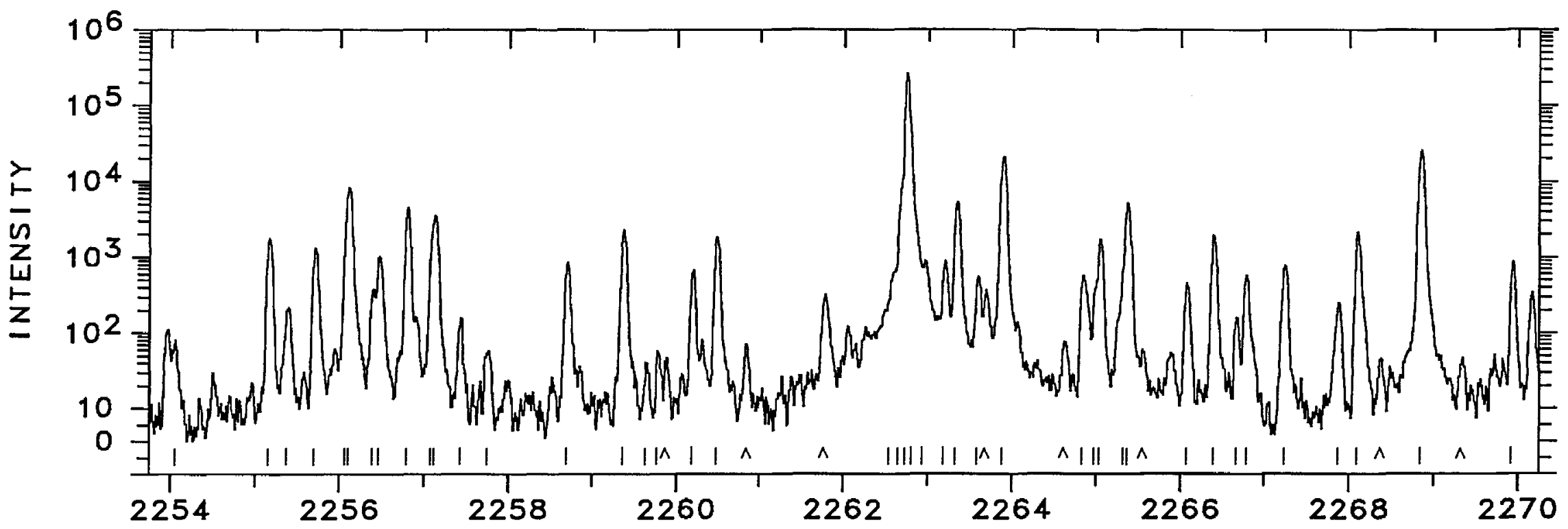

$\infty$

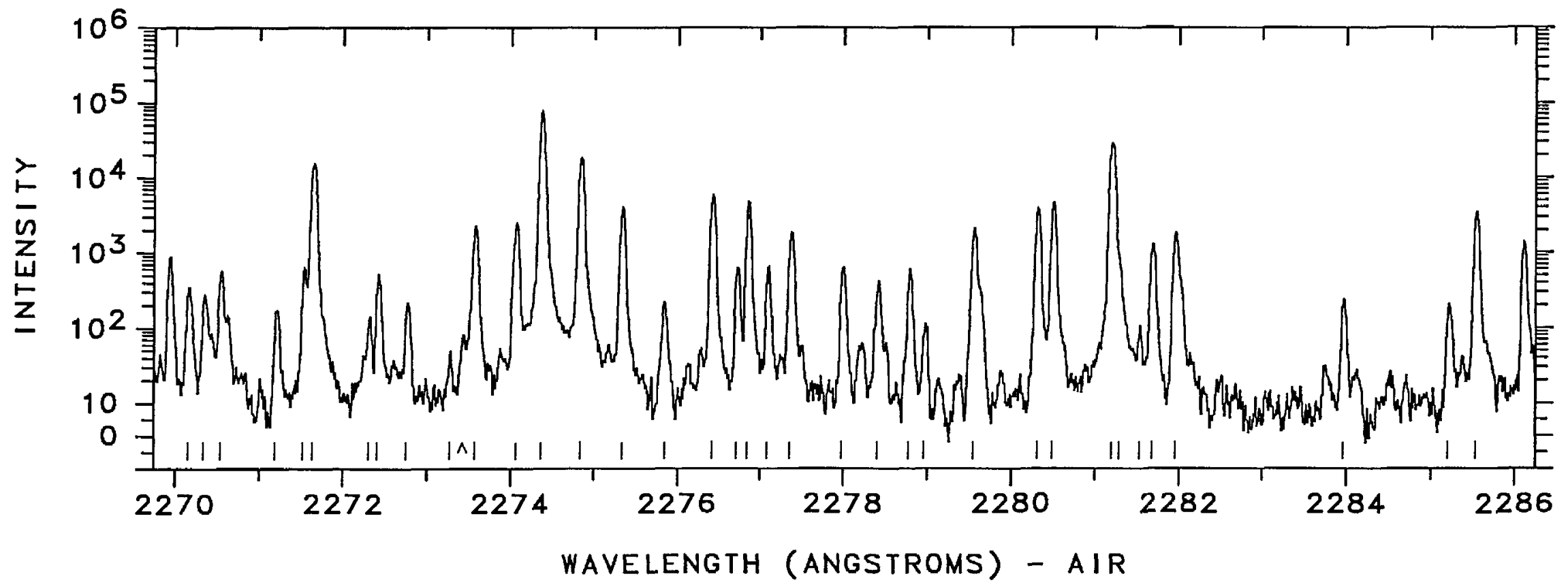


WAVELENGTH WAVE NUMBER INTENSITY

CLASSIF ICATION

CODE

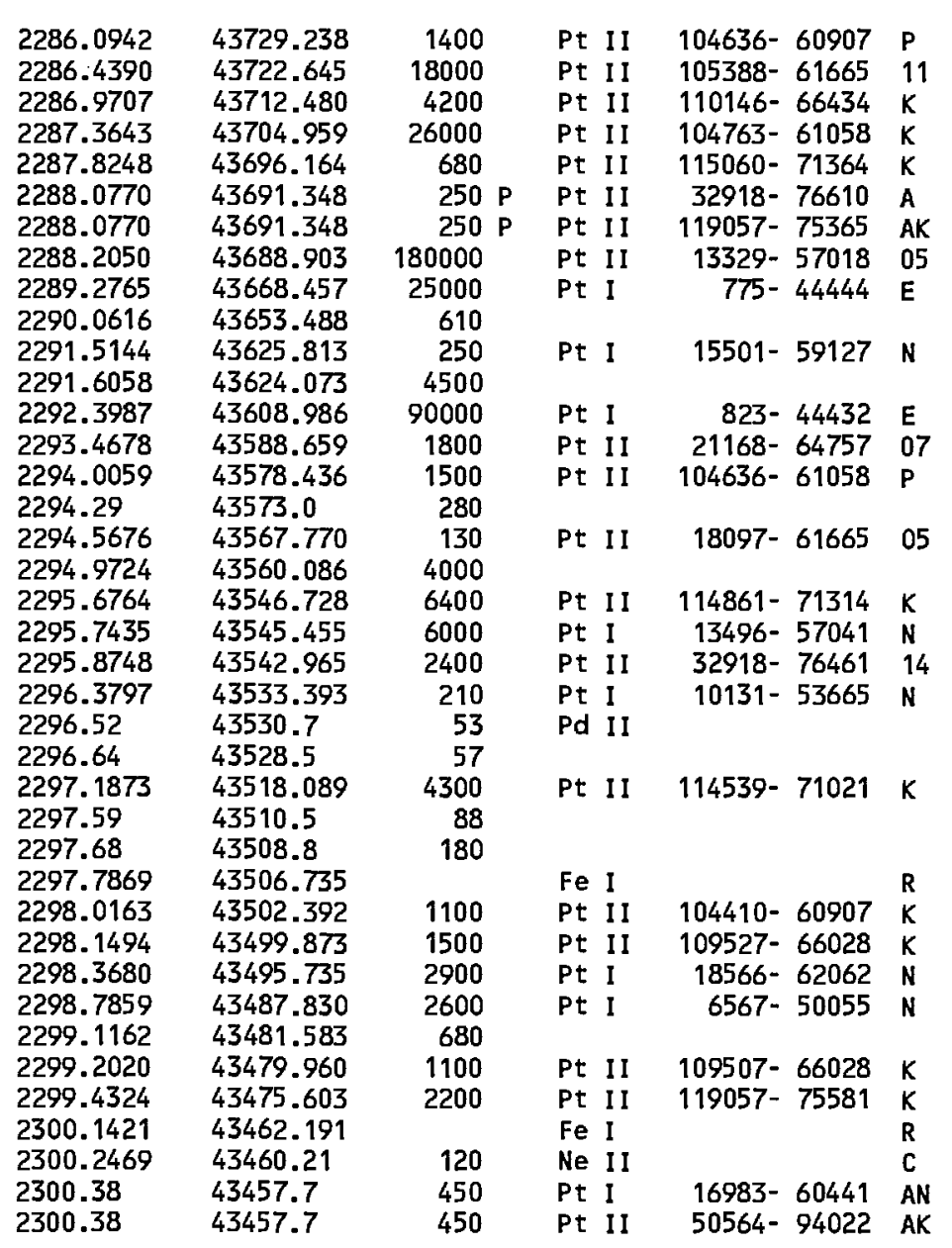

WAVELENGTH WAVE NUMBER INTENSITY

CLASSIFICATION

CODE

\begin{tabular}{|c|c|c|c|c|c|}
\hline $\begin{array}{l}2300.73 \\
2300.99 \\
2301.175 \\
2301.54\end{array}$ & $\begin{array}{l}43451.1 \\
43446.2 \\
43442.68 \\
43435.8\end{array}$ & $\begin{array}{r}130 \\
55\end{array}$ & $\mathrm{Fe}$ & & $\mathbf{s}$ \\
\hline 2301.69 & 43433.0 & 160 & Pt I I & $53749-97183$ & K \\
\hline 2302.3068 & 43421.330 & 11000 & Pt I I & $105086-61665$ & K \\
\hline 2302.42 & 43419.2 & 460 & Pt II & 54373- 97792 & K \\
\hline $\begin{array}{l}2303.2065 \\
2303.90\end{array}$ & $\begin{array}{l}43404.369 \\
43391.3\end{array}$ & $\begin{array}{r}7600 \\
34\end{array}$ & $\begin{array}{l}\text { Pt I } \\
\mathrm{Ne} \text { III }\end{array}$ & $6140-49544$ & E \\
\hline 2304.12 & 43387.2 & 54 & & & \\
\hline 2304.37 & 43382.5 & 72 & Pt II & $64003-107386$ & AK \\
\hline 2304.37 & 43382.5 & 72 & Pt II & $111162-67780$ & AK \\
\hline 2304.83 & 43373.8 & 99 & Ne III & & $L$ \\
\hline 2305.34 & 43364.2 & 110 & Pt I & 21967- 65331 & N \\
\hline 2305.6355 & 43358.646 & 1900 & & & \\
\hline $\begin{array}{l}2306.0122 \\
2306.58\end{array}$ & 43351.565 & $\begin{array}{r}2800 \\
160\end{array}$ & Pt II & $104410-61058$ & $k$ \\
\hline $\begin{array}{l}2300.28 \\
2307.78\end{array}$ & $\begin{array}{l}45340.9 \\
43318.4\end{array}$ & $\begin{array}{l}100 \\
130\end{array}$ & Pt II & $109346-66028$ & K \\
\hline 2308.0437 & 43313.411 & 35000 & Pt I & $6567-49880$ & $\ddot{E}$ \\
\hline 2308.63 & 43302.4 & 61 & Ne III & & $\mathrm{L}$ \\
\hline & & & & & \\
\hline
\end{tabular}




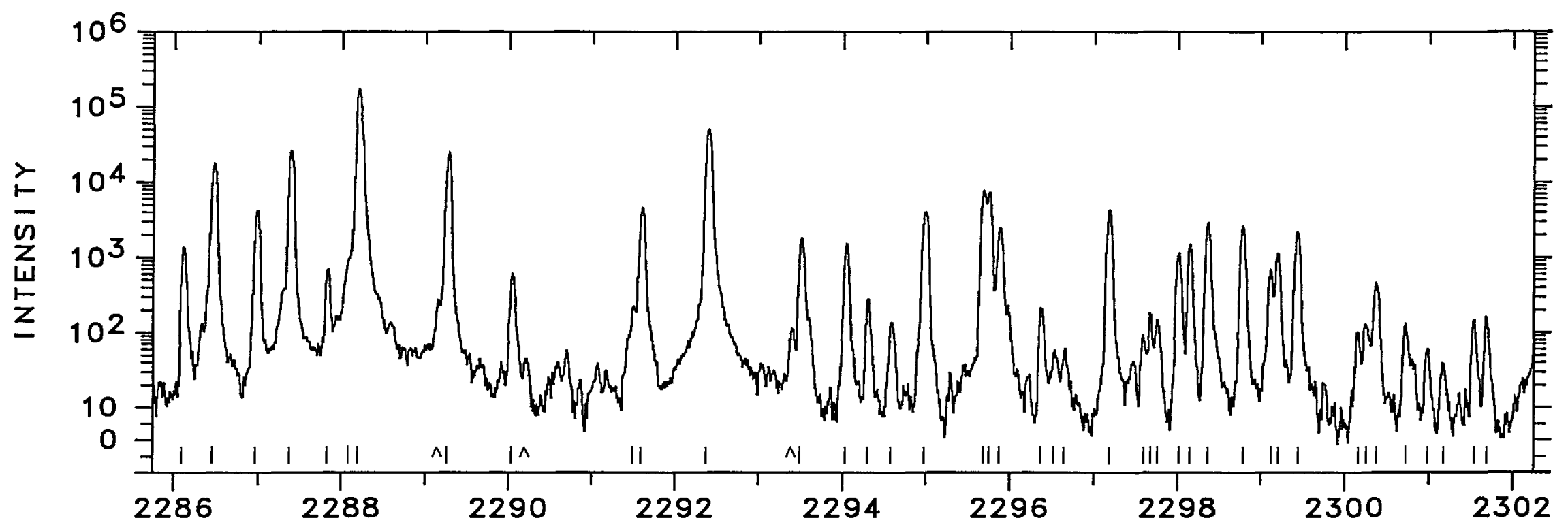

$\stackrel{\circ}{\circ}$

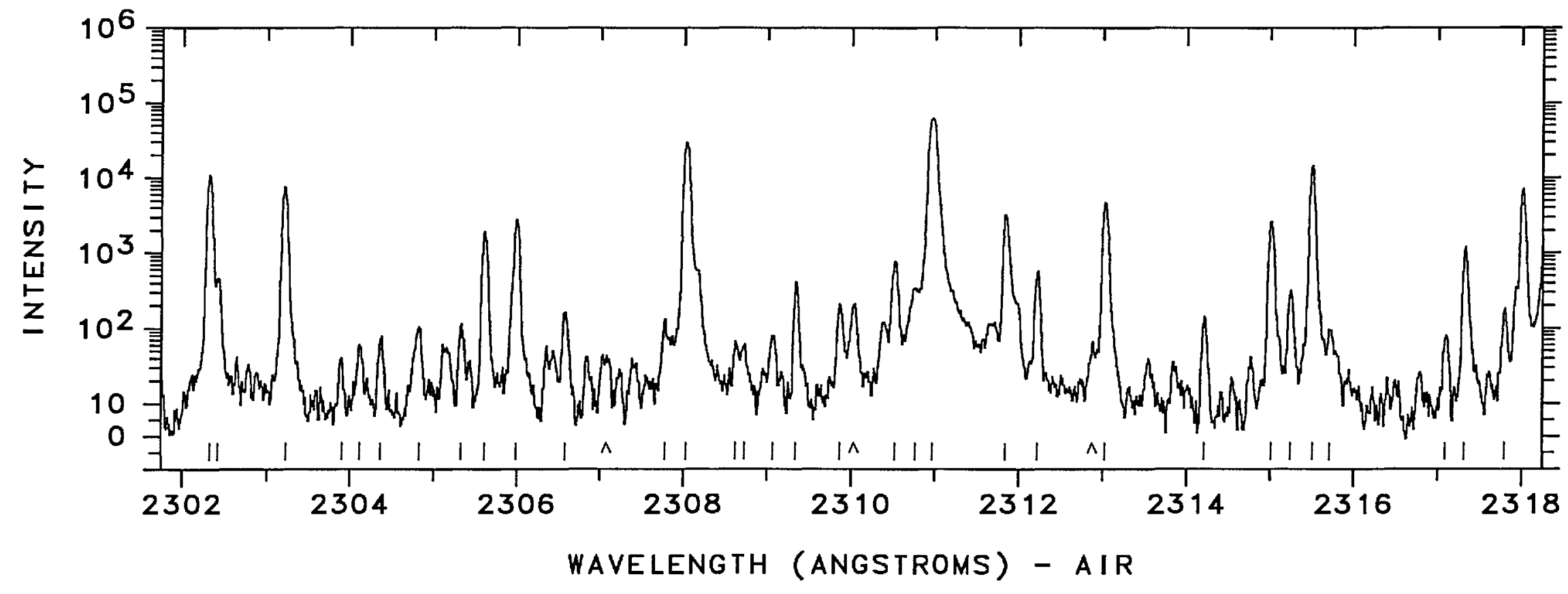


WAVELENGTH HAVE NUMBER INTENSITY

CLASSIFICATION

CODE

\begin{tabular}{|c|c|c|c|c|c|}
\hline 2318.0081 & 43127.235 & 7000 & Pt II & $58491-101618$ & K \\
\hline 2318.2969 & 43121.864 & 57000 & Pt I & $823-43945$ & E \\
\hline 2318.83 & $\begin{array}{l}43112.0 \\
43108.6\end{array}$ & $\begin{array}{r}54 \\
110\end{array}$ & Pt II & $115060-71948$ & K \\
\hline 2319.1251 & $\begin{array}{l}43708.6 \\
43106.466\end{array}$ & 620 & Pt I I & $114127-71021$ & $\mathrm{k}$ \\
\hline 2319.8215 & 43093.526 & 750 & Pt II & $109527-66434$ & $\mathrm{k}$ \\
\hline 2319.8869 & 43092.311 & 12000 & Pt II & $18097-61190$ & 06 \\
\hline 2320.23 & 43085.9 & 55 & & & \\
\hline $\begin{array}{l}2320.6133 \\
2320.85\end{array}$ & $\begin{array}{l}43078.823 \\
43074.4\end{array}$ & $\begin{array}{r}250 \\
77\end{array}$ & Pt I & $18566-61645$ & N \\
\hline 2321.7422 & 43057.879 & 2400 & Pt II & $58491-101549$ & K \\
\hline 2322.0304 & 43052.535 & 1100 & & & \\
\hline 2323.09 & 43032.9 & 75 & & & \\
\hline 2323.42 & 43026.8 & 190 & & & \\
\hline 2325.87 & 42981.5 & 110 & & & \\
\hline 2326.1053 & 42977.122 & 8600 & Pt I & $6567-49544$ & $\mathbf{E}$ \\
\hline 2326.3386 & 42972.812 & 13000 & Pt II & $23461-66434$ & 10 \\
\hline $\begin{array}{l}2326.4148 \\
2326.91\end{array}$ & $\begin{array}{l}42971.406 \\
42962.3\end{array}$ & $\begin{array}{r}750 \\
35\end{array}$ & Pt II & $104636-61665$ & $\mathrm{~K}$ \\
\hline 2328.0220 & 42941.741 & 780 & Pt II & $114256-71314$ & K \\
\hline 2328.57 & 42931.6 & 110 & Pt II & $117340-74409$ & K \\
\hline $\begin{array}{l}2328.79 \\
2328.88\end{array}$ & $\begin{array}{l}42927.6 \\
42925.9\end{array}$ & $\begin{array}{r}150 \\
71\end{array}$ & Pt II & $116689-73761$ & $\mathrm{~K}$ \\
\hline 2329.2862 & 42918.437 & 92 & Pt II & $29030-71948$ & 09 \\
\hline 2330.07 & 42904.0 & 89 & & & \\
\hline $\begin{array}{l}2330.2360 \\
2330.46\end{array}$ & $\begin{array}{l}42900.945 \\
42896.8\end{array}$ & $\begin{array}{r}830 \\
38\end{array}$ & Pt II & $24879-67780$ & $\mathrm{~K}$ \\
\hline 2330.9705 & 42887.428 & 3200 & Pt I & $10131-53019$ & $E$ \\
\hline 2331.7820 & 42872.503 & 280 & Pt II & $34647-77519$ & 13 \\
\hline 2331.9574 & 42869.279 & 950 & & & \\
\hline $\begin{array}{l}2332.21 \\
2332.7994\end{array}$ & $\begin{array}{l}42864.6 \\
42853.808\end{array}$ & 170 & & & $\mathbf{s}$ \\
\hline $\begin{array}{l}2332.7994 \\
2333.08\end{array}$ & $\begin{array}{l}42853.808 \\
42848.7\end{array}$ & 120 & & & \\
\hline 2333.48 & 42841.3 & 360 & Pt II & $58062-100903$ & $\mathrm{~K}$ \\
\hline
\end{tabular}

WAVELENGTH WAVE NUMBER INTENSITY CLASSIFICATION CODE

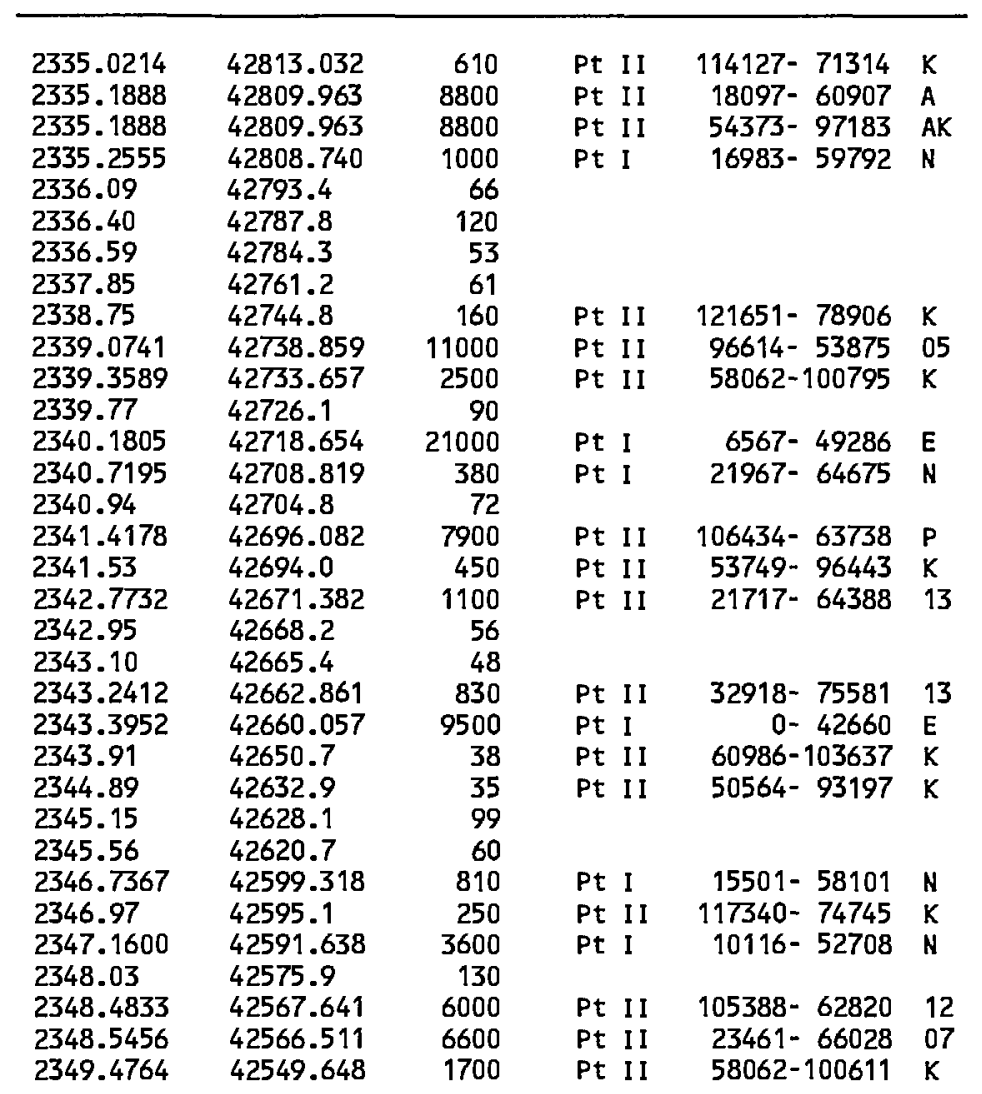




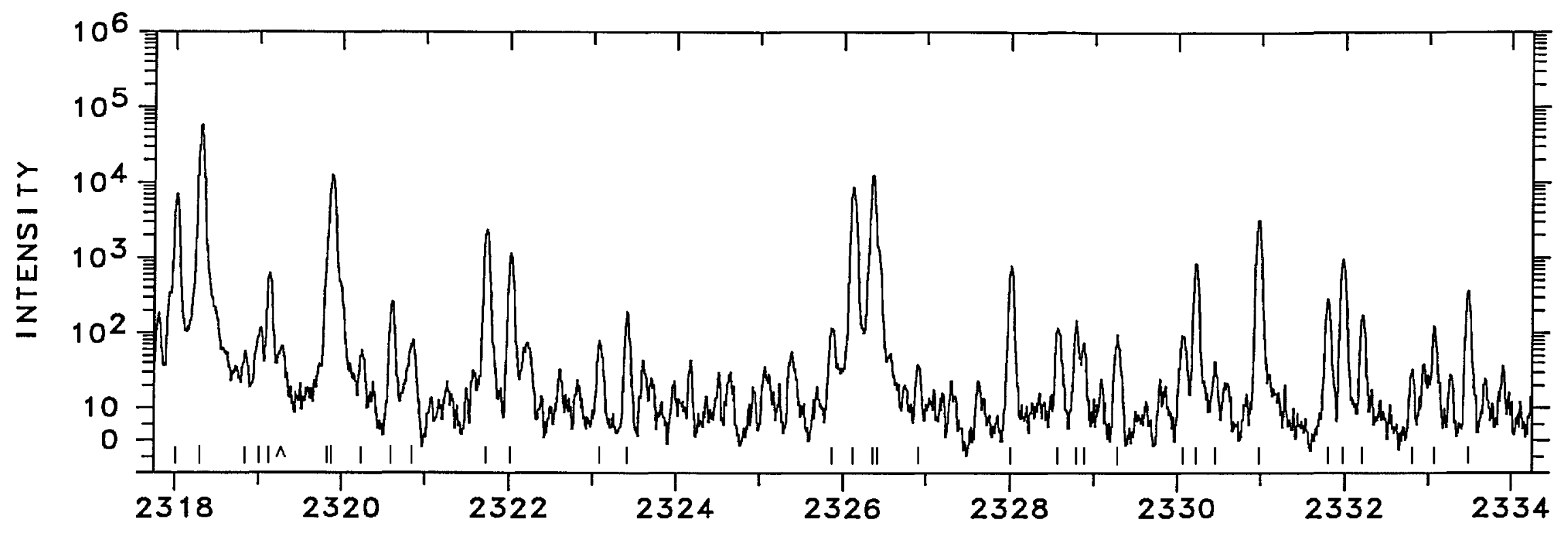

$\infty$

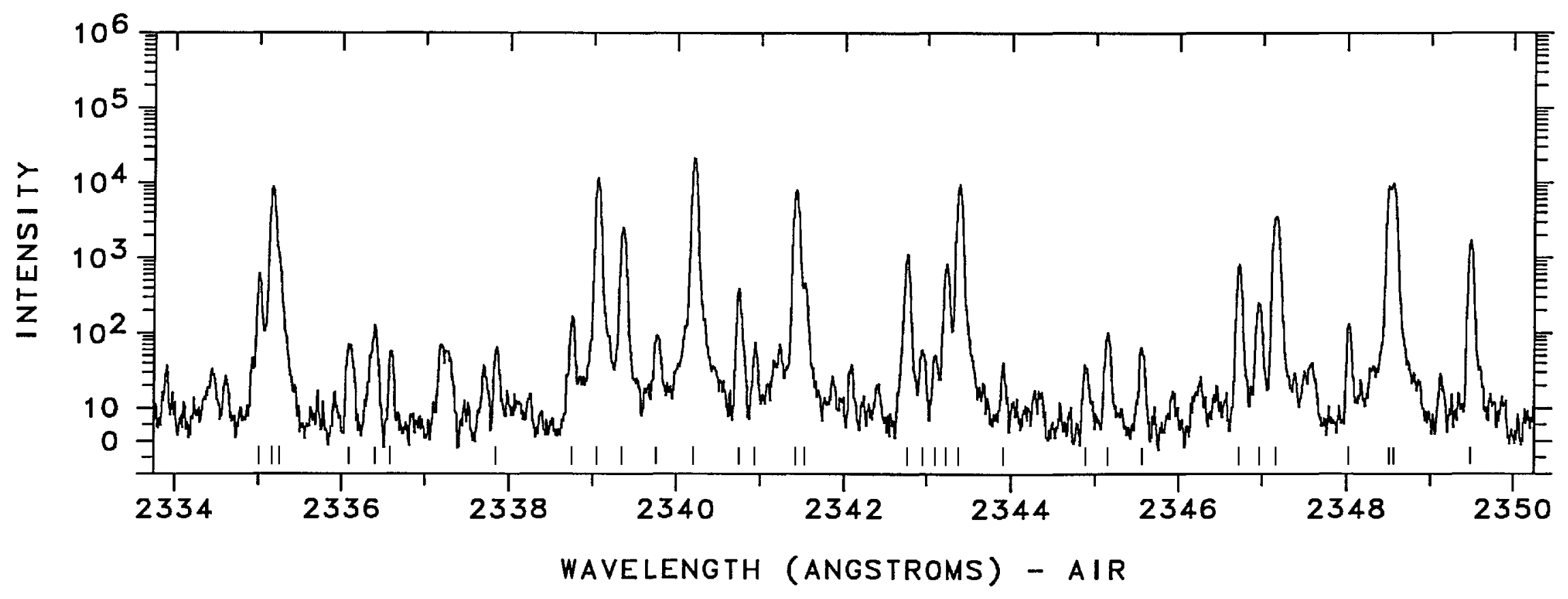


\begin{tabular}{llll} 
WAVELENGTH WAVE NUMBER INTENSITY CLASSIFICATION CODE \\
\hline
\end{tabular}

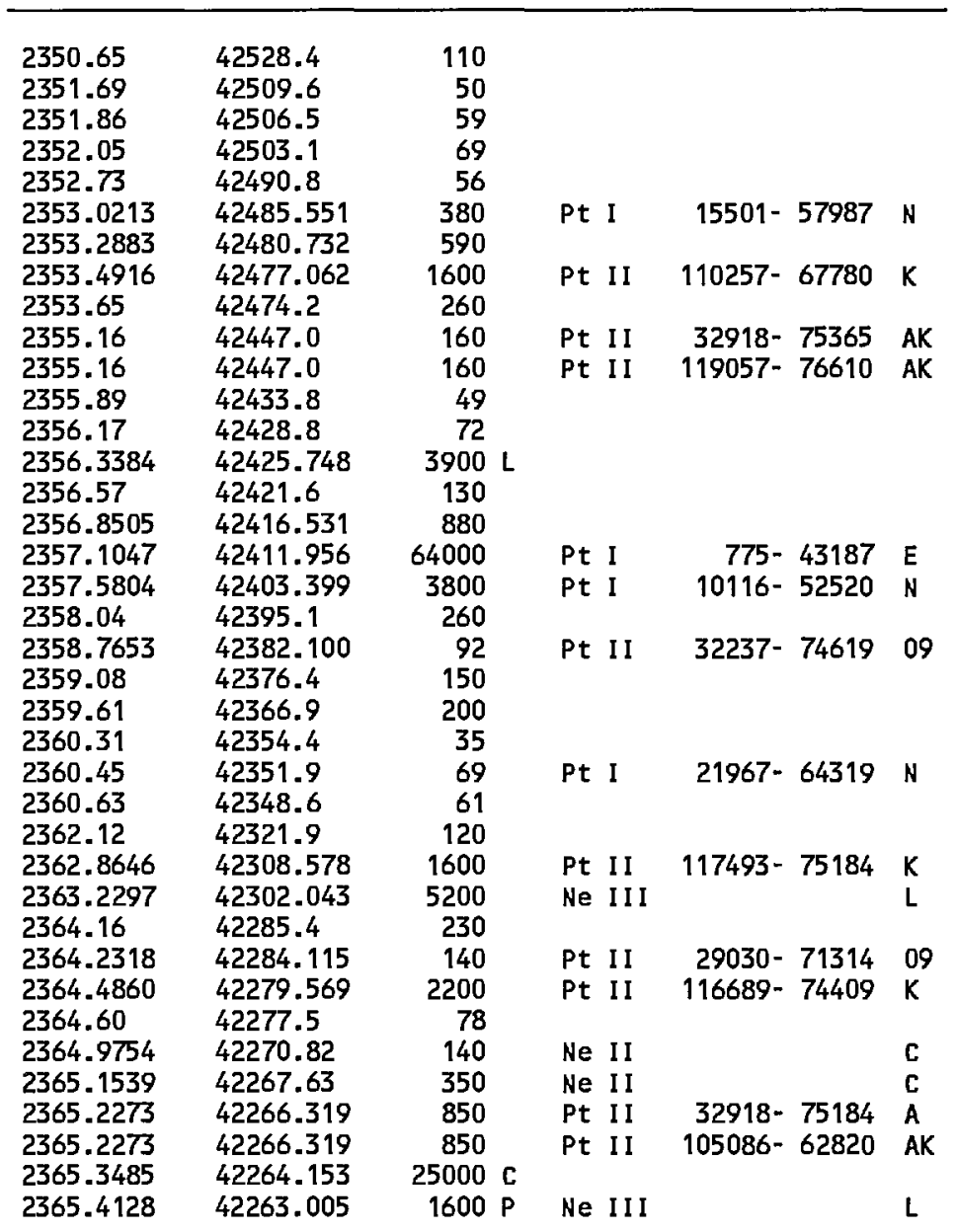

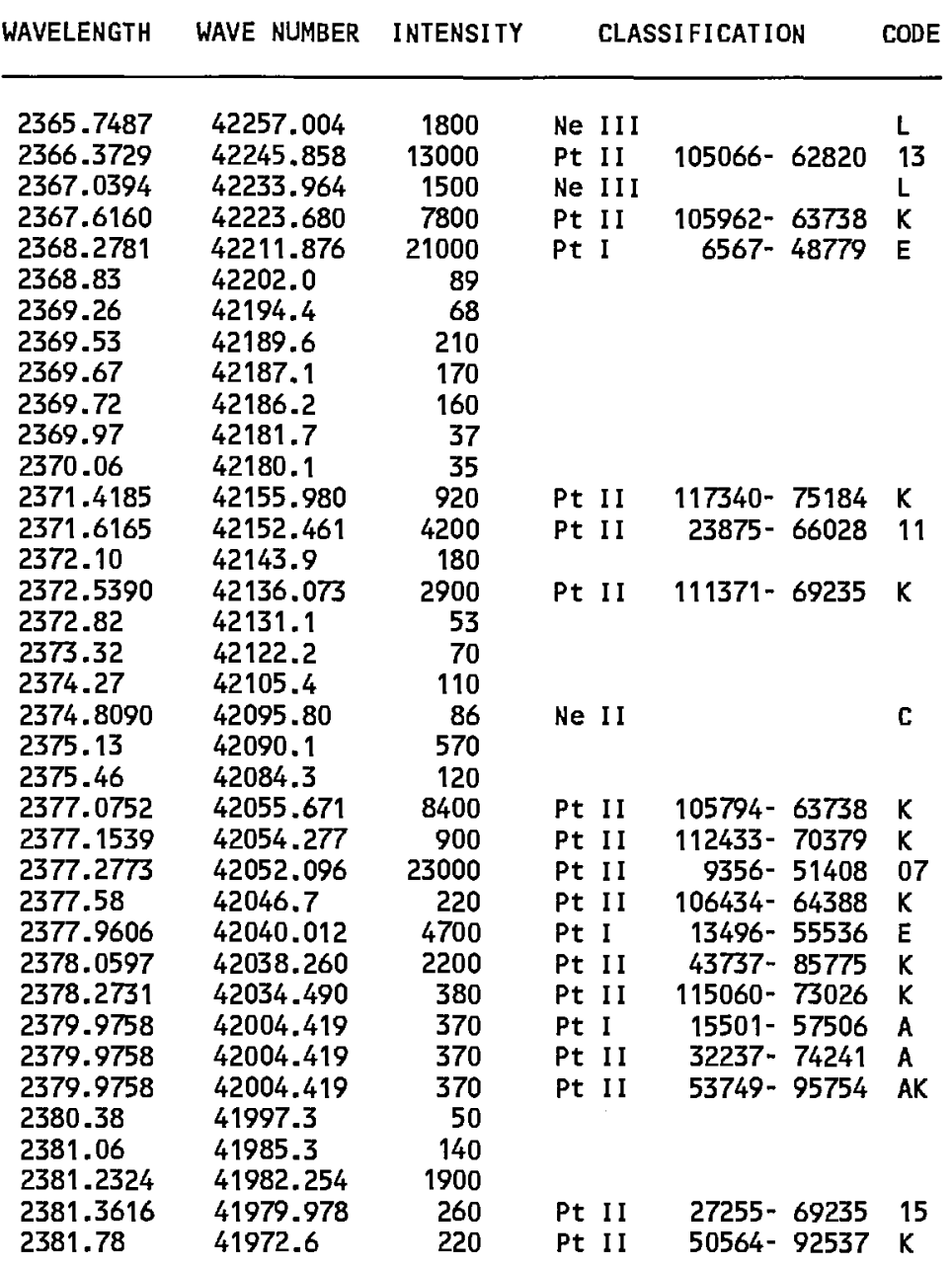



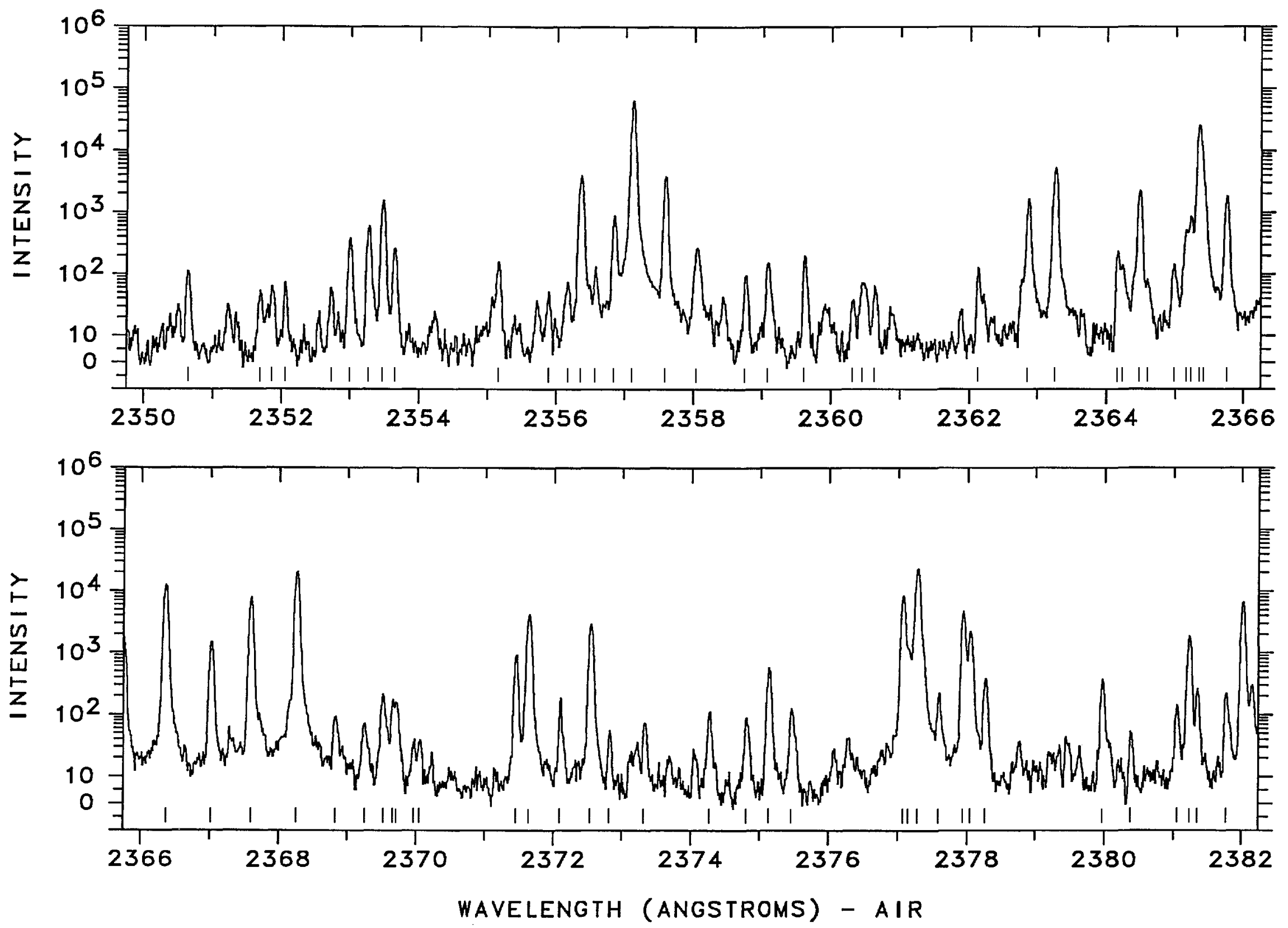


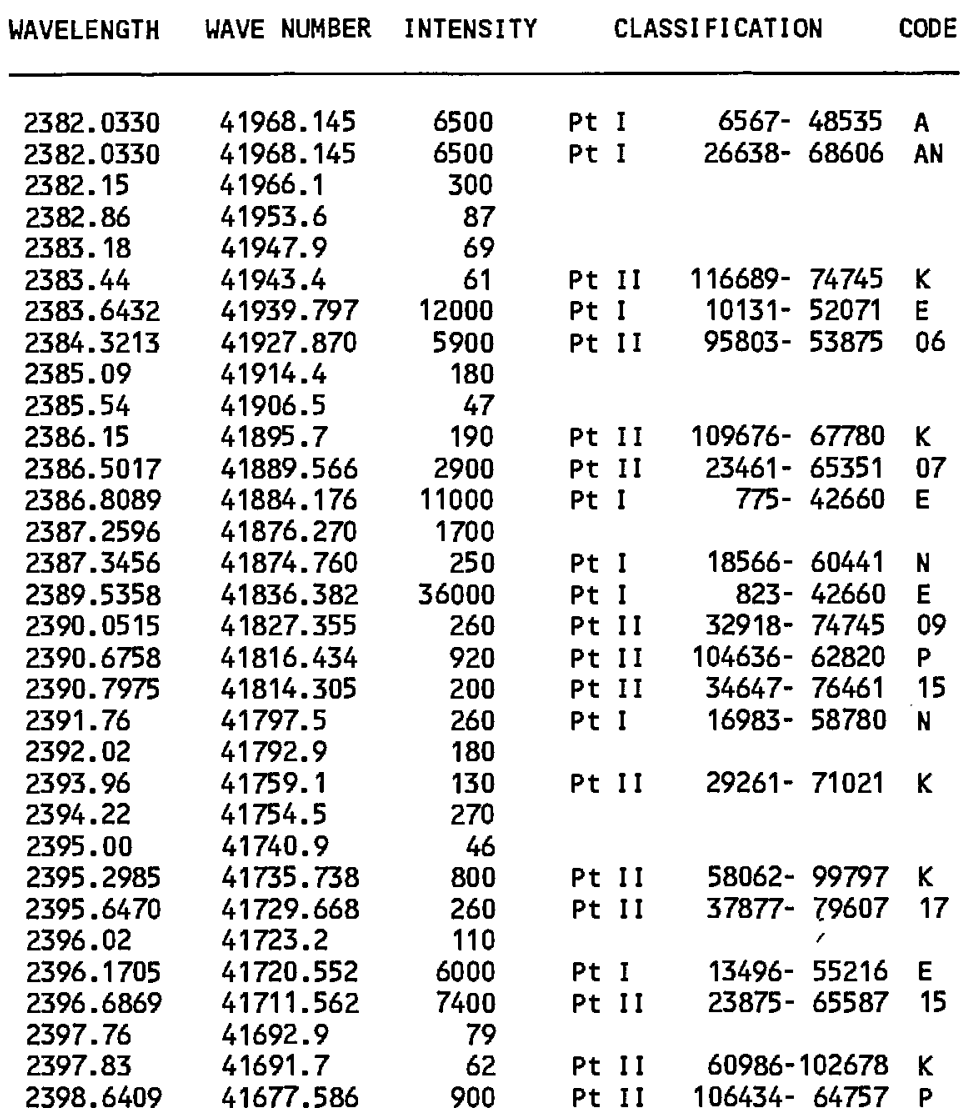

\begin{tabular}{|c|c|c|c|c|c|}
\hline AVELENGTH & AVE NUMBER & INTENSITY & CLAS & IF ICATION & CODE \\
\hline $\begin{array}{l}2398.73 \\
2399.2413 \\
2400.01 \\
2400.2707 \\
2400.56 \\
2400.75 \\
2401.0033 \\
2401.8773 \\
2402.3655 \\
2403.0918 \\
2403.50 \\
2403.7227 \\
2404.6239 \\
2405.7269 \\
2406.5926 \\
2407.29 \\
2407.59 \\
2407.82 \\
2408.65 \\
2409.41 \\
2409.63 \\
2410.3280 \\
2411.89 \\
2411.99 \\
2412.8173 \\
2412.90 \\
2413.0462 \\
2413.0462 \\
2413.18 \\
2413.54 \\
2413.76\end{array}$ & $\begin{array}{l}41676.0 \\
41667.158 \\
41653.8 \\
41649.289 \\
41644.3 \\
41641.0 \\
41636.581 \\
41621.432 \\
41612.974 \\
41600.398 \\
41593.3 \\
41589.480 \\
41573.895 \\
41554.835 \\
41539.889 \\
41527.9 \\
41522.7 \\
41518.7 \\
41504.4 \\
41491.3 \\
41487.5 \\
41475.516 \\
41448.7 \\
41446.9 \\
41432.731 \\
41431.3 \\
41428.800 \\
41428.800 \\
41426.5 \\
41420.3 \\
41416.5\end{array}$ & $\begin{array}{r}73 \\
57 \\
6500 \\
85 \\
100 \\
4700 \\
8500 \\
700 \\
17000 \\
110 \\
450 \\
2500 \\
15000 \\
740 \\
49 \\
57 \\
140 \\
160 \\
110 \\
160 \\
780 \\
110 \\
360 \\
410 \\
230 \\
850 \\
850 \\
86 \\
68 \\
100\end{array}$ & $\begin{array}{ll}\text { Fe } & \text { II } \\
\text { Pt } & \text { II } \\
\text { Ne III } & \\
\text { Pt I } \\
\text { Pt I } \\
\text { Pt II } \\
\text { Pt I } \\
\text { Pt II } \\
\text { Pt II } \\
\text { Pt II } \\
\text { Pt I }\end{array}$ & $\begin{array}{r}10116-51753 \\
10131-51753 \\
21168-62781 \\
6140-47740 \\
104410-62820 \\
105962-64388 \\
24879-66434 \\
15501-57041\end{array}$ & $\begin{array}{l}S \\
13 \\
L \\
E \\
E \\
09 \\
E \\
K \\
K \\
10 \\
N\end{array}$ \\
\hline
\end{tabular}




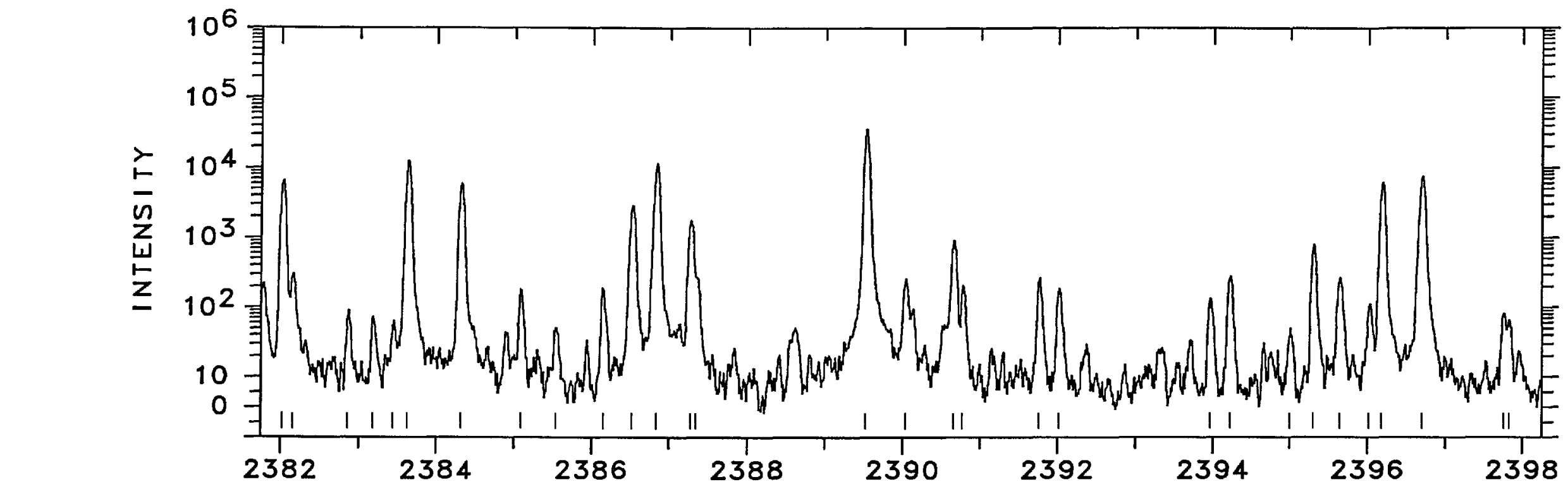

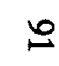

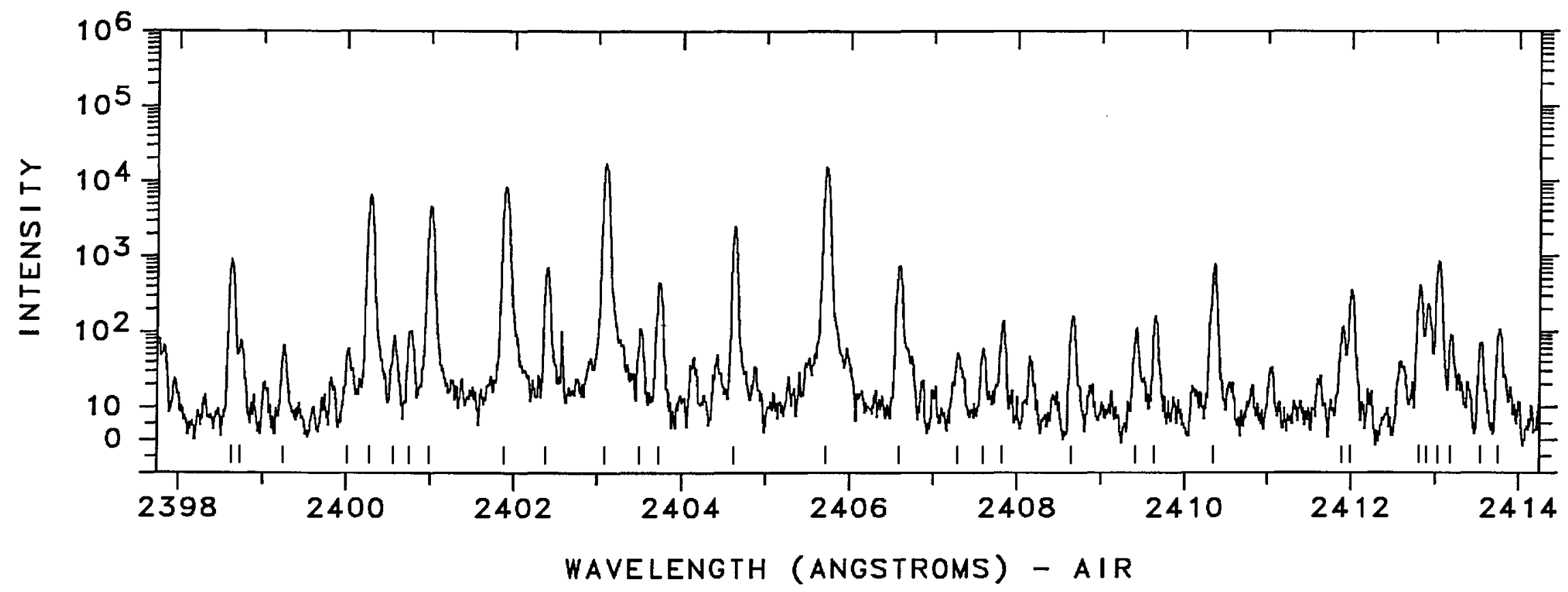




\begin{tabular}{llll} 
WAVELENGTH WAVE NUMBER INTENSITY CLASSIFICATION CODE \\
\hline
\end{tabular}

\begin{tabular}{|c|c|c|c|c|c|}
\hline WAVELENGTH & WAVE NUMBER & INTENSITY & CLA & SIF ICATION & CODE \\
\hline $\begin{array}{l}2429.7731 \\
2430.0176 \\
2430.1647 \\
2430.96 \\
2431.60 \\
2432.04 \\
2432.39 \\
2433.3064 \\
2433.49 \\
2433.49 \\
2433.54 \\
2434.14 \\
2434.2105 \\
2434.4128 \\
2434.4610 \\
2435.1545 \\
2435.6448 \\
2436.0764 \\
2436.6887 \\
2436.91 \\
2437.69 \\
2437.8887 \\
2439.1180 \\
2439.34 \\
2439.42 \\
2440.0608 \\
2441.24 \\
2441.4347 \\
2442.6261 \\
2442.91 \\
2443.0933 \\
2444.36 \\
2444.50 \\
2445.3359\end{array}$ & $\begin{array}{l}41143.62 \\
41139.48 \\
41136.99 \\
41123.5 \\
41112.7 \\
41105.3 \\
41099.4 \\
41083.882 \\
41080.8 \\
41080.8 \\
41079.9 \\
41069.8 \\
41068.624 \\
41065.210 \\
41064.398 \\
41052.705 \\
41044.44 \\
41037.169 \\
41026.858 \\
41023.1 \\
41010.0 \\
41006.664 \\
40986.00 \\
40982.3 \\
40980.9 \\
40970.165 \\
40950.4 \\
40947.11 \\
40927.139 \\
40922.4 \\
40919.314 \\
40898.1 \\
40895.8 \\
40881.790\end{array}$ & $\begin{array}{r}83 \\
200 \\
220 \\
37 \\
98 \\
290 \\
320 \\
10000 \\
160 \\
160 \\
160 \\
150 \\
430 \\
1000 \mathrm{P} \\
16000 \\
\\
250 \\
480 \\
35000 \\
180 \\
120 \\
440 \\
170 \\
120 \\
150 \\
170000 \\
87 \\
130 \\
9100 \\
140 \\
1400 \\
280 \\
110 \\
1100\end{array}$ & $\begin{array}{ll}\mathrm{Pt} & \text { II I } \\
\mathrm{Pt} & \mathrm{II} \\
\mathrm{Pt} & \mathrm{II} \\
\mathrm{Pt} & \mathrm{II} \\
\mathrm{Ne} & \mathrm{II} \\
\mathrm{Pt} & \mathrm{II} \\
\mathrm{Pt} & \\
\mathrm{Pt} & \mathrm{II} \\
\mathrm{Pt} & \mathrm{II} \\
\mathrm{Si} & \mathrm{I} \\
\mathrm{Ne} & \mathrm{II} \\
\mathrm{Pt} & \mathrm{II} \\
\mathrm{Pt} & \mathrm{I}\end{array}$ & $\begin{array}{r}50564-91669 \\
114861-73761 \\
106434-65351 \\
32918-73999 \\
53749-94829 \\
112433-71364 \\
21717-62781\end{array}$ & $\begin{array}{l}\mathrm{C} \\
13 \\
\mathrm{~K} \\
13 \\
\mathrm{~K} \\
\mathrm{~N} \\
11\end{array}$ \\
\hline
\end{tabular}



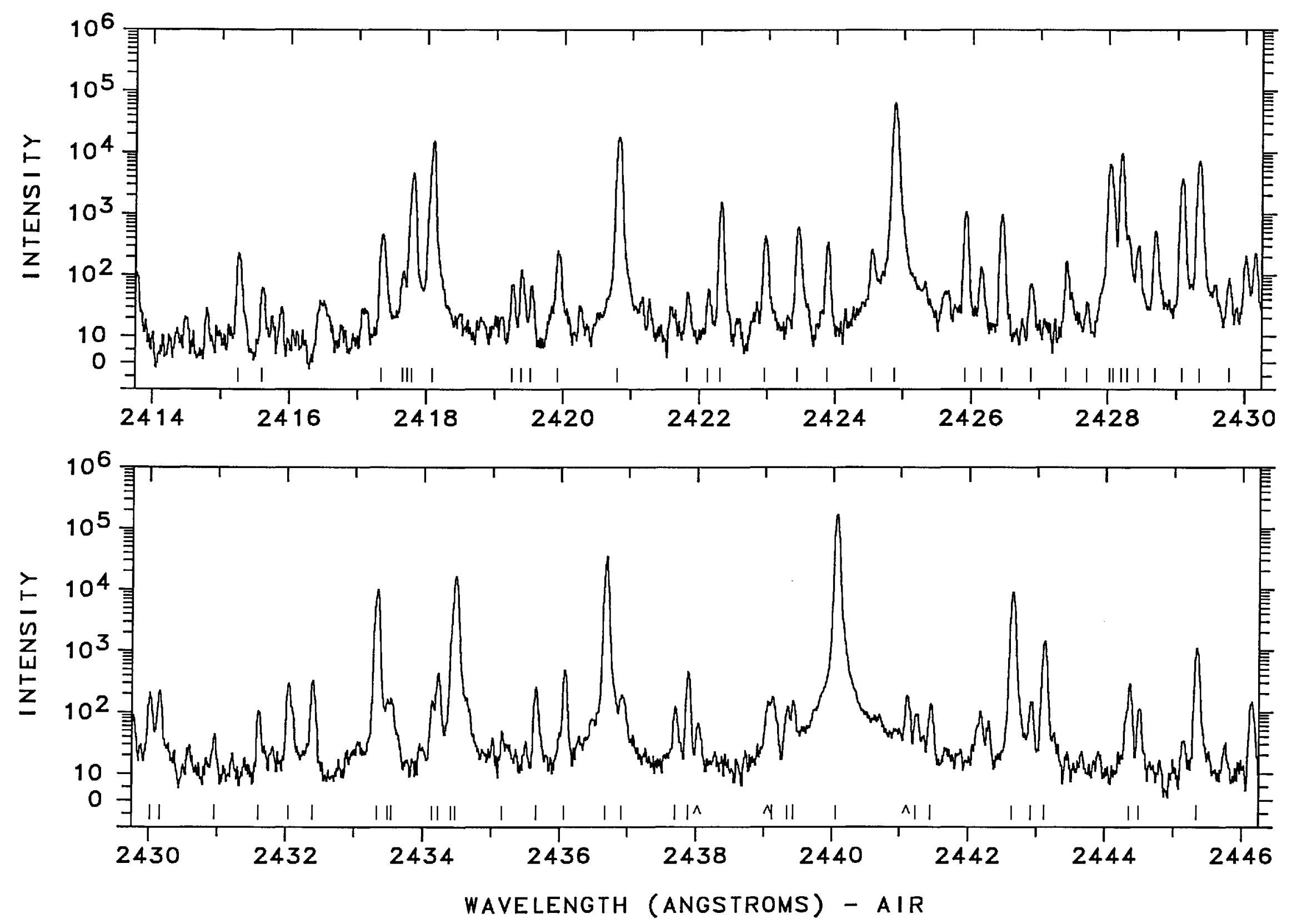


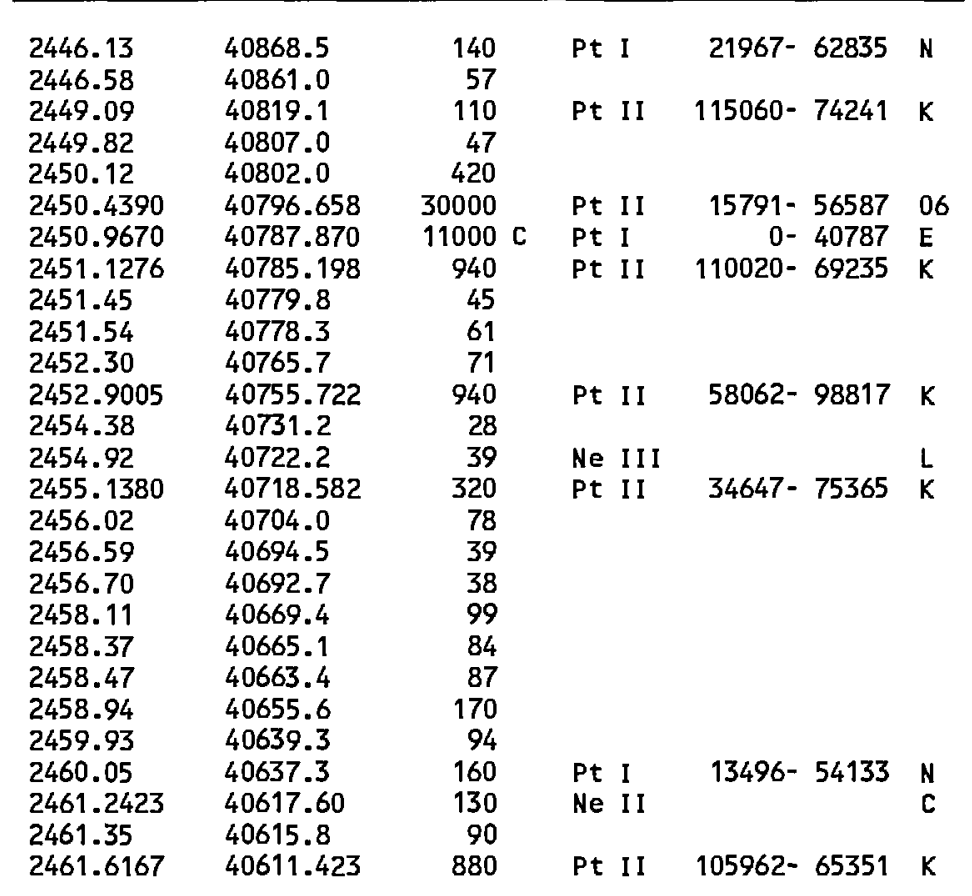

\begin{tabular}{|c|c|c|c|c|c|}
\hline $\begin{array}{l}2461.79 \\
2462.05 \\
2462.29 \\
2464.33\end{array}$ & $\begin{array}{l}40608.6 \\
40604.3 \\
40600.3 \\
40566.7\end{array}$ & $\begin{array}{r}18 \\
16 \\
81 \\
180\end{array}$ & Ne II I & & $L$ \\
\hline 2467.4003 & 40516.236 & $80000 \mathrm{P}$ & Pt I & $0-40516$ & $\mathbf{E}$ \\
\hline $\begin{array}{l}2467.4824 \\
2467.5920 \\
2468.27\end{array}$ & $\begin{array}{l}40514.888 \\
40513.089\end{array}$ & $\begin{array}{c}15000 \mathrm{P} \\
5800 \\
69\end{array}$ & $\begin{array}{ll}\text { Pt } & \text { I } \\
\text { Pt } & \text { I I }\end{array}$ & $\begin{array}{l}13496-54011 \\
23875-64388\end{array}$ & $\begin{array}{l}E \\
16\end{array}$ \\
\hline $\begin{array}{l}2468.72 \\
2469.33\end{array}$ & $\begin{array}{l}40494.6 \\
40484.6\end{array}$ & $\begin{array}{r}60 \\
300\end{array}$ & 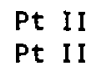 & $\begin{array}{l}114256-73761 \\
112433-71948\end{array}$ & $\begin{array}{l}K \\
K\end{array}$ \\
\hline 2469.41 & 40483.3 & 120 & Pt I & $26638-67121$ & N \\
\hline 2470.0003 & 40473.59 & 63 & Ne II & & C \\
\hline 2470.27 & 40469.2 & 69 & Pt II & $54373-94842$ & $\mathrm{~K}$ \\
\hline 2470.59 & 40463.9 & 130 & Pt II & $41434-81897$ & $\mathrm{~K}$ \\
\hline 2471.0073 & 40457.098 & 6400 & Pt I & $13496-53953$ & $\mathrm{E}$ \\
\hline 2471.1551 & 40454.678 & 700 & Pt II & $110408-69953$ & $\mathrm{~K}$ \\
\hline 2471.31 & 40452.1 & 83 & Pt II & $114861-74409$ & K \\
\hline 2471.39 & 40450.8 & 88 & & & \\
\hline 2471.8422 & 40443.433 & 4600 & Pt II & $105794-65351$ & K \\
\hline 2473.3856 & 40418.199 & 12000 & $\mathrm{Ne}$ III & & $\mathrm{L}$ \\
\hline 2474.0576 & 40407.221 & $1300 \mathrm{~L}$ & Pt II & $60986-101394$ & $\mathrm{~K}$ \\
\hline 2475.89 & 40377.3 & 130 & Pt II & $37877-78254$ & K \\
\hline $\begin{array}{l}2470.03 \\
2477.2734\end{array}$ & 40354.772 & $\begin{array}{r}04 \\
180\end{array}$ & Pt & $21967-62321$ & $\mathbf{N}$ \\
\hline & 40352.1 & 230 & Pt II & $104090-63738$ & $\mathrm{~K}$ \\
\hline 2477.5449 & 40350.35 & 100 & $\mathrm{Ne} I \mathrm{I}$ & & c \\
\hline
\end{tabular}




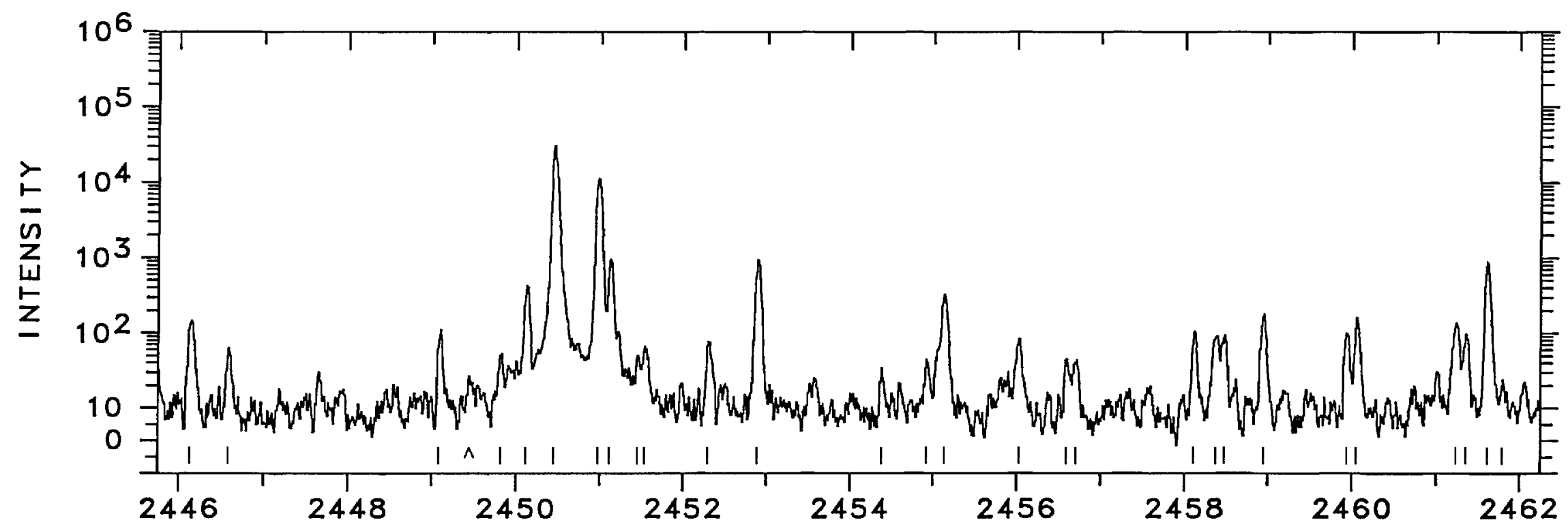

i

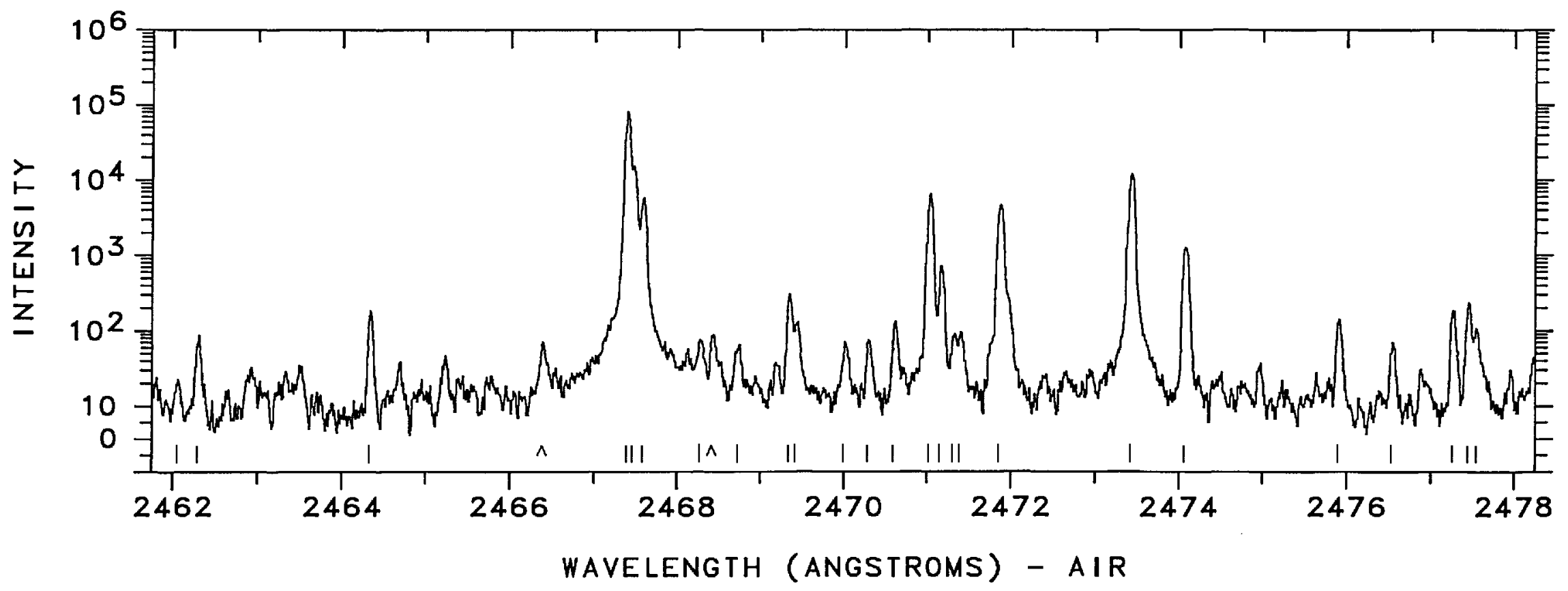




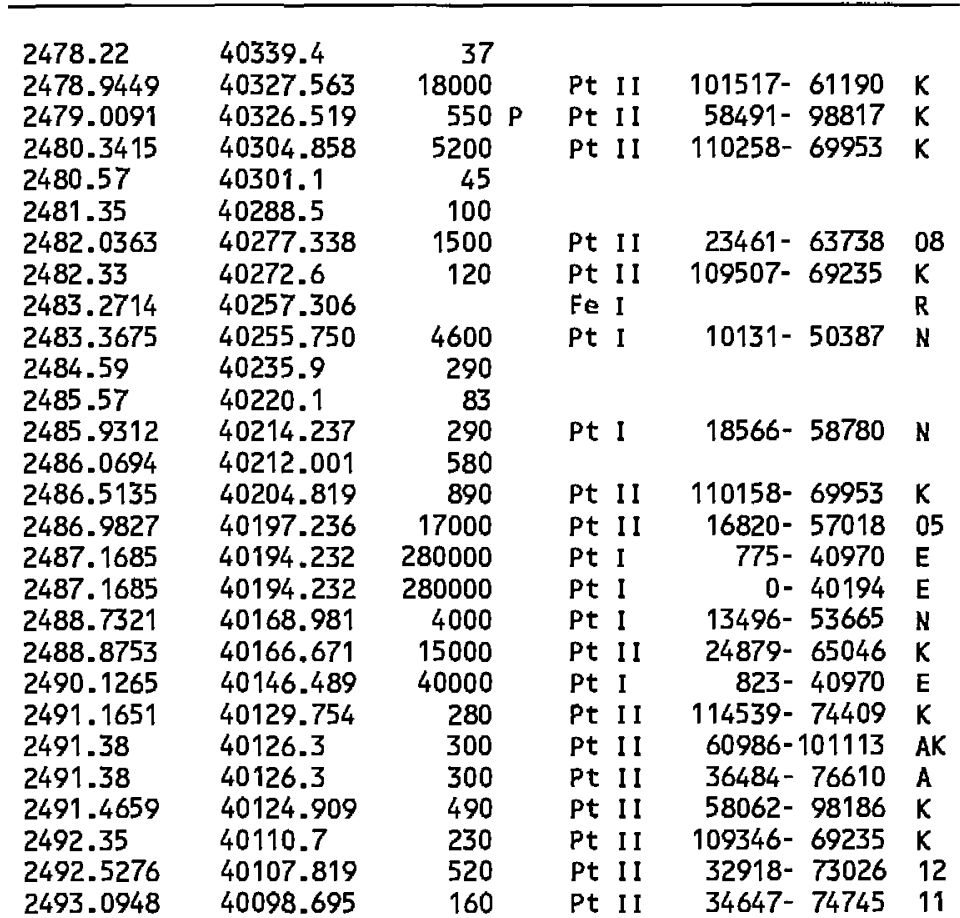

\begin{tabular}{|c|c|c|c|c|c|c|}
\hline $\begin{array}{l}2493.4332 \\
2494.12\end{array}$ & $\begin{array}{l}40093.252 \\
40082.2\end{array}$ & $\begin{array}{l}330 \\
100\end{array}$ & Pt I I & 113119- & 73026 & $k$ \\
\hline 2495.63 & 40058.0 & 160 & Pt I & 16983- & 57041 & N \\
\hline 2495.8126 & 40055.032 & 31000 & Pt I & $6567-$ & 46622 & $\mathrm{E}$ \\
\hline 2497.0968 & 40034.434 & 280 & Pt I & 15501- & 55536 & $\mathbf{N}$ \\
\hline 2497.9137 & 40021.342 & 680 & Pt II & 21168- & 61190 & 08 \\
\hline 2498.4996 & 40011.958 & 89000 & Pt I & 775- & 40787 & $E$ \\
\hline 2498.6806 & 40009.059 & 15000 & Pt II & 101199- & 61190 & 07 \\
\hline 2499.2092 & 40000.598 & 730 & & 106434- & 66434 & $P$ \\
\hline 2499.86 & 39990.2 & 46 & & & & \\
\hline 2500.20 & 39984.7 & 47 & & & & \\
\hline 2500.28 & 39983.5 & 36 & & & & \\
\hline 2500.67 & 39977.2 & 100 & & & & \\
\hline 2500.81 & 39975.0 & $\begin{array}{r}150 \\
31\end{array}$ & Pt I & 21967- & 61942 & $\mathbf{N}$ \\
\hline $\begin{array}{l}2502.97 \\
2503.3469\end{array}$ & $\begin{array}{l}39940.5 \\
39934.487\end{array}$ & 440 & Pt II & 105962- & 66028 & K \\
\hline 2504.0404 & 39923.427 & 1700 & Pt I & $10131-$ & 50055 & $\hat{E}$ \\
\hline 2504.34 & 39918.7 & 39 & & & & \\
\hline 2504.50 & 39916.1 & 38 & & & & \\
\hline 2505.09 & 39906.7 & 44 & & & & \\
\hline 2505.9225 & 39893.445 & 5000 & Pt I & 10116- & 50010 & $\mathrm{~N}$ \\
\hline 2506.8216 & 39879.138 & 540 & Pt II & $110258-$ & 70379 & K \\
\hline $\begin{array}{l}2506.8973 \\
2506.96\end{array}$ & 39877.934 & & si I & & & B \\
\hline $\begin{array}{l}2506.96 \\
2507.04\end{array}$ & $\begin{array}{l}39876.9 \\
30875\end{array}$ & 260 & & & & 1 \\
\hline 2507.69 & $\begin{array}{l}39875.7 \\
39865.3\end{array}$ & $\begin{array}{l}150 \\
140\end{array}$ & $\mathrm{Ne}$ I I I & & & $L$ \\
\hline 2508.4973 & 39852.500 & 11000 & Pt I & 6567- & 46419 & $E$ \\
\hline 2509.5 & 39836.1 & 89 & Pt II & 114455- & 74619 & K \\
\hline
\end{tabular}



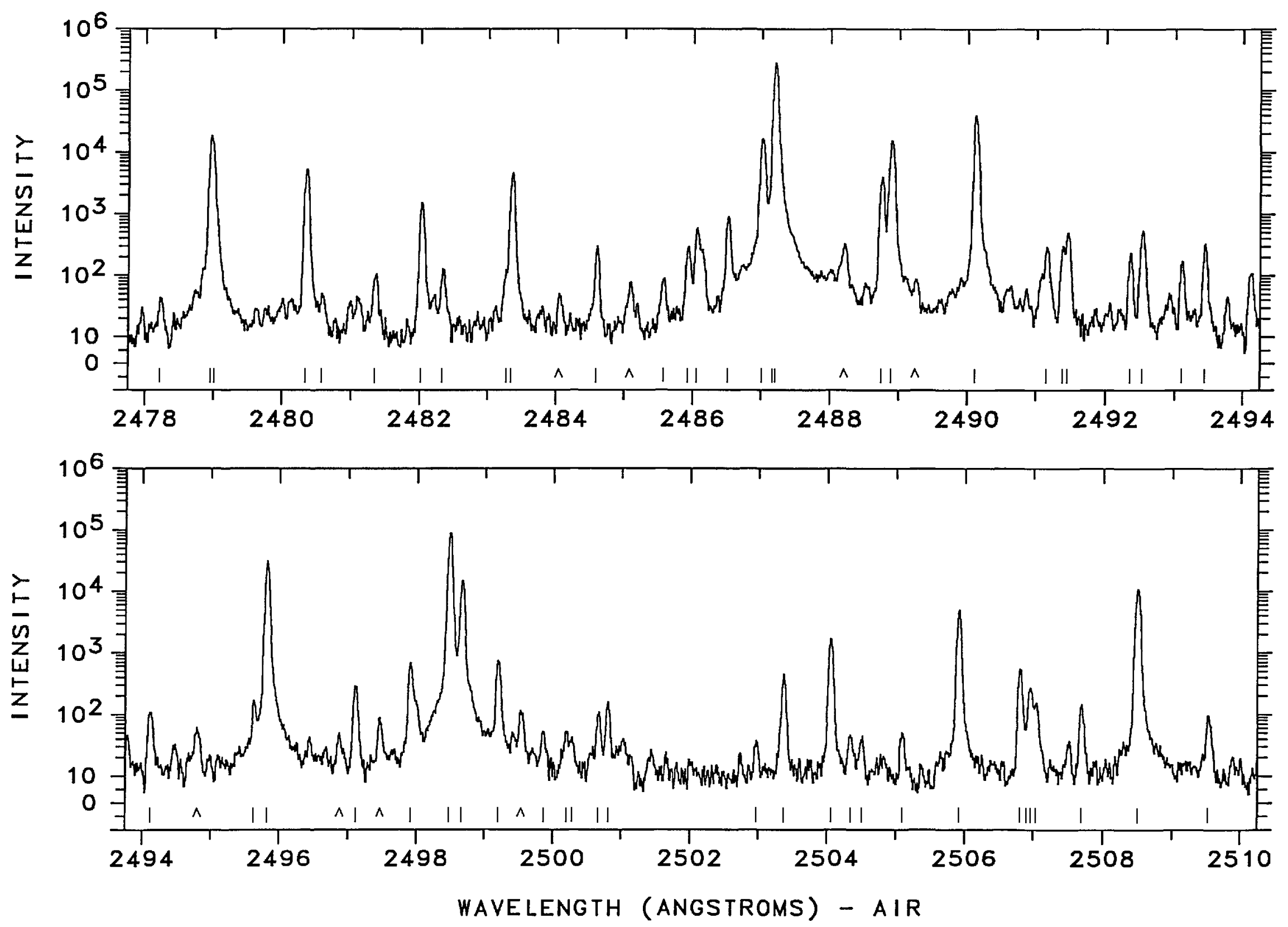


WAVELENGTH WAVE NUMBER INTENSITY CLASSIFICATION CODE

\begin{tabular}{|c|c|c|c|c|c|}
\hline WAVELENGTH & WAVE NUMBER & INTENSITY & \multicolumn{2}{|c|}{ CLASSIFICATION } & CODE \\
\hline 2524.8688 & 39594.11 & 78 & $\mathrm{Ne} I I$ & & c \\
\hline $\begin{array}{l}2525.09 \\
2525.31\end{array}$ & $\begin{array}{l}39590.6 \\
39587.2\end{array}$ & $\begin{array}{r}43 \\
110\end{array}$ & Pt II & $104636-65046$ & $\mathrm{k}$ \\
\hline 2525.8211 & 39579.183 & 2700 & Pt 11 & $104930-65351$ & $\mathrm{~K}$ \\
\hline 2526.15 & 39574.0 & 110 & Pt II & $109527-69953$ & $\mathrm{~K}$ \\
\hline $\begin{array}{l}2526.5031 \\
2528.26\end{array}$ & $\begin{array}{l}39568.499 \\
39541.0\end{array}$ & $\begin{array}{r}480 \\
71\end{array}$ & Pt II & $58062-97630$ & $\mathrm{~K}$ \\
\hline 2528.5086 & 39537.117 & & Si 1 & & B \\
\hline 2528.7336 & 39533.600 & 30000 & Pt II & $101199-61665$ & 05 \\
\hline 2529.0806 & 39528.176 & 1900 & Pt II & $105962-66434$ & K \\
\hline 2529.4100 & 39523.029 & 2500 & Pt I & $13496-53019$ & $\mathbf{E}$ \\
\hline 2529.74 & 39517.9 & 69 & & & \\
\hline 2530.23 & 39510.2 & 46 & $\mathrm{Ne} I \mathrm{I}$ & & A \\
\hline 2530.23 & 39510.2 & 46 & Pt II & $114256-74745$ & AK \\
\hline $\begin{array}{l}2531.32 \\
2532.02\end{array}$ & $\begin{array}{l}39493.2 \\
39482.3\end{array}$ & $\begin{array}{r}110 \\
37\end{array}$ & & & \\
\hline 2532.1535 & 39480.21 & 260 & $\mathrm{Ne} I I$ & & c \\
\hline $\begin{array}{l}2532.89 \\
2535.17\end{array}$ & $\begin{array}{l}39468.7 \\
39433.2\end{array}$ & $\begin{array}{l}50 \\
67\end{array}$ & & & \\
\hline 2535.9677 & 39420.834 & 1000 & Pt I & $18566-57987$ & E \\
\hline 2536.4932 & 39412.668 & 4500 & Pt I & $10131-49544$ & E \\
\hline 2536.89 & 39406.5 & 93 & & & \\
\hline 2537.7612 & 39392.976 & 410 & Pt II & $109346-69953$ & $\mathrm{k}$ \\
\hline 2538.25 & 39385.4 & 180 & Pt I & $21967-61352$ & $\mathbf{N}$ \\
\hline 2538.5033 & 39381.461 & 360 & Pt II & $114127-74745$ & $A K$ \\
\hline 2538.5033 & 39381.461 & 360 & $\mathrm{Ne} 11$ & & A \\
\hline 2539.2067 & 39370.552 & 46000 & Pt 1 & 823- 40194 & E \\
\hline $\begin{array}{l}2540.38 \\
2540.59\end{array}$ & $\begin{array}{l}39352.4 \\
39349.1\end{array}$ & $\begin{array}{l}49 \\
36\end{array}$ & Pt 11 & $34647-73999$ & K \\
\hline 2541.00 & 39342.8 & 69 & Pt 11 & $104930-65587$ & $k$ \\
\hline $\begin{array}{l}2541.3494 \\
2541.65\end{array}$ & $\begin{array}{l}39337.359 \\
39332.7\end{array}$ & $\begin{array}{r}480 \\
42\end{array}$ & Pt 1 & $15501-54839$ & $\mathbf{N}$ \\
\hline
\end{tabular}




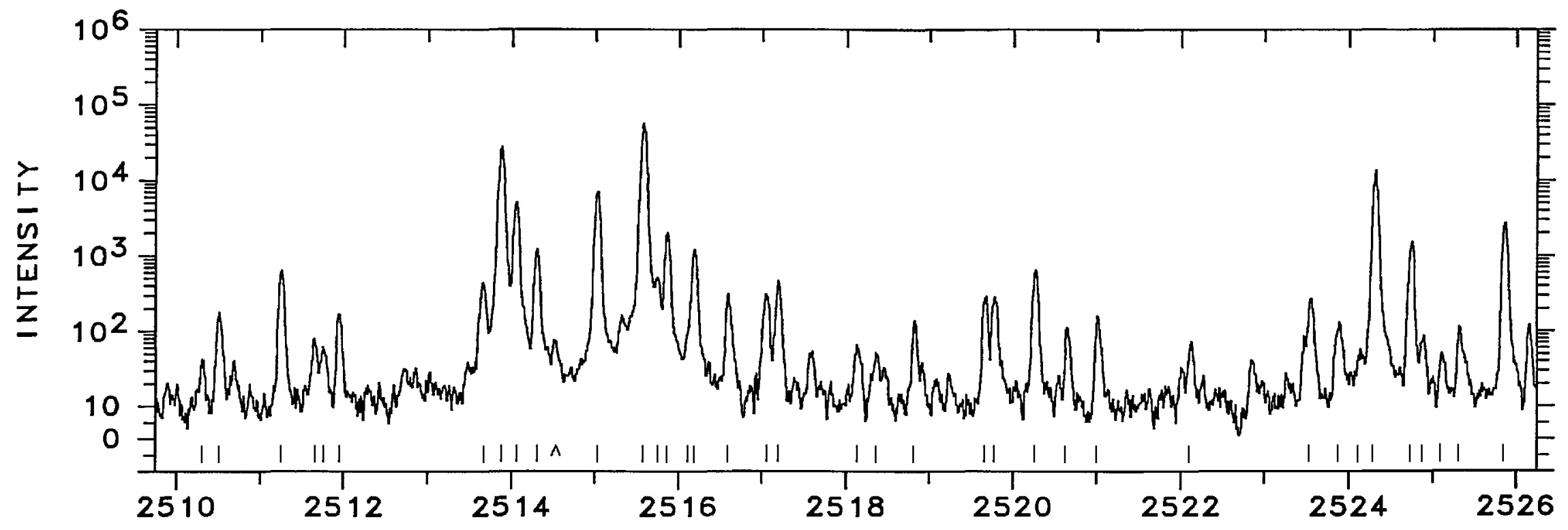

8

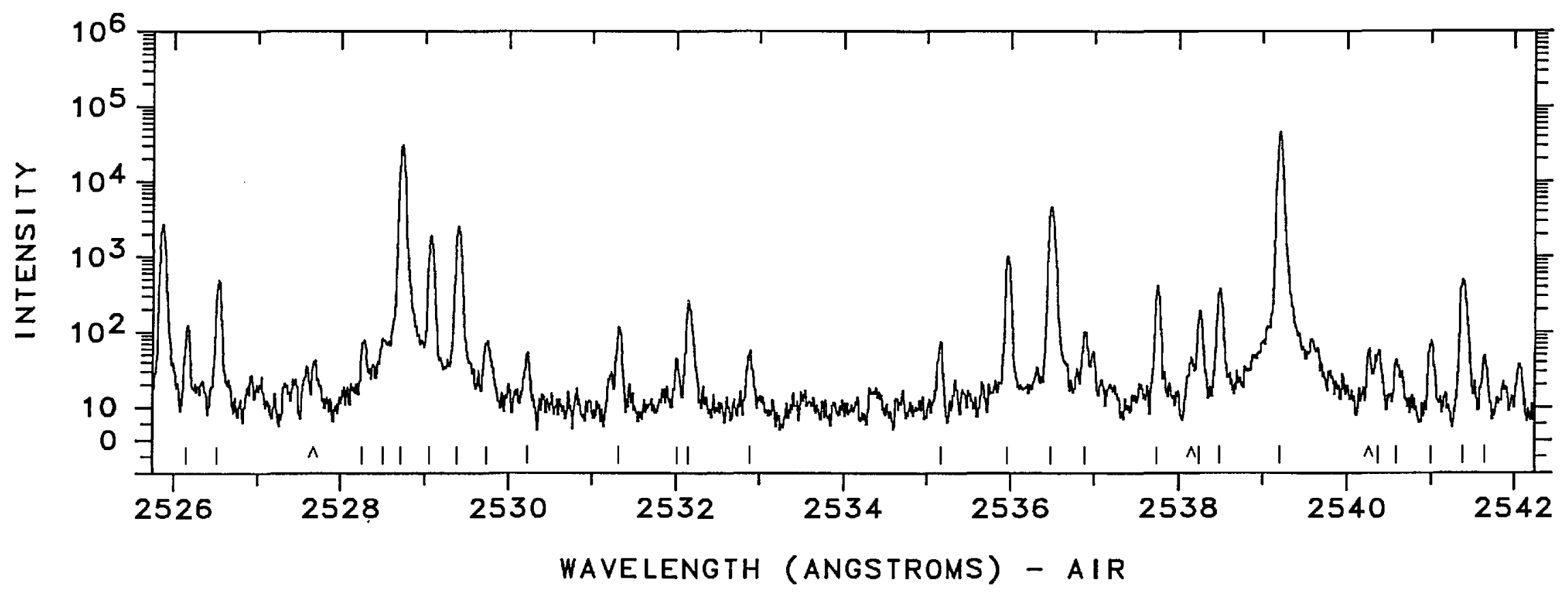




\section{WAVELENGTH WAVE NUMBER INTENSITY}

\section{CLASSIFICATION}

CODE

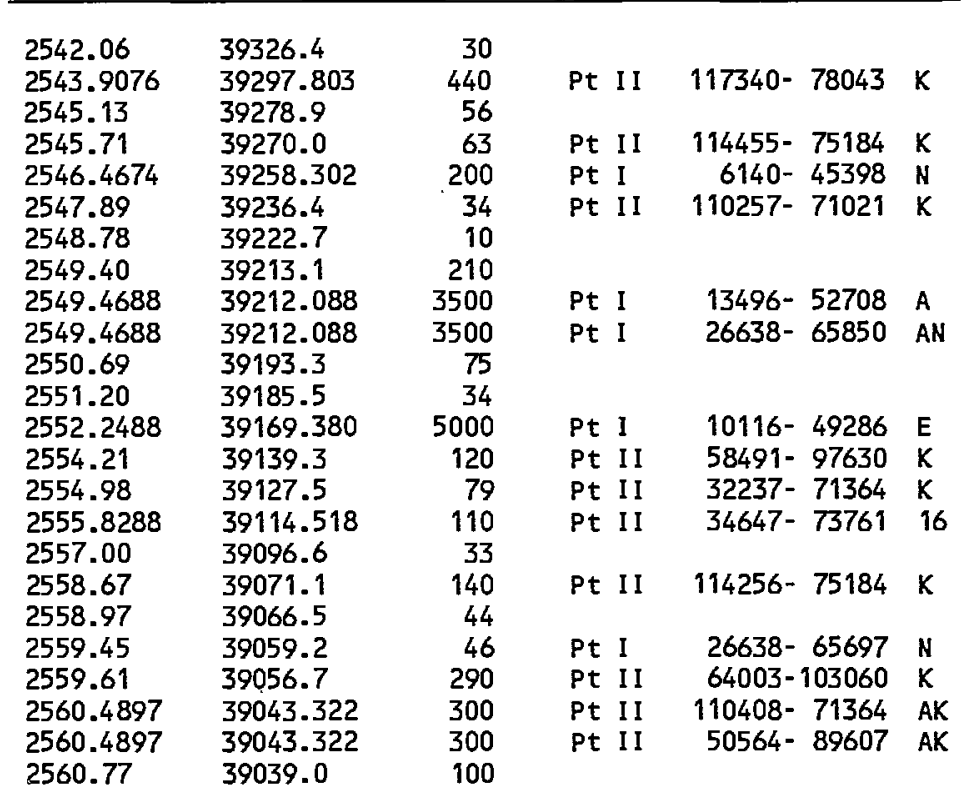

WAVELENGTH WAVE NUMBER INTENSITY

CLASSIFICATION

CODE

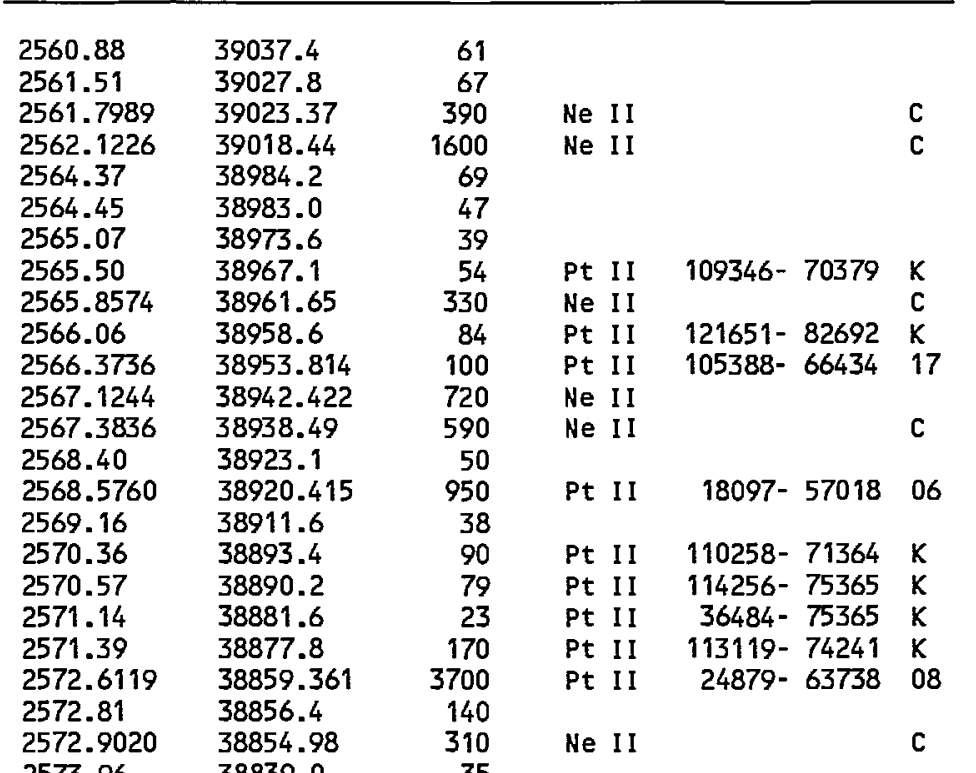




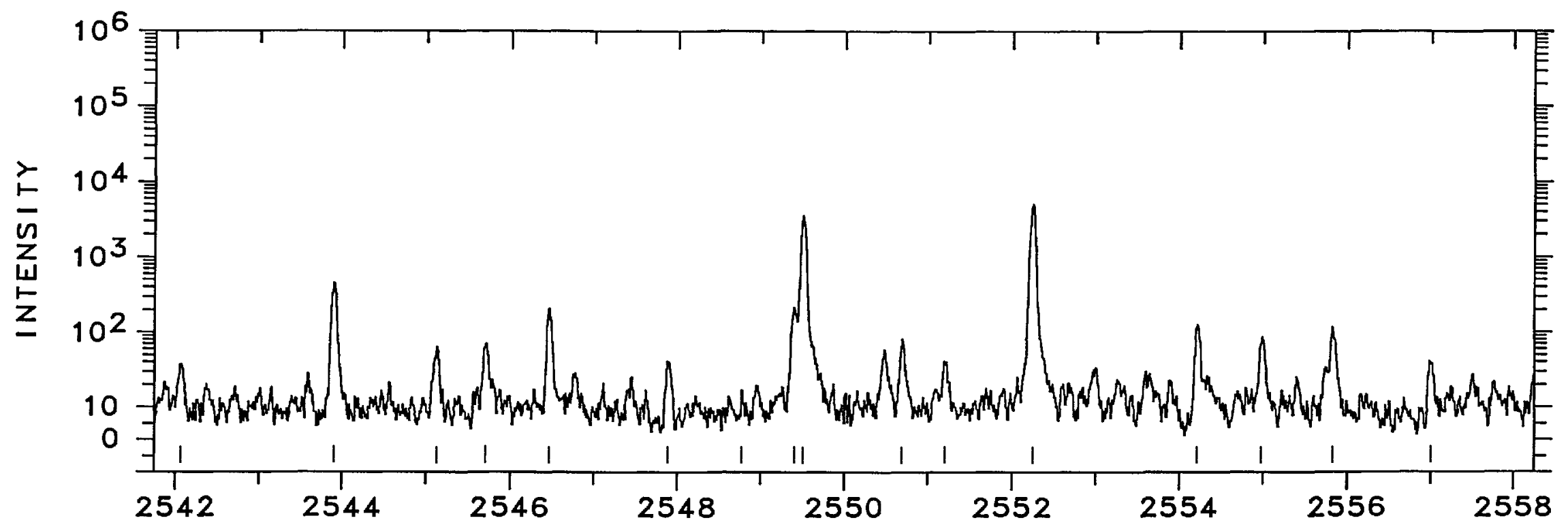

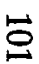

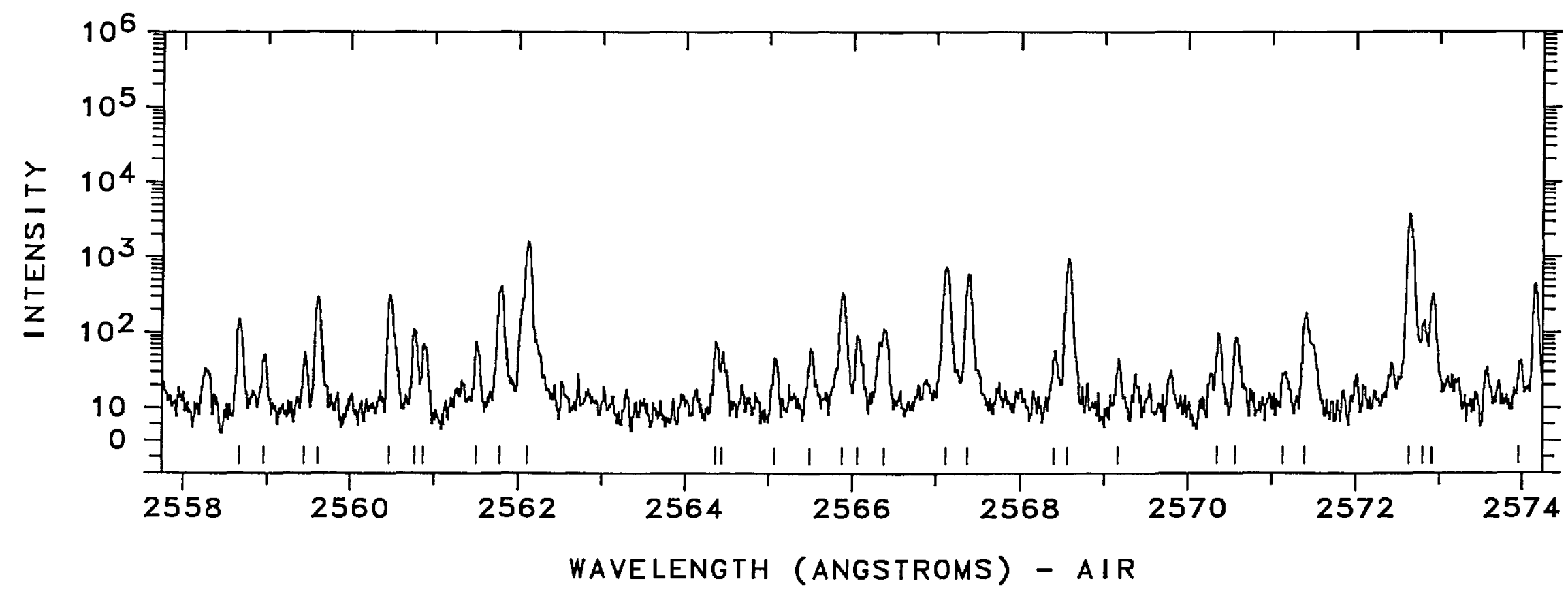




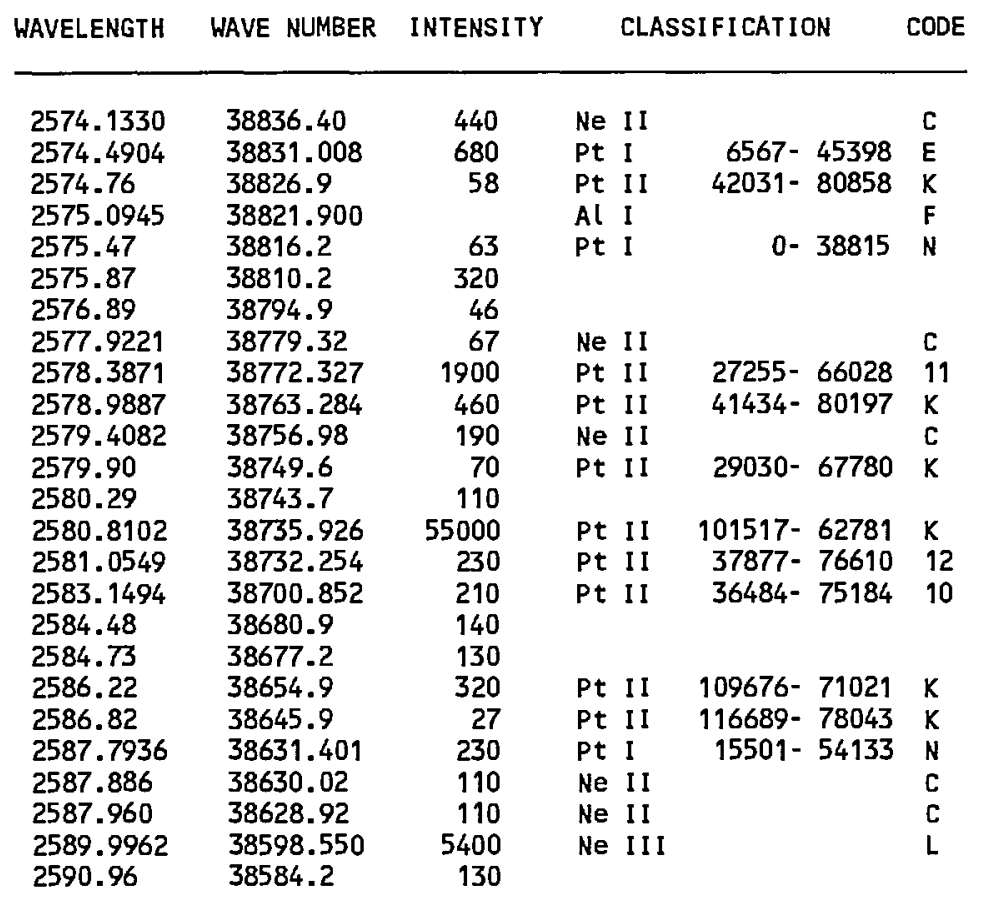

WAVELENGTH WAVE NUMBER INTENSITY

CLASSIFICATION

CODE

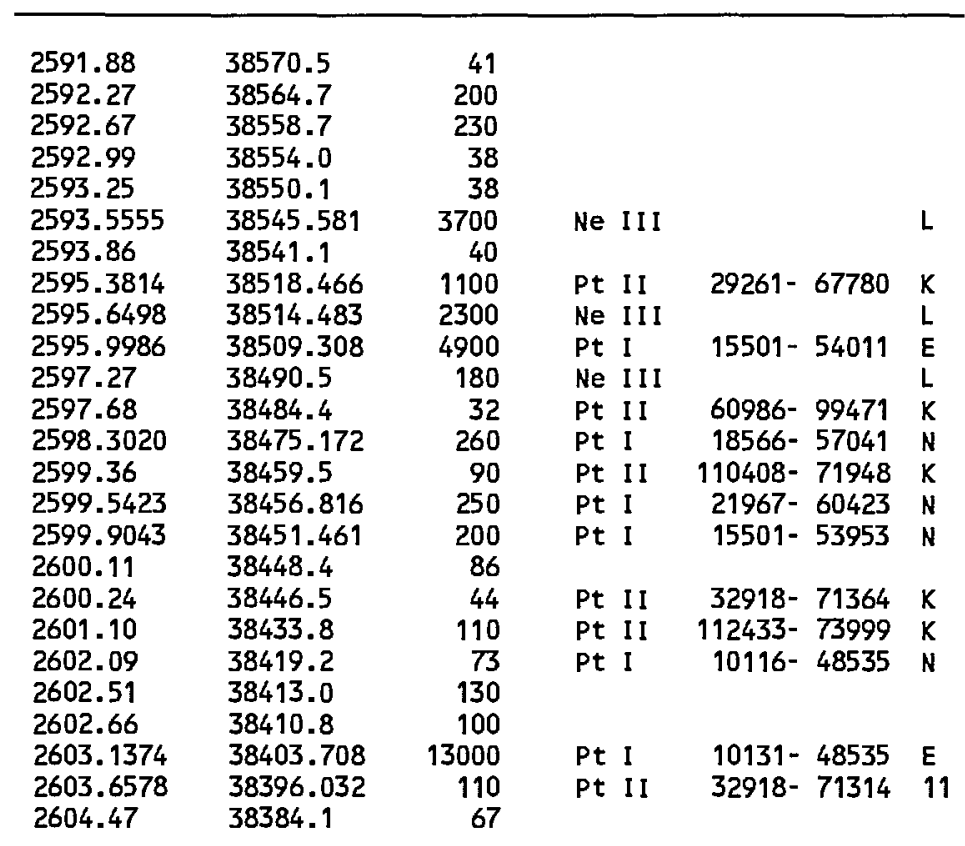




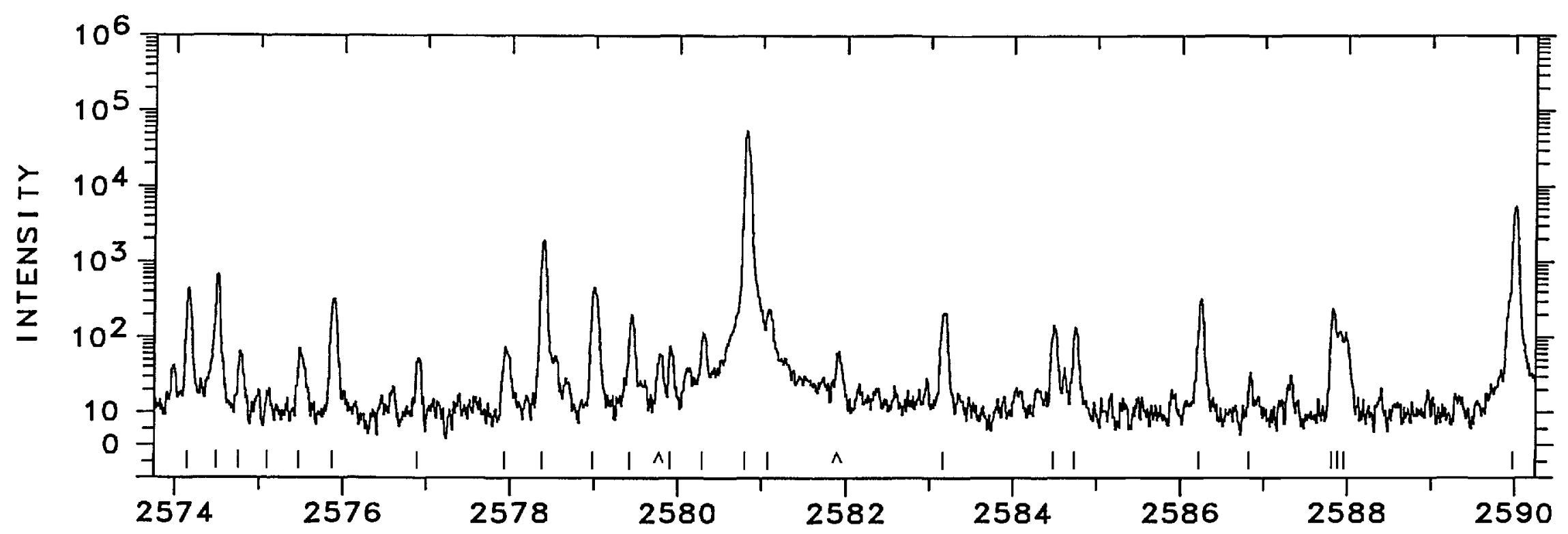

宫

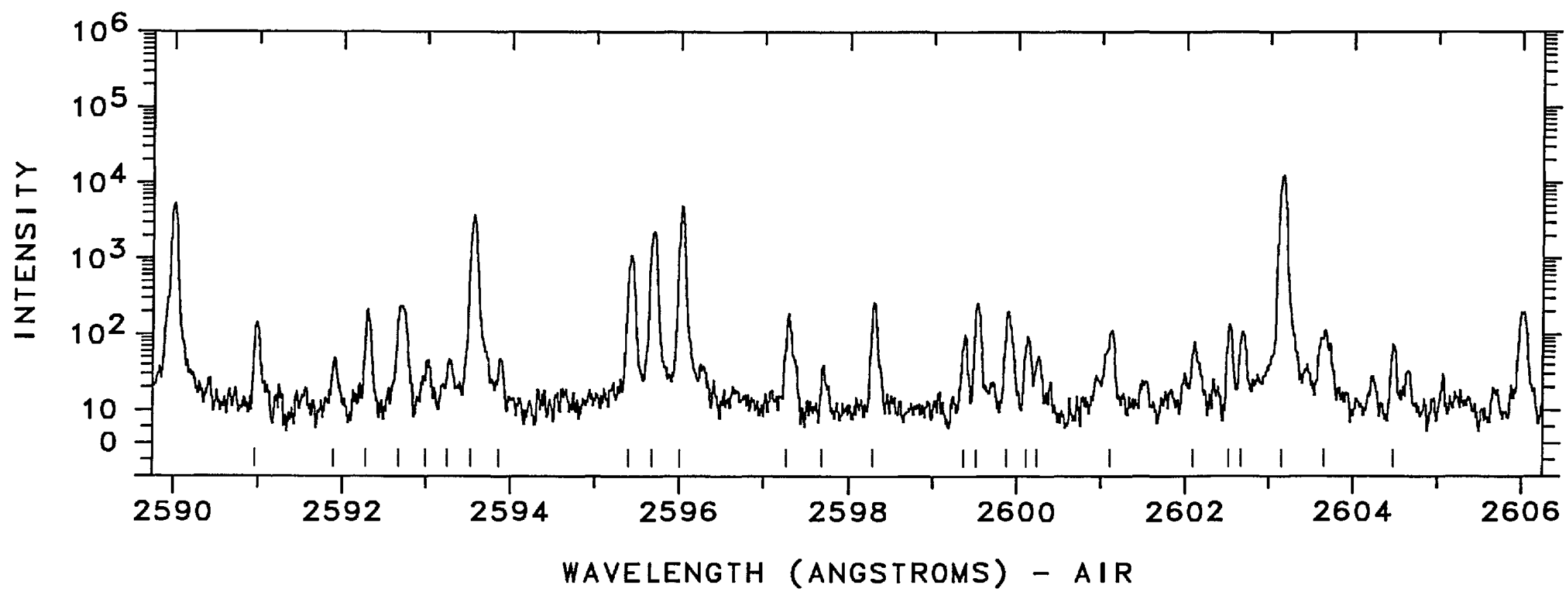




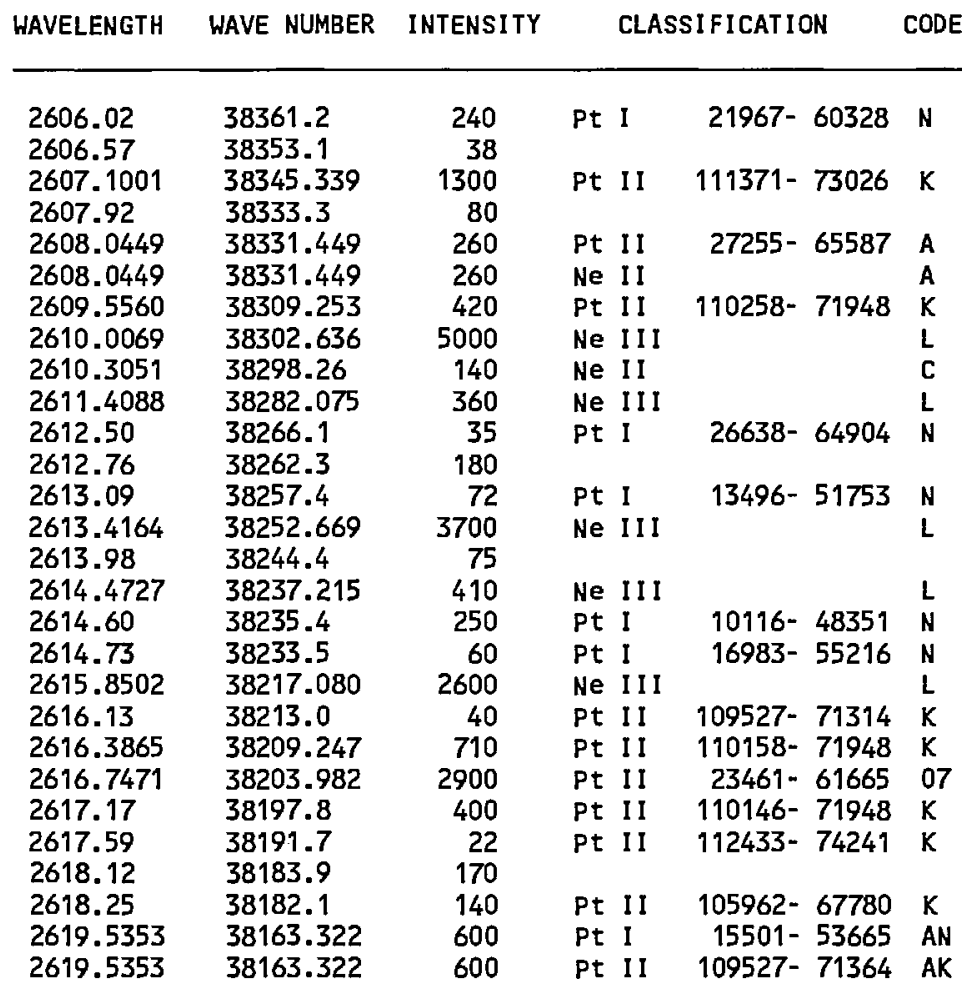

\begin{tabular}{llrlrl} 
HAVELENGTH & WAVE NUMBER & INTENSI TY & \multicolumn{2}{c}{ CLASSI FICATION } & CODE \\
& & & & & \\
2619.5674 & 38162.854 & 6700 & Pt I & $6567-44730$ & E \\
2619.72 & 38160.6 & 110 & Pt II & $43737-81897$ & K \\
2620.5386 & 38148.71 & 350 & Ne II & & C \\
2620.9986 & 38142.016 & 230 & Pt II & $32237-70379$ & 19 \\
2621.3906 & 38136.312 & 600 & Pt II & $111162-73026$ & K \\
2621.4754 & 38135.079 & 290 & Pt II & $36484-74619$ & 11 \\
2623.0193 & 38112.633 & 1100 & Pt II & $121651-83538$ & K \\
2623.1070 & 38111.36 & 1200 & Ne II & & C \\
2623.4567 & 38106.28 & 250 & Ne II & & C \\
2625.14 & 38081.8 & 130 & Pt II & $64003-102086$ & AK \\
2625.14 & 38081.8 & 130 & Pt II & $60986-99068$ & AK \\
2625.3264 & 38079.143 & 5100 & Pt II & $13329-51408$ & O6 \\
2625.9859 & 38069.58 & 290 & Ne II & & C \\
2626.68 & 38059.5 & 64 & & & \\
2627.3883 & 38049.262 & 1600 & Pt I & $13496-51545$ & E \\
2628.0269 & 38040.016 & 270000 & Pt I & $775-38815$ & E \\
2629.40 & 38020.2 & 110 & & & \\
2629.7211 & 38015.51 & 1000 & Ne II & & C \\
2629.8858 & 38013.13 & 2000 & Ne II & & C \\
2631.24 & 37993.6 & 68 & Pt II & $114455-76461$ & K \\
2631.9686 & 37983.05 & 260 & Ne II & & C \\
2632.11 & 37981.0 & 43 & Pt I & $26638-64619$ & N \\
2634.10 & 37952.3 & 69 & & & \\
2634.8852 & 37941.009 & 640 & Pt II & $24879-62820$ & 08 \\
2635.42 & 37933.3 & 69 & & & \\
2635.68 & 37929.6 & 26 & Ne III & & L \\
2636.0734 & 37923.907 & $2200 \mathrm{~S}$ & Ne II & & \\
2636.9466 & 37911.35 & 140 & Ne II & & C
\end{tabular}




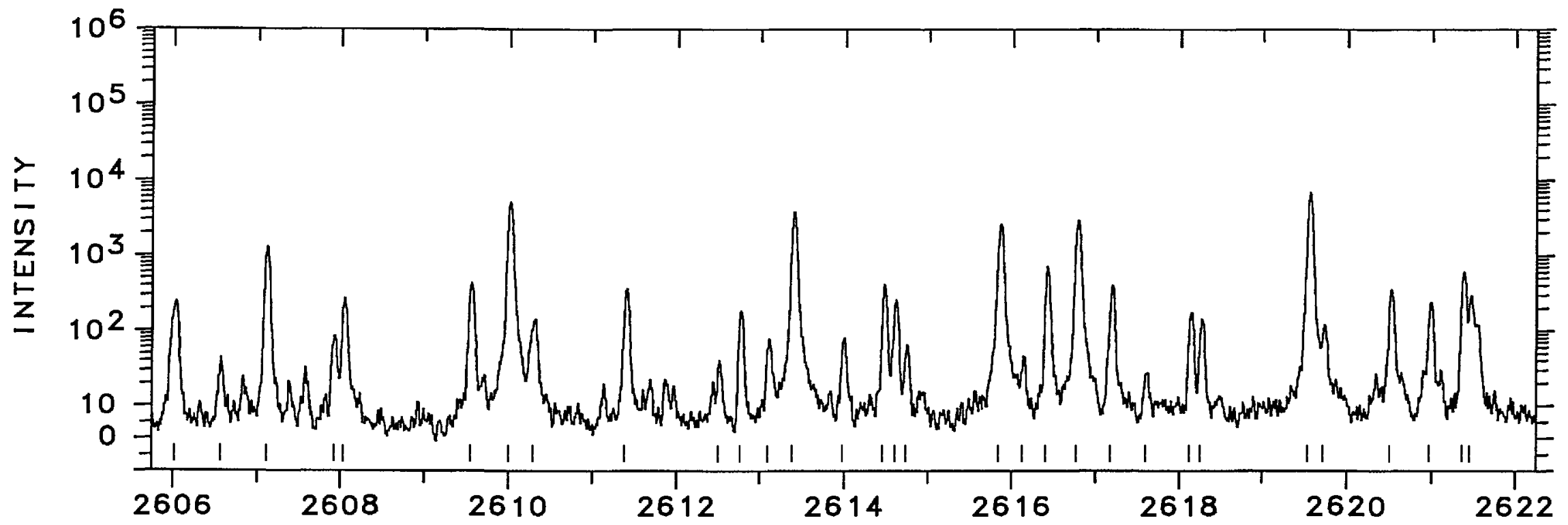

㤐

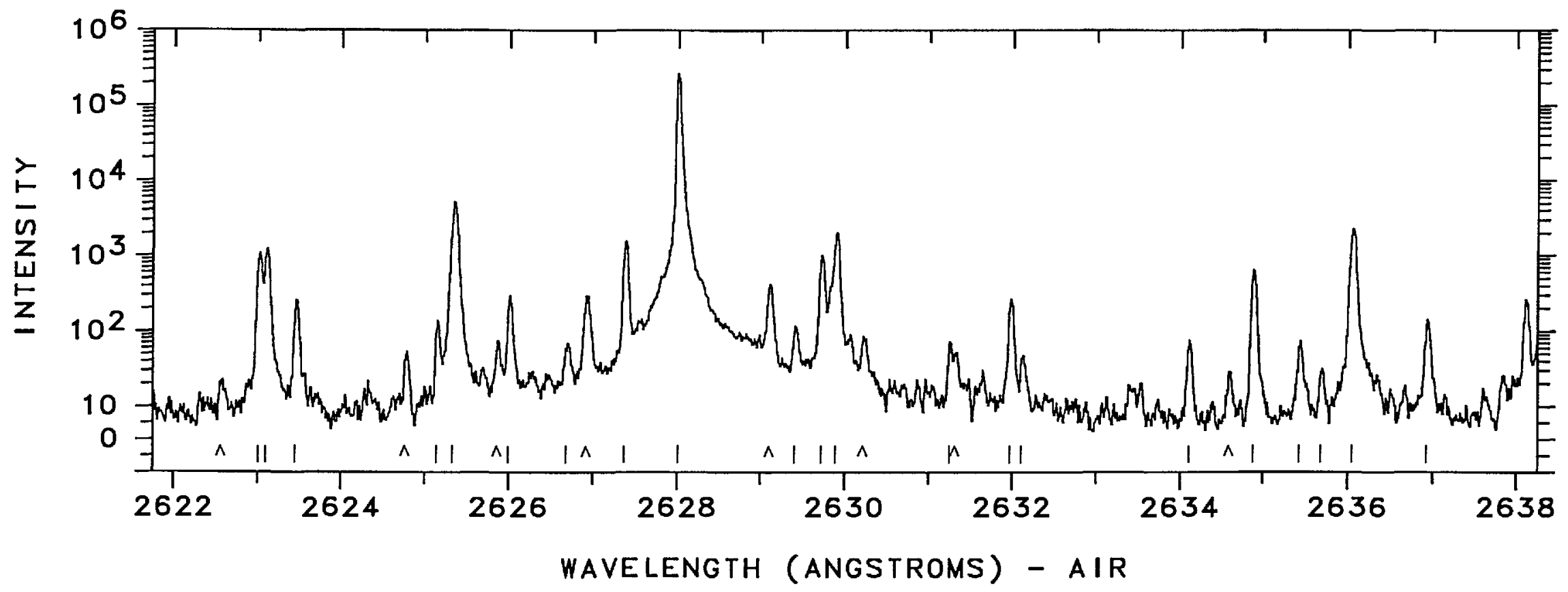




\section{WAVELENGTH WAVE NUMBER INTENSITY}

\section{CLASSIFICATION}

CODE

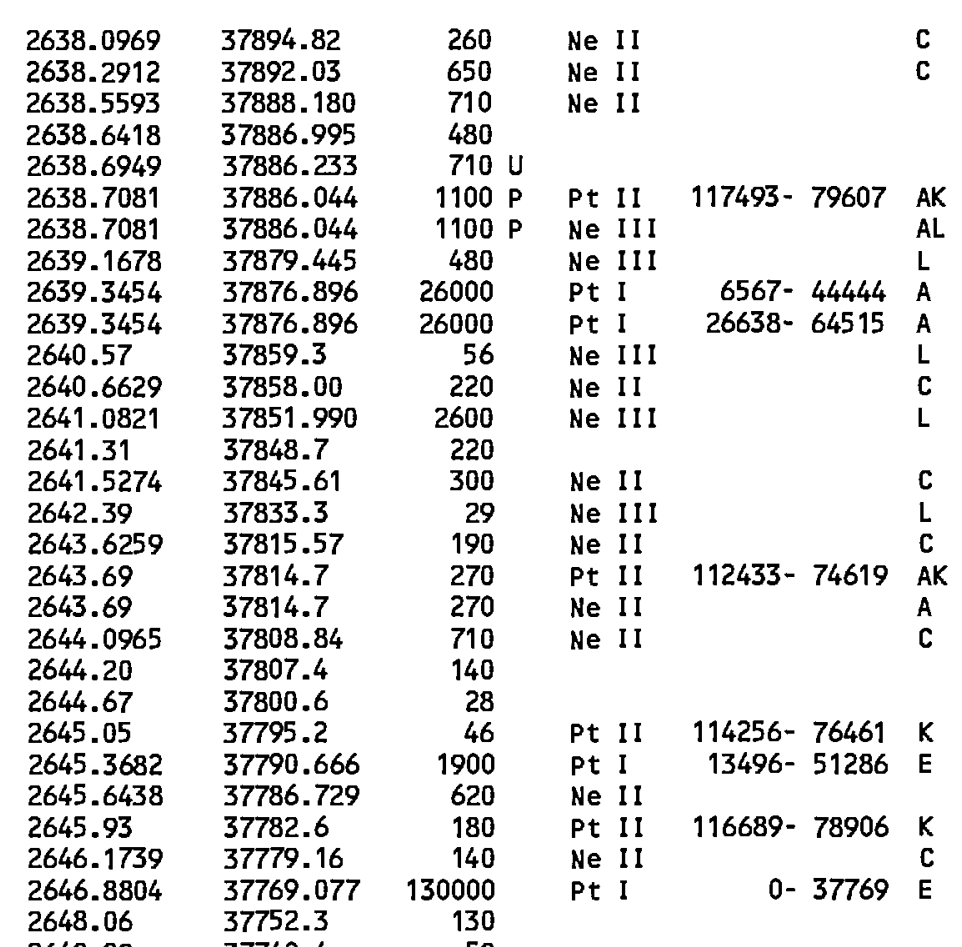

WAVELENGTH WAVE NUMBER INTENSITY

CLASSI FI CAT ION

CODE

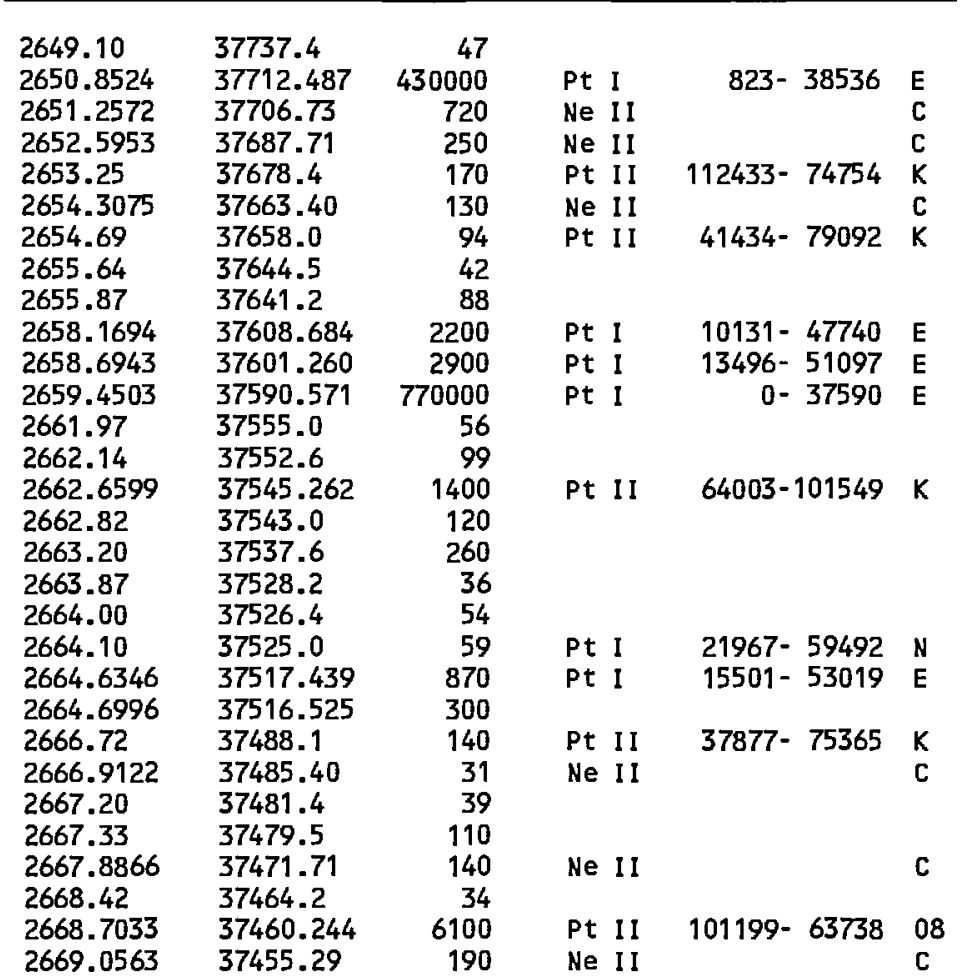




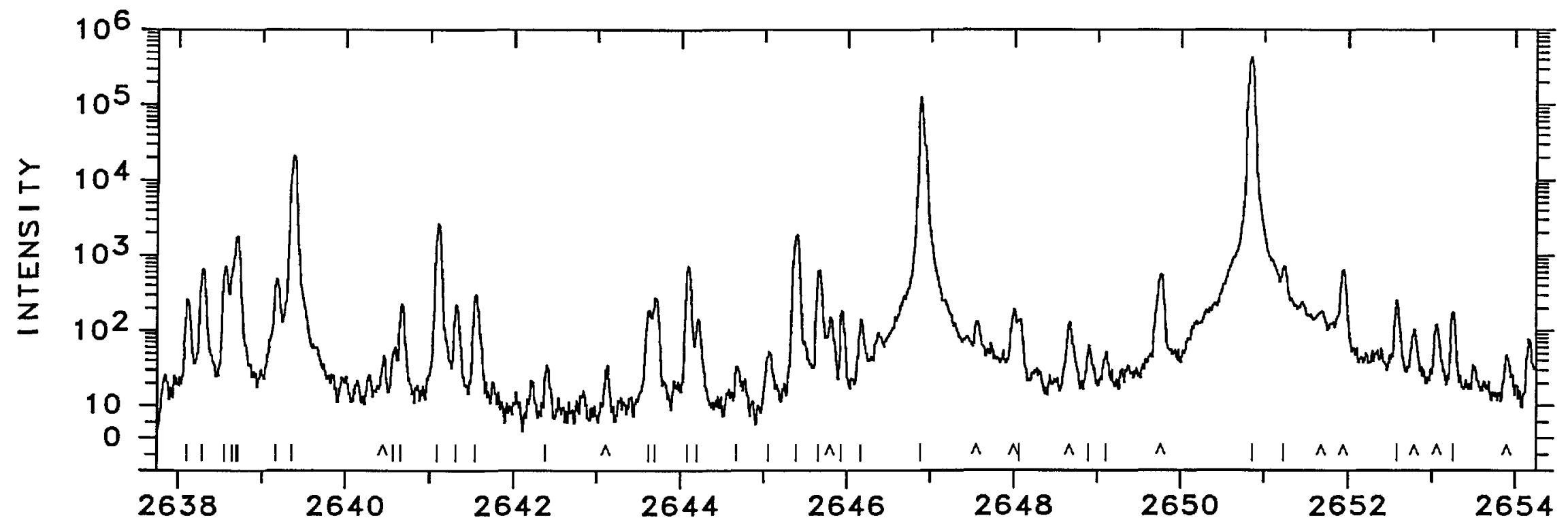

客

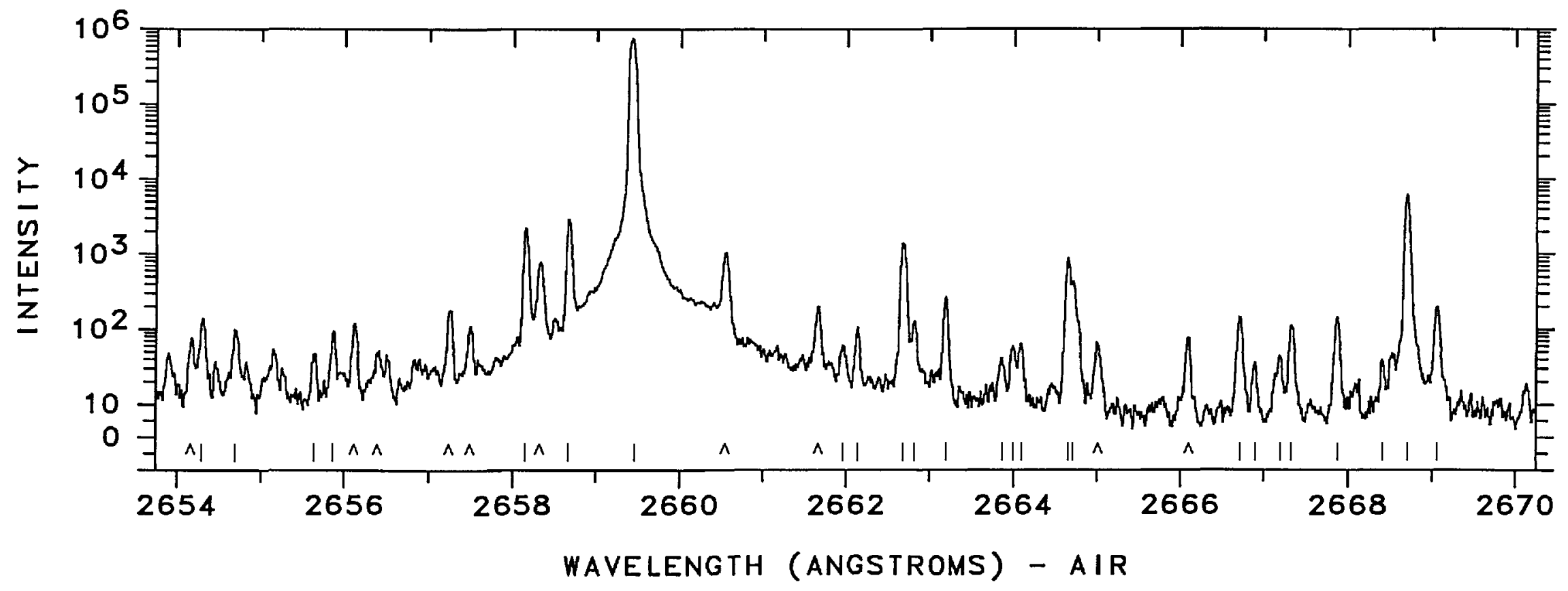




\section{WAVELENGTH WAVE NUMBER INTENSITY}

\section{CLASSIFICATION}

CODE

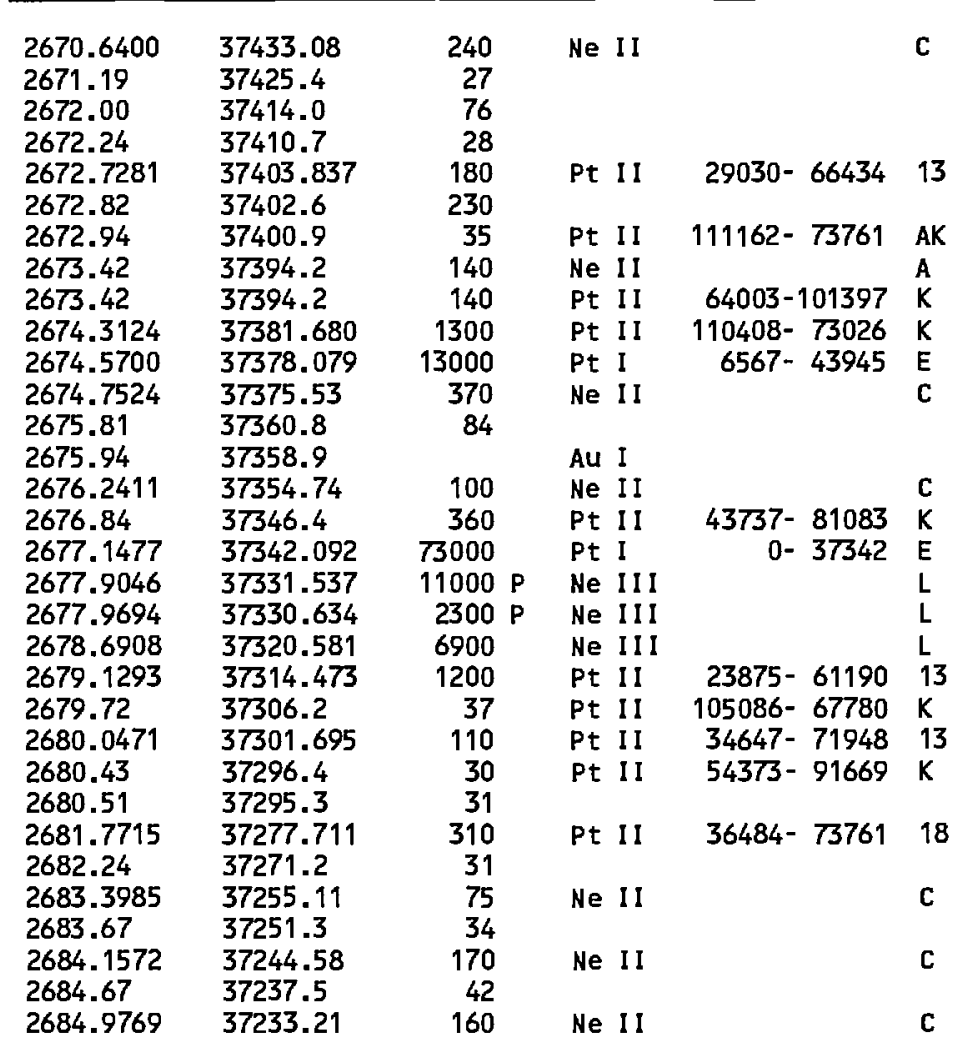

WAVELENGTH WAVE NUMBER INTENSITY CLASSIFICATION

CODE

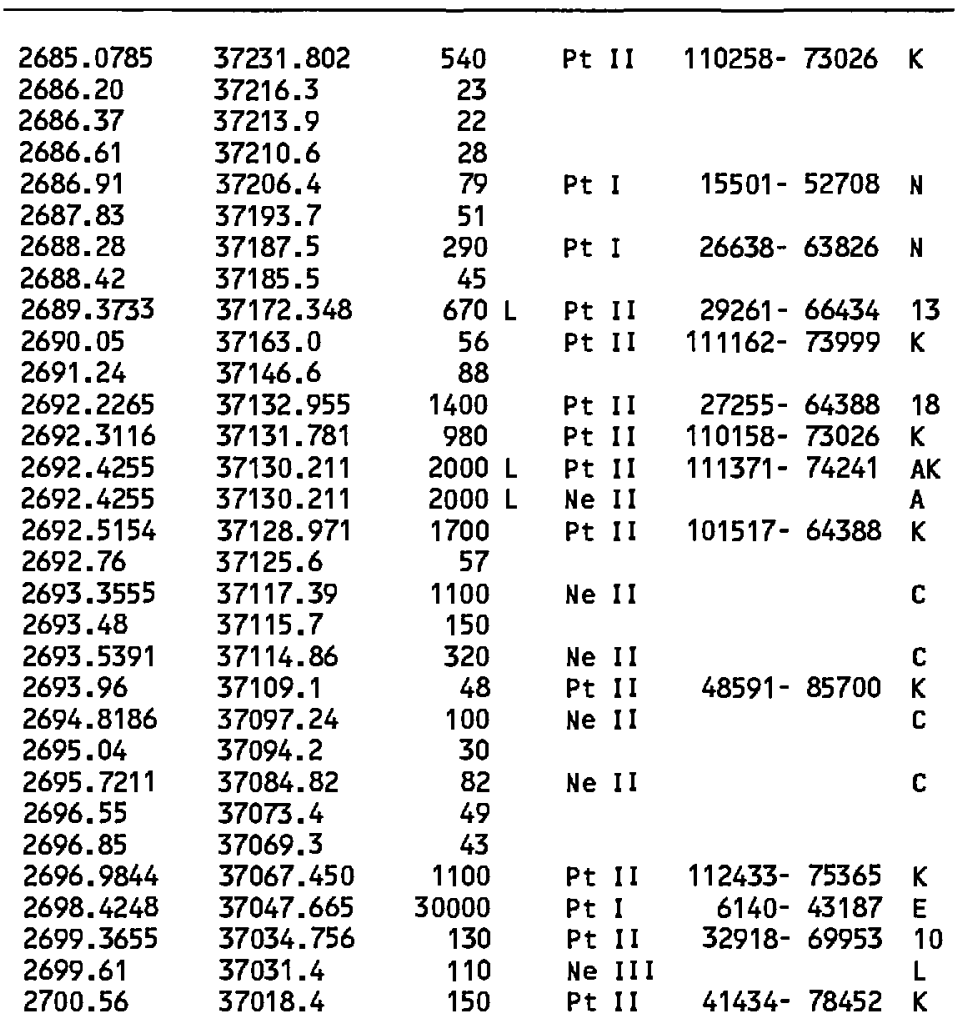




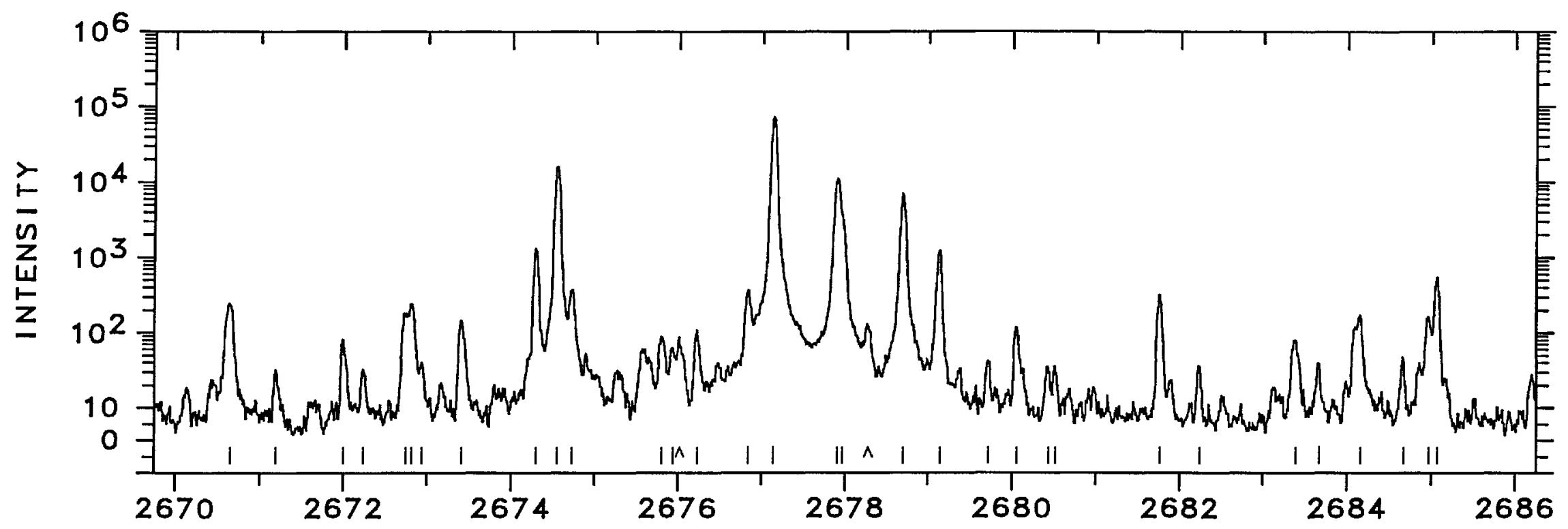

5

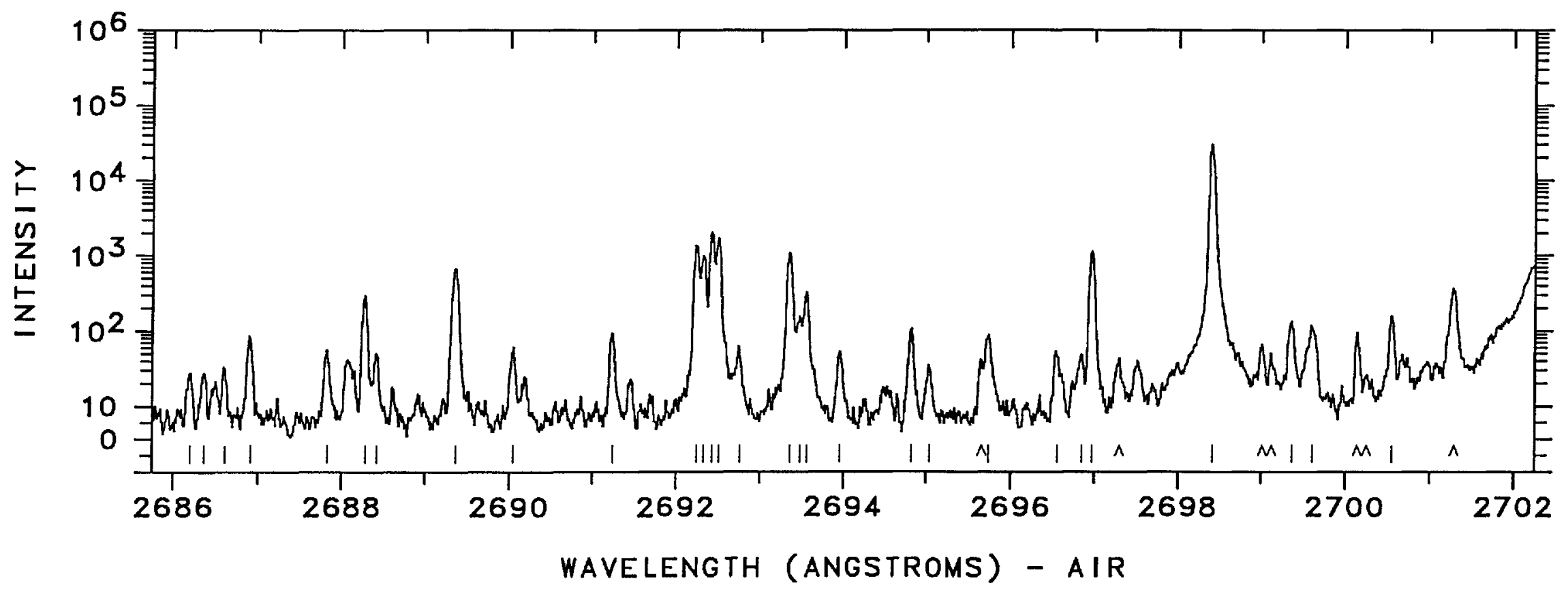




\begin{tabular}{|c|c|c|c|c|c|c|c|c|c|c|c|}
\hline WAVELENGTH & WAVE NUMBER & INTENSITY & CLA & IFICATION & CDDE & WAVELENGTH & WAVE NUMBER & I NTENSI TY & & I I I CAT ION & CODE \\
\hline $\begin{array}{l}2702.3995 \\
2705.8951 \\
2707.6694 \\
2708.21 \\
2708.68 \\
2709.40 \\
2710.9114 \\
2711.4534 \\
2711.7322 \\
2712.75 \\
2713.1254 \\
2713.8944 \\
2714.53 \\
2715.7683 \\
2715.8156 \\
2716.37 \\
2716.62 \\
2716.95\end{array}$ & $\begin{array}{l}36993.178 \\
36945.391 \\
36921.183 \\
36913.8 \\
36907.4 \\
36897.6 \\
36877.031 \\
36869.66 \\
36865.87 \\
36852.0 \\
36846.940 \\
36836.50 \\
36827.9 \\
36811.084 \\
36810.443 \\
36802.9 \\
36799.5 \\
36795.1\end{array}$ & $\begin{array}{c}330000 \\
240000 \\
550 \\
76 \\
260 \\
27 \\
420 \mathrm{~W} \\
140 \\
48 \\
240 \\
7900 \\
110 \\
130 \\
800 \mathrm{P} \\
4500 \\
56 \\
150 \\
64\end{array}$ & 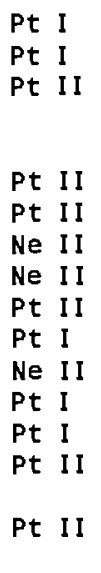 & $\begin{array}{r}54373-91271 \\
37877-74754 \\
\\
112433-75581 \\
10116-46963 \\
\\
26638-63466 \\
66967-30156 \\
101199-64388 \\
60986-97786\end{array}$ & $\begin{array}{l}K \\
23 \\
C \\
C \\
K \\
N \\
C \\
N \\
N \\
16\end{array}$ & $\begin{array}{l}2717.6199 \\
2718.4363 \\
2719.0333 \\
2719.5239 \\
2720.9024 \\
2721.29 \\
2722.1611 \\
2722.44 \\
2724.27 \\
2724.79 \\
2725.37 \\
2725.55 \\
2726.4128 \\
2727.8956 \\
2728.35 \\
2729.9123 \\
2733.6855 \\
2733.9567\end{array}$ & $\begin{array}{l}36786.005 \\
36774.958 \\
36766.883 \\
36760.251 \\
36741.628 \\
36736.4 \\
36724.64 \\
36720.9 \\
36696.2 \\
36689.2 \\
36681.4 \\
36679.0 \\
36667.373 \\
36647.444 \\
36641.3 \\
36620.372 \\
36569.829 \\
36566.201\end{array}$ & $\begin{array}{r}2100 \\
190000 \\
120000 \\
\\
92 \\
860 \\
41 \\
24 \\
150 \\
340 \\
26 \\
990 \\
740 \\
150 \\
19000 \\
4100 \\
420000\end{array}$ & 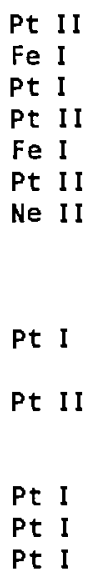 & $\begin{array}{r}24879-61665 \\
823-37590 \\
101517-64757 \\
114256-77519\end{array}$ & $\begin{array}{l}06 \\
M R \\
E \\
K \\
R \\
K \\
C\end{array}$ \\
\hline
\end{tabular}



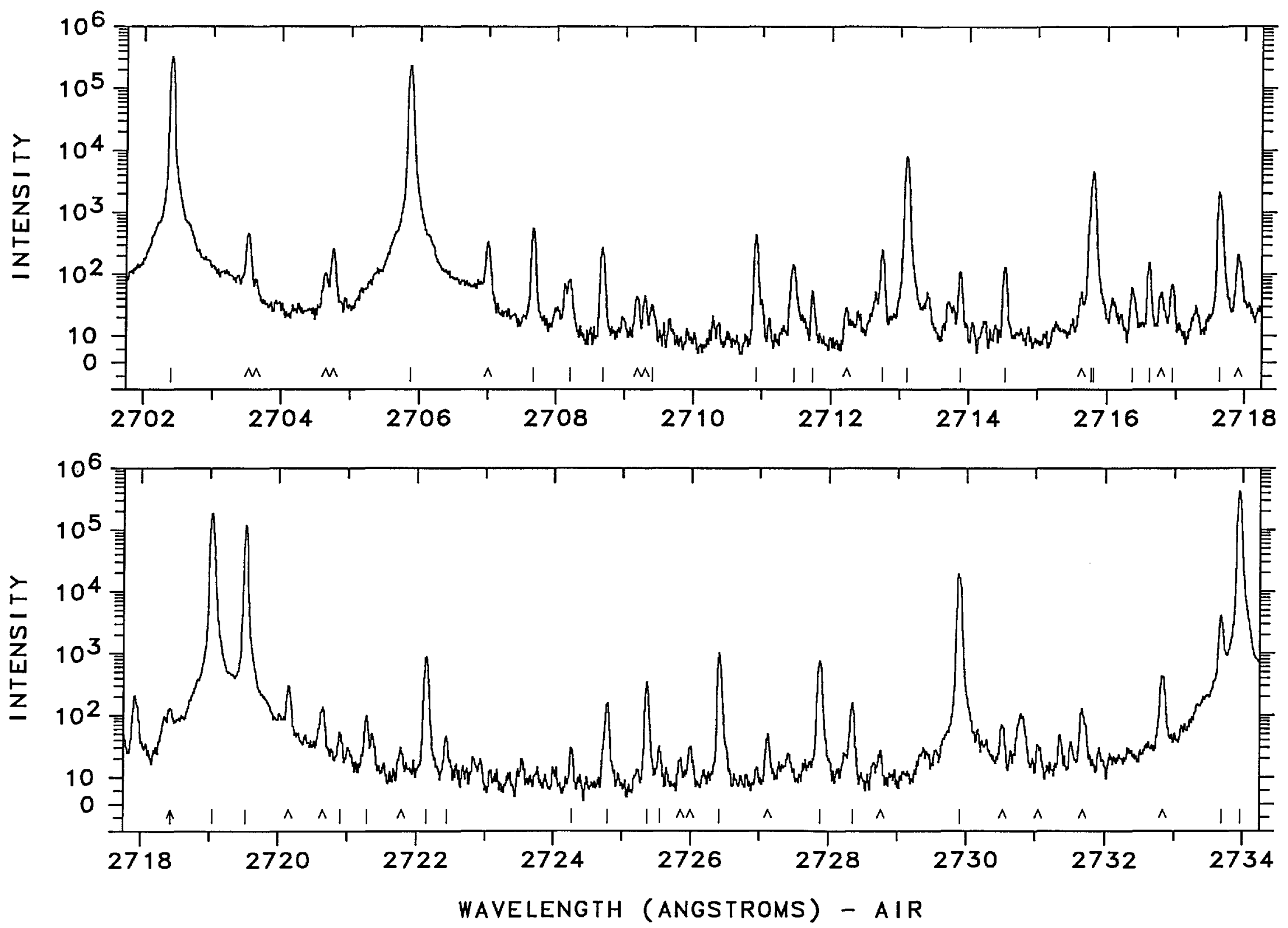


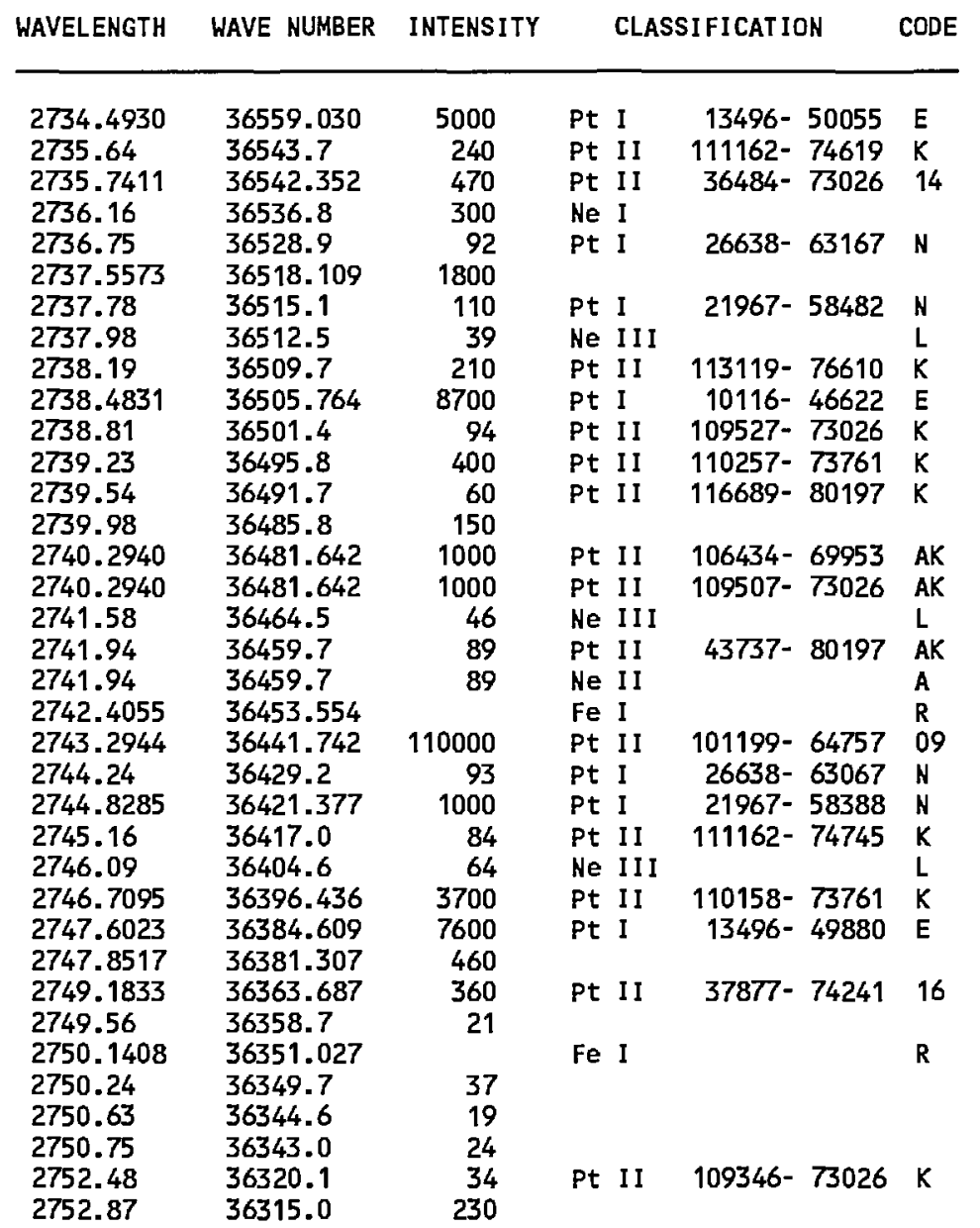

WAVELENGTH WAVE NUMBER INTENSITY

CLASSIFICATION

CODE

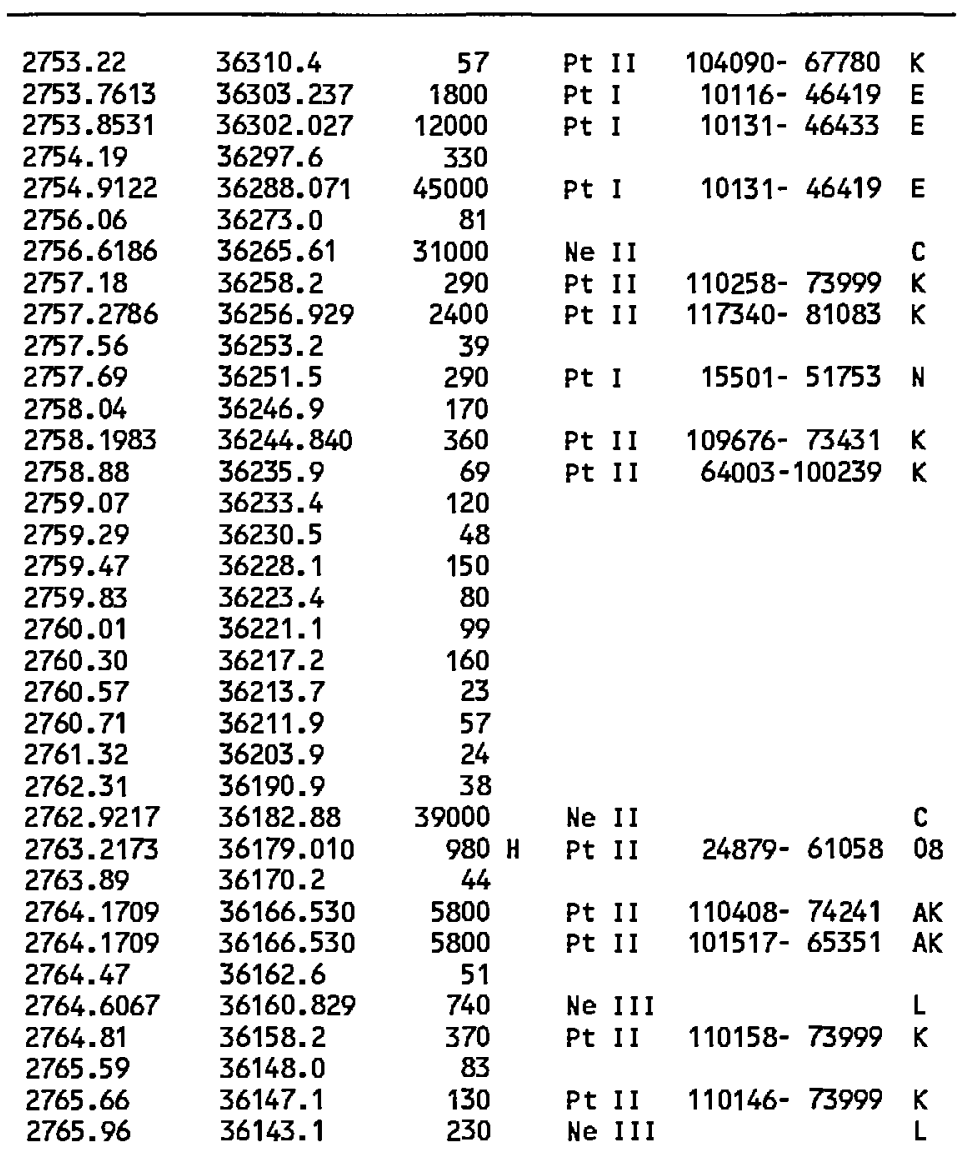




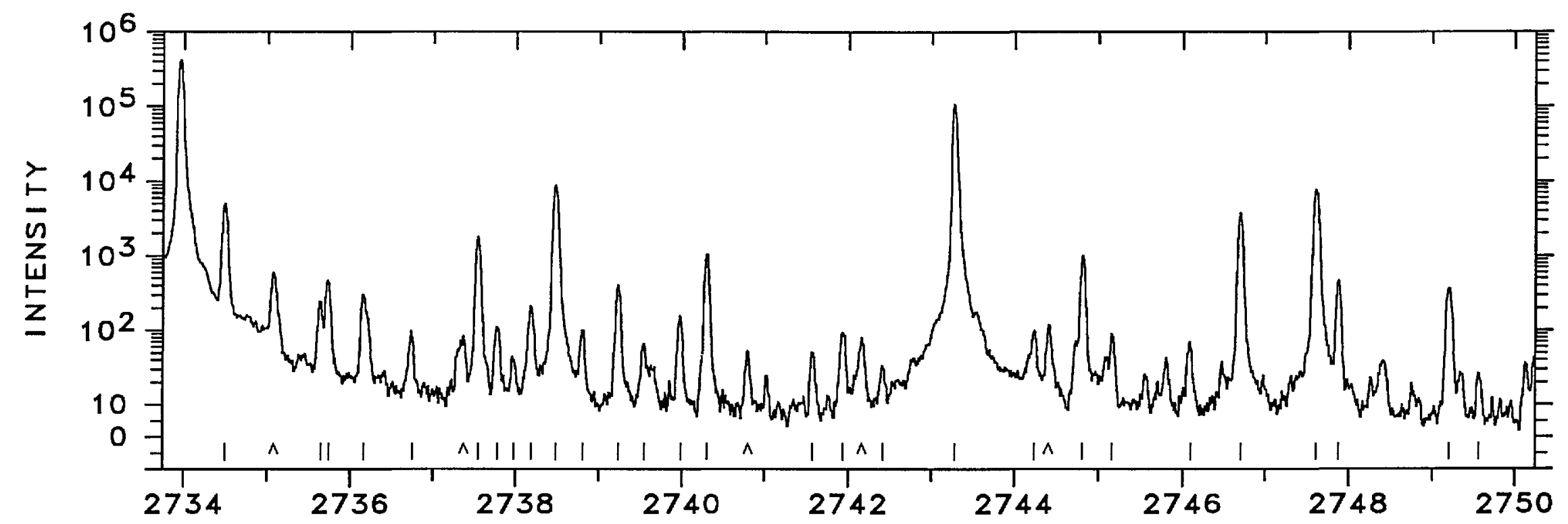

$\vec{\omega}$

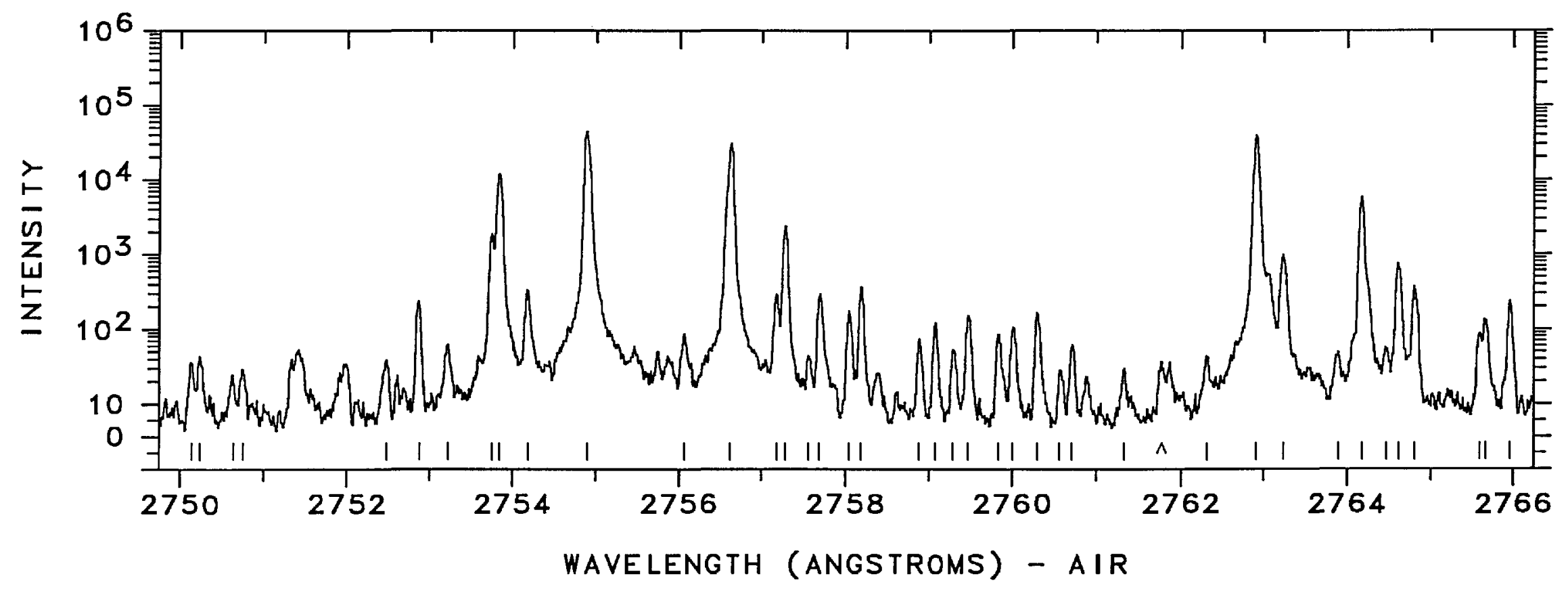




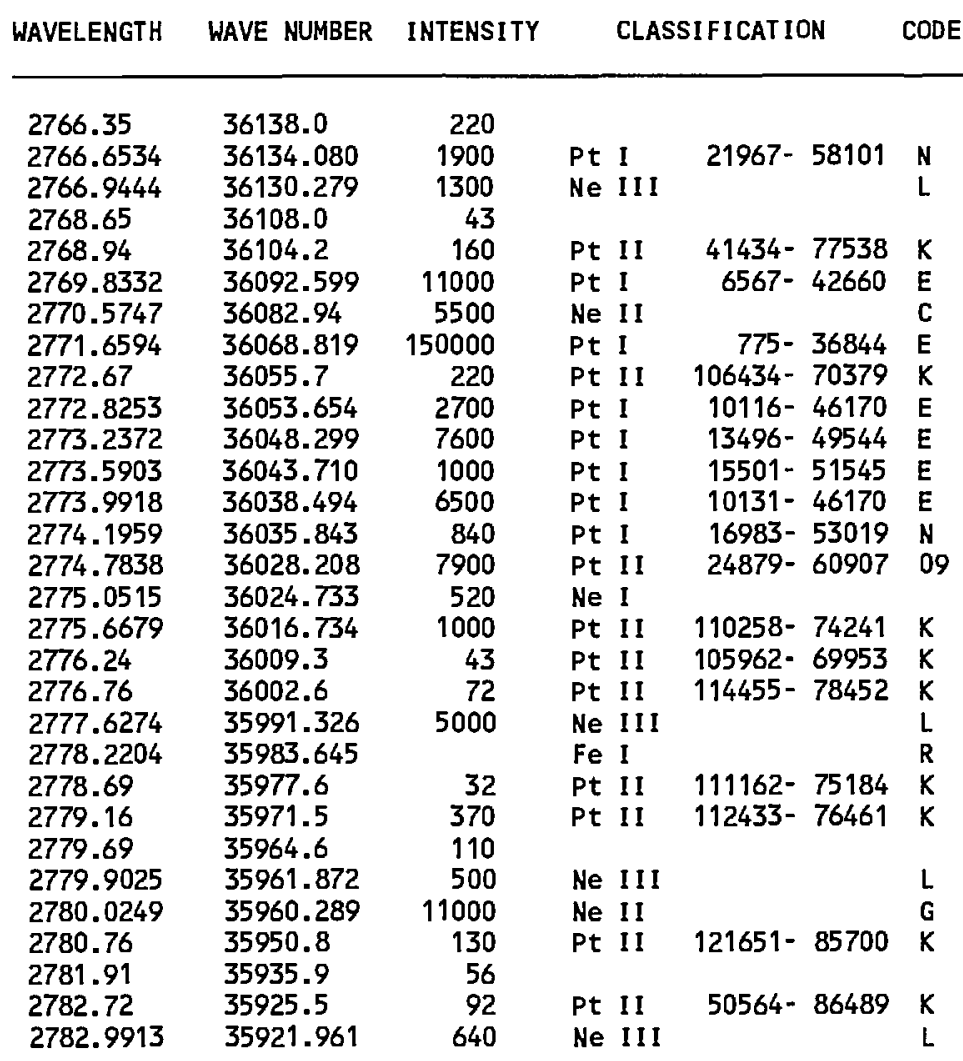

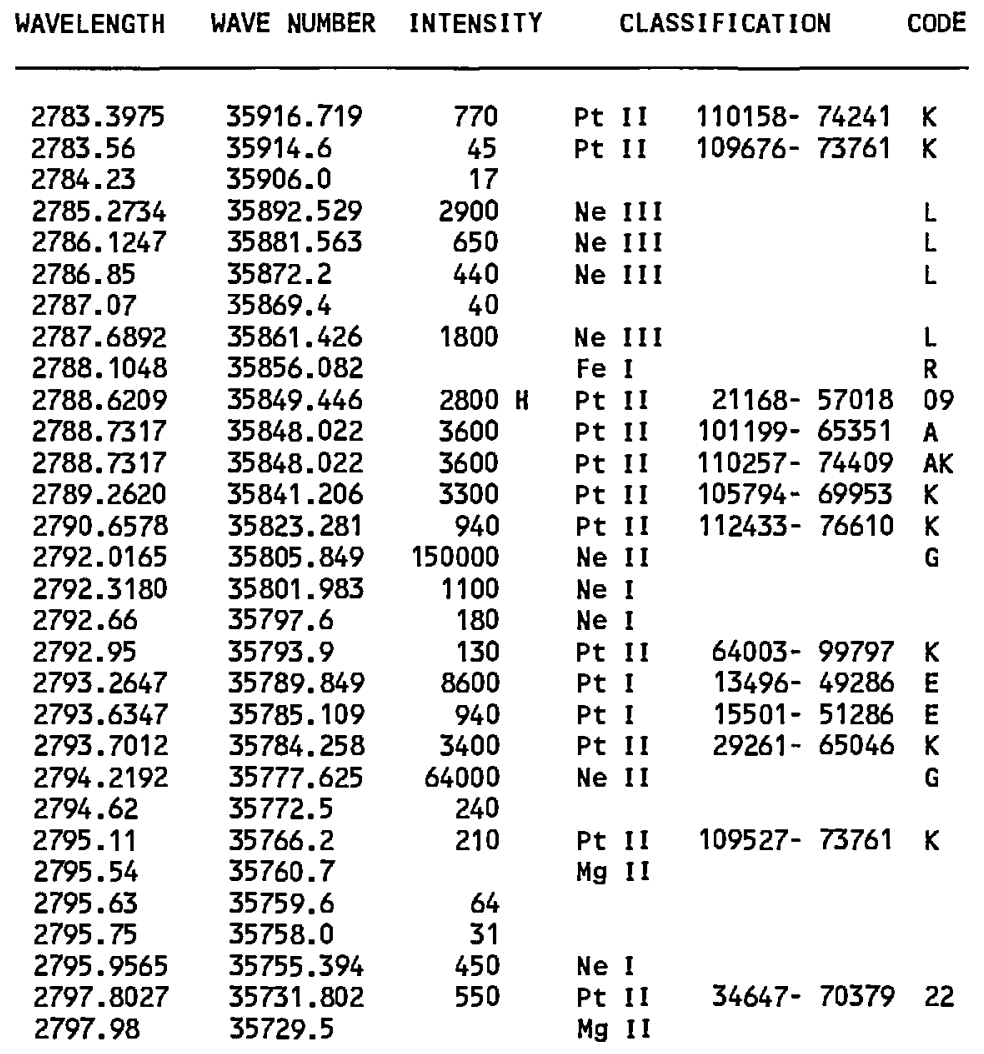




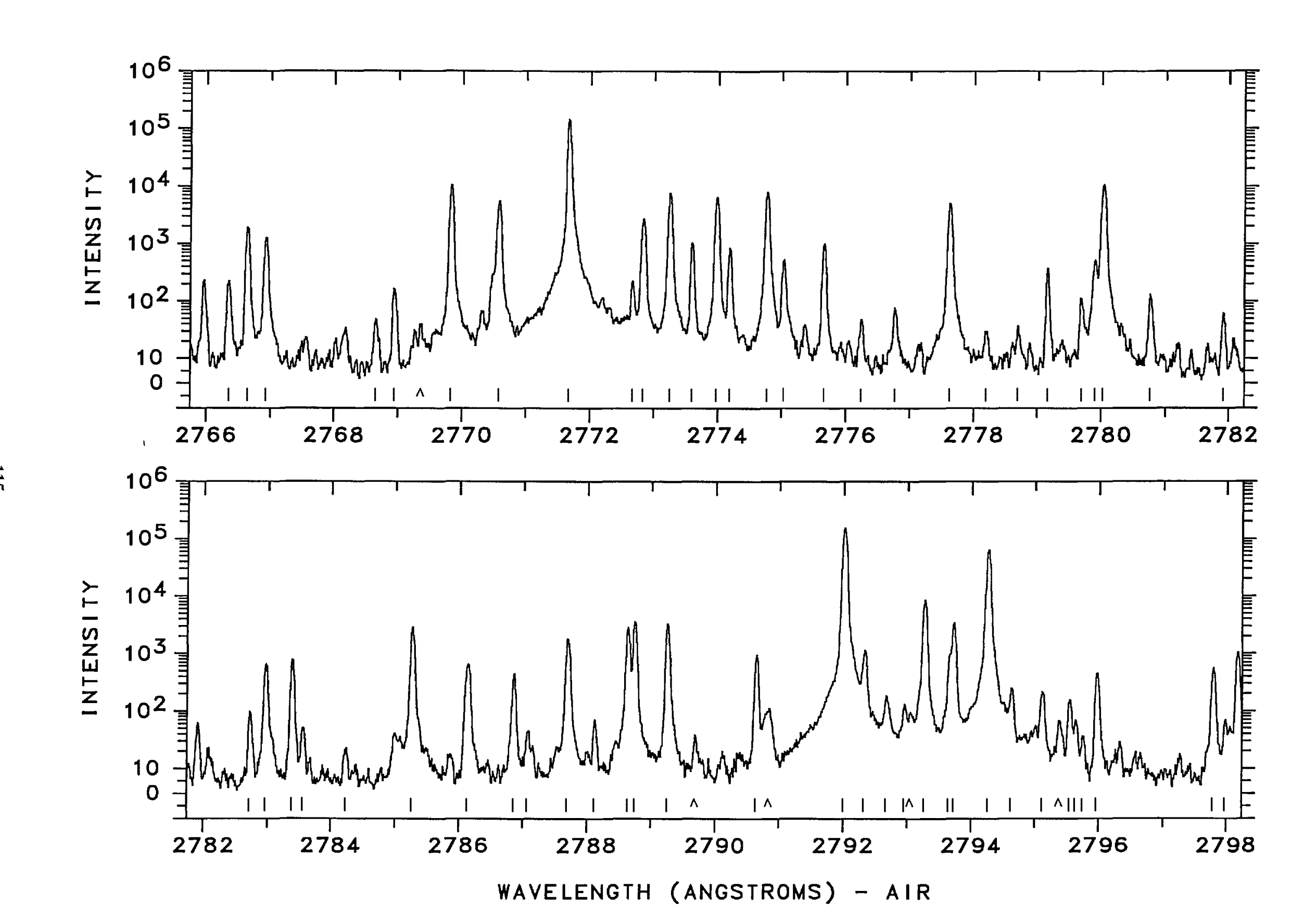




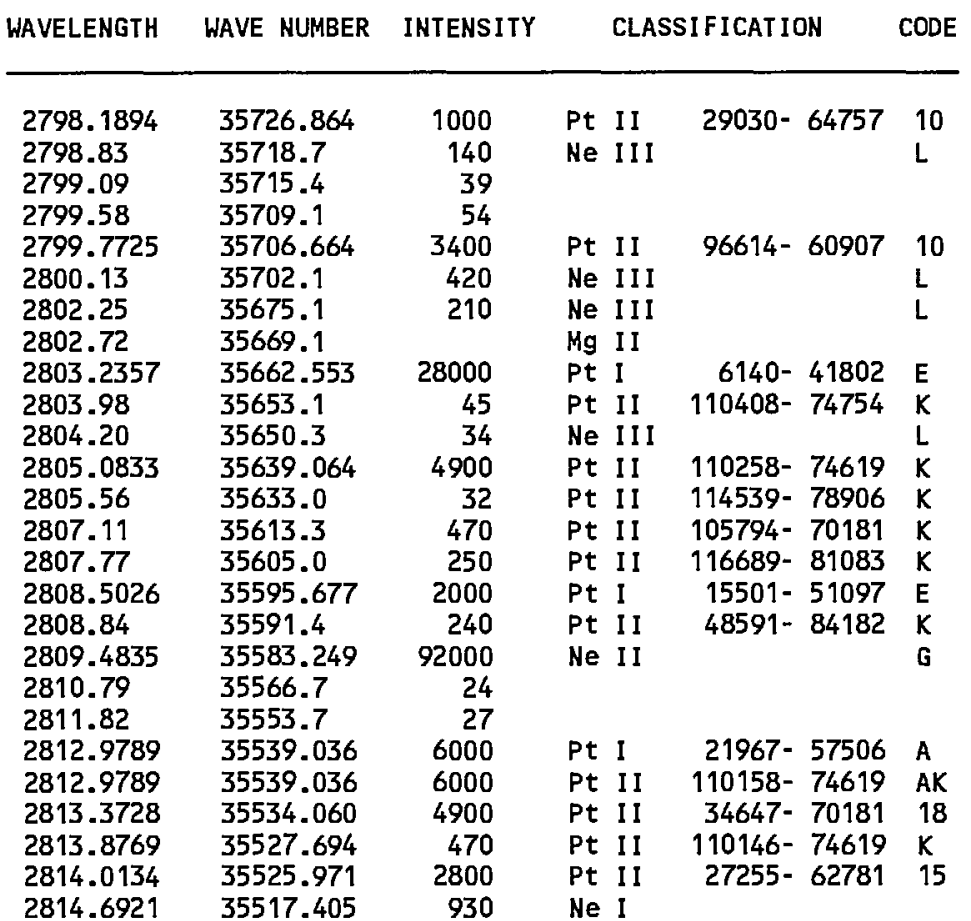

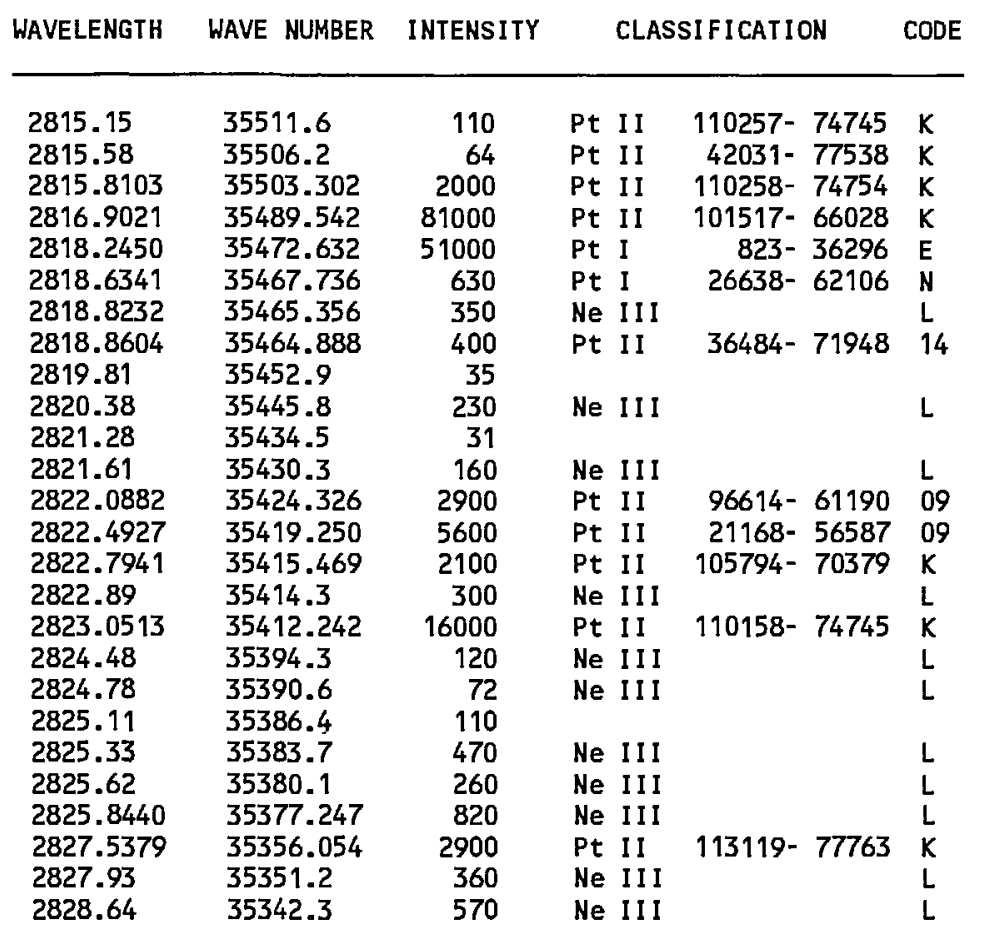




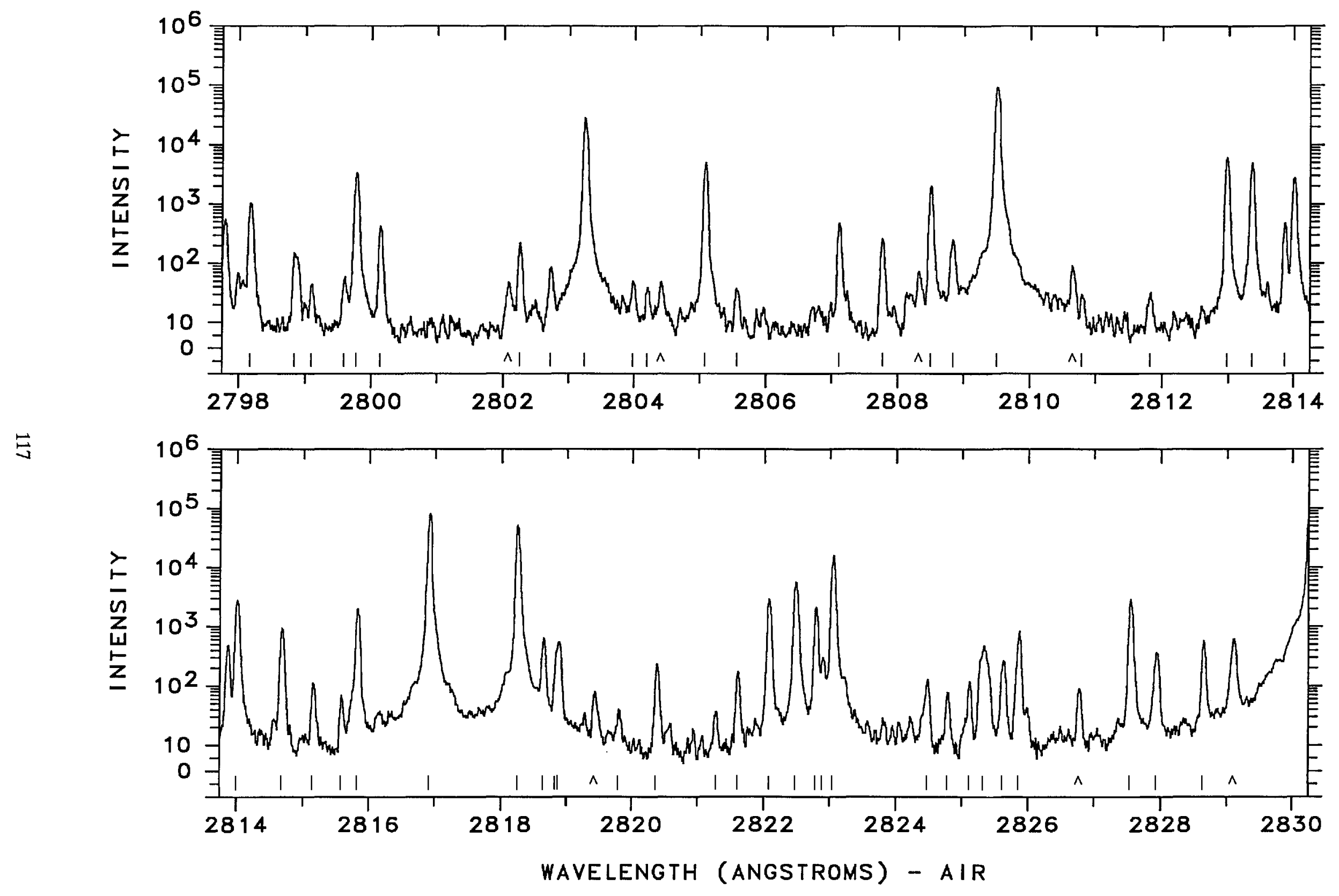




\begin{tabular}{|c|c|c|c|c|c|c|c|c|c|c|c|}
\hline WAVELENGTH & WAVE NUMBER & INTENSITY & CLAS & SIFICATION & CODE & WAVELENGTH & WAVE NUMBER & INTENSITY & CLAS & SIFICATION & CODE \\
\hline $\begin{array}{l}2830.2919 \\
2832.95 \\
2833.10 \\
2833.37 \\
2834.7107 \\
2835.2370 \\
2836.18 \\
2836.65 \\
2836.80 \\
2837.2284 \\
2837.37 \\
2837.86 \\
2838.57 \\
2839.16 \\
2839.5216 \\
2840.76 \\
2842.07 \\
2842.4101 \\
2843.27 \\
2844.05 \\
2844.2284 \\
2845.17 \\
2845.4468 \\
2846.34 \\
2846.52\end{array}$ & $\begin{array}{l}35321.653 \\
35288.5 \\
35286.6 \\
35283.3 \\
35266.596 \\
35260.049 \\
35248.3 \\
35242.5 \\
35240.6 \\
35235.302 \\
35233.5 \\
35227.5 \\
35218.6 \\
35211.3 \\
35206.848 \\
35191.5 \\
35175.3 \\
35171.071 \\
35160.4 \\
35150.8 \\
35148.588 \\
35137.0 \\
35133.538 \\
35122.5 \\
35120.3\end{array}$ & $\begin{array}{r}520000 \mathrm{C} \\
190 \\
180 \\
490 \\
26000 \\
800 \\
41 \\
68 \\
41 \\
610 \\
180 \\
62 \\
29 \\
35 \\
55 \\
540 \\
200 \\
12000 \\
67 \\
140 \\
120 \\
37 \\
1300 \\
34 \\
33\end{array}$ & $\begin{array}{l}\text { Pt I } \\
\text { Pt II } \\
\text { Pt I } \\
\text { Pt I } \\
\text { Ne I } \\
\text { Ne III } \\
\text { Pt I } \\
\text { Ne III }\end{array}$ & $\begin{array}{r}0-35321 \\
109527-74241 \\
13496-48779 \\
10131-45398\end{array}$ & $\begin{array}{l}23 \\
L \\
09 \\
N \\
18\end{array}$ & $\begin{array}{l}2846.86 \\
2848.32 \\
2849.15 \\
2849.94 \\
2850.41 \\
2850.60 \\
2851.16 \\
2851.23 \\
2852.1238 \\
2852.87 \\
2853.0972 \\
2853.3729 \\
2853.5092 \\
2853.84 \\
2854.14 \\
2855.79 \\
2858.0244 \\
2858.1879 \\
2858.2026 \\
2858.4846 \\
2859.77 \\
2860.4830 \\
2861.17 \\
2861.71\end{array}$ & $\begin{array}{l}35116.1 \\
35098.1 \\
35087.9 \\
35078.1 \\
35072.4 \\
35070.0 \\
35063.1 \\
35062.3 \\
35051.293 \\
35042.1 \\
35039.335 \\
35035.950 \\
35034.275 \\
35030.2 \\
35026.5 \\
35006.3 \\
34978.931 \\
34976.930 \\
34976.750 \\
34973.299 \\
34957.6 \\
34948.867 \\
34940.5 \\
34933.9\end{array}$ & $\begin{array}{r}120 \\
18 \\
150 \\
31 \\
110 \\
100 \\
78 \\
43 \\
\\
37 \\
3800 \\
810 \\
510 \\
190 \\
52 \\
74 \\
2200 \\
810 \quad P \\
650 \quad \text { U } \\
1800 \\
110 \\
6600 \\
230 \\
68\end{array}$ & $\begin{array}{l}\text { Pt II } \\
\text { Pt II } \\
\text { Ne I I I } \\
\text { Mg I } \\
\text { Pt I I } \\
\text { Pt I } \\
\text { Pt I }\end{array}$ & $\begin{array}{c}16983-52071 \\
110257-75184 \\
106434-71364\end{array}$ & $\begin{array}{l}\text { N } \\
\text { K } \\
\text { K } \\
L\end{array}$ \\
\hline
\end{tabular}




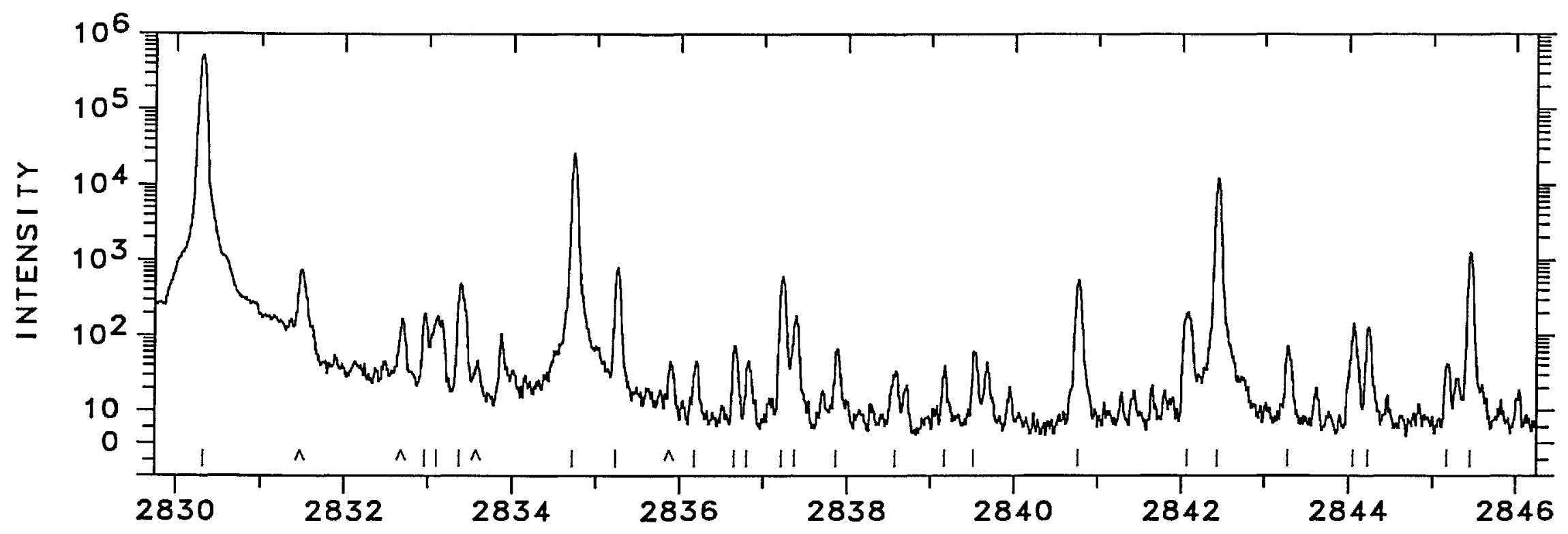

$\vec{\sigma}$

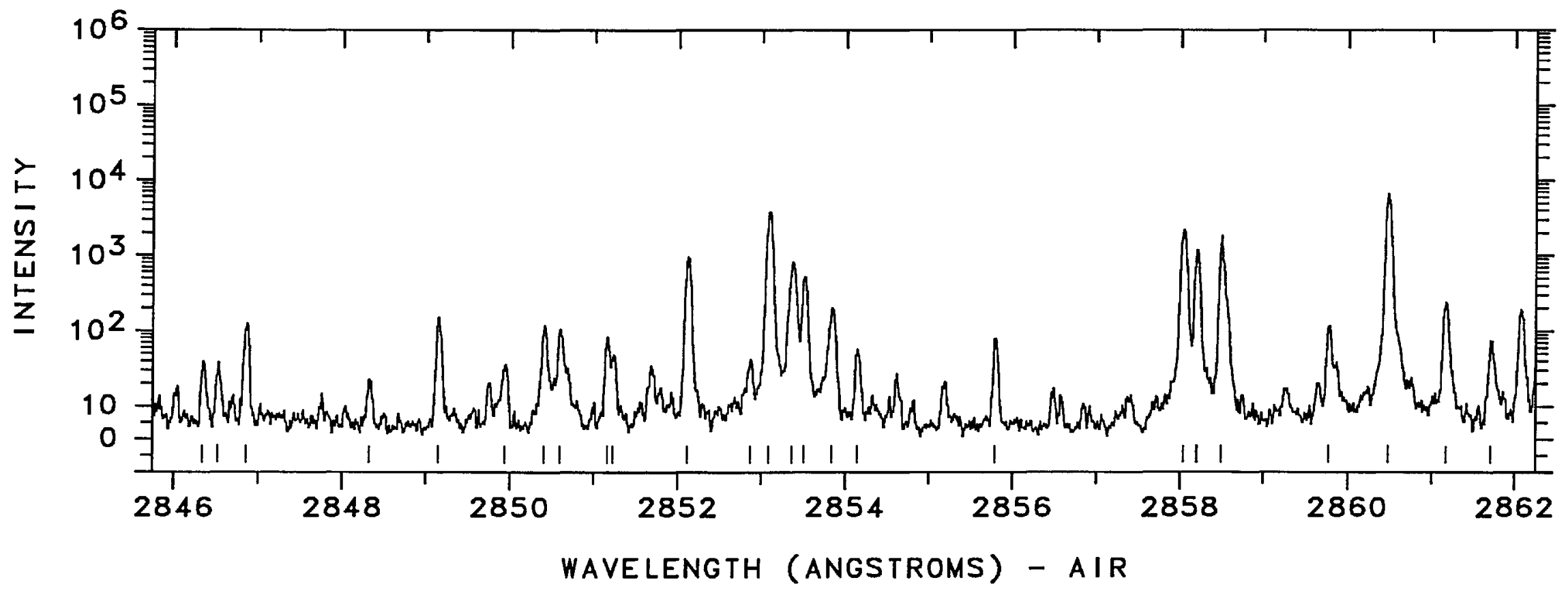




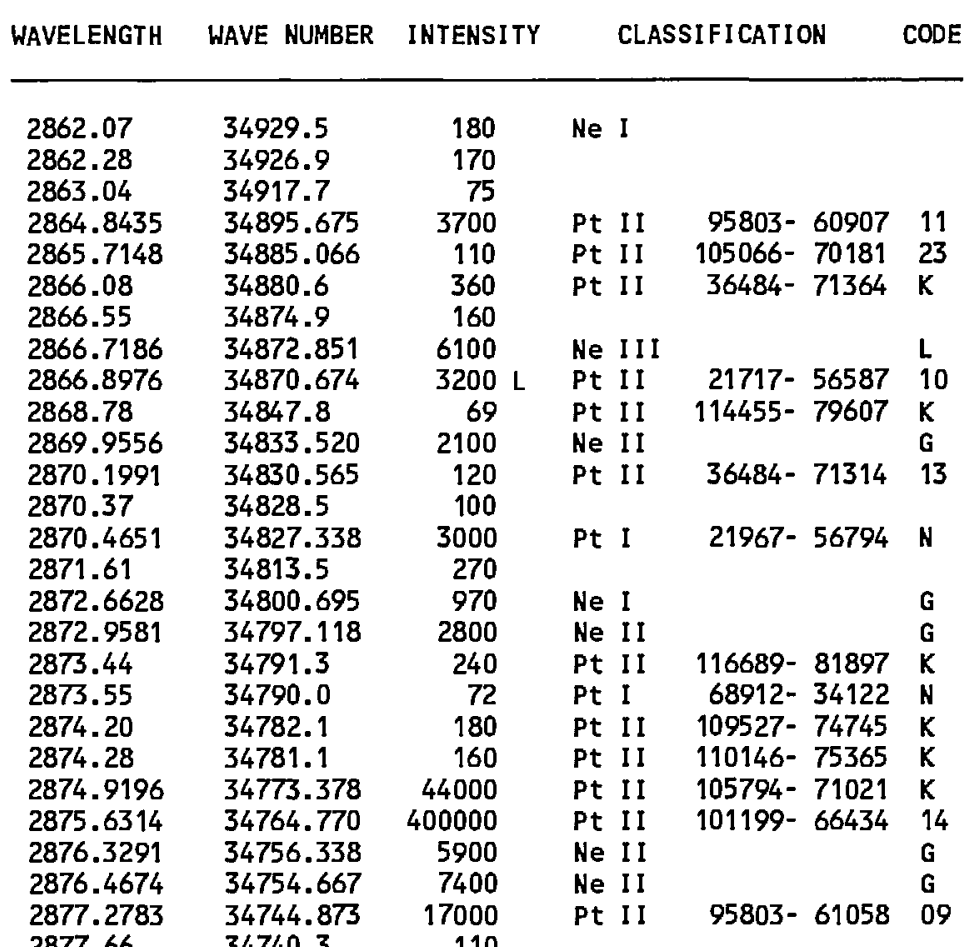

\begin{tabular}{|c|c|c|c|c|c|}
\hline & IAVE NUMBER & NTENSI TY & & IFICATION & CODI \\
\hline $\begin{array}{l}2878.1094 \\
2880.30 \\
2880.30 \\
2880.68 \\
2880.92 \\
2881.31 \\
2881.5792 \\
2881.85 \\
2882.38 \\
2882.9326 \\
2883.7321 \\
2883.98 \\
2885.3275 \\
2886.28 \\
2887.38 \\
2887.63 \\
2888.1924 \\
2888.4162 \\
2888.77 \\
2889.5096 \\
2890.3725 \\
2890.87 \\
2891.26 \\
2891.4581 \\
2893.2175 \\
2893.2881 \\
2893.8630\end{array}$ & $\begin{array}{l}34734.84 \\
34708.4 \\
34708.4 \\
34703.8 \\
34701.0 \\
34696.3 \\
34693.016 \\
34689.8 \\
34683.4 \\
34676.731 \\
34667.117 \\
34664.1 \\
34647.949 \\
34636.5 \\
34623.3 \\
34620.3 \\
34613.582 \\
34610.90 \\
34606.7 \\
34597.805 \\
34587.476 \\
34581.5 \\
34576.9 \\
34574.49 \\
34553.466 \\
34552.623 \\
34545.759\end{array}$ & $\begin{array}{r}450 \\
180 \\
180 \\
510 \\
37 \\
32 \\
\\
55 \\
28 \\
6000 \\
1200 \\
66 \\
6300 \\
71 \\
55 \\
68 \\
3900 \\
1300 \\
40 \\
4000 \\
2600 \\
180 \\
560 \\
430 \\
5600 \\
600 \mathrm{P} \\
200000\end{array}$ & $\begin{array}{ll}\text { Pt I } & \text { Ne II } \\
& \\
\text { Pt } & \text { II } \\
\text { Pt II } & \text { Pt I } \\
\text { Pt II } \\
\text { Ne II } \\
\text { Pt I } \\
\text { Pt II } \\
\text { Pt I }\end{array}$ & $\begin{array}{r}110258-75581 \\
113119-78452 \\
114861-80197 \\
105962-71314 \\
68759-34122\end{array}$ & $\begin{array}{l}\text { K } \\
07 \\
\text { N } \\
\text { K } \\
\text { C } \\
\text { E } \\
\text { K } \\
\text { E }\end{array}$ \\
\hline
\end{tabular}




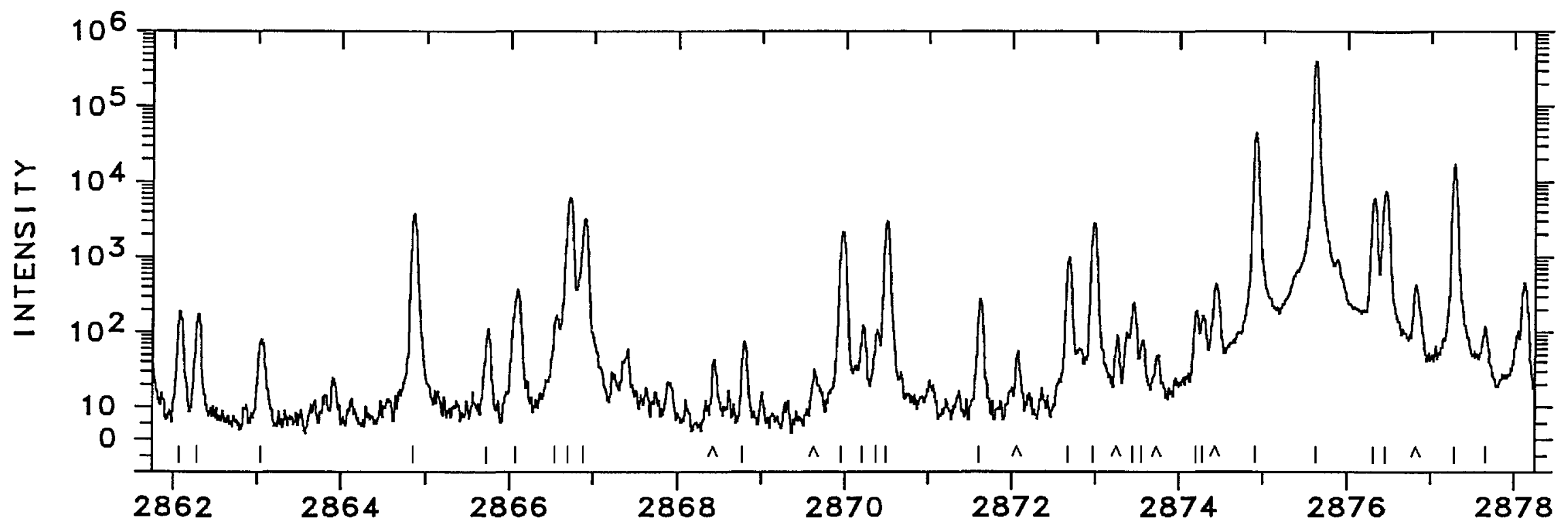

Nㅗㅁ

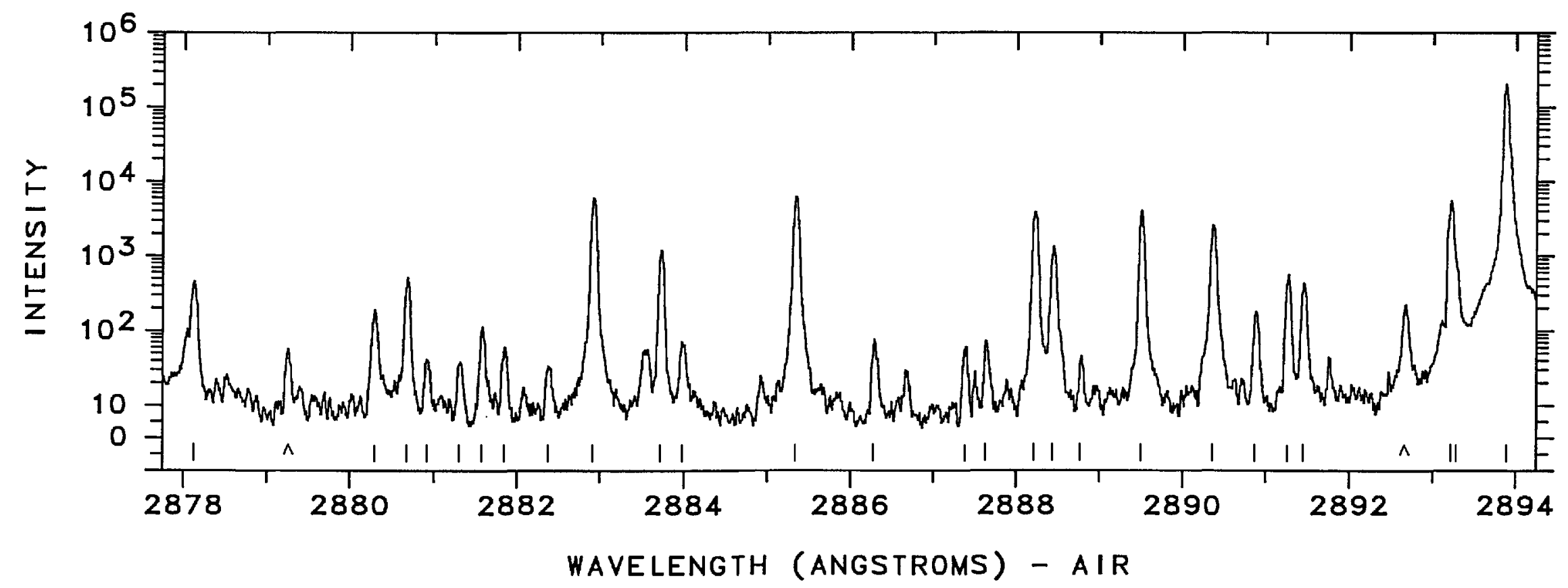




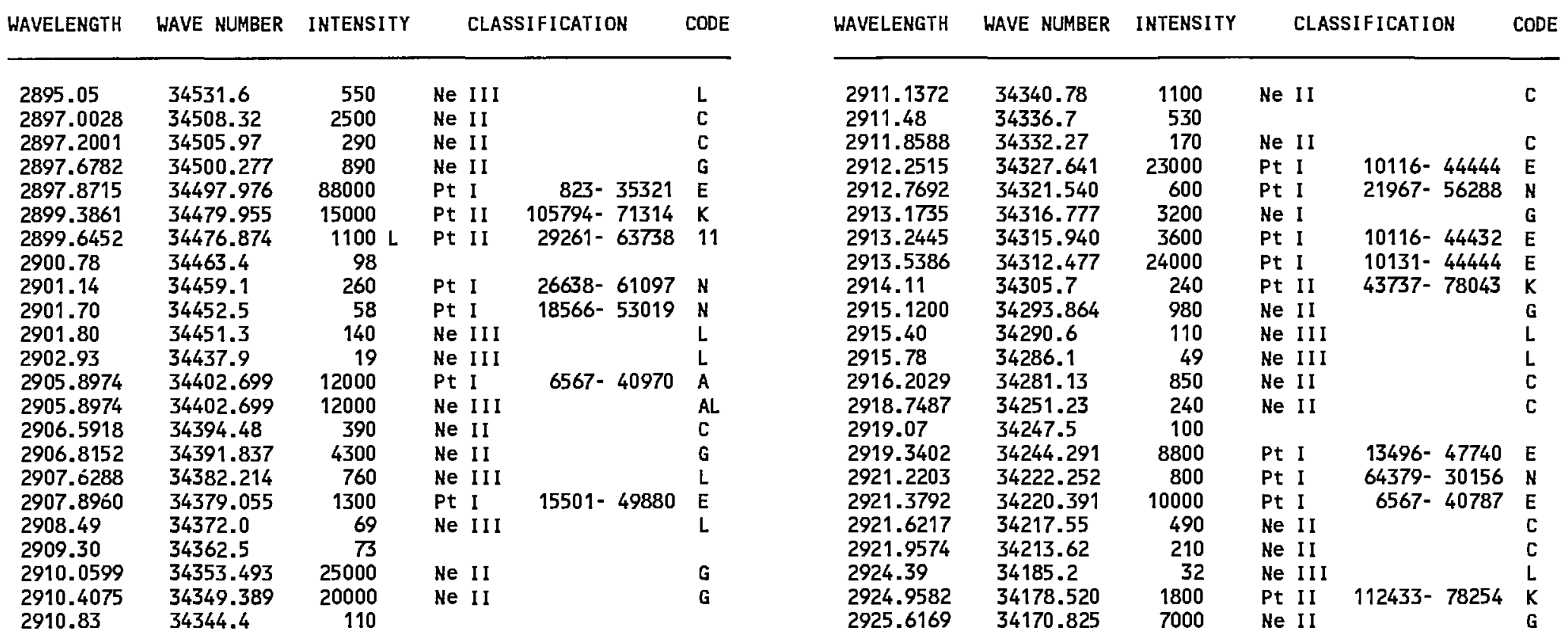



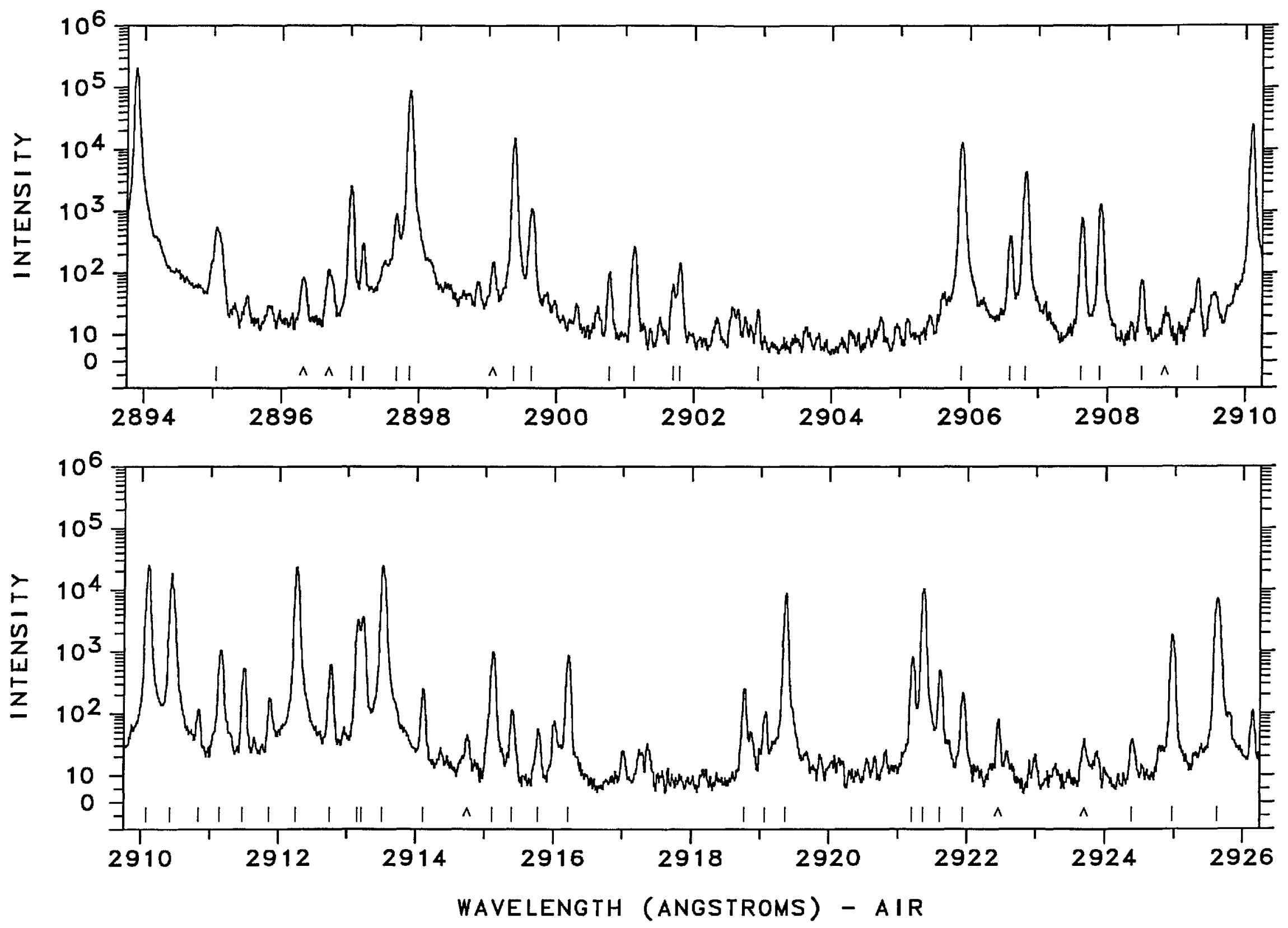


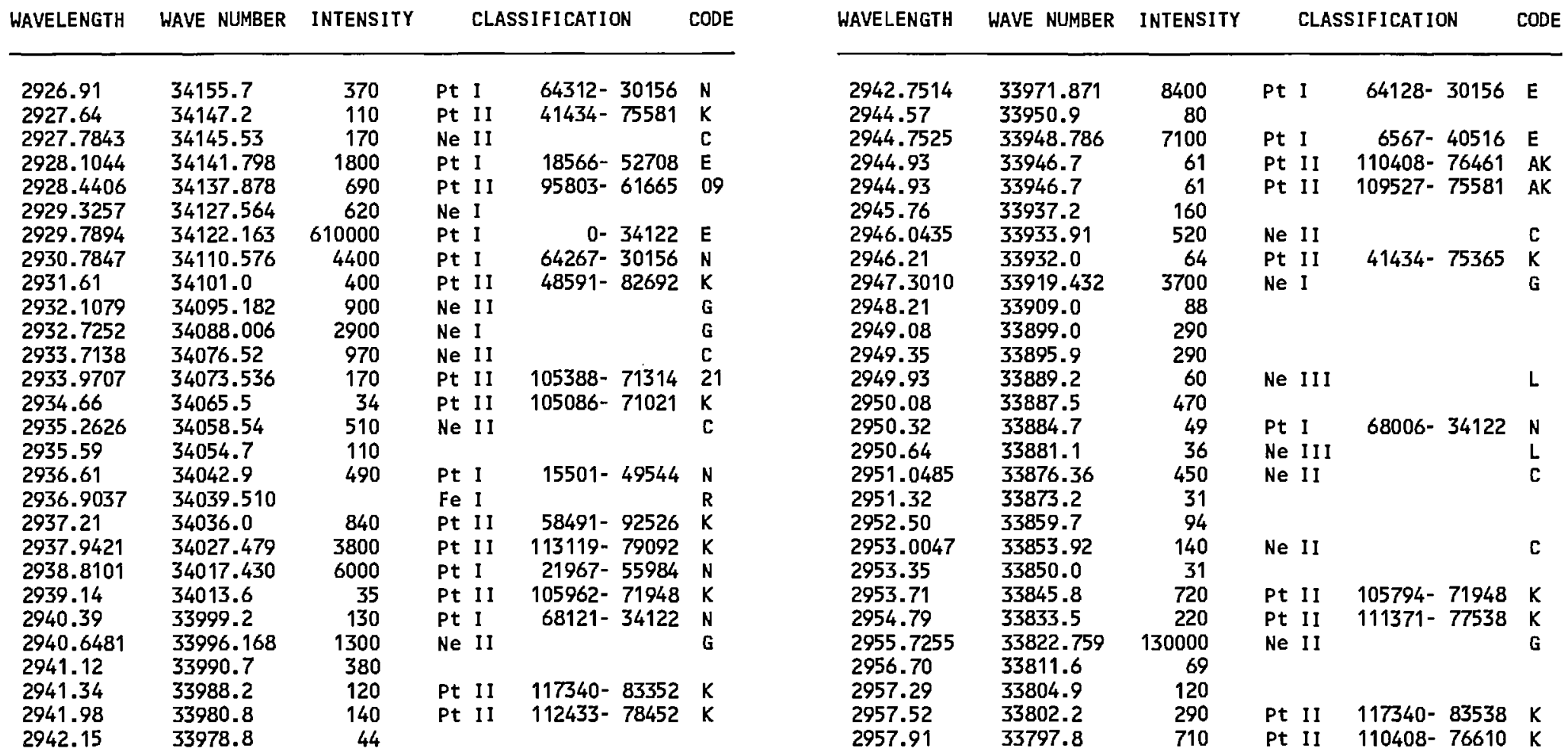




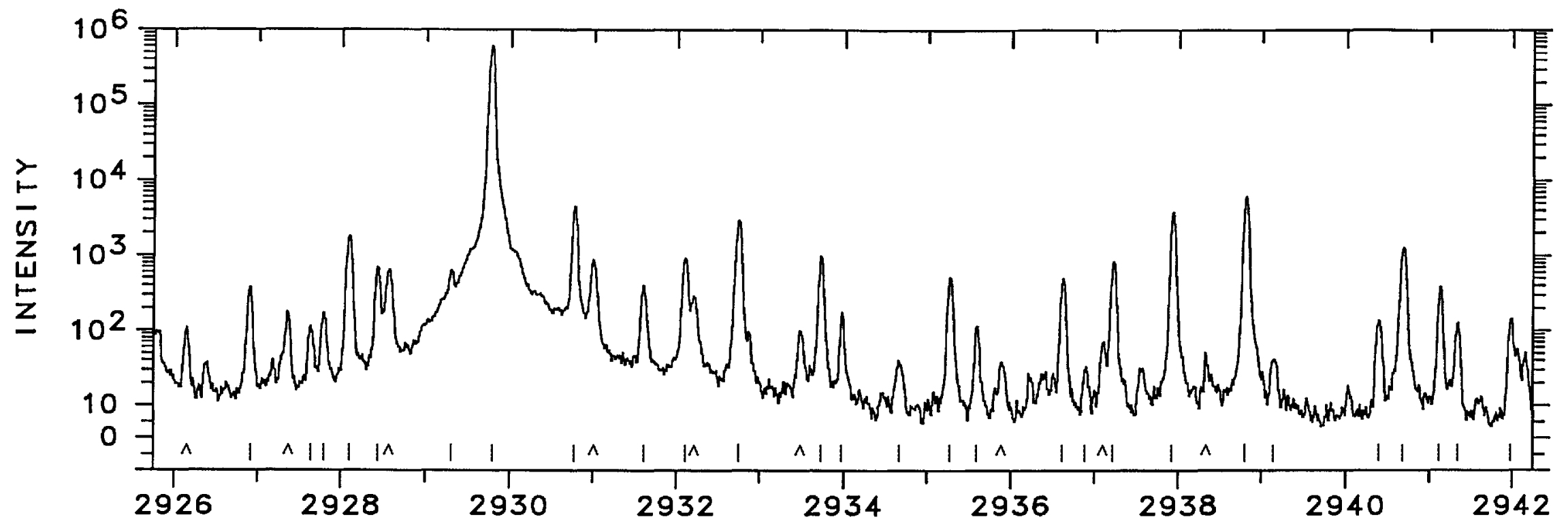

岕

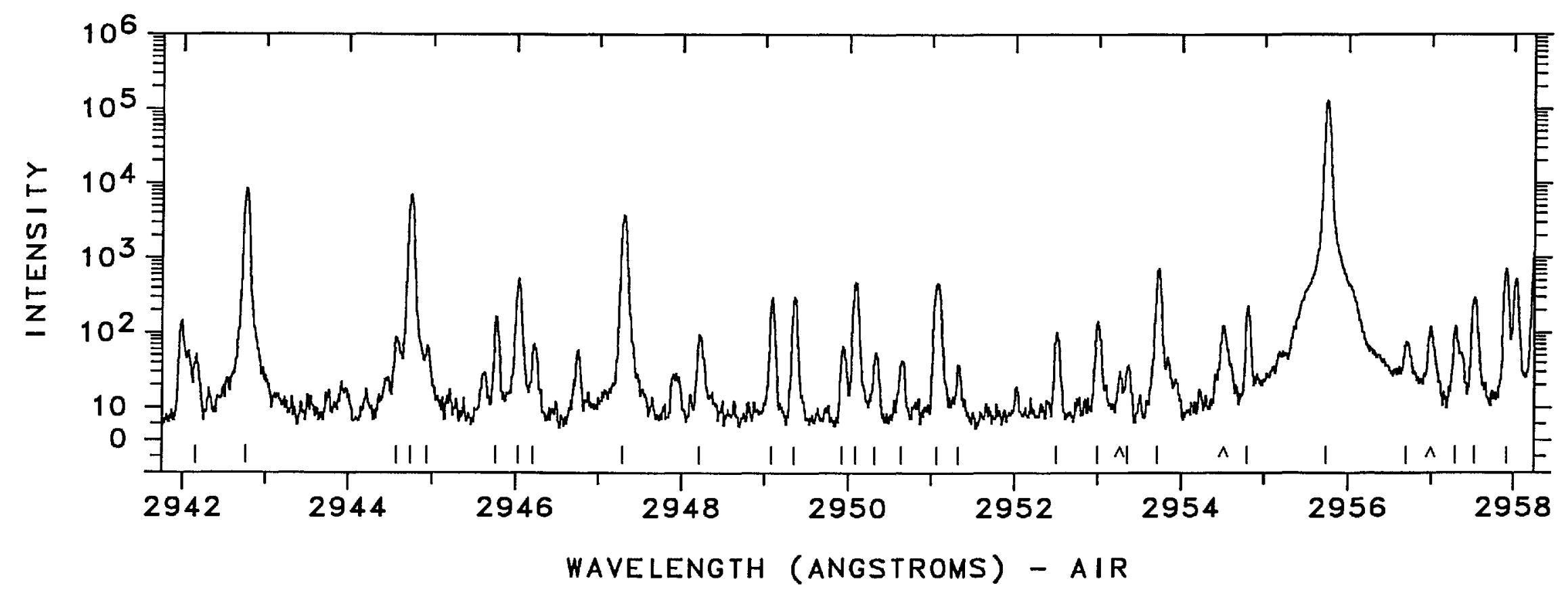




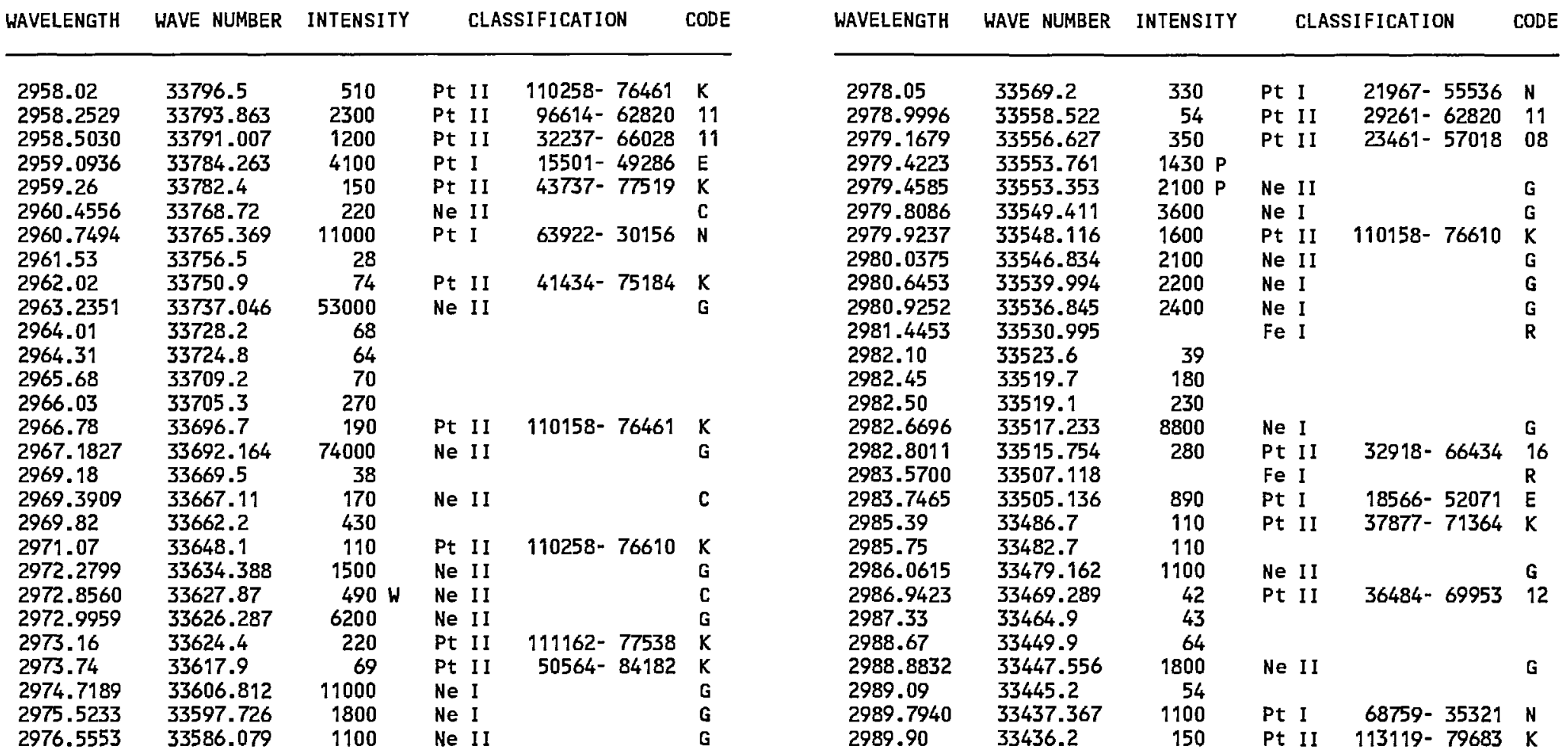



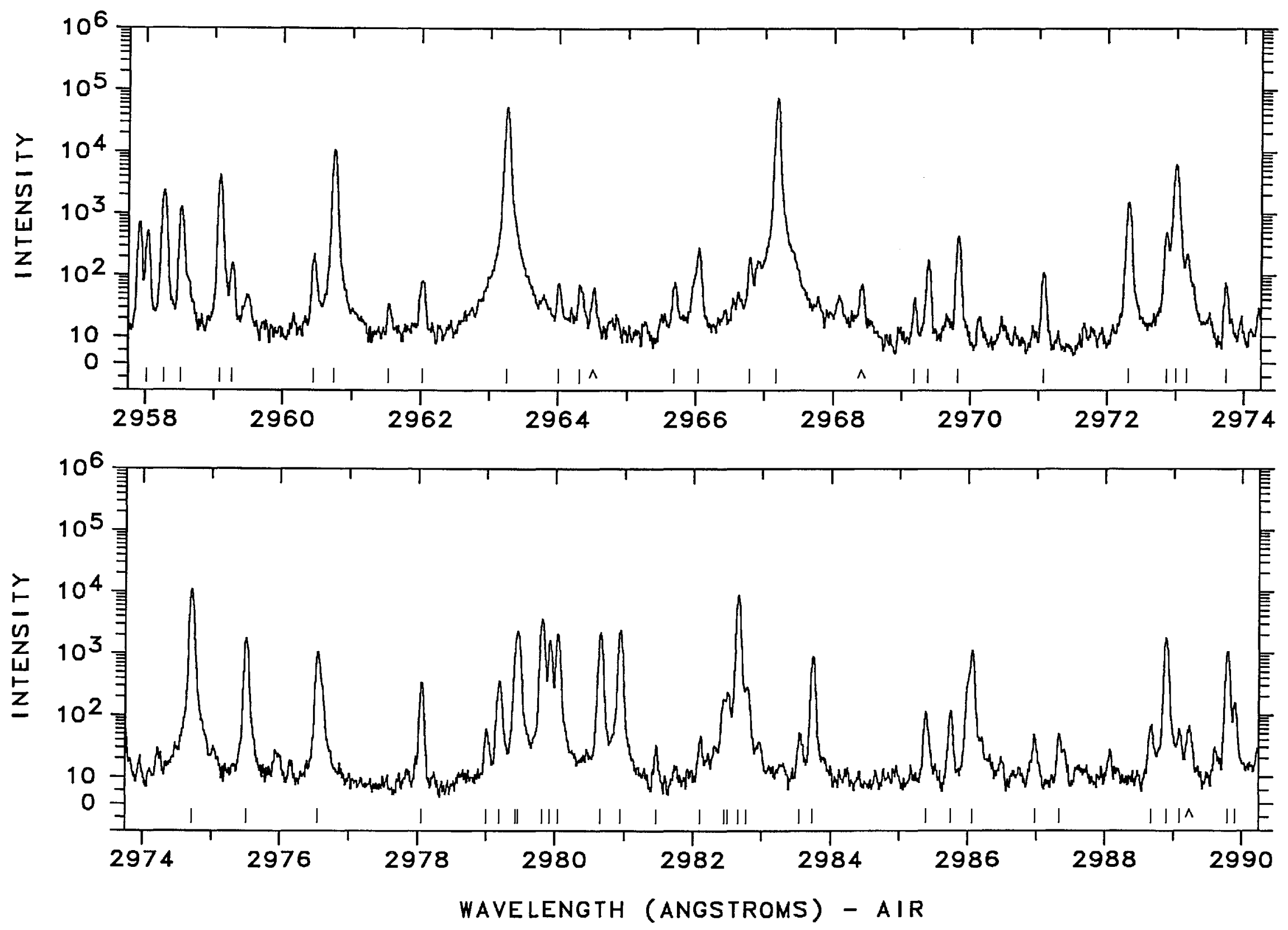


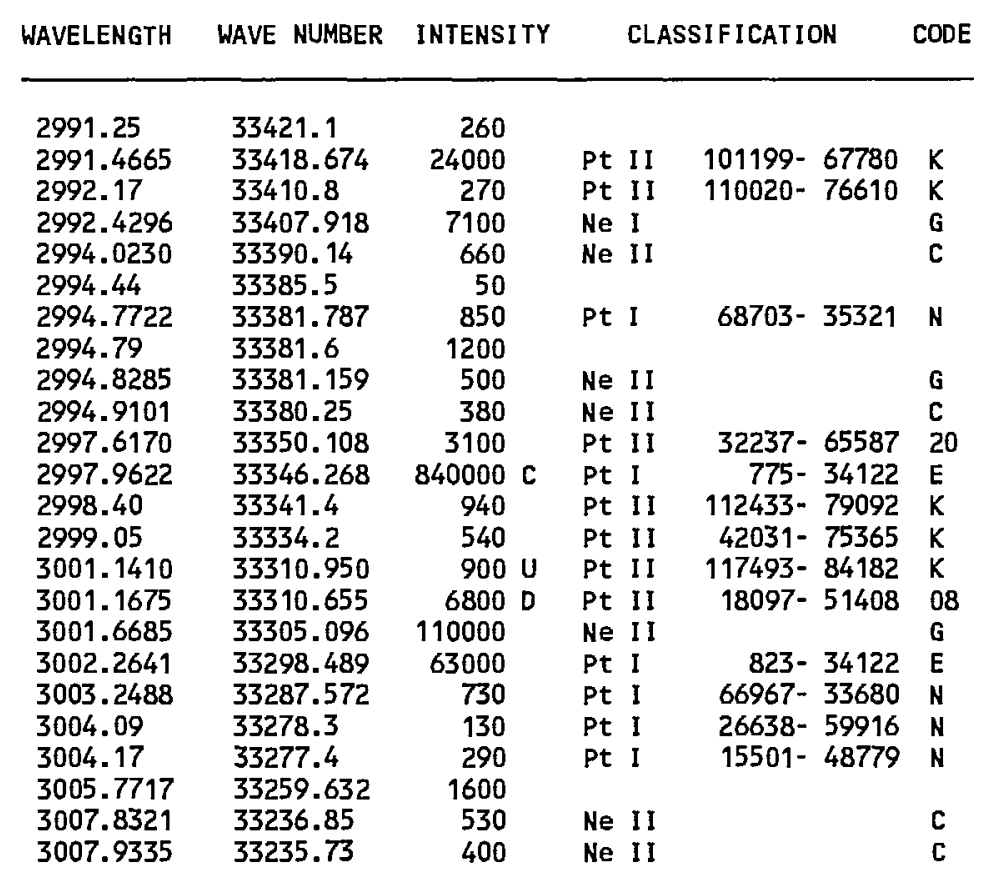

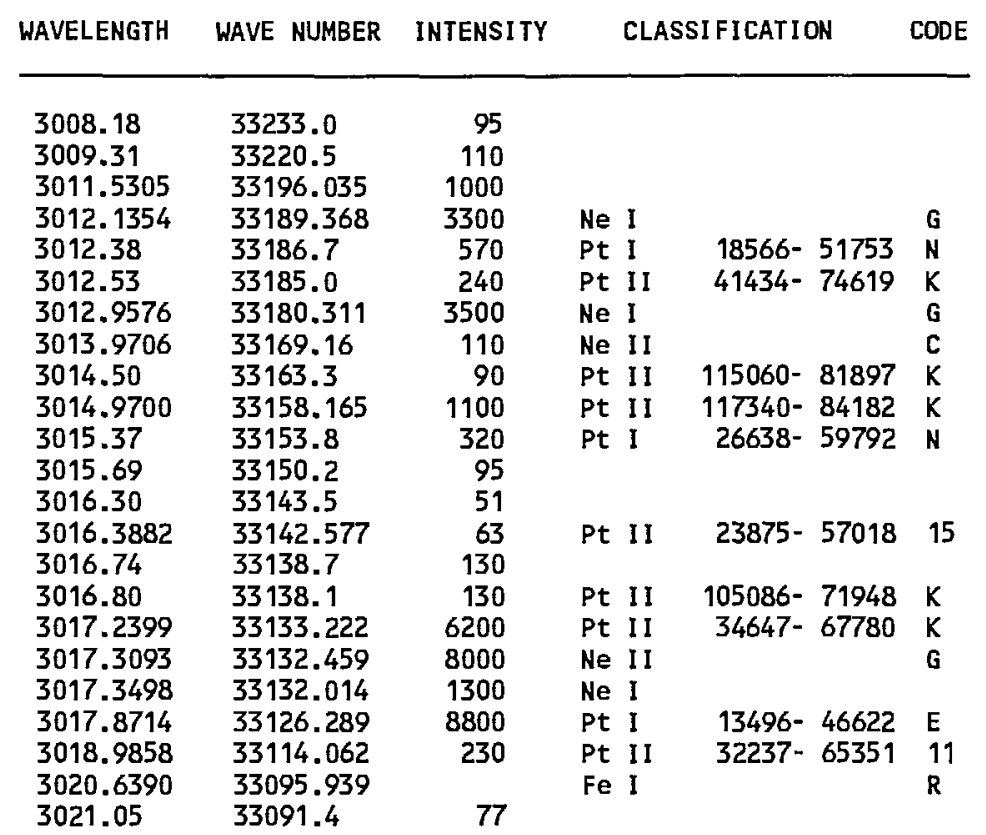




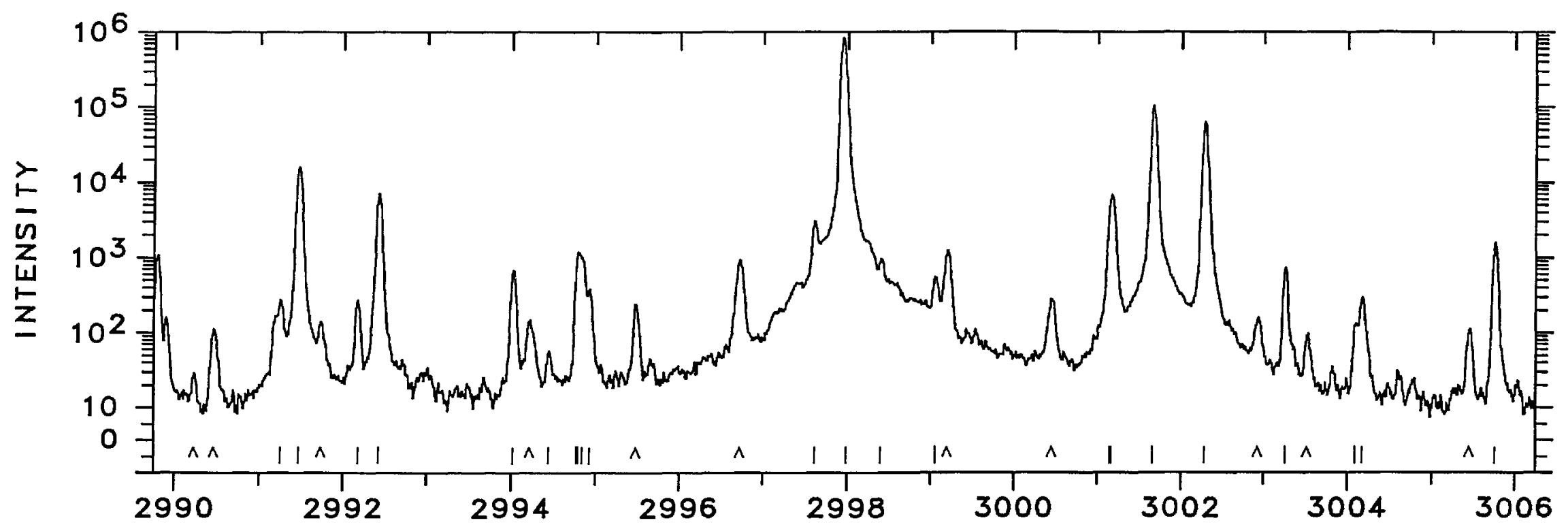

$\vec{U}$

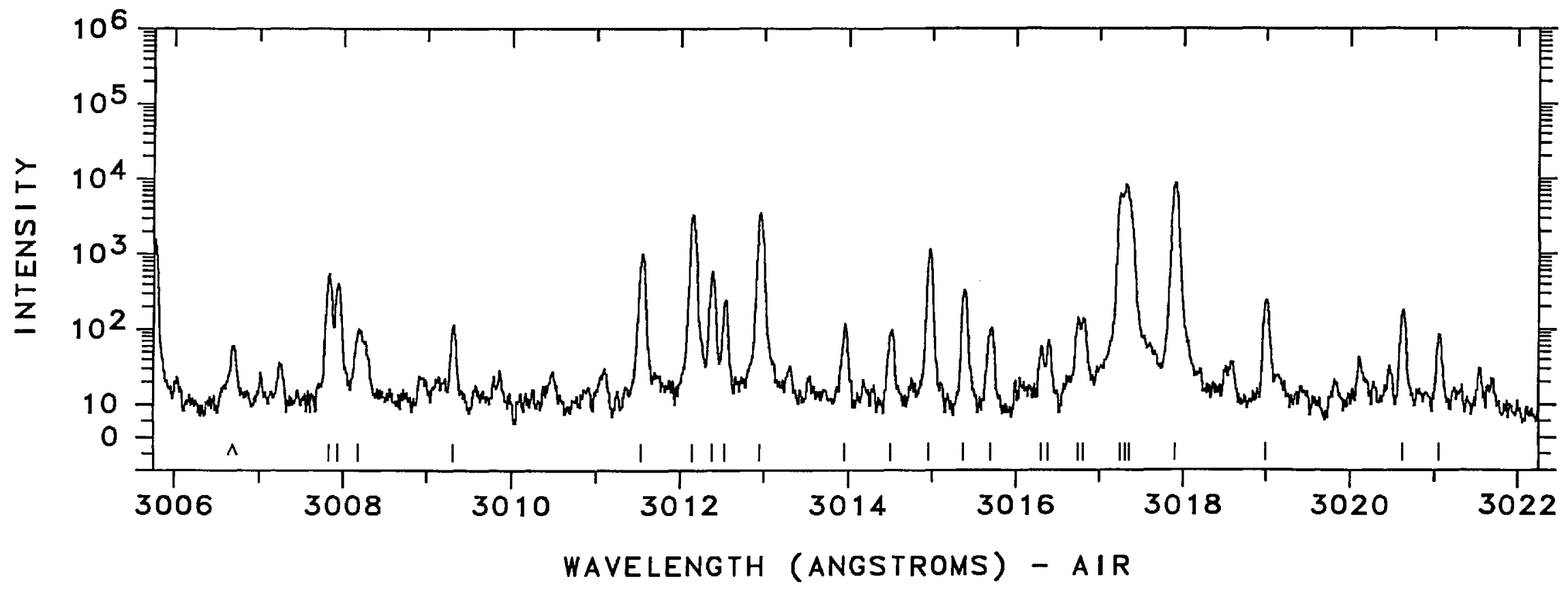


WAVELENGTH WAVE NUMBER INTENSITY CLASSIFICATION CODE

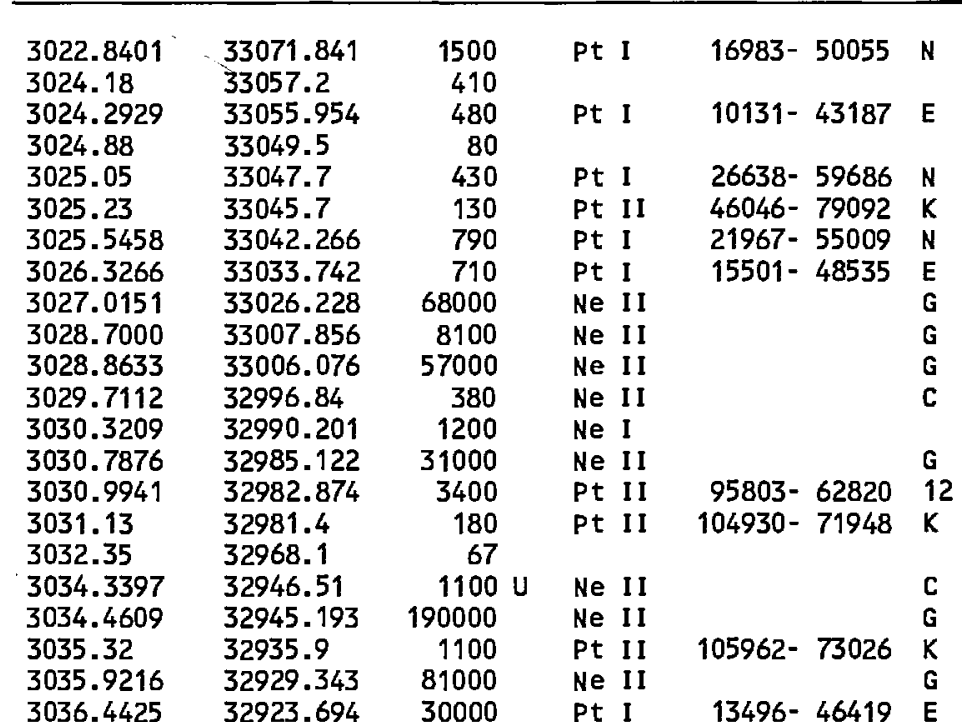

WAVELENGTH WAVE NUMBER INTENSITY

CLASSIFICATION

CODE

$\begin{array}{llrlrl}3036.6237 & 32921.73 & 750 & \text { Ne I I } & & \text { C } \\ 3036.84 & 32919.4 & 710 & \text { Pt II } & 111371-78452 & \text { K } \\ 3036.97 & 32918.0 & 160 & \text { Pt II } & 109527-76610 & \text { K } \\ 3037.7192 & 32909.858 & 62000 & \text { Ne II } & & \text { G } \\ 3038.8196 & 32897.941 & 1100 & \text { Pt II } & 109507-76610 & \text { K } \\ 3039.5855 & 32889.651 & 100000 & \text { Ne I I } & & \text { G } \\ 3040.8930 & 32875.511 & 3000 & \text { Pt I I } & 96614-63738 & 11 \\ 3041.2085 & 32872.100 & 830 & \text { Pt I } & 21967-54839 & \text { E } \\ 3042.6318 & 32856.724 & 1200000 \text { C } & \text { Pt I } & 823-33680 & \text { E } \\ 3044.0878 & 32841.009 & 26000 & \text { Ne II } & & \text { G } \\ 3045.5563 & 32825.174 & 30000 & \text { Ne I I } & & \text { G } \\ 3045.94 & 32821.0 & 210 & \text { Ne I } & & \\ 3046.79 & 32811.9 & 130 & & & \\ 3047.21 & 32807.4 & 340 & \text { Pt II } & 41434-74241 & \text { K } \\ 3047.5569 & 32803.627 & 91000 & \text { Ne II } & & \text { G } \\ 3048.66 & 32791.8 & 120 & & & \\ 3050.18 & 32775.4 & 230 & & & \text { G } \\ 3050.4724 & 32772.276 & 1800 & \text { Ne II } & & \text { C } \\ 3050.7662 & 32769.12 & 560 & \text { Ne II } & & \\ 3050.97 & 32766.9 & 200 & \text { Pt I } & 65387-32620 & \text { N } \\ 3052.54 & 32750.1 & 72 & \text { Pt II } & 112433-79683 & \text { K }\end{array}$

3052.54
Pt II 112433- $79683 \mathrm{~K}$ 

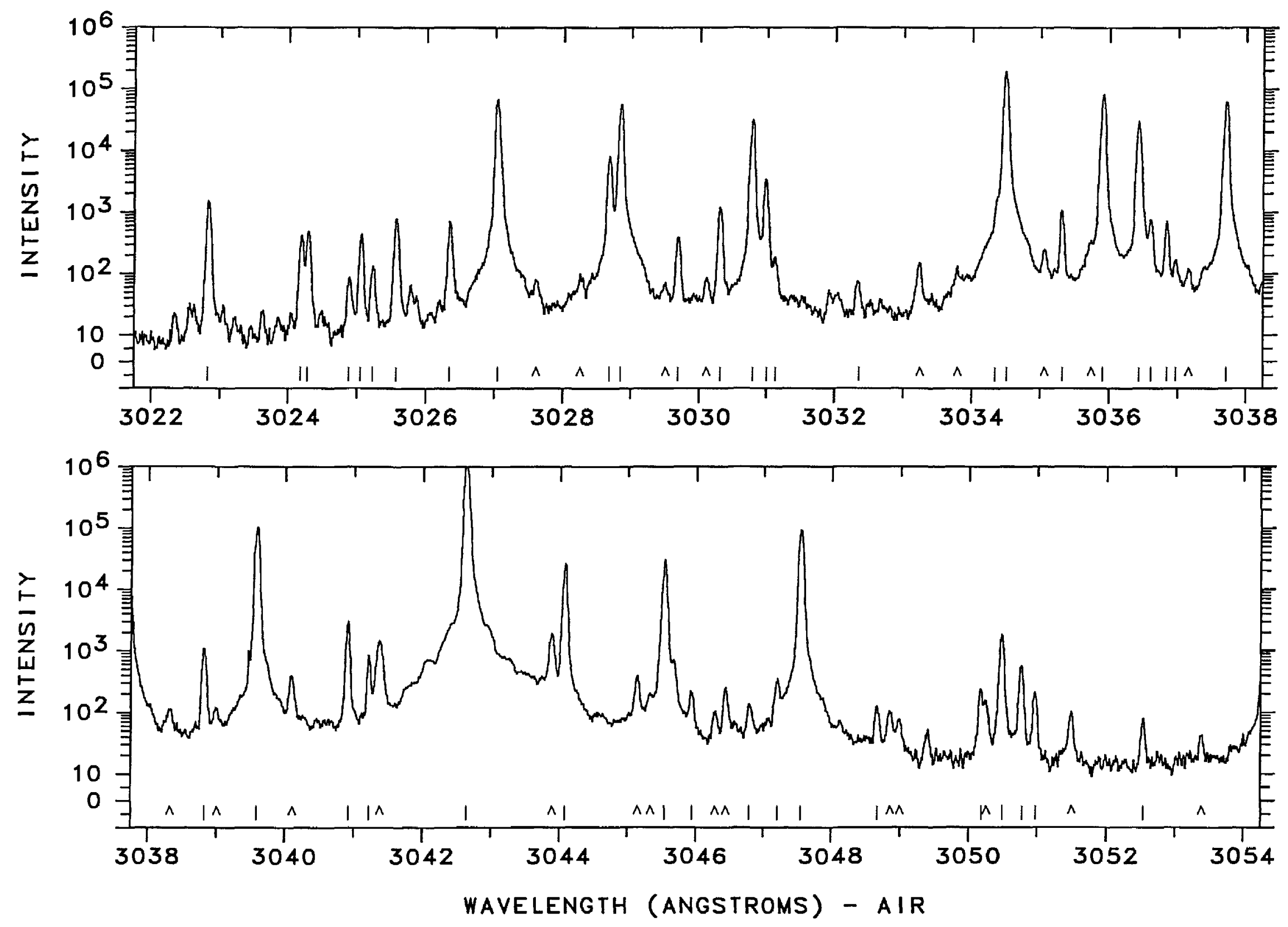


\section{WAVELENGTH WAVE NUMBER INTENSITY}

\section{CLASSI FI CATION}

CODE

\begin{tabular}{llrllll}
\hline & & & & & \\
3054.3449 & 32730.726 & 6600 & Ne II & & G \\
3054.4287 & 32729.828 & 1000 & Ne II & & G \\
3054.6747 & 32727.193 & 31000 & Ne II & & G \\
3054.7312 & 32726.588 & 850 U & & & \\
3054.8344 & 32725.483 & 1000 & & & \\
3055.3115 & 32720.372 & 1100 & Pt I & $18566-51286$ & E \\
3056.0579 & 32712.381 & 440 & Pt II & $23875-56587$ & 16 \\
3057.3907 & 32698.122 & 30000 & Ne I & & G \\
3057.8669 & 32693.030 & 1400 & Ne II & & G \\
3058.66 & 32684.6 & 47 & Pt II & $58062-90746$ & KM \\
3059.1050 & 32679.799 & 31000 & Ne II & & G \\
3059.6366 & 32674.121 & 5000 & Pt II & $13496-46170$ & E \\
3059.7250 & 32673.177 & 330 & Pt II & $106434-73761$ & A \\
3059.7250 & 32673.177 & 330 & Ne II & & A \\
3061.82 & 32650.8 & 290 & Pt I & $68947-36296$ & AN \\
3061.82 & 32650.8 & 290 & Ne II & & A \\
3062.4913 & 32643.664 & 9300 & Ne II & & G \\
3063.3015 & 32635.032 & 5400 & Ne II & & G \\
3063.6948 & 32630.842 & 1600 & Ne I & & \\
3064.7110 & 32620.023 & 1500000 & Pt I & $0-32620$ & E \\
3066.6875 & 32599.00 & 180 & Ne II & & C \\
3067.4494 & 32590.903 & 1500 & Ne II & & G \\
3068.85 & 32576.0 & 82 & & & \\
3069.79 & 32566.1 & 200 & Pt II & $41434-73999$ & $\mathrm{~K}$ \\
3070.23 & 32561.4 & 660 & Pt I & $16983-49544$ & N \\
3070.8916 & 32554.372 & 8300 & Ne II & & G \\
3071.0871 & 32552.300 & 8500 & Ne II & & G \\
3071.5310 & 32547.596 & 4200 & Ne II & & G \\
3071.9336 & 32543.331 & 33000 & Pt I & $10116-42660$ & E \\
3072.3009 & 32539.44 & 220 & Ne II & & C \\
3072.6543 & 32535.698 & 3400 & Ne II & & G \\
& & & & & &
\end{tabular}

WAVELENGTH WAVE NUMBER INTENSITY

CLASSIFICATION

CODE

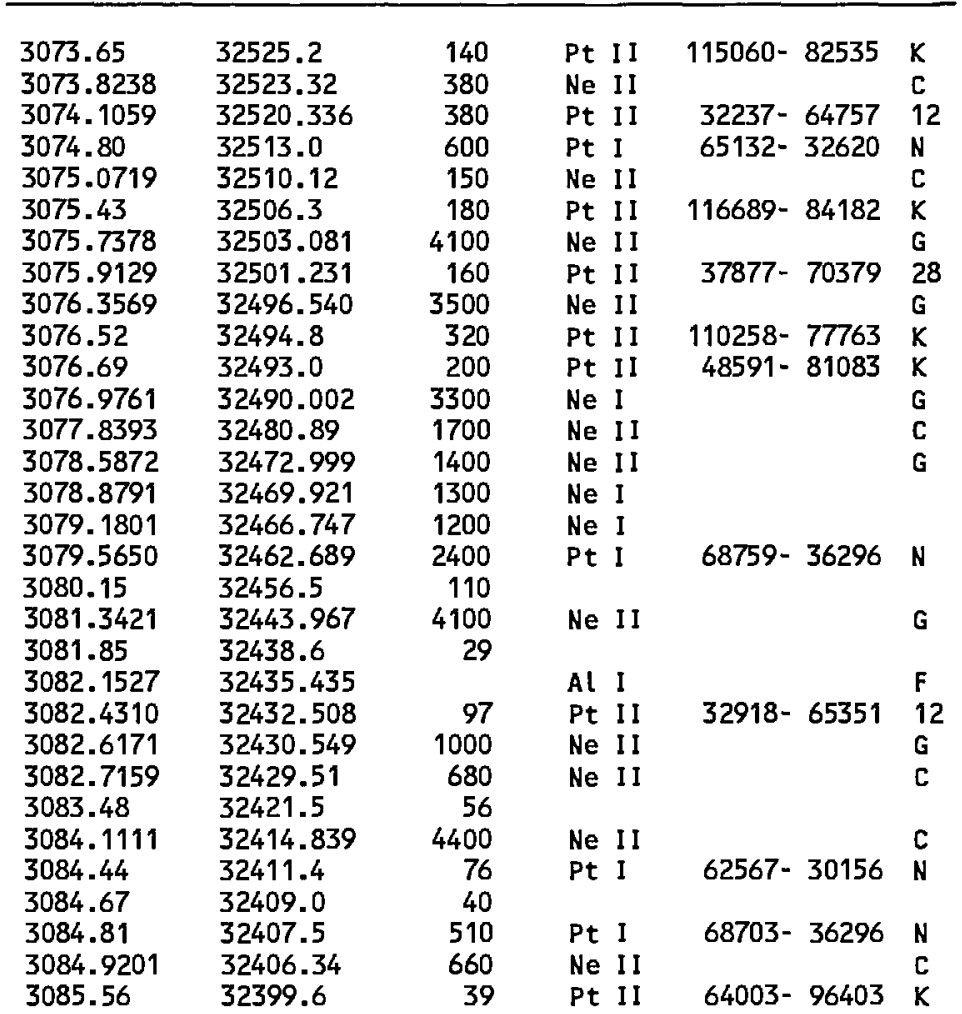




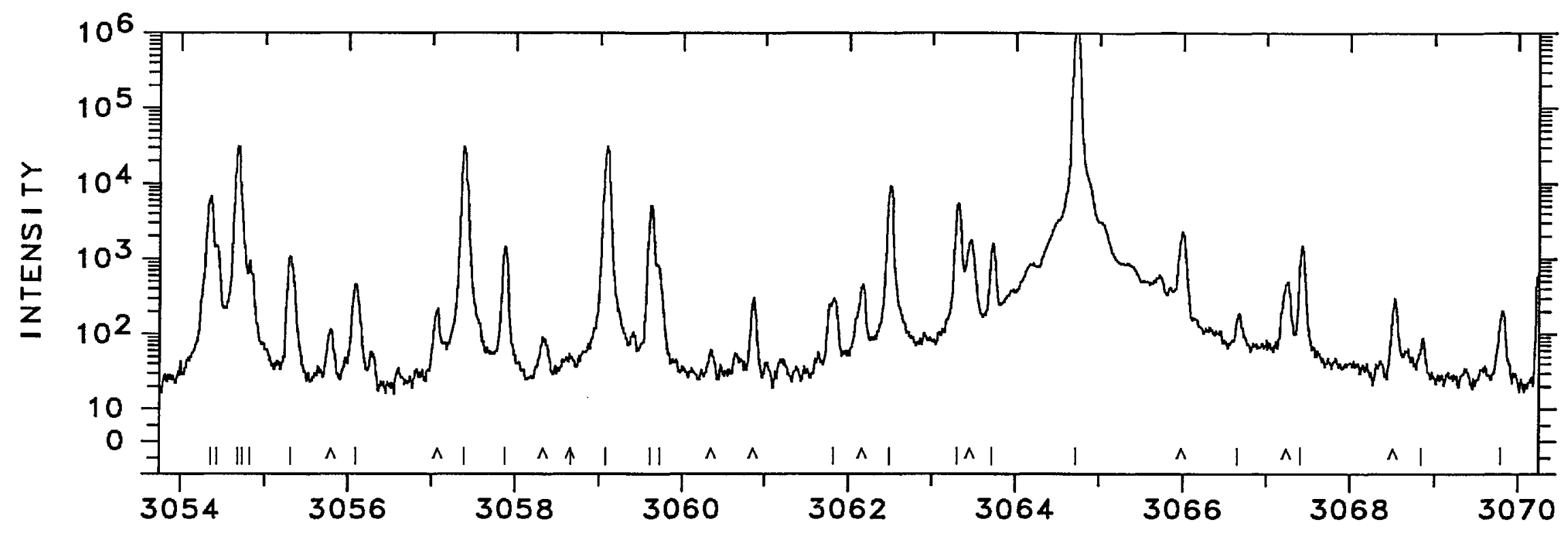

$\underset{\omega}{\omega}$

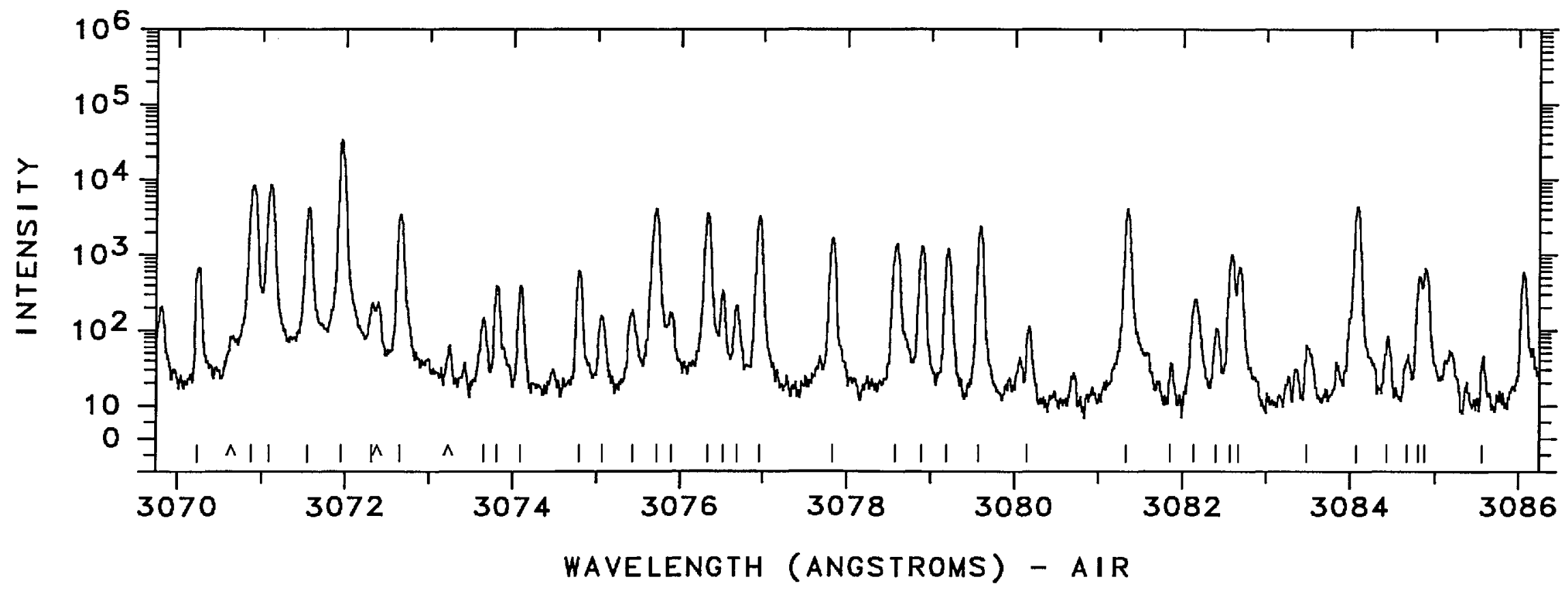




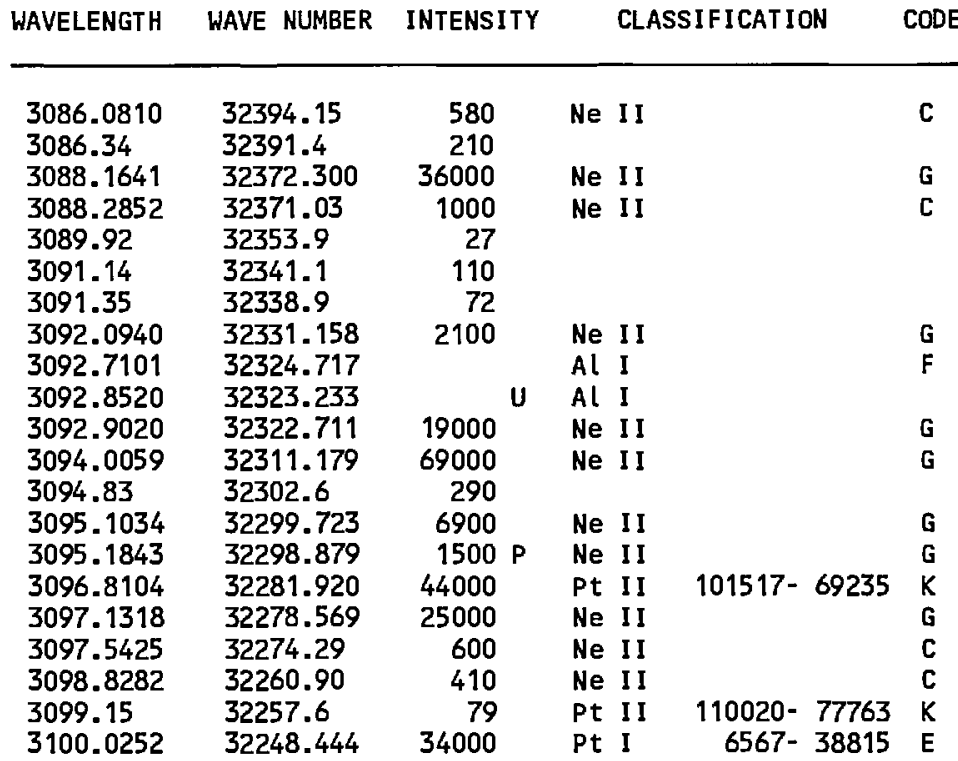

$\begin{array}{llrlrl}3100.9598 & 32238.725 & 2300 & \text { Pt I } & 15501-47740 & \text { E } \\ 3101.13 & 32237.0 & 120 & \text { Pt II } & 115060-82824 & \text { K } \\ 3101.41 & 32234.0 & 81 & & & \\ 3103.60 & 32211.3 & 260 & \text { Pt I } & 21967-54178 & \text { N } \\ 3103.77 & 32209.5 & 170 & \text { Pt II } & 42031-74241 & \mathrm{~K} \\ 3104.61 & 32200.8 & 220 & \text { Pt II } & 105962-73761 & \mathrm{~K} \\ 3105.30 & 32193.7 & 240 & \text { Pt II } & 106434-74241 & \mathrm{~K} \\ 3107.99 & 32165.8 & 39 & & & \\ 3108.71 & 32158.4 & 38 & & & \\ 3109.3597 & 32151.635 & 82 & \text { Pt II } & 32237-64388 & 21 \\ 3110.30 & 32141.9 & 49 & \text { Pt I } & 26638-58780 & \text { AN } \\ 3110.30 & 32141.9 & 49 & \text { Pt II } & 104090-71948 & \text { AK } \\ 3111.74 & 32127.0 & 82 & \text { Pt II } & 64003-96131 & \mathrm{~K} \\ 3112.80 & 32116.1 & 150 & \text { Pt II } & 54373-86489 & \mathrm{~K} \\ 3113.94 & 32104.3 & 42 & & & \\ 3115.64 & 32086.8 & 850 & \text { Ne II } & & \mathrm{C} \\ 3116.684 & 32076.08 & 1100 & \text { Ne II } & & \mathrm{C} \\ 3116.7380 & 32075.525 & 1300 & \text { Pt II } & 37877-69953 & 17 \\ 3117.155 & 32071.24 & 410 & \text { Ne II } & & \mathrm{C} \\ 3117.8076 & 32064.522 & 730 & \text { Pt II } & 95803-63738 & 12 \\ 3117.9807 & 32062.742 & 29000 & \text { Ne II } & & \mathrm{G}\end{array}$




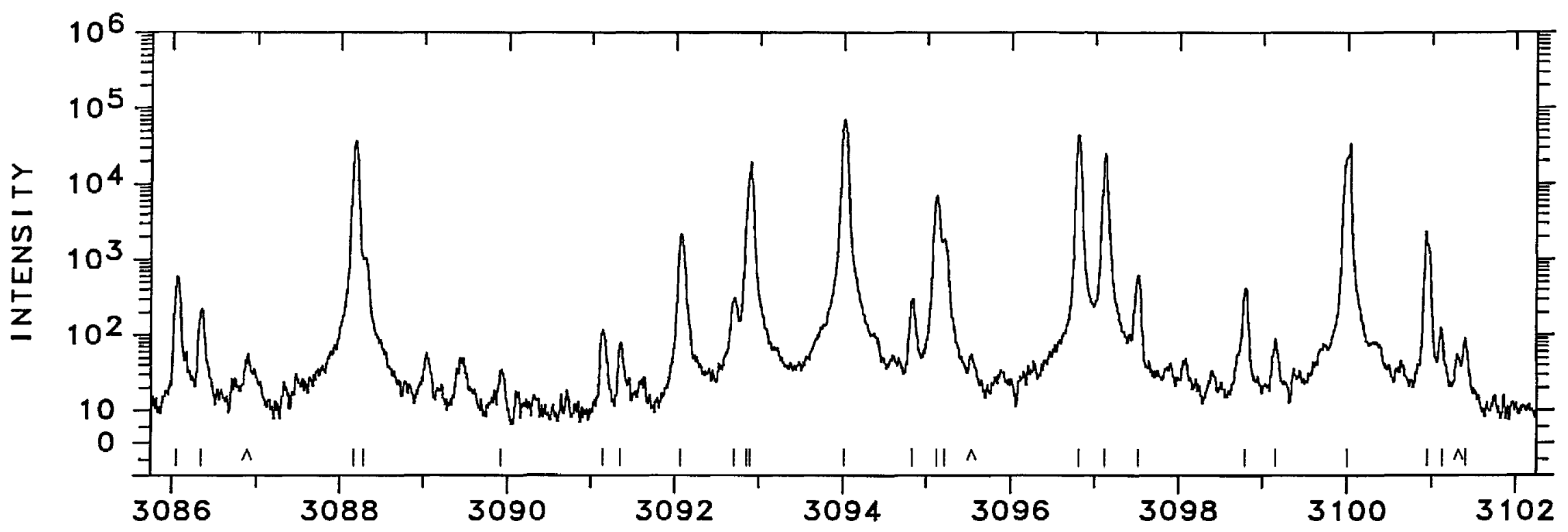

岕

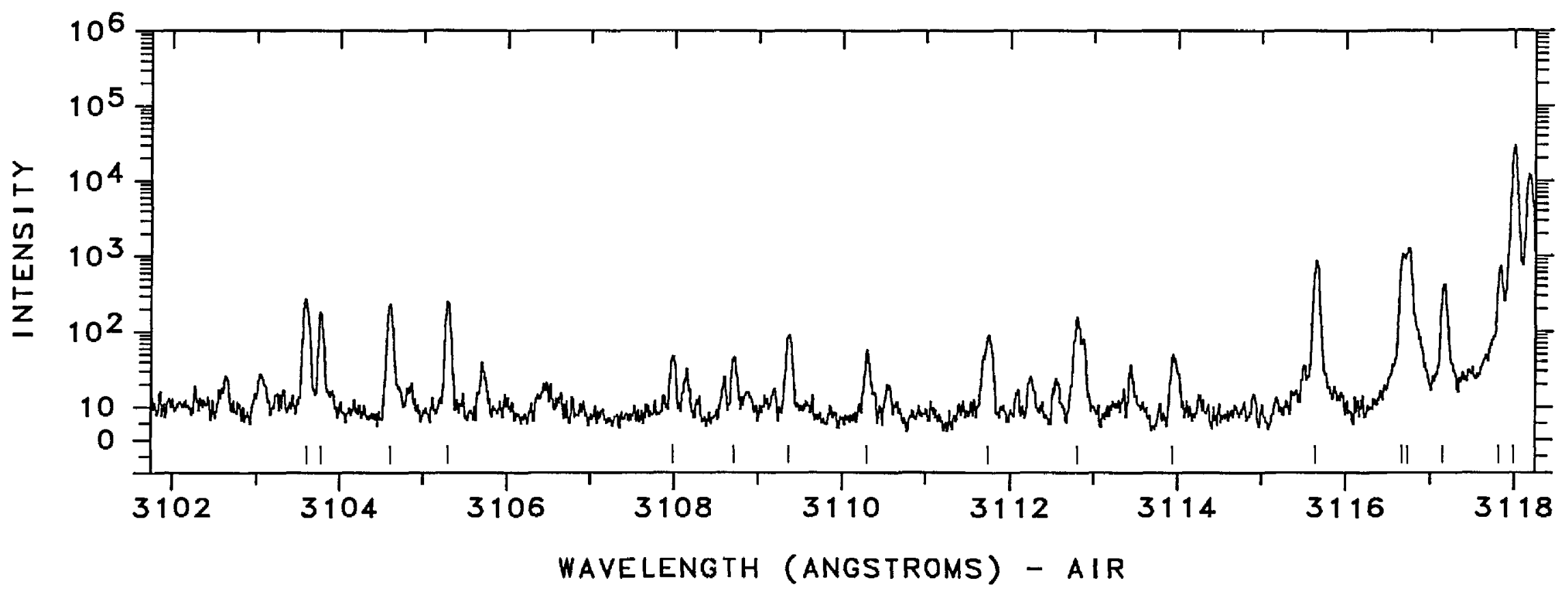




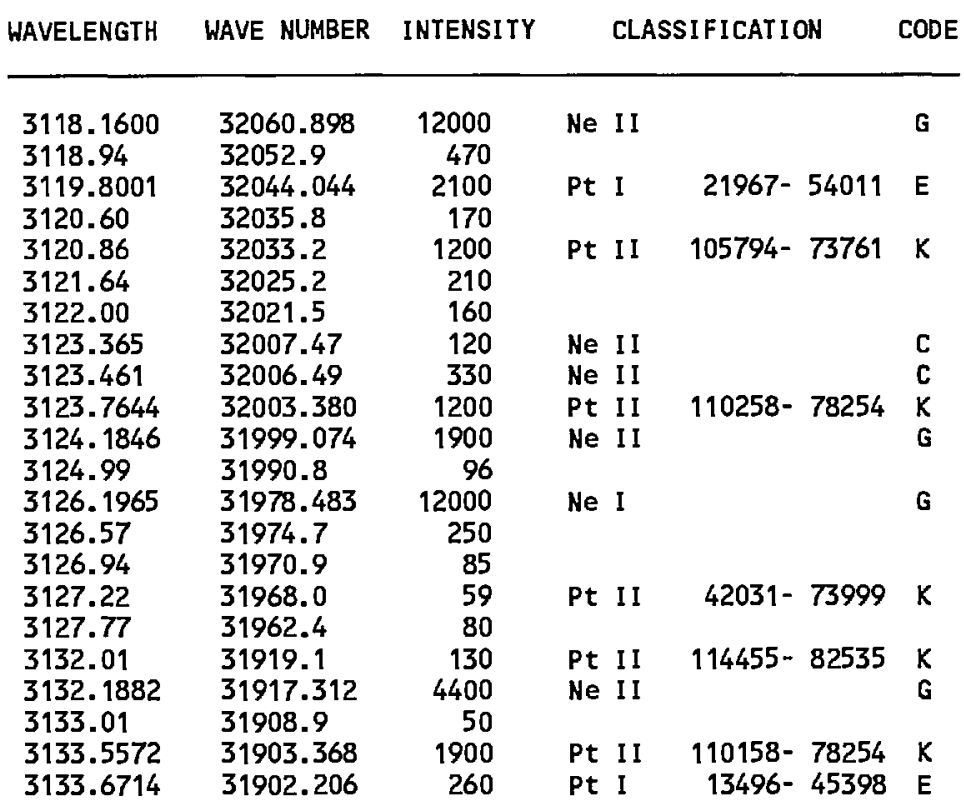

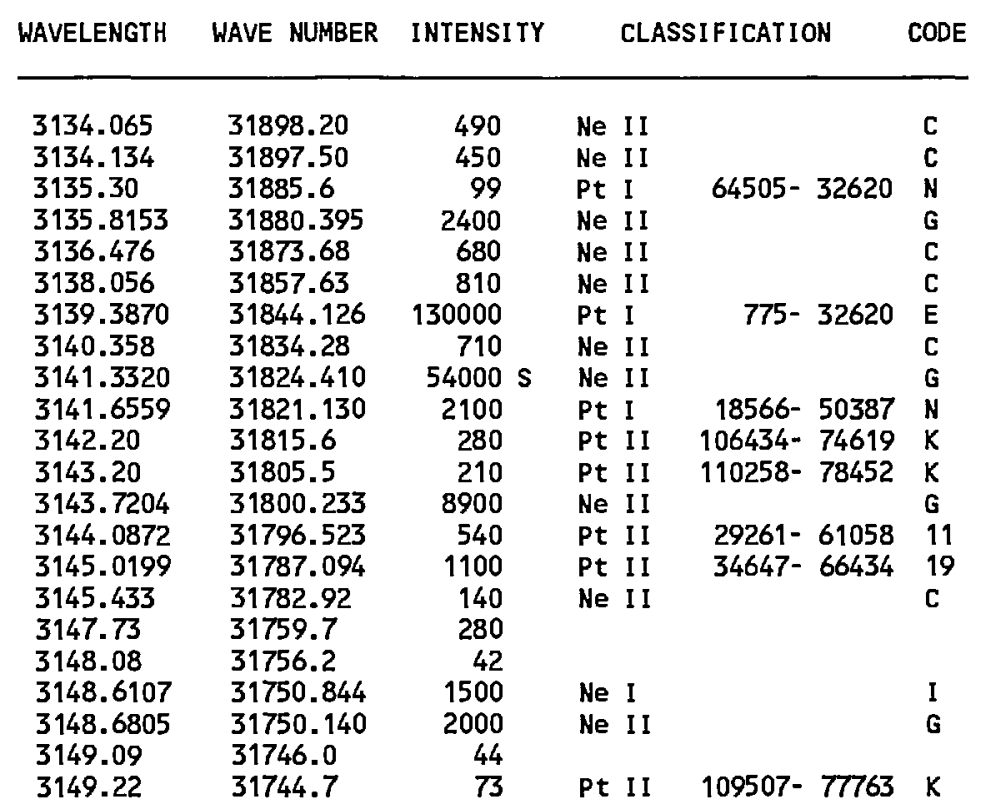




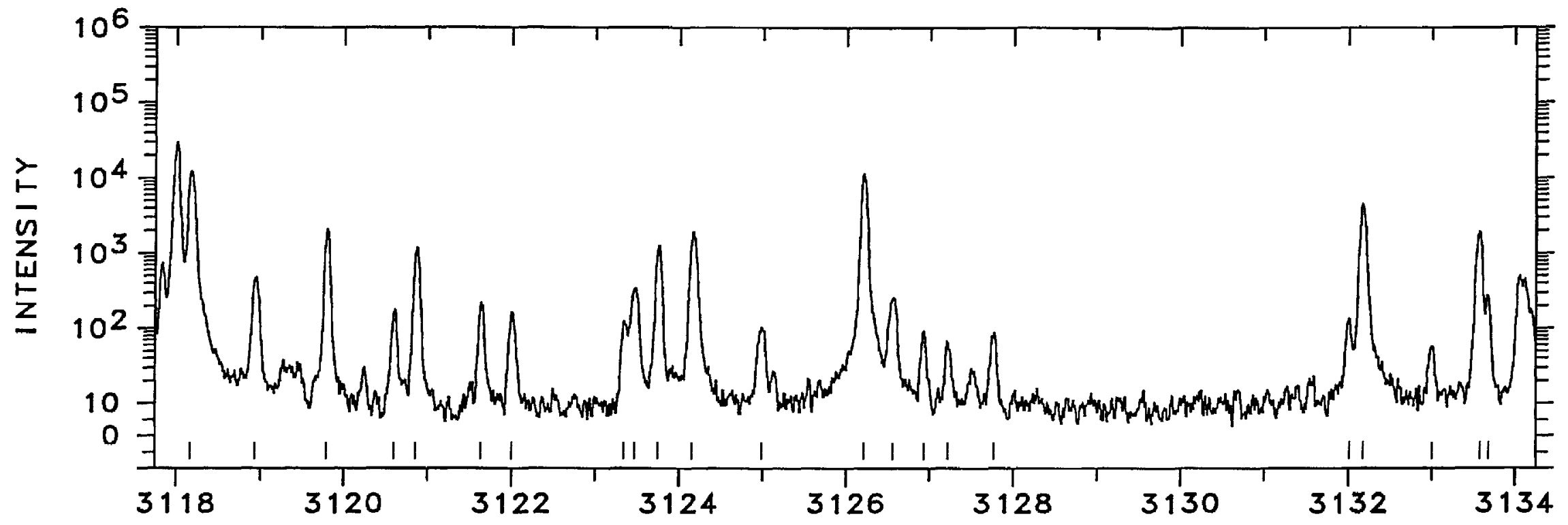

岕

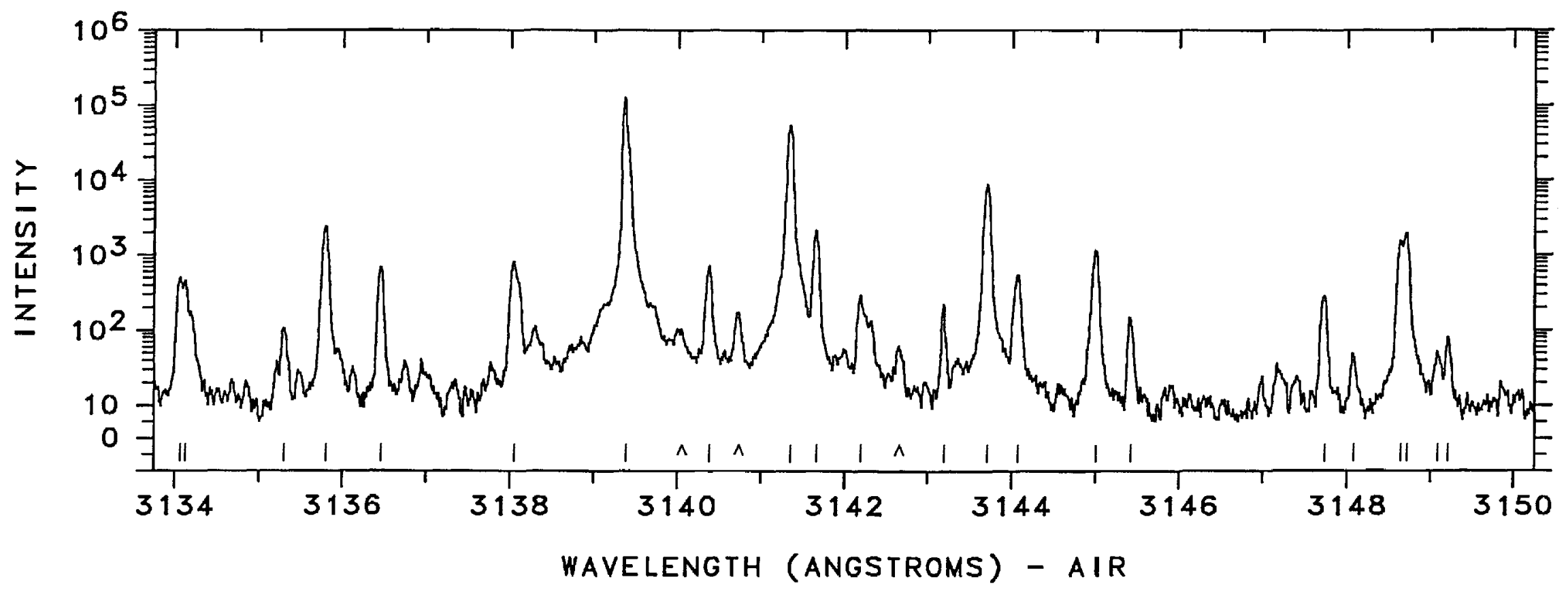




$\begin{array}{llrlrl}3151.1364 & 31725.396 & 6000 & \text { Ne II } & & \text { G } \\ 3151.58 & 31720.9 & 900 & \text { Pt II } & 105962-74241 & \text { AK } \\ 3151.58 & 31720.9 & 900 & \text { Ne II } & & \text { A } \\ 3151.58 & 31720.9 & 900 & \text { Pt II } & 114256-82535 & \text { AK } \\ 3151.95 & 31717.2 & 38 & \text { Pt II } & 46046-77763 & \text { K } \\ 3152.57 & 31711.0 & 63 & \text { Pt I } & 68006-36296 & \text { N } \\ 3153.09 & 31705.7 & 120 & \text { Pt II } & 110158-78452 & \text { K } \\ 3153.4107 & 31702.516 & 1300 & \text { Ne I } & & \text { I } \\ 3153.678 & 31699.83 & 620 & \text { Ne II } & & \text { C } \\ 3153.77 & 31698.9 & 100 & & & \\ 3154.51 & 31691.5 & 42 & & & \\ 3154.794 & 31688.62 & 1500 & \text { Ne II } & & \text { C } \\ 3156.5625 & 31670.862 & 53000 & \text { Pt I } & 10131-41802 & \text { E } \\ 3157.33 & 31663.2 & 76 & & & \\ 3157.87 & 31657.7 & 140 & & & \\ 3159.0704 & 31645.721 & 1800 & \text { Pt II } & 29261-60907 & 13 \\ 3159.57 & 31640.7 & 210 & \text { Pt II } & 117340-85700 & \text { K } \\ 3159.91 & 31637.3 & 140 & \text { Ne II } & & \text { A } \\ 3159.91 & 31637.3 & 140 & \text { Ne III } & & \text { AL } \\ 3160.28 & 31633.6 & 180 & & & \\ 3160.52 & 31631.2 & 46 & \text { Pt II } & 114455-82824 & \text { K } \\ 3161.0013 & 31626.390 & 56 & \text { Pt II } & 105388-73761 & 30 \\ 3162.46 & 31611.8 & 89 & & & \\ 3163.10 & 31605.4 & 51 & \text { Pt I } & 68947-37342 & \text { N } \\ 3163.578 & 31600.63 & 300 & \text { Ne II } & & \text { C } \\ 3164.231 & 31594.11 & 160 & \text { Ne II } & & \text { C } \\ 3164.4295 & 31592.129 & 12000 & \text { Ne II } & & \text { G } \\ 3164.6618 & 31589.810 & 31000 & \text { Ne II } & & \end{array}$

\begin{tabular}{llrlll}
\hline & & & & & \\
3165.01 & 31586.3 & 200 & Ne III & & L \\
3165.6479 & 31579.971 & 11000 & Ne II & & G \\
3166.180 & 31574.66 & 1300 & Ne II & & C \\
3166.79 & 31568.6 & 140 & Pt II & $110020-78452$ & K \\
3167.2244 & 31564.252 & 35000 & Pt II & $101517-69953$ & K \\
3167.55 & 31561.0 & 1100 & Ne I & & \\
3168.12 & 31555.3 & 90 & Pt II & $111162-79607$ & K \\
3169.304 & 31543.54 & 290 & Ne II & & C \\
3169.72 & 31539.4 & 47 & & & \\
3169.84 & 31538.2 & 120 & & & \\
3172.27 & 31514.0 & 45 & Pt II & $117340-85826$ & K \\
3172.474 & 31512.03 & 960 & Ne II & & C \\
3173.5726 & 31501.115 & 8600 & Ne II & & G \\
3174.37 & 31493.2 & 240 & & & \\
3174.49 & 31492.0 & 120 & Pt II & $46046-77538$ & $\mathrm{~K}$ \\
3174.8232 & 31488.707 & 660 & Pt I & $18566-50055$ & E \\
3175.12 & 31485.8 & 32 & Ne III & & L \\
3175.44 & 31482.6 & 31 & Pt II & $114455-82972$ & K \\
3175.61 & 31480.9 & 46 & Ne III & & L \\
3175.90 & 31478.0 & 440 & Pt II & $121651-90173$ & K \\
3176.1199 & 31475.852 & 5900 & Ne II & & G \\
3176.548 & 31471.61 & 1400 & Ne II & & C \\
3177.745 & 31459.76 & 200 & Ne II & & \\
3178.80 & 31449.3 & 29 & & & \\
3179.02 & 31447.1 & 150 & & & \\
3179.32 & 31444.2 & & Ca II & & \\
3181.58 & 31421.8 & 120 & & & \\
& & & & &
\end{tabular}




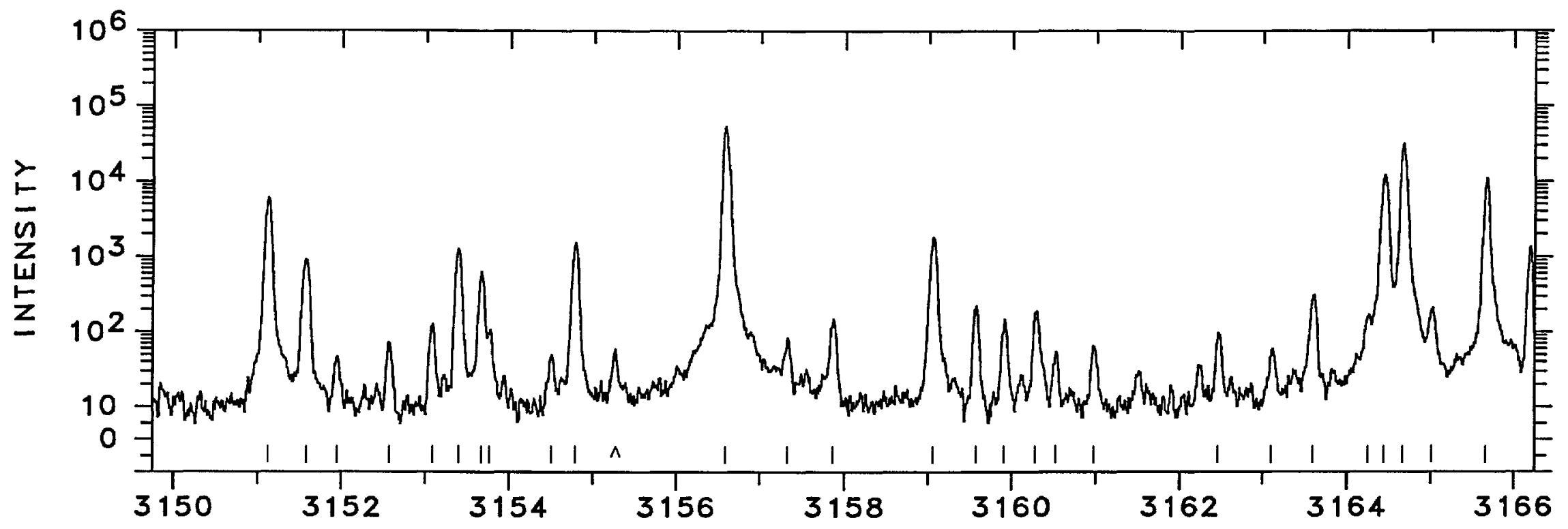

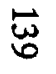

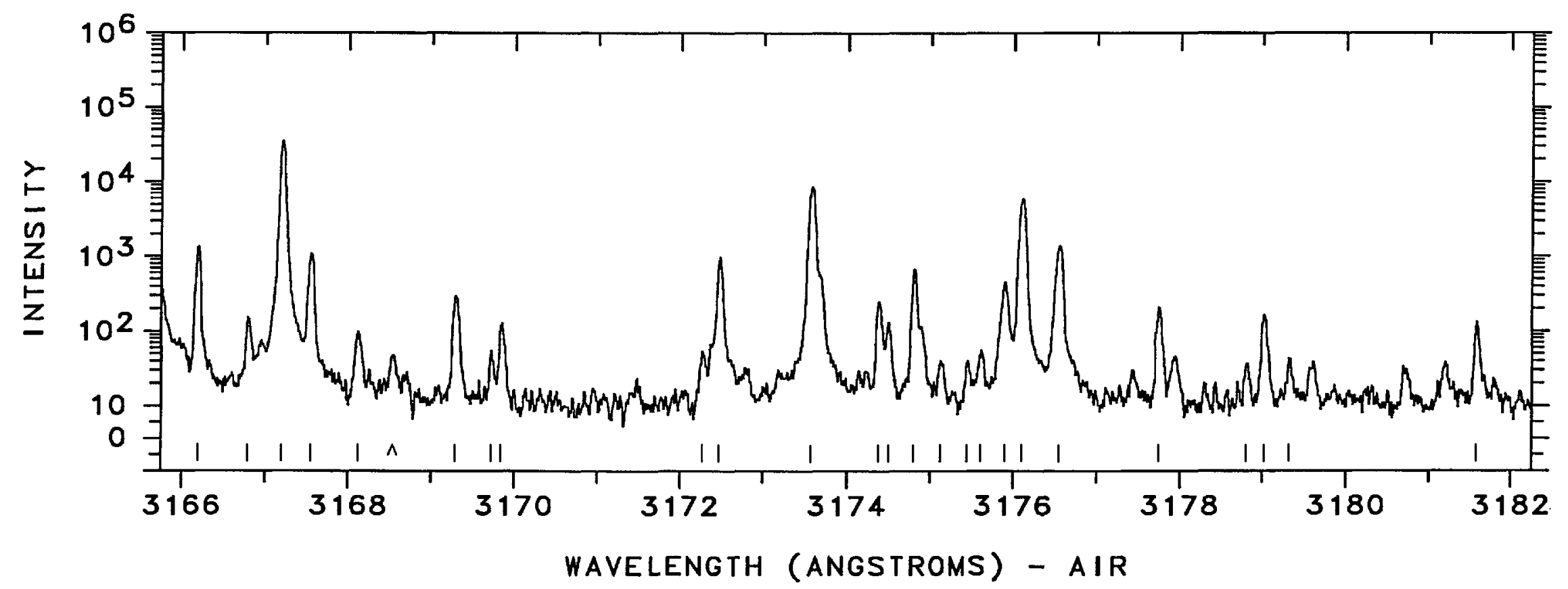


WAVELENGTH WAVE NUMBER INTENSITY

CLASSIFICATION

\begin{tabular}{llr}
\hline & & \\
3184.68 & 31391.3 & 72 \\
3184.88 & 31389.3 & 360 \\
3185.53 & 31382.9 & 38 \\
3187.42 & 31364.3 & 600 \\
3187.5763 & 31362.729 & 5700 \\
3188.0700 & 31357.873 & 450 \\
3188.7410 & 31351.275 & 17000 \\
3189.55 & 31343.3 & 750 \\
3190.60 & 31333.0 & 59 \\
3190.8630 & 31330.426 & 7600 \\
3191.10 & 31328.1 & 120 \\
3191.43 & 31324.9 & 340 \\
3191.43 & 31324.9 & 340 \\
3191.75 & 31321.7 & 89 \\
3192.5031 & 31314.331 & 1300 \\
3194.34 & 31296.3 & 520 \\
3194.5754 & 31294.018 & 28000 \\
3196.70 & 31273.2 & 160 \\
3197.7161 & 31263.283 & 4400 \\
3198.5862 & 31254.779 & 160000 \\
3198.916 & 31251.56 & 3100 \\
3199.06 & 31250.2 & 970
\end{tabular}

CODE

\section{Ne I I}

Pt II

$\mathrm{Ne}$ II

Pt II $105962-74619 \mathrm{~K}$

Pt II $50564-81897 \mathrm{~K}$

Ne II

Pt II 105086- 73761 AK

Pt I 68169- 36844 AN

Pt I 68912- $37590 \mathrm{~N}$

Pt I 18566- 49880

Pt II 36484- $67780 \mathrm{~K}$

Ne II

Pt II 109527- 78254 G

$\begin{array}{lrl}\text { Pt II } & 109527-78254 & K \\ \text { Pt II } & 96614-65351 & 12\end{array}$

Pt II

$\begin{array}{ll}\mathrm{Ne} & \text { I I } \\ \mathrm{Ne} & \text { I I }\end{array}$

Pt I

$68094-36844$ AN
WAVELENGTH WAVE NUMBER INTENSITY

CLASSIFICATION

CODE

3199.06

31250.2

3200.01

31250.2

970

Pt 11 106434- 75184 AK

48000
150
67000

31240.9

67000
140

$\begin{array}{ll}31225.8 & 140 \\ 31224.4 & 220\end{array}$

$\begin{array}{ll}3201.55 & 31225.8 \\ 3201.70 & 31224.4\end{array}$

220
1200

$3201.81 \quad 31223.3$

$3204.0364 \quad 31201.615 \quad 120000$

$3205.6023 \quad 31186.374 \quad 2800$

$3206.44 \quad 31178.2$

3207.43

31178.2

2800

31168.6

57
160

$3207.68 \quad 31166$.

3207.89

31164.1

$3208.27 \quad 31160.4$

3208.84

3208.9647

31154.9

$3209.3554-31149.905 \quad 37000$

$3210.52 \quad 31138.6 \quad 490$

$3210.52 \quad 31138.6$

$3211.994 \quad 31124.32$

3212.3775

31120.602
31107.459

3213.7348

Pt II 101199-69953 13

Pt I 68831- 37590

Pt I 13496- 44730 E

Pt I 6567- 37769 E

Pt I 65308- 34122 N

Pt I 68947- 37769 N

Pt I 68759- $37590 \mathrm{~N}$

Pt II 110258- 79092

$\begin{array}{lll}\text { Ne I I } & & \text { G } \\ \text { Ne I I } & & \text { G } \\ \text { Pt I I } & 101517-70379 & \text { K } \\ & & \\ \text { Ne I I } & & \text { C } \\ \text { Pt I } & 15501-46622 & \text { E } \\ \text { Ne II } & & \text { G }\end{array}$



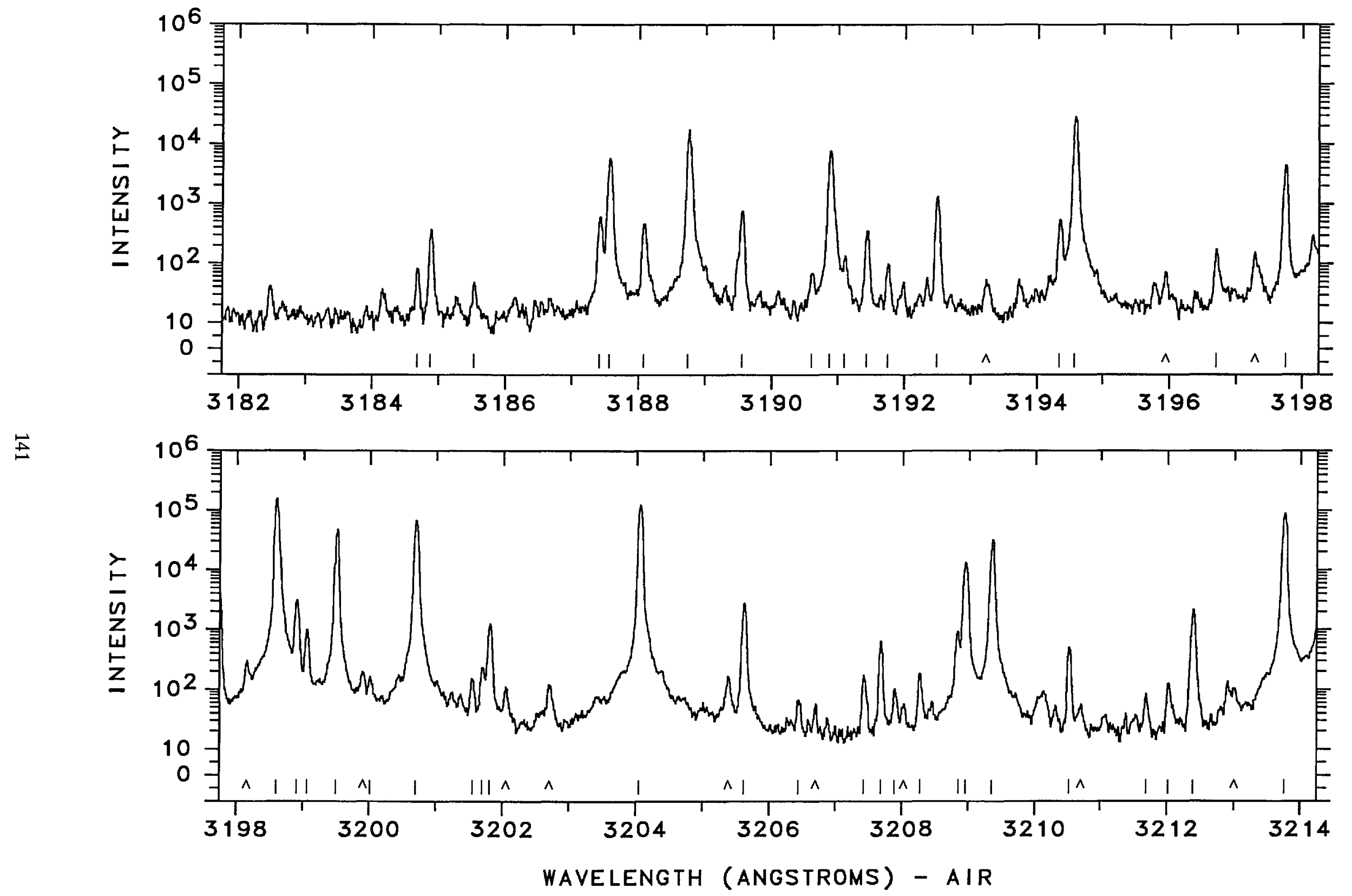


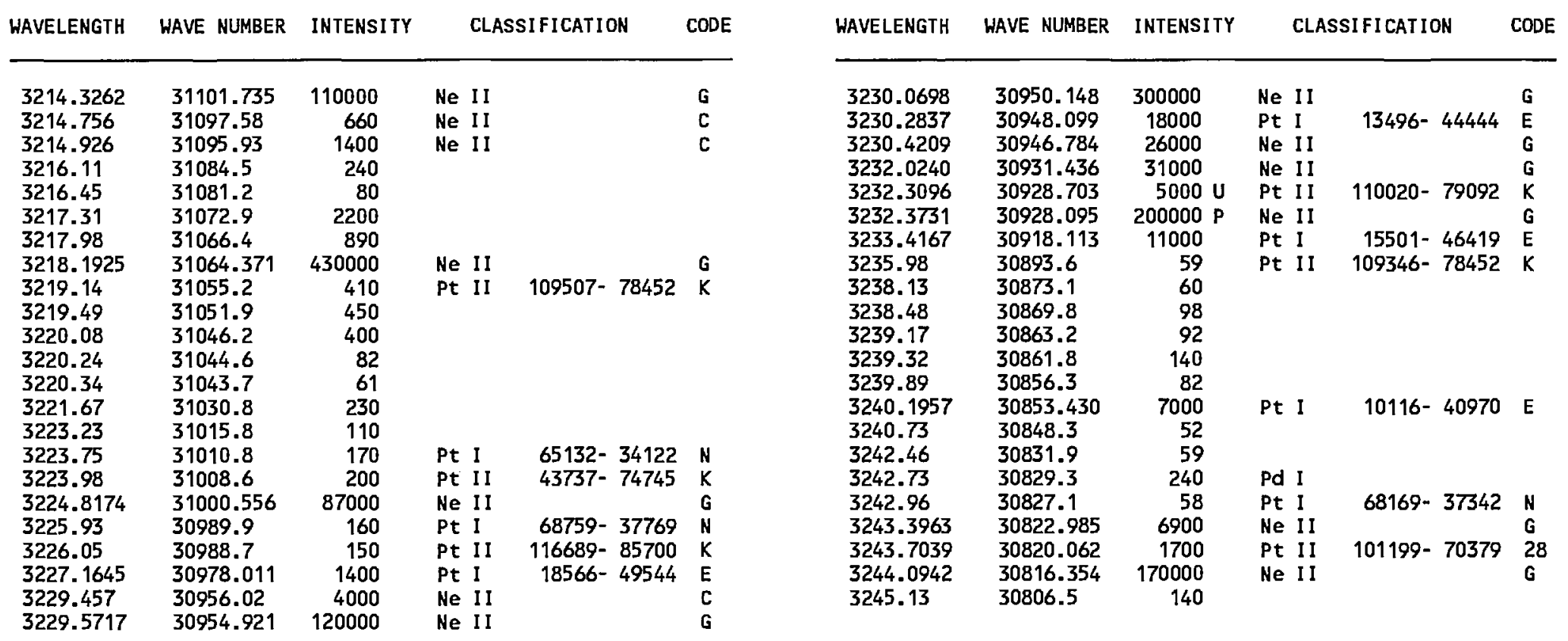




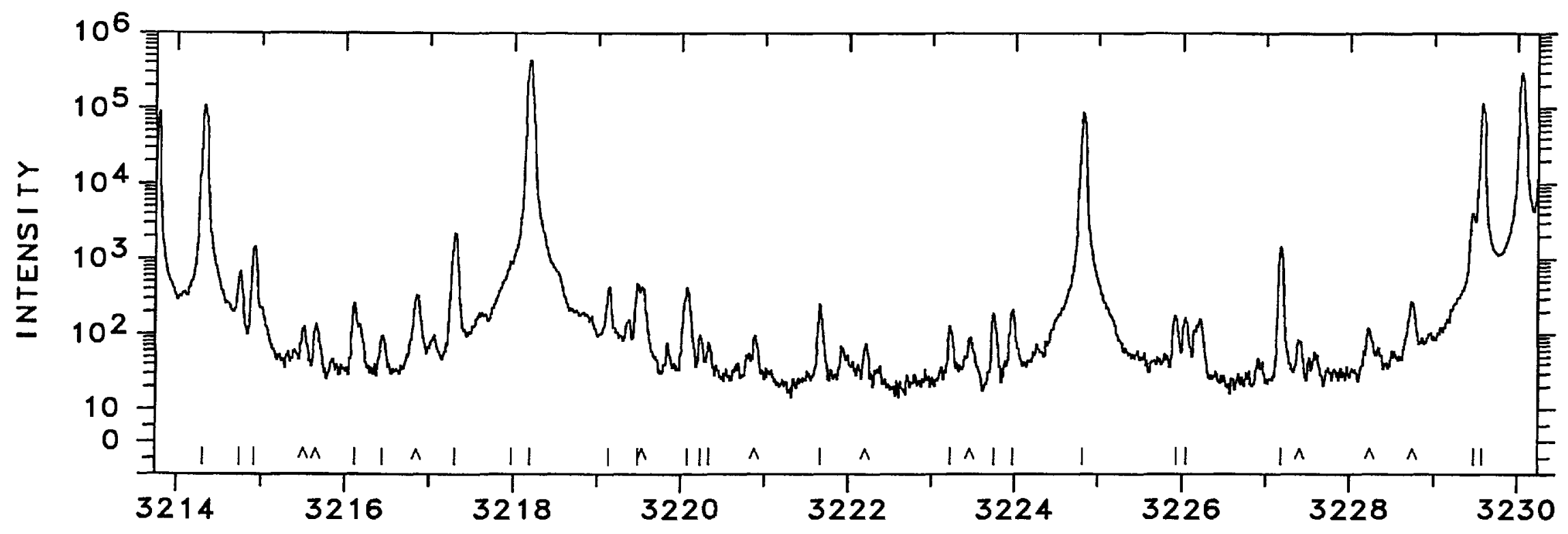

点

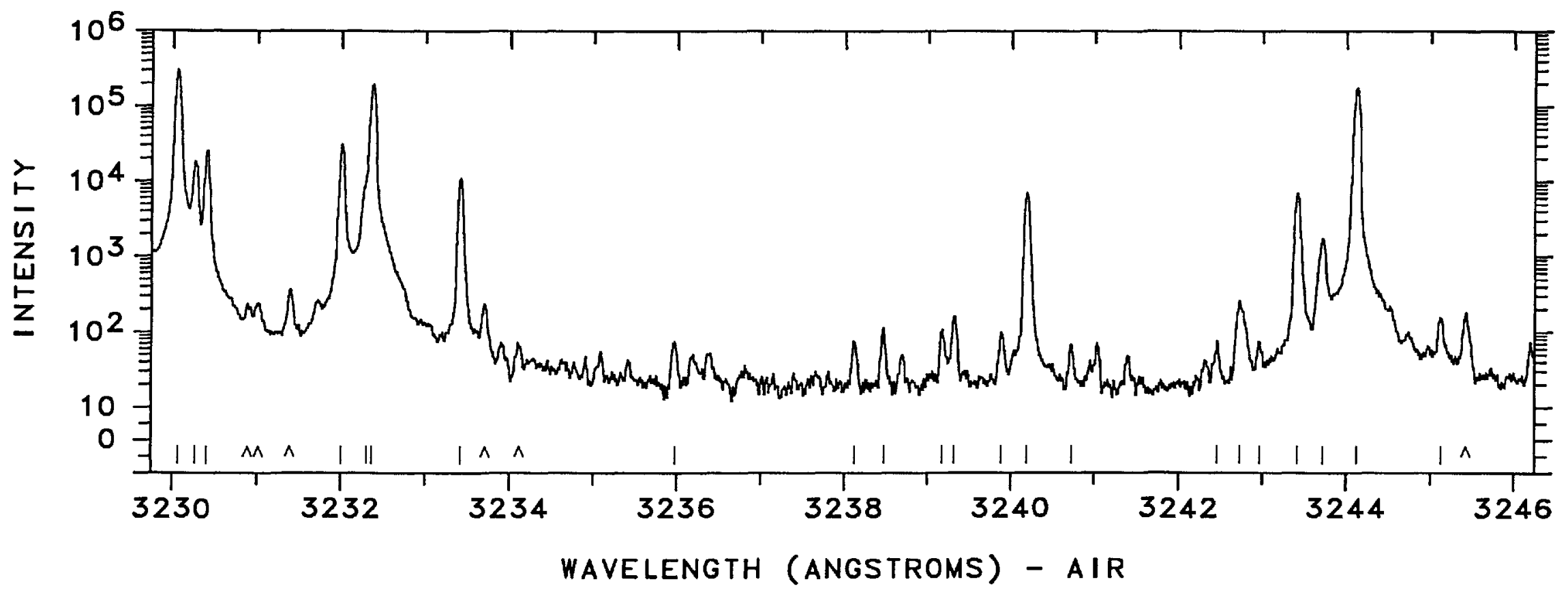




\begin{tabular}{llrlll} 
WAVELENGTH & WAVE NUMBER & INTENSITY & CLASSIFICATION & CODE \\
\hline 3246.21 & 30796.3 & 57 & & & \\
3246.77 & 30791.0 & 44 & & & \\
3247.28 & 30786.1 & 150 & & & \\
3247.53 & 30783.8 & & CU I & & \\
3248.1314 & 30778.053 & 5000 & Ne II & & G \\
3248.3449 & 30776.030 & 13000 P & Ne I I & & G \\
3248.3787 & 30775.710 & 3100 & Ne II & & G \\
3248.59 & 30773.7 & 290 & & & \\
3249.26 & 30767.4 & 76 & & & G \\
3250.3571 & 30756.978 & 29000 & Ne II & & \\
3250.82 & 30752.6 & 200 & Pt I & $68094-37342$ & N \\
3251.09 & 30750.0 & 200 & & & \\
3251.9787 & 30741.642 & 47000 & Pt I & $10131-40873$ & E \\
3253.18 & 30730.3 & 200 & Pt I & $68072-37342$ & N \\
3254.72 & 30715.8 & 69 & & & \\
3255.4223 & 30709.124 & 3100 & Ne II & & G \\
3255.9088 & 30704.536 & 72000 & Pt I & $6140-36844$ & E \\
3256.53 & 30698.7 & 930 & Pt I & $64379-33680$ & N \\
3257.26 & 30691.8 & 120 & & & \\
3257.84 & 30686.3 & 230 & & & \\
3259.7308 & 30668.536 & 8200 & Pt I & $15501-46170$ & E
\end{tabular}

\begin{tabular}{|c|c|c|c|c|c|}
\hline WAVELENGTH & WAVE NUMBER & INTENSITY & CLA & SIFICATION & CODE \\
\hline $\begin{array}{l}3260.14 \\
3261.0683 \\
3261.6887 \\
3262.5311 \\
3263.4128 \\
3263.58 \\
3263.92 \\
3266.02 \\
3268.4170 \\
3268.72 \\
3269.71 \\
3269.8705 \\
3270.8010 \\
3271.94 \\
3272.78 \\
3273.56 \\
3274.00 \\
3275.1810 \\
3276.54 \\
3277.19\end{array}$ & $\begin{array}{l}30664.7 \\
30655.958 \\
30650.127 \\
30642.213 \\
30633.935 \\
30632.4 \\
30629.2 \\
30609.5 \\
30587.034 \\
30584.2 \\
30574.9 \\
30573.438 \\
30564.741 \\
30554.1 \\
30546.3 \\
30539.0 \\
30534.9 \\
30523.866 \\
30511.2 \\
30505.2\end{array}$ & $\begin{array}{r}350 \\
1300 \\
8200 \\
410 \\
6400 \\
920 \\
94 \\
2600 \\
15000 \\
130 \\
470 \\
12000 \\
11000 \\
260 \\
1600 \\
46 \\
130 \\
11000 \\
130 \\
76\end{array}$ & 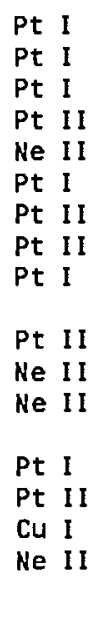 & $\begin{array}{r}68006-37342 \\
10131-40787 \\
64330-33680 \\
105388-74745 \\
\\
64312-33680 \\
64003-94633 \\
105794-75184 \\
64267-33680 \\
110258-79683\end{array}$ & $\begin{array}{l}N \\
E \\
N \\
26 \\
G \\
N \\
K \\
K \\
N \\
\\
K \\
G \\
G\end{array}$ \\
\hline
\end{tabular}




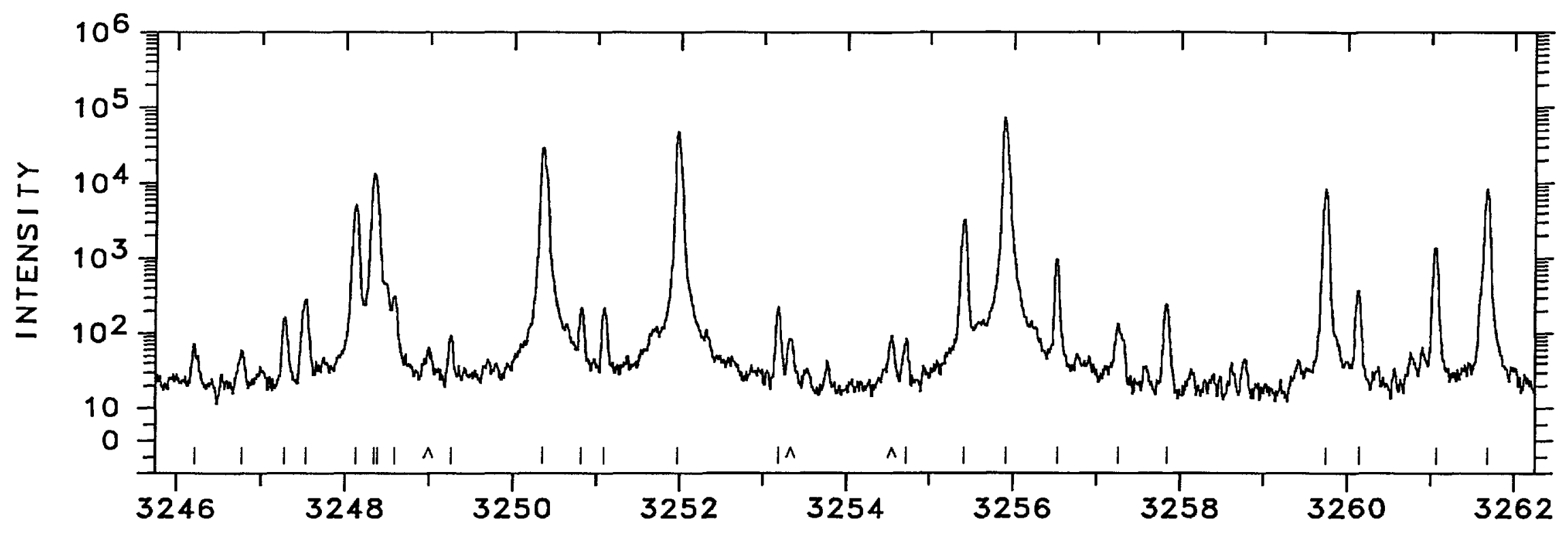

虫

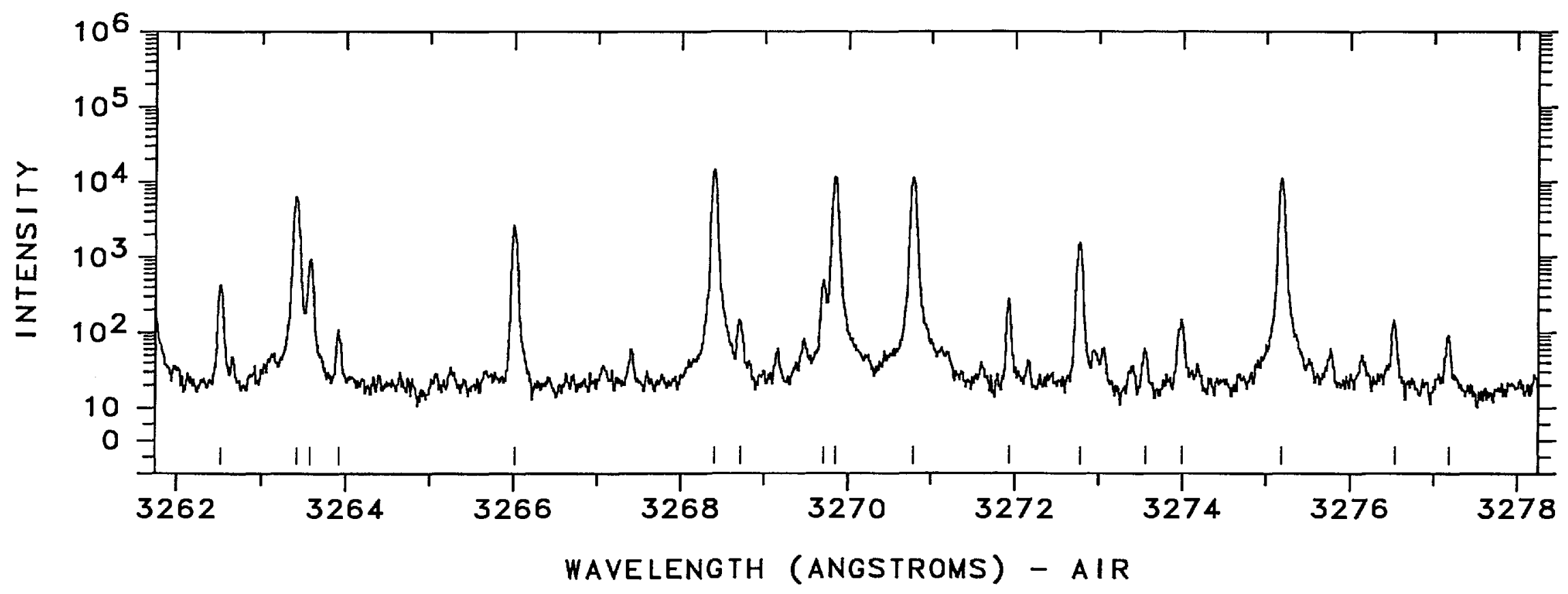




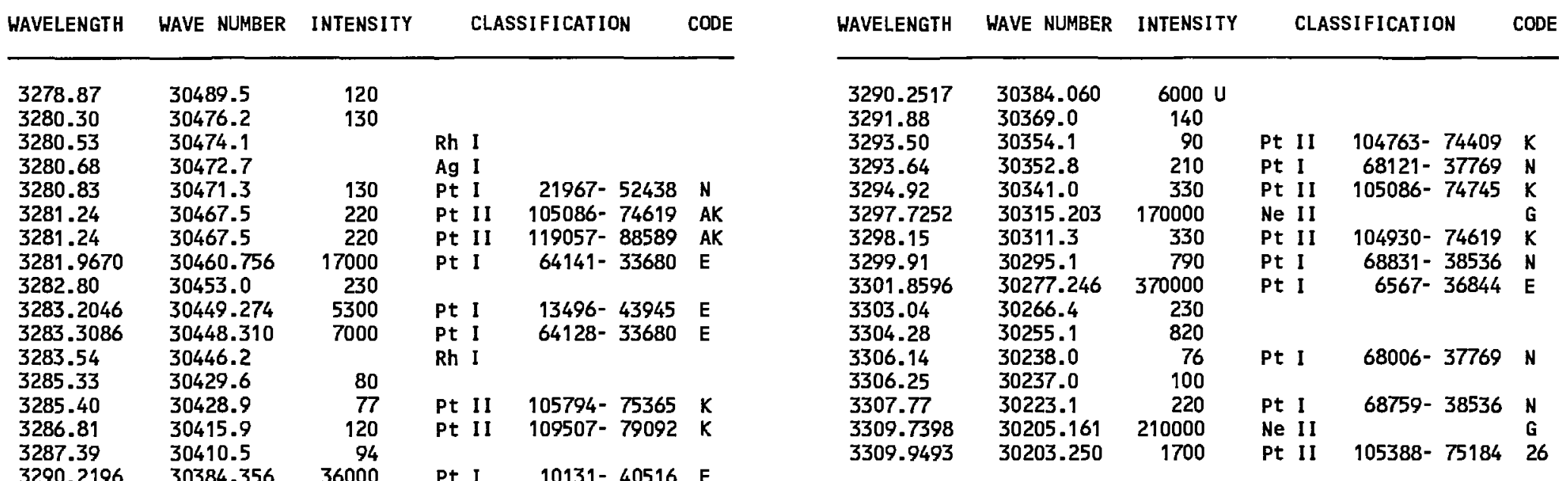




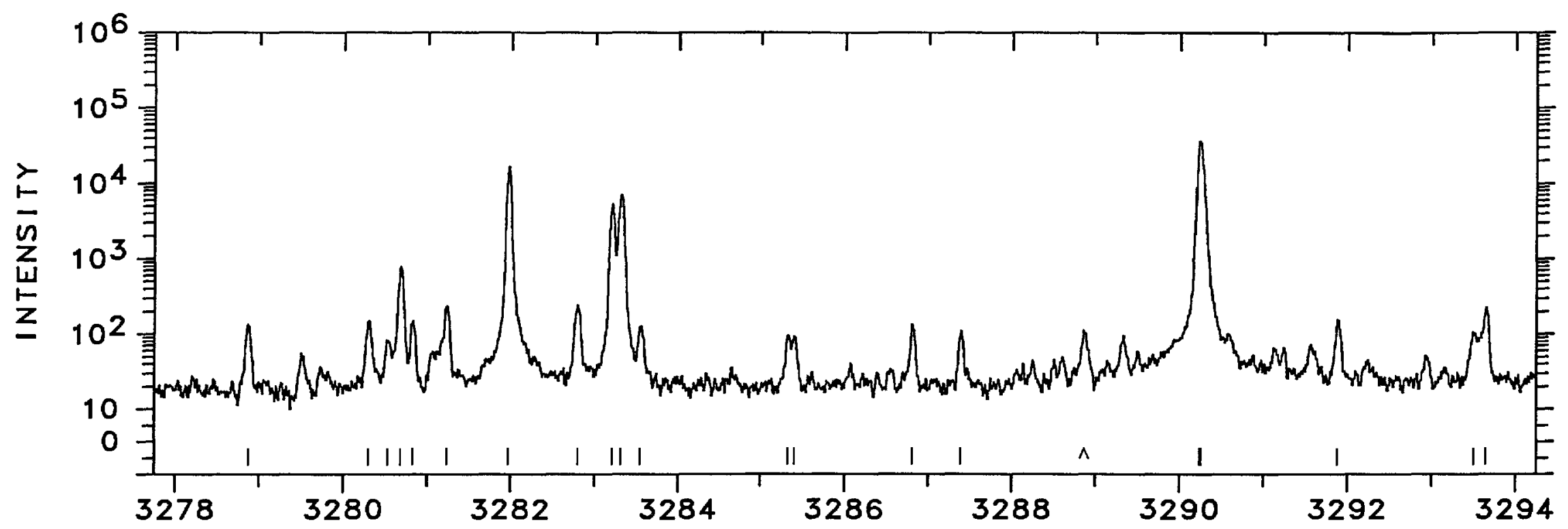

吾

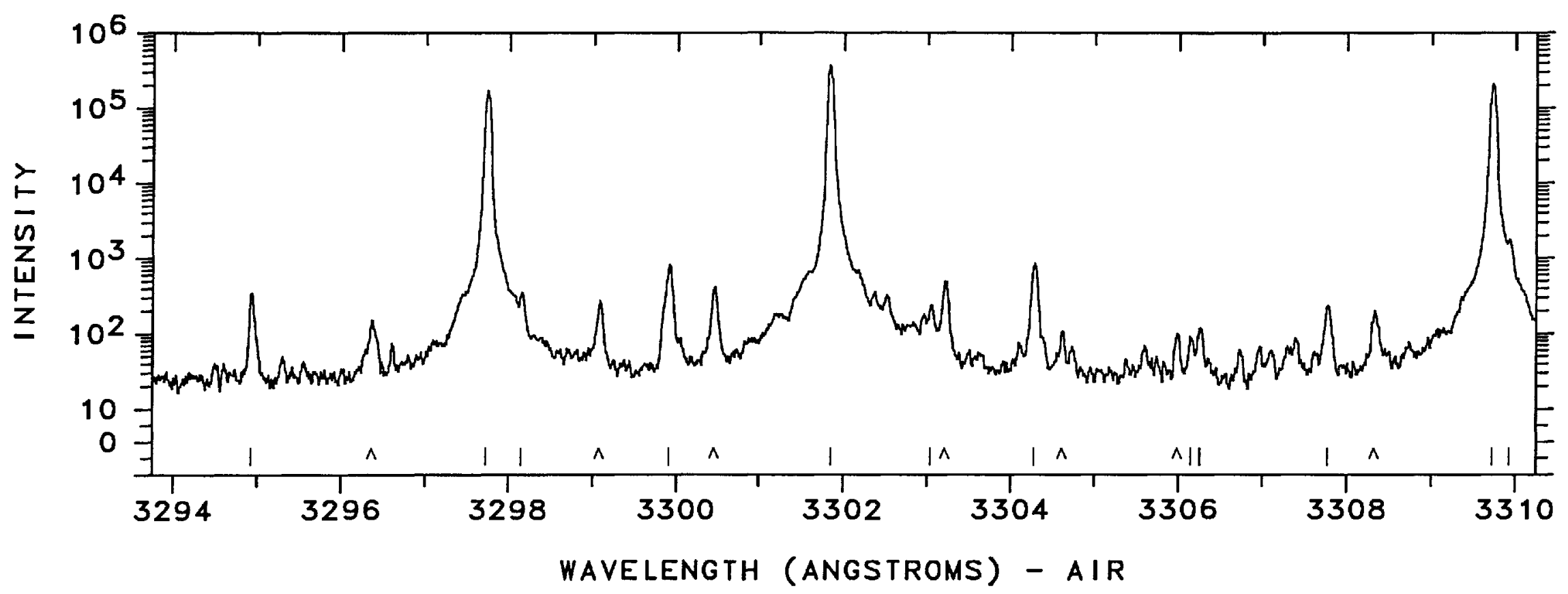




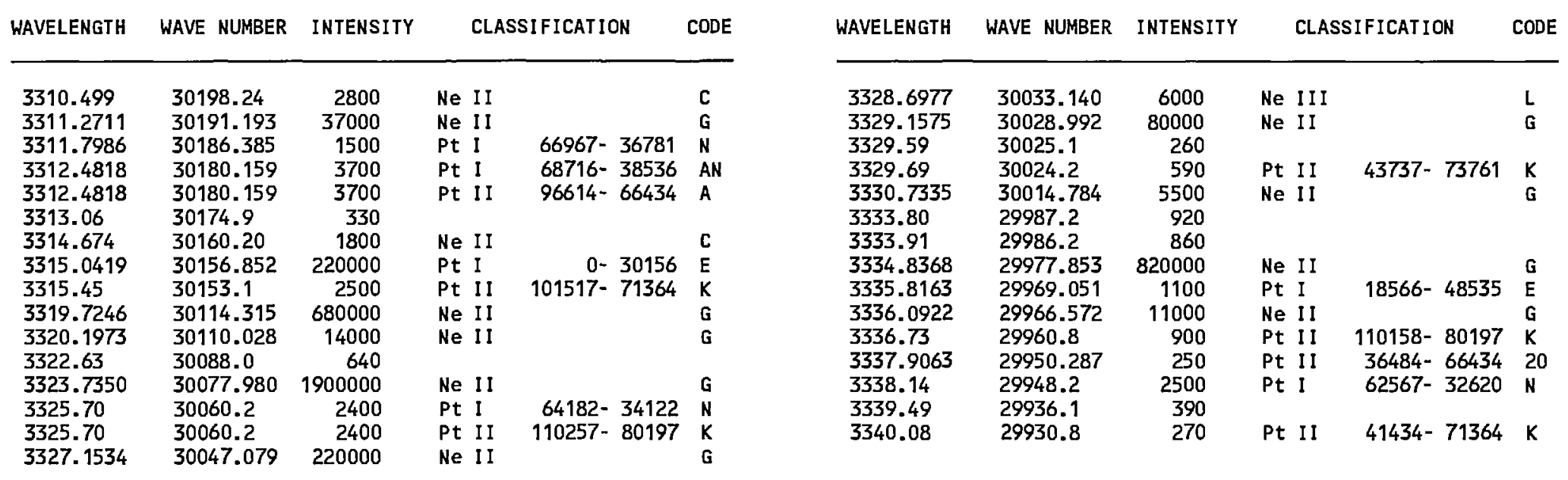




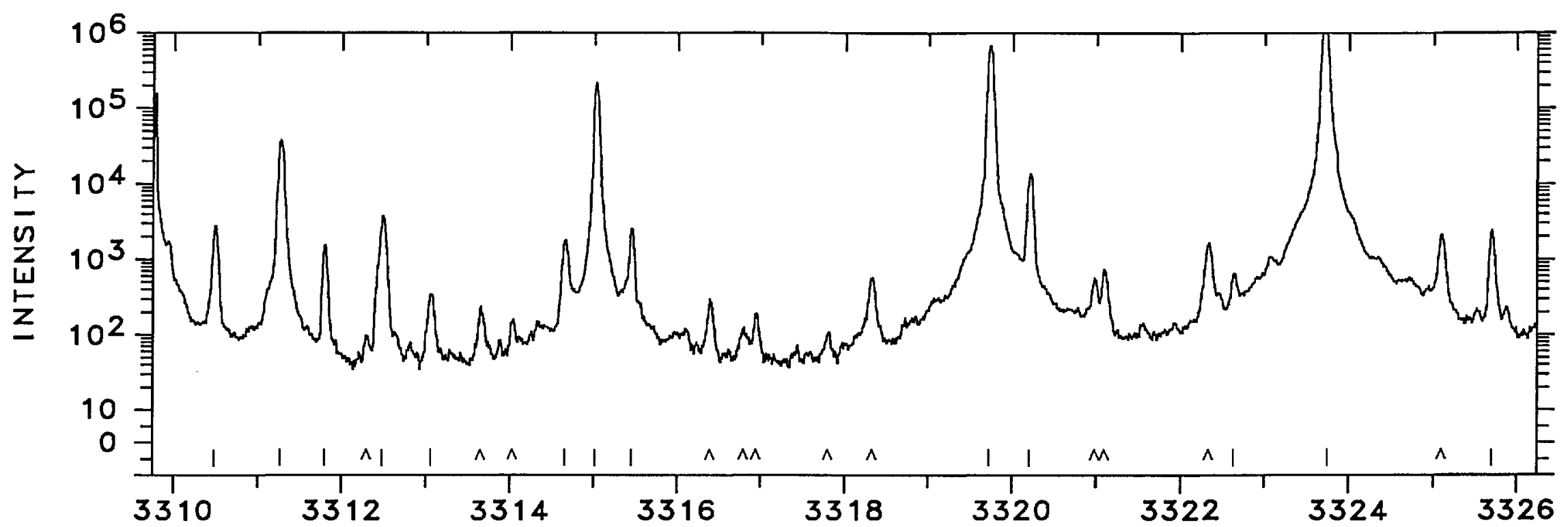

古

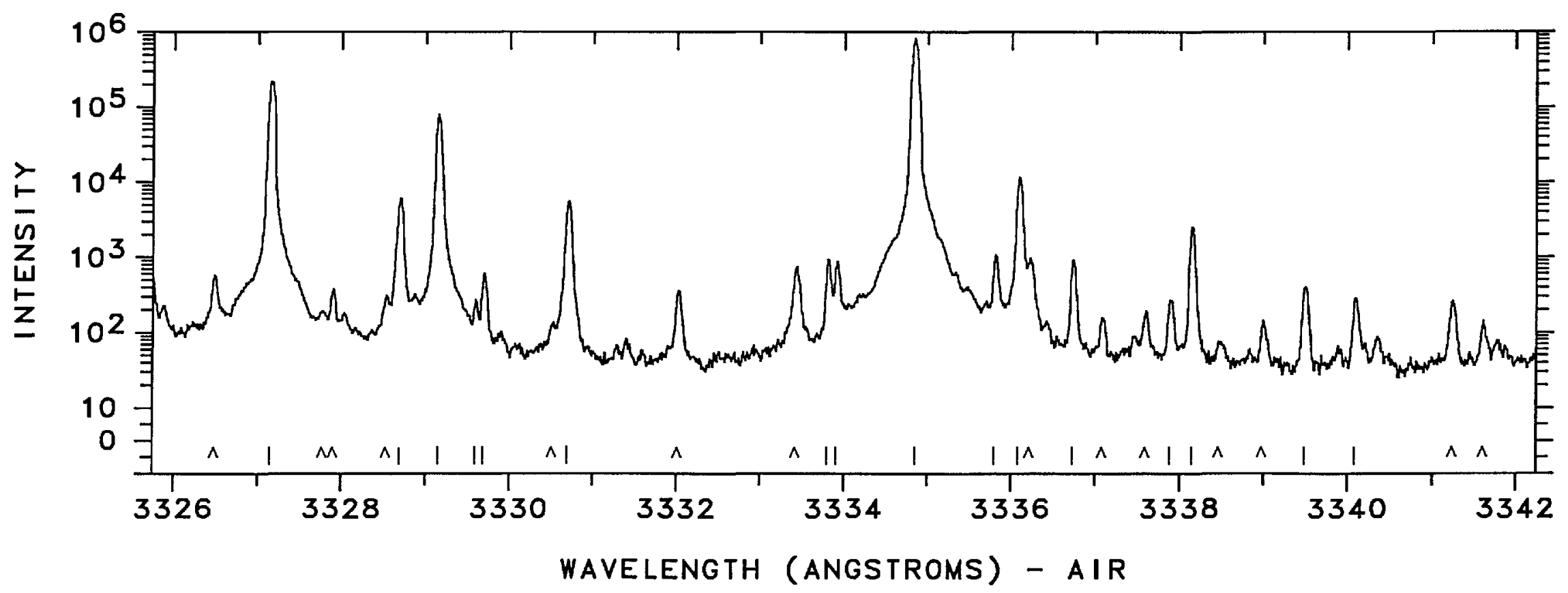




\begin{tabular}{llcllll} 
WAVELENGTH & WAVE NUMBER & INTENSITY & \multicolumn{2}{c}{ CLASSIFICATION } & CODE \\
\hline & & & & & \\
\hline 3343.30 & 29902.0 & 960 & Pt II & $105086-75184$ & $\mathrm{~K}$ \\
3343.8961 & 29896.640 & 14000 & Pt I & $15501-45398$ & E \\
3344.3956 & 29892.175 & 230000 & Ne II & & G \\
3345.2555 & 29884.491 & 10000 & Pt II & $101199-71314$ & 17 \\
3345.4544 & 29882.715 & 1200000 & Ne II & & G \\
3345.8304 & 29879.356 & 210000 & Ne II & & G \\
3345.8678 & 29879.023 & 15000 & & & & \\
3349.08 & 29850.4 & 190 & & & \\
3350.88 & 29834.3 & 3000 & Pt II & $101199-71364$ & $\mathrm{~K}$ \\
3351.7492 & 29826.595 & 320 & Ne I & & B \\
3351.94 & 29824.9 & 800 & Pt II & $106434-76610$ & $\mathrm{~K}$ \\
3353.567 & 29810.43 & 3400 & Ne II & & $\mathrm{C}$ \\
3355.0176 & 29797.539 & 550000 & Ne II & & $\mathrm{G}$ \\
3356.3078 & 29786.084 & 6800 & Ne II & & $\mathrm{G}$
\end{tabular}

\begin{tabular}{lcclll} 
WAVELENGTH & WAVE NUMBER & INTENSITY & CLASSIFICATION & CODE \\
\hline 3357.8190 & 29772.680 & 36000 & Ne I I & G \\
3360.2707 & 29750.958 & 6600 & Ne I I & G \\
3360.5977 & 29748.063 & 210000 & Ne II & G \\
3360.8058 & 29746.222 & 1000 & & & G \\
3362.1623 & 29734.220 & 26000 & Ne II & G \\
3362.7067 & 29729.407 & 14000 & Ne II & G \\
3362.9378 & 29727.363 & 9400 L & Ne II & A \\
3366.9903 & 29691.585 & 8900 & Pt I & $13496-43187$ & A \\
3366.9903 & 29691.585 & 8900 & Ne II & G \\
3367.2164 & 29689.592 & 200000 & Ne II & G \\
3369.8073 & 29666.766 & 49000 & Ne I & C \\
3369.9068 & 29665.890 & 150000 & Ne I & \\
3371.797 & 29649.26 & 39000 & Ne I I &
\end{tabular}




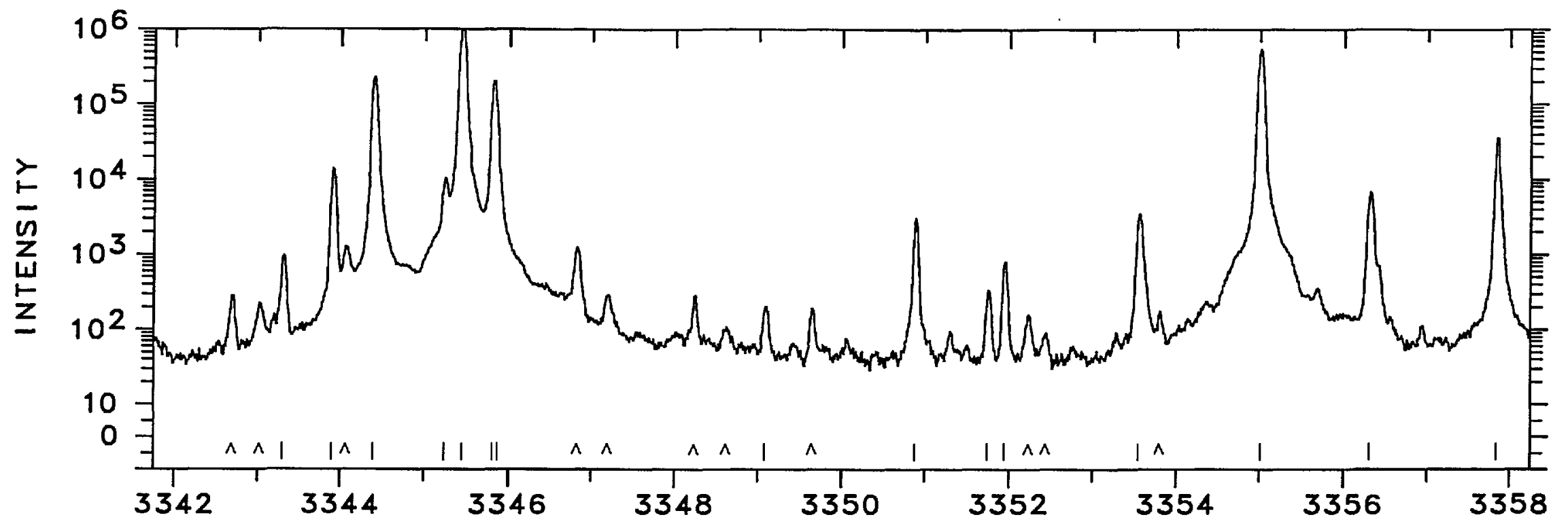

$\vec{s}$

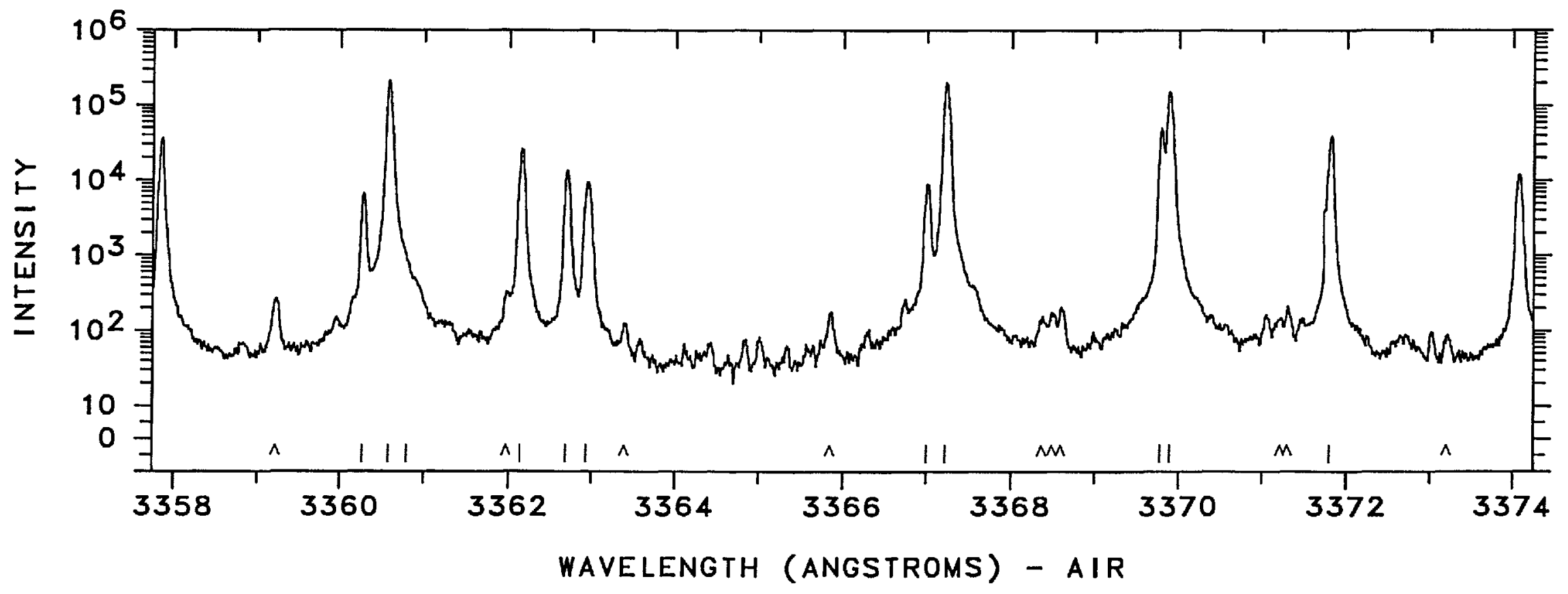




\begin{tabular}{llrlll} 
WAVELENGTH & HAVE NUMBER & INTENSITY & \multicolumn{2}{c}{ CLASSIFICATION } & CODE \\
\hline & & & & \\
3374.0607 & 29629.368 & 12000 & Ne II & & G \\
3375.6490 & 29615.427 & 5300 & Ne I & & B \\
3376.33 & 29609.5 & 330 & Pt II & $112433-82824$ & K \\
3377.1543 & 29602.228 & 28000 & Ne I I & & G \\
3378.2193 & 29592.895 & 1200000 & Ne I I & & G \\
3379.3209 & 29583.249 & 5500 & Ne I I & & G \\
3380.44 & 29573.5 & 200 & & & \\
3380.99 & 29568.6 & 5200 & Pt I I & $101517-71948$ & $\mathrm{~K}$ \\
3382.89 & 29552.0 & & Ag I & & \\
3383.8121 & 29543.986 & 670 & Pt II & $36484-66028$ & 15 \\
3384.82 & 29535.2 & 83 & Pt I I & $46046-75581$ & $\mathrm{~K}$ \\
3385.62 & 29528.2 & 130 & Ne I I & & C \\
3386.202 & 29523.13 & 4000 & Ne I I & & G \\
3388.4169 & 29503.837 & 150000 & Ne I & &
\end{tabular}

\begin{tabular}{|c|c|c|c|c|c|}
\hline HAVELENGTH & WAVE NUMBER & INTENSITY & $\mathrm{CL}$ & SIFICATION & CODE \\
\hline $\begin{array}{l}3388.77 \\
3388.9431 \\
3390.552 \\
3391.38 \\
3392.606 \\
3392.8006 \\
3393.1812 \\
3396.83 \\
3397.866 \\
3404.18 \\
3404.59 \\
3404.8208 \\
3405.89\end{array}$ & $\begin{array}{l}29500.8 \\
29499.256 \\
29485.26 \\
29478.1 \\
29467.41 \\
29465.717 \\
29462.412 \\
29430.8 \\
29421.79 \\
29367.2 \\
29363.7 \\
29361.696 \\
29352.5\end{array}$ & $\begin{array}{r}920 \\
12000 \\
3500 \\
550 \\
8700 \\
650000 \\
4400 \\
\\
3800 \\
140 \\
42000 \\
830\end{array}$ & $\begin{array}{l}\text { Pt I I } \\
\text { Ne I I } \\
\text { Ne I I } \\
\text { Pt I I } \\
\text { Ne I I } \\
\text { Ne I I } \\
\text { Ne I I } \\
\text { Rh I } \\
\text { Ne I I } \\
\text { Pd I } \\
\text { Ne I I } \\
\text { Pt I I }\end{array}$ & $\begin{array}{r}105962-76461 \\
64003-93482\end{array}$ & $\begin{array}{l}K \\
G \\
C \\
K M \\
C \\
G \\
G \\
C\end{array}$ \\
\hline
\end{tabular}




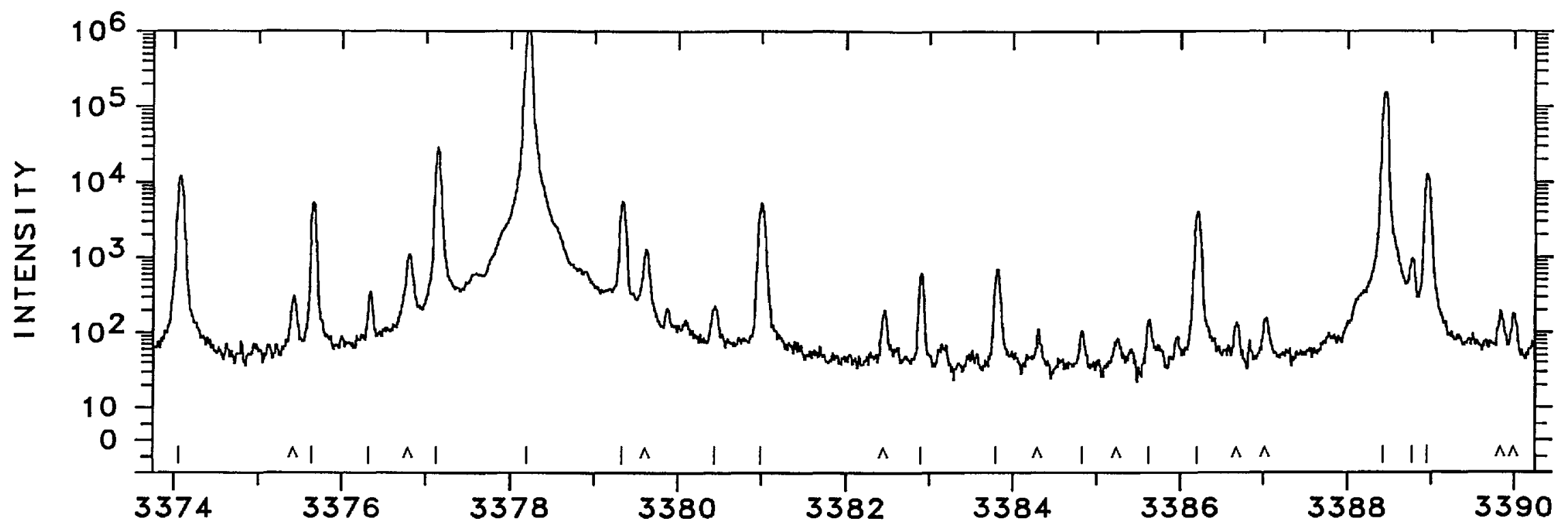

岛

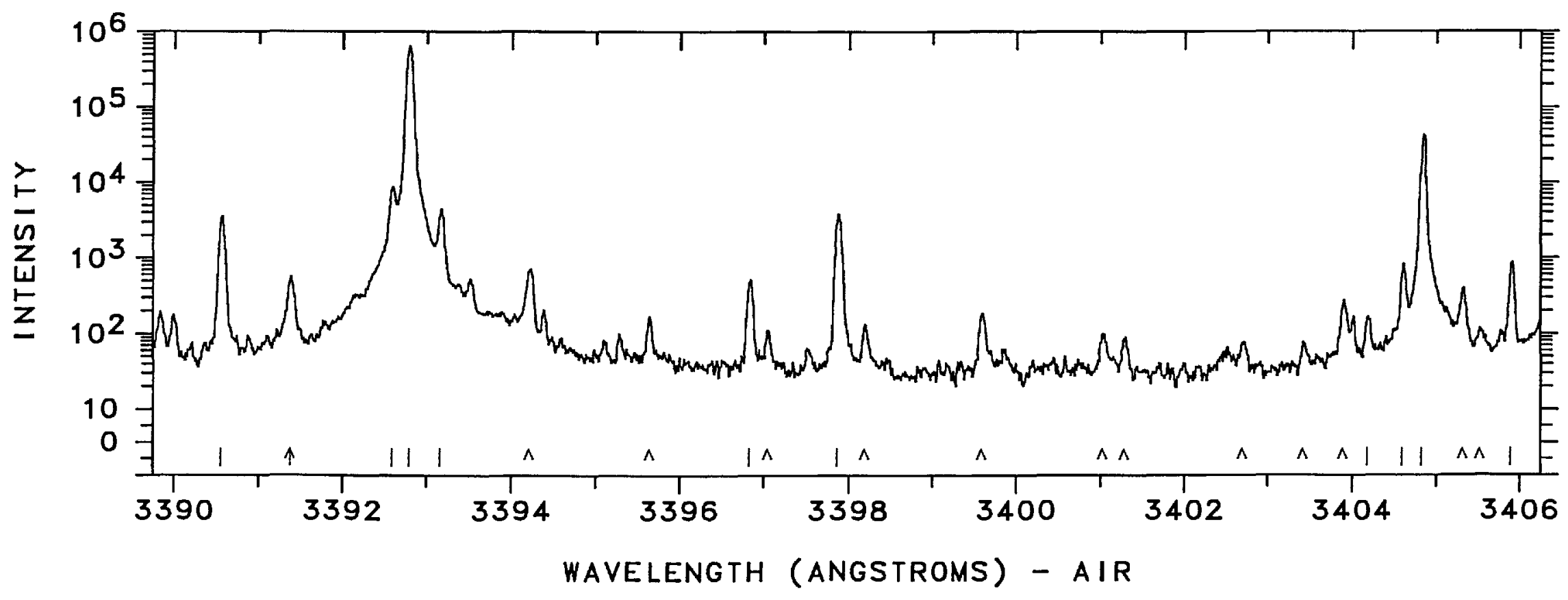




\section{WAVELENGTH WAVE NUMBER INTENSITY}

CLASSIFICATION

CODE

3406.52
3406.9451
3408.1308
3411.3604
3412.0248
3413.1453
3414.4564
3414.8886
3416.9126
3417.0828
3417.6870
3417.8034
3417.9029
3418.0052
3419.44
3420.3407

29347.12500

29333.181 1300000

Pt I

Ne II

64668- $35321 \mathrm{~N}$

$9305.412 \quad 3200$

Pt 1

823- 30156

299.705

350

Ne II

110158- $80858 \mathrm{~K}$

15000

$\mathrm{Ne} I 1$

$68094-38815$

1000

5000

33000

$29251.165 \quad 220000$

29250.169

Pt I

Ne II

Pt I 68072- 38815

$3249.318 \quad 320000$

Ne II

35000

Pt II

250

$29228.471 \quad 200$
WAVELENGTH WAVE NUMBER INTENSITY

CLASSIFI CATION

CODE

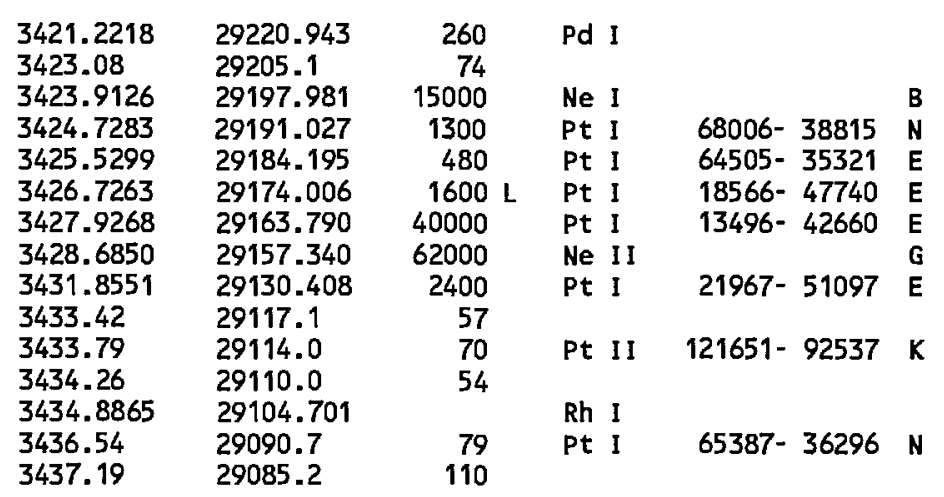




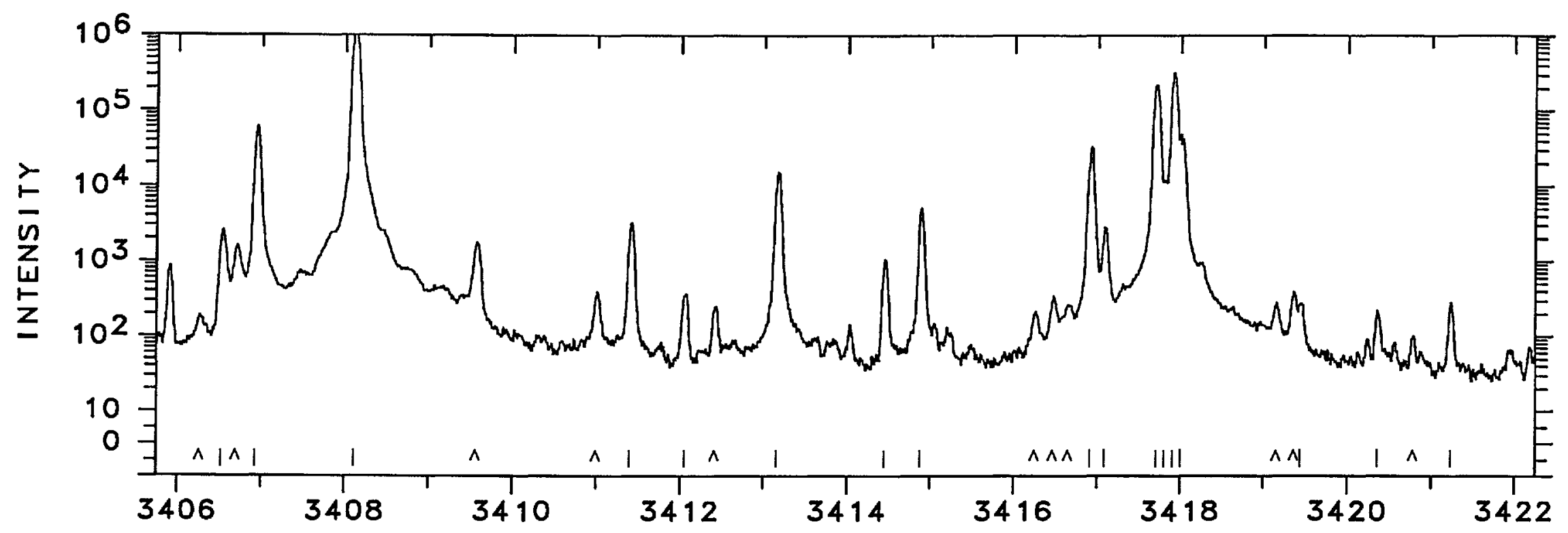

幽

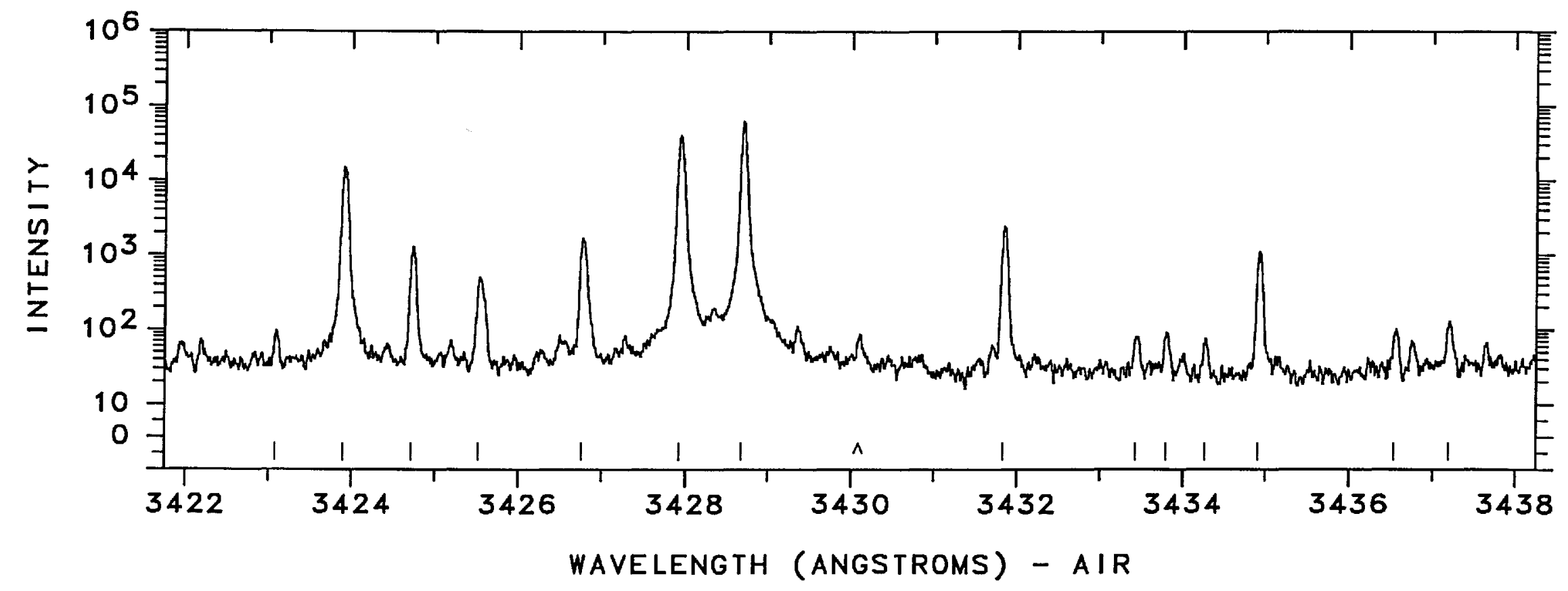




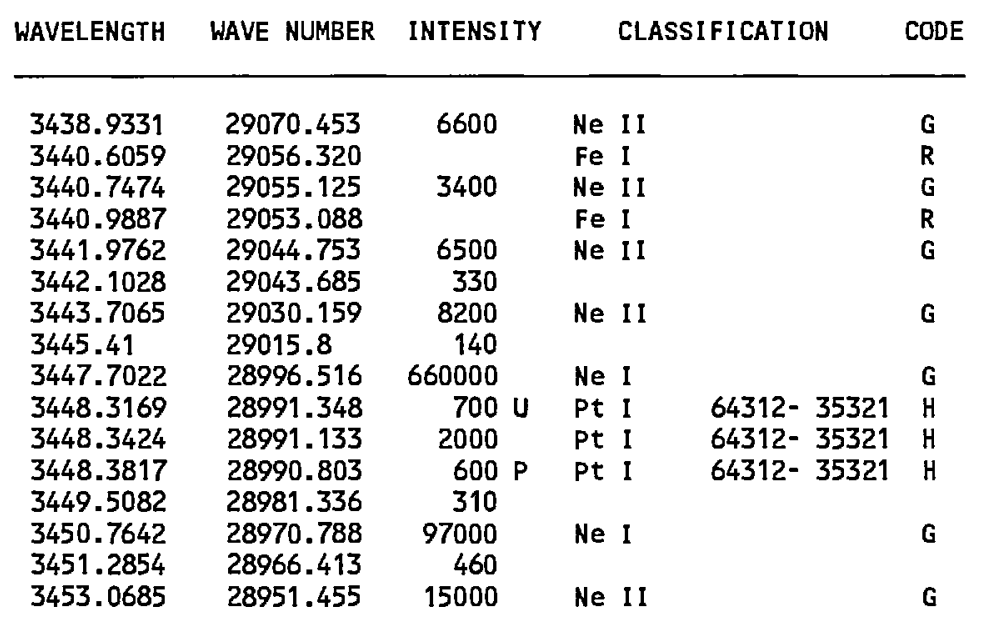

\begin{tabular}{lcclll} 
WAVELENGTH & WAVE NUMBER & INTENSITY & \multicolumn{2}{c}{ CLASSI I ICATION } & CODE \\
\hline & & & & & \\
3454.1940 & 28942.022 & 410000 & Ne I & & G \\
3454.7720 & 28937.180 & 30000 & Ne I I & & G \\
3455.7881 & 28928.672 & 190 & Pt I I & $48591-77519$ & K \\
3456.6081 & 28921.809 & 43000 & Ne II & & G \\
3457.0079 & 28918.464 & 3300 & Ne I I & & G \\
3457.084 & 28917.83 & 3300 & Ne II & & C \\
3459.3197 & 28899.140 & 50000 & Ne II & & G \\
3459.4946 & 28897.679 & 820 & Pt I & $26638-55536$ & E \\
3460.5233 & 28889.089 & 170000 & Ne I & & G \\
3462.03 & 28876.5 & & Rh I & & \\
3463.9094 & 28860.849 & 1200 U & Pt I & $64182-35321$ & H \\
3463.9340 & 28860.644 & 3600 P & Pt I & $64182-35321$ & H \\
3463.9873 & 28860.200 & 1000 & Pt I & $64182-35321$ & H \\
3464.3377 & 28857.281 & 240000 & Ne I & & G \\
3465.8607 & 28844.601 & & Fe I & & R \\
3466.5778 & 28838.634 & 290000 & Ne I & & G
\end{tabular}




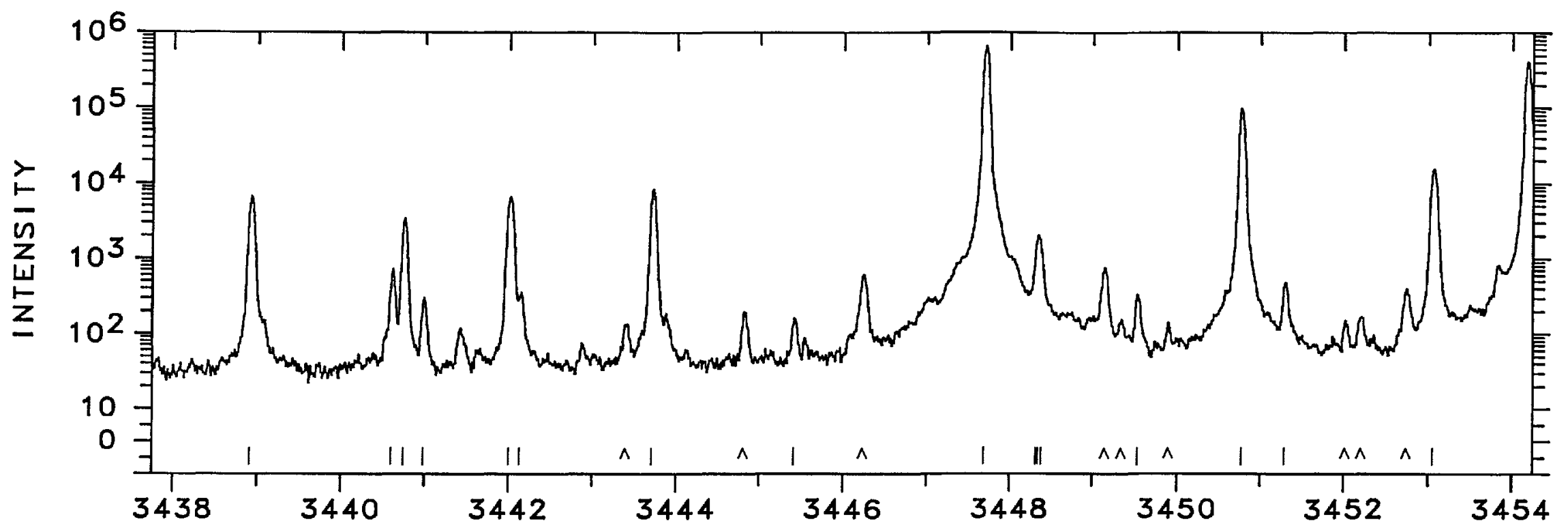

$\vec{y}$

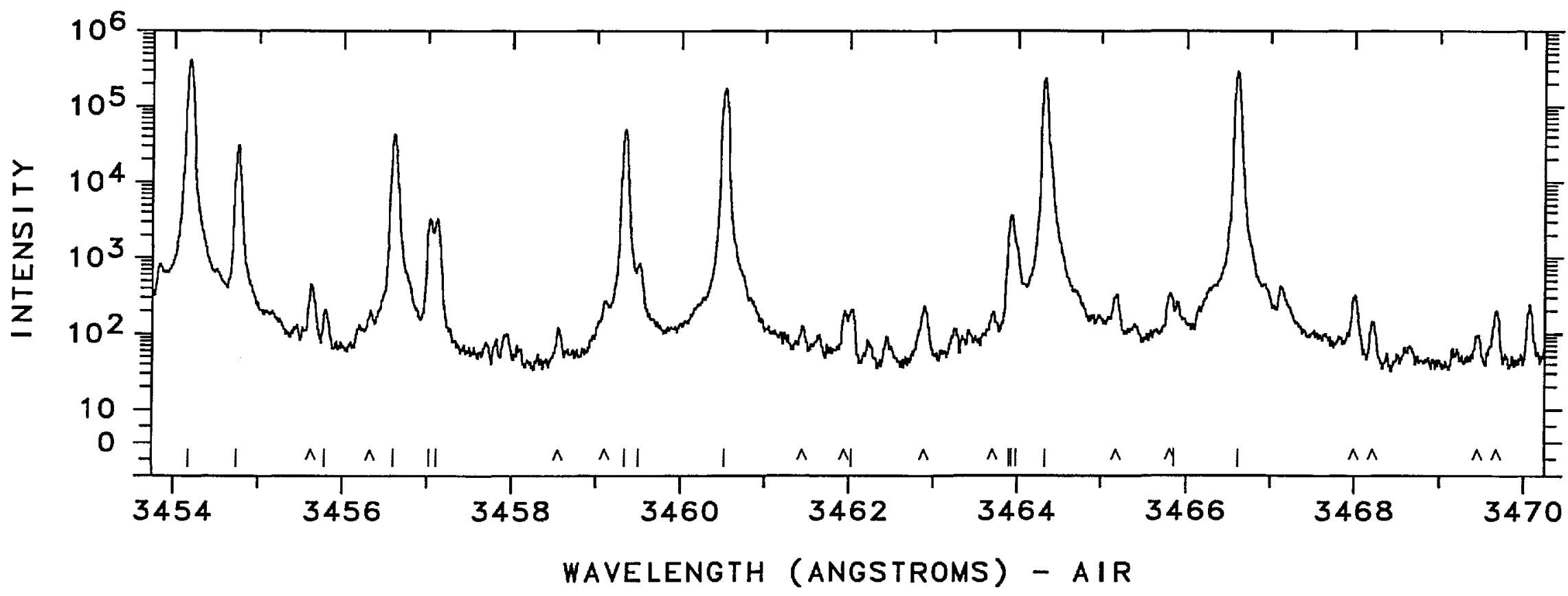




\begin{tabular}{|c|c|c|c|c|c|c|c|c|c|c|c|}
\hline WAVELENGTH & WAVE NUMBER & INTENSI TY & \multicolumn{2}{|c|}{ CLASSI FICATION } & $\begin{array}{c}\text { CODE } \\
G\end{array}$ & $\begin{array}{l}\text { WAVELENGTH } \\
\\
3481.1429 \\
3481.9337 \\
3483.4231 \\
3485.2641 \\
3490.5739\end{array}$ & $\begin{array}{c}\text { WAVE NUMBER } \\
28717.977 \\
28711.455\end{array}$ & $\begin{array}{l}\text { INTENSITY } \\
\\
360000 \\
18000 \\
92000\end{array}$ & \multicolumn{2}{|c|}{ CLASSIFICATION } & CODE \\
\hline $\begin{array}{l}3470.07 \\
3470.4444 \\
3470.6071 \\
3472.5701 \\
3474.78 \\
3475.241 \\
3475.4502 \\
3476.37 \\
3476.7600 \\
3477.6466 \\
3479.5193 \\
3480.7181 \\
3481.1429\end{array}$ & $\begin{array}{l}28809.6 \\
28806.505 \\
28805.154 \\
28788.871 \\
28770.6 \\
28766.75 \\
28765.015 \\
28757.4 \\
28754.179 \\
28746.848 \\
28731.377 \\
28721.482 \\
28717.977\end{array}$ & $\begin{array}{r}210 \\
1100 \\
530 \\
750000 \\
670 \\
\\
160 \\
8800 \\
14000 \\
130000 \\
250000 \\
2500\end{array}$ & 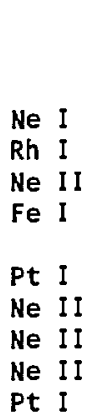 & $6567-35321$ & G & $\begin{array}{l}3481.1429 \\
3481.9337 \\
3483.4231 \\
3485.2641 \\
3490.5739 \\
3490.9998 \\
3497.5624 \\
3498.0635 \\
3498.1646 \\
3500.8873 \\
3501.2158 \\
3501.4968\end{array}$ & $\begin{array}{l}28717.977 \\
28711.455 \\
28699.179 \\
28684.020 \\
28640.388 \\
28636.894 \\
28583.163 \\
28579.068 \\
28578.243 \\
28556.017 \\
28553.338 \\
28551.047\end{array}$ & $\begin{array}{c}4700 \mathrm{C} \\
980 \\
180000 \\
2300 \mathrm{P} \\
600 \mathrm{P} \\
270000 \\
980\end{array}$ & 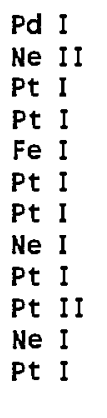 & $\begin{array}{r}68831-40194 \\
62705-34122 \\
\\
26638-55216 \\
114256-85700\end{array}$ & $\begin{array}{l}\text { A } \\
\text { G } \\
\text { E } \\
E \\
R \\
N \\
N \\
G \\
E \\
\text { K } \\
G \\
N\end{array}$ \\
\hline
\end{tabular}




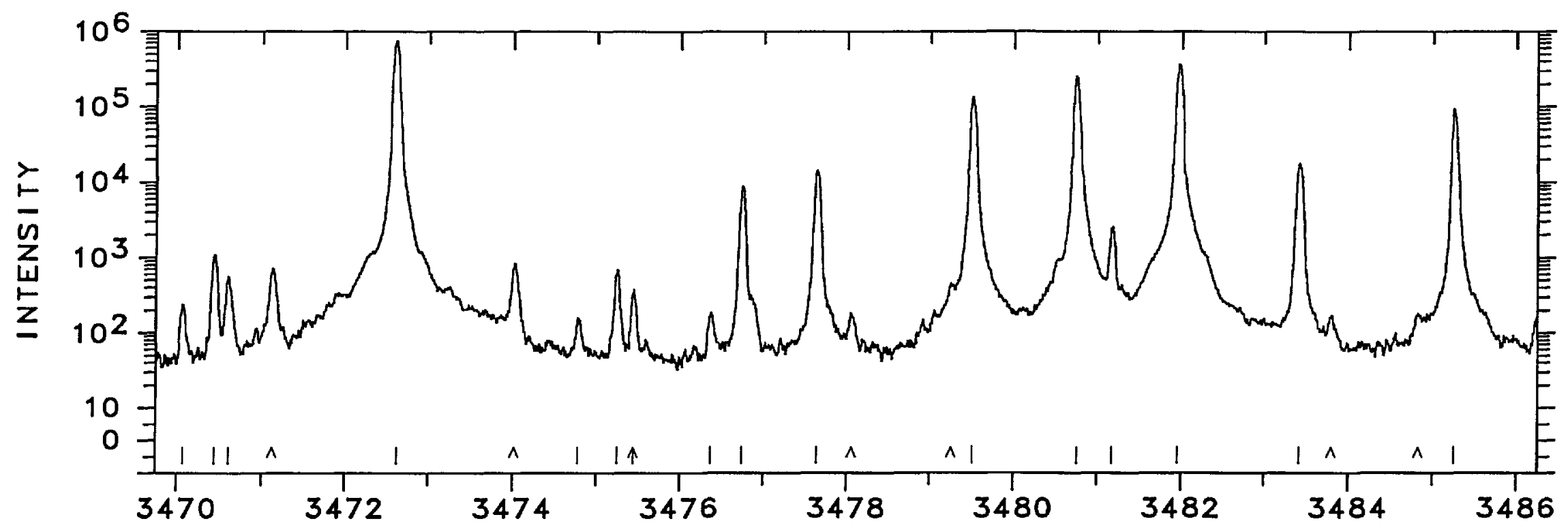

ज̆

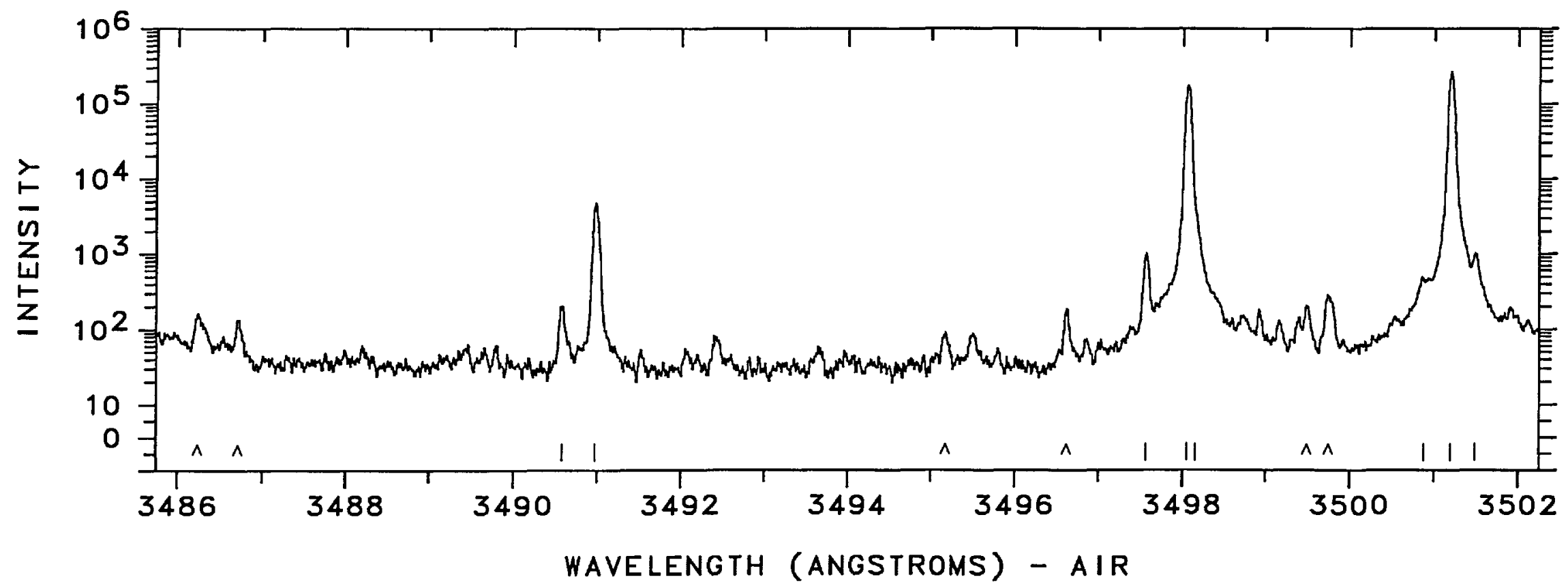




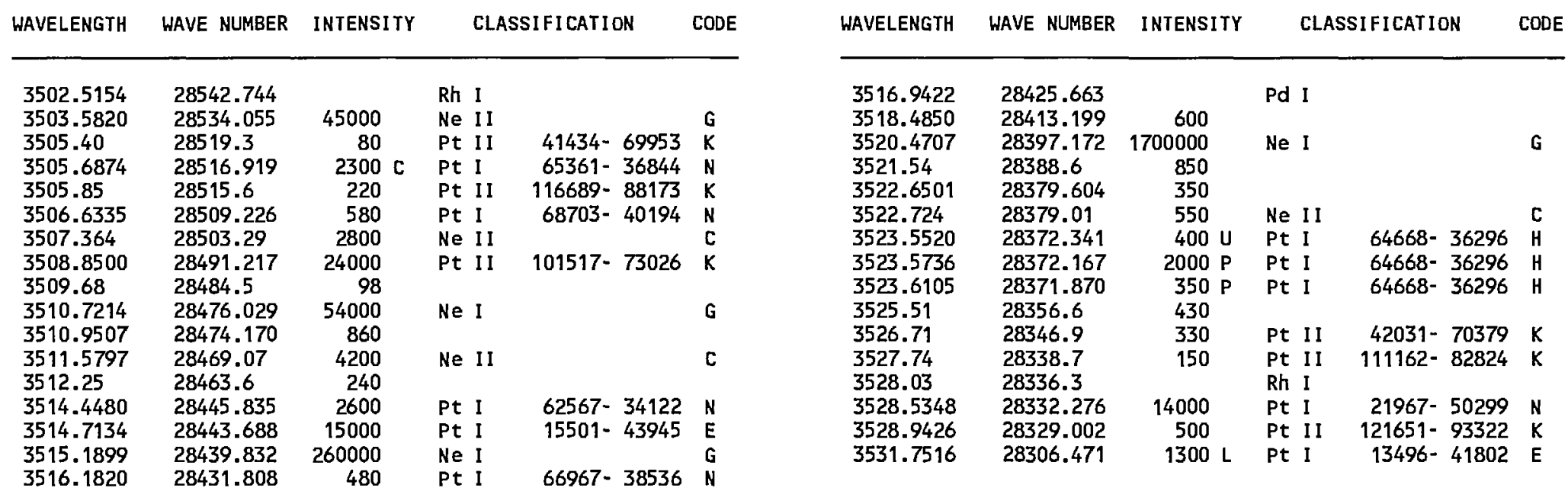




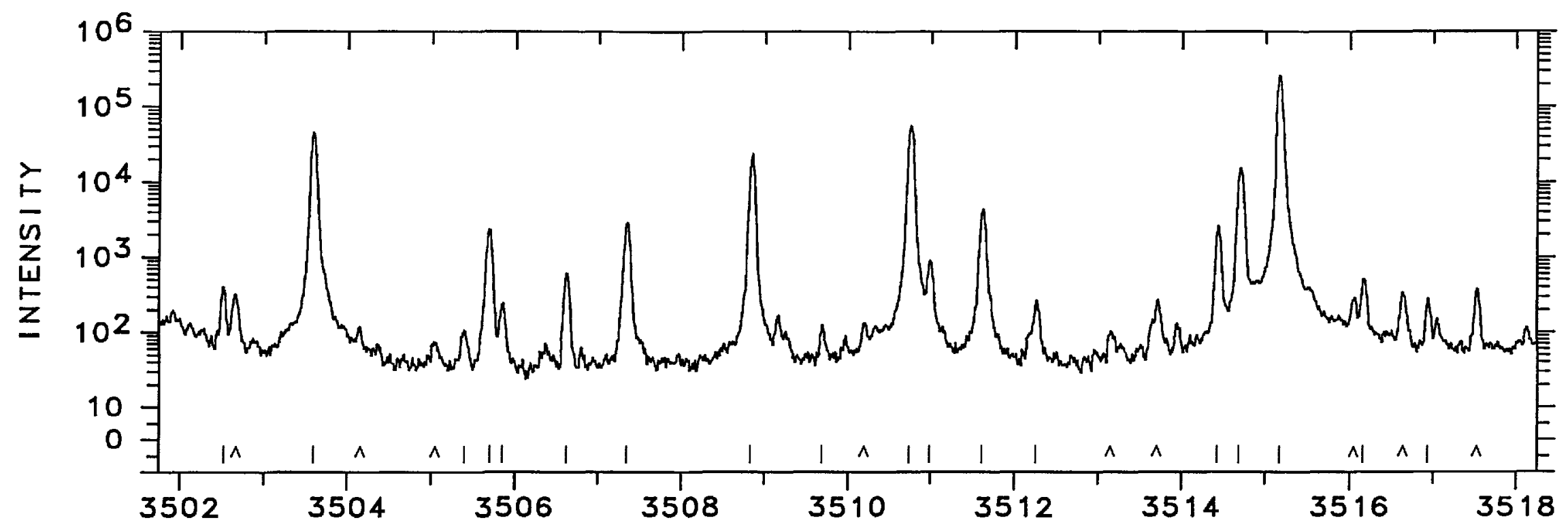

홍

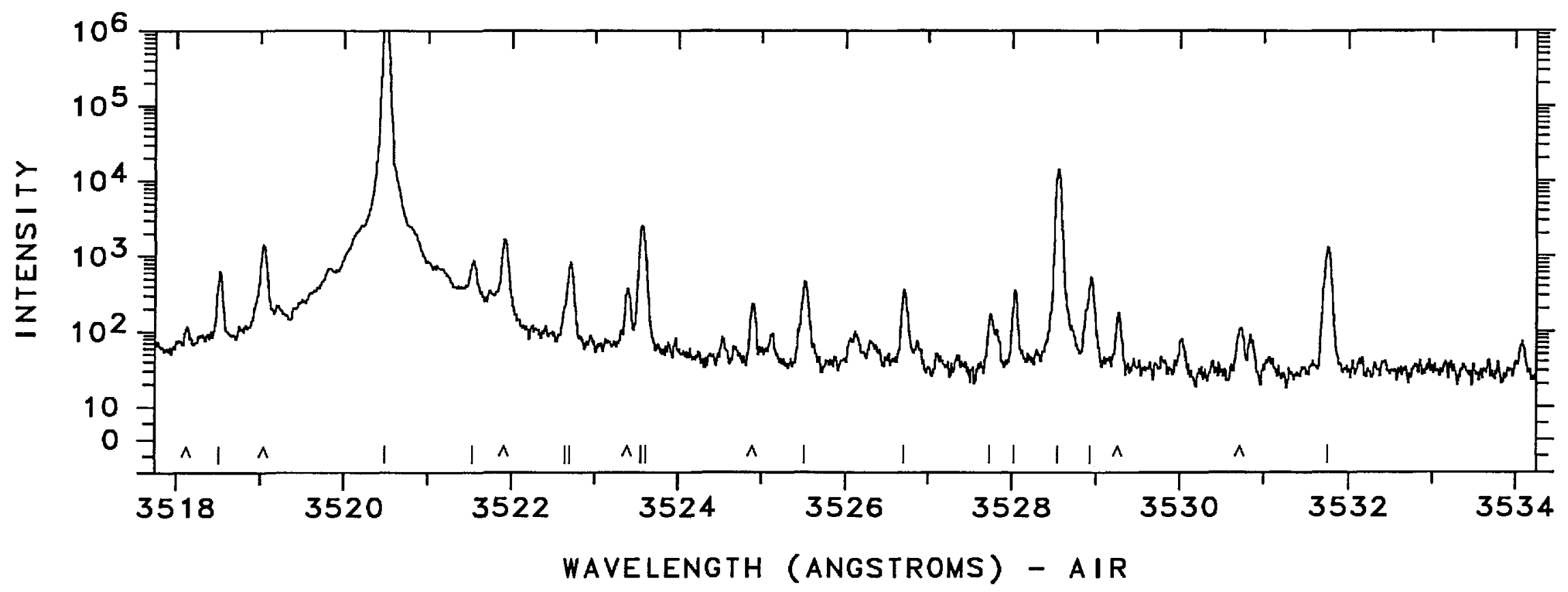


WAVELENGTH WAVE NUMBER INTENSITY CLASSIFICATION CODE

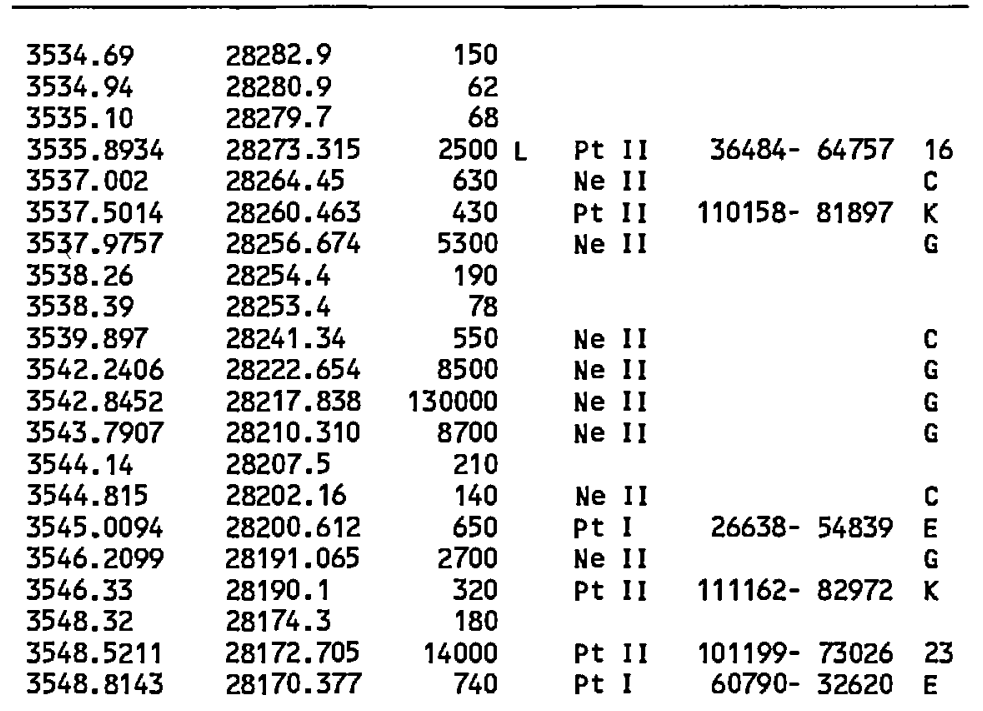

WAVELENGTH WAVE NUMBER INTENSITY CLASSIFICATION CODE

\begin{tabular}{llclll}
\hline 3548.9635 & 28169.193 & 430 & Pt II & $121651-93482$ & $\mathrm{~K}$ \\
3550.1696 & 28159.624 & 950 & Pt I & $68947-40787$ & $\mathrm{~N}$ \\
3551.3553 & 28150.222 & $3200 \mathrm{~L}$ & Pt II & $37877-66028$ & 22 \\
3551.557 & 28148.62 & $1600 \mathrm{H}$ & Ne II & & $\mathrm{C}$ \\
3553.07 & 28136.6 & & Pd I & & \\
3554.3563 & 28126.455 & 1300 & & & \\
3557.313 & 28103.08 & 750 & Ne II & & $\mathrm{C}$ \\
3557.8055 & 28099.188 & 64000 & Ne II & & $\mathrm{G}$ \\
3559.8455 & 28083.086 & $2500 \mathrm{P}$ & Ne I I & & $\mathrm{A}$ \\
3559.8455 & 28083.086 & $2500 \mathrm{P}$ & Pt I & $64379-36296$ & $\mathrm{AH}$ \\
3559.8748 & 28082.855 & $3500 \mathrm{P}$ & Pt I & $64379-36296$ & $\mathrm{H}$ \\
3559.9178 & 28082.515 & $1000 \mathrm{P}$ & Pt I & $64379-36296$ & $\mathrm{H}$ \\
3561.1990 & 28072.413 & 46000 & Ne II & & $\mathrm{G}$ \\
3562.9541 & 28058.584 & 1400 & Ne I & & $\mathrm{B}$ \\
3563.5851 & 28053.617 & 660 & Pt I & $65395-37342$ & $\mathrm{~N}$ \\
3564.6881 & 28044.936 & 1900 & Pt I & $65387-37342$ & $\mathrm{~N}$ \\
3564.9264 & 28043.062 & 760 & Pt I & $21967-50010$ & $\mathrm{~N}$ \\
3565.0472 & 28042.111 & 1800 & & & \\
3565.3790 & 28039.502 & & Fe I & & $\mathrm{R}$ \\
3565.8232 & 28036.008 & 34000 & Ne II & & $\mathrm{G}$
\end{tabular}



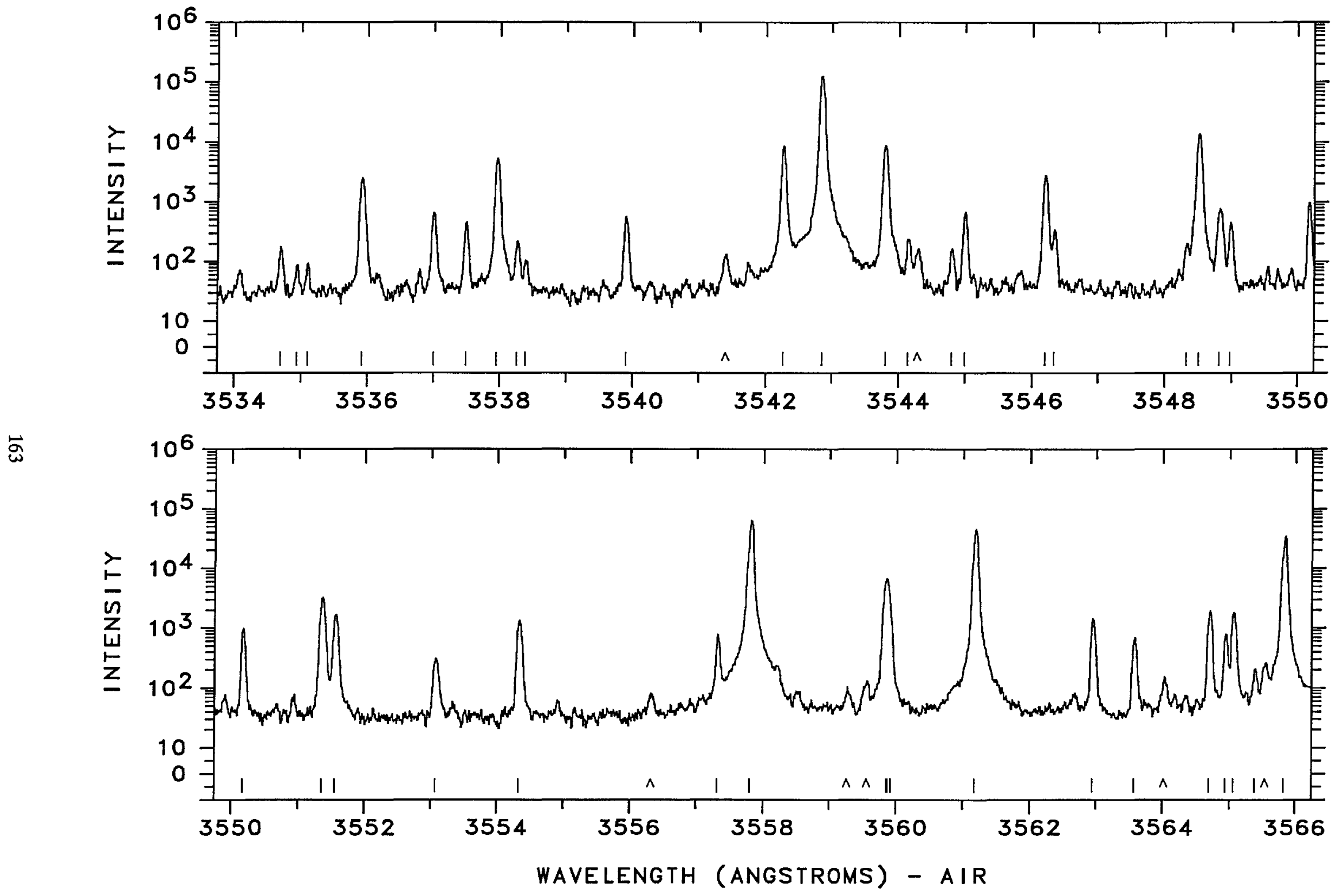


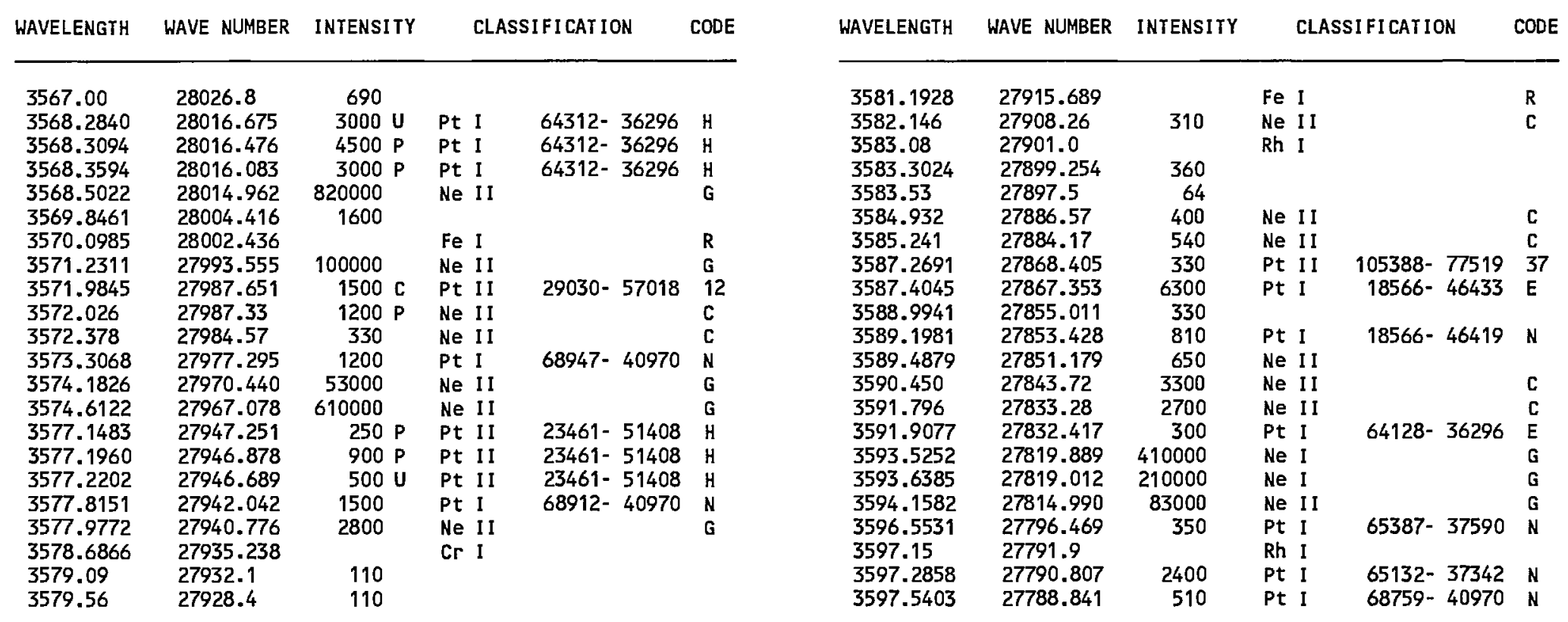




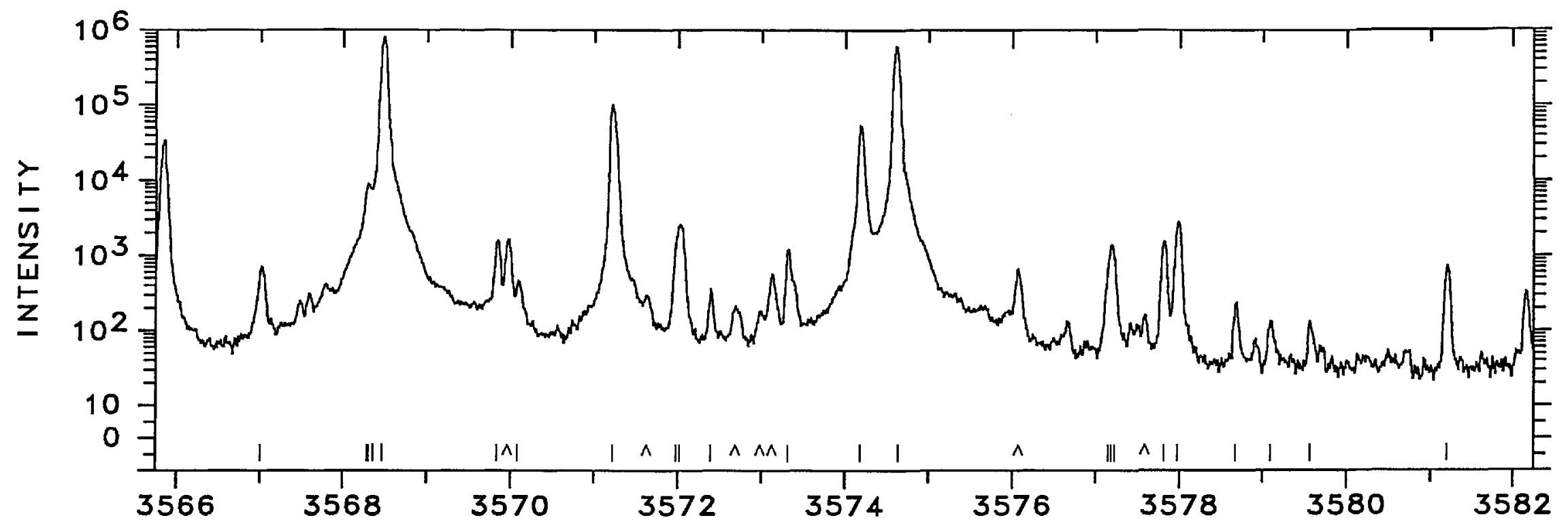

जั

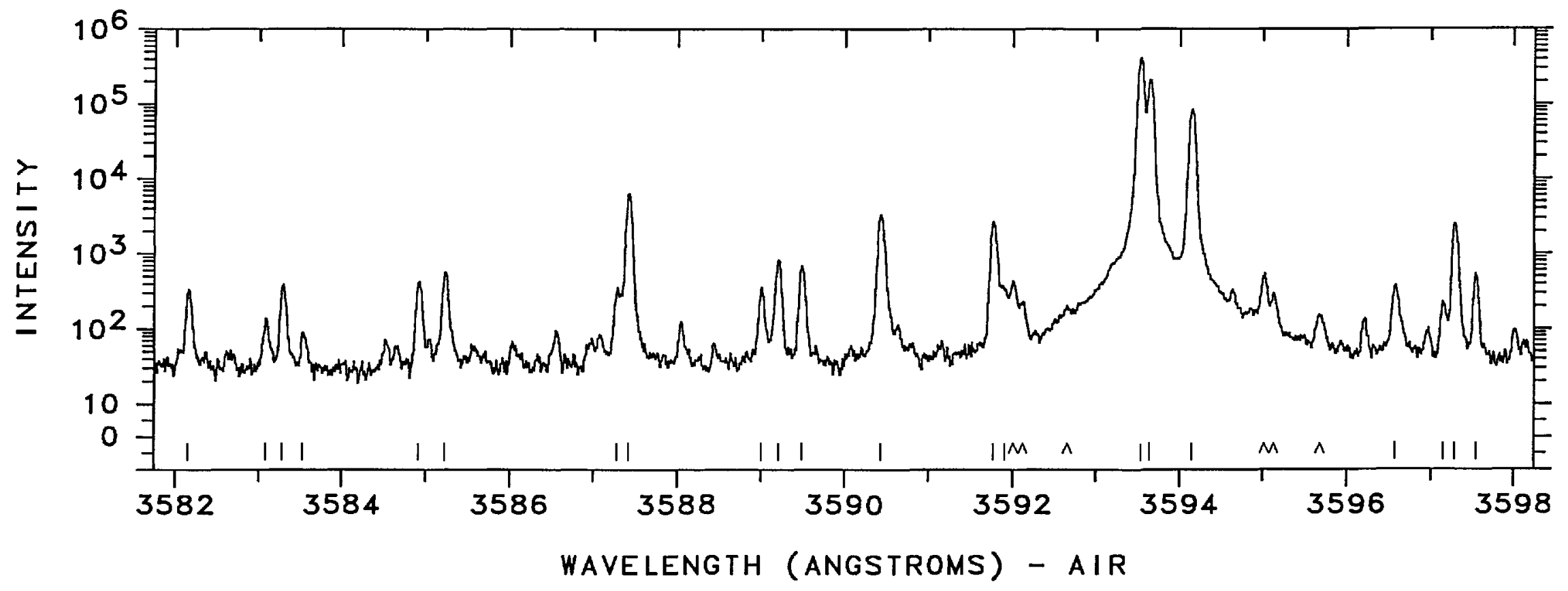


WAVELENGTH WAVE NUMBER INTENSITY

CLASSIFICATION

CODE

\begin{tabular}{llrlll}
\hline & & & & & \\
3598.02 & 27785.1 & 71 & & & G \\
3600.1682 & 27768.557 & 120000 & Ne I & & C \\
3601.056 & 27761.71 & 1300 & Ne II & & \\
3601.4005 & 27759.057 & 250 & Pt I & $68275-40516$ & N \\
3601.9340 & 27754.945 & 330 L & I & & \\
3602.3841 & 27751.477 & 420 L & Pt II & $105794-78043$ & K \\
3602.6582 & 27749.366 & 8100 & & & \\
3602.771 & 27748.50 & 630 & Ne II & & C \\
3603.236 & 27744.92 & 680 & Ne I I & & C \\
3604.1641 & 27737.772 & 330 & Pt I & $60357-32620$ & E \\
3604.76 & 27733.2 & 140 & Pt I & $68703-40970$ & N \\
3605.31 & 27729.0 & & Cr I & & \\
3606.12 & 27722.7 & 150 & & & \\
3606.7395 & 27717.966 & 840 & Pt I & $65308-37590$ & N \\
3607.504 & 27712.09 & 1400 & Ne I I & & C \\
3607.8646 & 277709.323 & 140 & Pt II & $37877-65587$ & 33 \\
3608.605 & 27703.64 & 290 & Ne II & & C \\
3608.8592 & 27701.686 & & Fe I & & R \\
3609.1783 & 27699.236 & 14000 & Ne I & & G \\
3609.5443 & 27696.428 & & Pd I & & \\
3610.9063 & 27685.982 & 2300 & Pt I & $15501-43187$ & E \\
3611.599 & 27680.67 & 650 & Ne I I & & C \\
3612.326 & 27675.10 & 11000 & Ne I I & & C \\
3612.606 & 27672.96 & 240 & Ne II & & C
\end{tabular}

WAVELENGTH WAVE NUMBER INTENSITY

CLASSI FI CATI ON

CODE

\begin{tabular}{llrlrl}
\hline 3613.84 & 27663.5 & 65 & & & \\
3614.33 & 27659.8 & 180 & & & \\
3615.17 & 27653.3 & 110 & Pt I & $68169-40516$ & $\mathrm{~N}$ \\
3615.28 & 27652.5 & 350 & Pt I & $10116-37769$ & $\mathrm{~N}$ \\
3615.40 & 27651.6 & 95 & & & \\
3615.99 & 27647.1 & 150 & & & \\
3616.348 & 27644.32 & 270 & Ne II & & $\mathrm{C}$ \\
3617.91 & 27632.4 & 78 & & & \\
3618.3806 & 27628.794 & 300 & Pt I I & $121651-94022$ & $\mathrm{~K}$ \\
3618.7603 & 27625.895 & 500 & Ne II & & $\mathrm{A}$ \\
3618.7603 & 27625.895 & & Fe I & & $\mathrm{A}$ \\
3619.2212 & 27622.377 & 780 & Pt I I & $110158-82535$ & $\mathrm{~K}$ \\
3619.8007 & 27617.955 & 1500 & Pt I & $65387-37769$ & $\mathrm{~N}$ \\
3620.5414 & 27612.305 & 3500 & & & \\
3621.6546 & 27603.818 & 2800 & Pt I & $18566-46170$ & $\mathrm{E}$ \\
3622.4709 & 27597.598 & 2200 & Pt I & $64379-36781$ & $\mathrm{~N}$ \\
3625.1223 & 27577.413 & $870 \mathrm{~L}$ & & & \\
3625.54 & 27574.2 & 140 & & & \\
3626.5363 & 27566.661 & 5000 & Ne I I & & $\mathrm{G}$ \\
3628.0329 & 27555.290 & 15000 & Ne I I & & $\mathrm{G}$ \\
3628.1107 & 27554.699 & 91000 & Pt I & $6567-34122$ & $\mathrm{E}$ \\
3628.8660 & 27548.965 & 20000 & Pt I & $64330-36781$ & $\mathrm{~N}$ \\
3628.9097 & 27548.632 & $400 \mathrm{U}$ & & & \\
3629.1744 & 27546.623 & $310 \mathrm{~W}$ & Pt II & $105066-77519$ & 38
\end{tabular}



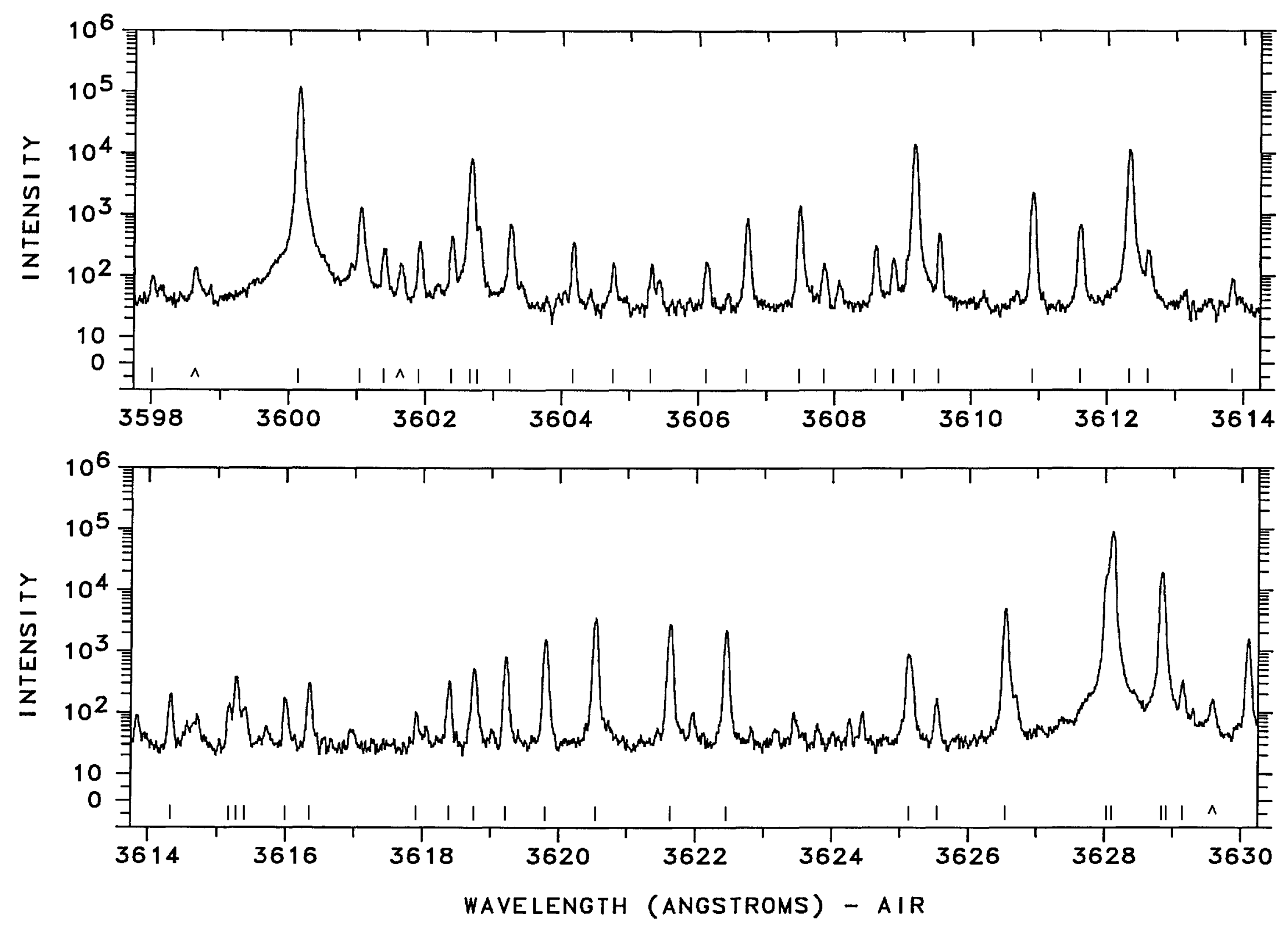


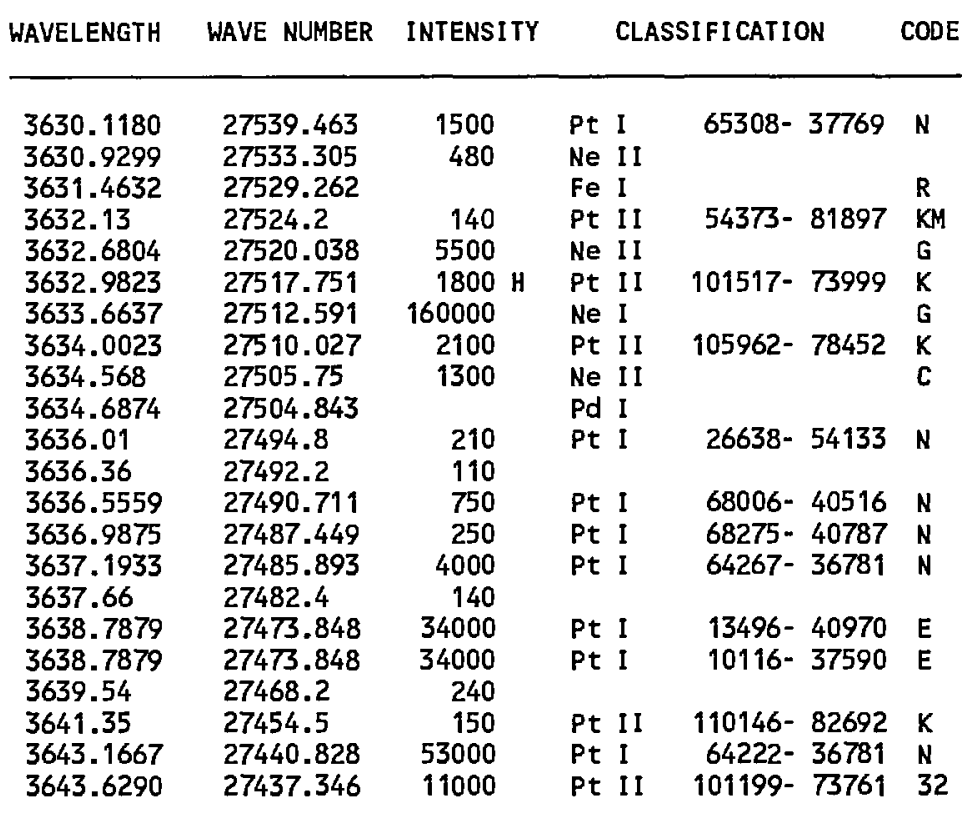

\begin{tabular}{|c|c|c|c|c|c|}
\hline ENGTH & IAVE NUMBER & INTENSITY & & SIFICATION & CODE \\
\hline $\begin{array}{l}3643.9291 \\
3644.0403 \\
3644.4680 \\
3644.8566 \\
3644.9425 \\
3647.8477 \\
3647.8477 \\
3650.7680 \\
3651.266 \\
3652.2552 \\
3652.812 \\
3653.9828 \\
3655.95 \\
3656.26 \\
3656.651 \\
3657.41 \\
3658.01 \\
3659.4131 \\
3659.8921 \\
3661.25 \\
3661.809\end{array}$ & $\begin{array}{l}27435.087 \\
27434.249 \\
27431.030 \\
27428.105 \\
27427.459 \\
27405.616 \\
27405.616 \\
27383.695 \\
27379.96 \\
27372.544 \\
27368.37 \\
27359.603 \\
27344.9 \\
27342.6 \\
27339.64 \\
27334.0 \\
27329.5 \\
27319.004 \\
27315.429 \\
27305.3 \\
27301.13\end{array}$ & $\begin{array}{c}170000 \\
1000 \mathrm{P} \\
730 \\
39000 \\
550 \mathrm{U} \\
\mathrm{W} \\
520 \mathrm{~W} \\
610 \\
280 \\
1700 \\
400 \\
3600 \\
210 \\
120 \\
570 \\
330 \\
\\
3100 \\
5200 \\
160 \\
650\end{array}$ & $\begin{array}{l}\text { Ne II } \\
\text { Pt II } \\
\text { Ne II } \\
\text { Fe I } \\
\text { Ne I I } \\
\text { Pt I } \\
\text { Ne II } \\
\text { Pt I } \\
\text { Ne I I } \\
\text { Pt I } \\
\text { Pt II } \\
\text { Pt I I } \\
\text { Ne I I } \\
\text { Pt I I } \\
\text { Rh I } \\
\text { Pt I } \\
\text { Ne I I } \\
\text { Pt I } \\
\text { Ne II }\end{array}$ & $\begin{array}{r}62705-35321 \\
26638-54011 \\
64141-36781 \\
105388-78043 \\
106434-79092 \\
110158-82824 \\
21967-49286 \\
68275-40970\end{array}$ & $\begin{array}{c}G \\
\mathbf{K} \\
\mathbf{G} \\
\mathbf{A} \\
\mathbf{A} \\
\mathbf{N} \\
\mathbf{C} \\
\mathrm{E} \\
\mathbf{C} \\
\mathrm{E} \\
\mathbf{X} \\
\mathbf{K} \\
\mathbf{C} \\
\mathrm{K}\end{array}$ \\
\hline
\end{tabular}




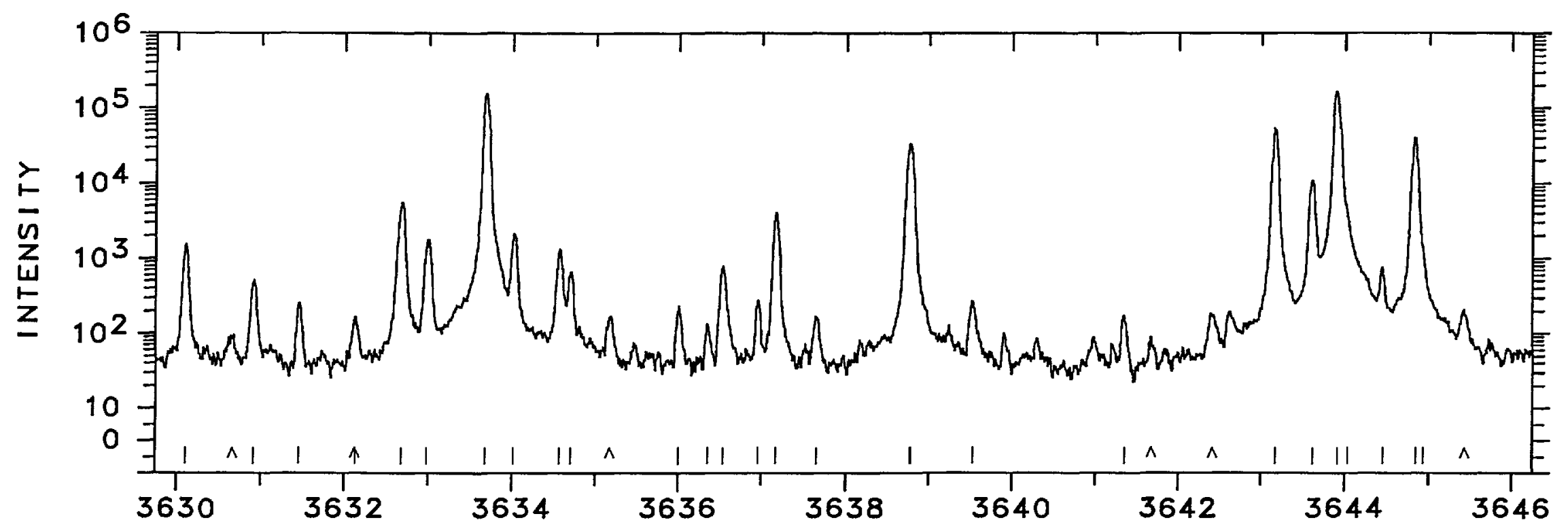

ิㅗㅇ

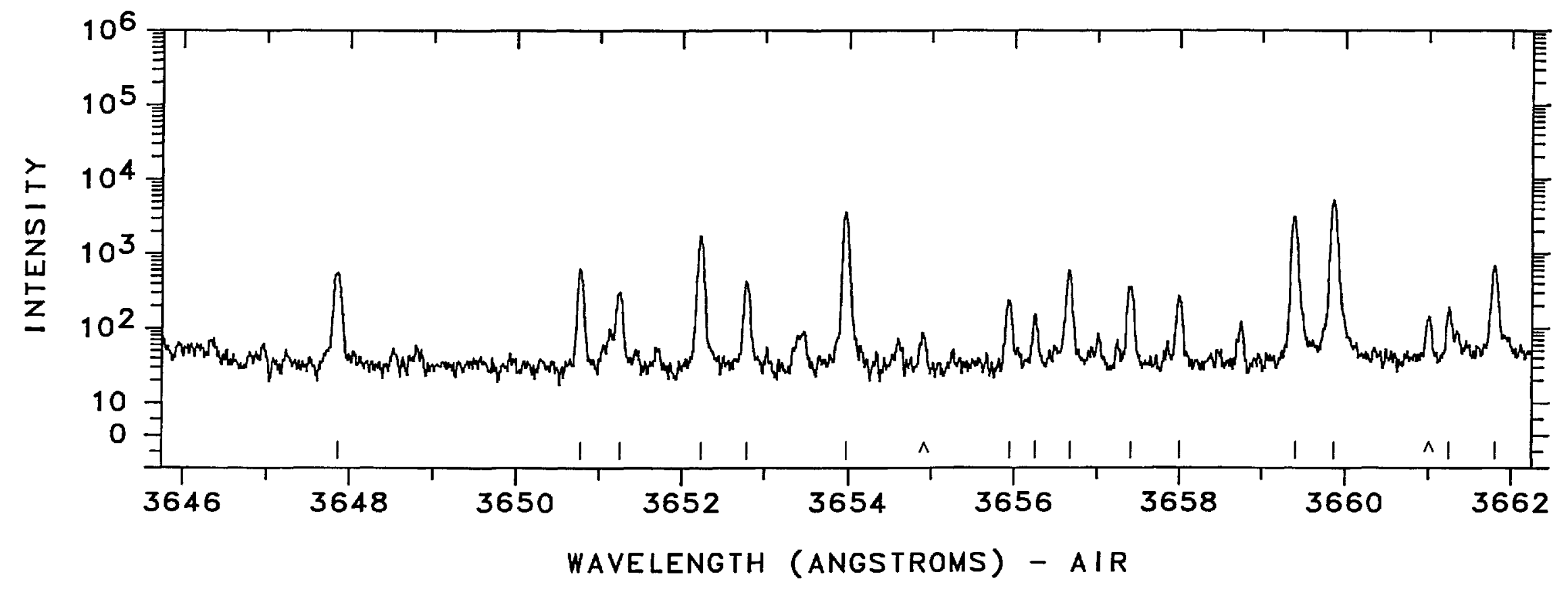


WAVELENGTH WAVE NUMBER INTENSITY

CLASSIFICATION

CODE

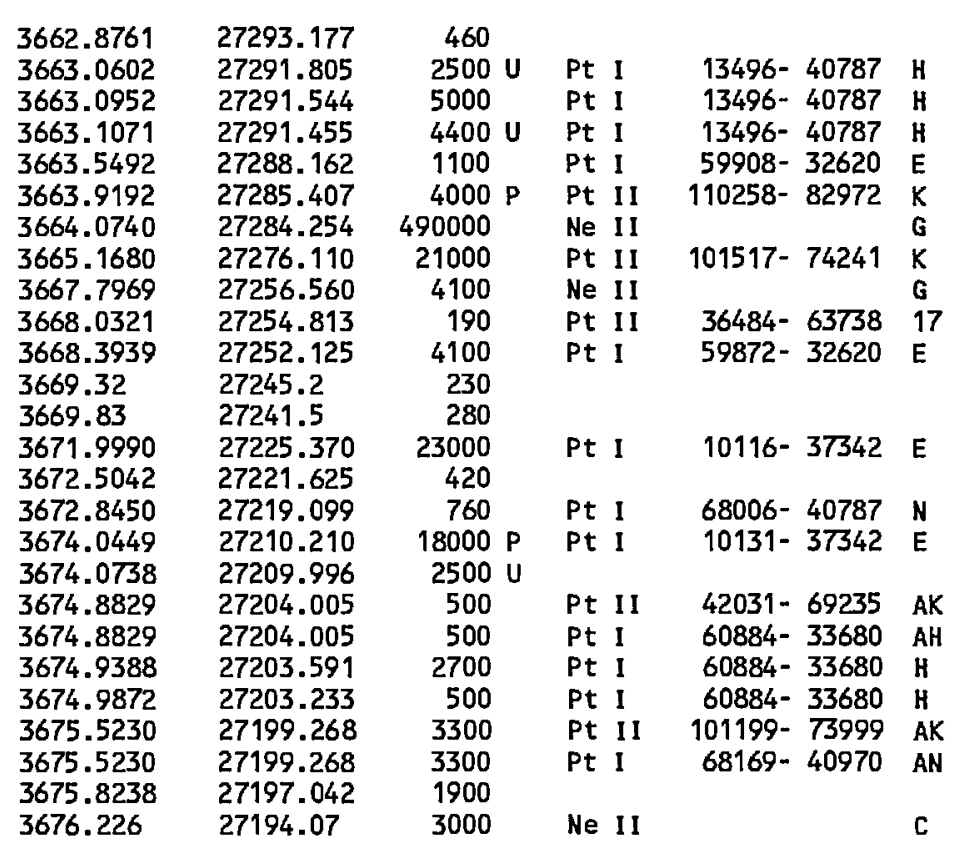

WAVELENGTH WAVE NUMBER INTENSITY CLASSIFICATION CODE

\begin{tabular}{llclll}
\hline 3676.804 & 27189.79 & 750 & Ne II & & C \\
3676.93 & 27188.9 & 270 & & & \\
3677.090 & 27187.68 & 700 & Ne II & & C \\
3677.3943 & 27185.427 & 730 & Pt II & $110158-82972$ & K \\
3679.8160 & 27167.536 & 6500 & Ne II & & G \\
3680.3319 & 27163.729 & 580 & Pt I & $64505-37342$ & N \\
3680.4520 & 27162.842 & 2200 & Pt I & $59782-32620$ & E \\
3681.0364 & 27158.530 & 2500 U & Ne II & & G \\
3681.0798 & 27158.210 & 7500 P & Pt I & $15501-42660$ & E \\
3681.941 & 27151.86 & 600 P & Ne II & & C \\
3682.0226 & 27151.256 & 2900 & Ne II & & G \\
3682.2418 & 27149.640 & 64000 & Ne I & & G \\
3682.9727 & 27144.252 & 15000 & Pt I & $59764-32620$ & E \\
3683.77 & 27138.4 & 170 & & & \\
3684.51 & 27132.9 & 150 & & & \\
3685.7349 & 27123.910 & 110000 & Ne I & & G \\
3687.4152 & 27111.550 & 28000 & Pt I & $59731-32620$ & E \\
3687.497 & 27110.95 & 350 U & Ne II & & C \\
3688.81 & 27101.3 & 180 & & & \\
3689.316 & 27097.58 & 330 & Ne II & & C \\
3690.32 & 27090.2 & 87 & Pd I & & \\
3691.98 & 27078.0 & 200 & Pt I & $64668-37590$ & N \\
3692.272 & 27075.89 & 500 & Ne II & & C \\
3692.3525 & 27075.299 & & Rh I & & \\
3693.389 & 27067.70 & 2900 & Ne II & & C
\end{tabular}




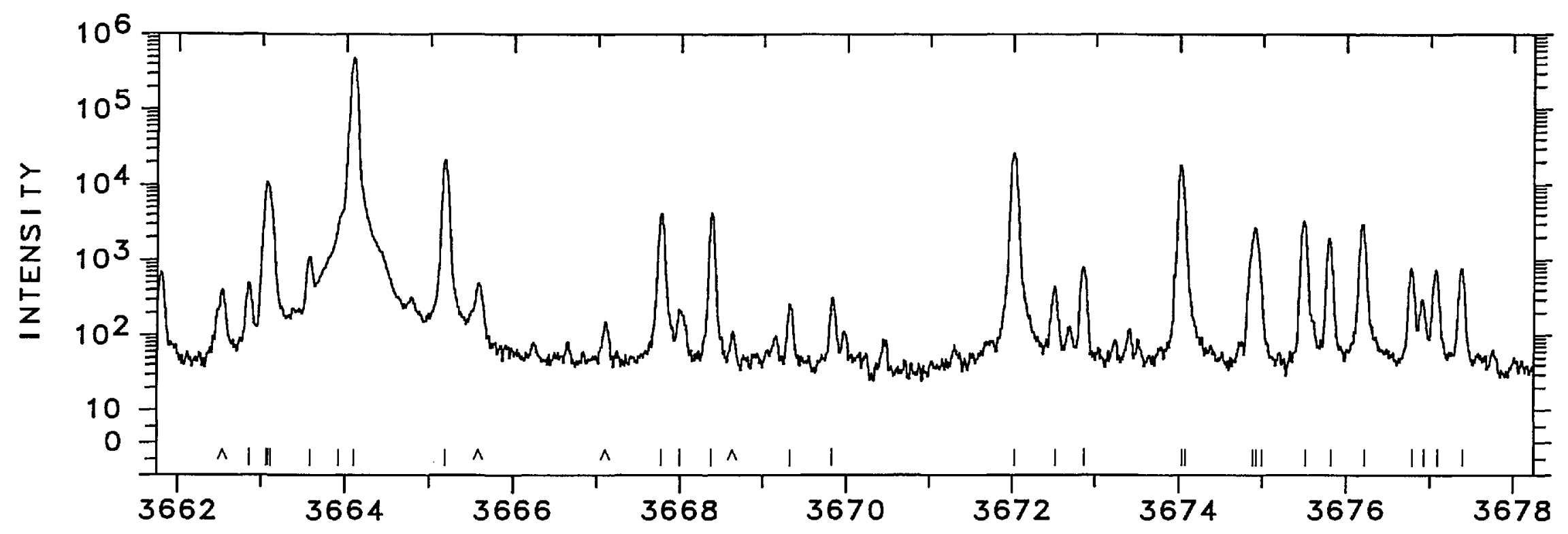

$\Xi$

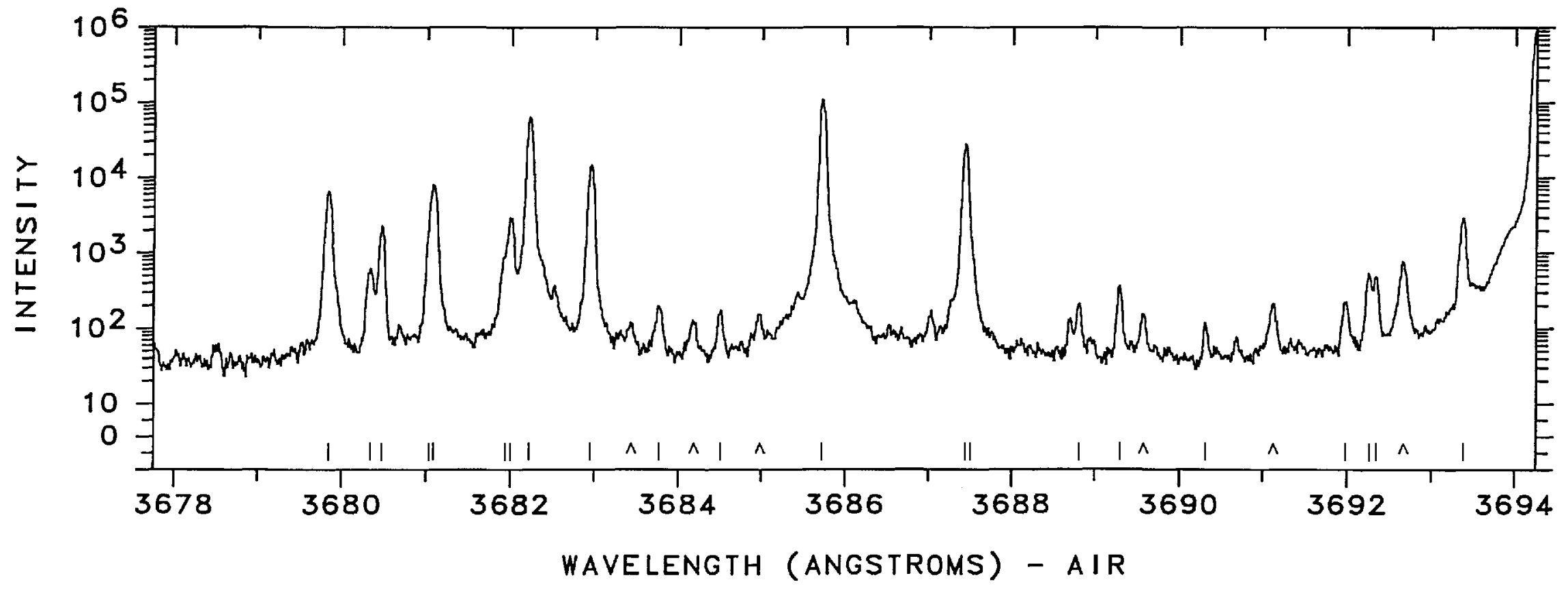




\begin{tabular}{llclll} 
WAVELENGTH & WAVE NUMBER & INTENSITY & \multicolumn{2}{c}{ CLASSIFICATION } & CODE \\
\hline & & & & \\
\hline 3694.2145 & 27061.652 & 910000 & Ne II & & G \\
3695.247 & 27054.09 & 860 & Ne II & & C \\
3696.6518 & 27043.810 & 430 & Pt II & $105086-78043$ & K \\
3697.1234 & 27040.360 & 5100 & Ne II & & G \\
3697.3787 & 27038.493 & 760 & & & \\
3698.067 & 27033.46 & 420 & Ne II & & C \\
3698.9960 & 27026.671 & 920 & Pt I & $26638-53665$ & N \\
3699.54 & 27022.7 & 180 & & & \\
3699.8649 & 27020.325 & $300 \mathrm{U}$ & Pt I & $13496-40516$ & H \\
3699.9126 & 27019.976 & 21000 & Pt I & $13496-40516$ & H \\
3699.9539 & 27019.675 & $350 \mathrm{U}$ & Pt I & $13496-40516$ & H \\
3700.1471 & 27018.264 & $450 \mathrm{~W}$ & Pt II & $34647-61665$ & 16 \\
3700.219 & 27017.74 & 1900 & Ne II & & C \\
3700.9064 & 27012.721 & 270 & & & G \\
3701.2242 & 27010.401 & 110000 & Ne I & & G \\
3701.7769 & 27006.368 & 31000 & Ne II & & \\
3702.2305 & 27003.060 & 400 & & & R \\
3705.5660 & 26978.754 & & Fe I & & C \\
3705.744 & 26977.46 & 1200 & Ne II & & \\
3706.5217 & 26971.798 & 22000 & Pt I & $59591-32620$ & N
\end{tabular}

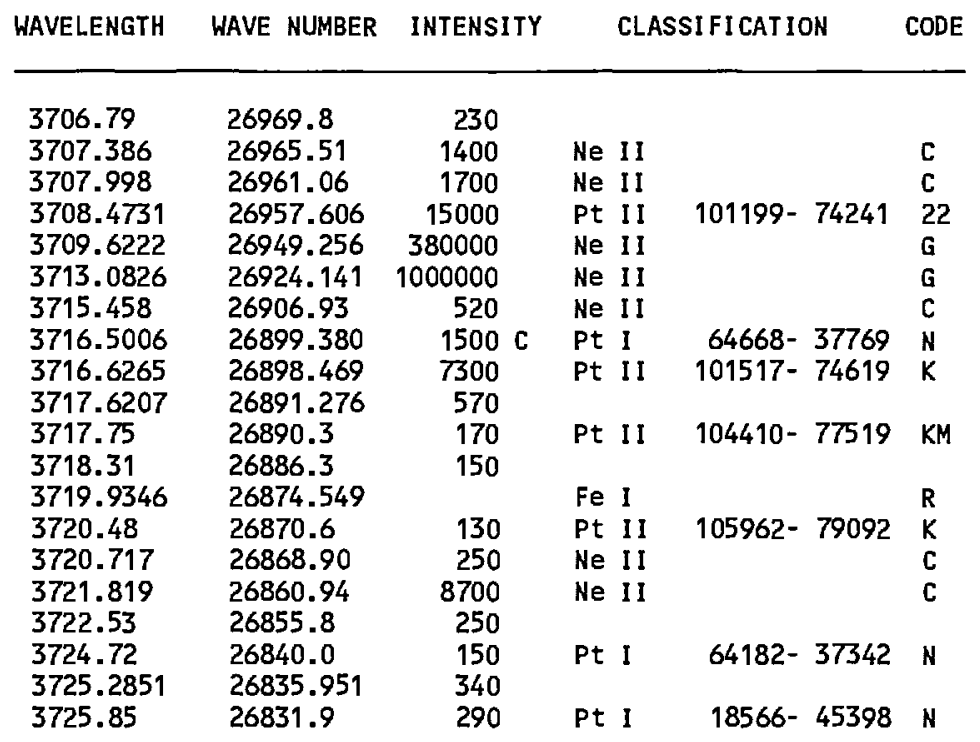




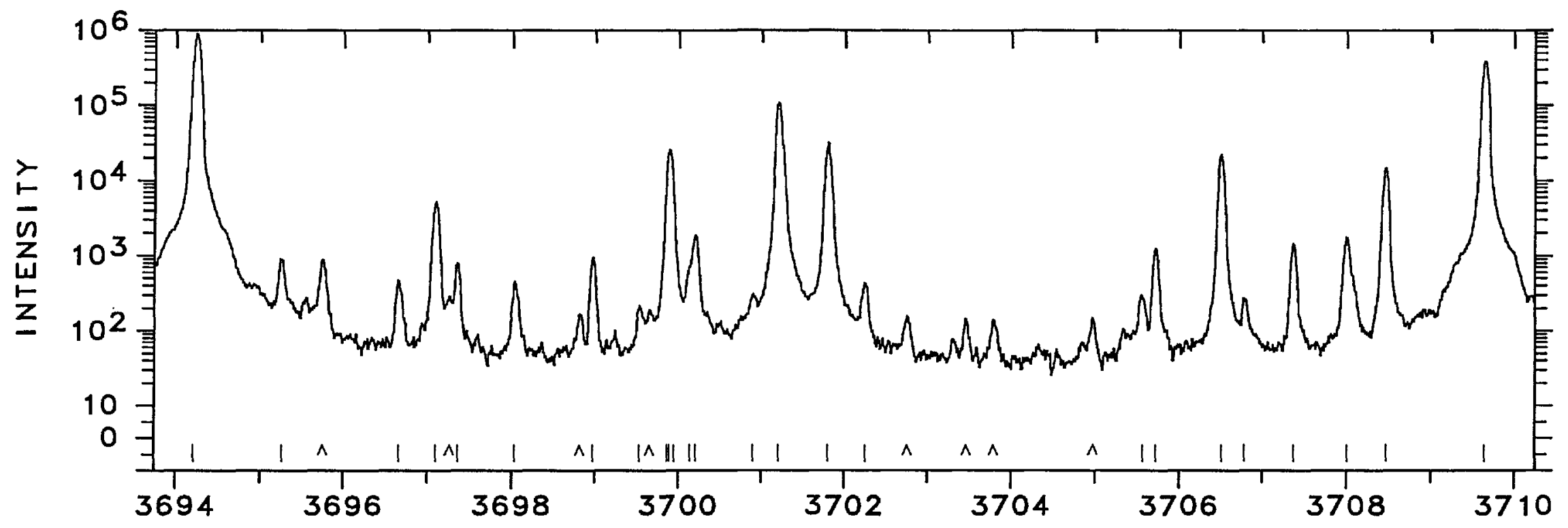

产

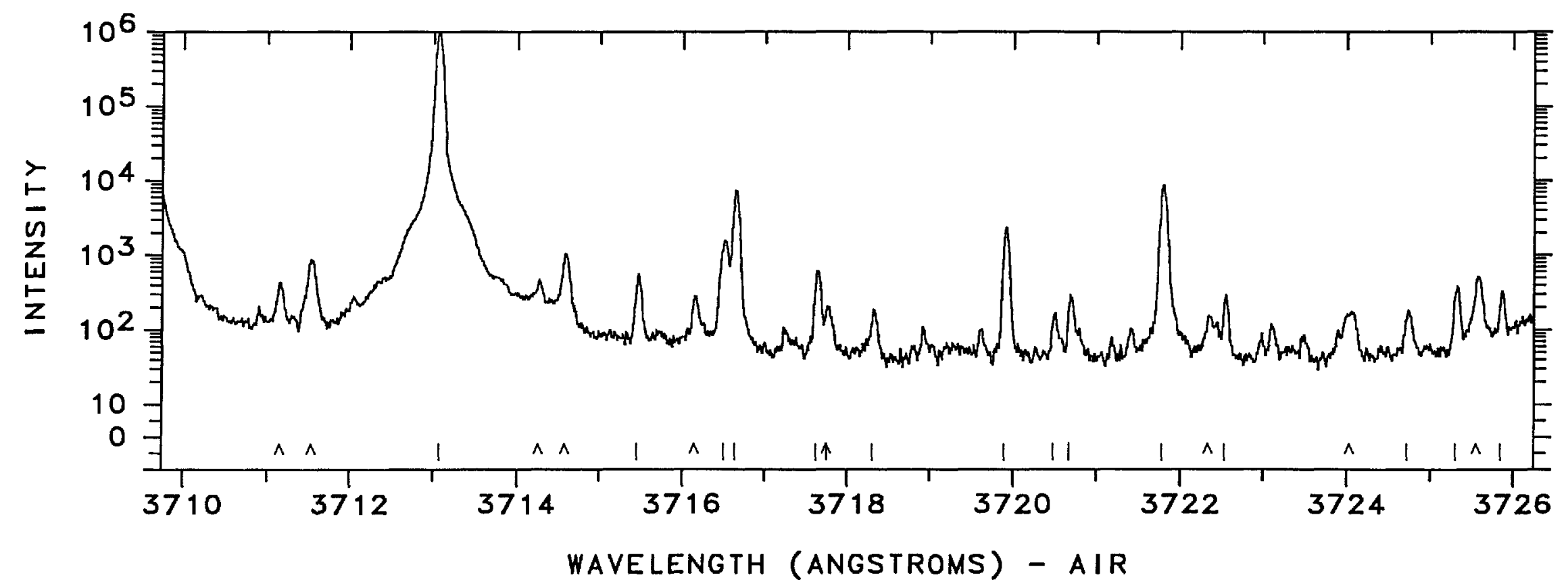




\begin{tabular}{|c|c|c|c|c|c|c|c|c|c|c|c|}
\hline WAVELENGTH & WAVE NUMBER & INTENSITY & CLA & SIFICATION & CODE & WAVELENGTH & WAVE NUMBER & INTENSITY & \multicolumn{2}{|c|}{ CLASSIFICATION } & CODE \\
\hline $\begin{array}{l}3726.871 \\
3727.1081 \\
3729.20 \\
3730.07 \\
3730.50 \\
3730.981 \\
3731.8721 \\
3732.346 \\
3732.777 \\
3733.8023 \\
3734.9388 \\
3735.4749 \\
3735.5740 \\
3735.6027 \\
3735.6221 \\
3737.1313 \\
3738.688 \\
3738.78 \\
3739.1037 \\
3740.5987 \\
3740.967 \\
3741.1392 \\
3742.4511 \\
3742.67 \\
3743.69 \\
3744.6245 \\
3745.5613\end{array}$ & $\begin{array}{l}26824.53 \\
26822.825 \\
26807.8 \\
26801.5 \\
26798.4 \\
26794.98 \\
26788.585 \\
26785.18 \\
26782.09 \\
26774.737 \\
26766.589 \\
26762.749 \\
26762.039 \\
26761.833 \\
26761.694 \\
26750.887 \\
26739.75 \\
26739.1 \\
26736.776 \\
26726.090 \\
26723.46 \\
26722.229 \\
26712.862 \\
26711.3 \\
26704.0 \\
26697.358 \\
26690.681\end{array}$ & $\begin{array}{c}6800 \mathrm{P} \\
550000 \\
480 \\
200 \\
410 \\
950 \\
900 \mathrm{~s} \\
350 \\
650 \\
190 \\
150000 \\
420 \\
1200 \mathrm{U} \\
3500 \mathrm{P} \\
1300 \mathrm{U} \\
\\
150 \\
420 \\
1100 \mathrm{~s} \\
3000 \\
130 \\
800 \\
160 \\
170 \\
100 \\
11000\end{array}$ & $\begin{array}{l}\mathrm{Ne} \text { II } \\
\mathrm{Pt} \text { I } \\
\mathrm{Ne} \text { II } \\
\mathrm{Ne} \text { II } \\
\mathrm{Ne} \text { II } \\
\mathrm{Pt} \text { I I } \\
\mathrm{Pt} \text { I } \\
\mathrm{Pt} \text { I } \\
\mathrm{Pt} \text { I } \\
\mathrm{Fe} \\
\mathrm{Ne} \\
\mathrm{NI} \\
\mathrm{Pt} \\
\mathrm{Ne} \text { II } \\
\mathrm{Ne} \text { II } \\
\mathrm{Pt} \\
\mathrm{Pt} \\
\mathrm{Pt} \\
\mathrm{Pt} \\
\mathrm{Ne} \text { II } \\
\mathrm{Fe} \text { I }\end{array}$ & $\begin{array}{r}101517-74754 \\
60884-34122 \\
60884-34122 \\
60884-34122\end{array}$ & $\begin{array}{l}C \\
\text { N } \\
C \\
C \\
C \\
G \\
K \\
H \\
H \\
H \\
R \\
C \\
\\
E \\
G \\
C \\
N \\
E\end{array}$ & $\begin{array}{l}3745.8995 \\
3746.4653 \\
3747.53 \\
3747.849 \\
3748.2622 \\
3748.7156 \\
3748.7469 \\
3749.4853 \\
3749.7263 \\
3750.588 \\
3751.2459 \\
3751.6678 \\
3751.7200 \\
3751.9754 \\
3752.1269 \\
3752.6447 \\
3753.6755 \\
3753.7792 \\
3754.2143 \\
3754.2685 \\
3754.4527 \\
3754.92 \\
3755.0849 \\
3756.393 \\
3756.88 \\
3757.8940\end{array}$ & $\begin{array}{l}26688.271 \\
26684.241 \\
26676.7 \\
26674.39 \\
26671.449 \\
26668.223 \\
26668.000 \\
26662.749 \\
26661.035 \\
26654.91 \\
26650.235 \\
26647.238 \\
26646.868 \\
26645.054 \\
26643.978 \\
26640.301 \\
26632.986 \\
26632.250 \\
26629.163 \\
26628.779 \\
26627.473 \\
26624.2 \\
26622.990 \\
26613.72 \\
26610.3 \\
26603.090\end{array}$ & $\begin{array}{c}950 \\
350 \\
450 \\
\\
1700 \\
400 \mathrm{U} \\
\\
2900 \\
920 \mathrm{~W} \\
68000 \\
310 \\
250 \\
300 \\
140 \\
300 \\
310 \\
35000 \\
23000 \\
1000 \mathrm{P} \\
3400 \\
460 \\
280 \\
410 \\
260 \\
480\end{array}$ & $\begin{array}{ll}\mathrm{Fe} & \text { I } \\
\mathrm{Ne} & \mathrm{II} \\
\mathrm{Pt} & \mathrm{I} \\
\mathrm{Ne} & \mathrm{II} \\
\mathrm{Fe} & \mathrm{I} \\
\mathrm{Pt} & \mathrm{I} \\
& \\
\mathrm{Fe} & \mathrm{I} \\
\mathrm{Pt} & \mathrm{II} \\
\mathrm{Ne} & \mathrm{II} \\
\mathrm{Ne} & \mathrm{II}\end{array}$ & $60790-34122$ & $\begin{array}{l}R \\
N \\
\text { C } \\
R \\
\text { E } \\
\\
R \\
18 \\
C \\
\text { G }\end{array}$ \\
\hline
\end{tabular}




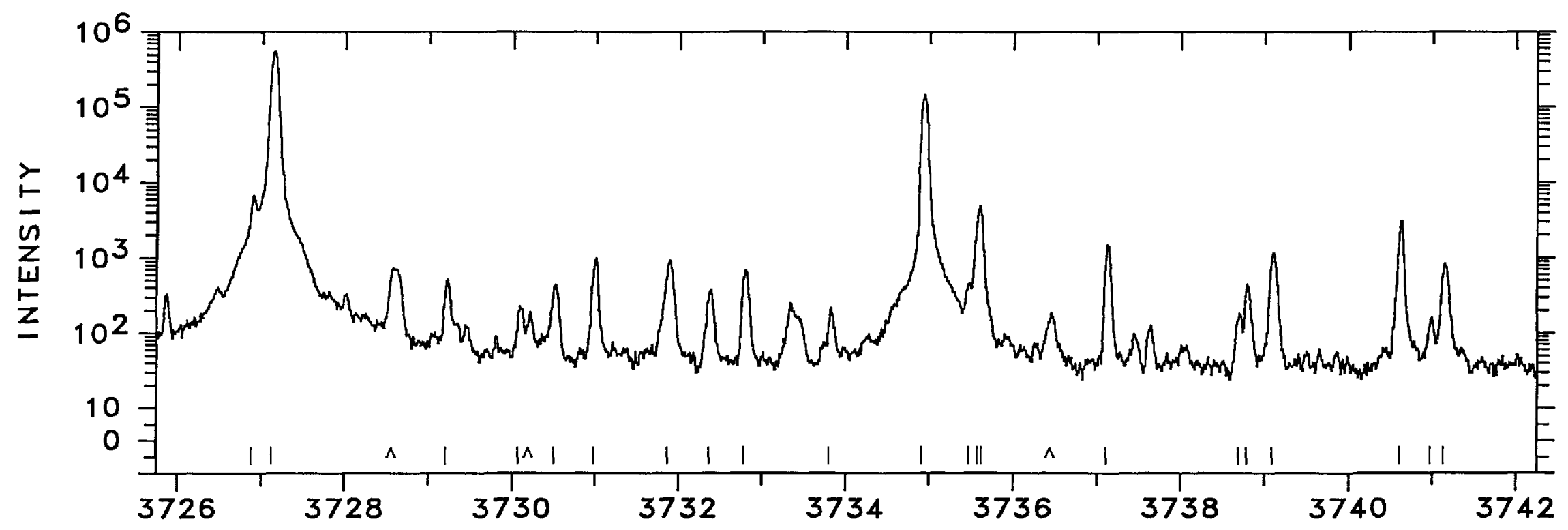

जั

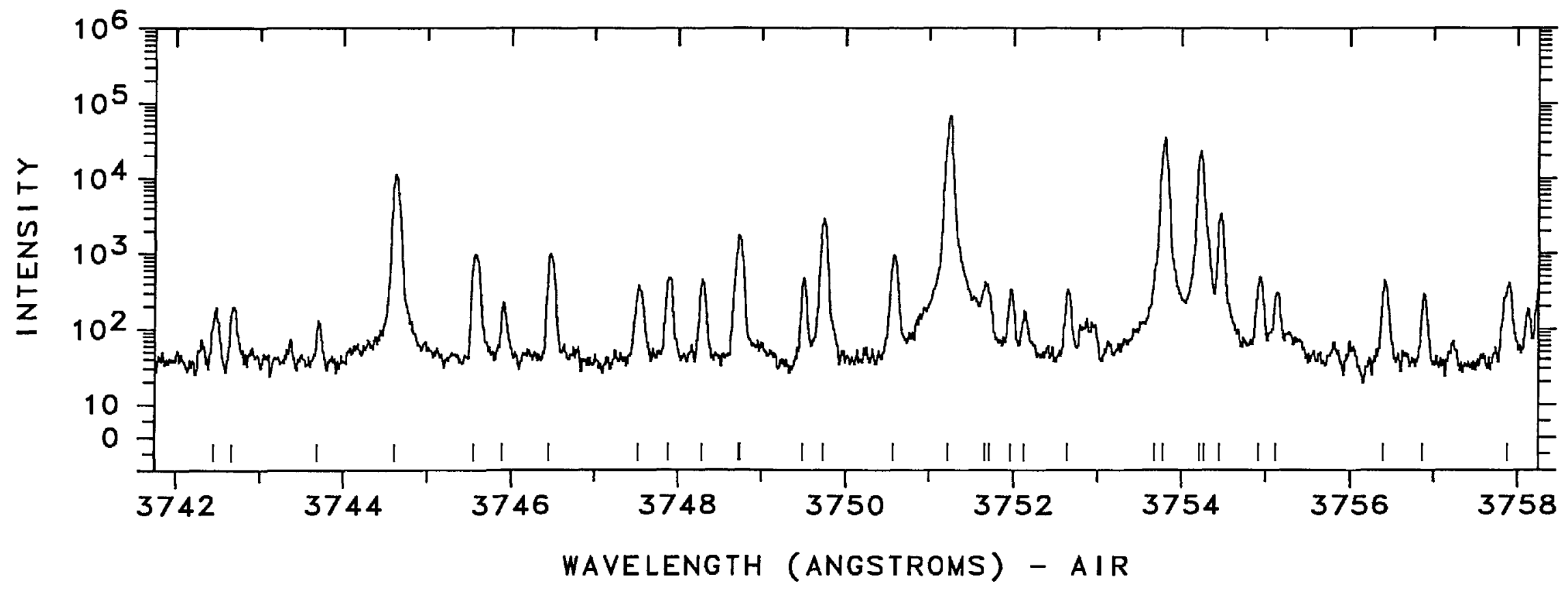




\begin{tabular}{|c|c|c|c|c|c|c|c|c|c|c|c|}
\hline WAVELENGTH & WAVE NUMBER & INTENSITY & CLA & SIFICATION & CODE & WAVELENGTH & WAVE NUMBER & INTENSITY & CLAS & SIFICATION & CODE \\
\hline $\begin{array}{l}3758.12 \\
3758.2330 \\
3758.29 \\
3758.97 \\
3759.12 \\
3760.51 \\
3760.71 \\
3761.1616 \\
3761.51 \\
3762.40 \\
3762.67 \\
3763.16 \\
3763.646 \\
3763.7891 \\
3764.708 \\
3764.9789 \\
3766.260 \\
3766.4078 \\
3766.810 \\
3767.4986 \\
3767.6446 \\
3767.6959 \\
3768.1727 \\
3768.29 \\
3768.4048 \\
3768.7573\end{array}$ & $\begin{array}{l}26601.5 \\
26600.690 \\
26600.3 \\
26595.5 \\
26594.4 \\
26584.6 \\
26583.2 \\
26579.978 \\
26577.5 \\
26571.2 \\
26569.3 \\
26565.9 \\
26562.43 \\
26561.423 \\
26554.94 \\
26553.029 \\
26544.00 \\
26542.956 \\
26540.12 \\
26535.271 \\
26534.242 \\
26533.881 \\
26530.524 \\
26529.7 \\
26528.890 \\
26526.409\end{array}$ & $\begin{array}{c}140 \\
380 \\
240 \\
290 \\
160 \\
220 \\
12000 \\
270 \\
490 \\
160 \\
80 \\
1100 \\
\\
840 \mathrm{H} \\
200 \\
260000 \\
2500 \mathrm{U} \\
390 \\
140 \\
150 \\
120 \\
270 \\
340 \\
610 \mathrm{C} \\
430 \mathrm{~W}\end{array}$ & $\begin{array}{l}\text { Pt II } \\
\text { Pt I } \\
\text { Ne II } \\
\text { Fe I } \\
\text { Ne II } \\
\text { Ne II } \\
\text { Ne II } \\
\text { Pt II }\end{array}$ & $\begin{array}{r}101199-74619 \\
65387-38815\end{array}$ & $\begin{array}{l}\mathrm{C} \\
\mathrm{R} \\
\mathrm{C} \\
\mathrm{C} \\
\mathrm{C} \\
\mathrm{K}\end{array}$ & $\begin{array}{l}3769.8806 \\
3770.27 \\
3770.54 \\
3770.9691 \\
3771.6504 \\
3771.7806 \\
3772.10 \\
3774.06 \\
3774.17 \\
3774.73 \\
3775.7464 \\
3775.86 \\
3776.4251 \\
3777.1359 \\
3779.1920 \\
3780.0762 \\
3782.38 \\
3782.65 \\
3783.88 \\
3784.7698 \\
3784.9106 \\
3785.19 \\
3786.35 \\
3789.5705 \\
3789.8282\end{array}$ & $\begin{array}{l}26518.505 \\
26515.8 \\
26513.9 \\
26510.851 \\
26506.062 \\
26505.147 \\
26502.9 \\
26489.1 \\
26488.4 \\
26484.4 \\
26477.308 \\
26476.5 \\
26472.550 \\
26467.568 \\
26453.169 \\
26446.981 \\
26430.9 \\
26429.0 \\
26420.4 \\
26414.184 \\
26413.201 \\
26411.3 \\
26403.2 \\
26380.723 \\
26378.930\end{array}$ & $\begin{array}{c}890 \\
150 \\
150 \\
220 \\
900 \\
430 \\
150 \\
60 \\
66 \\
200 \\
430 \\
250 \\
2500 \\
290000 \\
1800 \\
450 \\
83 \\
85 \\
130 \\
410 \\
1800 \mathrm{~s} \\
100 \\
200 \\
640 \\
420\end{array}$ & $\begin{array}{l}\text { Pt I } \\
\text { Ne II } \\
\text { Pt I I } \\
\text { Ne II I } \\
\text { Ne III } \\
\text { Pt I }\end{array}$ & $\begin{array}{r}60640-34122 \\
116689-90173 \\
37877-64388\end{array}$ & $\begin{array}{l}\text { N } \\
\text { G } \\
22 \\
\text { L } \\
\text { L }\end{array}$ \\
\hline
\end{tabular}




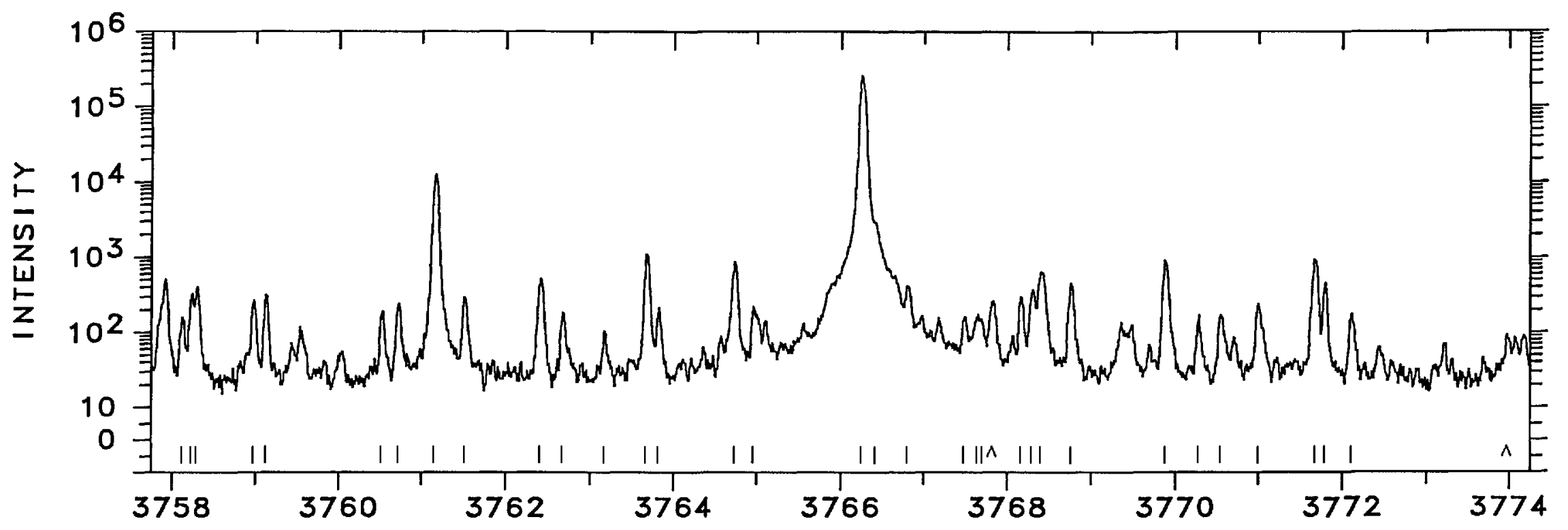

$\Xi$

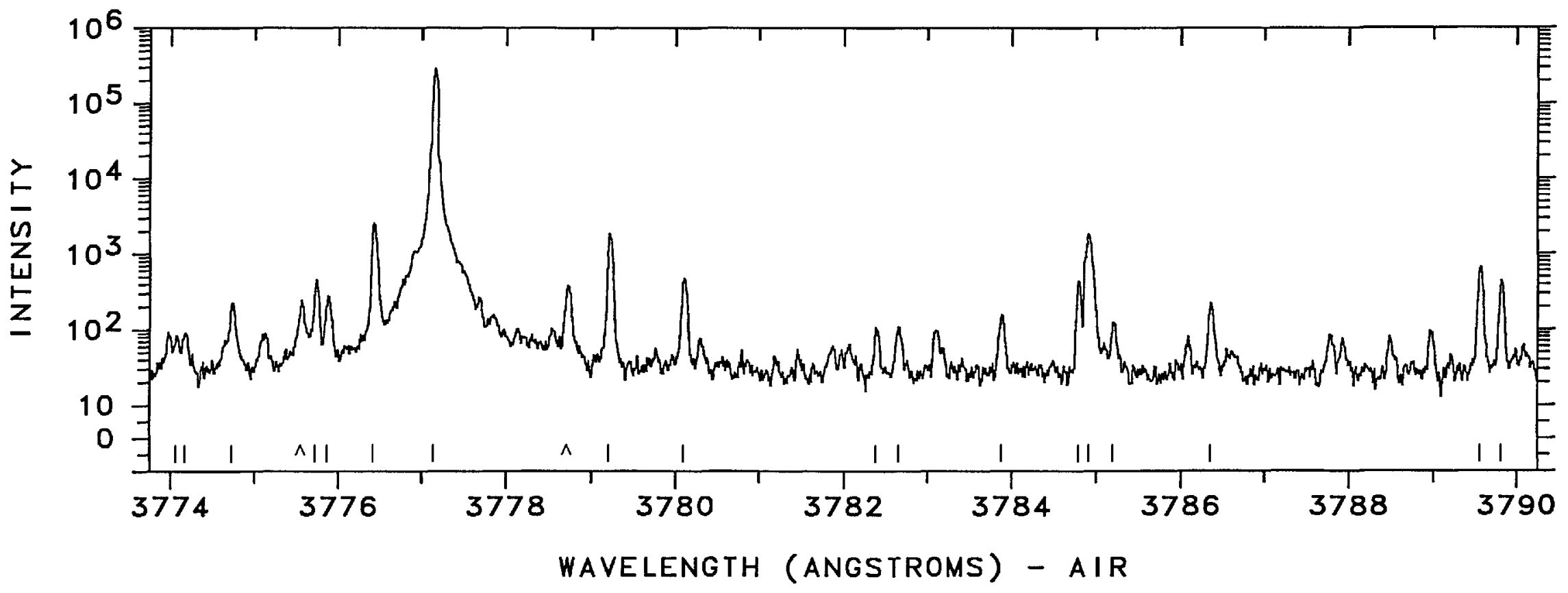




\begin{tabular}{llrlll} 
WAVELENGTH & WAVE NUMBER & INTENSITY & \multicolumn{2}{l}{ CLASSIFICATION } & CODE \\
\hline 3790.50 & 26374.3 & 98 & & & \\
3790.918 & 26371.35 & 1500 & Ne I I & & C \\
3791.59 & 26366.7 & 580 & Pt I & $68169-41802$ & N \\
3792.161 & 26362.70 & 500 & Ne I I & & C \\
3793.2055 & 26355.443 & & Rh I & & \\
3793.26 & 26355.1 & 260 & Pt II & $105962-79607$ & K \\
3793.55 & 26353.0 & 300 & & & \\
3794.5166 & 26346.337 & 460 & Pt II & $41434-67780$ & K \\
3795.01 & 26342.9 & 55 & & & \\
3795.2677 & 26341.123 & 370 & & & \\
3795.54 & 26339.2 & 95 & & & \\
3798.2534 & 26320.418 & 1000 & & & G \\
3798.3227 & 26319.937 & 1500 & Ne II & & \\
3798.74 & 26317.0 & 160 & Pt I & $65132-38815$ & N \\
3798.88 & 26316.1 & 180 & & & \\
3799.32 & 26313.0 & & Rh I & & \\
3799.64 & 26310.8 & 180 & & & G \\
3799.9645 & 26308.566 & 17000 & Ne II & & \\
3800.456 & 26305.16 & 870 & Ne II & & \\
3800.80 & 26302.8 & 270 & & & \\
3801.0723 & 26300.899 & 20000 D & Pt I & $15501-41802$ & E
\end{tabular}

\begin{tabular}{llrllll} 
WAVELENGTH & WAVE NUMBER & INTENSITY & \multicolumn{2}{l}{ CLASSIFICATION } & CODE \\
\hline 3802.3589 & 26291.999 & 1400 & Pt I & $68094-41802$ & N \\
3802.83 & 26288.7 & 90 & & & & \\
3803.0226 & 26287.412 & 1600 & Pt I & $68947-42660$ & N \\
3803.17 & 26286.4 & 510 & & & & \\
3804.40 & 26277.9 & 130 & & & & \\
3805.2973 & 26271.698 & 500 & Pt I & $62567-36296$ & N \\
3805.8569 & 26267.835 & 1500 & & & \\
3806.249 & 26265.13 & 2300 & Ne II & & C \\
3806.9248 & 26260.467 & 240 & Pt II & $34647-60907$ & 21 \\
3807.2422 & 26258.278 & 320 & & & \\
3808.1298 & 26252.157 & 2600 & Pt I & $68912-42660$ & N \\
3813.8174 & 26213.008 & 250 & & & & \\
3815.0673 & 26204.420 & 2700 & Pt I & $16983-43187$ & N \\
3815.8403 & 26199.1112 & & Fe I & & R \\
3815.88 & 26198.8 & 200 & & & \\
3817.5962 & 26187.062 & 1000 & Pt I I & $105794-79607$ & K \\
3817.8859 & 26185.075 & 330 & Ne II & & \\
3818.4236 & 26181.388 & 34000 & Ne I & $10116-36296$ & E \\
3818.6874 & 26179.579 & 380000 & Pt I & 10116 & & R \\
3820.4254 & 26167.670 & & Fe I & &
\end{tabular}




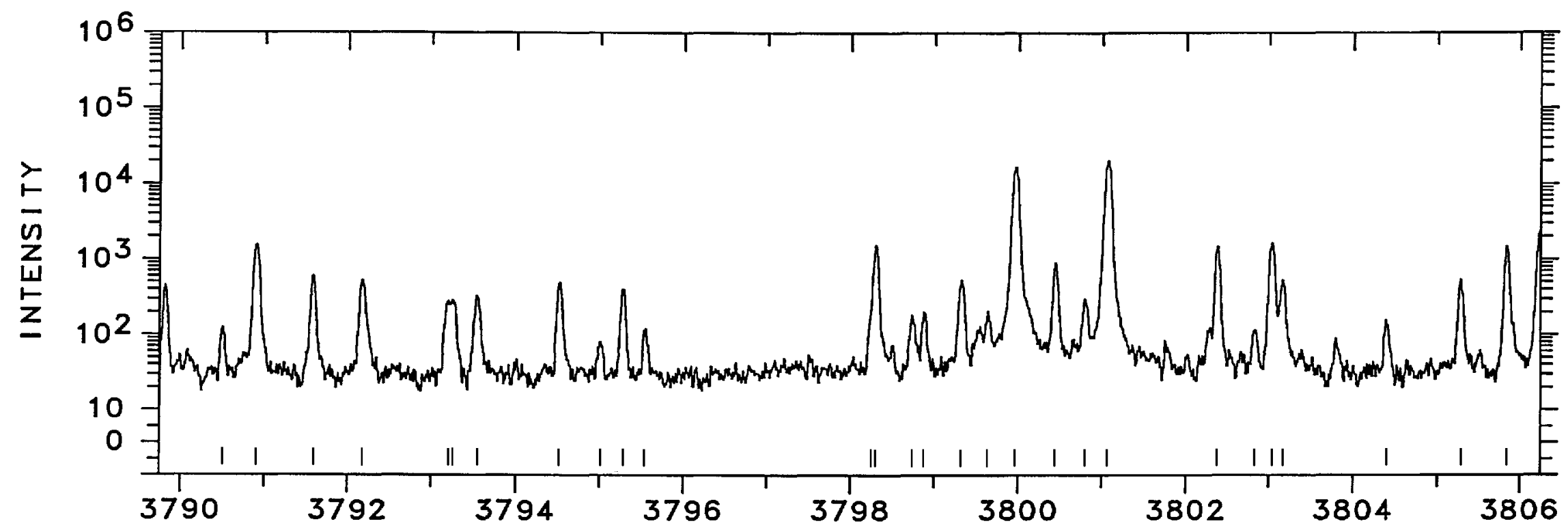

공

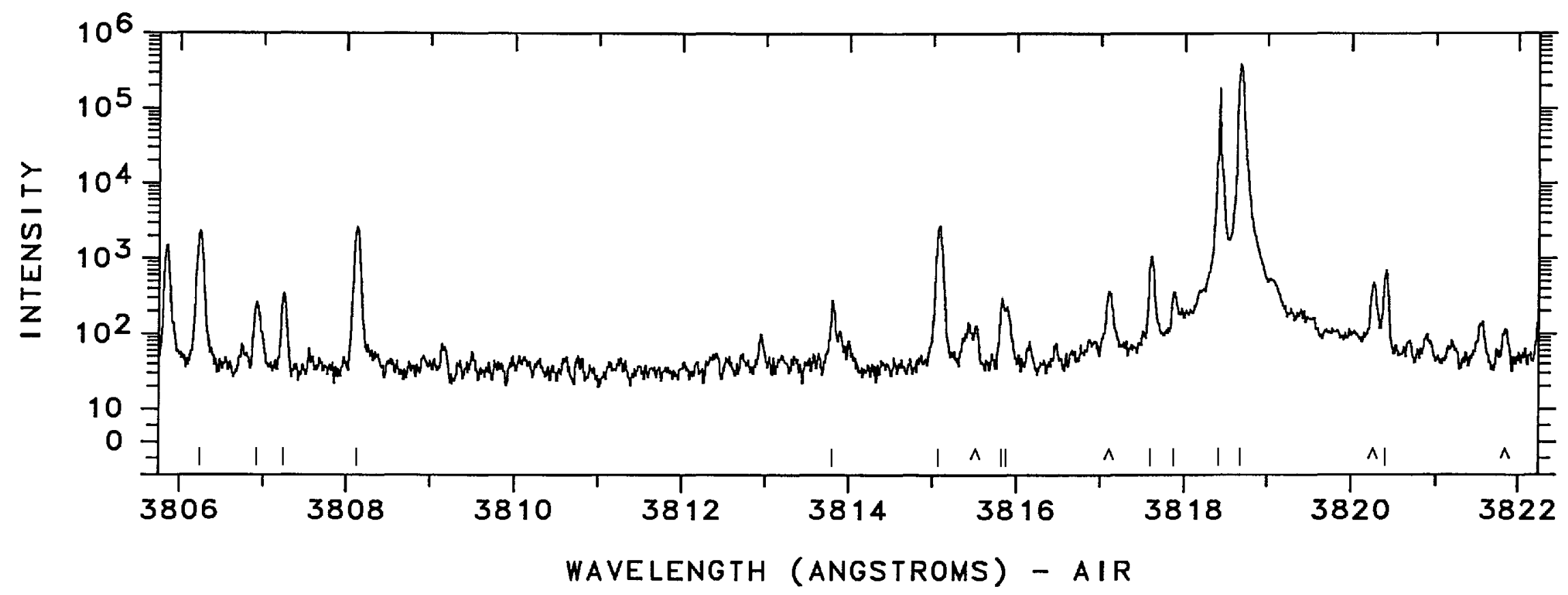




\begin{tabular}{|c|c|c|c|c|c|c|c|c|c|c|c|}
\hline WAVELENGTH & WAVE NUMBER & INTENSITY & CLA & SIFICATION & CODE & WAVELENGTH & WAVE NUMBER & INTENSITY & \multicolumn{2}{|c|}{ CLASSI FI CATION } & CODE \\
\hline $\begin{array}{l}3822.25 \\
3822.7531 \\
3823.152 \\
3824.4436 \\
3825.8814 \\
3827.8227 \\
3828.46 \\
3829.34 \\
3829.7503 \\
3832.31 \\
3834.2224 \\
3835.20\end{array}$ & $\begin{array}{l}26155.2 \\
26151.736 \\
26149.01 \\
26140.177 \\
26130.354 \\
26117.102 \\
26112.8 \\
26106.8 \\
26103.957 \\
26086.5 \\
26073.511 \\
26066.9\end{array}$ & $\begin{array}{r}180 \\
69000 \\
190\end{array}$ & $\begin{array}{ll}\mathrm{Rh} & \mathrm{I} \\
\mathrm{Pt} & \mathrm{II} \\
\mathrm{Ne} & \mathrm{II} \\
\mathrm{Fe} & \mathrm{I} \\
\mathrm{Fe} & \mathrm{I} \\
\mathrm{Fe} & \mathrm{I} \\
\mathrm{Rh} & \mathrm{I} \\
& \\
\mathrm{Ne} & \mathrm{II} \\
\mathrm{Mg} & \mathrm{I} \\
\mathrm{Fe} & \mathrm{I}\end{array}$ & $101517-75365$ & $\begin{array}{l}K \\
C \\
Q \\
Q \\
Q\end{array}$ & $\begin{array}{l}3837.29 \\
3838.2891 \\
3838.6561 \\
3839.4742 \\
3840.4953 \\
3841.0480 \\
3842.9636 \\
3843.24 \\
3844.88 \\
3847.78 \\
3850.41 \\
3852.13\end{array}$ & $\begin{array}{l}26052.7 \\
26045.886 \\
26043.396 \\
26037.847 \\
26030.924 \\
26027.179 \\
26014.205 \\
26012.3 \\
26001.2 \\
25981.6 \\
25963.9 \\
25952.3\end{array}$ & $\begin{array}{r}350 \\
\\
670 \\
500 \\
3900 \\
\\
980 \\
130 \\
160 \\
220 \\
340 \\
110\end{array}$ & $\begin{array}{l}\text { Pt I } \\
\text { Mg I } \\
\text { Pt I } \\
\text { Ne I I } \\
\text { Fe I } \\
\text { Pt I I } \\
\text { Ne I I I } \\
\mathrm{Ne} \text { I I I } \\
\mathrm{Ne} \text { I I I } \\
\text { Pt I I }\end{array}$ & $\begin{array}{r}6567-32620 \\
68703-42660\end{array}$ & $\begin{array}{l}\text { N } \\
\text { N } \\
\text { C } \\
Q \\
20 \\
\text { L } \\
\text { L } \\
\text { K }\end{array}$ \\
\hline
\end{tabular}




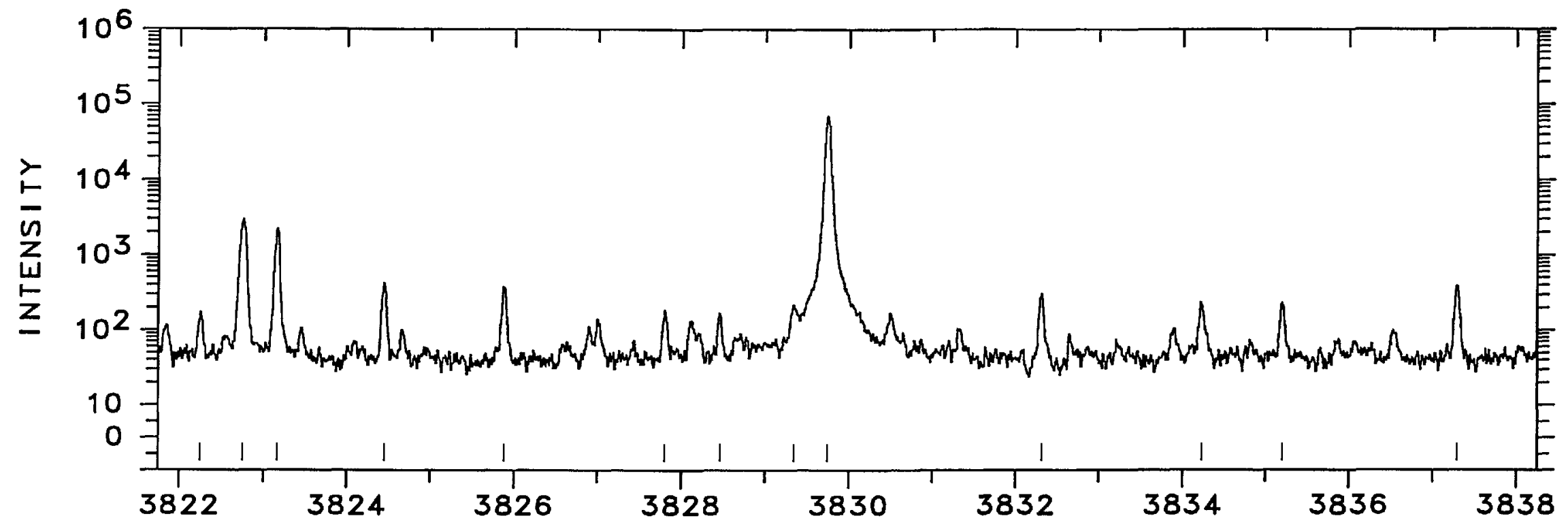

$\stackrel{\infty}{\infty}$

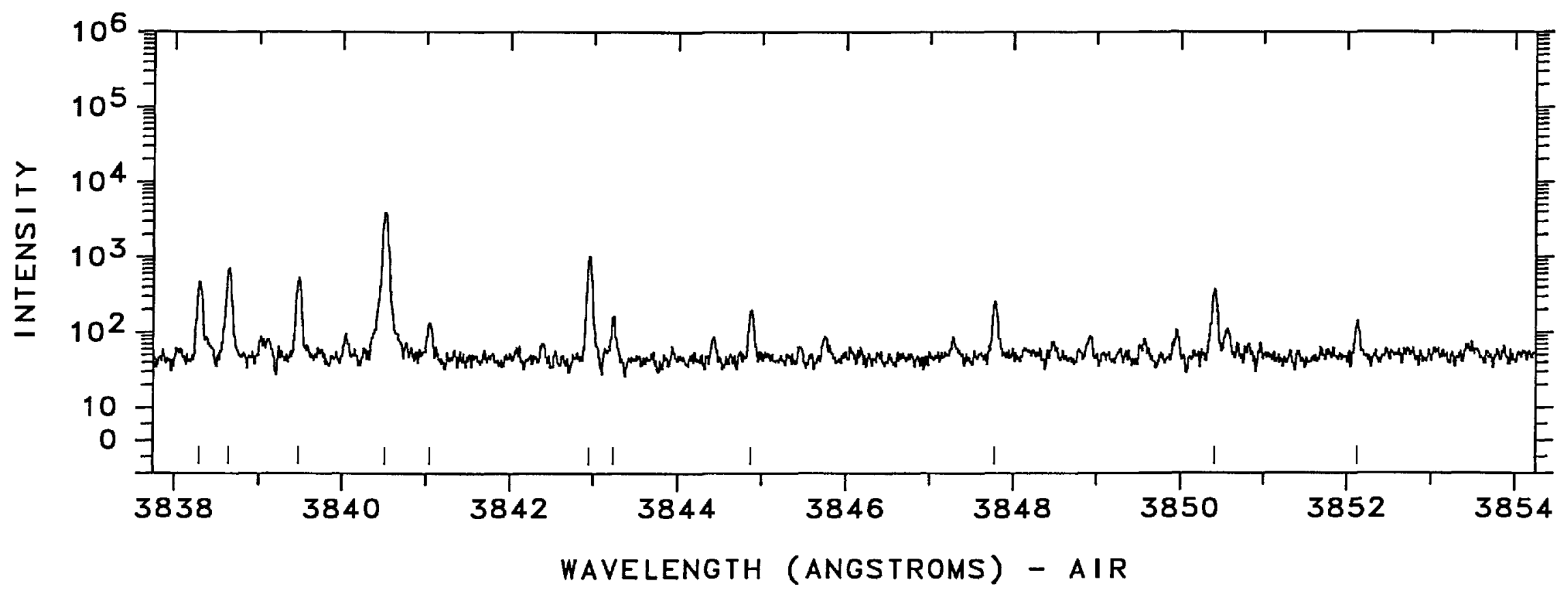




\begin{tabular}{llrlll} 
WAVELENGTH & WAVE NUMBER & INTENSITY & \multicolumn{2}{c}{ CLASSIFICATION } & CODE \\
\hline 3854.5252 & 25936.178 & 2000 & Pt I I & $101517-75581$ & $\mathrm{~K}$ \\
3855.24 & 25931.4 & 180 & & & \\
3856.3716 & 25923.760 & & Fe I & & Q \\
3856.53 & 25922.7 & 160 & Rh I & & \\
3856.78 & 25921.0 & 960 & Ne II & & C \\
3857.817 & 25914.05 & 960 & & & R \\
3858.07 & 25912.3 & 280 & & & \\
3859.9115 & 25899.986 & & Fe I & &
\end{tabular}

WAVELENGTH WAVE NUMBER INTENSITY

CLASSIFICATION

CODE

$\begin{array}{llllll}3865.7875 & 25860.619 & 530 & \text { Pt I } & 62705-36844 & N\end{array}$

$3868.420925843 .015 \quad 22000$ Pt I $64379-38536 \mathrm{~N}$

3868.93

3869.8816

25839.6

640

3876.9749

3878.3549

3878.5733

$\begin{array}{lllll}25785.997 & 22000 & \text { Pt I } & 64330-38536 & \mathrm{~N}\end{array}$

3880.8488

1000

Pt

59908- 34122 E

3882.3976

25775.371

(5)

2600

25760.258

$\mathrm{Fe}$

1500

2100

Pt I 59882- 34122

59872- 34122

Pt I $\quad 64267-38536 \mathrm{~N}$ 


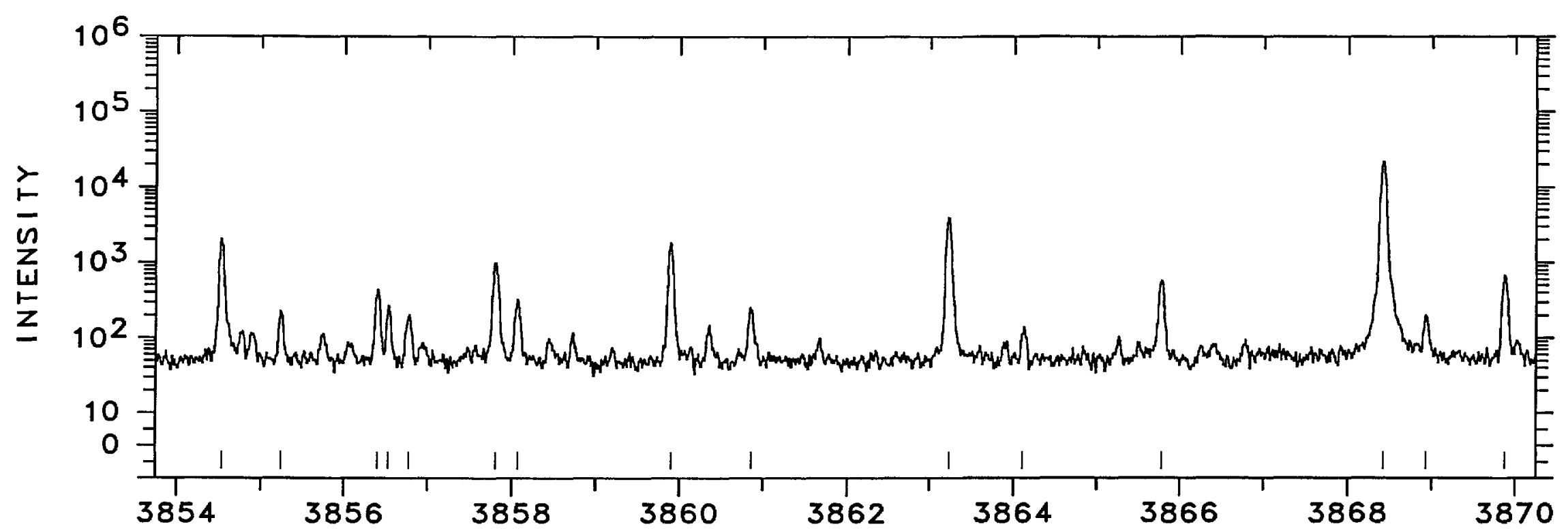

㐫

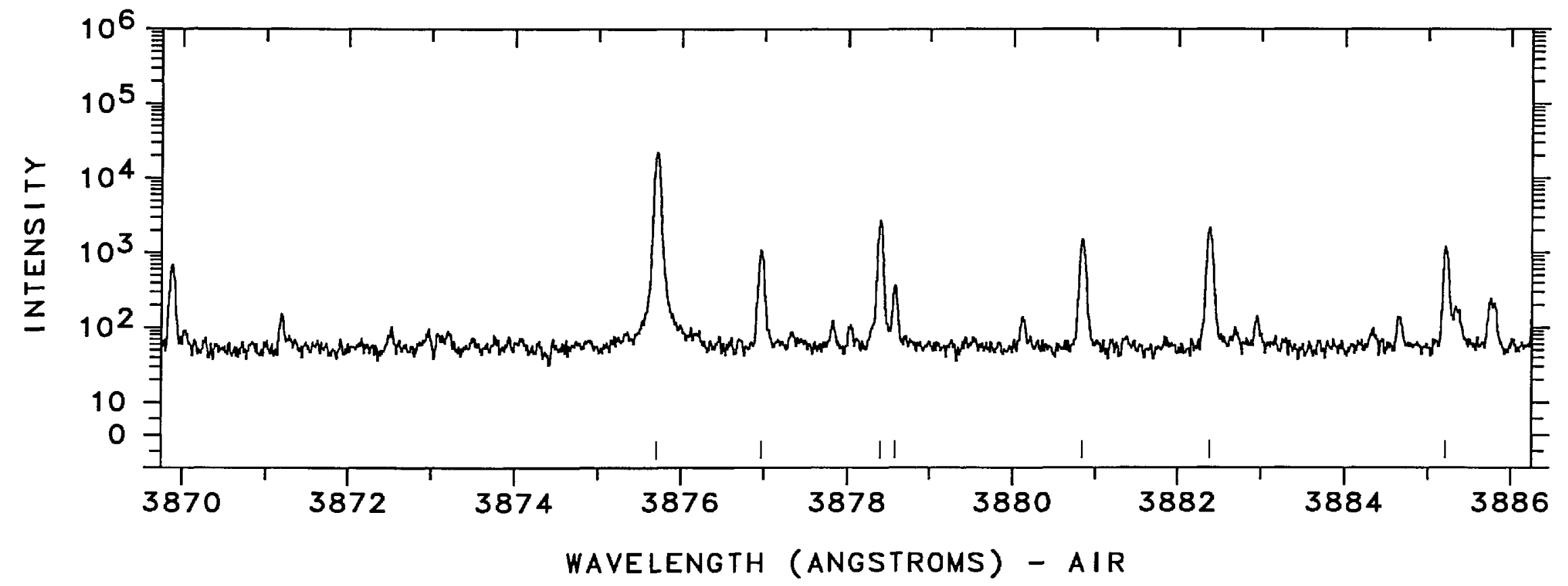




\begin{tabular}{lccllll} 
WAVELENGTH & WAVE NUMBER & INTENSITY & \multicolumn{2}{c}{ CLASSIFICATION } & CODE \\
\hline & & & & \\
\hline 3886.2823 & 25724.243 & & Fe I & & \\
3894.33 & 25671.1 & 190 & Pt II & $117340-91669$ & K \\
3895.6564 & 25662.344 & & Fe I & & Q \\
3896.846 & 25654.51 & 940 & Ne II & & C \\
3898.7316 & 25642.103 & $23000 \mathrm{~S}$ & Pt I & $59764-34122$ & E \\
3899.7074 & 25635.687 & & Fe I & & $Q$ \\
3900.7228 & 25629.014 & $51000 \mathrm{~S}$ & Pt I & $59751-34122$ & E \\
3902.4512 & 25617.663 & 3400 & Pt II & $101199-75581$ & 36
\end{tabular}

WAVELENGTH WAVE NUMBER INTENSITY

CLASSIFICATION

$\begin{array}{llrlrl}3903.7085 & 25609.412 & 7100 & \text { Pt I } & 59731-34122 & \text { E } \\ 3904.3823 & 25604.993 & 18000 & \text { Pt I } & 64141-38536 & \text { E } \\ 3905.57 & 25597.2 & 350 & \text { Pt II } & 105794-80197 & \text { K } \\ 3906.2788 & 25592.562 & 5500 & \text { Pt I } & 64128-38536 & \text { E } \\ 3908.75 & 25576.4 & 300 & \text { Pt II } & 106434-80858 & \text { K } \\ 3910.8955 & 25562.351 & 20000 & \text { Pt I } & 60884-35321 & \text { E } \\ 3911.98 & 25555.3 & 230 & & & \\ 3913.47 & 25545.5 & 180 & & & \end{array}$




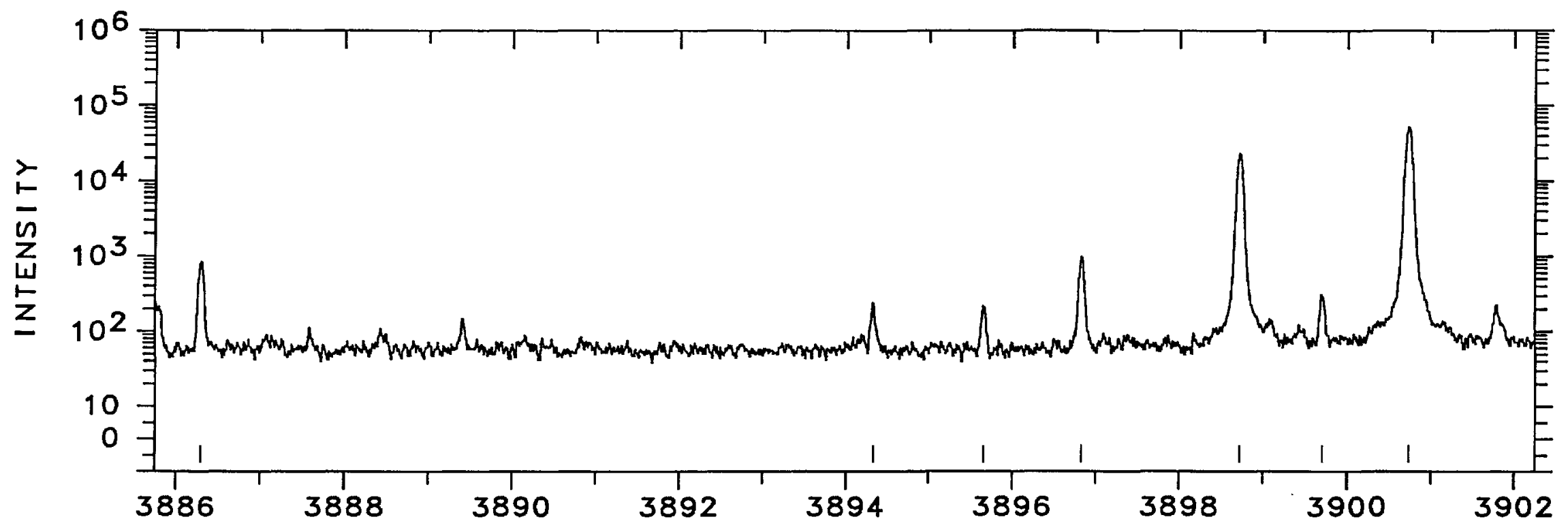

品

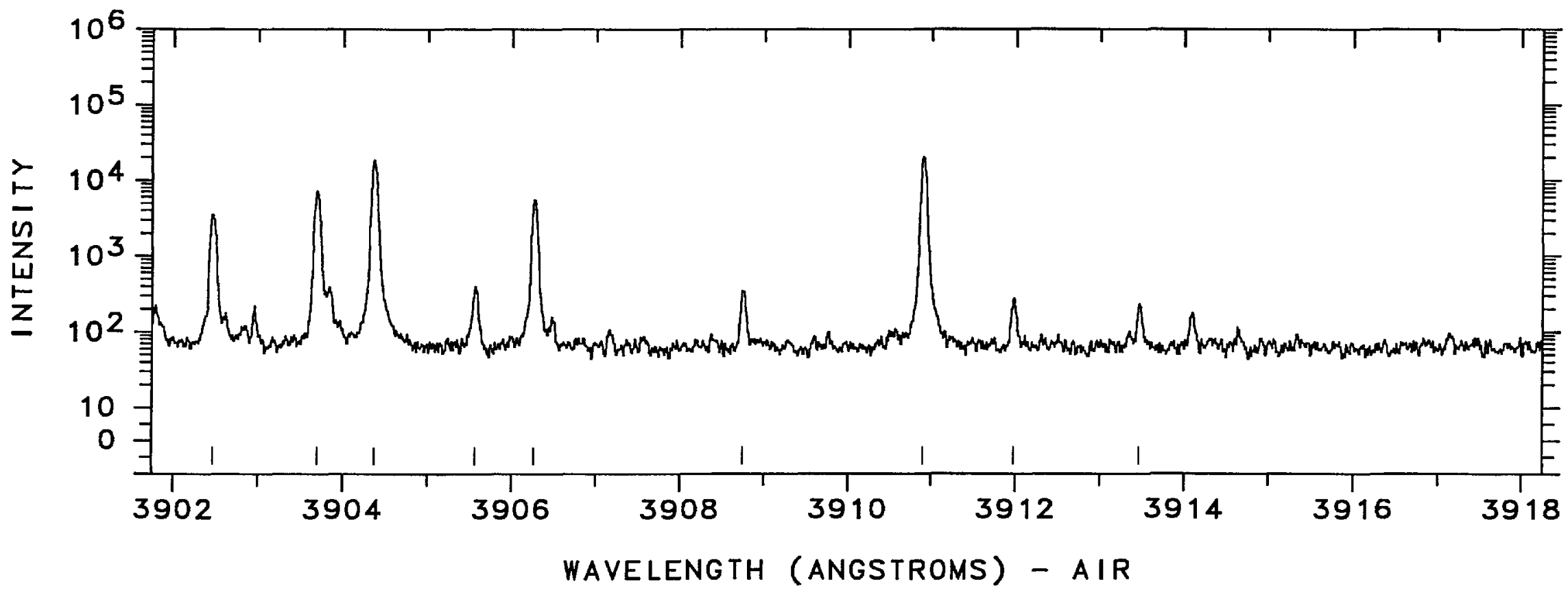




\begin{tabular}{|c|c|c|c|c|c|c|c|c|c|c|c|}
\hline WAVELENGTH & WAVE NUMBER & INTENSITY & & I FICATI ON & CODE & WAVELENGTH & WAVE NUMBER & INTENSI TY & \multicolumn{2}{|c|}{ CLASSIFICATION } & CODE \\
\hline $\begin{array}{l}3919.50 \\
3920.2580 \\
3920.63 \\
3920.78 \\
3921.79 \\
3922.9559 \\
3923.0660 \\
3925.2718 \\
3925.3359 \\
3926.3831 \\
3927.9199 \\
3930.2967\end{array}$ & $\begin{array}{l}25506.2 \\
25501.304 \\
25498.9 \\
25497.9 \\
25491.3 \\
25483.766 \\
25483.051 \\
25468.731 \\
25468.315 \\
25461.523 \\
25451.562 \\
25436.170\end{array}$ & $\begin{array}{r}210 \\
\\
220 \\
190 \\
170 \\
86000 \mathrm{C} \\
370 \mathrm{U} \\
3500 \\
22000 \\
1700\end{array}$ & $\begin{array}{ll}\text { Fe I } \\
\\
\text { Pt I } \\
\text { Pt } \\
\text { Pt } & \text { I } \\
\text { Pt } & \text { I } \\
\text { Fe } & \text { I } \\
\text { Fe } & \text { I }\end{array}$ & $\begin{array}{l}55640-30156 \\
60790-35321 \\
15501-40970 \\
68121-42660\end{array}$ & $\begin{array}{l}E \\
E \\
E \\
N \\
Q \\
Q\end{array}$ & $\begin{array}{l}3931.83 \\
3933.465 \\
3933.66 \\
3941.5998 \\
3942.262 \\
3943.99 \\
3944.11 \\
3946.63 \\
3948.3325 \\
3948.3881 \\
3948.4117 \\
3948.59\end{array}$ & $\begin{array}{l}25426.3 \\
25415.68 \\
25414.4 \\
25363.230 \\
25358.97 \\
25347.9 \\
25347.1 \\
25330.9 \\
25319.981 \\
25319.625 \\
25319.474 \\
25318.3\end{array}$ & $\begin{array}{c}480 \\
3700 \\
2100 \\
8600 \\
\\
180 \\
170 \\
3000 \mathrm{P} \\
14000 \mathrm{P} \\
4000 \mathrm{U} \\
240\end{array}$ & $\begin{array}{ll}\mathrm{Ne} & \text { II } \\
\mathrm{Ca} & \mathrm{II} \\
\mathrm{Pt} & \mathrm{I} \\
\mathrm{Ne} & \mathrm{II} \\
\mathrm{Al} & \mathrm{I} \\
\mathrm{Pt} & \mathrm{I}\end{array}$ & $\begin{array}{l}68006-42660 \\
13496-38815 \\
13496-38815 \\
13496-38815 \\
46046-71364\end{array}$ & $\begin{array}{l}\mathrm{C} \\
\mathrm{N} \\
\mathrm{C} \\
\mathrm{N}\end{array}$ \\
\hline
\end{tabular}




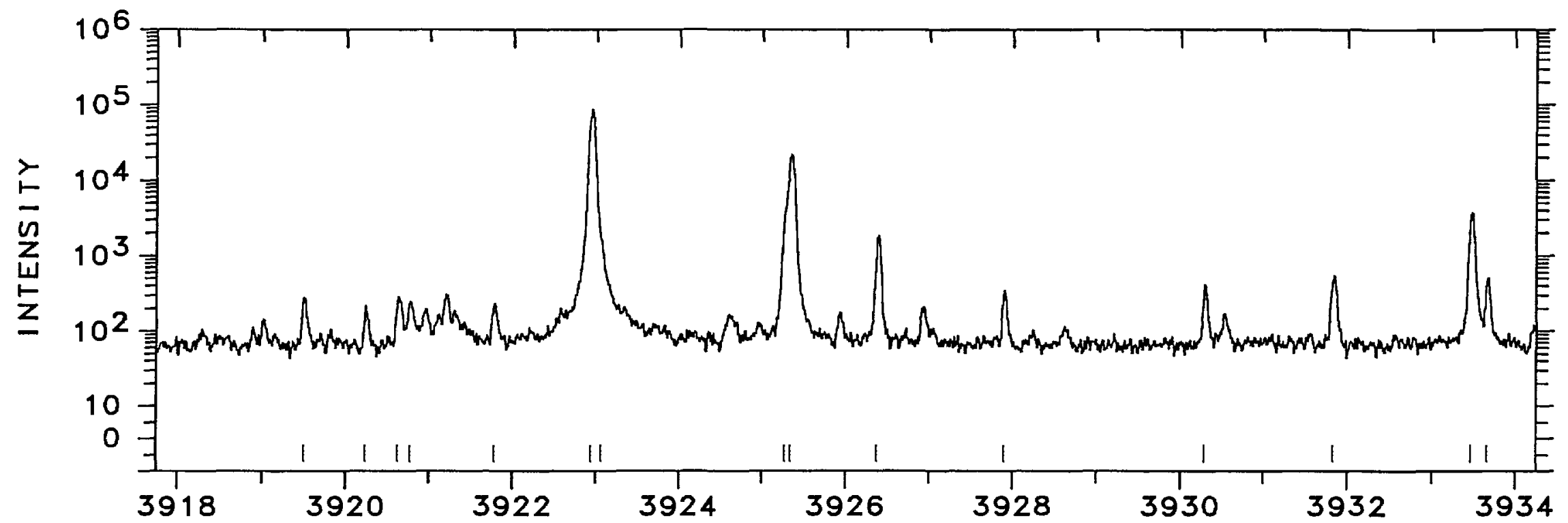

$\stackrel{\infty}{⿶}$

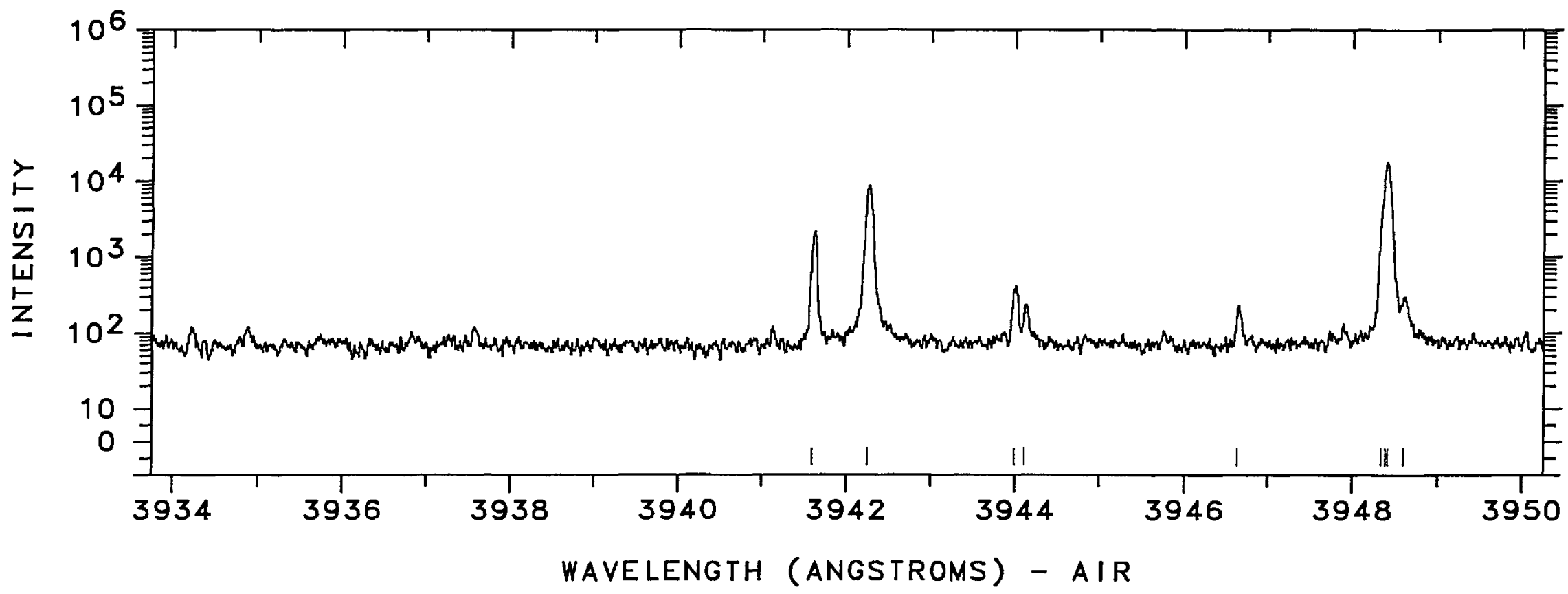




\begin{tabular}{|c|c|c|c|c|c|c|c|c|c|c|c|}
\hline WAVELENGTH & WAVE NUMBER & INTENSITY & CLA & IF ICATION & CODE & WAVELENGTH & WAVE NUMBER & INTENSITY & CLA & IFICATION & CODE \\
\hline $\begin{array}{l}3953.6375 \\
3961.51 \\
3963.04 \\
3966.3570 \\
3968.44\end{array}$ & $\begin{array}{l}25286.008 \\
25235.8 \\
25226.0 \\
25204.921 \\
25191.7\end{array}$ & $\begin{array}{r}1800 \\
170 \\
150000\end{array}$ & $\begin{array}{ll}\text { Pt } & \text { I } \\
\text { Al } & \text { I } \\
\text { Pt } & \text { I } \\
\text { Pt } & \text { I } \\
\text { Ca } & \text { I I }\end{array}$ & $\begin{array}{l}15501-40787 \\
62567-37342 \\
10116-35321\end{array}$ & $\begin{array}{l}\mathrm{E} \\
\mathrm{N} \\
\mathrm{E}\end{array}$ & $\begin{array}{l}3970.0530 \\
3971.40 \\
3973.458 \\
3975.69 \\
3980.6010\end{array}$ & $\begin{array}{l}25181.457 \\
25172.9 \\
25159.88 \\
25145.8 \\
25114.731\end{array}$ & $\begin{array}{r}1800 \\
190 \\
410 \\
640 \\
1400\end{array}$ & $\begin{array}{l}\text { Pt II } \\
\text { Ne II } \\
\text { Pt I }\end{array}$ & $26638-51753$ & $\begin{array}{l}16 \\
\mathrm{C} \\
\mathrm{E}\end{array}$ \\
\hline
\end{tabular}



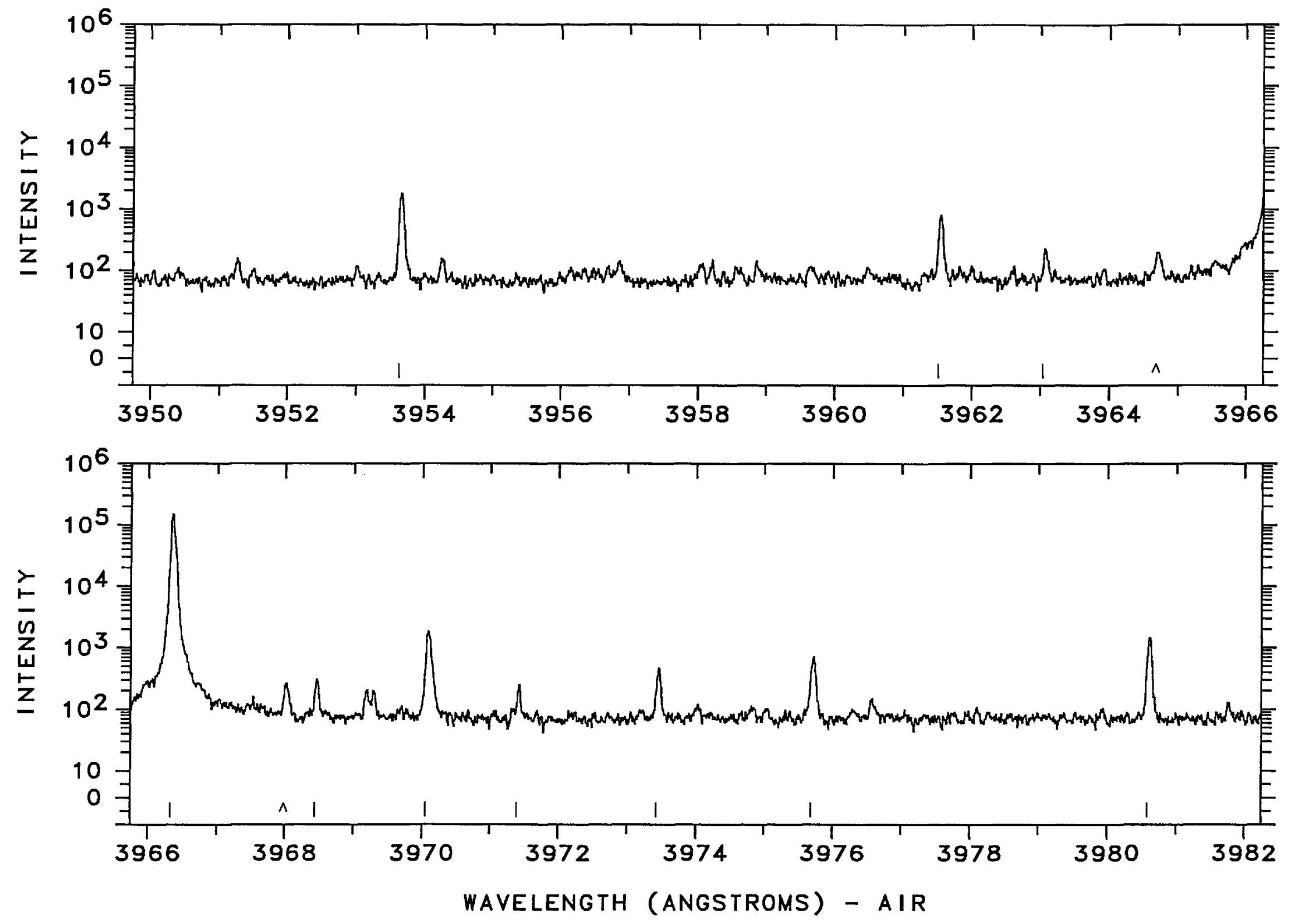


\begin{tabular}{llrllll} 
WAVELENGTH & WAVE NUMBER & INTENSI TY & \multicolumn{2}{c}{ CLASSIFICATION } & CODE \\
\hline & & & & & \\
\hline 3982.26 & 25104.3 & 230 & Pt I I & $105962-80858$ & $\mathrm{~K}$ \\
3982.87 & 25100.4 & 200 & & & & \\
3984.94 & 25087.4 & 300 & Pt I & $68275-43187$ & N \\
3985.723 & 25082.46 & 1300 & Ne I I & & C \\
3985.85 & 25081.7 & 170 & & & & \\
3990.27 & 25053.9 & 270 & & & & \\
3996.5674 & 25014.399 & 17000 & Pt I & $15501-40516$ & E \\
3998.79 & 25000.5 & 250 & Pt I I & $41434-66434$ & K
\end{tabular}

$\begin{array}{llrlll}3999.468 & 24996.26 & 1700 & \text { Ne I I } & & \text { C } \\ 4001.80 & 24981.7 & 220 & \text { Pt I } & 68169-43187 & \text { N } \\ 4002.4834 & 24977.427 & 6100 & \text { Pt I } & 62567-37590 & \text { N } \\ 4004.69 & 24963.7 & 260 & & & \\ 4009.0950 & 24936.236 & 2700 & \text { Pt I } & 62705-37769 & \text { N } \\ 4009.94 & 24931.0 & 160 & & & \\ 4010.68 & 24926.4 & 210 & & & \\ 4013.7145 & 24907.536 & 23000 & \text { Pt II } & 101517-76610 & \text { K }\end{array}$



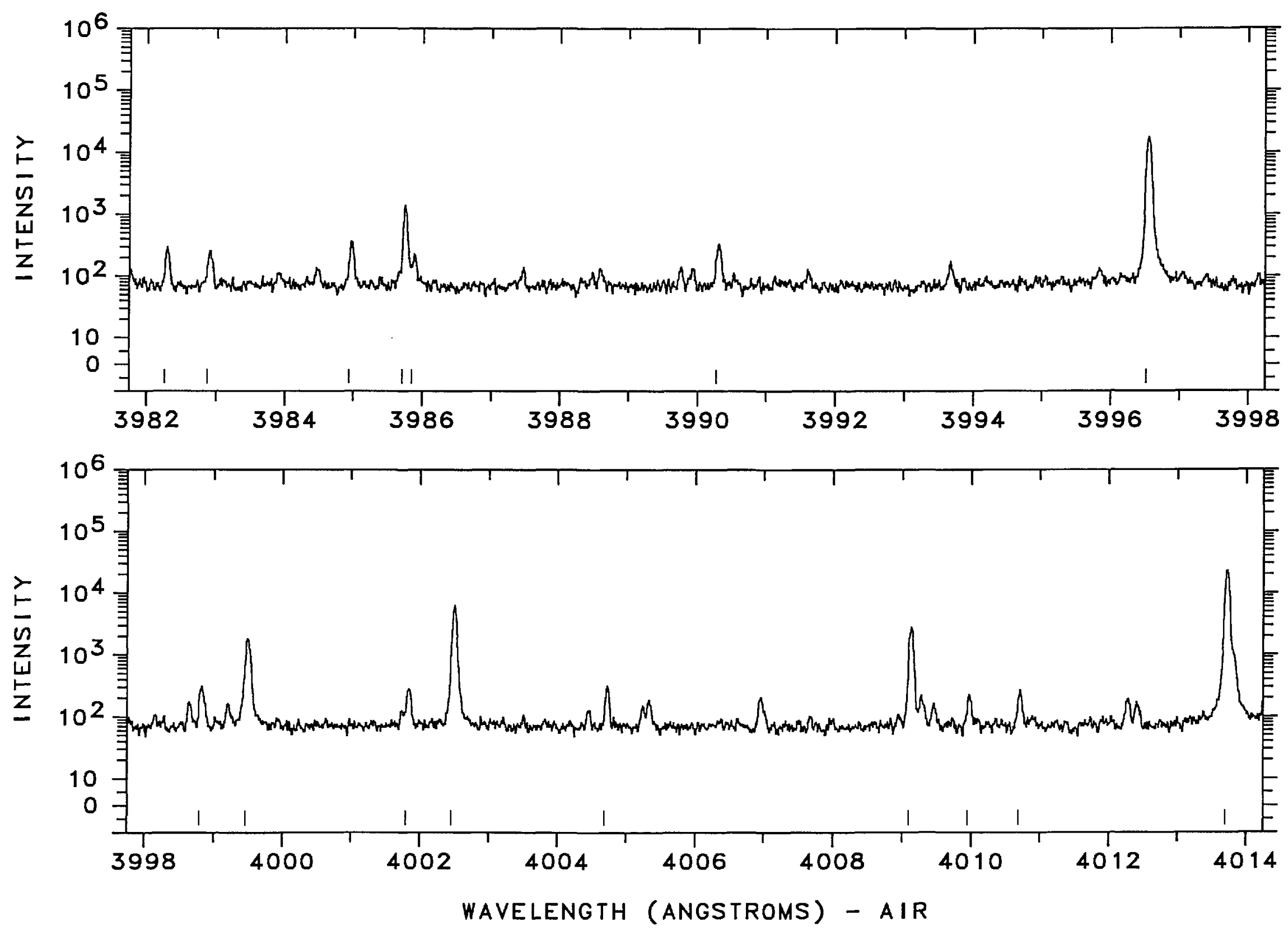


\begin{tabular}{llclll} 
HAVELENGTH & WAVE NUMBER & INTENSITY & \multicolumn{2}{c}{ CLASSIFICATION } & CODE \\
\hline 4014.3061 & 24903.866 & 360 & Pt II & $37877-62781$ & 30 \\
4014.75 & 24901.1 & 160 & & & \\
4018.21 & 24879.7 & 480 & Pt I & $65395-40516$ & $\mathrm{~N}$ \\
4019.61 & 24871.0 & 460 & Pt I & $65387-40516$ & $\mathrm{~N}$ \\
4020.48 & 24865.6 & 200 & & & \\
4022.42 & 24853.6 & 110 & & & \\
4022.73 & 24851.7 & 130 & & & \\
4023.11 & 24849.4 & 190 & & & \\
4023.8153 & 24845.014 & 230 & Pt II & $29030-53875$ & 17 \\
4024.041 & 24843.62 & 370 & Ne II & & C
\end{tabular}

WAVELENGTH WAVE NUMBER INTENSITY

CLASSIFICATION

CODE

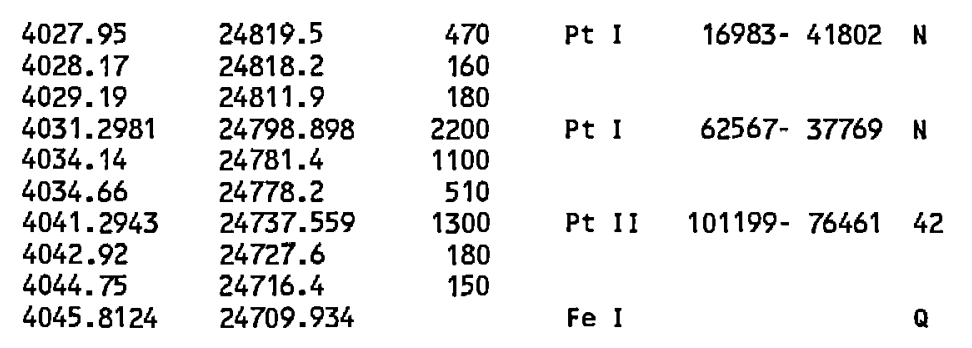




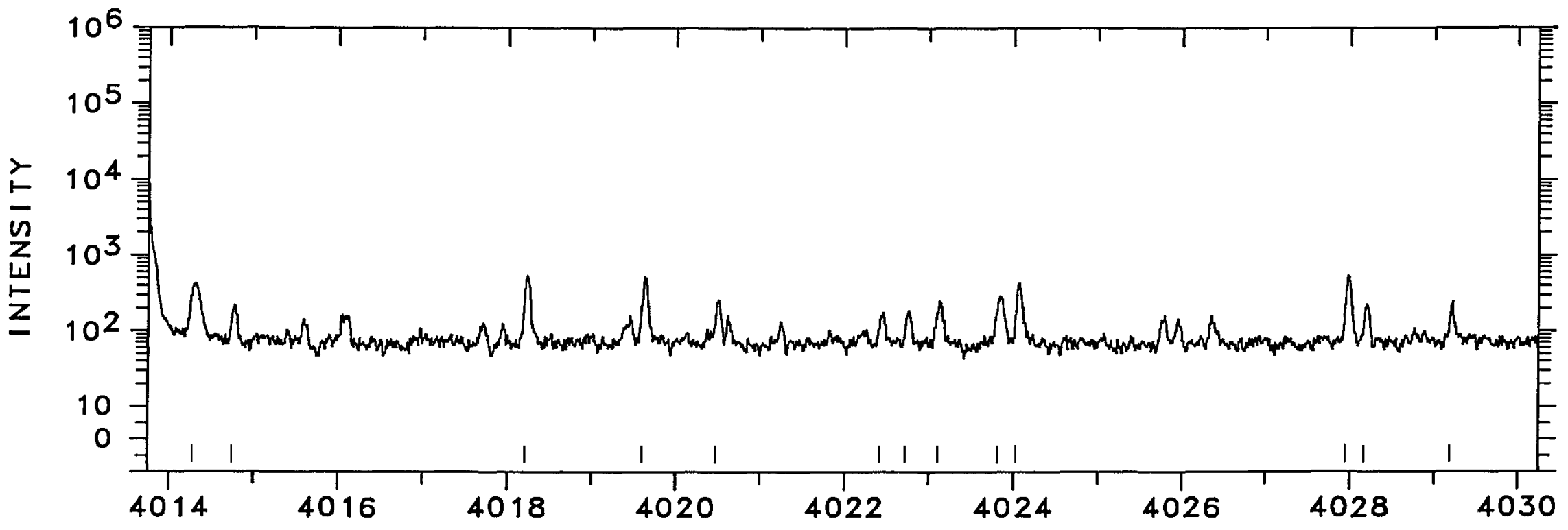

$\vec{w}$

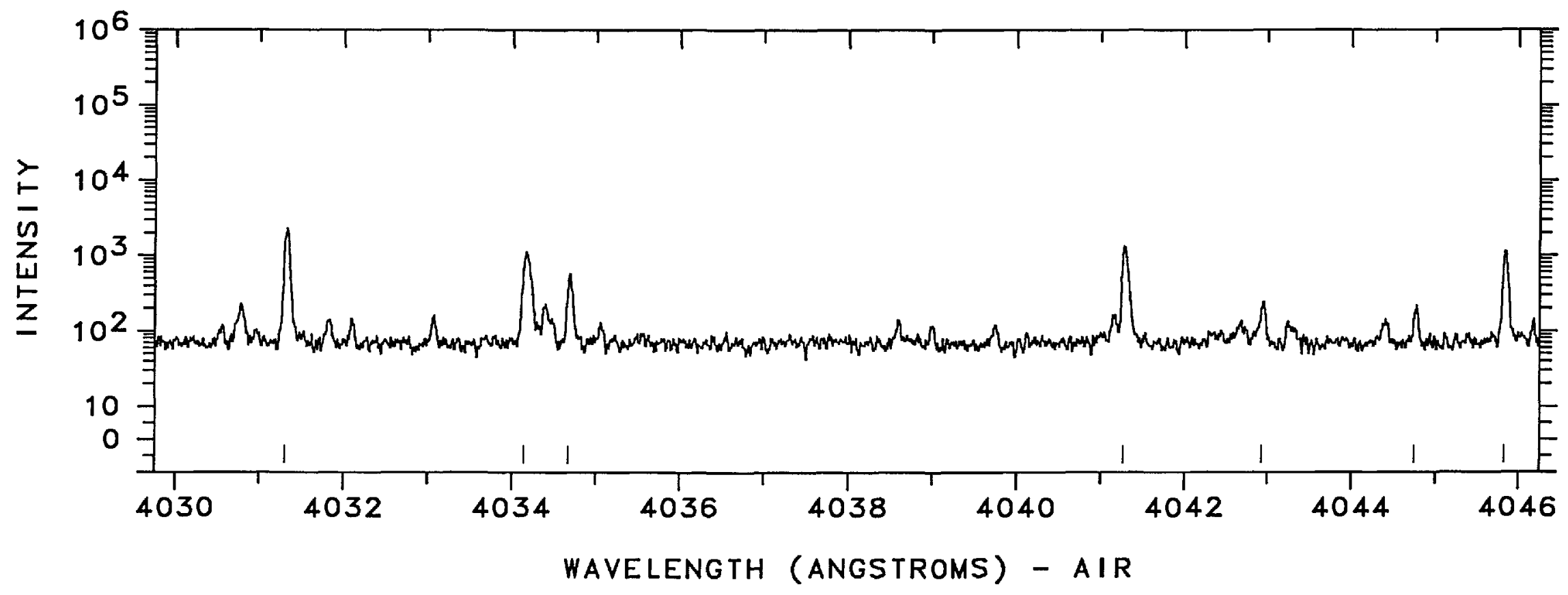


WAVELENGTH WAVE NUMBER INTENSITY

CLASSI FICATION

CODE

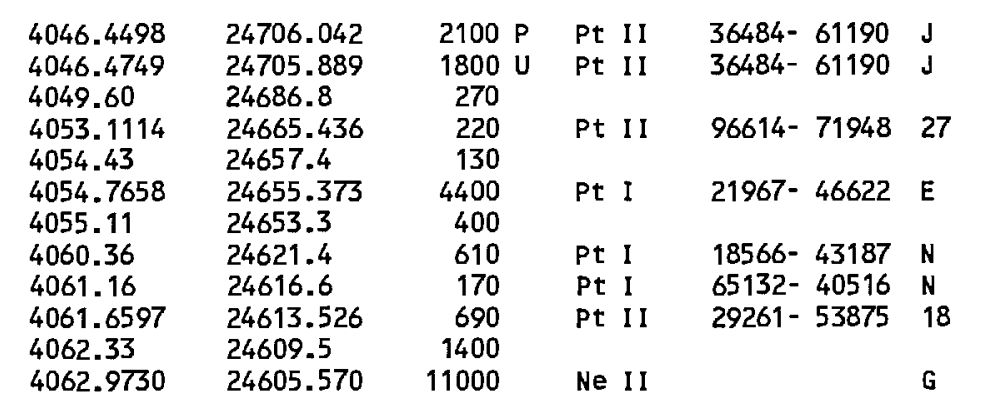

WAVELENGTH

WAVE NUMBER INTENSITY

CLASSI FICATION

CODE

4063.5940

4065.8895

24589.039

24589.039

Fe I

11000

101199- 76610 Q

$4065.9283 \quad 24587.686 \quad 16000 \mathrm{P}$ Pt I 60884- $36296 \mathrm{H}$

$4066.09 \quad 24586.7 \quad 460$ Pt I $59908-35321 \mathrm{~N}$

$\begin{array}{llrlll}4066.63 & 24583.4 & 220 & & & \\ 4070.3844 & 24560.769 & 1900 & \text { Pt I } & 59882-35321 \quad \text { E }\end{array}$

$4071.55 \quad 24553.7$

$4071.7379 \quad 24552.605$

4071.85

$\begin{array}{ll}4071.85 & 24551.9 \\ 4072.1002 & 24550.420\end{array}$

560

Fe I

200

210

$\mathrm{Pt}$

$59872-35321 \quad \mathrm{~N}$ 

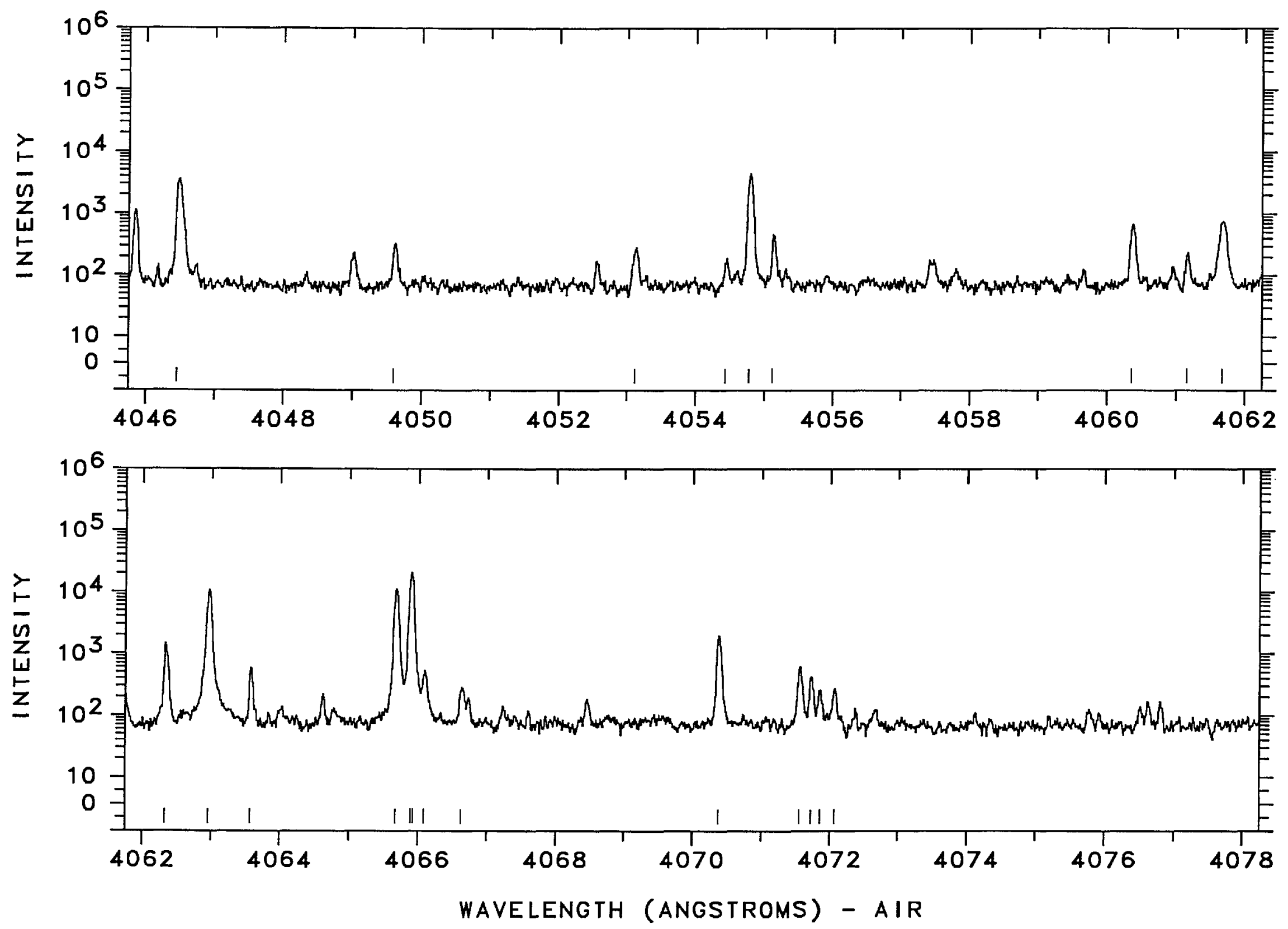


\begin{tabular}{|c|c|c|c|c|c|c|c|c|c|c|c|}
\hline \multirow[b]{2}{*}{$\begin{array}{l}\text { WAVELENGTH } \\
\\
4080.516 \\
4081.4669 \\
4083.9285 \\
4084.7775 \\
4085.21 \\
4086.769 \\
4087.3313 \\
4090.0628 \\
4092.2522 \\
4092.92 \\
4094.36 \\
4095.31 \\
4095.5370 \\
4097.48 \\
4098.1807 \\
4098.77\end{array}$} & \multirow[b]{2}{*}{$\begin{array}{l}\text { WAVE NUMBER } \\
\\
24499.79 \\
24494.080 \\
24479.316 \\
24474.229 \\
24471.6 \\
24462.30 \\
24458.937 \\
24442.603 \\
24429.526 \\
24425.5 \\
24416.9 \\
24411.3 \\
24409.933 \\
24398.4 \\
24394.187 \\
24390.7\end{array}$} & $\frac{\text { INTENSI TY }}{5900}$ & \multicolumn{2}{|c|}{ CLASSIFICATION } & $\begin{array}{c}\mathrm{CODE} \\
\mathrm{C} \\
\mathrm{E}\end{array}$ & $\begin{array}{c}\text { WAVELENGTH } \\
4098.864\end{array}$ & $\begin{array}{c}\text { WAVE NUMBER } \\
24390.12\end{array}$ & $\begin{array}{c}\text { I NTENS ITY } \\
13000\end{array}$ & \multicolumn{2}{|c|}{ CLASSIFICATION } & CODE \\
\hline & & $\begin{array}{c}5900 \\
14000 \mathrm{c} \\
330 \\
2900 \\
220 \\
3100 \\
2800 \\
5100 \\
19000 \\
400 \\
490 \\
430 \\
2800 \\
1700 \\
280 \\
590\end{array}$ & 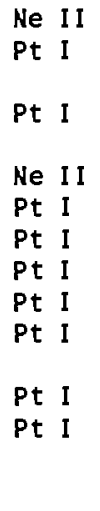 & $\begin{array}{l}26638-51097 \\
59764-35321 \\
59751-35321 \\
65395-40970 \\
65387-40970\end{array}$ & $\begin{array}{l}\mathrm{N} \\
\mathrm{C} \\
\mathrm{E} \\
\mathrm{E} \\
\mathrm{E} \\
\mathrm{N} \\
\mathrm{N}\end{array}$ & $\begin{array}{l}4098.864 \\
4100.354 \\
4100.95 \\
4101.36 \\
4101.928 \\
4104.36 \\
4104.68 \\
4104.73 \\
4105.4613 \\
4106.61 \\
4107.60 \\
4108.05 \\
4109.05 \\
4109.61 \\
4109.88\end{array}$ & $\begin{array}{l}24390.12 \\
24381.26 \\
24377.7 \\
24375.3 \\
24371.90 \\
24357.5 \\
24355.6 \\
24355.3 \\
24350.927 \\
24344.1 \\
24338.2 \\
24335.6 \\
24329.7 \\
24326.3 \\
24324.7\end{array}$ & $\begin{array}{r}13000 \\
3900 \\
190 \\
260 \\
320 \\
400 \\
490 \\
490 \\
820 \\
220 \\
150 \\
180 \\
550 \\
450 \\
250\end{array}$ & $\begin{array}{l}\text { Pt II } \\
\text { Pt I }\end{array}$ & $\begin{array}{l}32237-56587 \\
65308-40970\end{array}$ & $\begin{array}{l}\mathrm{N} \\
\mathrm{N}\end{array}$ \\
\hline
\end{tabular}




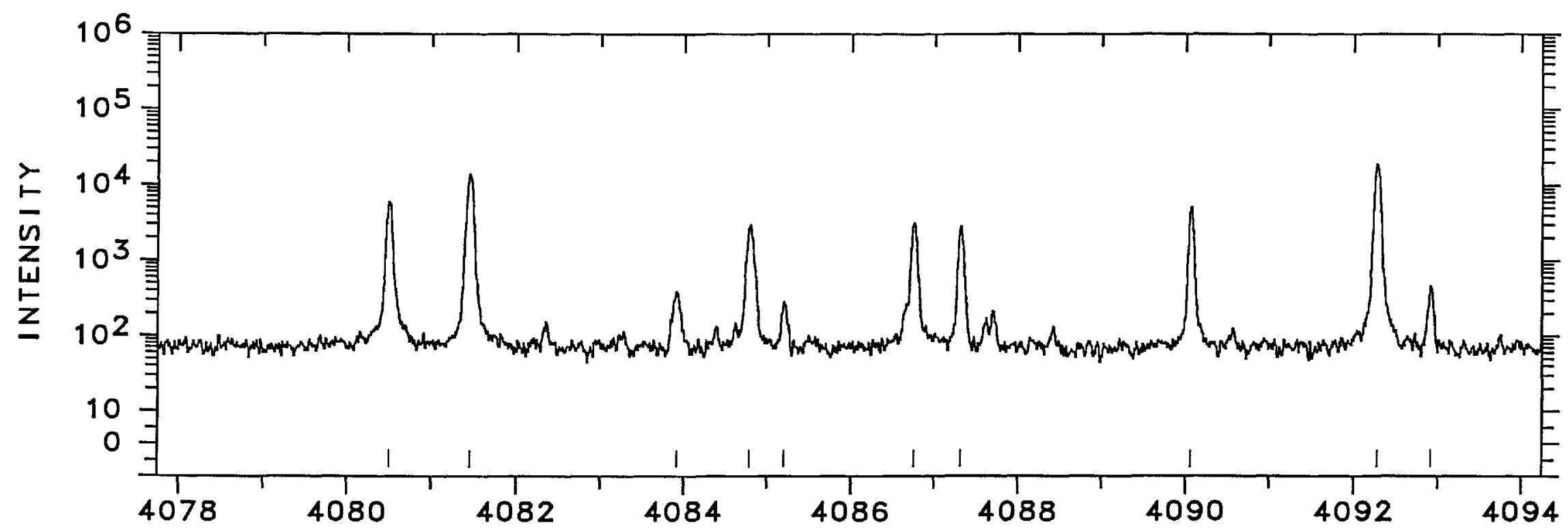

6

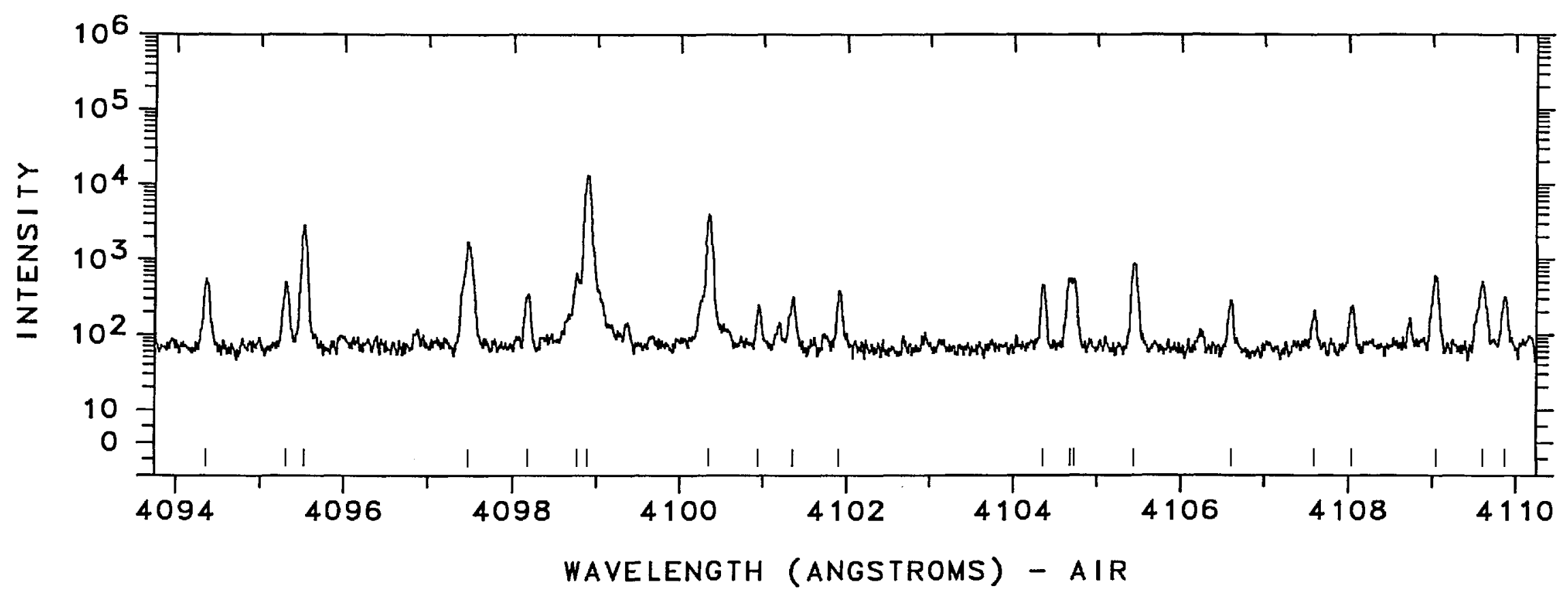




\begin{tabular}{|c|c|c|c|c|c|c|c|c|c|c|c|}
\hline WAVELENGTH & WAVE NUMBER & INTENSITY & CLA & IFICATION & CODE & WAVELENGTH & WAVE NUMBER & INTENSITY & \multicolumn{2}{|c|}{ CLASSIF ICATION } & CODE \\
\hline $\begin{array}{l}4112.12 \\
4112.395 \\
4114.13 \\
4115.24 \\
4116.97 \\
4118.199 \\
4118.6745 \\
4119.20 \\
4120.41 \\
4120.56 \\
4127.00\end{array}$ & $\begin{array}{l}24311.5 \\
24309.87 \\
24299.6 \\
24293.1 \\
24282.9 \\
24275.61 \\
24272.808 \\
24269.7 \\
24262.6 \\
24261.7 \\
24223.8\end{array}$ & $\begin{array}{r}300 \\
3000 \\
330 \\
160 \\
270 \\
2200 \\
89000 \\
350 \\
120 \\
130 \\
140\end{array}$ & $\begin{array}{l}\text { Pt I } \\
\text { Ne II } \\
\text { Ne II } \\
\text { Pt I }\end{array}$ & 13496- 37769 & $\begin{array}{l}C \\
E\end{array}$ & $\begin{array}{l}4128.09 \\
4131.09 \\
4132.38 \\
4133.691 \\
4133.871 \\
4135.18 \\
4137.08 \\
4137.47 \\
4138.34 \\
4139.75\end{array}$ & $\begin{array}{l}24217.4 \\
24199.9 \\
24192.3 \\
24184.63 \\
24183.58 \\
24175.9 \\
24164.8 \\
24162.5 \\
24157.5 \\
24149.2\end{array}$ & $\begin{array}{r}420 \\
280 \\
250 \\
17000 \\
2000 \\
710 \\
230 \\
2700 \\
320 \\
250\end{array}$ & $\begin{array}{l}\text { Ne I I } \\
\text { Ne I I } \\
\text { Pt I } \\
\text { Pt I } \\
\text { Pt I }\end{array}$ & $\begin{array}{l}68121-43945 \\
65132-40970 \\
68094-43945\end{array}$ & $\begin{array}{l}\mathrm{C} \\
\mathrm{C} \\
\mathrm{N} \\
\mathrm{N} \\
\mathrm{N}\end{array}$ \\
\hline
\end{tabular}




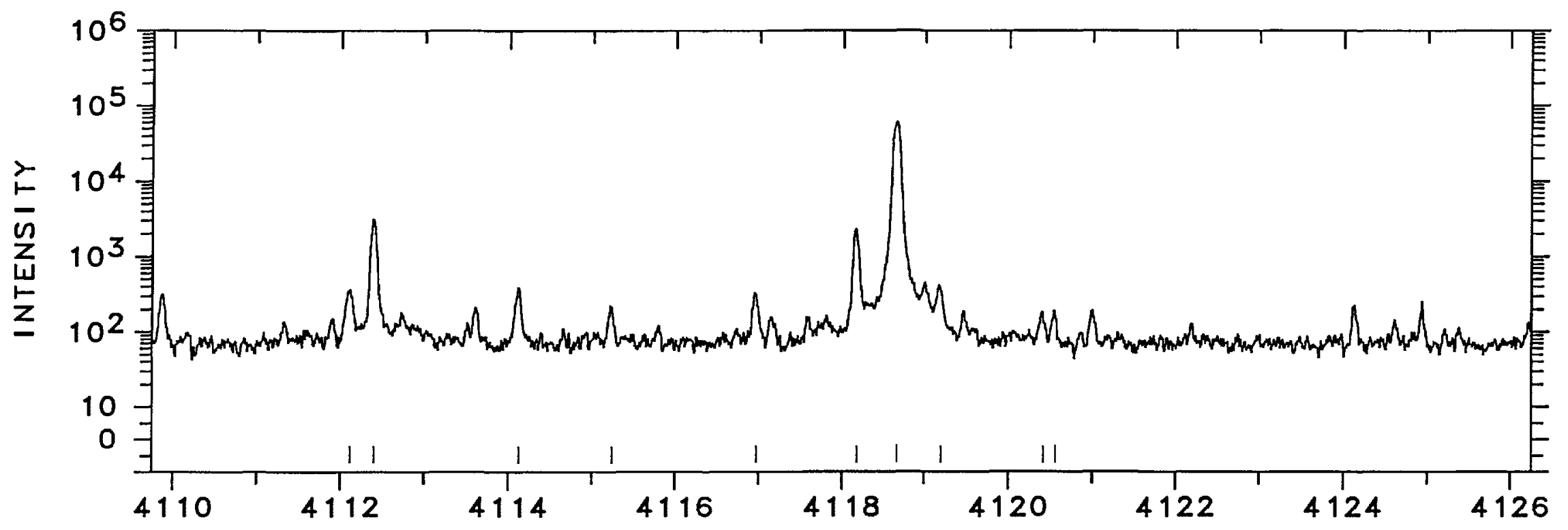

훙

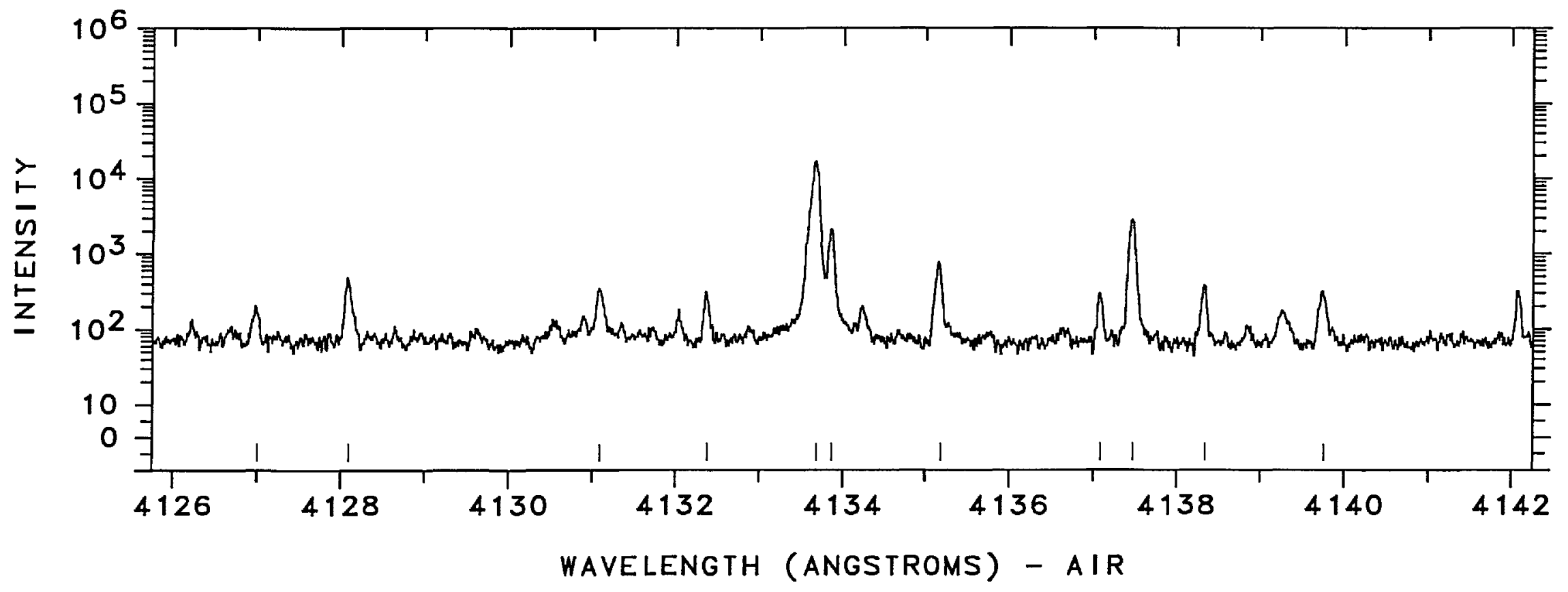




\begin{tabular}{|c|c|c|c|c|c|c|c|c|c|c|c|}
\hline HAVELENGTH & WAVE NUMBER & INTENSITY & $\mathrm{CL}$ & IFICATION & CODE & WAVELENGTH & WAVE NUMBER & INTENSITY & \multicolumn{2}{|c|}{ CLASSI FICATI ON } & CODE \\
\hline $\begin{array}{l}4142.09 \\
4142.83 \\
4143.61 \\
4143.8680 \\
4145.03 \\
4147.50 \\
4148.2820 \\
4150.6893\end{array}$ & $\begin{array}{l}24135.6 \\
24131.3 \\
24126.7 \\
24125.240 \\
24118.5 \\
24104.1 \\
24099.569 \\
24085.592\end{array}$ & $\begin{array}{r}260 \\
150 \\
840 \\
\\
6300 \\
260 \\
770 \\
20000\end{array}$ & $\begin{array}{l}\text { Pt I } \\
\text { Fe I } \\
\text { Pt I } \\
\text { Pt II } \\
\text { Ne II }\end{array}$ & $\begin{array}{l}68072-43945 \\
64312-40194 \\
32918-57018\end{array}$ & $\begin{array}{l}N \\
Q \\
N \\
16 \\
G\end{array}$ & $\begin{array}{l}4152.84 \\
4153.30 \\
4154.84 \\
4159.45 \\
4164.5491 \\
4167.30 \\
4169.08 \\
4172.89\end{array}$ & $\begin{array}{l}24073.1 \\
24070.5 \\
24061.5 \\
24034.9 \\
24005.436 \\
23989.6 \\
23979.3 \\
23957.5\end{array}$ & $\begin{array}{r}920 \\
240 \\
270 \\
200 \\
78000 \\
5300 \\
3100 \\
180\end{array}$ & 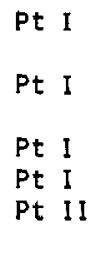 & $\begin{array}{r}64267-40194 \\
68006-43945 \\
\\
10116-34122 \\
64505-40516 \\
101517-77538\end{array}$ & $\begin{array}{l}\mathrm{N} \\
\mathrm{N} \\
\mathrm{E} \\
\mathrm{N} \\
\mathrm{K}\end{array}$ \\
\hline
\end{tabular}




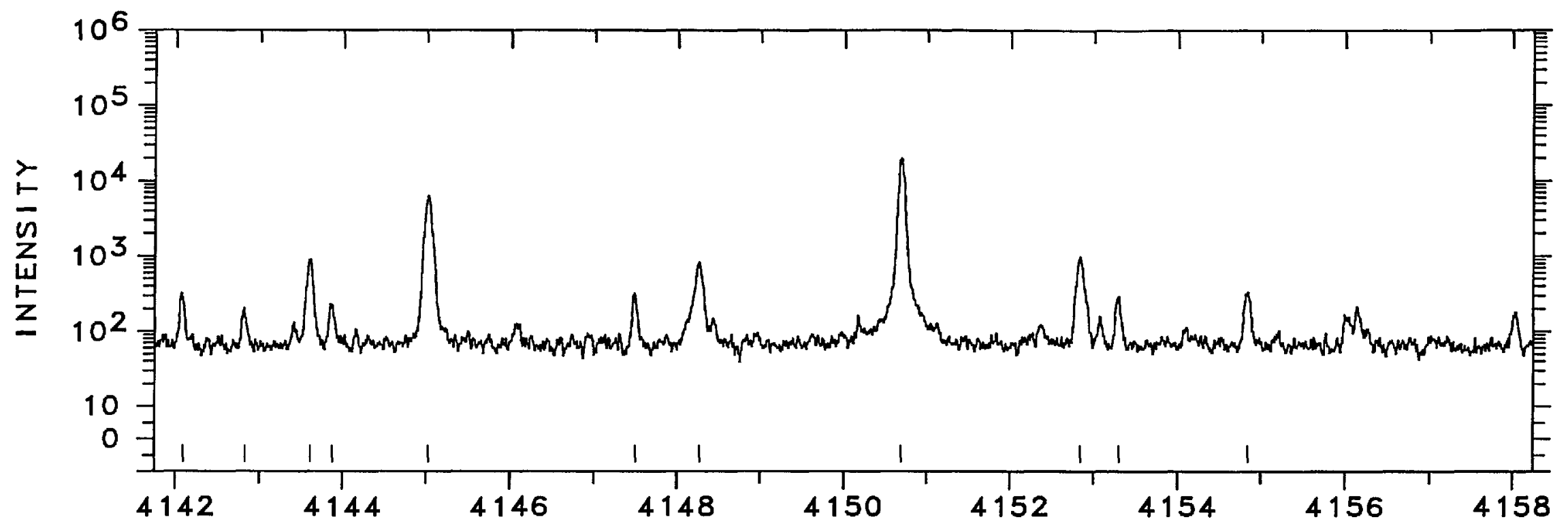

吕

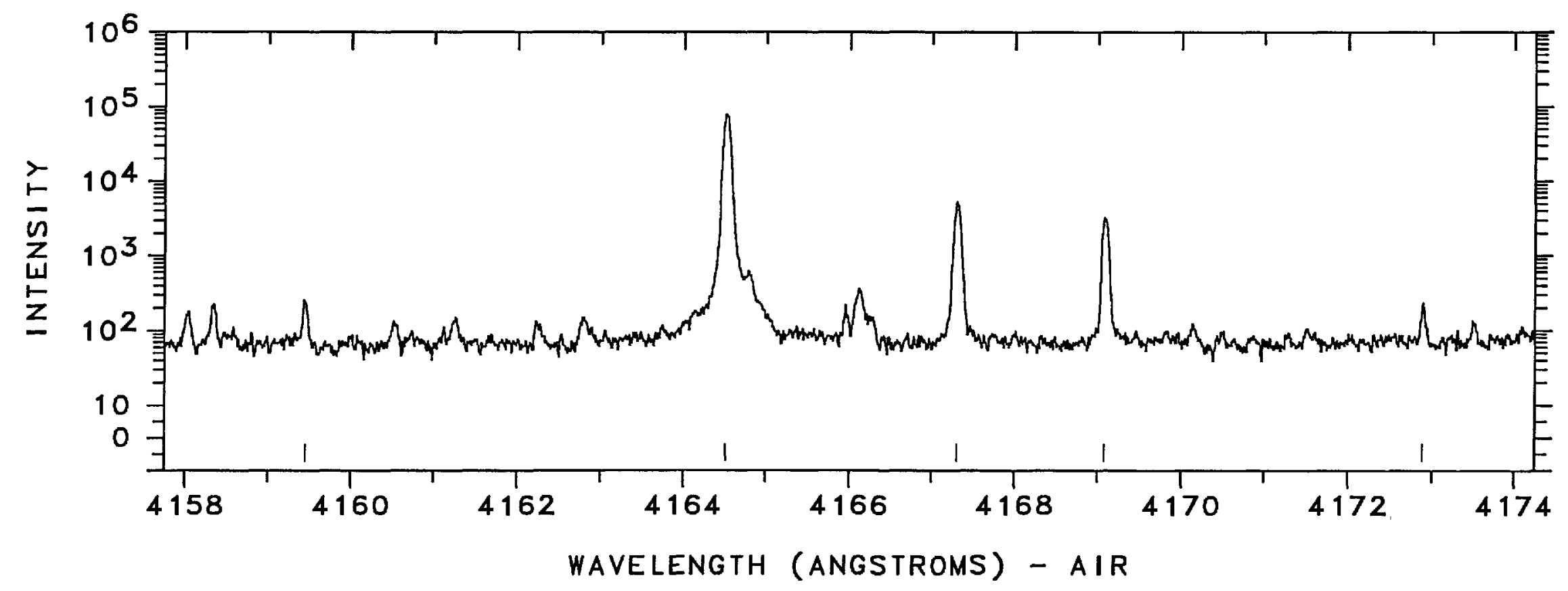




\begin{tabular}{|c|c|c|c|c|c|c|c|c|c|c|c|}
\hline WAVELENGTH & WAVE NUMBER & INTENSITY & CLA & SIFICATION & CODE & WAVELENGTH & WAVE NUMBER & INTENSITY & $C L$ & SIFICATION & CODE \\
\hline $\begin{array}{l}4174.96 \\
4183.07 \\
4184.75 \\
4185.39 \\
4186.662\end{array}$ & $\begin{array}{l}23945.6 \\
23899.2 \\
23889.6 \\
23885.9 \\
23878.65\end{array}$ & $\begin{array}{l}440 \\
220 \\
780 \\
200 \\
230\end{array}$ & $\begin{array}{l}\text { Pt II } \\
\text { Pt I } \\
\text { Ne II }\end{array}$ & $\begin{array}{r}106434-82535 \\
62705-38815\end{array}$ & $\begin{array}{l}\mathrm{K} \\
\mathrm{N}\end{array}$ & $\begin{array}{l}4190.14 \\
4192.4231 \\
4201.2102 \\
4205.5937\end{array}$ & $\begin{array}{l}23858.8 \\
23845.835 \\
23795.961 \\
23771.159\end{array}$ & $\begin{array}{r}300 \\
35000 \\
9200 \\
4700\end{array}$ & $\begin{array}{ll}\text { Pt } & \text { I I } \\
\text { Pt } & \text { I } \\
\text { Pt } & \text { I } \\
\text { Ne } & \text { I I }\end{array}$ & $\begin{array}{r}117340-93482 \\
13496-37342 \\
60640-36844\end{array}$ & $\begin{array}{l}K \\
E \\
E \\
G\end{array}$ \\
\hline
\end{tabular}




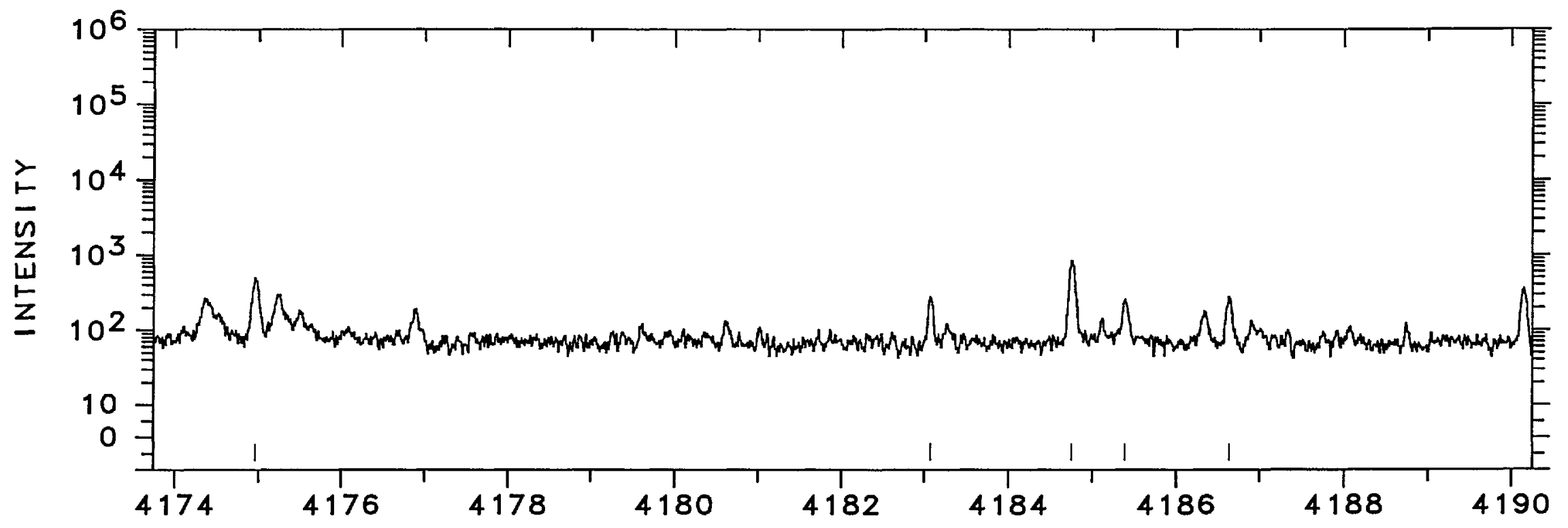

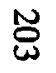

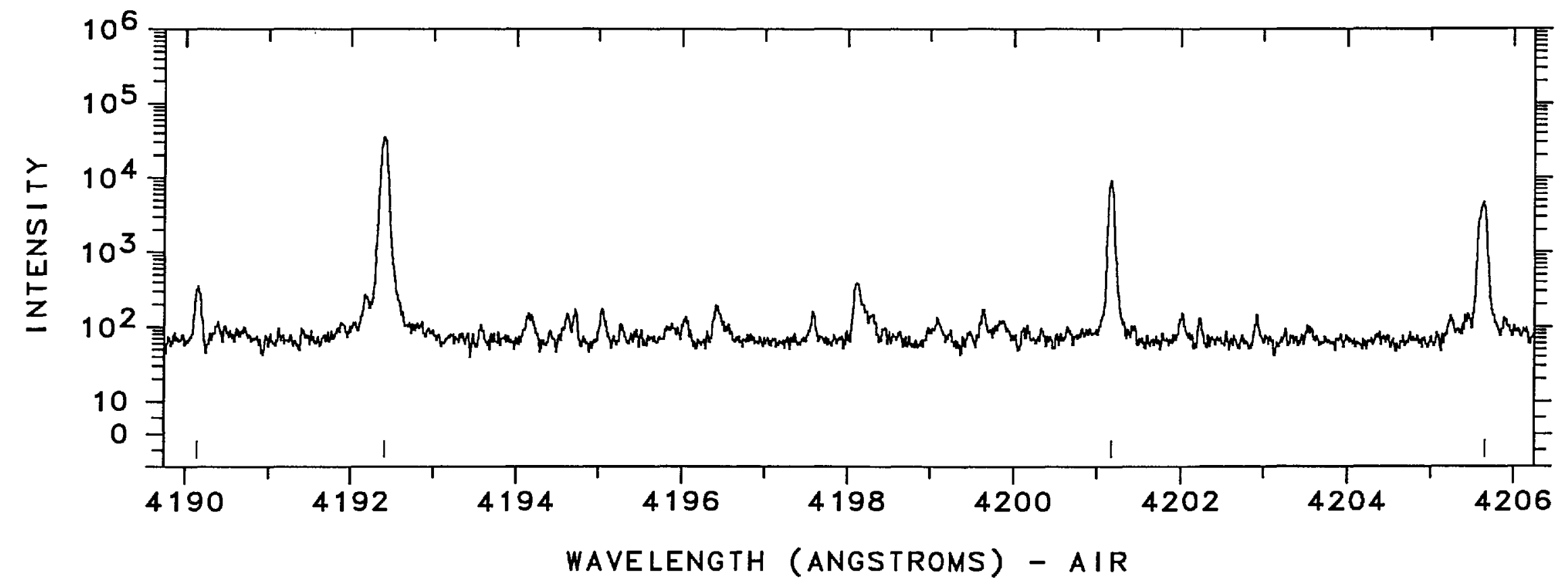




\begin{tabular}{llrllll} 
WAVELENGTH & HAVE NUMBER & INTENSITY & \multicolumn{2}{c}{ CLASSIF ICAT IDN } & CODE \\
\hline & & & & & \\
4206.5022 & 23766.025 & 3000 & Ne I I & & G \\
4208.60 & 23754.2 & 1400 & Pt II & $101517-77763$ & K \\
4213.09 & 23728.9 & 5100 & Pt I & $60573-36844$ & N \\
4213.77 & 23725.0 & 2600 & Pt I & $68169-44444$ & N \\
4215.02 & 23718.0 & 970 & Pt I & $64505-40787$ & N \\
4217.171 & 23705.90 & 26000 & Ne II & & C \\
4218.52 & 23698.3 & 1100 & Pt I & $64668-40970$ & N \\
4219.369 & 23693.55 & 7300 & Ne II & & C \\
4219.7457 & 23691.438 & 120000 & Ne II & & G \\
4220.8932 & 23684.997 & 20000 & Ne II & & G \\
4221.0827 & 23683.933 & 4500 & Ne II & & G \\
4223.43 & 23670.8 & 180 & & & & \\
4223.6790 & 23669.376 & 480 & Pt II & $32918-56587$ & A \\
4223.6790 & 23669.376 & 480 & Pt II & $54373-78043$ & AK
\end{tabular}

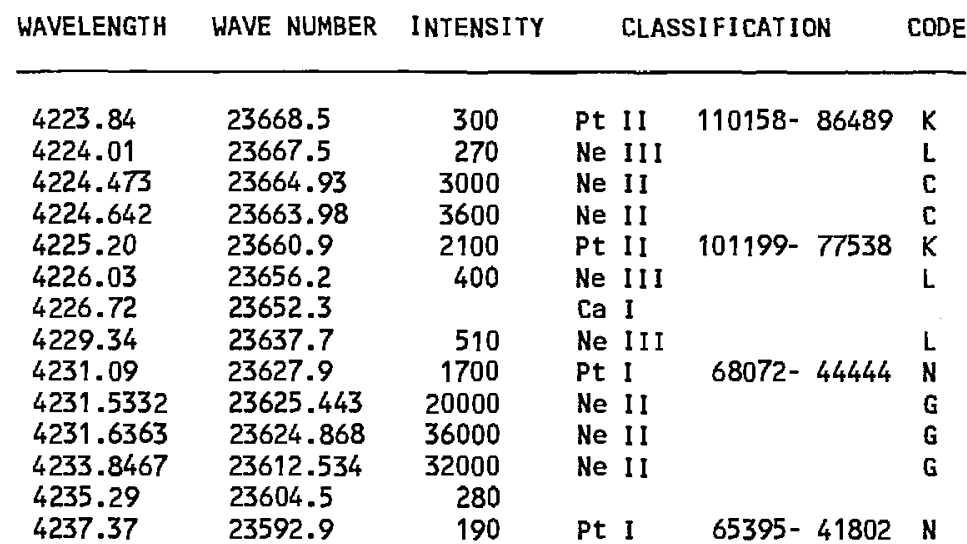




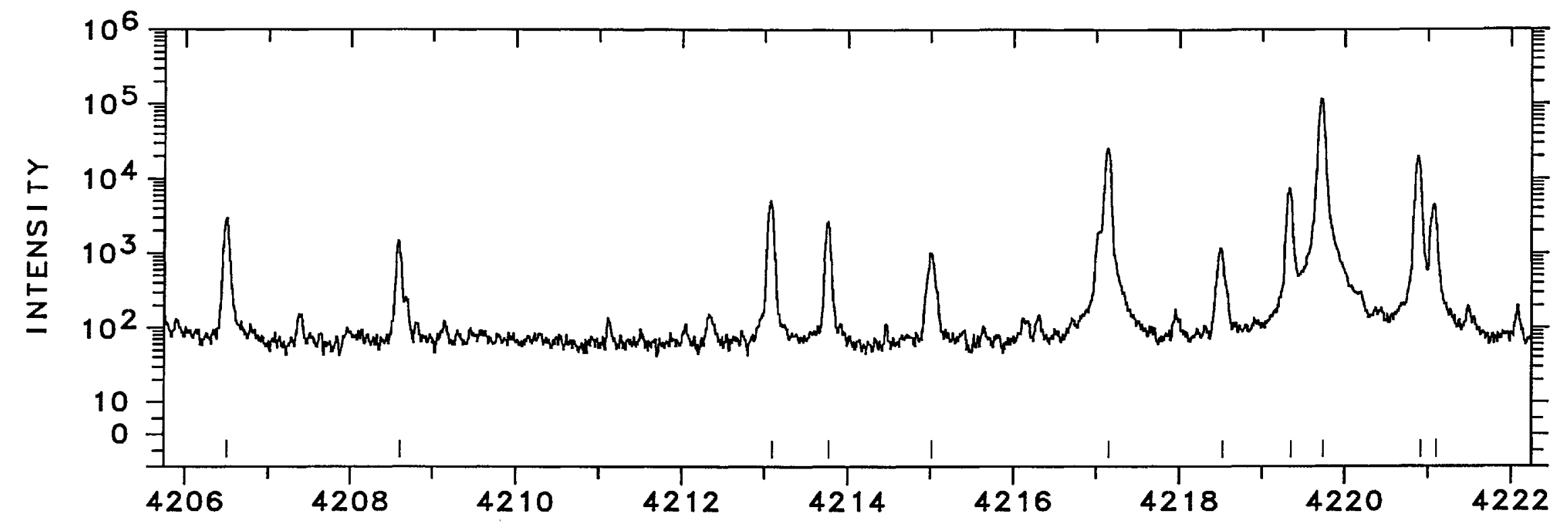

苔

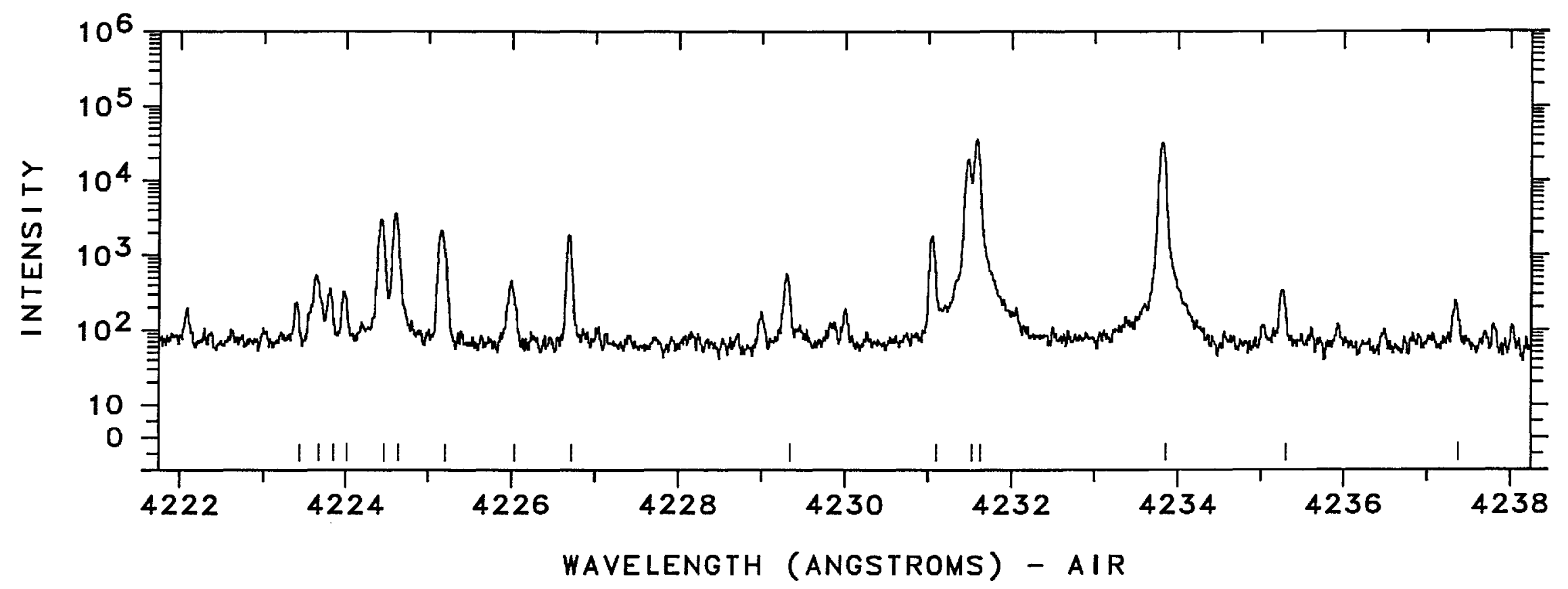




\begin{tabular}{|c|c|c|c|c|c|c|c|c|c|c|c|}
\hline WAVELENGTH & WAVE NUMBER & INTENSITY & CLA & I FICATION & CODE & WAVELENGTH & WAVE NUMBER & INTENSITY & \multicolumn{2}{|c|}{ CLASSIFICATION } & CODE \\
\hline $\begin{array}{l}4239.9190 \\
4240.1049 \\
4240.72 \\
4241.59 \\
4242.040 \\
4242.2094 \\
4242.86 \\
4244.10 \\
4244.17 \\
4245.42 \\
4245.85 \\
4245.99 \\
4246.99 \\
4247.6735 \\
4248.83 \\
4250.6462 \\
4251.17 \\
4251.36 \\
4253.87 \\
4254.32 \\
4254.51 \\
4254.59\end{array}$ & $\begin{array}{l}23578.717 \\
23577.683 \\
23574.3 \\
23569.4 \\
23566.93 \\
23565.987 \\
23562.4 \\
23555.5 \\
23555.1 \\
23548.2 \\
23545.8 \\
23545.0 \\
23539.5 \\
23535.673 \\
23529.3 \\
23519.214 \\
23516.3 \\
23515.3 \\
23501.4 \\
23498.9 \\
23497.9 \\
23497.4\end{array}$ & $\begin{array}{r}7300 \\
21000 \\
190 \\
1200 \\
2100 \\
4400 \\
180 \\
390 \\
1100 \\
200 \\
310 \\
500 \\
450 \\
3200 \\
430 \\
31000 \\
5100 \\
390 \\
210 \\
\\
250 \\
270\end{array}$ & $\begin{array}{l}\mathrm{Ne} \text { II } \\
\mathrm{Ne} \\
\mathrm{Pt}\end{array}$ & $\begin{array}{l}68006-44444 \\
42031-65587\end{array}$ & $\begin{array}{l}\mathbf{G} \\
\mathbf{G} \\
\mathbf{N} \\
\mathbf{C} \\
\mathbf{G} \\
\mathbf{N} \\
\mathrm{K}\end{array}$ & $\begin{array}{l}4256.85 \\
4257.180 \\
4257.395 \\
4257.8028 \\
4258.60 \\
4259.43 \\
4259.9310 \\
4260.99 \\
4261.09 \\
4261.91 \\
4263.02 \\
4263.24 \\
4263.5022 \\
4265.16 \\
4265.16 \\
4265.95 \\
4267.46 \\
4268.05 \\
4268.36 \\
4268.70 \\
4269.2490 \\
4269.72\end{array}$ & $\begin{array}{l}23484.9 \\
23483.12 \\
23481.93 \\
23479.683 \\
23475.3 \\
23470.7 \\
23467.953 \\
23462.1 \\
23461.6 \\
23457.1 \\
23450.9 \\
23449.7 \\
23448.296 \\
23439.2 \\
23439.2 \\
23434.8 \\
23426.5 \\
23423.3 \\
23421.6 \\
23419.7 \\
23416.733 \\
23414.1\end{array}$ & $\begin{array}{r}900 \\
1700 \\
1700 \\
19000 \\
270 \\
470 \\
3800 \\
200 \\
220 \\
250 \\
400 \\
290 \\
4600 \\
750 \\
750 \\
400 \\
440 \\
520 \\
260 \\
220 \\
1900 \\
930\end{array}$ & $\begin{array}{l}\text { Pt I } \\
\text { Ne III } \\
\text { Pt I } \\
\text { Pt II } \\
\text { Ne III }\end{array}$ & $\begin{array}{r}60790-37342 \\
68169-44730 \\
105962-82535\end{array}$ & $\begin{array}{l}E \\
K \\
L\end{array}$ \\
\hline
\end{tabular}




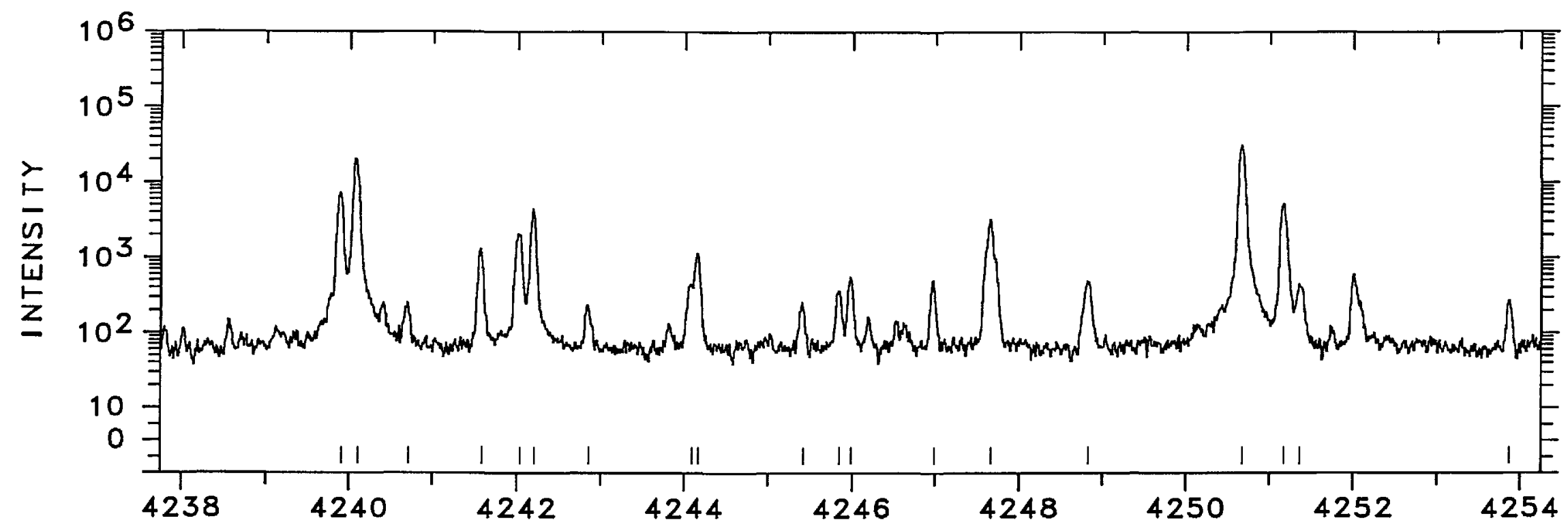

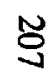

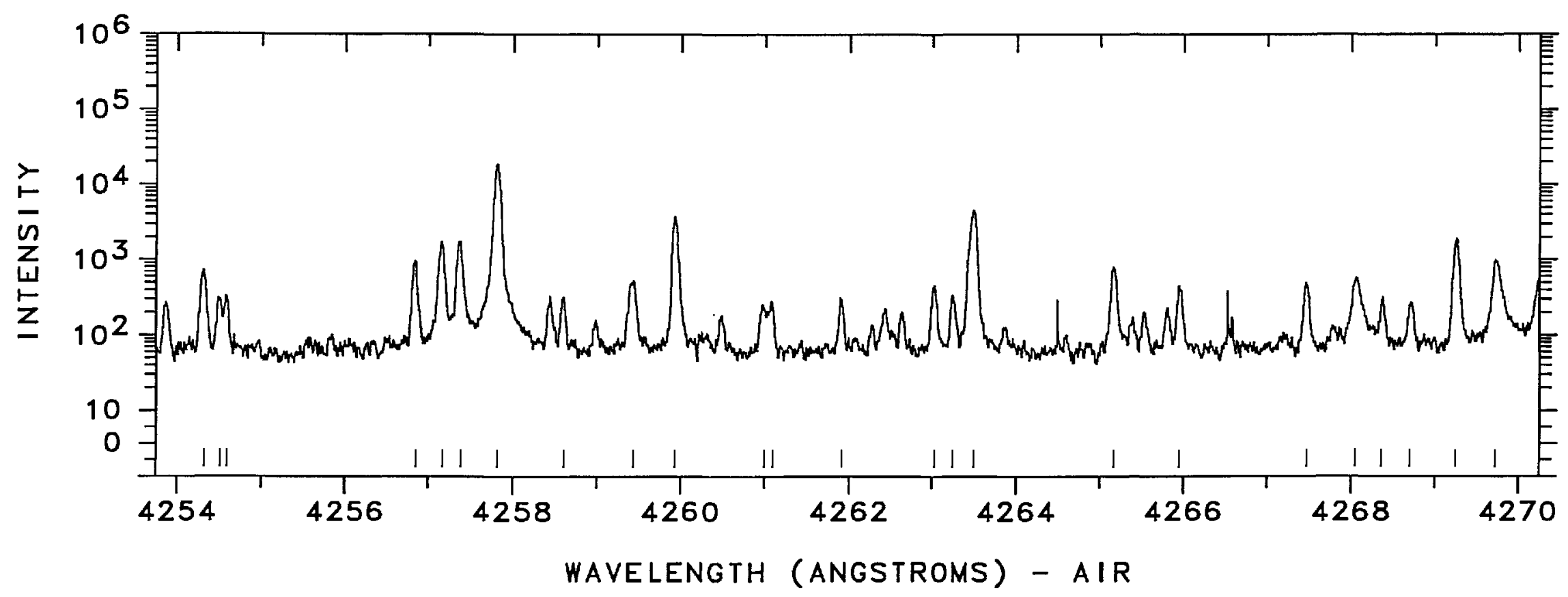




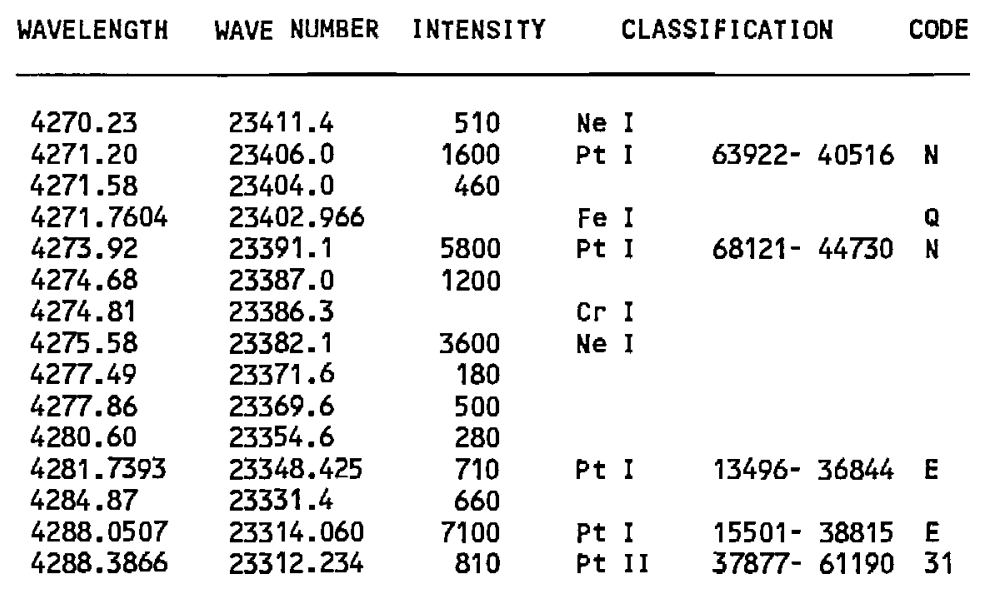

\begin{tabular}{llrlll} 
WAVELENGTH & WAVE NUMBER & INTENSITY & \multicolumn{2}{c}{ CLASSIFICATION } & CODE \\
\hline 4289.57 & 23305.8 & 170 & & & \\
4289.73 & 23304.9 & & Cr I & & \\
4290.374 & 23301.44 & 11000 & Ne I I & & C \\
4290.602 & 23300.20 & 8000 & Ne I I & & C \\
4290.8991 & 23298.584 & 4700 & Pt I & $60640-37342$ & E \\
4292.60 & 23289.4 & 410 & & & \\
4294.27 & 23280.3 & 400 & & & \\
4294.92 & 23276.8 & 670 & Pt I & $68006-44730$ & N \\
4296.86 & 23266.3 & 710 & & & \\
4297.01 & 23265.5 & 1900 & & & C \\
4298.096 & 23259.57 & 1500 & Ne II & & \\
4301.02 & 23243.8 & 290 & & & \\
4301.09 & 23243.4 & 320 & & & \\
4301.27 & 23242.4 & 400 & Pt I & $26638-49880$ & N
\end{tabular}




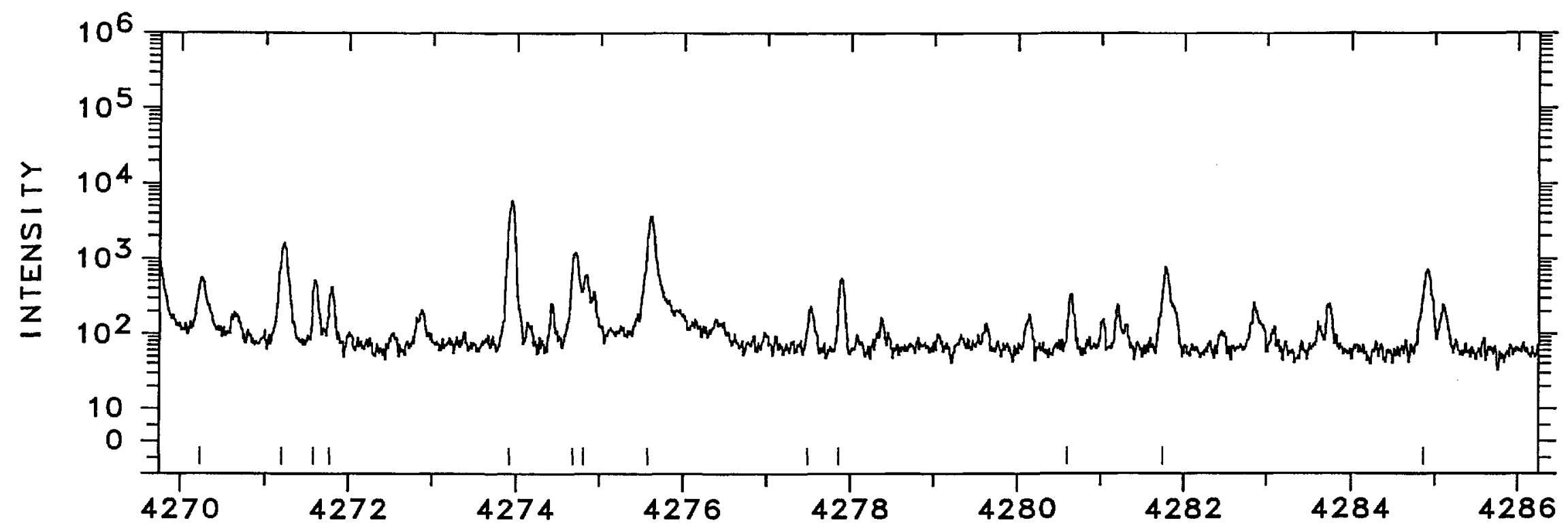

:

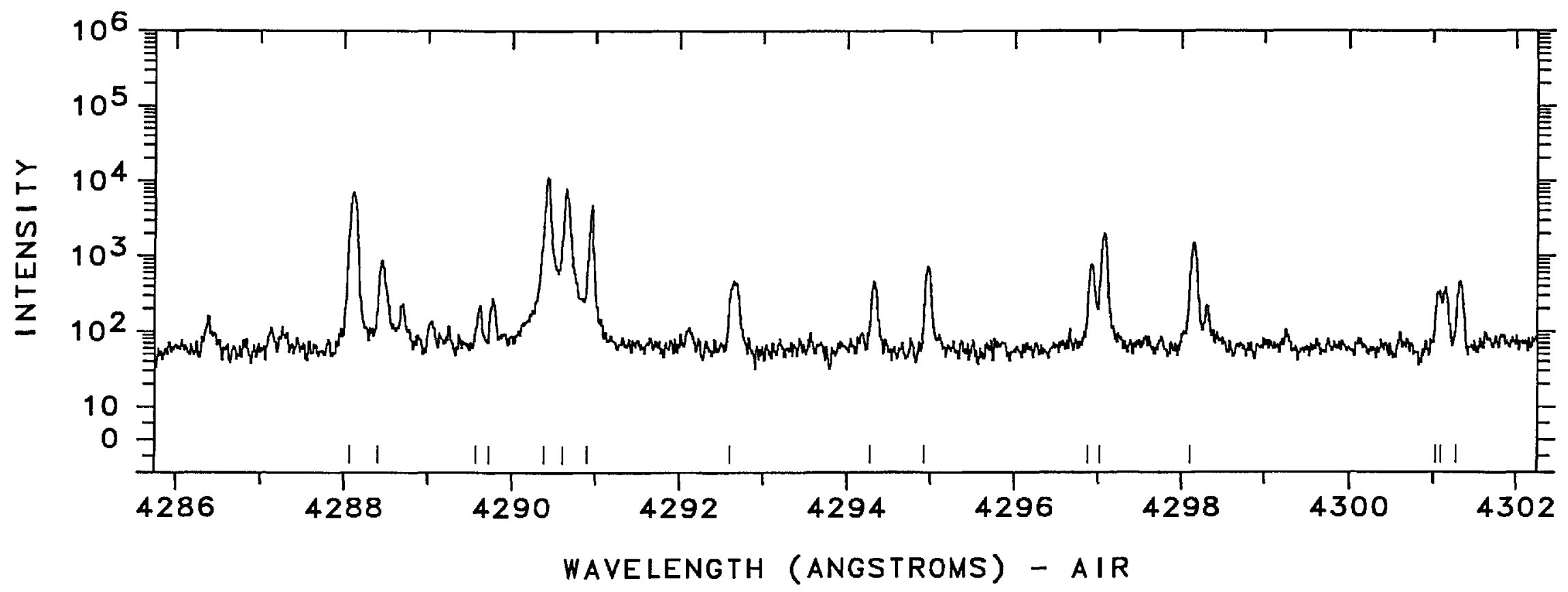




\begin{tabular}{|c|c|c|c|c|c|c|c|c|c|c|c|}
\hline WAVELENGTH & WAVE NUMBER & INTENSITY & CLA & SIFICATION & CODE & WAVELENGTH & WAVE NUMBER & INTENSITY & $\mathrm{CL} /$ & SIFICATION & CODE \\
\hline $\begin{array}{l}4302.4207 \\
4303.26 \\
4305.87 \\
4306.22 \\
4307.80 \\
4307.9021 \\
4309.1759 \\
4310.16 \\
4310.31 \\
4311.15 \\
4311.15 \\
4312.59 \\
4317.30 \\
4317.56\end{array}$ & $\begin{array}{l}23236.193 \\
23231.7 \\
23217.6 \\
23215.7 \\
23207.2 \\
23206.628 \\
23199.768 \\
23194.5 \\
23193.7 \\
23189.1 \\
23189.1 \\
23181.4 \\
23156.1 \\
23154.7\end{array}$ & $\begin{array}{r}430 \\
430 \\
160 \\
1300 \\
440 \\
\\
3600 \\
340 \\
300 \\
150 \\
150 \\
470 \\
720 \\
91\end{array}$ & $\begin{array}{l}\text { Pt I } \\
\text { Ne I } \\
\text { Pt I I } \\
\text { Fe I } \\
\text { Pt I }\end{array}$ & $\begin{array}{r}116689-93482 \\
60790-37590\end{array}$ & $\begin{array}{l}K \\
Q \\
E\end{array}$ & $\begin{array}{l}4320.10 \\
4320.33 \\
4320.59 \\
4321.33 \\
4321.74 \\
4322.3727 \\
4322.7409 \\
4324.62 \\
4324.99 \\
4325.235 \\
4325.7618 \\
4327.0533 \\
4330.74 \\
4331.08\end{array}$ & $\begin{array}{l}23141.1 \\
23139.9 \\
23138.5 \\
23134.5 \\
23132.3 \\
23128.937 \\
23126.967 \\
23116.9 \\
23114.9 \\
23113.63 \\
23110.816 \\
23103.919 \\
23084.3 \\
23082.4\end{array}$ & $\begin{array}{r}280 \\
260 \\
1100 \\
250 \\
150 \\
5800 \\
5700 \\
650 \\
560 \\
2900 \\
\\
17000 \\
70 \\
65\end{array}$ & $\begin{array}{l}\text { Pt II } \\
\text { Pt I } \\
\text { Ne II } \\
\text { Ne II } \\
\text { Pt I } \\
\text { Ne II } \\
\text { Fe I } \\
\text { Pt I } \\
\text { Pt II }\end{array}$ & $\begin{array}{r}60884-37769 \\
56784-33680 \\
106434-83352\end{array}$ & $\begin{array}{l}K \\
N \\
G \\
G \\
G \\
N \\
C \\
Q \\
E\end{array}$ \\
\hline
\end{tabular}




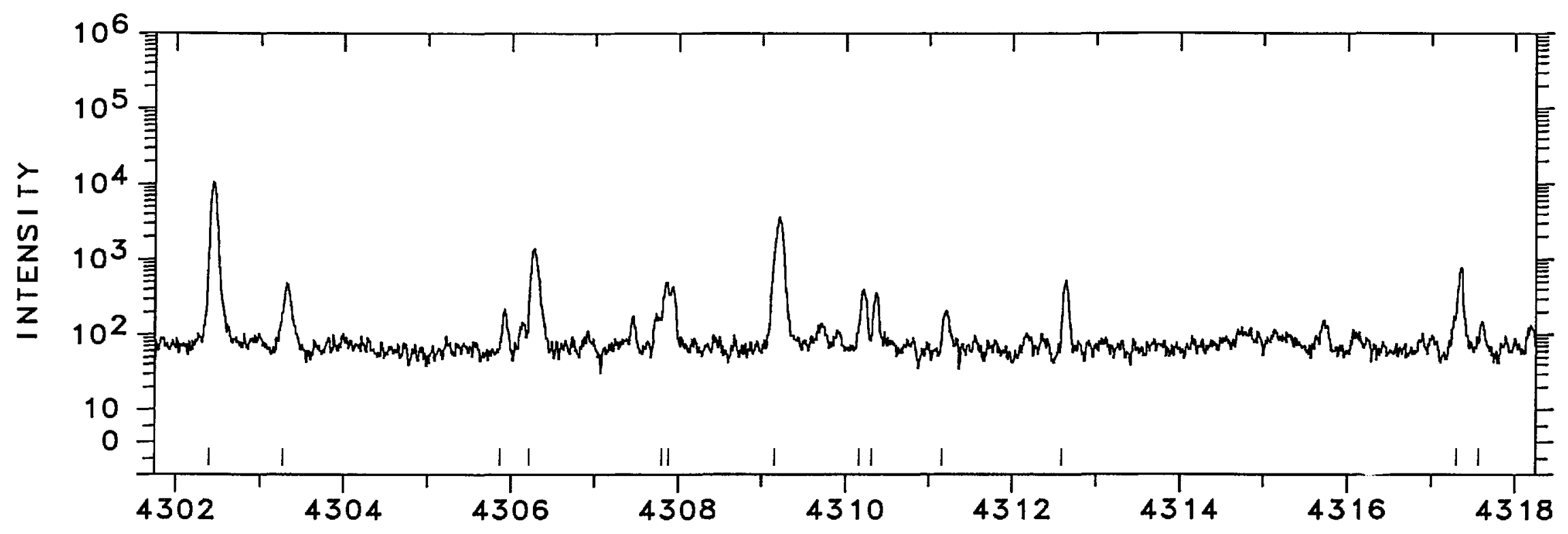

$\stackrel{N}{\Xi}$

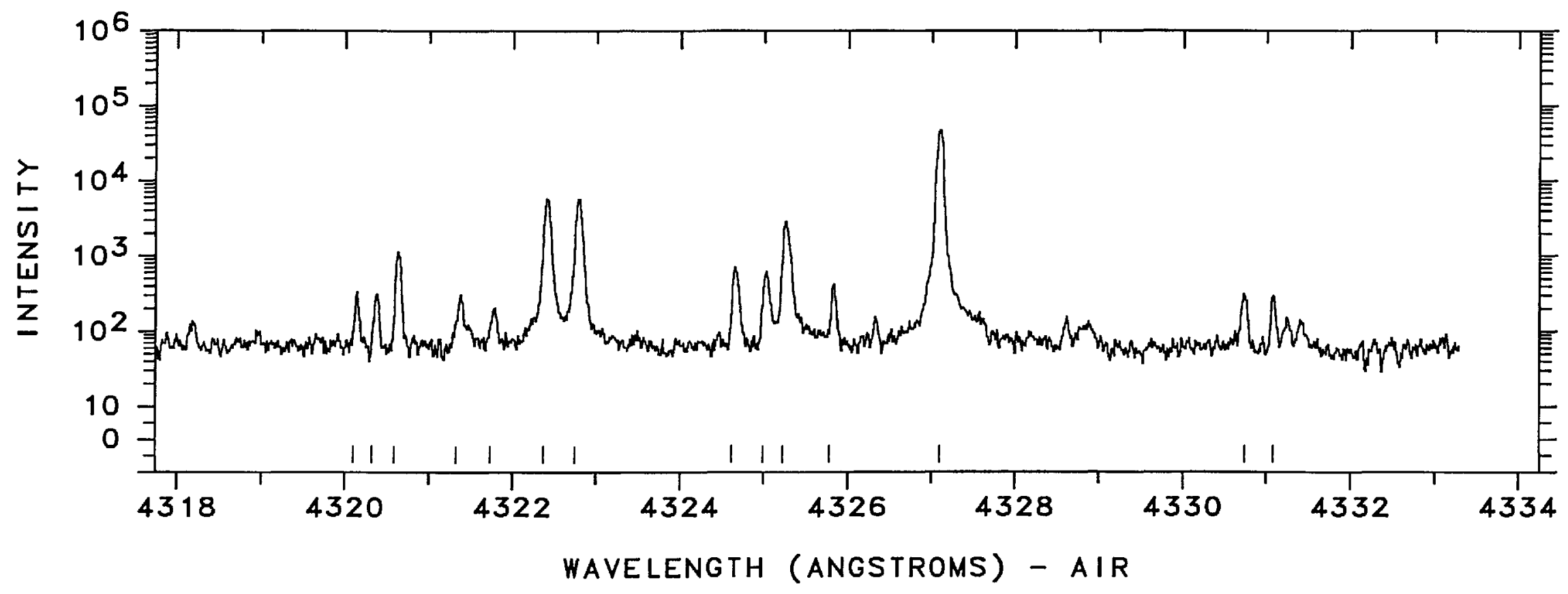

